ACTIGRAPHY SIGNAL ANALYSIS SYSTEM FOR

SLEEP AND WAKE STUDIES

by

Yashodhan Rajiv Athavale

B.Tech, VNIT, Nagpur, India, 2007

MASc., Ryerson University, Toronto, Canada, 2010

A DISSERTATION

PRESENTED TO RYERSON UNIVERSITY

IN PARTIAL FULFILLMENT OF THE REQUIREMENTS FOR THE

DEGREE OF

DOCTOR OF PHILOSOPHY

IN THE PROGRAM OF

ELECTRICAL AND COMPUTER ENGINEERING

TORONTO, ONTARIO, CANADA

OCTOBER, 2019

(c) Yashodhan Rajiv Athavale 2019 


\section{Author's Declaration}

I hereby declare that I am the sole author of this dissertation. This is a true copy of the dissertation, including any required final revisions, as accepted by my examiners.

I authorize Ryerson University to lend this dissertation to other institutions or individuals for the purpose of scholarly research.

I further authorize Ryerson University to reproduce this dissertation by photocopying or by other means, in total or in part, at the request of other institutions or individuals for the purpose of scholarly research. I understand that my dissertation may be made electronically available to the public. 


\title{
Actigraphy Signal Analysis System for Sleep and Wake Studies
}

Doctor of Philosophy, 2019

Yashodhan Rajiv Athavale

Electrical and Computer Engineering, Ryerson University

\begin{abstract}
The Internet of Things (IoT) is a trending model in the wake of recent advancements in ubiquitous sensors and smart devices, and is rapidly being deployed in communications, infrastructure, transportation and healthcare services. The Internet of Medical Things (IoMT) is a subset of the IoT and provides a layered architecture for connecting individuals with mobile devices and wearables, such that their vital physiological data can be captured and analyzed non-invasively using smart sensors embedded within these devices.

Currently available wearables have embedded sensing modules for measuring movement, direction, light and pressure. Actigraphs are one such type of wearables which exclusively employ the use of accelerometers for capturing human movement-based vibration data. The main intention of this research work is the analysis of unstructured, non-stationary actigraphy signals. This study aims to address three key objectives: (i) enabling compression and denoising of actigraphy data during acquisition; (ii) extracting regions of interest from the actigraphy data, and; (iii) deriving actigraphy specific features for improving the activity classification accuracy. These have been achieved through three key contributions, namely: (A) a signal encoding framework for data compression and denoising, (B) two novel adaptive segmentation schemes which help in extracting specific movement information from actigraphy data, and (C) two key actigraphy specific features, which quantify limb movements, and hence provide a better classification accuracy using machine learning algorithms. The outcome of this research work is a device-independent actigraphy analysis application for estimating the severity of neuromuscular diseases, identifying types of daily activity, and classify joint degeneration severity, which has been tested on five different actigraphy datasets from wake and sleep states.

Compared to traditional signal processing methods, the proposed algorithms ensured a $20-90 \%$ increase in SNR (signal-to-noise ratio), 50-80\% reduction in bit rate, $50-90 \%$ data compression, and an increase in activity recognition accuracy by over 5-20\%. Results from this systematic investigation indicate that data analysis conducted at the acquisition source, optimizes signal denoising, memory and power usage, and activity recognition, thereby promoting an edge computing approach to physiological signal analysis using wearables in a low resource environment.
\end{abstract}




\section{Acknowledgments}

I would like to express my sincere gratitude to my supervisor Dr. Sri Krishnan who has been a strong source of inspiration throughout my graduate studies. Dr. Krishnan's constant support and guidance helped me achieve my career goals and succeed in all my endeavours. I would also like to thank Dr. Mark Boulos and Dr. Brian Murray for guiding me and providing relevant materials in our ongoing collaborative research with Sunnybrook Health Sciences Center. Also, I would like to thank Ryerson University and NSERC for funding this research work and ensuring that my graduate studies are completed smoothly.

I would also like to acknowledge my supervisory committee - Dr. April Khademi, Dr. Ebrahim Bagheri and Dr. Amirnaser Yazdani for overseeing my doctoral studies and providing their valuable inputs to improve my research outcomes.

Also I would like to express my sincere thanks to all friends and colleagues in the SAR (Signal Analysis Research) lab for supporting me in every aspect during my studies. And last but not least I would like to thank everyone at Ryerson University for giving me a superb educational experience in world-class facilities. 


\section{Dedication}

I lovingly dedicate this dissertation to the love of my life Shruti Athavale, my parents Rajiv Athavale $\mathcal{E}$ Chhaya Athavale and my sister, Tanvi Athavale, as a token of humble and sincere appreciation for their invaluable love, enormous support and continuous encouragement. . . 


\section{Dedication}

To my wife Shruti and my son Aayush - Without you this journey would not be possible. . . 


\section{Table of Contents}

$\begin{array}{lll}\text { Abstract } & \text { iii }\end{array}$

Table of Contents $\quad$ viii

List of Tables $\quad x$

List of Figures $\quad$ xiv

Epigraph $\quad$ xv

1 Introduction 1

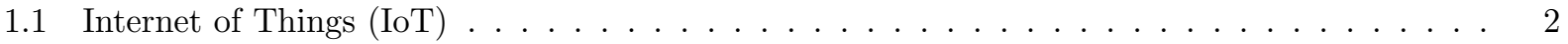

1.2 Internet of Medical Things $(\mathrm{IoMT}) \ldots \ldots \ldots \ldots \ldots \ldots$

1.3 Wearables for Health Monitoring . . . . . . . . . . . . . . . . . . . . 18

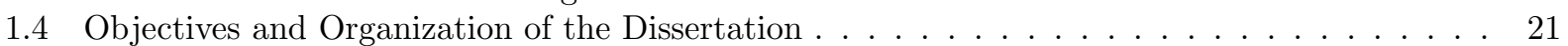

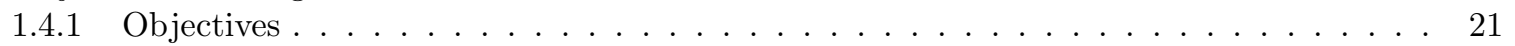

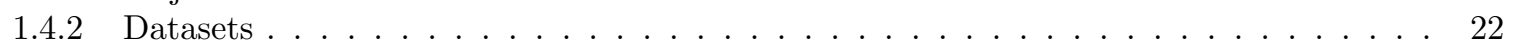

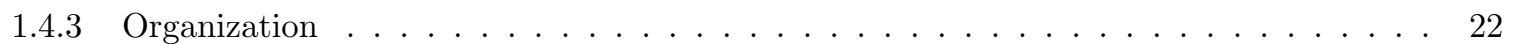

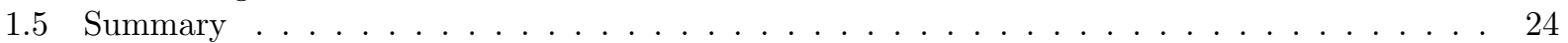

2 Understanding Actigraphy 26

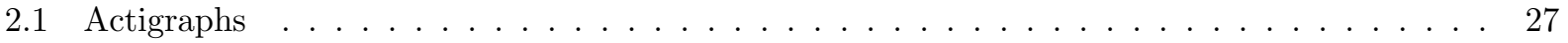

2.2 Understanding Actigraphy Data $\ldots \ldots \ldots \ldots \ldots \ldots \ldots \ldots$

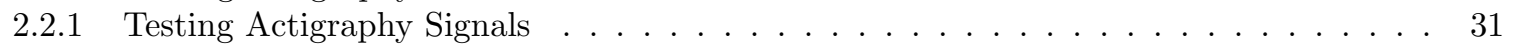

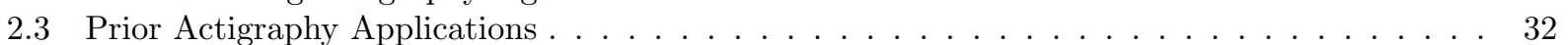

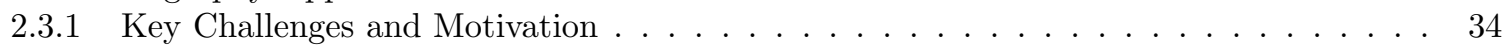

2.4 Research Contribution: Actigraphy Analysis Application . . . . . . . . . . . . . . . . . 36

2.5 Summary . . . . . . . . . . . . . . . . . . . . . . . . 39

3 Actigraphy Signal Analysis $\quad 40$

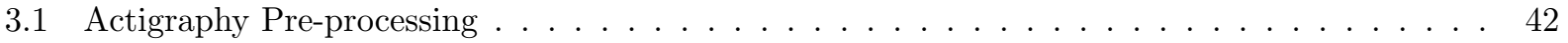

3.1.1 Known Pre-processing Methods . . . . . . . . . . . . . . . . . . . . . . 43

3.1.2 Research Contribution: Signal Encoding . . . . . . . . . . . . . . . . . . . . . . 51

3.2 Actigraphy Signal Segmentation _. . . . . . . . . . . . . . . . . . . . 58

3.2 .1 Fixed Segmentation _. . . . . . . . . . . . . . . . . . . . 60

3.2 .2 Adaptive Segmentation _. . . . . . . . . . . . . . . . . . . . 64

3.2.3 Research Contribution: The Athavale-Krishnan (AK) segmentation

algorithm . . . . . . . . . . . . . . . . . . . 66

3.2.4 Research Contribution: Mobility-based adaptive segmentation algorithm . . . . . . . . 74

3.3 Actigraphy Feature Extraction $\ldots \ldots \ldots \ldots \ldots \ldots \ldots$

3.3 .1 Time-domain Features . . . . . . . . . . . . . . . . . . . . 78

3.3 .2 Frequency-domain Features . . . . . . . . . . . . . . . . . . . 82

3.3.3 Demographic and Domain-specific Features: . . . . . . . . . . . . . . . . . . . . 83 
3.3.4 Research Contribution: Actigraphy-specific Features . . . . . . . . . . . . . . . 86

3.3.5 Research Contribution: Auto-correlation Kernel for Actigraphy Modeling . . . . . . . 88

3.3.6 Summary of modalities and features used in this research . . . . . . . . . . . . . . 94

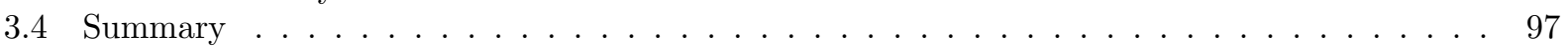

4 Sleep Actigraphy $\quad \mathbf{9 8}$

4.1 Sleep Disorders . . . . . . . . . . . . . . . . . . . . . . . . . . . . . . . . . . 99

4.1 .1 Periodic Limb Movements in Sleep (PLMS) . . . . . . . . . . . . . . . . . 101

4.2 Preliminary Experiments using Database A . . . . . . . . . . . . . . . . . . 109

4.2 .1 Materials and Methods . . . . . . . . . . . . . . . . . . . . 110

4.2 .2 Results from Preliminary Experiments . . . . . . . . . . . . . . . . . . 115

4.3 Sleep Actigraphy Analysis using Database B . . . . . . . . . . . . . . . . . . . . 117

4.3 .1 Materials and Methods . . . . . . . . . . . . . . . . . . . 118

4.3.2 Results from Experiments on Database B . . . . . . . . . . . . . . . . . . . . 127

4.4 Sleep Actigraphy Analysis for Stroke Subjects using

Database C . . . . . . . . . . . . . . . . . . . . . 150

4.4 .1 Materials and Methods . . . . . . . . . . . . . . . . . . . . 152

4.4 .2 Results from Experiments on Database C . . . . . . . . . . . . . . . 156

4.5 Observations from Experiments on Datasets A, B and C . . . . . . . . . . . 166

4.5.1 Observations from Database A experiments . . . . . . . . . . . . . 166

4.5.2 Observations from Database B experiments . . . . . . . . . . . . . . . 167

4.5 .3 Observations from Database $\mathrm{C}$ experiments $\ldots \ldots \ldots \ldots \ldots$

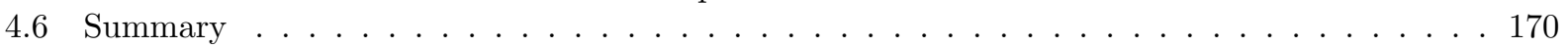

5 Wake Actigraphy $\quad 171$

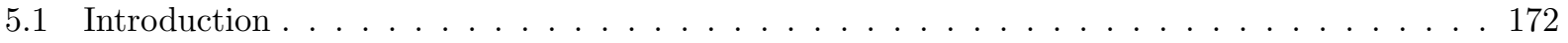

5.2 Experiments with Database D . . . . . . . . . . . . . . . . . . 174

5.2 .1 Materials and Methods . . . . . . . . . . . . . . . . . . . . 175

5.2 .2 Results from Experiments on Database D . . . . . . . . . . . . . . . . . 180

5.3 Experiments with Database E . . . . . . . . . . . . . . . . . . . . . 199

$5.3 .1 \quad$ Joint Degeneration Disorders _ . . . . . . . . . . . . . . . . . . 199

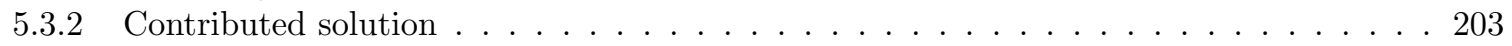

5.3 .3 Materials and Methods . . . . . . . . . . . . . . . . . . . . . 204

5.3 .4 Results from Experiments on Database E . . . . . . . . . . . . . . . . . . . . 209

5.4 Observations from Experiments on Datasets D and E . . . . . . . . . . . . . . 220

5.4 .1 Observations from Database $\mathrm{D}$ experiments $\ldots \ldots \ldots \ldots \ldots \ldots$

5.4 .2 Observations from Database E experiments . . . . . . . . . . . . . . 221

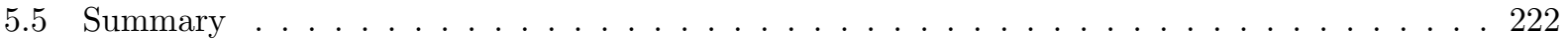

6 Discussions, Conclusions and Future Works $\quad 223$

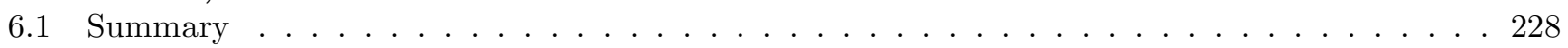

$\begin{array}{ll}\text { Bibliography } & 233\end{array}$ 


\section{List of Tables}

1.1 Datasets Used . . . . . . . . . . . . . . . . . . . . . . . . . . . 22

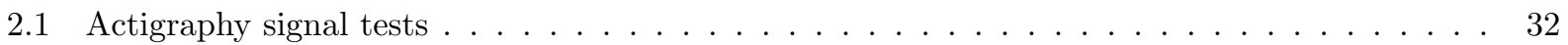

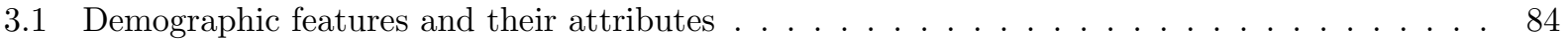

3.2 Features used in this actigraphy study $\ldots \ldots \ldots \ldots \ldots \ldots \ldots$

4.1 Neurological disorder statistics for North America $[1-4] \ldots \ldots$. . . . . . . . . . . . . . . 100

4.2 Survey of prior studies in actigraphy for PLM estimation . . . . . . . . . . . . . 108

4.3 Demographics and PSG parameters - Actical Data, Database A [5] . . . . . . . . . . . . 111

4.4 Features extracted from Actigraphy Signals in Database A . . . . . . . . . . . . . . . . . 114

4.5 PLM Classification results using Database A . . . . . . . . . . . . . . . . . 116

4.6 Demographics and PSG parameters - Tri-Axial X16 ${ }^{\mathrm{TM}}$ Data, Database B [6] . . . . . . 121

4.7 Features captured from Groups $\operatorname{Tr}_{F}$ and $\operatorname{Tr}_{E}$ signals $\ldots \ldots \ldots \ldots$. . . . . . . . . 125

4.8 Parametric comparison between raw, filtered and encoded signals . . . . . . . . . . . 128

4.9 Fixed, AK and Mobility-based segments for sample actigraphy . . . . . . . . . . . . 132

4.10 Classification of PLMs using 14 Features . . . . . . . . . . . . . . . . . . . . . . 135

4.11 Classification of PLMs using 23 features . . . . . . . . . . . . . . . . 136

4.12 Classification of PLMs using top 5 mRMR features for encoded signals . . . . . . . . . . . 138

4.13 Classification of PLMs using 22 features, excluding periodicity index . . . . . . . . . . . 139

4.14 Classification of PLMs using 10 proposed features . . . . . . . . . . . . . . . . . 140

4.15 Classification of PLMs using 9 proposed features . . . . . . . . . . . . . . . . . . 141

4.16 Classification of PLMs based on Gender using 24 features - Left Leg . . . . . . . . . . . . . 144

4.17 Classification of PLMs based on Gender using 24 features - Right Leg . . . . . . . . . . . 145

4.18 Classification of PLMs using 24 features including Age - Left Leg . . . . . . . . . . . . 147

4.19 Classification of PLMs using 24 features including Age - Right Leg . . . . . . . . . . . 148

4.20 Demographics - Single Axial Actical ${ }^{\mathrm{TM}}$ Data, Database C . . . . . . . . . . . . . . 152

4.21 Features extracted from Groups $\operatorname{Str}_{R}$ and $\operatorname{Str}_{E}$ actigraphy signals . . . . . . . . . 156

4.22 Parametric comparison between raw and encoded stroke actigraphy signals . . . . . . . 157

4.23 Fixed, AK and Mobility-based segments for sample actigraphy . . . . . . . . . . . . . 161

4.24 Classification of Stroke subjects using 22 features . . . . . . . . . . . . . . . . . . 161

4.25 Classification of Stroke Subjects using top $5 \mathrm{mRMR}$ features for encoded signals . . . . . 163

4.26 Classification of Stroke subjects using 15 features . . . . . . . . . . . . . . . . . . 164

4.27 Classification of Stroke subjects using 9 features $\ldots \ldots \ldots \ldots$

5.1 Demographics - ADL Data, Database D . . . . . . . . . . . . . . . . . . . 175

5.2 ADL Dataset with Class labels for classification $[7,8] \ldots \ldots \ldots \ldots \ldots \ldots$

5.3 Features extracted from Groups $A D L_{R}$ and $A D L_{E}$ activity signals $\ldots \ldots \ldots \ldots$

5.4 Parametric comparison between raw and encoded ADL signals . . . . . . . . . . . . 181

5.5 No. of Segments between AK and Mobility algorithms on ADL data . . . . . . . . . . 190

5.6 Activity Recognition using 22 features . . . . . . . . . . . . . . . . . . . . 192

5.7 Activity Recognition using top 5 mRMR features for encoded signals . . . . . . . . . . . 194

5.8 Activity Recognition using 9 features . . . . . . . . . . . . . . . . . . . . . 195

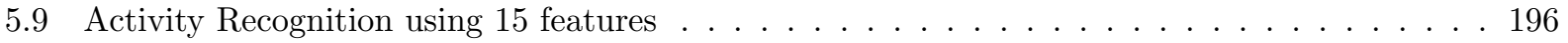


5.10 Demographics - VAG Data, Database E . . . . . . . . . . . . . . . . . . . 204

5.11 Features extracted from Groups $V A G_{R}$ and $V A G_{E}$ VAG signals . . . . . . . . . . . . . . . . 209

5.12 Parametric comparison between raw and encoded VAG signals . . . . . . . . . . . . . . . . . 210

5.13 Fixed, AK and Mobility-based segments for sample VAG signals . . . . . . . . . . . . . . . 213

5.14 Classification of VAG data using 21 features . . . . . . . . . . . . . . . . . . . . . . 213

5.15 Classification of VAG Data using top 5 mRMR features for encoded signals . . . . . . . . . 215

5.16 Classification of VAG data using 9 features . . . . . . . . . . . . . . . . . . . 216

5.17 Classification of VAG data using 14 features . . . . . . . . . . . . . . . . . . . 217 


\section{List of Figures}

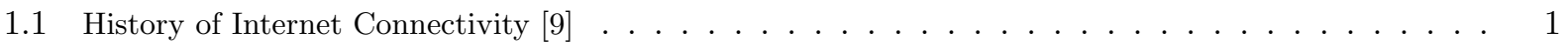

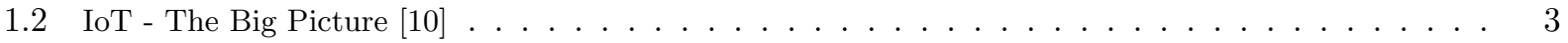

1.3 IoT Architecture $[9][11] \ldots \ldots \ldots \ldots . \ldots \ldots$

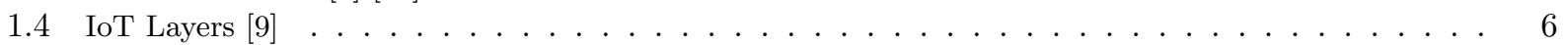

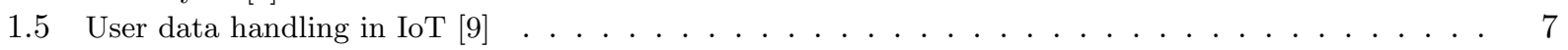

1.6 Opportunities in IoT $[11][12] \ldots \ldots \ldots \ldots$

1.7 IoMT services $[13] \ldots \ldots \ldots \ldots \ldots$

1.8 IoMT framework [13] . . . . . . . . . . . . . . . . . . . . . . . . . . . . . 12

1.9 Remote health monitoring via IoMT $[13]$. . . . . . . . . . . . . . . . . . . . . . 13

1.10 Use of smart phone applications [13] . . . . . . . . . . . . . . . . . . . . . . . . 13

1.11 Hardware aspects of IoMT [13] $[14]$. . . . . . . . . . . . . . . . . . . . . . . . . . . . . . 14

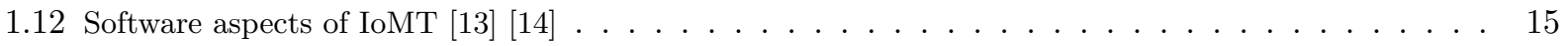

1.13 Networking and Computing aspects of IoMT $[13] \ldots \ldots$. . . . . . . . . . . . . . 16

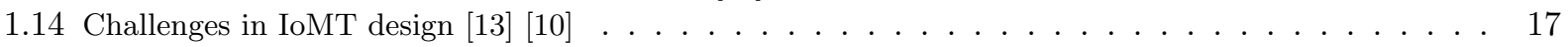

1.15 The Connected Human $[15]$. . . . . . . . . . . . . . . . . . . . . . . . . . . . . . 19

1.16 Organization of Dissertation . . . . . . . . . . . . . . . . . . . . . . . 23

1.17 Key Contributions of this research . . . . . . . . . . . . . . . . . . . . . . . 23

2.1 Organization of Dissertation . . . . . . . . . . . . . . . . . . . . . 26

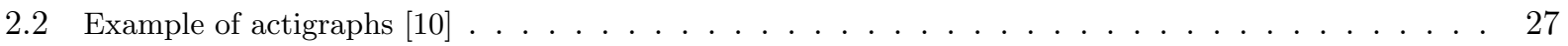

2.3 Actigraph circuitry . . . . . . . . . . . . . . . . . . . . . . . . . . . . 28

2.4 Actigraph recording . . . . . . . . . . . . . . . . . . . . . . . . . . . 29

2.5 (A) Tri-axial [16], and (B) Single-axial actigraphy [17] signals from two different actigraphs . . . . 30

2.620 -second segment of a single-axial actigraphy signal . . . . . . . . . . . . . . . . . . 30

2.7 Flowchart of the Actigraphy Analysis System . . . . . . . . . . . . . . . . . . . . . 37

3.1 Organization of Dissertation . . . . . . . . . . . . . . . . . . . . . . . 40

3.2 Actigraphy Signal Normalization . . . . . . . . . . . . . . . . . . . . . . . 45

3.3 Actigraphy signal detrending . . . . . . . . . . . . . . . . . . . . . . . . 46

3.4 Spectral plots of detrended and low pass filtered actigraphy signals . . . . . . . . . . . . . . . . 47

3.5 Comparison of filtered actigraphy signal with its detrended version . . . . . . . . . . . . . . . . . 47

3.6 Haar mother wavelet (left), and corresponding scaling function (right) $[18,19]$. . . . . . . . . . . . 48

3.7 Variations in Haar transform with respect to scale, phase and timeshift $[18,19]$. . . . . . . . . . . . 49

3.8 Haar wavelet denoising of actigraphy signals . . . . . . . . . . . . . . . . . . . . . . . 50

3.9 (A) Tri-axial signal [16], and (B) its Vector Compounded version . . . . . . . . . . . . . . . . . 51

3.10 Pre-processing steps on a sample tri-axial sleep actigraphy signal . . . . . . . . . . . . . . . . . 51

3.11 Signal Quantization and Encoding using PCM-DM . . . . . . . . . . . . . . . . . 53

3.12 Signal Encoding Scheme $[10,20]$. . . . . . . . . . . . . . . . . . . . . . . . . . 54

3.13 Application of 3-bit encoding to a small patch of sleep actigraphy signal . . . . . . . . . . . . . 56

3.14 Application of the encoding scheme to actigraphy . . . . . . . . . . . . . . . . . . 56

3.15 Example of 3-bit encoding of actigraphy data . . . . . . . . . . . . . . . . . . . . . 57

3.16 Pre-processing of a sample long-duration tri-axial sleep actigraphy signal . . . . . . . . . . . . . . 57

3.17 Pre-processing of a subset of the signal used in Figure 3.16 . . . . . . . . . . . . . . . . . 58 
3.18 Window function variation in an STFT plane . . . . . . . . . . . . . . . . . . . . . 62

3.19 Example of morphological thresholding . . . . . . . . . . . . . . . . . . . . . . . . . 62

3.20 Peak detection based fixed segmentation . . . . . . . . . . . . . . . . . . . . . 63

3.21 Zero-crossings based fixed segmentation . . . . . . . . . . . . . . . . . . . . 63

3.22 Flowchart of AK Algorithm . . . . . . . . . . . . . . . . . . . . . . . . . . . . . 67

3.23 Actigraphy Segmentation using AK Algorithm . . . . . . . . . . . . . . . . . . . . . . 68

3.24 Pseudocode for AK Segmentation Algorithm . . . . . . . . . . . . . . . . . . . . . . . . . . . . . 72

3.25 Segmenting a synthetic signal using AK Algorithm . . . . . . . . . . . . . . . . . . . . . . 73

3.26 Testing AK Algorithm using a synthetic signal . . . . . . . . . . . . . . . . . . . . . . . . . 74

3.27 Pseudocode for Mobility Segmentation Algorithm . . . . . . . . . . . . . . . . . . . . . . 76

3.28 Testing Mobility-based segmentation on a synthetic signal . . . . . . . . . . . . . . . . . . 77

3.29 Periodicity Index as a classification feature $[6]$. . . . . . . . . . . . . . . . . . . 85

3.30 Computation of RCF . . . . . . . . . . . . . . . . . . . . . . . . . . . 87

3.31 Thresholding Actigraphy Data using RCF . . . . . . . . . . . . . . . . . . . . . 88

3.32 Dimension transformation using kernel methods $[21]$. . . . . . . . . . . . . . . . . . . . . . 89

3.33 Overall Flowchart of Actigraphy Analysis Application . . . . . . . . . . . . . . . . . . . . 96

4.1 Organization of Dissertation . . . . . . . . . . . . . . . . . . . . . . . 98

4.2 PSG Setup in a sleep clinic $[22]$. . . . . . . . . . . . . . . . . . . . . . . . 100

4.3 A Patient experiencing PLMs in lower left limb [23] . . . . . . . . . . . . . . . . 103

4.4 Sample PSG Signal taken from lower left leg . . . . . . . . . . . . . . . . . . . . . . . 104

4.5 A Patient experiencing PLMs in lower left limb [23] . . . . . . . . . . . . . . . . . . . 110

4.6 Actigraph Data Acquisition Setup . . . . . . . . . . . . . . . . . . . . . . . . . 111

4.7 Visual correlation between PSG and Actigraphy . . . . . . . . . . . . . . . . . . . . . . . 112

4.8 Algorithm for preliminary studies . . . . . . . . . . . . . . . . . . . . . . . 113

4.9 Plots of PIs for normal and abnormal patients . . . . . . . . . . . . . . . . . . . . . 115

4.10 ROC Curve for Group 1 Classification . . . . . . . . . . . . . . . . . . . . . . . . 116

4.11 ROC Curve for Group 2 Classification . . . . . . . . . . . . . . . . . . . . . . . . . . . 117

4.12 Sleep Actigraphy Analysis System . . . . . . . . . . . . . . . . . . . . . . . . . . 118

$4.13 \mathrm{CSV}$ format data from X16-mini ${ }^{\mathrm{TM}}$ Actigraph . . . . . . . . . . . . . . . . . . . . . . 119

4.14 Model X16-mini ${ }^{\mathrm{TM}}$ Actigraph . . . . . . . . . . . . . . . . . . . . . . . . . . 119

4.15 Sample Tri-axial actigraphy signal from a healthy subject's left leg . . . . . . . . . . . . . . 120

4.16 Low-pass filtering of tri-axial sleep actigraphy . . . . . . . . . . . . . . . . . . . . . . 127

4.17 3-bit Encoding of tri-axial sleep actigraphy . . . . . . . . . . . . . . . . . . . . . . 128

4.18 3-bit Encoding of tri-axial sleep actigraphy . . . . . . . . . . . . . . . . . . . . . . . . 129

4.19 AK Segmentation of left actigraphy signal in sleep . . . . . . . . . . . . . . . . . . . . 130

4.20 AK Segmentation of right actigraphy signal in sleep . . . . . . . . . . . . . . . . . . . . 130

4.21 Mobility-based Segmentation of left actigraphy signal in sleep . . . . . . . . . . . . . . . . . 131

4.22 Mobility-based Segmentation of right actigraphy signal in sleep . . . . . . . . . . . . . . . . . . . . 131

4.23 Left Leg PLM Index v/s No. of Segments . . . . . . . . . . . . . . . . . . . . . . . . . . . 132

4.24 Right Leg PLM Index v/s No. of Segments . . . . . . . . . . . . . . . . . . . . . . . . . 132

4.25 Combined PLM Index v/s No. of Segments . . . . . . . . . . . . . . . . . . . . . . . . 133

4.26 Left Leg Actigraphy segregation of patients using sleep efficiency . . . . . . . . . . . . . . . . . . 133

4.27 Right Leg Actigraphy segregation of patients using sleep efficiency . . . . . . . . . . . . . . . . . 134

4.28 ROC Curves for Group Tri $i_{F}$ in Table 4.10: (A) Naive-Bayes and LDA, (B) SVM . . . . . . . . . . 135

4.29 ROC Curves for Group Tri $i_{E}$ in Table 4.10: (A) Naive-Bayes and LDA, (B) SVM . . . . . . . . . 136

4.30 ROC Curves for Group Tri $i_{F}$ in Table 4.11: (A) Naive-Bayes and LDA, (B) SVM . . . . . . . . . . 137

4.31 ROC Curves for Group Tri $i_{E}$ in Table 4.11: (A) Naive-Bayes and LDA, (B) SVM . . . . . . . . . . 137

4.32 Top 3 Features Classifying PLMs . . . . . . . . . . . . . . . . . . . . . . . . . . . . 138

4.33 ROC Curves for Group Tri $i_{F}$ in Table 4.13: (A) Naive-Bayes and LDA, (B) SVM . . . . . . . . . . 139

4.34 ROC Curves for Group Tri $i_{E}$ in Table 4.13: (A) Naive-Bayes and LDA, (B) SVM . . . . . . . . . . 139

4.35 ROC Curves for Group Tri $i_{F}$ in Table 4.14: (A) Naive-Bayes and LDA, (B) SVM . . . . . . . . . . 140

4.36 ROC Curves for Group Tri $i_{E}$ in Table 4.14: (A) Naive-Bayes and LDA, (B) SVM . . . . . . . . . 140

4.37 ROC Curves for Group $\operatorname{Tr}_{F}$ in Table 4.15: (A) Naive-Bayes and LDA, (B) SVM . . . . . . . . . . 141 
4.38 ROC Curves for Group Tri $i_{E}$ in Table 4.15: (A) Naive-Bayes and LDA, (B) SVM . . . . . . . . . 142

4.39 Visualizing PI for Normal and Abnormal Subjects . . . . . . . . . . . . . . . . . . . . . . . . . . 142

4.40 Classification Accuracy vs Encoding Levels . . . . . . . . . . . . . . . . . . . . . . . . . . . . . . 143

4.41 ROC Curves for Group Tri $T_{F}$ in Table 4.16: (A) LDA, (B) SVM . . . . . . . . . . . . . . . . . . . 144

4.42 ROC Curves for Group Tri $i_{E}$ in Table 4.16: (A) LDA, (B) SVM . . . . . . . . . . . . . . . . . . . 145

4.43 ROC Curves for Group $T r i_{F}$ in Table 4.17: (A) LDA, (B) SVM . . . . . . . . . . . . . . . . . . 146

4.44 ROC Curves for Group $T_{r} i_{E}$ in Table 4.17: (A) LDA, (B) SVM . . . . . . . . . . . . . . . . . . . 146

4.45 ROC Curves for Group Tri $T_{F}$ in Table 4.18: (A) LDA, (B) SVM . . . . . . . . . . . . . . . . . . . 147

4.46 ROC Curves for Group Tri $i_{E}$ in Table 4.18: (A) LDA, (B) SVM . . . . . . . . . . . . . . . . . . . 148

4.47 ROC Curves for Group $T r i_{F}$ in Table 4.19: (A) LDA, (B) SVM . . . . . . . . . . . . . . . . . . . 149

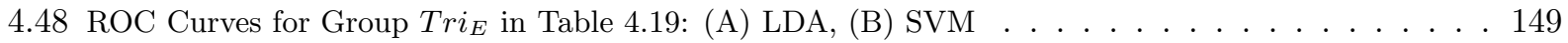

4.49 Ischemic Stroke $[24]$. . . . . . . . . . . . . . . . . . . . . . . . . 150

4.50 Hemorrhagic Stroke $[24]$. . . . . . . . . . . . . . . . . . . . . . . . 151

4.51 Transient Ischemic Attack [24] . . . . . . . . . . . . . . . . . . . . . . . . . . . . . 151

4.52 Raw and Encoded sleep actigraphy signal from stroke recovery subject . . . . . . . . . . . . . . . 157

4.53 3-bit encoding of stroke recovery actigraphy . . . . . . . . . . . . . . . . . . . . . . 158

4.54 AK Segmentation of Left Leg Stroke Actigraphy . . . . . . . . . . . . . . . . . . . . . . . . . . 159

4.55 AK Segmentation of Right Leg Stroke Actigraphy . . . . . . . . . . . . . . . . . . . . . . . . . 159

4.56 Mobility-based Segmentation of Left Leg Stroke Actigraphy . . . . . . . . . . . . . . . . . . . . . 160

4.57 Mobility-based Segmentation of Right Leg Stroke Actigraphy . . . . . . . . . . . . . . . . . . . . . 160

4.58 ROC Curves for Group $\operatorname{Str}_{R}$ in Table 4.24: (A) Naive-Bayes \& LDA, (B) SVM . . . . . . . . . . . 162

4.59 ROC Curves for Group $\operatorname{Str}_{E}$ in Table 4.24: (A) Naive-Bayes \& LDA, (B) SVM . . . . . . . . . . 162

4.60 Top 3 Features Classifying Stroke Subjects . . . . . . . . . . . . . . . . . . . . . . . . 163

4.61 ROC Curves for Group $\operatorname{Str}_{R}$ in Table 4.26: (A) Naive-Bayes \& LDA, (B) SVM . . . . . . . . . . . 164

4.62 ROC Curves for Group $\operatorname{Str}_{E}$ in Table 4.26: (A) Naive-Bayes \& LDA, (B) SVM . . . . . . . . . . . 164

4.63 ROC Curves for Group $\operatorname{Str}_{R}$ in Table 4.27: (A) Naive-Bayes \& LDA, (B) SVM . . . . . . . . . . 165

4.64 ROC Curves for Group $\operatorname{Str}_{E}$ in Table 4.27: (A) Naive-Bayes \& LDA, (B) SVM . . . . . . . . . . 165

4.65 Classification Accuracy vs Encoding Levels . . . . . . . . . . . . . . . . . . . . . 166

5.1 Organization of Dissertation . . . . . . . . . . . . . . . . . . . . . 171

5.2 Proposed Daily Activity Analysis System . . . . . . . . . . . . . . . . . . . . . . . . 174

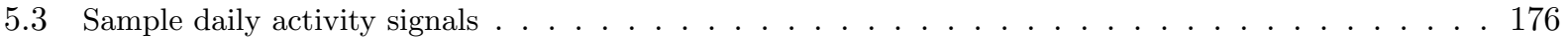

5.4 Example of 3-bit encoding of ADL signals . . . . . . . . . . . . . . . . . . . . . . . . 181

5.53 -bit encoding of ADL actigraphy . . . . . . . . . . . . . . . . . . . . . . . . . . 182

5.6 Adaptive Segmentation: Brushing Teeth . . . . . . . . . . . . . . . . . . . . . . 183

5.7 Adaptive Segmentation: Climbing Stairs . . . . . . . . . . . . . . . . . . . . . . . . . 183

5.8 Adaptive Segmentation: Combing Hair . . . . . . . . . . . . . . . . . . . . . . . . . . . . . . . 184

5.9 Adaptive Segmentation: Descending Stairs . . . . . . . . . . . . . . . . . . . . . . . . . . . 184

5.10 Adaptive Segmentation: Drink from Glass . . . . . . . . . . . . . . . . . . . . . . . . . . . 185

5.11 Adaptive Segmentation: Eating Meat . . . . . . . . . . . . . . . . . . . . . . . . . . . 185

5.12 Adaptive Segmentation: Eating Soup . . . . . . . . . . . . . . . . . . . . . 186

5.13 Adaptive Segmentation: Get up from bed . . . . . . . . . . . . . . . . . . . . . . . . 186

5.14 Adaptive Segmentation: Lie Down on bed . . . . . . . . . . . . . . . . . . . . . . . . 187

5.15 Adaptive Segmentation: Pouring water . . . . . . . . . . . . . . . . . . . . 187

5.16 Adaptive Segmentation: Sit Down . . . . . . . . . . . . . . . . . . . . . . . . 188

5.17 Adaptive Segmentation: Stand up . . . . . . . . . . . . . . . . . . . . . . . . 188

5.18 Adaptive Segmentation: Telephone Talk . . . . . . . . . . . . . . . . . . . . . . . . . . 189

5.19 Adaptive Segmentation: Walking . . . . . . . . . . . . . . . . . . . . . . . . . . 189

5.20 AK Algorithm applied to a synthetic signal of 11 concatenated activities . . . . . . . . . . . . . . . 191

5.21 Mobility-based Algorithm applied to a synthetic signal of 14 concatenated activities - (A) Ground truth signal, (B) Segmented signal . . . . . . . . . . . . . . . . . . . . . . . . . 191

5.22 ROC Curves for Group $A D L_{R}$ in Table 5.6: (A) ROC for LDA, (B) Confusion Matrix for LDA, (C) ROC for SVM and (D) Confusion Matrix for SVM . . . . . . . . . . . . . . . . . . 193 
5.23 ROC Curves for Group $A D L_{E}$ in Table 5.6: (A) ROC for LDA, (B) Confusion Matrix for LDA, (C) ROC for SVM and (D) Confusion Matrix for SVM . . . . . . . . . . . . . . . . . . . . . . . 194

5.24 ROC Curves for Group $A D L_{R}$ in Table 5.8: (A) ROC for LDA, (B) Confusion Matrix for LDA, (C) ROC for SVM and (D) Confusion Matrix for SVM . . . . . . . . . . . . . . . . . . . . 195

5.25 ROC Curves for Group $A D L_{E}$ in Table 5.8: (A) ROC for LDA, (B) Confusion Matrix for LDA, (C) ROC for SVM and (D) Confusion Matrix for SVM . . . . . . . . . . . . . . . . . . . . 196

5.26 ROC Curves for Group $A D L_{R}$ in Table 5.9: (A) ROC for LDA, (B) Confusion Matrix for LDA, (C) ROC for SVM and (D) Confusion Matrix for SVM . . . . . . . . . . . . . . . . . . . . . 197

5.27 ROC Curves for Group $A D L_{E}$ in Table 5.9: (A) ROC for LDA, (B) Confusion Matrix for LDA, (C)

ROC for SVM and (D) Confusion Matrix for SVM . . . . . . . . . . . . . . . . . . . . . 198

5.28 Classification Accuracy vs Encoding Levels for ADL Dataset . . . . . . . . . . . . . . . . . . . . 199

5.29 Effects of Osteoarthritis [25] . . . . . . . . . . . . . . . . . . . 200

5.30 X-ray of OA joints $[26] \ldots \ldots . \ldots \ldots \ldots$

5.31 VAG signal acquisition $[27]$. . . . . . . . . . . . . . . . . . . . . . . . . 202

5.32 Proposed Knee Vibroarthrography Analysis System . . . . . . . . . . . . . . . . . . . . . . . . . . 204

5.33 Sample VAG signals $[27]$. . . . . . . . . . . . . . . . . . . . . . . . . . . 205

5.34 Sample VAG signal and its 3-bit encoded version . . . . . . . . . . . . . . . . . . . . . . . 210

5.35 3-bit encoding of VAG Data . . . . . . . . . . . . . . . . . . . . . . . . . . . 211

5.36 Adaptive Segmentation of a sample normal VAG signal . . . . . . . . . . . . . . . . . . . . . . . . 212

5.37 Adaptive Segmentation of a sample abnormal VAG signal . . . . . . . . . . . . . . . . . . . . . . 212

$5.38 V A G_{R}$ ROC Curves-21 Features: (A) Naive-Bayes, (B) LDA, (C) SVM . . . . . . . . . . . . . . . 214

$5.39 V A G_{E}$ ROC Curves-21 Features: (A) Naive-Bayes, (B) LDA, (C) SVM . . . . . . . . . . . . . . 215

$5.40 V A G_{R}$ ROC Curves-9 Features: (A) Naive-Bayes, (B) LDA, (C) SVM . . . . . . . . . . . . . . . 216

$5.41 V A G_{E}$ ROC Curves-9 Features: (A) Naive-Bayes, (B) LDA, (C) SVM . . . . . . . . . . . . . . . 217

$5.42 V A G_{R}$ ROC Curves-14 Features: (A) Naive-Bayes, (B) LDA, (C) SVM . . . . . . . . . . . . . . 218

$5.43 V A G_{E}$ ROC Curves-14 Features: (A) Naive-Bayes, (B) LDA, (C) SVM . . . . . . . . . . . . . . . 219

5.44 Classification Accuracy vs Encoding Levels for VAG Dataset . . . . . . . . . . . . . . . . . . . . . 220

6.1 Organization of Dissertation . . . . . . . . . . . . . . . . . . . . . 223 


\section{Epigraph}

Take up one idea. Make that one idea your life - think of it, dream of it, live on that idea. Let the brain, muscles, nerves, every part of your body, be full of that idea, and just leave every other idea alone. This is the way to success.

- Swami Vivekananda 


\section{Chapter 1}

\section{Introduction}

The idea of connecting human civilization on a global scale gave birth to telephony in the 1920s. This was also augmented by advancements in other communication modalities such as Morse code, telegraphy, radar and sonar techniques. Though communication forms one aspect of human civilization, it is indeed the most primal medium of survival, and through the $20^{\text {th }}$ and $21^{\text {st }}$ centuries, communication technologies have boosted a rapid growth in most verticals including infrastructure, transportation, food and healthcare. Up until the late 1960s, most communication methods which were applied for commercial and military applications involved extensive manual labor, in order to collect, store, analyze and transmit information or decisions.

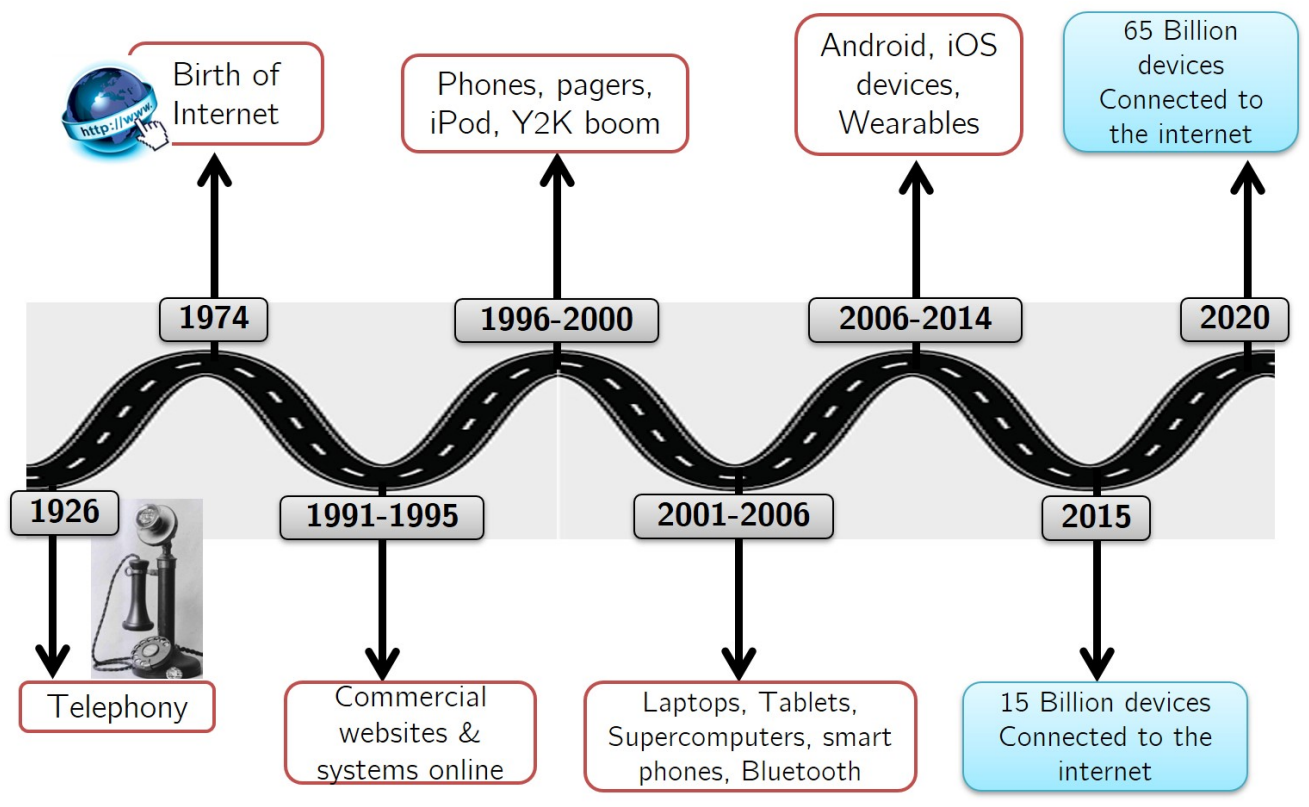

Figure 1.1: History of Internet Connectivity [9] 
With the evolution of the Internet in early 1970s, human civilization has immensely progressed in developing advanced wireless communication protocols, cloud analytics and services, smart phones, tablets, computers and wearables. Following the development of commercial websites and systems in early 90s, we have witnessed rapid growth in the development of mobile phones, music players, laptops, tablets, smart phones and their related data encoding and communication protocols. This has also started a trend to standardize communication pathways between devices and users, and sensor design, depending on specific applications.

In a recent survey conducted by Wollschlaeger et al. [28], it was found that by the year 2015, 15 billion devices were already connected to the internet, and this number is now projected to grow over 65 billion towards the end of this decade. Development of new communication protocols such as Bluetooth ${ }^{\mathrm{TM}} 4.0$, IEEE $^{\circledR} 802.11 \mathrm{a} / \mathrm{b} / \mathrm{c} / \mathrm{g} / \mathrm{n}$, LTE $^{\mathrm{TM}}$ (Long Term Evolution), NFC ${ }^{\mathrm{TM}}$ (near field communication) and wireless sensor networks (WSN), has extensively promoted the development of customer-specific smart devices for usage in communications, health monitoring, home automation, transportation, and, virtual and augmented environments. To cite a few examples:

- Smart phones, computers, tablets, laptops and wearables

- Cloud computing services and Smart energy grids

- Smart home appliances for temperature control, cleaning, cooking and refrigeration

- Advanced gaming consoles, VR (virtual reality) and AR (augmented reality) devices

- Autonomous (or self-driving) transportation

- Advanced healthcare services such as digital pathology, robotic surgeries, physiological data analysis using wearables

The Internet of Things (IoT) framework ensures a seamless connectivity and friendly interaction of the users with their surroundings, devices and sensors. The model addresses gaps in device inter-operability, internet connectivity, data analytics, and ensures the development of a smart, connected environment to the end user. Following section describes the IoT in more details.

\section{$1.1 \quad$ Internet of Things (IoT)}

The IoT can be defined as a complex, intelligent, interconnection of computers, phones, wearables, home appliances, vehicles and infrastructure via the Internet, to collect, store and exchange data. In simple terms, 
any device or equipment with an ON-OFF switch can be connected to the IoT network [11] [9]. This framework allows an individual to engage in a friendly interaction with the surroundings, thereby improving quality of life. A one-on-one interaction with different devices within an IoT structure, generates structured and unstructured big data, which could be then further analyzed to develop user-specific feedback and decisions. In order to enable this features, it is imperative that all the connected devices or appliances are embedded with sensors, actuators and communication protocols. Figure 1.2 illustrates the overall structure of an IoT model.

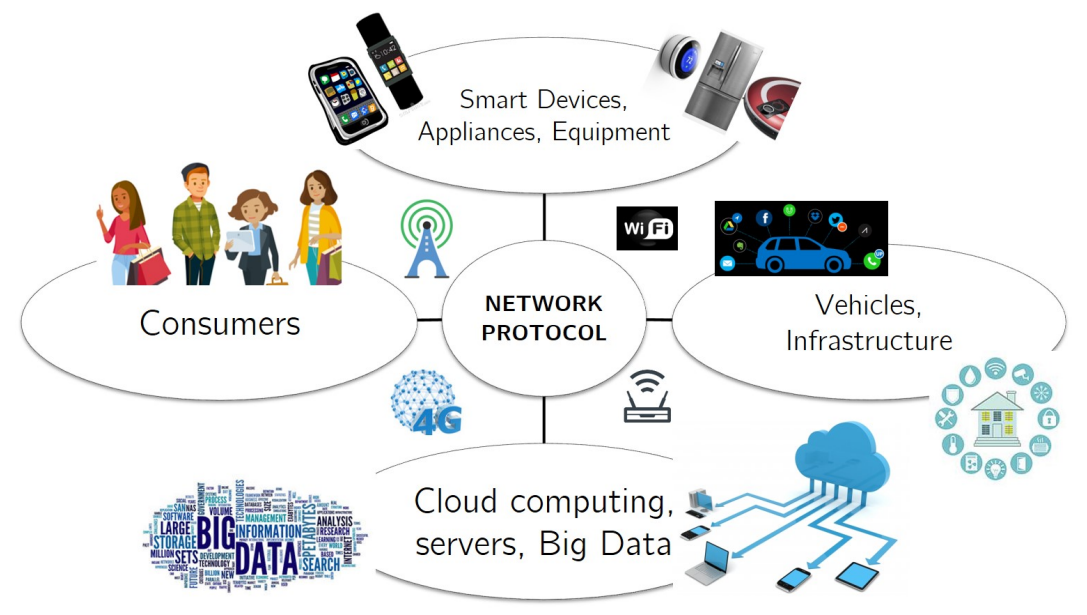

Figure 1.2: IoT - The Big Picture [10]

In order to understand the IoT framework, two main aspects of this internet-connectivity model along with the functioning of its layered have been covered. Figure 1.3 depicts two aspects of the IoT architecture, namely - hardware and software. 


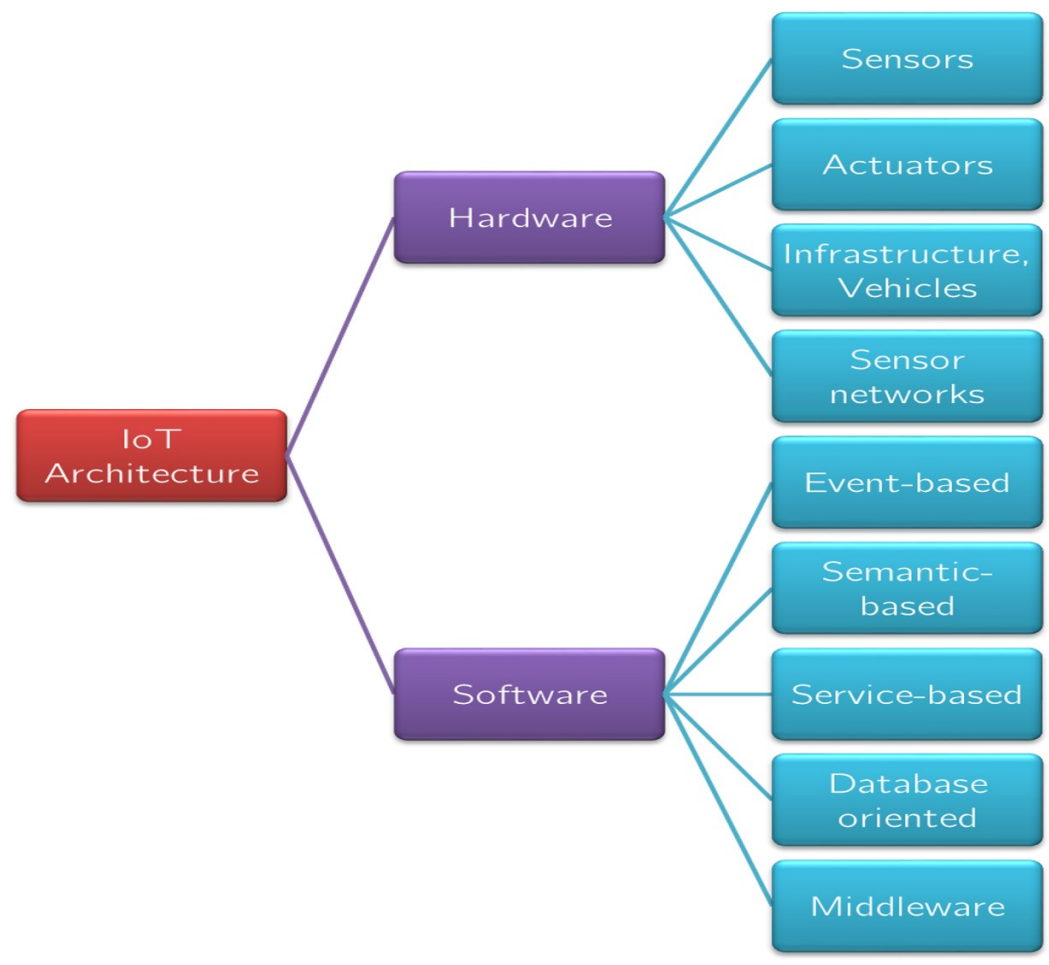

Figure 1.3: IoT Architecture [9] [11]

\section{HARDWARE:}

- Sensors: These include sensing chips embedded within smart devices and appliances for capturing various types of data such as - vibrations, motion, direction, orientation, light, temperature and pressure. Sensors could be in the form of accelerometers, gyroscopes, pressure films, magnetometers, altimeters and light detectors. These modules capture analog information from the user and surroundings, denoise and digitize it, and transmit it to embedded micro-controllers for further analysis. In a lot of cases, the sensed data is also transmitted to cloud-based services for analysis [9] [29].

- Actuators: These components are primarily responsible for controlling a mechanism or system within an appliance or device. Unlike a sensor, an actuator requires the triggering of a control signal and voltage to start performing a specific function. Examples of actuators include - energy grids, water supply, temperature control units, vehicle gears and heating sources. Actuators could be pneumatic, electric or mechanical in nature, depending on the equipment and its application. It should be noted that sensors and actuators could be directly connected to the internet within a device and are usually not included as secondary or supporting components of the IoT structure [9] [11]. 
- Secondary Components: These are the modules which are indirectly affected or controlled in an IoT-based mechanism. For example, a garage door which is controlled by a mobile application via the internet, or a power supply outlet to a bulb which controls the intensity of light in a room [9] [11].

- Sensor Networks: These networks could be wired or wireless in nature depending on the type of device or appliance used. For example, home appliance such as refrigerators could have Ethernet based internet connectivity to control cooling and water supply, or door entry points could be controlled by wireless, closed-loop NFC or RFID (radio-frequency identification) tags. Another example would be a smart home whose individual users, devices and appliances are connected either using wired or wireless connections, governed by device-specific as well as universal communication protocols to store and exchange information [9] [11].

\section{SOFTWARE:}

- Event-based: These applications are user-specific and cater to small tasks such as calendars, reminders, notes and alarms.

- Semantics-based: These applications are usually deployed for enabling interoperability between devices. For example, Google's Chromecast ${ }^{\mathrm{TM}}$ plug-in device, when connected to a TV enable wireless video playback via an Android or iOS device having the YouTube ${ }^{\mathrm{TM}}$ application installed.

- Service-based: Service oriented applications usually cater towards billing a user for accessing different applications such as music, movies, mobile phone plans, internet subscriptions and cable TV. These applications do not interact much with user inputs, and mostly work in background for subscription validation and tracking. Service-oriented applications can also be free-of-cost and could include services for healthcare, emergencies and police [9].

- Database-oriented: These applications are generally not visible to the end user, and function in the background within the device, external storage or cloud services. The primary role of these applications is to store user and device-generated data in tabular formats in order to ensure integrity and easy access to analytical applications. For example, physiological data captured using wearables is stored on the device's on-board memory or onto a supporting cloud storage in the form of standard formats such as CSV (comma separated values) files [11].

- Middleware: These applications could have multiple functionalities such as [12] [9]:

- Create and deploy new IoT services between devices to exchange and process data.

- Enable device-interoperability using a single universal code, protocol or small application. 
- Define standard networking protocols.

Middelware applications usually act as a bridge between a device's operating system, and its supporting applications with the user and other equipment in a smart environment.

In order to ensure a seamless functioning of various hardware and software applications within an IoT model, one must follow a layered architecture model as illustrated in Figure 1.4.

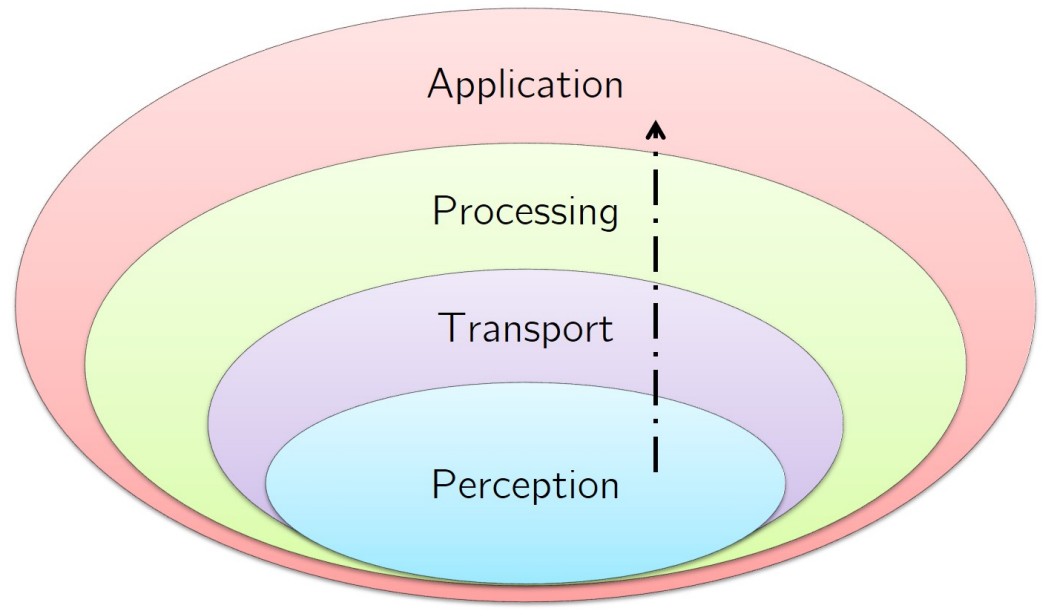

Figure 1.4: IoT Layers [9]

Each layer has been designed to meet specific functions and inter-operates with layers above and below it, depending on user interaction and information generation. The the functioning of each layer has been briefly described as follows [9] [11]:

1. Physical or Perception Layer: This layer enables the hardware connectivity of users with surrounding devices and equipment using operating systems and middleware applications. It also includes sensors and actuators which gather data for information processing and analytics.

2. Network Layer: The Transport and Processing layers together form the networking layer of the IoT model, and include various functions such as:

- Connecting devices using networking protocols to the internet

- Data transmission and exchange from sensors

- Store and analyze incoming data using DBMS (database management systems), cloud computing and big data modules

- Enable semantic and middleware applications for inter- and intra-device operability and connectivity. 
3. Application Layer: This layer is usually visible to the user in the form of service-based, event-based and user-specific applications. It includes software installed on devices to interact with the user, as well as gather, process and transmit sensory information in an IoT environment.

With regards to user data processing acquired from sensors, applications and devices, IoT provides many sources such as (see Figure 1.5:

1. User's personal computer

2. Personal mobile device for local storage and small analysis

3. Enabling gateway for a network server which stores data from different connected devices

4. Cloud storage and computing services for big data analytics and feedback generation to the user via personal computer or mobile device

5. Fog Computing algorithms and applications which enable on-device data processing and analysis, thereby reducing computational constraints on local computer or cloud.

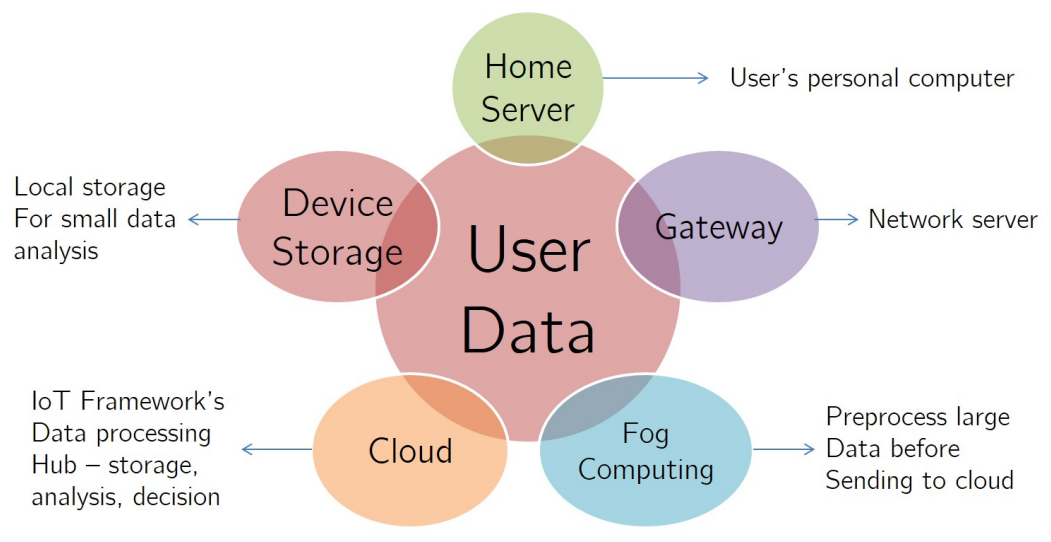

Figure 1.5: User data handling in IoT [9]

It is evident that for a seamless functioning of the IoT framework, its three components, namely hardware, software and supporting architecture layers must coordinate and function in synchronization with each other. This being said, current niche markets retailing smart devices for IoT environments pose one or many of the following challenges in order to ensure the robustness and integrity of a connected environment [10]:

- Ensuring user data privacy, security and confidentiality. As per a recent survey, although currently available tools and devices for an IoT model do support this clause, there still exists a huge gap in de- 
signing data formatting, security and standardization, which must address inter- and intra-operability concerns.

- An IoT model must be able to handle smart device data traffic in a mobile environment without any significant information loss, which could impact the intelligence and thereby the feedback given to a user.

- IoT frameworks must ensure reliability and real-time actuation with respect to user inputs and data.

- The cloud services employed for big data analytics must address the scalability factor by being able to handle multiple device requests for data storage, transmission and processing.

- IoT models must be designed by considering power and memory requirements of each connected device, as well that of available computing resources such as local computers or cloud services.

- The communication and networking protocols must adhere to multi-hop methods in order to ensure secure and robust inter-device connectivity.

- As a layered model, it also expected in an IoT model to have middleware applications which could be used by an individual for remote device management.

- Lastly, it is highly imperative that sensor and device design must focus on context detection, and the associated data processing resources must include machine learning models for analytics and feedback generation.

Meeting these challenges will not only help in developing a robust IoT environment for an individual, but a conglomeration of many such models could potentially lead to development of a connected human population. Recent developments in smart technologies coupled with ubiquitous sensor design, has provided a significant number of opportunities in the IoT domain, as highlighted in Figure 1.6. 

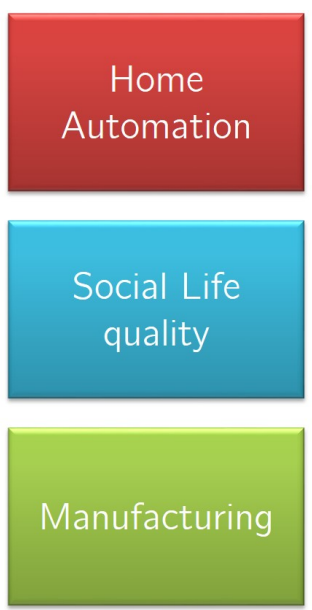
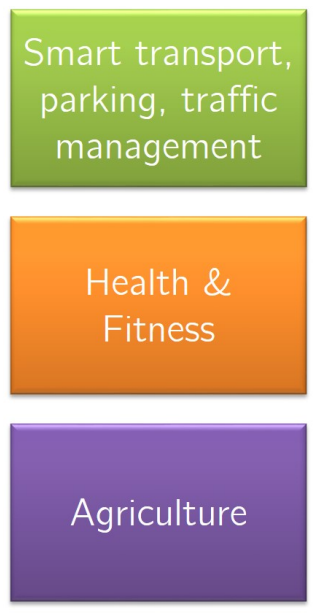
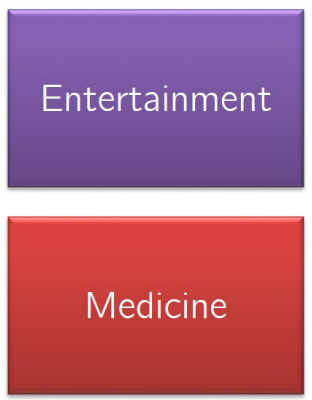

Logistics, supply

chain

management

Figure 1.6: Opportunities in IoT [11] [12]

As evident from these opportunities, current IoT models enable the networking of devices with limited $\mathrm{CPU}$, memory and power with the Internet and end user. Extensive literature review indicates that home automation, advanced healthcare and autonomous transportation are some of the hot niche markets within the IoT context, and have spurred a massive retailing of smart home appliances, wearables, devices for security, lighting and air conditioning, and self-driven vehicles. A classic example of one such technology would be the Nest ${ }^{\mathrm{TM}}$, which is a self-learning, Wi-Fi enabled, self-driven, intelligent thermostat which optimizes home temperatures by gathering and analyzing human inputs and presence data. This device has a built-in machine learning capability for analyzing streaming temperature and ambient data, in order to make appropriate heating or cooling adjustments. It is one of the few devices in the market to employ a fog or edge computing approach to data analytics [30], thereby reducing the need to maintain contant connectivity with its cloud services for feedback generation.

IoT-based home automation technologies have greatly enabled and motivated a healthy-life style adoption for elderly citizens, disabled individuals, chronically ill subjects and the general population as well. Home automation tools include devices which provide assistive living, thus improving the quality of life. This being said, it is evident that apart from infrastructure and home appliances, an IoT-based home would also feature the use of specific devices for better living, such as [10],

- Voice based security and temperature control

- Assistive devices such as Google's Home ${ }^{\mathrm{TM}}$ or Amazon's Echo ${ }^{\mathrm{TM}}$ for setting reminders, playing music, news and general interaction with the user

- Smart cooking and refrigeration systems 
- Wearables for continuous monitoring of daily activities of a individual. These would be helpful for remote health consultations, fitness and alarm generation.

In the next section, various aspects of a sub-structure of the IoT framework - the Internet of Medical Things (IoMT) model will be covered, which will address the use of remote health monitoring technologies in a connected healthcare and smart environment context.

\subsection{Internet of Medical Things (IoMT)}

Before delving into the working of an IoMT framework, it is imperative to understand the basics of Telehealth and how IoMT fits in it. Telehealth refers to the remote delivery of clinical and non-clinical healthcare services in a given geographical area. In olden times, when there were no computing or connectivity technologies, telehealth was practiced subtly in the form of visiting doctors and home-care nurses who would monitor a patient's health and prescribe medicine accordingly. Over the years of immense technological advancements, healthcare technologies have evolved, and so has the concept of telehealth. In today's context, telehealth networks not only deal with patient care and drug delivery, but also control non-clinical services such as maintaining electronic patient records, providing medical education to service providers, administrative meetings and consultations. Additionally, a telehealth framework also supports the use of novel health monitoring technologies such as wearables which capture heterogeneous physiological data for generating informed decisions on a individual's daily health [14] [31] [13].

The clinical service aspect of a telehealth model is also termed as Telemedicine [10], and it applies to delivery of healthcare services via existing telecommunication infrastructure. Prior studies have indicated that telemedicine technologies significantly reduce consultation time and frequency of doctor visits, by enabling anytime patient-doctor communication via the Internet. Additionally, the use of clinically validated and approved devices such as Holter monitors, sleep apnea test kits, blood pressure monitor, glucometers and actigraphs, leverage this framework by remote monitoring of vital health signs and daily activity in elderly, disable and chronically ill individuals. Although telemedicine does not support other aspects such as prescribing medications or training healthcare providers on using mobile monitoring equipment, it has nevertheless motivated people for healthier life choices by making healthcare accessible to remote locations, in recent times [14] [13].

The IoMT framework is a subset of the IoT model, and aims to deliver remote or home-based healthcare services to an individual via the use of smart devices, wearables, clinically approved equipment and infrastructure [13]. The model employs the use of local computers, grid computing techniques and edge computing approaches for biomedical data analysis before transmission to a healthcare provider for disease diagnosis. 
As shown in Figures 1.7 and 1.8, the IoMT framework takes a five-pronged approach for remote healthcare delivery, namely [31] [14] [10]:

1. Remote health monitoring using mobile, clinically validated equipment

2. Use of smartphones and related applications

3. Ambient assisted living

4. Use of wearables for mobile physiological data collection and analysis, and lastly,

5. Providing emergency care for critically ill individuals

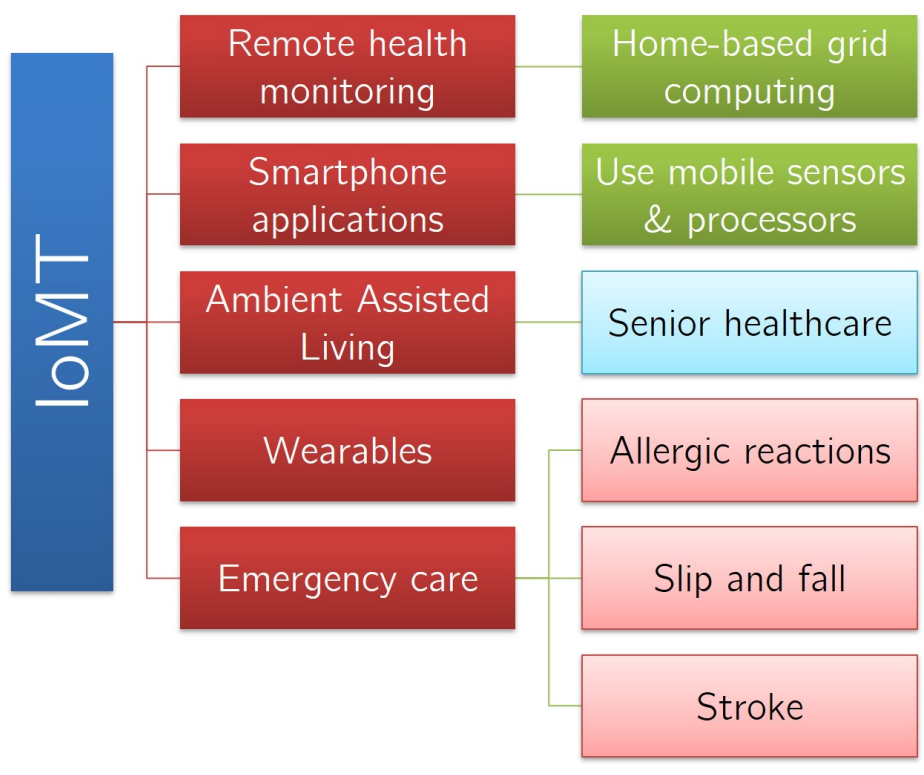

Figure 1.7: IoMT services [13] 


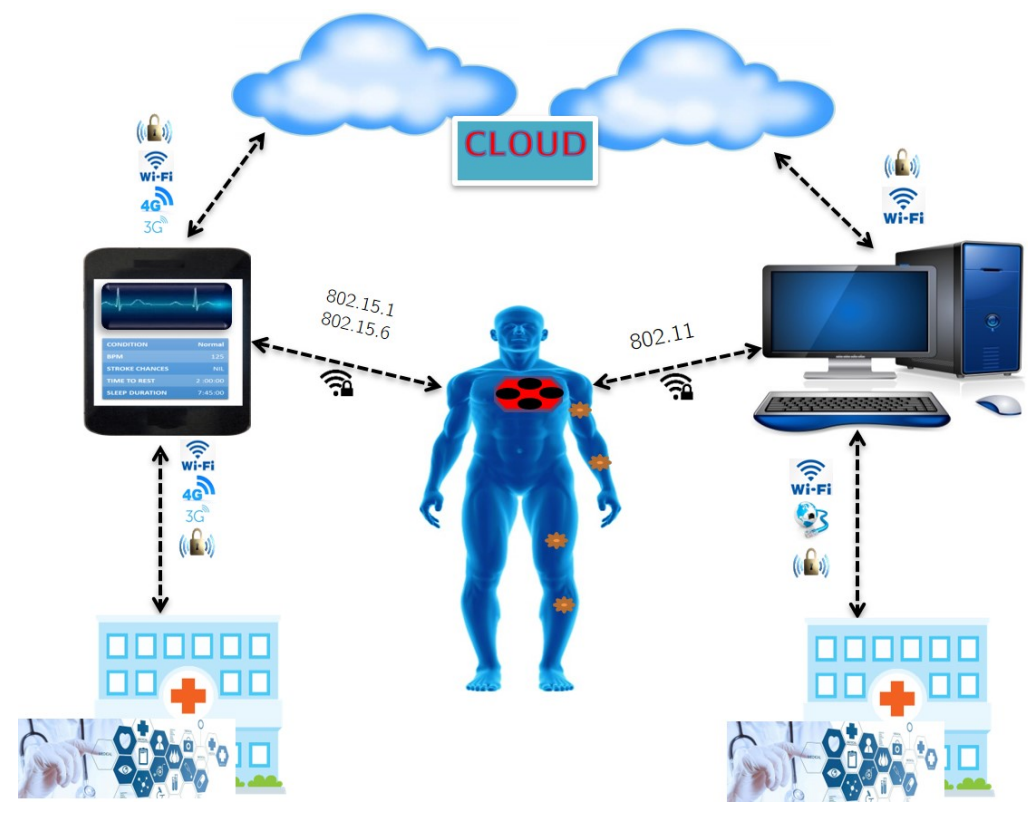

Figure 1.8: IoMT framework [13]

Two of the afore-mentioned IoMT applications will describe this further in detail as follows:

\section{Remote health monitoring:}

This aspect of IoMT deals with the remote monitoring of chronic, terminal or disabled individuals using home monitoring equipment given by the health care provider. The IoMT model allows for maintaining the inter-operability of this equipment via middleware applications and cloud-based services [11] [13]. As shown in Figure 1.9 In order to build such a setting, three components are essential:

- Friendly user interface and data processing via computers and phones

- Use of electrode-based equipment which captures clinically relevant physiological information

- Seameless internet and telephone connectivity to maintain contant communication with the healthcare provider 


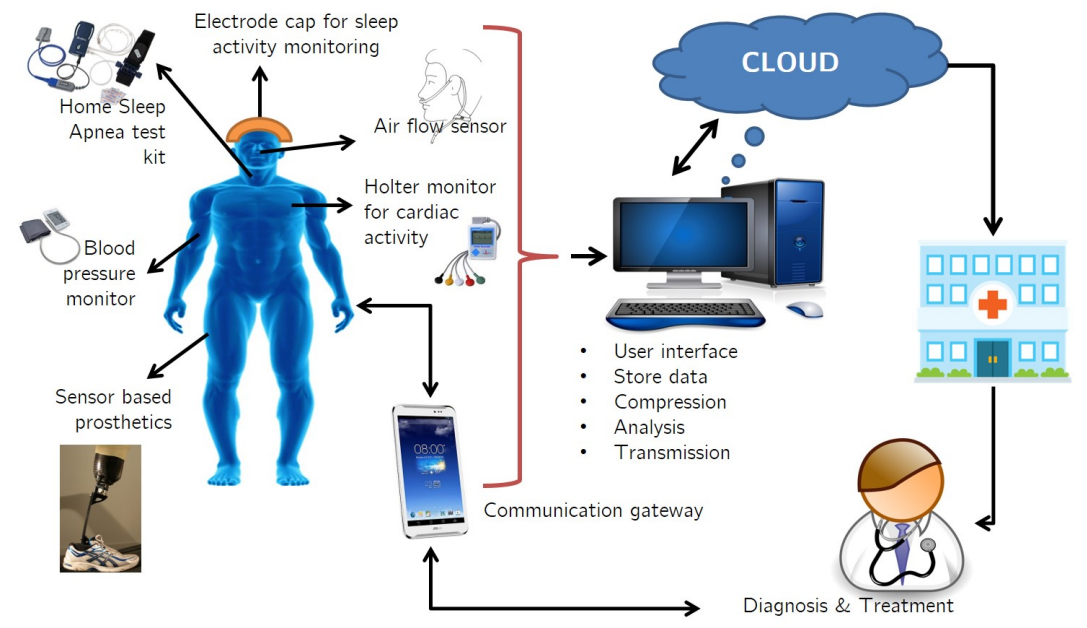

Figure 1.9: Remote health monitoring via IoMT [13]

\section{Smart phone applications:}

Within an IoMT environment, the embedded sensors within smart phones can be used to analyze small bursts of physiological data to monitor user habits such as food intake, calories burnt, fitness levels, sleep times and prescription reminders. Sensors such as accelerometers, gyroscopes and camera could be used to develop user-friendly mobile applications for activity monitoring and injury assessment [13].

Figure 1.10 shows how smart phone data fits in the IoMT model.

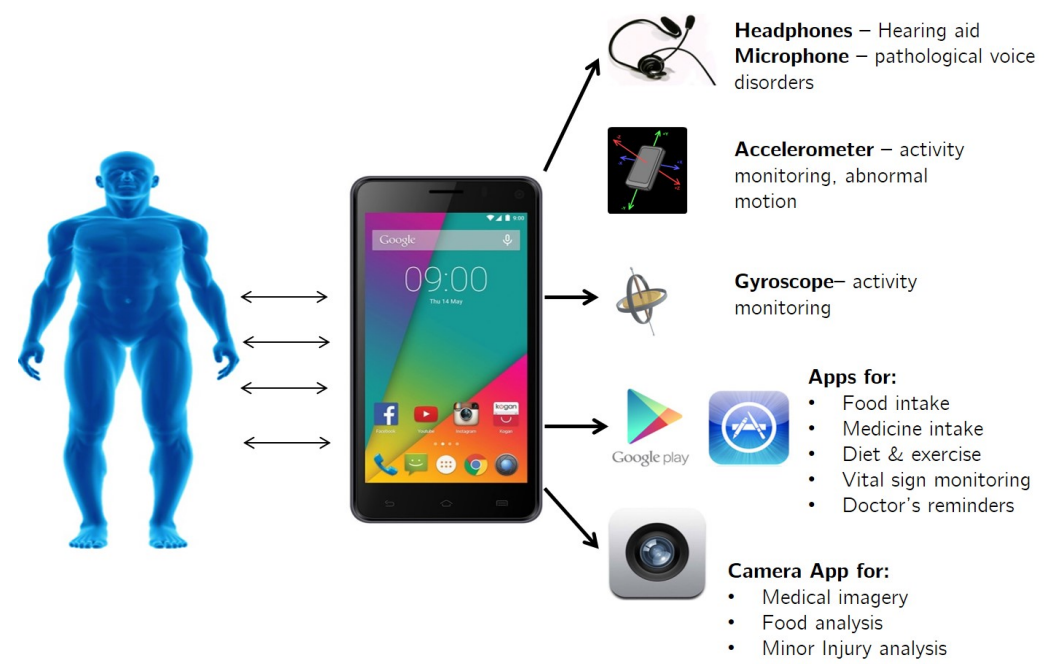

Figure 1.10: Use of smart phone applications [13]

Similar to the IoT structure, various hardware, software and networking components of an IoMT model show how a connected healthcare environment is developed. Considering the use of mobile equipment, 
computers, smart phones and wearables in a IoMT framework, two main hardware components could be identified, namely - sensors and processors. While the sensors can be used for gathering user generated data from movements, temperature, pressure and proximity, the processors employ the use of signal processing techniques to denoise, digitize, segment and analyze the collected data, to generate feedback to the user, via a friendly interface. Figure 1.11 illustrates the hardware aspects of IoMT.

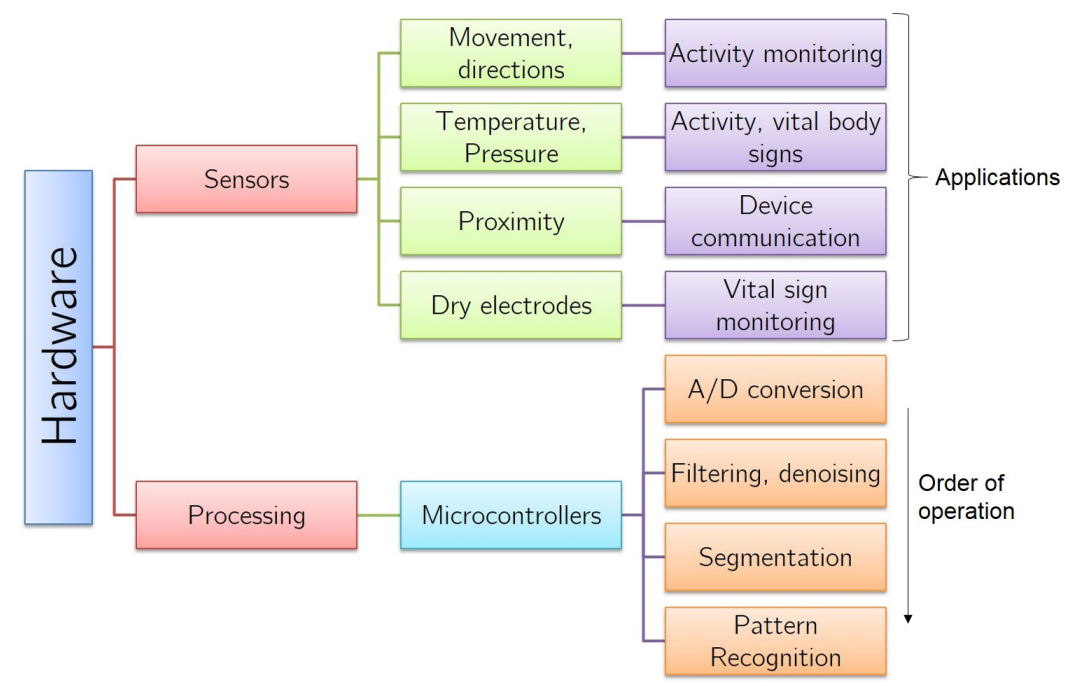

Figure 1.11: Hardware aspects of IoMT [13] [14]

Further to this, from a software perspective, three types of applications for a device to function in an IoMT environment can be identified as shown in Figure 1.12. While the analytical software handles the physiological data pre-processing and analysis via machine learning techniques, the middleware applications enable user interaction and alarm generation. Parallel to these, the networking applications provide communication protocols for effective data exchange and interaction of user with device, as well as the device and the Internet. 


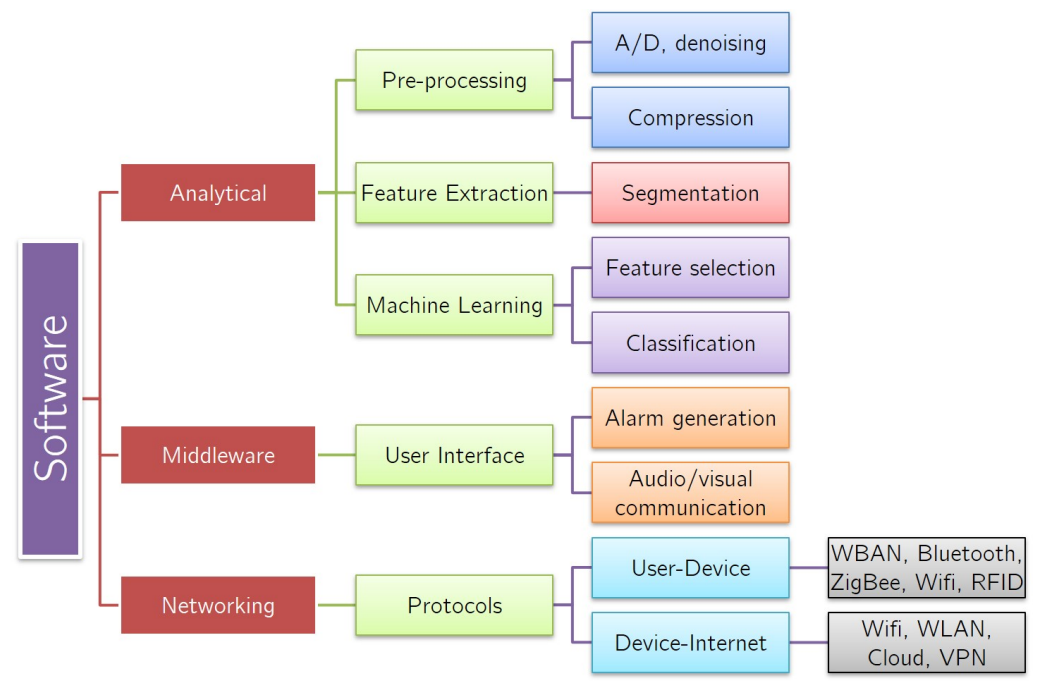

Figure 1.12: Software aspects of IoMT [13] [14]

Based on the hardware and software available, IoMT framework also defines various networking and computing aspects which enable device inter-operability, data exchange and intelligent analytics. As shown in Figure 1.13, the networking protocols could be user-based, location-based or environment-based, and their application could vary accordingly. For example, for a user-based networking protocol, employing a low power WSN can effectively reduce bandwidth and power usage when transmitting data between user and device. This data could also be augmented by location-based protocols which define geographical location. Additionally, this data can also be transmitted to a cloud server via a wired internet or WiFi connection. The computing aspects of an IoMT model, on the other hand, could be device-based or server-based. While the device-based applications enable data encoding, compression, visualization and edge computing approaches, the server-based applications can handle intelligent, cloud analytics, and data processing via high-end computers. 


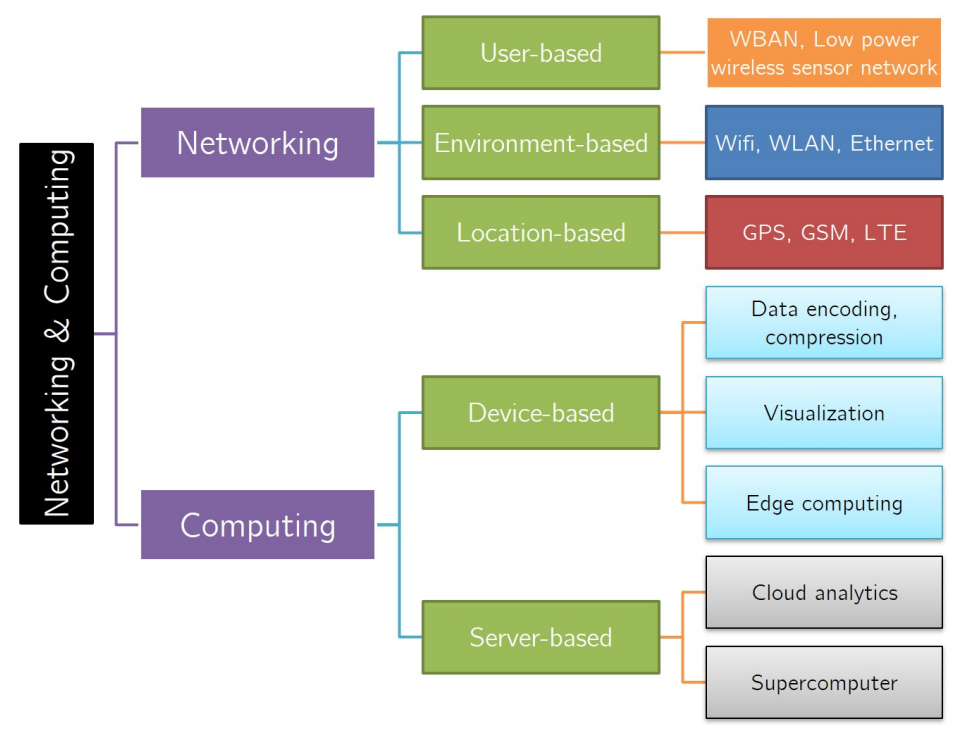

Figure 1.13: Networking and Computing aspects of IoMT [13]

Although, these aspects present a promising future for IoMT-friendly tools and technologies for personalized healthcare, a review of prior works indicates that developing a full-scale IoMT framework still needs to overcome some crucial hurdles. Some of these include [10]:

1. Developing health monitoring smart devices and applications, which are clinically approved and validated. It should be noted that clinical approvals require exhaustive data collection, analysis and ground truth benchmarking for different geographical regions. Only then the resultant outcomes could be translated into rules for home-based health monitoring applications.

2. Designing comfortable, safe and non-invasive devices and wearables, embedded with ubiquitous sensors for collecting and analyzing physiological data in real-time. This also addresses the aspects of computations, memory and power usage.

3. An IoMT framework must ensure seamless connectivity of users, devices, infrastructure and services to the Internet and cloud servers.

4. Physiological data collection algorithms must have embedded functionality to ensure privacy, security and confidentiality of user data. In other words, apart from network encryption, user data encryption is a must, and should be done right at the data acquisition source, that is at the sensors and microcontroller level.

5. Lastly and most importantly, the underlying physiological data analysis algorithms must be focused on employing edge computing approaches. That is, the data collection, denoising, preliminary analysis 
and segmentation must be done at the acquisition source without creating the need to transmit longduration real-time data to cloud servers. This would be highly beneficial in ensuring data privacy and optimizing computational resource usage, on-device memory and battery power. It should be noted that edge computing techniques aim to increase operating efficiency and throughput of smart devices and wearables in an IoMT environment.

In addition to these, Figure 1.14 highlights other challenges which must be met when designing an IoT-IoMT model.

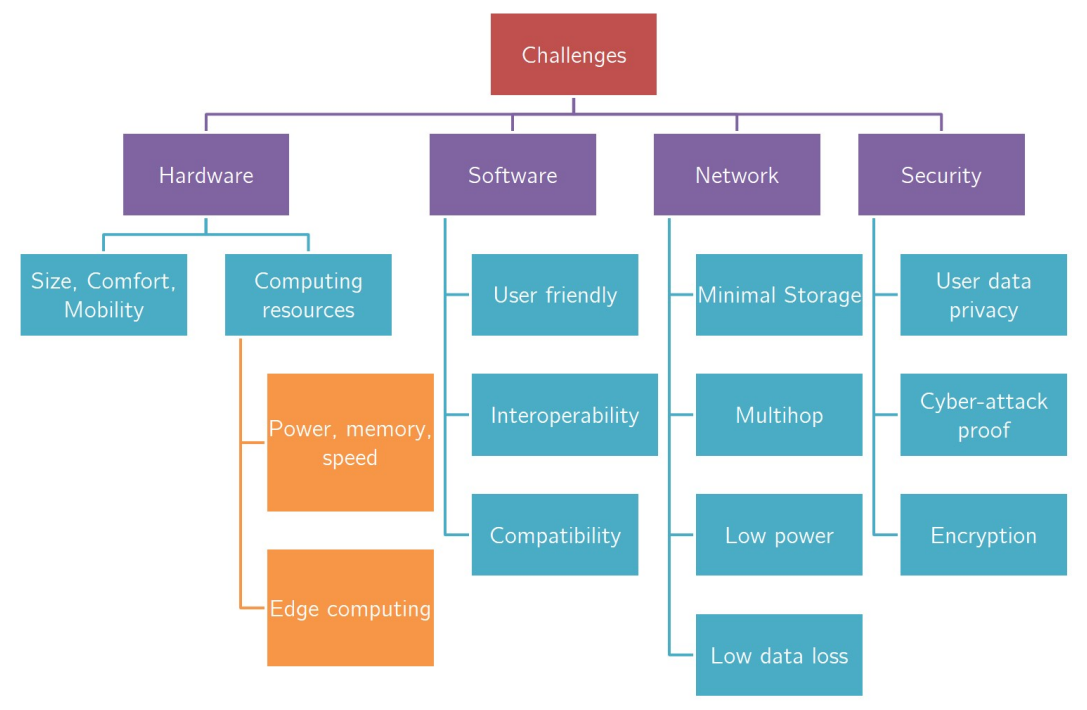

Figure 1.14: Challenges in IoMT design [13] [10]

Despite these challenges, IoMT models do present numerous opportunities with respect to personalized health monitoring and networking. Some of these are [10]:

- Vital sign monitoring using wearables

- Textile computing - smart clothing embedded with sensors for physiological data analysis

- Developing universal electronic health records which maintain transparency between an individual and healthcare provider

- Scope for developing tools for data security, privacy, network encryption and edge computing

Keeping these challenges and opportunities in mind, the next section narrows down on understanding the wearables aspect of the IoMT framework and how they could be used for vital health and physiological signal monitoring applications. 


\subsection{Wearables for Health Monitoring}

As mentioned in the beginning of this Chapter, with the evolution of the Internet, a plethora of technological advancements in sensor design, data frameworks, smart devices and connected environments have taken place. The rising public awareness in leading a better quality of life has given birth to a niche market of wearable smart devices or wearables in general, which are being used various facets of daily life. Health monitoring for wellness and fitness has become a de facto trend in the millennial generation, and the use of wearables helps individuals track their physiological parameters [32].

In simple words, wearables are smart devices embedded with miniature data acquisition sensors, microcontrollers and supporting data analysis algorithms and operating systems, which can worn comfortably on the human body, and provide an intuitive interface to the individual to obtain feedback on certain physiological parameters. As shown in Figure 1.11, wearables could have one or more sensors depending on their application [32] [10]. These sensors capture large amounts of physiological signal data from an individual's daily activities, which is then analyzed using signal processing algorithms for various applications such as fitness monitoring, calories burnt, blood pressure, glucose levels, behavior and vital sign monitoring such as chances of stroke or a neuromuscular abnormality (see Figure 1.15).

A prior review study indicates that until the last decade, most wearables were made available only after clinical approvals and validations. Some of these are Holter monitors, sleep apnea test kits, stethoscope and blood pressure monitors. Although this continues even today, smart device manufacturers have found novel ways for recording and analyzing physiological signals without the need to go through regulatory approvals. Though the validity of using wearables for continuous health monitoring remains a debatable topic amongst clinicians, researchers and manufacturers, many prior studies and surveys indicate that use of wearable technology could potentially fill the gaps in telehealth and IoMT frameworks [32].

An investigation into various technology news and forums, found that many popular wearables such as FitBit ${ }^{\mathrm{TM}}$, Apple Watch ${ }^{\mathrm{TM}}$, Samsung Gear ${ }^{\mathrm{TM}}$ and $\mathrm{Nymi}^{\mathrm{TM}}$ band are able to capture and generate health information such as pulse rate, step count, calories burnt, food intake requirements and sleep efficiency to the consumer. Although these metrics have not been clinically validated extensively, empirical results from consumer surveys indicate that their usage has improved people's quality of life in certain demographic regions. An exploration of these devices' specifications and software development kits (SDKs), described how the physiological data is collected, pre-processed and analyzed to generate feedback to the user. For example, an investigation into Thalmic Labs' MYo ${ }^{\mathrm{TM}}$ controller's SDK revealed how surface-EMG (electromyography), accelerometry and gyroscopy data was captured and analyzed to generate feedback on the user's arm activity for controlling a toy drone [32]. Similarly, a validation study was also conducted using actigraphs, to test 
the efficiency of using wearables to track sleep-related movement disorders $[5,10,32]$.

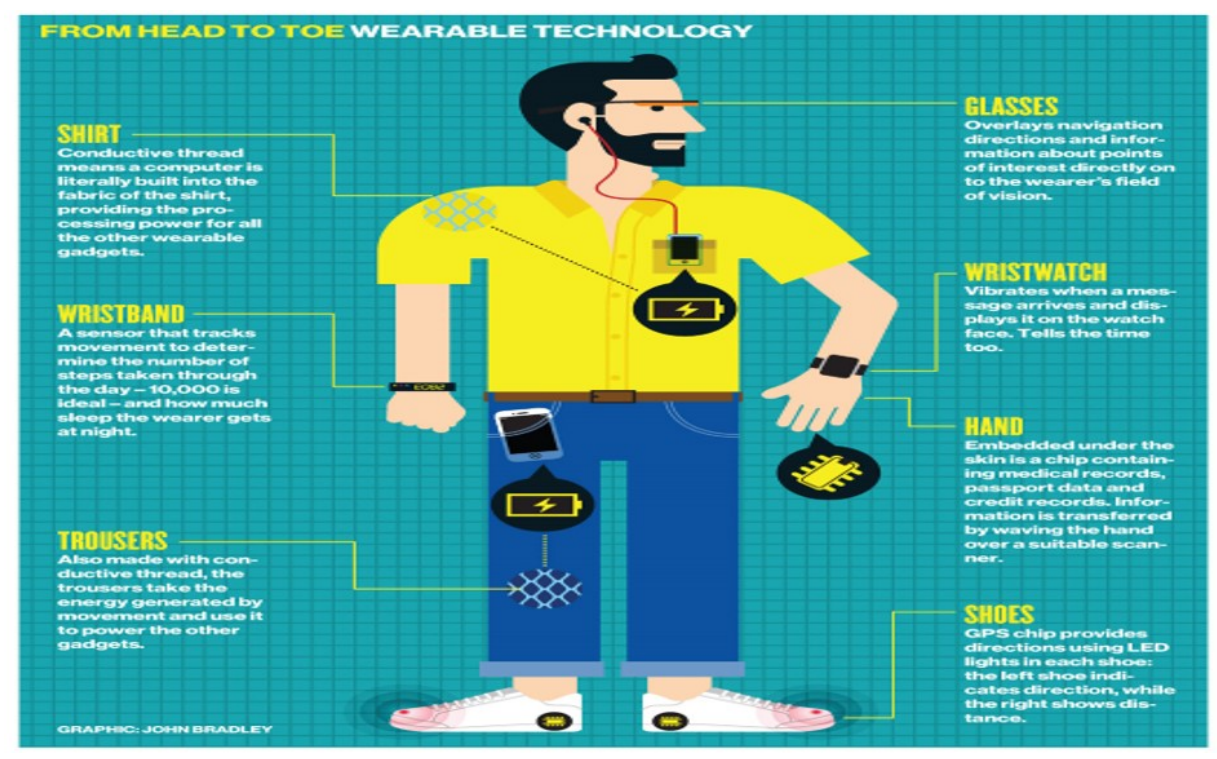

Figure 1.15: The Connected Human [15]

A recent survey conducted by a wearables database organization, Vandrico, pointed that only about 90 of the currently retailed 600 wearables, could be used for clinically relevant health monitoring applications [33]. Additionally, popular news magazine, Business Insider ${ }^{\mathrm{TM}}$ projected that the wearables market could expand to about 400 million users in North America alone, which also supports another survey which indicated that the healthcare system in this continent could profit by over a billion dollars in the next couple of decades, solely through IoMT frameworks and wearable technology [32] [34] [35]. These trends only provide further motivation to investigate into exploring the feasibility of using wearables for physiological signal analysis.

As described in previous section, Figures 1.11, 1.12 and 1.13 are also applicable to wearable device design and its underlying signal analysis algorithm development. As evident from Figure 1.11, sensors form a primal part of the wearable design, and gather data as soon as human interaction begins with the device or surroundings. Sensors installed in a wearable could be one of the following types [13] [32]:

- Accelerometer for sensing user movements and vibrations. For example, an accelerometer data could be used to detect patterns in different human activities like walking or holding and using various objects.

- Gyroscope for sensing direction of activity in three dimensions. For example, a gyroscope changes screen resolution and orientation from portrait to landscape when a smart phone is rotated.

- Temperature sensor for estimating skin temperature of the user and ambient surroundings. 
- Pressure sensor for estimating the pressure applied by an individual while performing specific physical tasks. For example, pressure sensors in shoe soles measure intensity of gait patterns.

- Proximity sensors which trigger a mechanism or application within a wearable or its connected device such as a smart phone, when it detects a human presence nearby.

The data acquired using these sensors is then forwarded to a micro-controller for analysis. Before generating feedback, the sensor data must undergo the following key signal processing operations, which are [32] [36]:

- Analog-to-digital conversion

- Digital filtering and denoising to remove redundant information, artifacts, dc drift and ambient noise

- Signal segmentation to identify events or regions of interest

- Feature extraction to identify key properties exhibited in different phenomena of human activity

- Machine learning and pattern classification to generate feedback to the user. The outputs are further used by the same learning tool to build ground truth information, specific to each user.

The applications or software governing a wearable design could be divided into the following three categories [13] [11]:

- Analytical algorithms and applications for processing sensor data and generating user friendly feedback.

- Middleware applications which enable interfacing between user and the device, along with other tasks such as scheduling, alarm generation and communication with accessories.

- Networking protocols and applications which ensure robust connectivity between users, wearables and internet within a connected environment.

While it is true that physiological data from sensors could be analyzed efficiently using signal analysis algorithms, designing and implementing appropriate sensors in the first place is equally important. A review of prior studies indicates that the following criteria are important with respect to sensor design and signal analysis [32] [36]:

- Context Awareness - What data is being acquired and for what purpose

- Sensor size, power usage, memory and cost

- Understanding physiological signal structure and morphology

- Extracting robust features which exhibit maximum relevance to a corresponding human activity 
- Piece-wise evaluation of sensor data in order to address its non-linearity and non-stationarity properties

- It should also be kept in mind that unlike clinically relevant data such as ECG (electrocardiogram), wearable sensor data may not have characteristic structure and may require the use of other signal processing methods to derive meaningful information.

Keeping these hardware, software and algorithmic aspects in mind, prior studies indicated that there still exists a huge potential in developing wearable-based signal analysis systems, which operate efficiently, irrespective of manufacturer specifications. With regards to sensor usage, it was found that the accelerometer is the most commonly used IMU (Inertial Motion Unit) component in wearables and accounts for nearly $90 \%$ of currently retailed devices [10]. This motivates further, the need to conduct a systematic investigation into developing a novel signal analysis system for monitoring actigraphy wearable data for activity assessment applications in daily sleep and wake states. In order to test the robustness of the signal analysis system developed through this research, it was implemented on different types of actigraphy signals acquired using various actigraphs, from test subjects performing movements in wake and sleep states. In the following chapters of this dissertation, various aspects of actigraphy signal analysis and applications have been described, and how this study's contributions would prove to be a significant addition to ongoing studies in wearable health monitoring applications. Following section concludes this chapter, which describes the objectives and organization of this dissertation.

\subsection{Objectives and Organization of the Dissertation}

\subsubsection{Objectives}

The research goal is to develop a state-of-art, IoMT-friendly, device-independent actigraphy signal analysis technique which meets the following objectives:

1. Perform on-the-fly signal compression and denoising

2. Signal quantization or encoding using optimized bit resolution

3. Conduct adaptive segmentation of the encoded data by removing redundant activity markers and highlighting regions of peak movements, specific to each human activity

4. Develop "handcrafted" features which are specific to actigraphy signals, and are able to characterize human movements. 


\subsubsection{Datasets}

The system developed in the study has been applied and tested successfully on the following actigraphy datasets as indicated in Table 1.1.

Table 1.1: Datasets Used

\begin{tabular}{cllccc}
\hline Dataset & Placement & \multicolumn{1}{c}{ Activity } & No. of Participants & No. of Signals & Actigraph type \\
\hline A & Bilateral ankle & Sleep - PLMs & 96 & 191 & Single-axial \\
B & Bilateral ankle & Sleep - PLMs & 166 & 332 & Tri-axial \\
C & Bilateral ankle & Sleep - Stroke Recovery & 46 & 92 & Single-axial \\
D & Wrist & Wake - Daily activity & 16 & 274 & Tri-axial \\
E & Knee & Wake - Vibroarthrography & 89 & 89 & Single-axial \\
\hline
\end{tabular}

Before proceeding further, the reader may note some critical aspects of this study. Firstly, it may be noted that the primary focus of this research work is to analyze sleep actigraphy data captured using tri-axial and omni-directional accelerometers. It should also be noted that in this dissertation, the terms acceleration, vibrations and motions have been interchangeably used, and they signify the same measure, which is the magnitude of activity counts captured per second by the accelerometer.

\subsubsection{Organization}

Before proceeding to further details of this research work, a detailed overview of the organization of this dissertation will give the reader a clear picture of the research objectives and their potential outcomes. The block diagram in the following Figure 1.16 illustrates the structure of this dissertation. 


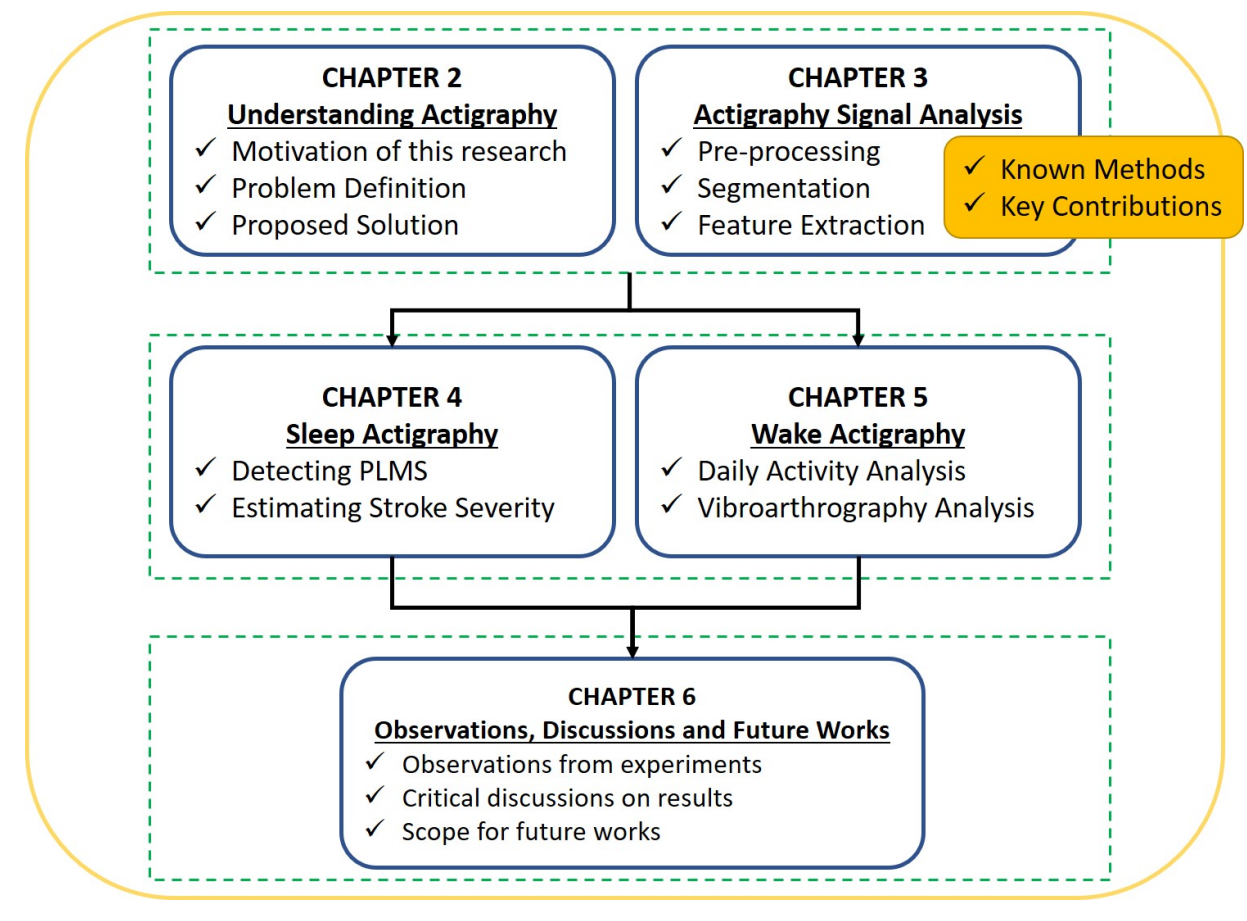

Figure 1.16: Organization of Dissertation

Additionally Figure 1.17 also highlights the key contributions to actigraphy signal analysis research.

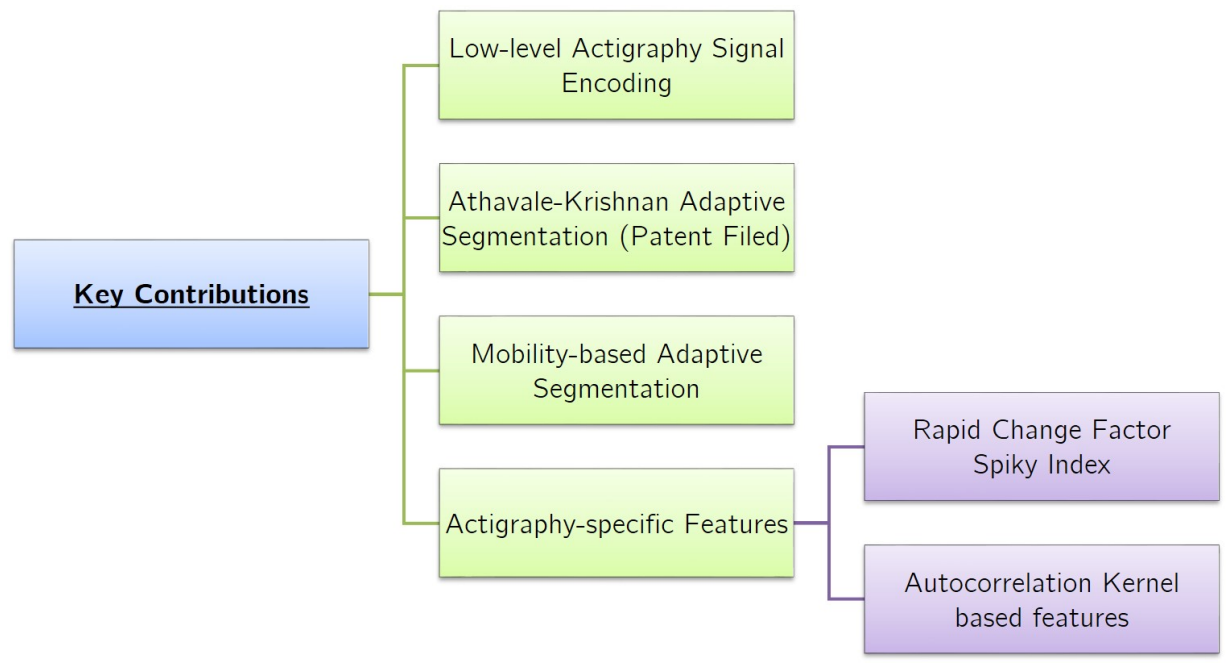

Figure 1.17: Key Contributions of this research

Further to this, each Chapter has been summarized as follows:

- Chapter 2 will cover a basic understanding of actigraphy and its applications. It will start with the functioning and composition of an actigraph, followed by understanding accelerometry or actigraphy signals. Next, some standard signal tests will be applied on various sample actigraphy data used in this 
research, and their properties would be recorded with respect to linearity, stationarity, Gaussianity and sparsity. Following this, some prior works in actigraphy signal analysis and their applications in homebased health monitoring will be discussed. This will be then concluded by highlighting the motivation for this research, defining a problem statement, and describing the solution developed in this study.

- Chapter 3 continues the discussion on actigraphy signals from previous chapter and proceeds with understanding different methods currently available for pre-processing, segmenting and feature extraction from actigraphy signals. Additionally, a survey of prior studies that have applied these methods has also been included. This chapter also describes in detail the key contributions to this research in actigraphy signal analysis. These include signal encoding, adaptive segmentation and actigraphy-specific feature extraction.

- Next, in Chapters 4 and 5, a combination of known and newly developed signal processing methods has been applied to different types of actigraphy datasets as mentioned earlier in this section. These also include various illustrations and discussions on key findings from various experiments, and show the efficiency of the actigraphy analysis system for each dataset developed in this research.

- Finally, in Chapter 6 will conclude this dissertation with some experimental observations, critical discussions and a scope for future works. Using the results from the experiments, it will be explained how the contributions to this research could help in addressing key challenges in the IoMT-Wearables sector. Further to this, the limitations of the developed system will also be cited and how they could be overcome to take this study further.

- At the end of this dissertation, a list of refereed and patented contributions to this research will be highlighted.

\subsection{Summary}

This chapter gives the reader a preface to the research study conducted in this dissertation. Considering that this research is based on actigraphy-based wearables, we first explore the basic concepts of Internet of Things (IoT), examine its definition, structure and describe various layers of its architecture. Next, different challenges and opportunities posed to the research community with regards to developing robust IoT environments are discussed. Following this, a zoom into the healthcare domain of IoT by providing a detailed description of the Internet of Medical Things (IoMT) has been done. The IoMT encapsulates various aspects of remote health monitoring using smart devices and wearables. A few key hurdles which 
must be crossed in order to implement home-based health monitoring tools have also been discussed further. This is then followed by a detailed discussion on the use of wearables for health monitoring applications, their software-hardware aspects, and list of criteria for sensor design which would suit physiological data collection and analysis using wearables. Finally, in the last section, the objectives of this research work have been listed, along with how the actigraphy analysis system has been developed, which would be useful for activity monitoring in wake and sleep states in daily life. We conclude this Chapter by providing a detailed organization of this dissertation, and highlight the key contributions to this research in wearable signal analytics. 


\section{Chapter 2}

\section{Understanding Actigraphy}

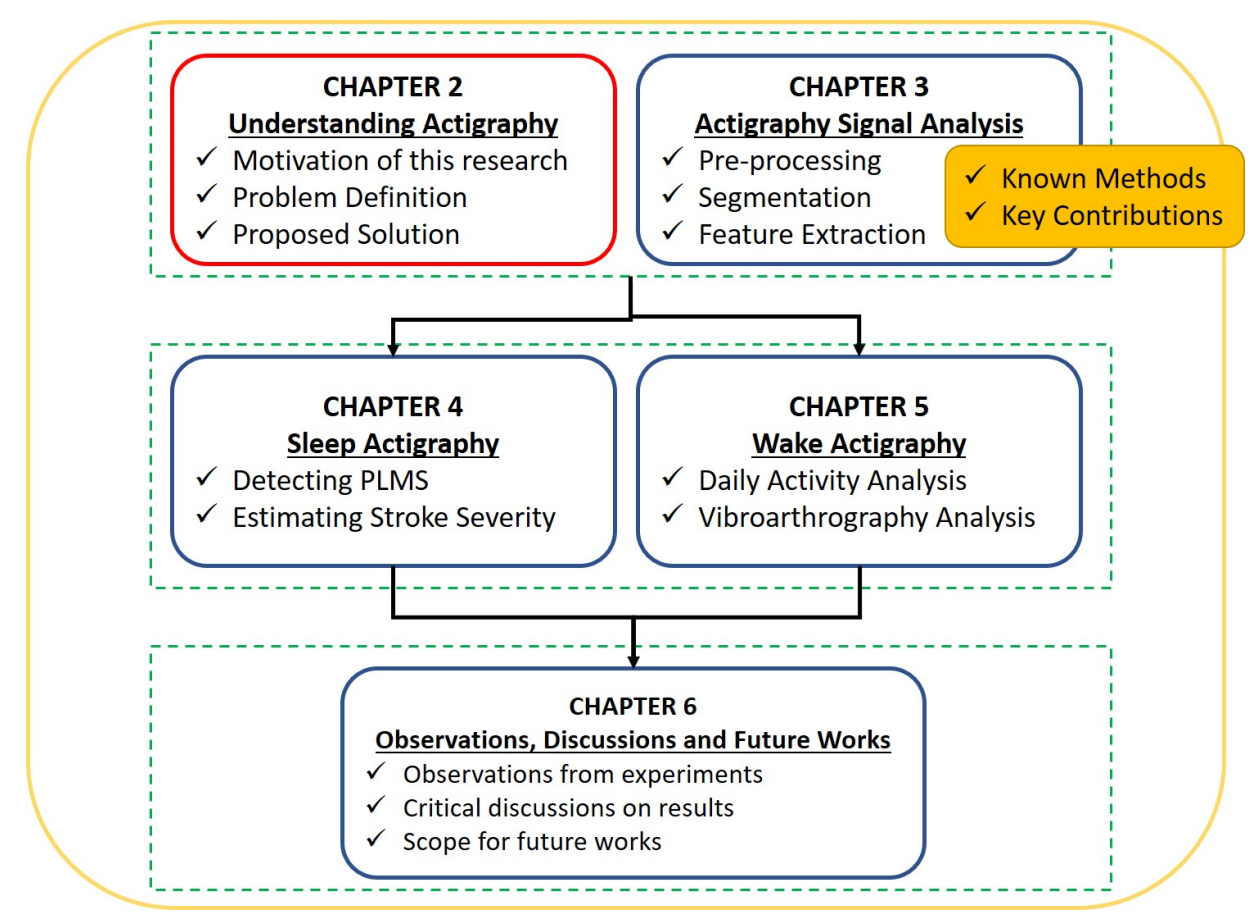

Figure 2.1: Organization of Dissertation

In this chapter, a discussion on actigraphy signal analysis will be conducted in detail, along with a survey of prior applications in human activity monitoring. Following this, the motivation behind this research will be described and the solution to improve actigraphy signal analysis methods for human activity analysis in wake and sleep. 


\subsection{Actigraphs}

To begin with, Actigraphs are miniature, strapable wearable devices, which are used for capturing movementbased vibrations from different areas of the human body. These devices measure the displacement of human body using embedded accelerometer sensors, either in a single or tri-axial $(\mathrm{X}, \mathrm{Y}, \mathrm{Z})$ directions, and register them as vibration or displacement amplitudes. The process of capturing these movements is hence termed as Actigraphy [37].
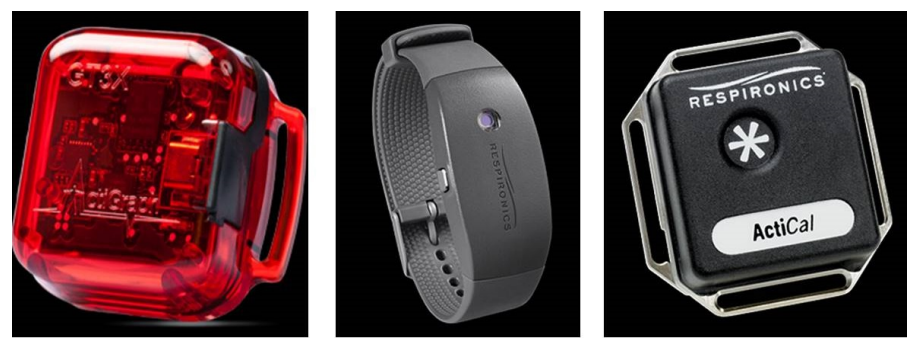

Figure 2.2: Example of actigraphs [10]

The reader must note that actigraphs are wearables which exclusively employ accelerometers for motion sensing, and do not include any other IMU sensors for physiological data acquisition. This being said, many currently available, popular wearables such as FitBit ${ }^{\mathrm{TM}}$ and Apple Watch ${ }^{\mathrm{TM}}$, do have accelerometer sensors in them, and even these could be used towards actigraphy-based studies for analyzing daily activity $[10,32,34,38]$. Currently available actigraphs are mostly wrist-watch shaped (see Figure 2.2), weigh about 20-30 grams depending on their design, and are designed to be used on either the wrist, ankle, waist or thighs for capturing movements [37]. Unlike the afore-mentioned smart wearables, actigraphs do not feature a user interface, but are rather used as simple motion recording devices.

Depending on the manufacturer specifications and battery power, an actigraph can record data for over 24 hours, and can store it on a flash memory for many days or weeks. Typically, these devices can be connected to a local computer via generic connections such as $\mathrm{USB}^{\mathrm{TM}}$, Bluetooth ${ }^{\mathrm{TM}}$, Wi-Fi or even a serial port. Typically actigraphs sample data between $16-3200 \mathrm{~Hz}$, and digitize the movement vibrations with a bit resolution in the range of 6-16 bits per sample [10]. Usually an actigraph maintains a record of zero-crossings and minimal threshold, and uses these values to generate raw values from movements. Currently available actigraphs usually differ in two important aspects:

- Their method of data acquisition, and,

- The settings available to end-user for device customization

From this, it can be inferred that the analysis of motion data may differ from one device to another, 
even if both are manufactured by the same company. This further provides a motivation to investigate into designing device and signal independent actigraphy analysis algorithms. Actigraphs are usually sold as comprehensive packages comprising of the actigraphy module, user manual, strap bands, charging and data transfer cables, and an SDK for research purposes [37-40]. This makes it easier to kick start any activity monitoring project and generate quick analysis results using the manufacturer provided software. That being said, there are also generic actigraphy devices, which do not include many accessories, and in order to analyze their recorded data, users must rely on third-party applications.

Typically, an actigraph is manufactured and embedded with the following components, as illustrated in Figure 2.3:

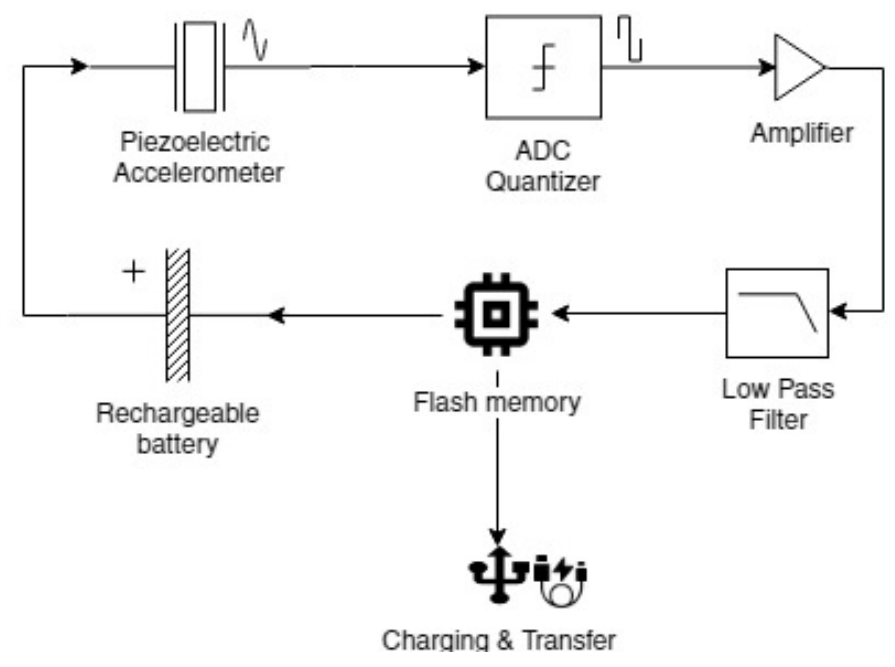

Figure 2.3: Actigraph circuitry

1. Piezoelectric accelerometer which captures vibrations. This type of accelerometer generates a digitized electrical signal, when the device itself experiences "proper" acceleration. "Proper" acceleration indicates a displacement of the device from its initial resting state, and does not account for relative acceleration with respect to earth's gravity [39] [37]. It is in fact because of this reason, why most actigraphs include a computation routine, which normalizes accelerometer's amplitudes with respect to earth's gravity, in order to output the true data to the user. The motion data is further summed using a pre-defined epoch period (typically 1 second), and is therefore measured in terms of counts per epoch, wherein a higher count means higher activity intensity [41]. Therefore, lifting a glass would register a lower intensity as compared to throwing a ball, when captured by an actigraph. Based on this information, it should be noted that in this research work, all the actigraphy signals have been measured with units as counts/g, where $g$ is the earth's acceleration due to gravity $[16,17]$. 
2. The accelerometry data is then digitized using an ADC (analog-to-digital converter), and further amplified using a signal amplifier.

3. The actigraph also includes a low pass filter to remove ambient white noise, and external vibrations.

4. The sampled and filtered data is then stored on a flash-memory.

5. The device also embeds a capacitive, rechargeable battery and a data transfer output port for cable connections.

The actigraphy recording process takes place through the following stages, as illustrated in Figure 3.9.

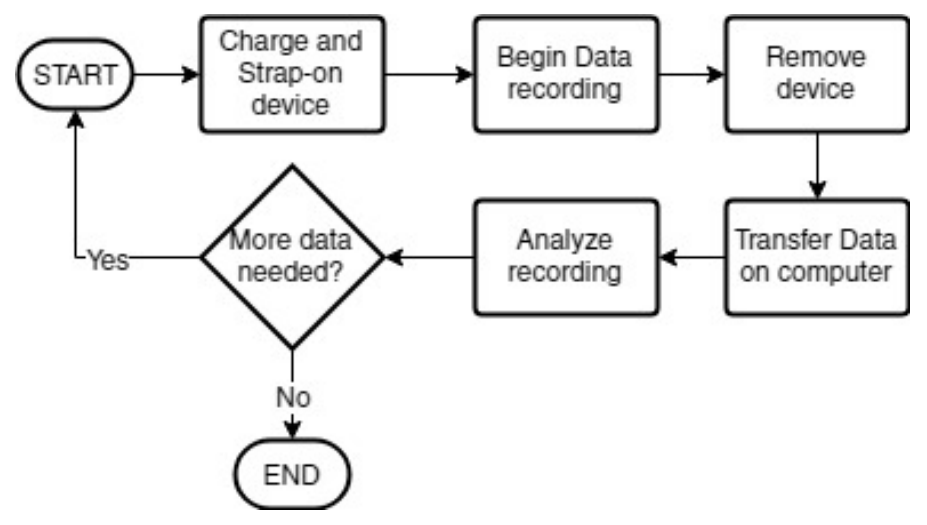

Figure 2.4: Actigraph recording

- Charge the actigraph

- Strap-on and initialize device to begin data acquisition

- Remove device and download recorded data onto the computer

- Analyze using user designed or proprietary software, and decide if enough data has been collected.

Following these basics on actigraphs, the next section describes and analyzes actigraphy signals and their properties.

\subsection{Understanding Actigraphy Data}

Depending on the device, user-specifications or application, the actigraphy data could be single or tri-axial. From a market survey, it was found that generally tri-axial actigraphs tend to be more sensitive when recording movements, as compared to their single-axial counterparts, and are mostly used in applications which require simultaneous recording and analysis [39] [42]. 
A single-axial actigraph records and visualizes vibration data in the form of transient spikes occurring only when an activity exceeds a certain threshold value. When the vibration intensity is less than the threshold, the final output is usually registered as zero. In case of tri-axial actigraphs, motion is captured in the X, $\mathrm{Y}$ and $\mathrm{Z}$ directions, and represented various aspects of acceleration, intensity and activity angle [39] [37]. Figure 2.5 below illustrates tri- and single-axial actigraphy signals captured from two different actigraphs.
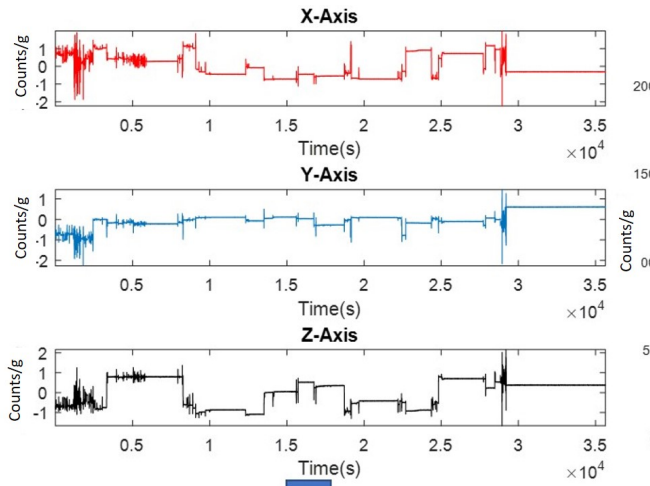

A

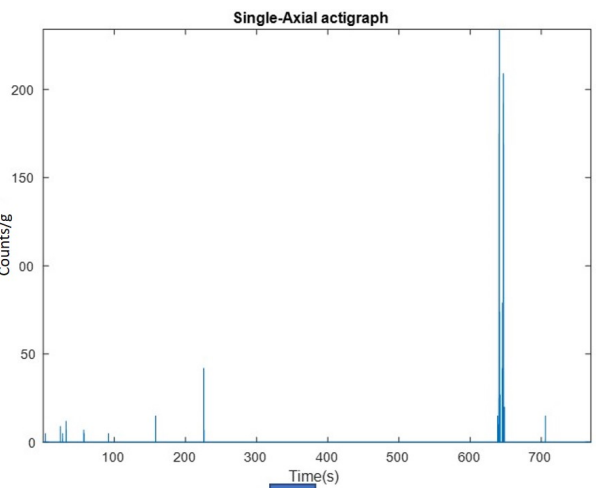

B

Figure 2.5: (A) Tri-axial [16], and (B) Single-axial actigraphy [17] signals from two different actigraphs

To explain this further, a "zoomed-in" version of a sample single-axial actigraphy signal in Figure 3.9. The actigraphy data illustrated in Figure, is a 20-second segment of a 6-hour long signal. It is evident that how an accelerometer captures and registers acceleration data in the form of random, transient spikes.

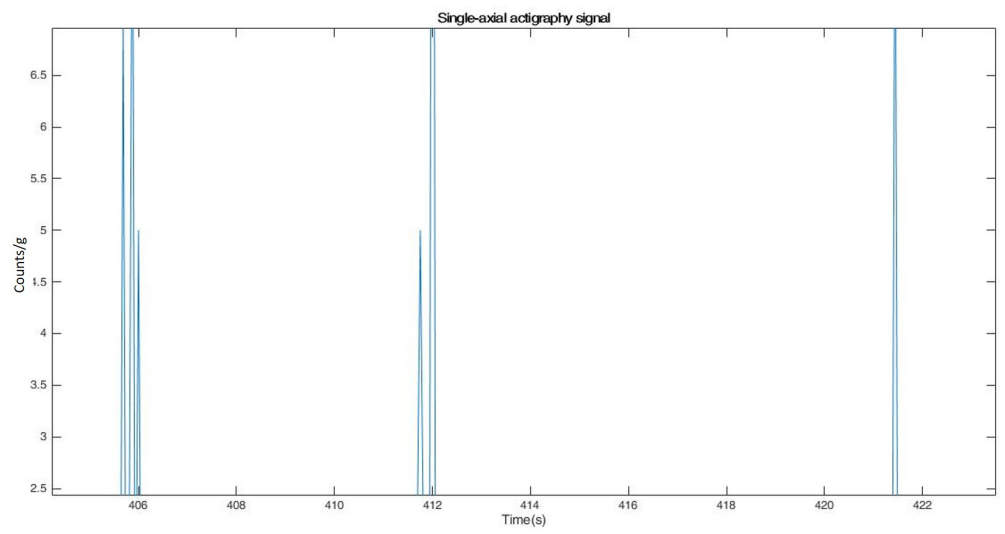

Figure 2.6: 20-second segment of a single-axial actigraphy signal 


\subsubsection{Testing Actigraphy Signals}

Prior to applying suitable signal processing methods for pattern analysis, the properties of any given signal must be taken into account. This can be done by applying simple statistical property tests and using their outcomes to determine further analysis approach. The key property tests are described as follows $[10,36]$ :

\section{Visual Inspection:}

The first step for any signal analysis researcher is to simply look at a sample signal, and deduce certain observations about its patterns and behavior. These observations are usually very helpful in designing a first set of experiments for signal analysis.

\section{Stationarity:}

In simple terms, a stationary process is a stochastic process whose first and second order statistics do not change with time. That is, its mean and variance remain constant. Generally speaking, when it comes to analyzing non-stationary data such as physiological or biomedical signals, it is assumed that their mean and variance do not change within a given time-frame, and accordingly these signals are segmented for analysis [43]. The assumption made in such cases is that the underlying signal generating process is wide-sense stationary (WSS), that is, its mean and autocorrelation function do not change with time.

\section{Linearity:}

Linearity in signals means that the system producing the data shows two key properties - homogeneity and additivity. Homogeneity indicates that a change in the system's input results in a corresponding change in its output signal. That is, given an input $x$, if the output of the system is $y$, then for an input $c x$, the output must be $c y$. Similarly, in terms of additivity, given that inputs $x_{1}$ and $x_{2}$ result in outputs $y_{1}$ and $y_{2}$ respectively, then the output for a combination $\left(x_{1}+x_{2}\right)$ should be $\left(y_{1}+y_{2}\right)$. When both of these conditions are satisfied simultaneously, then the given system and its output signal are termed as linear in nature [44].

\section{Gaussianity:}

Gaussianity indicates a normal distribution for a given signal or dataset. Most commonly called as bell curve, it represents the probability distribution of many random variables or samples (in a signal), with the most frequently occurring value towards the middle of the curve. As the frequency of occurrence goes down, the signal values are distributed more towards the extreme ends of the curve [45]. It should be noted that the mean, mode and median are all the same in a normal or Gaussian distribution.

\section{Sparsity:}


A signal $S$ containing $n$ samples, is said to be sparse if it contains $k(k \ll n)$ very few significant and large sample values or coefficients, and many small or zero coefficients. When the $(n-k)$ small coefficients are discarded and only the $k$ samples are retained, a sparse representation of the signal $S$ is obtained. Sparsity of the signal is then defined as [46],

$$
\text { Sparsity }=\frac{(n-k)}{n}
$$

In this study, the afore mentioned tests were conducted on various actigraphy datasets using standard functions and methods available in MATLAB ${ }^{\mathrm{TM}}$, and the following results were obtained as highlighted in Table 2.1.

Table 2.1: Actigraphy signal tests

\begin{tabular}{|c|c|}
\hline Property Test & Observations \\
\hline Visual inspection & $\begin{array}{l}\text { Spiky data with a large amounts of transient information randomly distributed. } \\
\text { Movements or peaks seem uncorrelated when separated by significant time period. }\end{array}$ \\
\hline Stationarity - KPSS test [43] & Actigraphy signals are non-stationary \\
\hline $\begin{array}{l}\text { Linearity - } \\
\text { Augmented Dickey-Fuller test [44] }\end{array}$ & Actigraphy signals exhibit non-linearity \\
\hline Gaussianity - KS test $[45]$ & Non-normal distribution in most cases, since human motion is random. \\
\hline Sparsity test - Gini Index [46] & $\begin{array}{l}\text { Actigraphy signals are sparse in short windows. In case of tri-axial data, vector } \\
\text { compounding and additional quantization may be needed. }\end{array}$ \\
\hline
\end{tabular}

The next section will present a review of some noted prior works in actigraphy-based activity monitoring applications. This will be followed by the motivation behind this research, the problem definition and the solution to improving actigraphy signal analysis.

\subsection{Prior Actigraphy Applications}

After reviewing numerous works in wearable applications it was found that actigraphs have been extensively used for human activity monitoring purposes. It must be noted that, being exclusively recording devices actigraphs have been highly useful in this research as well as prior works pertaining to long-duration human activity data acquisition and analysis. Actigraph-based activity monitoring applications have been exten- 
sively conducted in clinical and non-clinical research studies. From a non-clinical perspective, actigraph-based studies were mostly conducted on fitness monitoring and simple activity monitoring applications [38,40]. For example, Bruno et al. [7] performed human activity recognition and modeling using an actigrapy dataset which included various activities of daily life performed by healthy volunteers. Apart from this, numerous wearable manufacturers such as FitBit ${ }^{\mathrm{TM}}$ have conducted closed-loop studies in fitness monitoring using consumer data, and generating individual-level longitudinal insights into exercise levels, calories burnt and step count [47].

From a survey of literature it was found that, most actigraphy-based activity monitoring applications were conducted as part of clinical validation studies. The primary objective of these works was to assess the feasibility of using actigraphs as remote or home-based activity monitoring devices, as screening tools for various physical and mental disorders, as well as for rehabilitation purposes. To describe further, following are some noted clinical studies conducted using actigraphs:

- Home-based sleep staging: Actigraphy-based studies have been conducted for detecting sleep and wake states in healthy individuals as well as those experiencing sleep disorders. Some studies also included determining sleep stages and comparing the detection accuracy with PSG (polysomnography) standards [48-54].

- Sleep disorder detection: Many prior works have been conducted in detecting or screening individuals suffering from insomnia, depression, restless leg syndrome and periodic limb movements. Some studies were also focused on assessing daily activity in subjects experiencing Parkinson's and Alzheimer's disease $[5,6,55-66]$.

- Activity in children: Actigraphy based activity monitoring has been conducted in prior studies with children suffering from autistic spectral disorder and attention deficit hyperactivity disorder (ADHD) $[67,68]$.

- Rehabilitation: In few studies, actigraphs were used for monitoring daily activity in military personnel undergoing rehabilitation from joint related injuries, and assess their progress [69-71].

- Demographic studies: Actigraphy has been used to conduct community based studies for determine sleeping patterns along with age, gender and ethnicity in a given geographical area. These studies were conducted to determine sleep disorder prevalence as well [72].

A review of prior literature indicated that clinical and non-clinical actigraphy application studies were mostly conducted using one or more of the following methodologies [59,64,65, 73, 74]: 
- Manual actigraphy data analysis using human expertise. This involved scheming through vast amounts of actigraphy data per test subject and identifying regions of interest or peak activity for a specific application. For example, clinical studies were conducted for detecting periodic limb movements in sleep, by comparing a periodic limb index (PLMSI) score computed using conventional PSG (polysomnography) readings with actigraphy data.

- Conducting subjective questionnaires with individuals or patients.

- Statistical analyses of actigraphy data. This involved computing simple first and second order parameters such as mean, variance and standard deviation, and using them to assess activity levels.

- Demography based assessments on determining the efficacy of using actigraphs for home-based activity monitoring studies. For example, an actigraphy-based assessment was conducted to determine sleeping patterns with respect to age and gender in a given community [72].

- Applying signal processing methods and mathematical models to analyze actigraphy data, and determine patterns with respect to specific activity. For example, a prior study involved classifying mild and severe patients experiencing periodic limb movements in sleep from actigraphy signals recorded in sleep $[5,75]$.

As evident from afore described review, actigraphs have been used in a plethora of applications and despite their extensive usage in activity monitoring, few gaps have been identified which could be addressed in improving actigraphy data analysis using signal processing techniques. The next section will discuss the key challenges in actigraphy data analysis and the motivation for this research work.

\subsubsection{Key Challenges and Motivation}

Generally, before an actigraphy signal is analyzed for identifying activity patterns, it undergoes following signal processing operations [32] [36] [37]:

- Analog-to-digital conversion which assigns discrete real or integer values to movement amplitudes.

- Filtering digitized actigraphy signal to retain true activity movements. A review of prior works and experiments with different actigraphy datasets revealed that most human activity is captured between 0.3-6 Hz $[10,39,42]$. Therefore, applying a first order low pass Butterworth filter to actigraphy data can help capture this activity and remove ambient noise along with redundant peaks.

- Some prior works have also employed the use of additional band pass filters to remove low and high frequency artifacts, and noise. 
- Depending on specific applications, actigraphy data may also be truncated. For example, some studies performed clipping sleep actigraphy signals based on "lights-on" and "lights-off" times $[5,75]$.

A review of prior works indicated that actigraphs usually sample data at higher frequencies (typically $50 \mathrm{~Hz}$ and above) and sometimes couple this with a high quantization rate (about 12-16 bits per sample). This leads to higher memory consumption, and may cause battery usage limitations while recording [10]. Although currently available actigraphs have good specifications, a detailed investigation into prior works and experiments with actigraphy data revealed that there are some potential challenges, which need to be met in order to improve their efficiency as standalone devices, or even in smart wearables $[37,76]$. These key challenges are:

1. Actigraphs that sample data at higher frequencies (typically $100 \mathrm{~Hz}$ and above) along with a high quantization rate (typically 12-16 bits per sample), often lead to memory leakage and under-utilization of battery life during recording [10].

2. Manufacturer-based variability in sampling and quantization. This limits algorithms from being designed as device-independent tools $[10,37,76]$. Some actigraphs tend to sample movement data too infrequently, thus leading to information loss in the output raw signal.

3. Many prior studies have been conducted on short-duration actigraphy datasets and did not require extensive memory and computational resources for analysis $[48,58]$. Translating these studies into longterm activity monitoring solutions is not feasible unless the actigraphy data is subjected to significant compression and segmentation at the source.

4. Increased use of computational resources (local or cloud) during offline processing of long-term recordings. Conventionally, actigraphy data is captured and entirely transferred to a local computer or cloud for analysis. A prior literature review indicates that in most studies, no prior data processing is done at the source to retain only meaningful information and discard redundant values.

5. Despite a plethora of prior studies conducted for clinical validation of actigraphs as potential tools for home-based monitoring, very few have been successfully implemented in the wearable market. The main cause for this is the methodology opted in these studies, which focused on deriving statistical and subjective parameters with aid of clinical experts, rather performing extensive analysis of the actigraphy data.

These challenges motivated this research into performing a systematic investigation into actigraphy signal analysis, and develop a system which is capable of performing the following core tasks: 
1. Data compression and denoising at the source.

2. Adaptively segment actigraphy data at the source and extract regions of interest.

3. Extract actigraphy-specific and activity-specific features which provide maximum relevance to the application.

4. Apply light-weight machine learning tools to classify activity or patterns, without imposing computational load on cloud or local computer $[30,77,78]$.

The next section will illustrate the developed solution to various challenges in actigraphy signal analysis, and describe in the detail the algorithms developed through this study.

\subsection{Research Contribution: Actigraphy Analysis Application}

A review of previous studies indicates that the benefits of using actigraphs or accelerometer-based wearables as home-based activity monitoring devices are numerous. Apart from tracking neuromuscular and sleep disorders, they could also be used for monitoring joint disorders, assessing rehabilitation and post-treatment effects and for overall wellness purposes. Considering these applications, this research study has led to the development of a novel actigraphy analysis system which would be device-independent, IoMT-friendly, userfriendly and self-learning in nature. Figure 2.7 illustrates the developed system in the form of an algorithmic flowchart. 


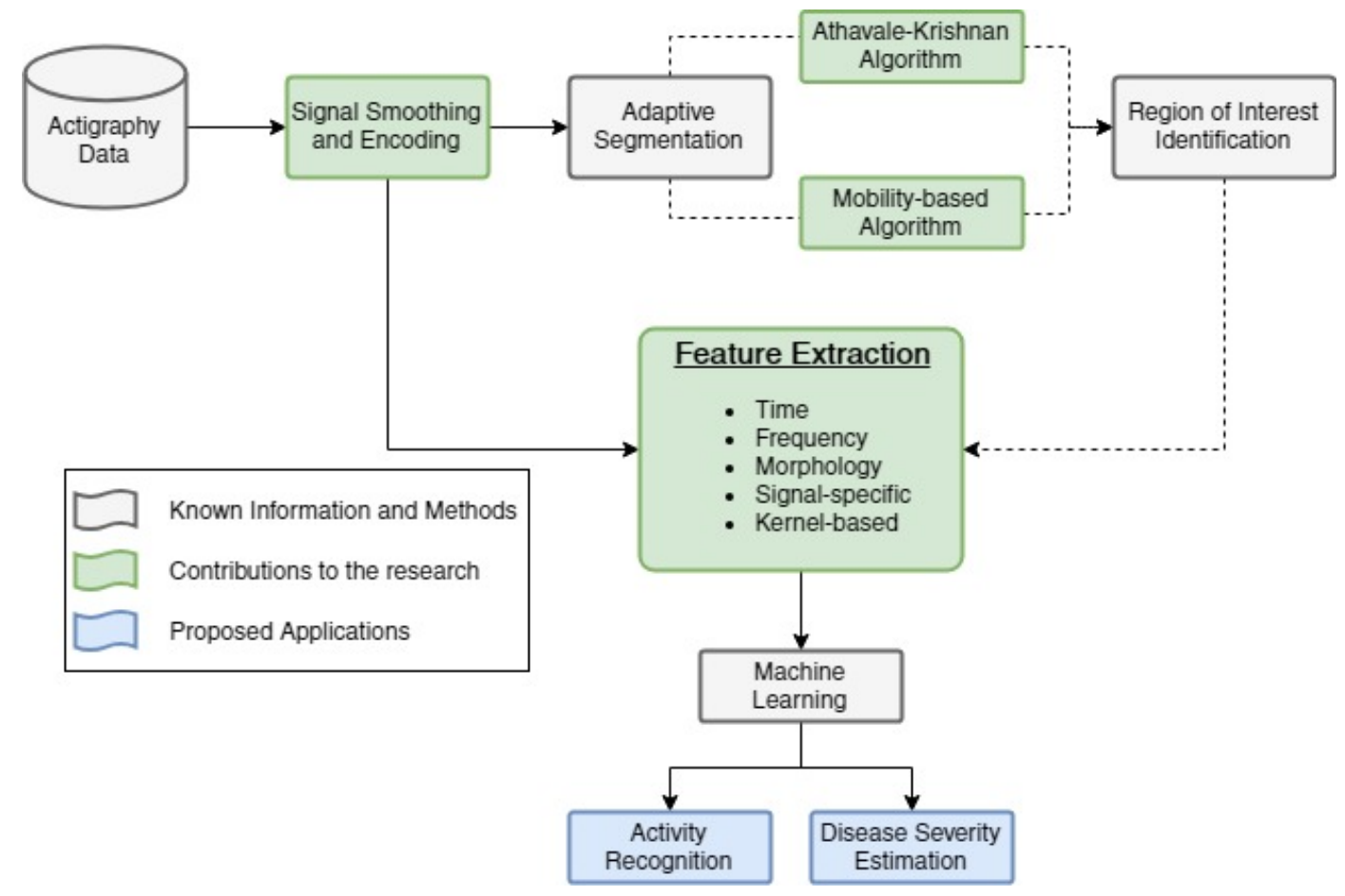

Figure 2.7: Flowchart of the Actigraphy Analysis System

The key contributions to this research, as highlighted in Figure 2.7, have been summarized as follows along with the specific challenge they intend to address:

\section{- Signal Smoothing and Encoding:}

In this study an actigraphy-specific signal smoothing and encoding module has been developed, which performs on-the-fly signal compression and denoising at the source. Unlike conventional pre-processing techniques, this module comprises of three basic operations:

1. Vector compounding of accelerometry data in case of tri-axial signals

2. Signal denoising and artifact removal

3. Bit-rate reduction, and

4. Signal compression

This module has been targeted towards addressing challenges in actigraph-specific variability in sampling rate and bit-resolution, by ensuring a uniform, very low $m$-bit encoding which would lead to optimizing memory and battery usage. Additionally, this would also help in developing actigraphy analysis algorithms as device independent tools, since the data could be uniformly encoded, denoised and compressed despite of varying sampling rate or bit resolution. 


\section{- Encoding-based Adaptive Segmentation Algorithm:}

This is a patent-protected algorithm developed in this research, and intends to perform adaptive segmentation of actigraphy signals using the signal encoding operation and activity-specific information. The algorithm tends to discard redundant and unwanted movement components from the signal, and only extracts regions of interest for further analysis. This would overcome the challenge of conducting long-term monitoring using cloud-based resources for analysis. A compression based adaptive segmentation of actigraphy data would ensure that most processing happens at the device level thereby reducing computational overheads.

\section{- Mobility-based Adaptive Segmentation Algorithm:}

Similar to the Athavale-Krishnan algorithm, an alternative mobility-based algorithm was also developed, which uses Hjorth's mobility parameter $[79,80]$ to compute intra-segment similarity within a signal for its adaptive segmentation. This algorithm also considers activity-specific information before generating regions of interest for pattern analysis. The idea of developing an alternative segmentation algorithm is to address challenges when it may not be feasible to apply encoding-based segmentation. For example, this algorithm could also be used for clinical data such as EEG or EMG signals collected during polysomnography.

\section{- Feature Extraction:}

This study also resulted in the extraction of two actigraphy-specific features, namely - Rapid Change Factor and Spiky Index. These parameters represent the instantaneous change in an actigraphy signal, and would be useful to detect movement patterns from the data. In addition to this, a new kernel model based on the autocorrelation function was introduced. This function performs a kernel-based feature extraction, and reduces the dimensionality of an actigraphy signal to four distinct parameters based on the mean and variance of the signal. The inclusion of actigraphy-specific features can definitely address the challenge of characterizing actigraphy signals without the use of statistical or subjective parameters, which otherwise might need clinical expertise for estimation. Additionally, this would also help better quantification and characterization of different human movements.

Emanating from this research study, the developed actigraphy analysis system could be foreseen to be used in two specific applications as shown in Figure 2.7:

\section{- Disease Severity Estimation:}

Many people suffer from certain neurological disorders such as Alzheimer's disease or Parkinson's disease, and neuromuscular disorders such as Restless leg syndrome (RLS) or periodic limb movements 
in sleep (PLMs). Using actigraphs in their daily life would not only track their activity, but would also be useful in detecting sleeping patterns, abnormal movements and treatment effects for a better quality of life.

\section{- Activity Recognition:}

The system could be used to develop applications which are able to detect different activities of daily life, and provide feedback to the user on their wellness and fitness. Additionally, these applications could also be useful in an assistive or rehabilitation environment by helping individuals perform routine activities with ease.

With regards to afore-mentioned verticals, the reader must note at this juncture that the core domain of this research study has been sleep actigraphy analysis; whereas the purpose of including wake actigraphy data has been done to show the feasibility of applying the contributed methods through this research to a myriad of actigraphy applications. Therefore, it may occur that some aspects of wake actigraphy may not be covered in detail, but would rather give the reader a flavour of different actigraphy applications.

After covering the basics of actigraphs, their signals, prior applications and the devised solution, the next chapter proceeds towards discussing various actigraphy signal analysis methods. This chapter will provide the reader a detailed outlook of existing methods in actigraphy signal analysis, along with a focus on the key contributions to this research work.

\subsection{Summary}

The second chapter of this dissertation zooms into the core topic of this research work - Actigraphy. To give the reader a well rounded idea, this chapter begins with a detailed discussion on actigraphs, their construction, and how they capture movement data using accelerometers. This is followed by delving into the structure and properties of actigraphy signals by doing a detailed analysis of some sample raw actigraphy signals. Using standard signal property tests in MATLAB ${ }^{\mathrm{TM}}$, the key behavior of actigraphy signals is unraveled with respect to visual perception, linearity, stationarity, Gaussianity and sparsity. Next, Section 2.3, conducts a brief literature review of prior actigraphy applications in various health monitoring verticals, type of studies conducted, and also lists down certain key challenges and limitations from prior works. These challenges then provide a motivation to develop the actigraphy analysis solution described in Section 2.4. This chapter then concludes by illustrating and describing the actigraphy analysis solution, thus giving the reader a brief idea about the contributions to this research study. 


\section{Chapter 3}

\section{Actigraphy Signal Analysis}

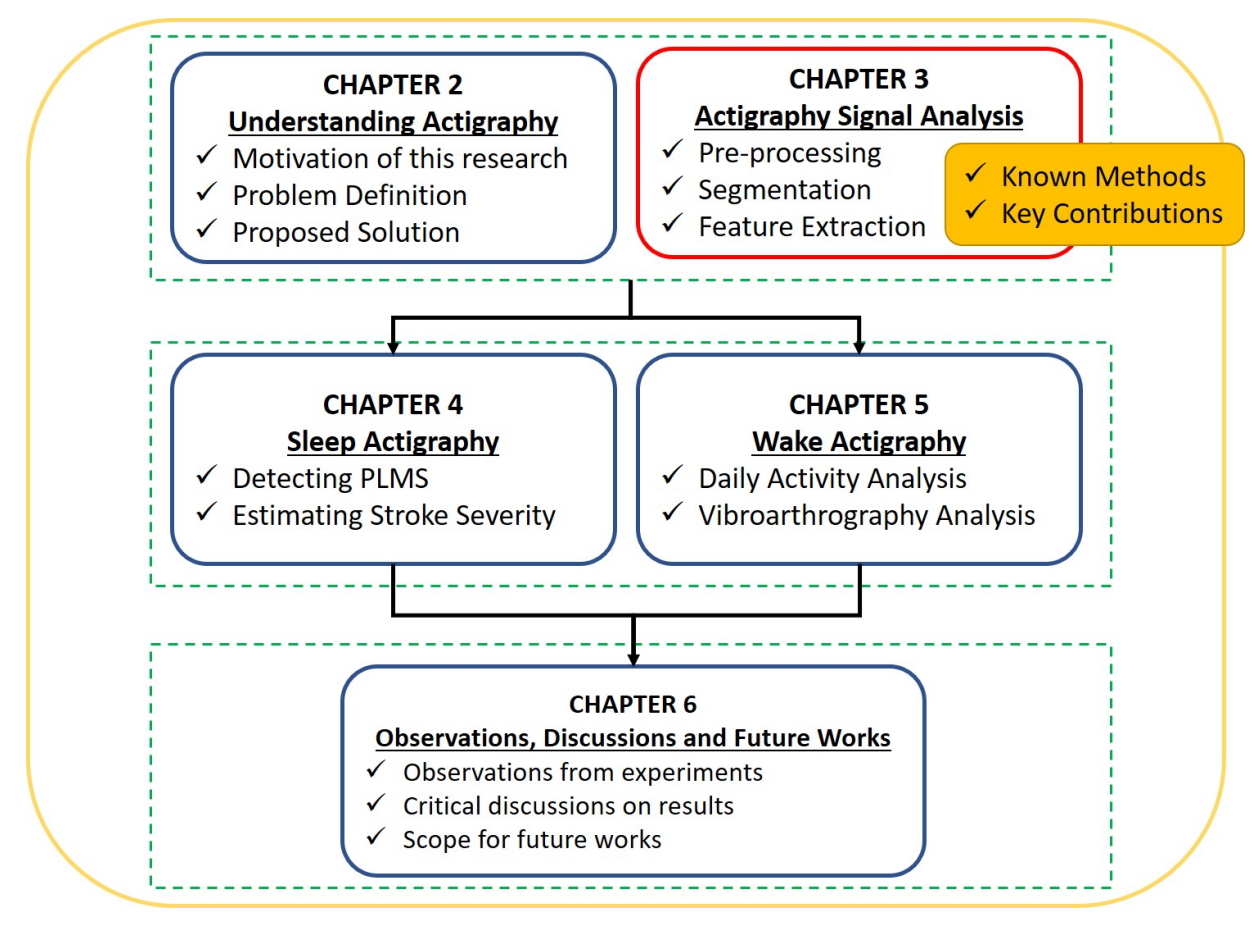

Figure 3.1: Organization of Dissertation

In today's world, huge amounts of signal data gets captured by artificial and natural sensors, which is then used in numerous applications such as analysis, decision making or simple transmission and reception. This captured data is mainly redundant in two aspects: (a) there could be multiple correlated versions of the same information in the signal, and (b) each signal is usually densely sampled [36]. It is surprising to know that the relevant information which causes signal generation in a specific pattern, if extracted efficiently, is usually of much smaller dimensions as compared to the original data. This causal information helps in signal 
analysis and reconstruction, and discrimination within signal classes. This would also mean that there exists a dimensionality gap between the physical processes and their causal observations, which would imply the difference between their respective representations by the sensor and in the physical space [36].

Any signal processing problem involves identifying signal-source behavior, extracting characteristic properties, applying mathematical models to increase or decrease dimensions and identifying patterns. Physiological signal analysis from clinical and non-clinical sources not only deals with understanding the disease/disorder morphology, or specific human activity, but also presents a problem of deciphering instantaneous hidden information from the non-linear and non-stationary signal patterns $[32,36]$.

Considering the rapid evolution in signal processing techniques, in today's date a plethora of options for analyzing signals are available. To begin with a simple time-domain or frequency-domain based approach could be taken to understand a signal, or even a joint time-frequency representation to transform it into two dimensions (TF plane) for simplification [36]. Recent advances in information sensing have made it easier to decipher instantaneous information from non-stationary signals using a minimalist approach [32]. The objective behind any of these methods remains the same - efficient feature extraction and pattern classification. In most cases, the flow of analyzing physiological signals occurs in six stages [36],

- Understanding the source and signal through visual inspection. This covers the morphological aspect of physiological data.

- Pre-processing for A-to-D (analog to digital) conversion, denoising, filtering, smoothing and artifact removal.

- Segmenting or truncating the pre-processed signal into sizable portions for analysis. The segmentation is performed either by taking fixed-window approach, or an adaptive approach wherein the signal behavior is considered and it is segmented based on certain key events observed in a given time period.

- Next, a mathematical model is applied to change the dimensionality (usually reduce) of the signal for simplification during analysis. This generates key parameters or features from the signal which are useful in identifying signal-source behavior and its patterns.

- The features generated from the signal modeling are then used for training and testing machine learning tool for predicting and identifying patterns from the signal.

- Finally, a feedback structure is created which helps in correcting misclassified patterns and fine tuning the machine learning tool.

The reader must note that not all physiological signals exhibit specific structure or patterns. For example, an ECG (electrocardiogram) would show characteristic PQRST peaks and troughs in a typical heart beat, 
but the corresponding EMG (electromyography) would exhibit a very random and sparse structure with respect to the heart muscle activity $[32,36]$. Though most biomedical signals are captured on the basis of the electrical activity of nerves and muscles using electrodes or similar sensors, there are some signals which are captured using different modalities [32]. For example, eye movements and facial expressions can be analyzed using camera data, neural activity in brain can be identified using infrared imaging, and movement activity can be collected using accelerometer and gyroscope-based devices [32]. This being said, the key to strong signal analysis is to develop or extract robust features which are invariant to device or signal source changes. This would then ensure a seamless application of machine learning tools to identify patterns.

In this research work, the focus has been on understanding and analyzing actigraphy signals captured in daily sleep and wake activity $[5,6,10,20,75]$. With regards to this, Chapter 2 first built a foundation on actigraphs and understanding their signal behavior. This chapter now proceeds with a detailed discussion on processing actigraphy signals for pattern recognition in activity monitoring applications. The first section will start this by describing the various approaches in pre-processing raw actigraphy signals, and how each method helps in removing noise, redundant data and artifacts. Next, approaches to how to effectively segment actigraphy signals in order to extract regions of interest or peak movements will be explored, which would be then useful for extracting robust features for analysis. Also, different feature extraction methods will be discussed, in order to cite which would be best suited for actigraphy signal analysis. This chapter is then concluded by providing the reader a master-table of features and segmentation methods used in this research study of actigraphy signal analysis.

\subsection{Actigraphy Pre-processing}

The first step to analyzing any signal is to clean and condition it before delving into pattern analysis. This conditioning of signals to remove unwanted information is termed as signal pre-processing and could be performed using various mathematical methods [36]. Usually, before proceeding to extract signal information, it first digitized using an A-to-D converter. This way it becomes easier to identify signal properties over discrete time intervals rather scheming the entire analog signal for instantaneous information. At the grass root level, the simplest way is to apply digital filters which remove unwanted artifacts from the signal such as third party components, ambient noise, DC drift, redundant signal values and overlapping white noise. Selecting a pre-processing method is not trivial, and one may approach the problem in two ways [36]:

1. Designing signal specific filters if the properties of unwanted artifacts are known, or,

2. Taking a trial-and-error approach to filtering signals if the artifact information is unknown. 
As described and tested in Chapter 2, actigraphy signals are non-linear, non-stationary, non-Gaussian and sparse in short windows [10]. Upon visual inspection of various actigraphy signals, it was also observed that the data tends to be spiky and transient in nature, occurring at random time intervals depending on the movement activity recorded by the accelerometer sensor. From a review of prior studies in actigraphy analysis, it was found that human motion is mostly captured in the $0.3-6 \mathrm{~Hz}$ range $[10,39]$, and tends to get distorted by high frequency noise around the sampling frequency.

In addition, actigraphy data is also affected by DC drift which needs to be removed using de-trending techniques. Implementing suitable low pass and band pass filters to denoise actigraphy recordings could be useful for short-duration data. But when analyzing long-term actigraphy, these additional pre-processing components could increase the computational overhead, or even the circuitry design on a wearable device embedded with accelerometer for activity recording [10,20,32]. A literature review indicates that actigraphy signals are inherently quantized with a 6-16 bit resolution depending on the manufacturer specifications $[10,20]$. Using these test results and observations, further investigation into various known actigraphy preprocessing methods has been conducted in the following sections.

\subsubsection{Known Pre-processing Methods}

Any given raw actigraphy signal $S_{r}$ can be pre-processed through one or more of the following sequential operations. Note that this is a de facto model as per prior studies for actigraphy signal processing, and could be modified as per the application.

\section{Normalization:}

When an accelerometer sensor senses movement, it registers data or peaks which include the effect of earth's gravitational force. In the absence of activity, the accelerometer senses only the acceleration due to gravity and registers the same in the memory. In order to remove signal components which have been amplified or caused due to earth's gravitational effect, the device manufacturer usually provides a mathematical formula which is then applied to raw acceleration data for normalization. In this study, actigraphy datasets were collected from four different actigraphs, which came with their manufacturerspecific data normalization formulae. This would also give a specific unit to each actigraphy signal in the form of counts $/ g$. Given a raw actigraphy signal $S_{r}$, its normalized version can be computed as follows:

- For the single-axial sleep actigraphy signals captured using the Philips ${ }^{\circledR}$ Actical $^{\mathrm{TM}}$ device, the normalized actigraphy data was directly obtained from the device's SDK (software development kit), without the need to manually remove the $g$ factor [17]. Note that here $g=9.8 \mathrm{~m} / \mathrm{s}^{2}$, the 
acceleration due to gravity.

- In case of tri-axial sleep actigraphy signals captured using the GC Dataconcepts Model X-16 $6^{\mathrm{TM}}$ actigraph, the movement data was normalized using the relation [16],

$$
S_{\text {norm }}=\frac{S_{r}}{2048 \text { counts } / g}
$$

- For the wrist-actigraphy signals captured using a generic accelerometry device in the ADL (Activities of Daily Life) dataset [8], the normalization was performed as per the instruction manual cited in [8] and included with the dataset folder,

$$
s_{n o r m}=-1.5 g+\frac{S_{r}}{63} \times 3 g
$$

- For the vibroarthrography dataset focused on detecting knee-joint disorders, the signal was normalized using the expression [27],

$$
S_{n o r m}=\frac{\left(\max _{S_{r_{i}}}\left(S_{r}\right)-S_{r}\right)}{\left(\max _{S_{r_{i}}}\left(S_{r}\right)-\min _{S_{r_{i}}}\left(S_{r}\right)\right)}
$$

where $\max _{S_{r_{i}}}\left(S_{r}\right)$ and $\min _{S_{r_{i}}}\left(S_{r}\right)$ denote the maximum and minimum amplitudes in the raw signal $S_{r}$, and $i=1,2, \ldots, n$ represents the number of samples present in the actigraphy signal.

In case of tri-axial signals the normalization operation is applied to each axial data before performing vector compounding of tri-axial information. The normalization of an actigraphy signal has been shown in following Figure 3.2. 

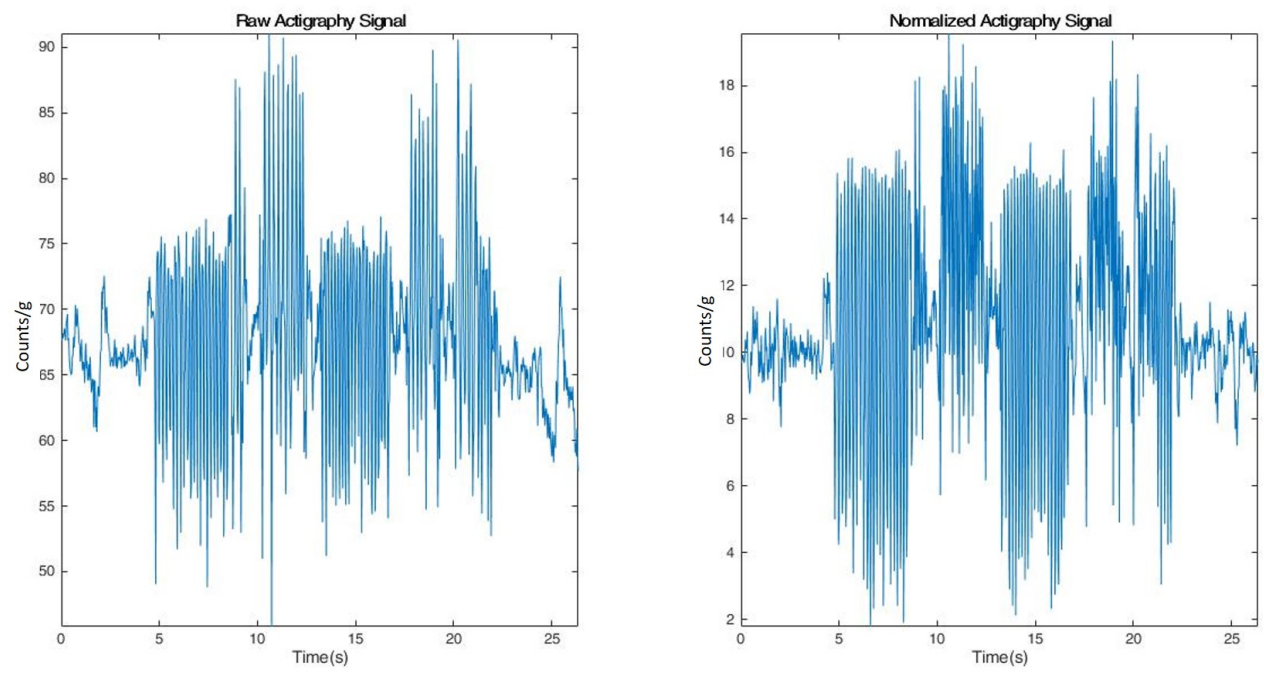

Figure 3.2: Actigraphy Signal Normalization

\section{Detrending:}

Detrending refers to the removal of DC drift or DC bias from a signal whose mean amplitude is not zero. This means, in case the mean amplitude is zero, there would be no DC bias. De facto standards indicate that the simplest way to remove DC drift is to subtract each sample of the actigraphy signal from the mean value [36]. For example, given a normalized actigraphy signal $S_{\text {norm }}$, its detrended version would be,

$$
S_{d c}=S_{\text {norm }_{i}}-\left(\frac{\sum_{i=1}^{n} S_{\text {norm }_{i}}}{n}\right)
$$

It must be noted that in case of tri-axial data, detrending should be performed for each axis. Detrending actigraphy signals is highly useful in denoising data, as it helps remove redundant values and noise around the mean amplitude value. As illustrated in Figure 3.3, it can be observed that upon detrending different types of actigraphy signals, the single-sided magnitude spectrum shifts up positively, thereby indicating the presence of true movement amplitudes. Figure 3.3 illustrates the effect of detrending actigraphy signals. Notice how the magnitude range of the detrended version is lower than the normalized signal. 

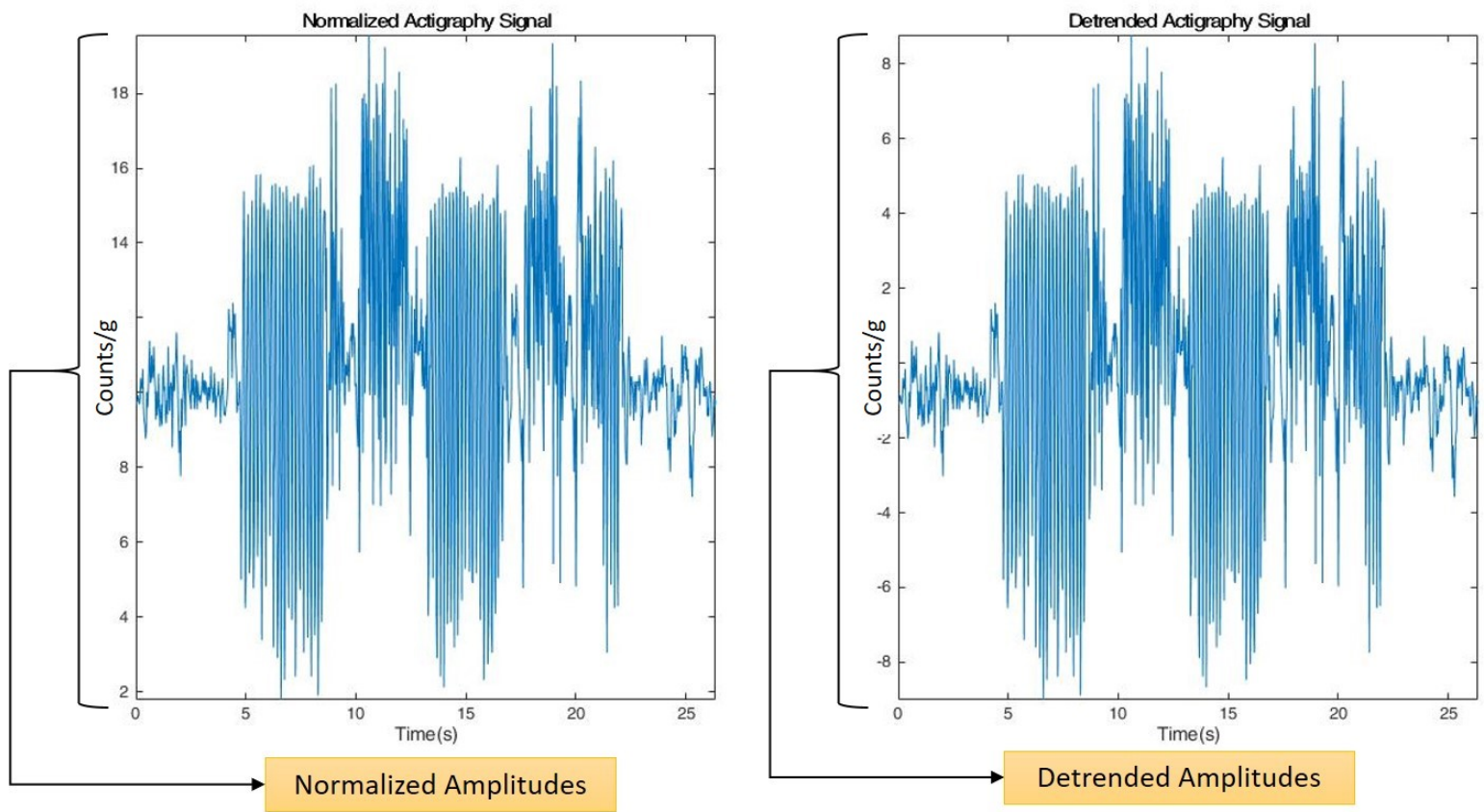

Figure 3.3: Actigraphy signal detrending

\section{Filtering:}

Another approach to denoising actigraphy data and retaining vital movement information, is application of digital filters which restrict the signal within a frequency range [37, 51,81]. In most prior applications, actigraphy signals have been filtered either using a second order low pass Butterworth filter, or a band-pass Butterworth filter [51,81]. As mentioned previously, since it is known that human movement information is usually captured within $0.3-6 \mathrm{~Hz}$ frequency range, a second-order low pass filter can be applied with a pass-band of $0.4 \mathrm{~Hz}$ and a stop-band of $1.6 \mathrm{~Hz}$ using the relation,

$$
S_{f}(t)=S_{d c}(t) \circledast h_{l p f}(t)
$$

where, $h_{l p f}$ denotes the impulse response of the filter. Contrary to prior literature which indicated that human movements could be captured up to $6 \mathrm{~Hz}$, in this research the 0.4-1.6 Hz band was selected for filtering, as the actigraphy signals obtained from different datasets in this study, exhibited maximum movement information within this frequency range, and a second-order low-pass filter gave a better coverage of spectral peaks, thereby retaining vital movement information. Figures 3.4 and 3.5 illustrate the application of a second-order low pass Butterworth filter to the $\mathrm{X}$-axis component of a sample triaxial actigraphy signal captured in sleep. 

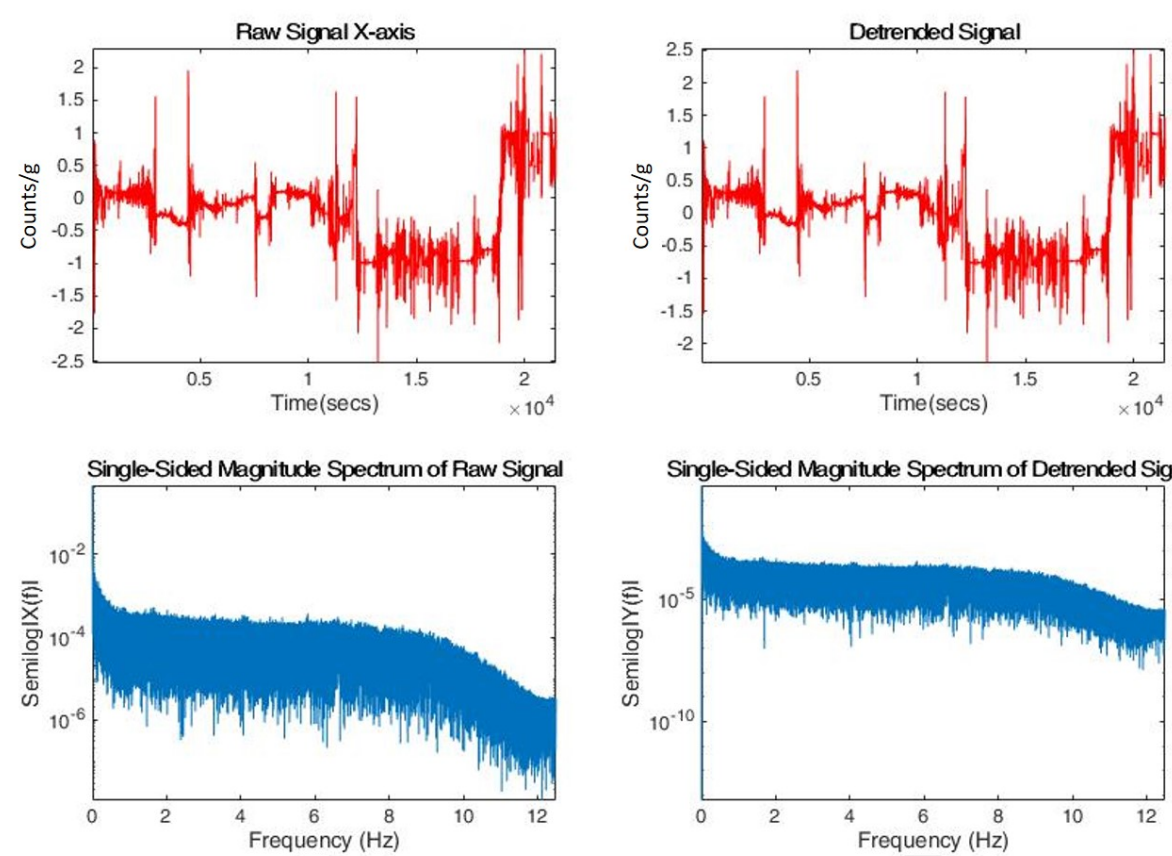

A

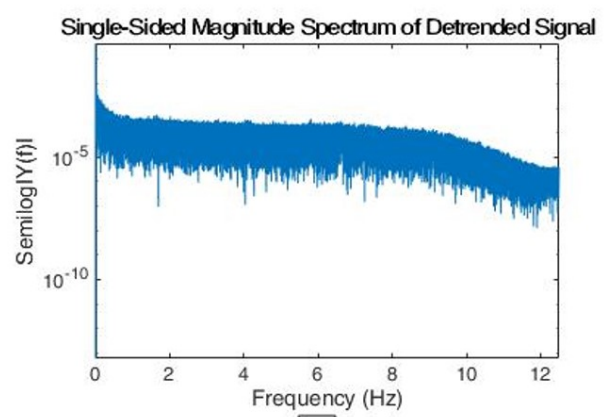

$B$

Figure 3.4: Spectral plots of detrended and low pass filtered actigraphy signals

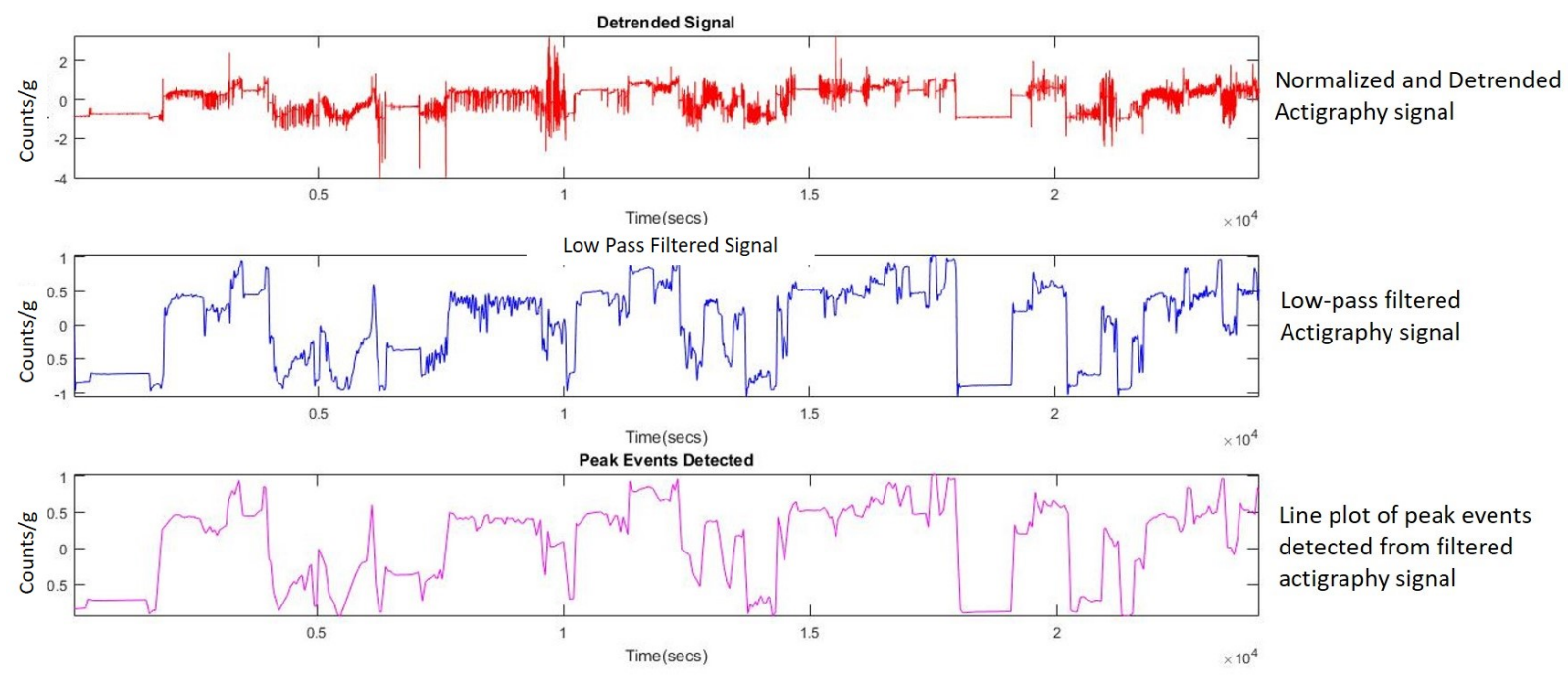

Figure 3.5: Comparison of filtered actigraphy signal with its detrended version

Figure 3.5 depicts the low-pass filtering of a sample, long-duration sleep actigraphy signal. As illustrated, the first plot shows a detrended version of the raw actigraphy signal, which is then filtered to obtain the second sub-plot in the figure. The third plot is a line-plot of all the peak events detected in 
the filtered signal.

The reader must note here that in this research study, an alternative approach to signal filtering has been taken, in the form of a lower bit encoding procedure, which would ensure denoising and compression at acquisition source. Further to this, it may also be noted that in this study, the aspect of filtering has been covered to compare its performance against the signal encoding method.

\section{Wavelet-based denoising:}

Another way of removing unwanted artifacts and smoothen an actigraphy signal is the application of Haar-wavelets. Haar wavelets belong to the class of discrete wavelet transforms (DWT). They can be used as a sequence of square shaped functions, which when applied to a non-stationary signal, detect local features through their orthogonality [18]. Additionally, the square nature of a Haar wavelet helps in denoising the signal by smoothing out noisy components, redundant values and insignificant peaks. This property would be useful in denoising actigraphy data which exhibits similar behavior in the form of spurious peaks and noisy data. Another reason for using Haar wavelets for denoising actigraphy signals is that it is able to generate highly discriminative coefficients which could help in improving the feature space visulaization and pattern classification [82]. A Haar wavelet's basis function, also known as the mother wavelet is given by the expression [18],

$$
\psi_{(s, p, \tau)}(t)=\psi_{p}\left(2^{d} t-n\right)
$$

where, $s$ represents the scaling parameter, $p$ is the phase parameter of the frequency shift in the signal and $\tau$ is the shift in time parameter. Figure 3.6 depicts the mother wavelet and its corresponding scaling function.
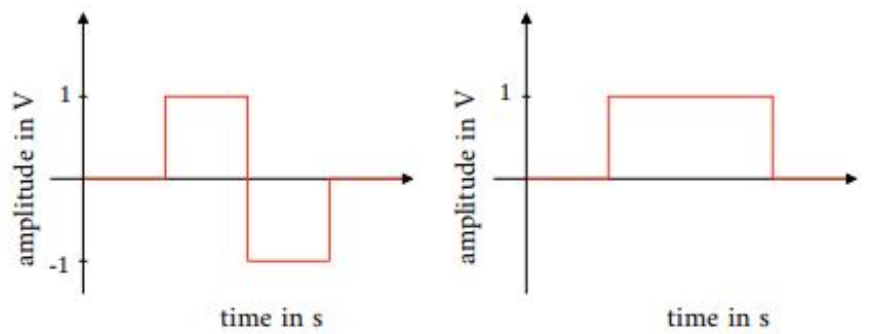

Figure 3.6: Haar mother wavelet (left), and corresponding scaling function (right) $[18,19]$ 
The square structure of the mother wavelet is then expressed using the dependencies as,

$$
\psi^{h}(t)= \begin{cases}1 & \text { if } 0 \leq t<\frac{1}{2} \\ -1 & \text { if } \frac{1}{2} \leq t<1 \\ 0 & \text { for all other } t\end{cases}
$$

Using the mother wavelet, different versions of the Haar wavelet transform can be created by changing scales, phase and timeshift. Figure 3.7 illustrates this effect.

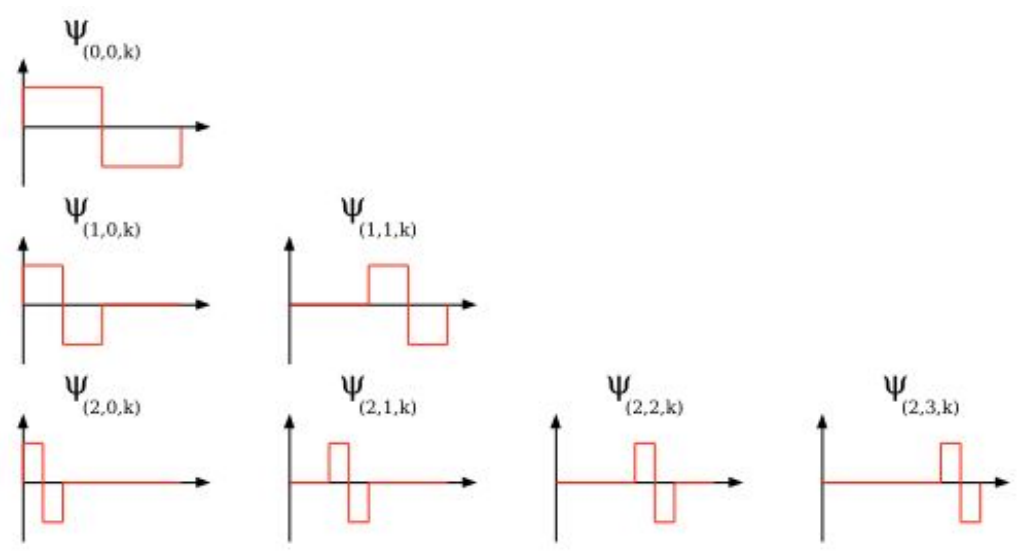

Figure 3.7: Variations in Haar transform with respect to scale, phase and timeshift [18,19]

Then for a zero phase shift and considering the presence of noise components in a signal $f(t)=$ $s(t)+e(t)$, where $s(t)$ is the actual signal and $e(t)$ is the noisy component, the Haar wavelet coefficients can be computed as,

$$
w_{s, p, \tau}=\sum_{t} f(t) \psi_{s, p, \tau}{ }^{h}(t)
$$

In this study, the Haar wavelet for denoising actigraphy signals was applied using Eqns.3.6-3.8 in MATLAB $^{\mathrm{TM}}$. Figure 3.8 depicts how different levels of Haar wavelet could be used for denoising actigraphy data between $0.4-1.6 \mathrm{~Hz}$ frequency range for 11 decomposition levels. 

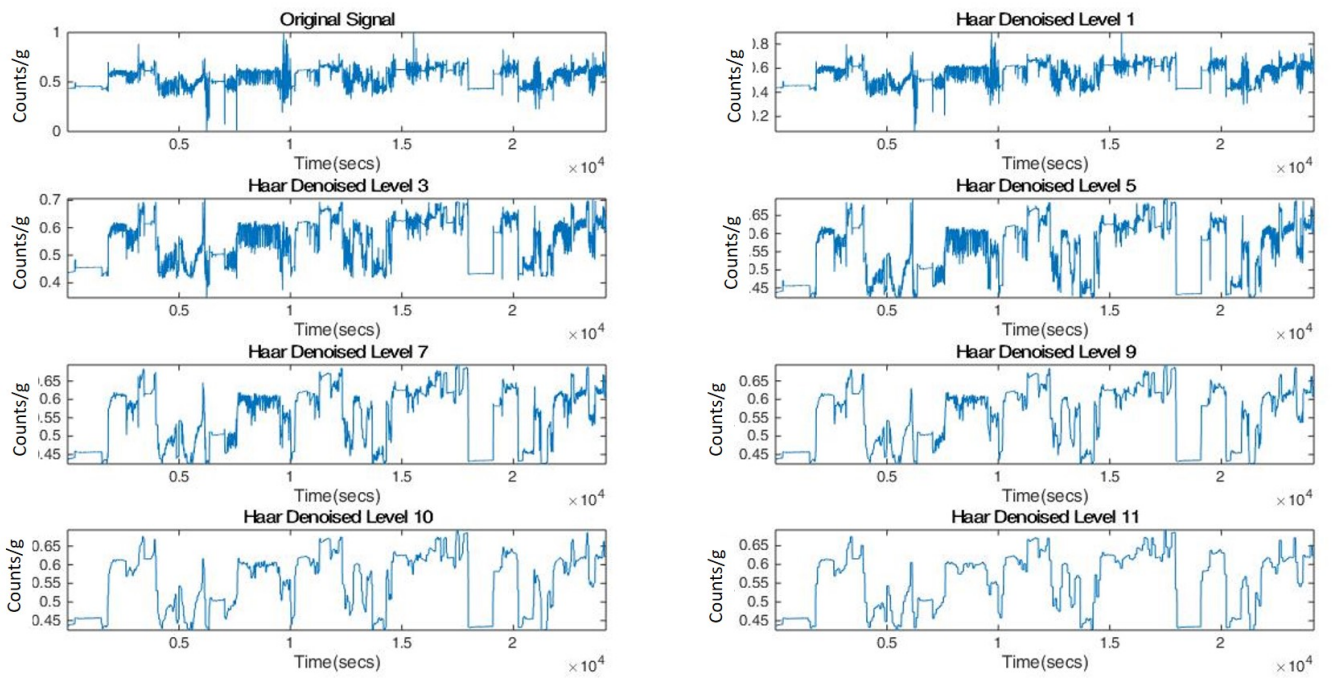

Figure 3.8: Haar wavelet denoising of actigraphy signals

\section{Vector Compounding:}

This operation is only applied to tri-axial accelerometry data, and its purpose is to combine the effect of acceleration, direction and intensity of each recorded movement. In order to analyze a triaxial activity, the experiments in this study and a survey of prior works indicate that performing a vector compounding of individual axial data, ensures data integrity and avoids information loss. It should be noted that some device manufacturers recommend this operation for effective activity data analysis $[10,39,42]$. The vector compounding is a non-linear operation which encapsulates movement information from all the three axes. Assume a low pass filtered tri-axial actigraphy signal denoted by $S_{f}=\left(x_{n}, y_{n}, z_{n}\right)$, where $n$ is the number of samples and each axis represents one of the three components, namely - direction, acceleration and orientation in space. Considering the fact that it is needed to evaluate the overall intensity of a human activity, the signal $S_{f}$ 's vector magnitude is computed as $[10,39,42]$,

$$
S_{v}=\sqrt{x^{2}+y^{2}+z^{2}-g}
$$

Note that the $g$ factor can be eliminated, when the manufacturer specifies on how to normalize the raw actigraphy data based on the specific accelerometer's properties. To illustrate this effect further, Figure 3.9 shows a sample tri-axial signal and its corresponding vector compounded version. 

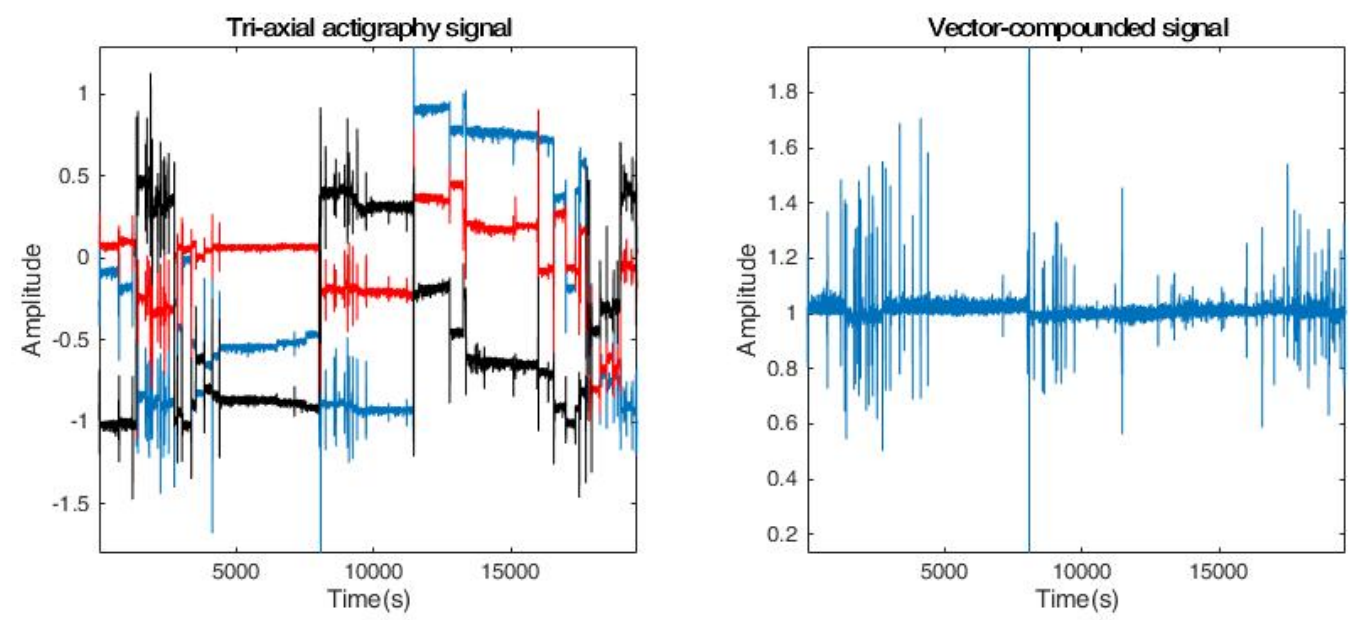

Figure 3.9: (A) Tri-axial signal [16], and (B) its Vector Compounded version

To summarize all the known pre-processing methods, following Figure 3.10 would give the reader a clearer picture of the process.
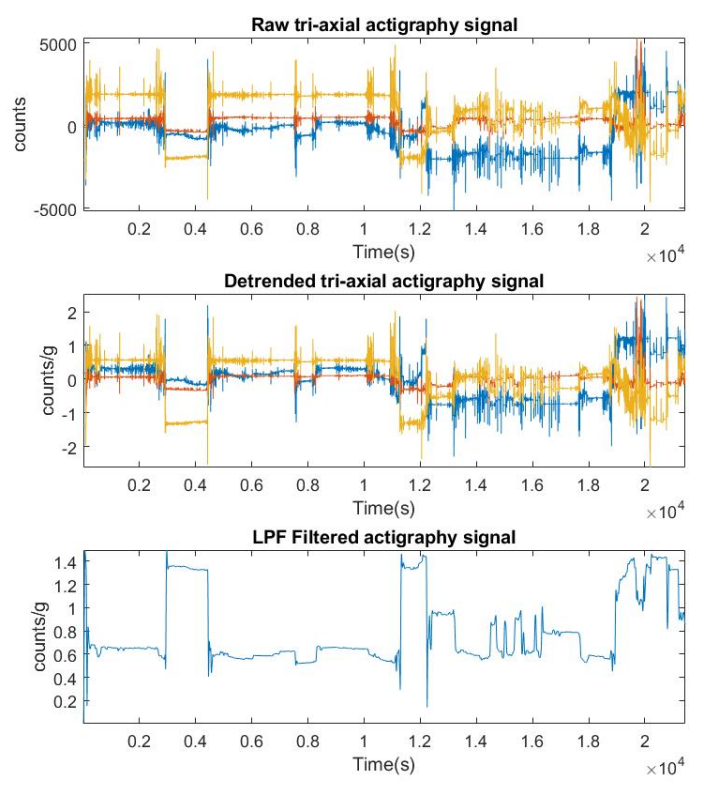
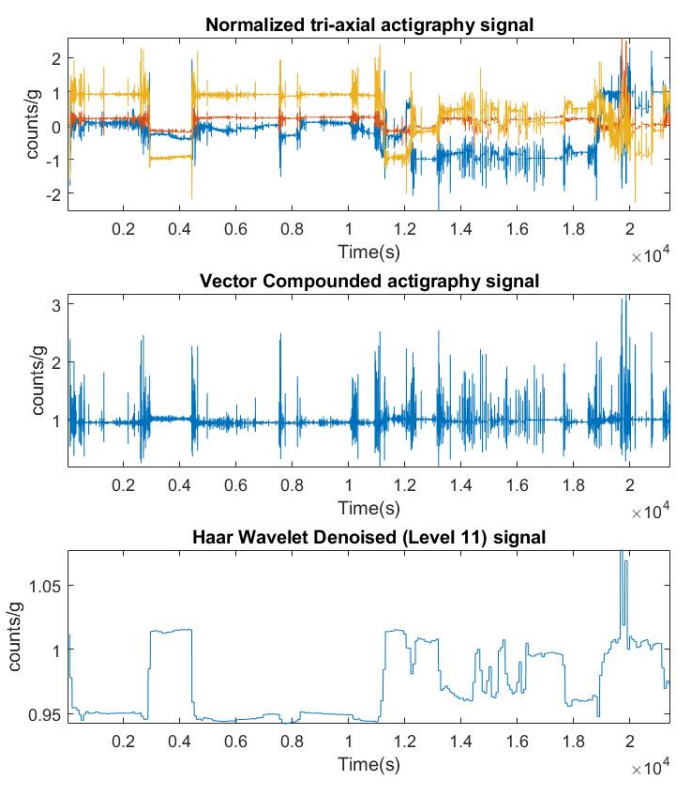

Figure 3.10: Pre-processing steps on a sample tri-axial sleep actigraphy signal

\subsubsection{Research Contribution: Signal Encoding}

Following these pre-processing methods, this section will now discuss in detail the novel contribution to actigraphy pre-processing. As discussed in previous chapters, although many studies have been conducted 
on long term monitoring applications using actigraphy wearables in an IoMT paradigm, there are a few key challenges which must be met for their viable implementation [10,32]. These are:

- Manufacturer-based variability in device sampling rate and quantization resolution.

- Inefficient power consumption and memory leakage due to over-sampling of information.

- Higher computational cost to analyze long-duration data when processed offline, without any prior compression or segmentation at the source.

A simpler approach to denoising and compressing actigraphy signal would be to further encode the digitized actigraphy data using concepts derived from communications theory. Pulse code modulation (PCM) and Delta modulation (DM) are commonly used techniques in sampling and encoding analog signals in wireless communications $[83,84]$. Pulse code modulation is a method of quantizing or digitally representing a sampled analog signal. It is a commonly used technique in digital audio and telephony communications. A pulse code modulator basically encodes uncompressed digital signals. To begin with, an analog signal is first sampled or its amplitude measured at regular time intervals. Following this, each amplitude is then discretly quantized using a $r$-bit quantization. That is, if each amplitude of the analog signal is assigned $r$ bits, then there would be $2^{r}$ possible quantized values. For example, in case of a 6-bit quantization, we would have $2^{6}=64$ possible values to represent each amplitude. Usually the quantization is conducted within the dynamic range of the sampled signal, i.e. the full scale amplitude limit of allowable input to the analog-to-digital converter. Following the quantization, each amplitude which is now represented by one of the $2^{r}$ levels, is then converted to a binary stream of 0 s and 1 s (for example in a 6-bit resolution, a sample amplitude could be 011001). This process is known as coding, and completes the process of pulse code modulation. It should be noted that in order to avoid amplitude saturation, i.e. when the sampled amplitude is outside the dynamic range of the input signal or when one of the amplitudes reach extreme values, the number of quantization steps could be reduced by 1 step such that each amplitude would have a $r$-bit resolution but would be mapped to one of the $\left(2^{r}-1\right)$ levels [85].

Delta modulation on the other hand takes this coding further by representing each amplitude sample by only one bit, which is either 0 or 1 . Delta modulation transmits only the difference between consecutive samples rather than sending each sample itself in a communication channel. It allows for simplified signal quantization, encryption, transmission and reconstruction $[83,86]$. In other words, delta modulation could be considered as a 1-bit PCM technique. But delta modulation could suffer when the variation in the amplitude of the signal is large. That is, if the sampled signal rapidly changes its amplitude, then it would be difficult for the quantizer to catch up with matching each amplitude correctly to one of the $\left(2^{r}-1\right)$ levels, thereby 
causing delay or lag in encoding. In other words, if an input signal changes more than half of the step size within a sampling interval, there will be distortion in the encoding process. This phenomenon is called Slope Overload distortion. A solution to this would be the application of a limiting condition to the first order difference of the input signal as, $\left.\frac{d s(t)}{d t}\right|_{\max } \leq \frac{s}{t_{s}}$, where $\frac{d s(t)}{d t}$ is the first order difference of the input signal $s$ and $t_{s}$ is the sampling period [87]. This is holds true when the encoding is binary. In case the coding is more than two bits, the condition could be imposed as $\left.\frac{d s(t)}{d t}\right|_{\max } \leq \frac{s}{m \times t_{s}}$, where $m$ is the number of bits used for encoding. Figure 3.11 illustrates the quantization and encoding of a signal using PCM and DM methods [86]. For additional information on PCM and DM techniques, the reader may refer to one of $[83,84,88]$

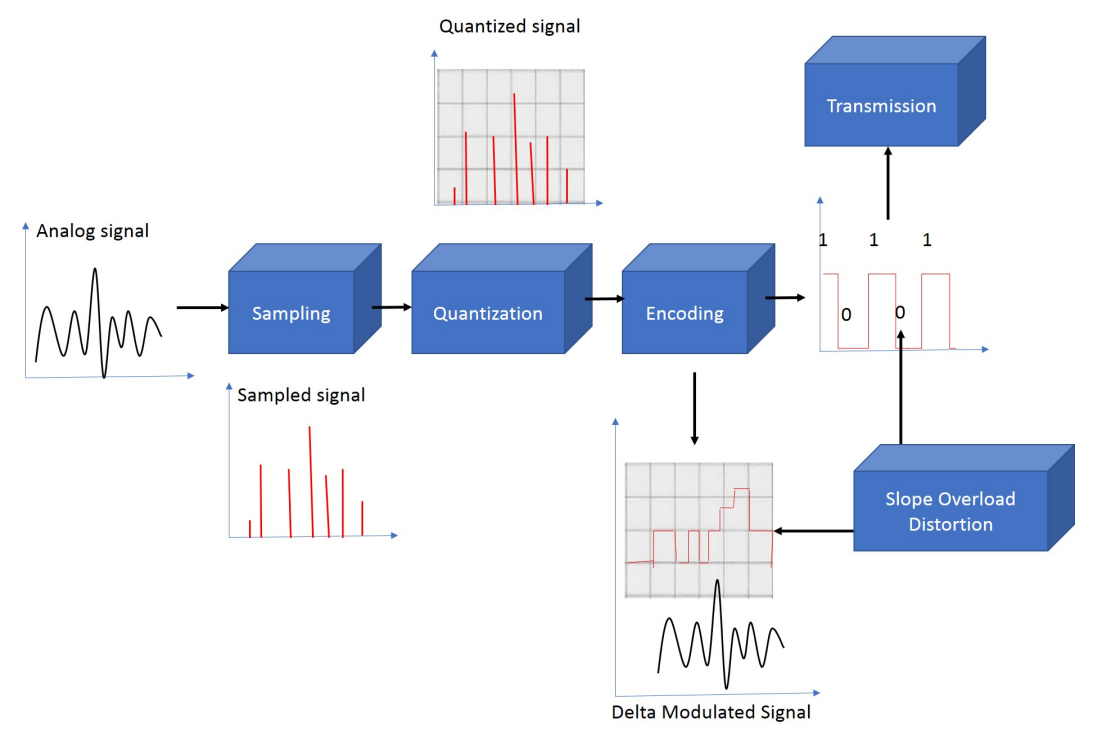

Figure 3.11: Signal Quantization and Encoding using PCM-DM

Using these concepts, a modified $m$-bit signal encoding technique was developed $[10,20]$ based on the concepts of Pulse Code and Delta modulation, and intended to reduce the bit-rate of the actigraphy signal by mapping it to lower-steps of quantization [86] with higher amplitudes. The encoding scheme avoids uniform quantization of peak movements or activity and retains vital information in the form of a lower number of discretized steps. Following steps briefly describe the encoding procedure for actigraphy signals shown in Figure 3.12: 


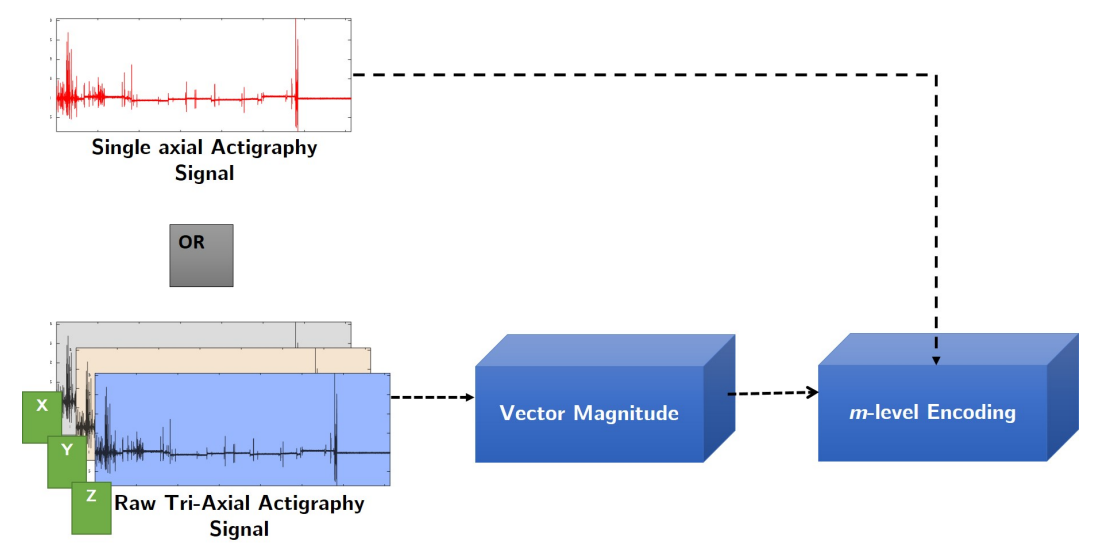

Figure 3.12: Signal Encoding Scheme [10,20]

1. The raw actigraphy signal $S_{r}$ is first normalized as described by Eqns.3.1-3.3 in section 3.1.1. This eliminates the effect of $g$ from the actigraphy signal. The reader may note here that the range of each actigraphy signal would be different as it may have different amplitude strengths depending on the participant's recorded movements. Therefore, it should be noted that the range of each signal would define its encoding, thereby making the process unique for each signal.

2. Next, in case of tri-axial signals vector compounding of normalized data is performed as per Eqn.3.9. This step can be skipped in case of single-axial normalized signals.

3. Assuming that $r$ bits/sample is the current quantization resolution of the normalized actigraphy signal, the intention is to further encode it into a $m$ bits/sample resolution, such that $m \ll r$. The new encoding factor can be computed as [89],

$$
q_{f}=\frac{\left(2^{m}-1\right)}{2}
$$

As indicated previously, the quantization factor accounts for clipping or saturation of extreme actigraphy signal values, and in order to avoid them from going beyond the mapping level, it limits the quantization steps to $\left(2^{m}-1\right)$. Generally speaking, without accounting for signal saturation, an encoding factor could be considered as $\frac{2^{m}}{2}$.

4. Using the factor $q_{e}$, the normalized actigraphy signal $S_{\text {norm }}$ is encoded using the floor operation $[88,89]$,

$$
\begin{gathered}
S_{e}=\left\lfloor\left(S_{\text {norm }} \times \frac{\left(2^{m}-1\right)}{2}+\frac{\left(2^{m}-1\right)}{2}\right)\right\rfloor O R \\
S_{e}=\left\lfloor\left(S_{n o r m} \times q_{f}+q_{f}\right)\right\rfloor
\end{gathered}
$$


The floor operation shown in Eqns.3.11 and 3.12 approximates each sample of $\left(S_{\text {norm }} \times q_{f}+q_{f}\right)$ to the greatest integer less than or equal to it. For example, a sample value of $\left(S_{n o r m} \times q_{f}+q_{f}\right)=5.4$ would be approximated to $S_{e}=5$ in the encoded signal.

5. The quantization error is then computed as $[85,87]$,

$$
q_{\text {err }}=S_{\text {norm }}-S_{e}
$$

6. The SNR of the encoded signal $S_{e}$ is calculated using the expression $[85,90]$,

$$
S N R_{S_{e}}=20 \log \left(\frac{R M S_{S_{n o r m}}}{R M S_{q_{e r r}}}\right) d B
$$

where, $R M S_{S_{\text {norm }}}$ and $R M S_{q_{e r r}}$ are the root mean square (RMS) values of the normalized signal and the quantization error respectively. Eqn.3.14 can be further elaborated as,

$$
S N R_{S_{e}}=20 \log \left(\frac{\sqrt{\frac{1}{n}\left(S_{\text {norm }_{1}}^{2}+S_{\text {norm }}^{2}+\ldots+S_{\text {norm }}^{2}\right)}}{\sqrt{\frac{1}{n}\left(q_{\text {err }}^{2}+q_{\text {err }}^{2}+\ldots+q_{\text {err }}^{2}\right)}}\right) d B
$$

where $n$ is the number of samples present in the actigraphy signal.

The encoding scheme has been intended to be applied directly at the data acquisition source in order to enable on-the-fly data compression and denoising. Additionally, as explained in Section 2.4, the encoding process aims to create a standardized method of acquiring, denoising and compressing data through an optimal number of encoding bits. This would allow to develop subsequent analysis algorithms to be deviceindependent. Figure 3.13 shows the encoding process applied to a truncated sleep actigraphy signal of about 5000 samples. The figure highlights the differences between the vector compounded, encoded and the quantization error signals through means of their respective signal-to-noise ratios (SNR). The reader may note the large difference between the SNRs of the vector compounded signal and its encoded version. Also it may be noted that there are different amplitude ranges for the vector compounded, encoded and quantization error (or noise) signals as shown in Figure 3.13. Further to this Figure 3.14 illustrates the encoding scheme applied to different actigraphy signals. In Figure 3.14, the left panel shows the original actigraphy signal, and the right panel shows its encoded version. Notice the difference in amplitude ranges (units of counts $/ g$ ). 

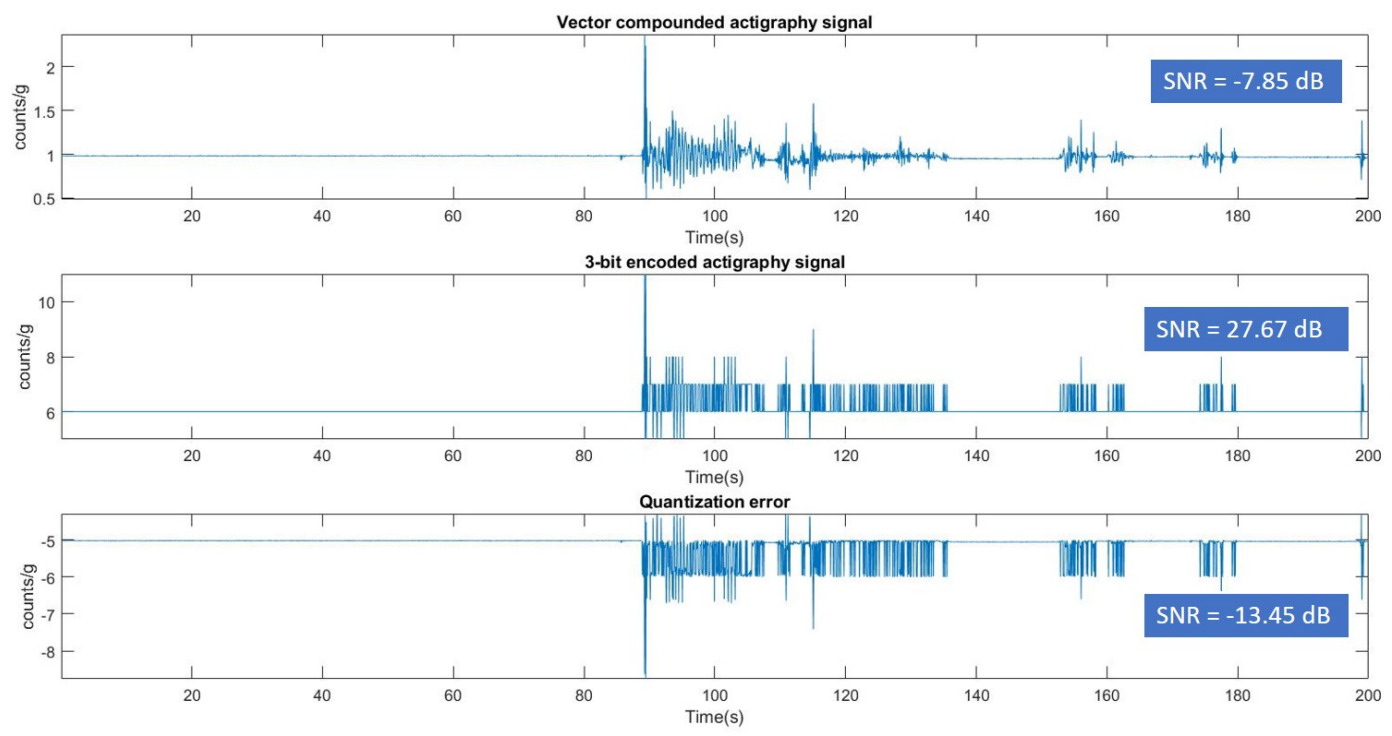

Figure 3.13: Application of 3-bit encoding to a small patch of sleep actigraphy signal
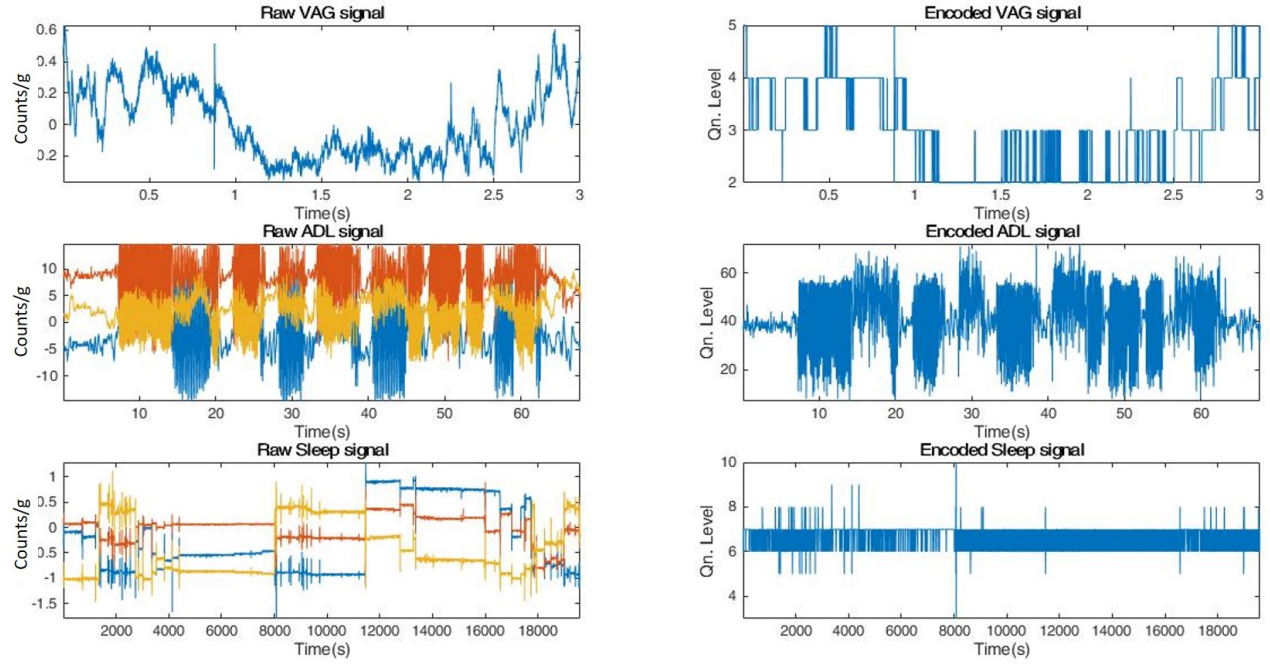

Figure 3.14: Application of the encoding scheme to actigraphy

To explain the encoding effect further, consider the example of a 3-bit encoding scheme shown in Figure 3.15. Notice how each sample is mapped on to one of the $\left(2^{3}-1\right)=7$ steps. 


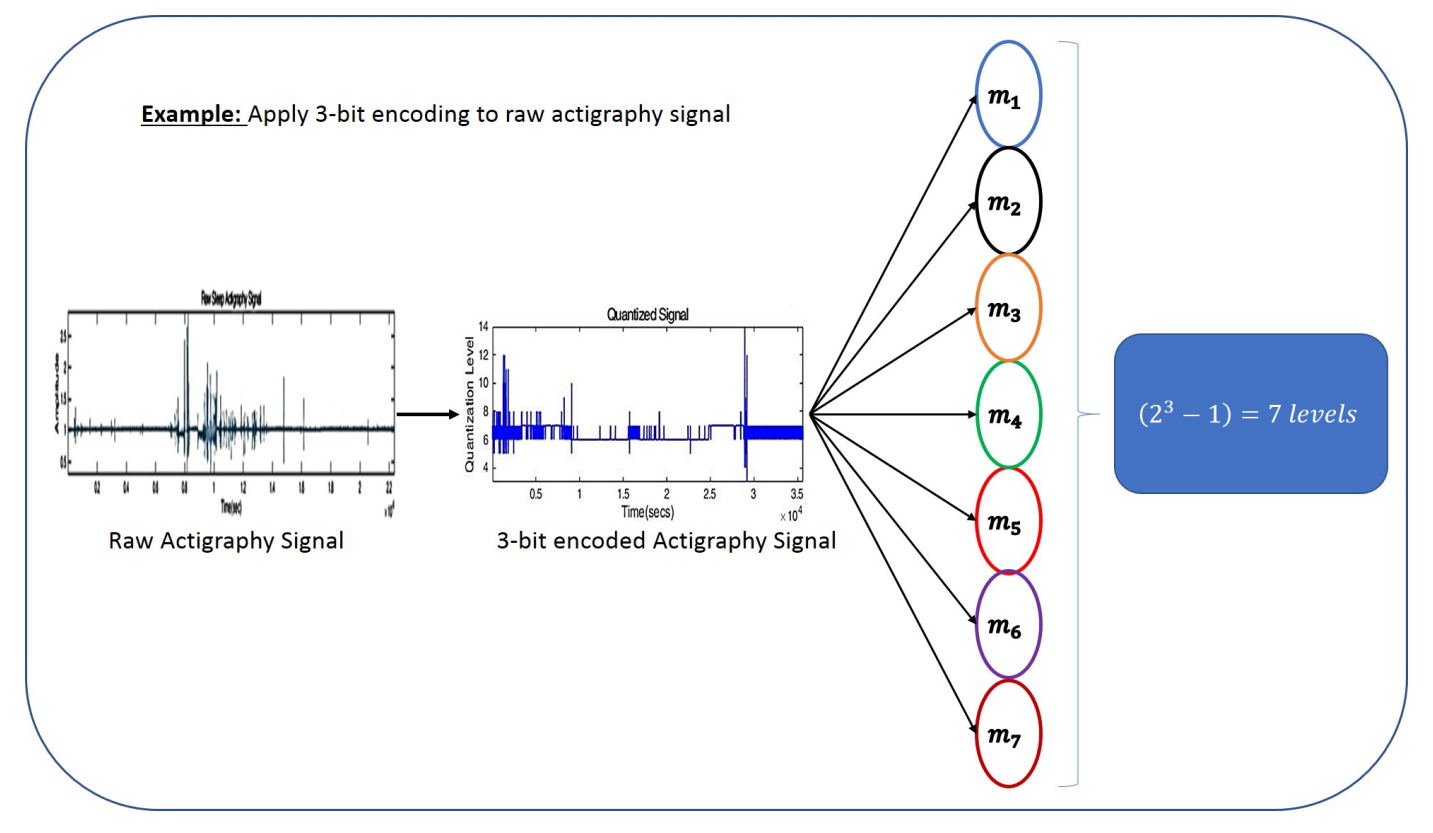

Figure 3.15: Example of 3-bit encoding of actigraphy data

In order to encapsulate all the known methods of signal pre-processing and compare them with the novel signal encoding method, following Figures 3.16 and 3.17 have been illustrate to visually explain each pre-processing method, which would be useful to the reader at this juncture.
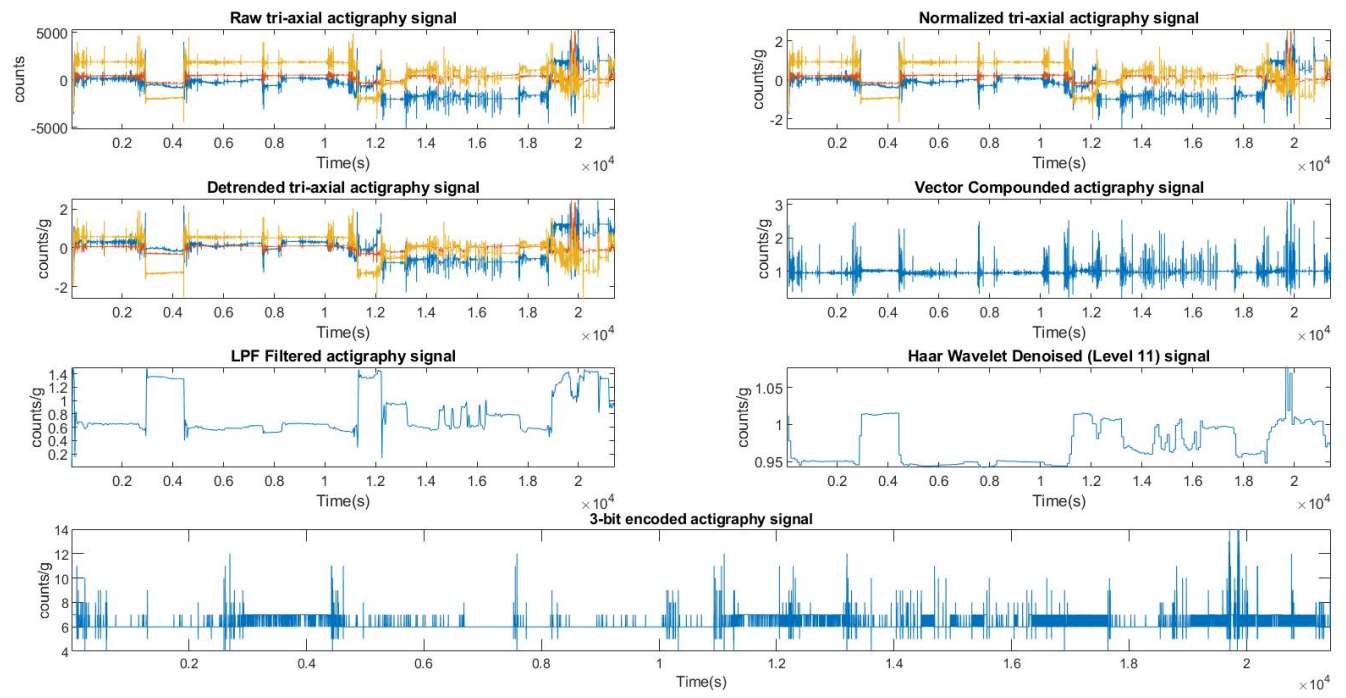

Figure 3.16: Pre-processing of a sample long-duration tri-axial sleep actigraphy signal 

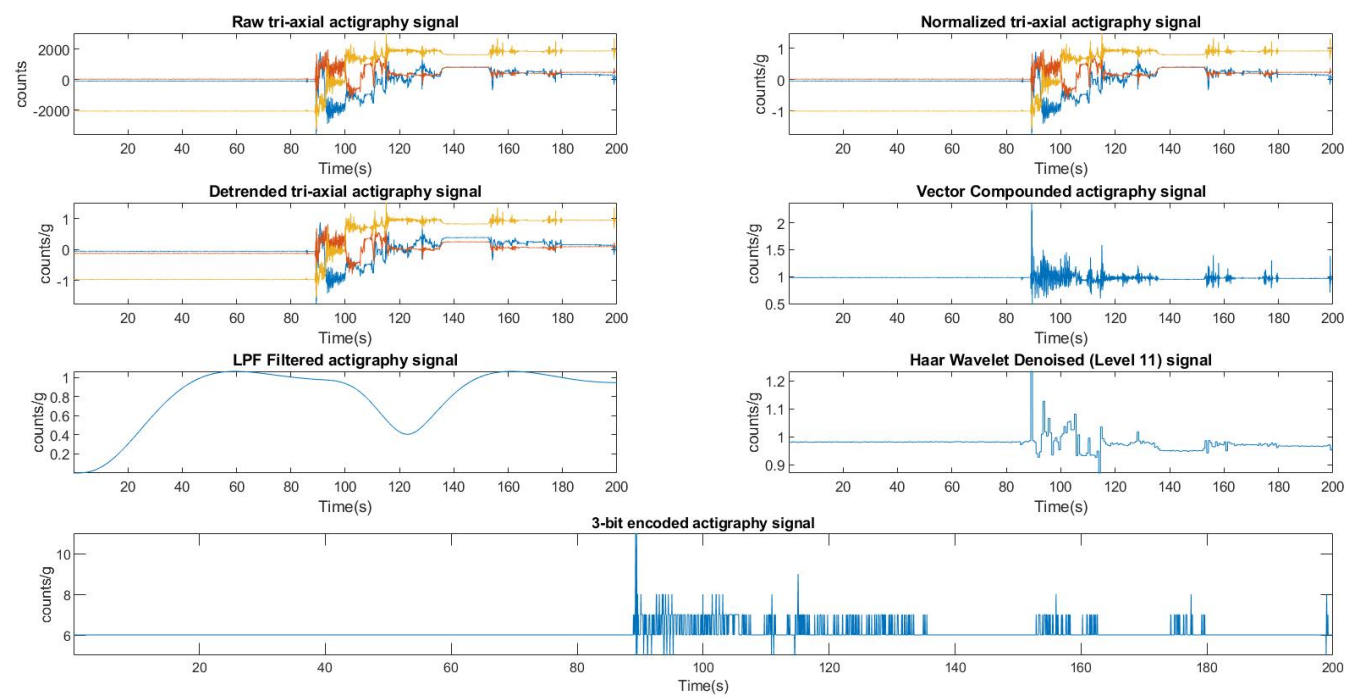

Figure 3.17: Pre-processing of a subset of the signal used in Figure 3.16

In this study of various actigraphy applications, the encoding scheme has been applied to different datasets and remarkable results have been obtained with respect to reduced file size, better signal clarity and a higher pattern classification accuracy. These results have been highlighted in the next few chapters of this dissertation. In the next section, different aspects of signal segmentation, known methods and this study's contributions in adaptive signal segmentation based on the afore-described $m$ - bit signal encoding scheme, will be discussed.

\subsection{Actigraphy Signal Segmentation}

Once a raw signal has been, the next step is to extract regions of interest from the signal. This can be done by segmentation. Before delving in the details of different segmentation methods, one must first understand the basics of signal segmentation.

The process of breaking down a signal into multiple, fixed or variable-sized, non-overlapping windows or frames for analysis, is termed as segmentation. Most signal segmentation algorithms are based on detecting regions of interest or finding samples which exhibit drastic changes in the signal, depending on the type of signal-source and application $[27,36]$. Segmentation helps in simplifying signal analysis, by unveiling hidden information depicted by instantaneous parameters. The advantages of segmenting any non-stationary signal are four-fold as follows [27]:

- Its an effective treatment for non-stationary signal analysis, by splitting it into quasi-stationary frames 
for analysis.

- Segmentation expedites feature extraction by restricting the process only to the frames extracted from a segmented signal. It should be noted that some methods also employ the use of signal-specific features for signal segmentation.

- It helps in removing redundant information and unwanted artifacts from the signal.

- Signal segmentation also helps in dimensionality reduction and signal compression at the source. This effectively reduces the computational cost of running a feature extraction and pattern classification algorithm.

Biomedical signals are typically generated from dynamic and time-variant physiological systems or sources $[32,36]$. It should be noted that most physiological signals exhibit one or more of the following properties:

- Non-linearity: Output of the physiological system is usually not proportional to the input or evoke potential.

- Non-stationarity: First and second order statistics (mean and variance) change with time.

- Gaussian or non-Gaussian distribution: Distribution of signal samples could be normal in nature, in the shape of a bell curve such that most sample values occur around the central region, and least occurring values are found towards the curve's extremities.

- Sparsity: Some biomedical signals may have very few significant values, and a large number of insignificant or close to zero values [46].

- Multi-modality: A single signal could be generated by the effect of multiple physiological systems.

- Multi-channel: Some biomedical signals such as EEG (electroencephalogram) are composed of different channels depending on the frequency and intra-region within a specific brain lobe.

- Multi-source: Biomedical signals can also be multi-source, i.e. a same signal can be generated from different sources.

Considering the fact that any given physiological signal is non-linear and non-stationary in nature, it could be analyzed by segmenting it into smaller frames using a moving window approach. In each window various statistical measures can be obeserved, which could be used for characterizing signal-source behavior. These characteristics could be identified as follows [27,36]: 
- Mean: The mean value of a signal is the average or DC value of all the signal samples in a given short-time window. For a non-stationary signal, a dynamic mean value indicates the presence of a wandering baseline or low frequency artifact, which can be removed using detrending or through a high pass filter.

- Variance: This second order statistics parameter indicates signal variability by showing the number of peaks and troughs in the analysis window. Generally, variance indicates the average signal power of each window.

- Activity Measures: These define the busyness of a signal, and usually indicate the zero-crossing rate of the signal. That is, how many times a signal crosses the baseline on the $\mathrm{X}$-axis.

- Autocorrelation Function (ACF): The autocorrelation of a signal is a function of the signal compared with its own time-shifted version. The ACF of a non-stationary signal varies with time, and is usually decaying in nature. Therefore it can be assumed that the signal is wide-sense stationary (WSS) or quasi-stationary in short windows.

- Power Spectral Density (PSD): The Fourier transform of the ACF of a signal yields its power spectral density. When visualized on a time-frequency plane, the PSD highlights regions of high energy in the signal, thereby indicating signal samples of high frequency.

- Higher order statistics: A non-stationary signal can further be characterized by its third and fourth order moments, namely - skewness and kurtosis respectively. The skewness indicates how signal samples are distributed, and how this distribution deviates from a normal or Gaussian distribution of the signal. Similarly, the kurtosis defines the shape of the probability distribution curve of the signal data.

Analyzing physiological signals using these characteristics, simplifies the segmentation problem, and helps understand the signal behavior better. Before proceeding with a detailed description of the novel segmentation methods in this research, it would be imperative to first quickly understand different segmentation approaches.

\subsubsection{Fixed Segmentation}

Fixed segmentation refers to the splitting of a non-stationary signal into short frames of fixed time duration for analysis. Usually, these frames are of equal width, and their segmentation is done under two assumptions:

- For a given frame or signal window, its first and second order statistics do not change, and,

- The signal is assumed to be stationary, linear or non-linear within that frame 
Mathematically, given a signal $s=\left[s_{1}, s_{2}, \ldots, s_{n}\right]$ of sample length $n$, it can be equally divided into $k$ frames, each with $m$ samples, such that $m \ll n$, and $k=\frac{n}{m}$. Therefore, each segment could be generalized as $[27]$,

$$
s_{k}(i)=s[i+(k-1) m], 0 \leq i \leq(m-1), \text { and } 1 \leq k \leq \frac{n}{m}
$$

Although Eqn.3.16 denotes a simplification of fixed segmentation, it should be noted that in most cases the division of a signal into smaller segments is governed by a decision rule or windowing function. Windowing functions can be designed by the user, and are usually applied considering the signal structure and properties. Some key fixed segmentation methods can be discussed in brief as follows:

\section{Short-time Fourier Transform (STFT):}

Also known as windowed Fourier transform, the STFT segments a signal into fixed-width frames using a moving window function $h(n-m)$. For a given signal frame, at a given time instant $m$, it can be represented using the window function as [91],

$$
s_{k}(n)=s(n) h(n-m)
$$

And its Fourier transform would be,

$$
S_{k}(\omega)=\sum_{n=0}^{m-1}[s(n) h(n-m)] \exp (-j \omega n)
$$

Here,

$$
h(n)=\left\{\begin{array}{cc}
1 & \text { for } 0 \leq n \leq(m-1) \\
0 & \text { otherwise }
\end{array}\right.
$$

The windowing function $h(n)$ is compactly supported and is usually non-overlapping. In case of continuous time analysis, the windowed signal frame can be represented as,

$$
S_{k}(\omega)=\int_{-\infty}^{\infty} s(t) h(t-\tau) \exp (-j \omega t) d t
$$

$S_{k}(\omega)$ represents the time-frequency distribution of the signal segment, and it can be visualized using $\left|S_{k}(\omega)\right|^{2}$, which is the energy density spectrum or spectrogram of the segment [27]. It should be noted that there is no need to compute Fourier transform for each window, and the overlapping can be avoided by advancing the analysis window by $\frac{m}{2}$ samples. The window function must be short enough to impose stationarity as well as extract robust features. Figure 3.18 illustrates how the window function can be 
expanded or contracted using time-frequency variations.

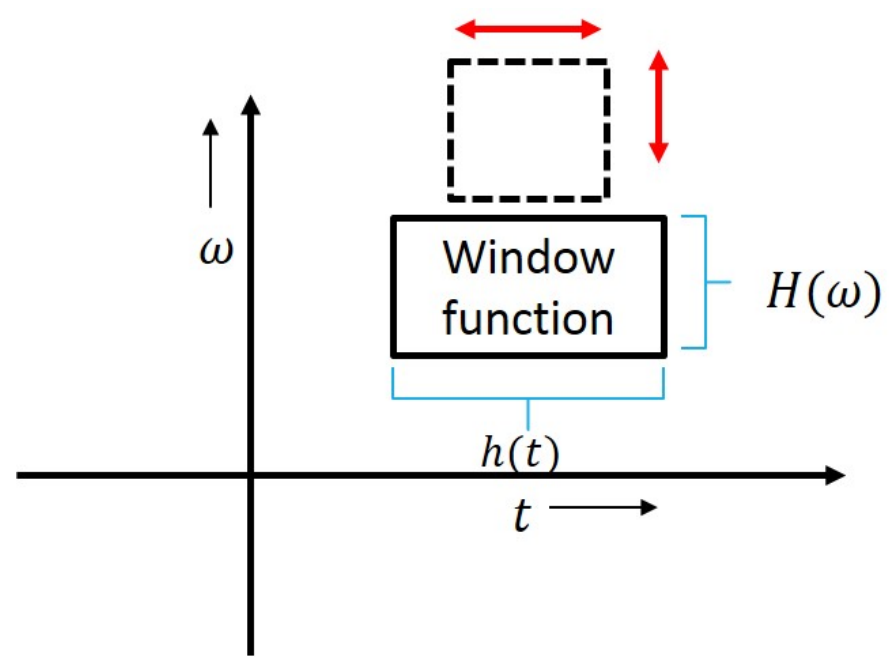

Figure 3.18: Window function variation in an STFT plane

\section{Morphological Thresholding:}

It is known that physiological systems and signals exhibit specific characteristics with respect to the morphology of the body source. Each physiological source behaves differently under varied conditions. For example the Gait pattern at an age of 25 years would be different from that at an age of 55 years for the same individual or two different individuals. This is because of the aging changes in the physiological source, i.e. the knee joints and connecting muscles. Using morphological thresholding, such ground truth or clinical information about the physiological source can be used to generate fixed threshold levels to segment the signal into quasi-stationary segments. Figure 3.19 illustrates the example of segmenting a gait signal from a test subject who moves 2 steps every 5 seconds. Here, the threshold is $t=5$ seconds, assuming that each sample is of 1 second duration. Therefore, $s_{k}(n)=s(n-5)$.

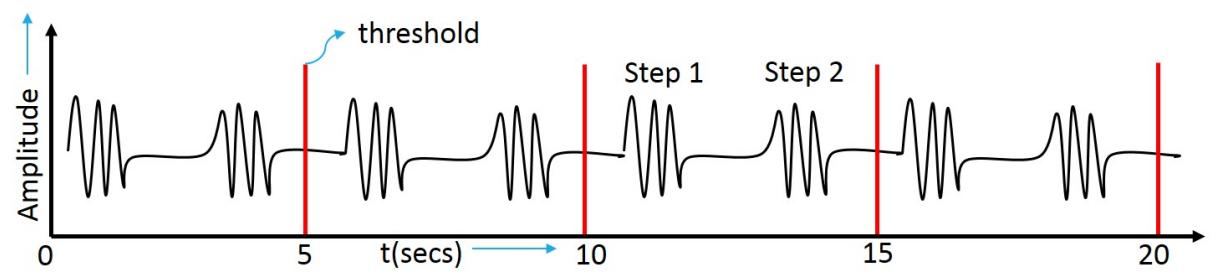

Figure 3.19: Example of morphological thresholding

\section{Peak Detection and Zero Crossings:}

Fixed segmentation of a non-stationary signal can also be performed by identifying peaks and zero- 
crossings in the signal. In order to do so, the signal can be scanned throughout to find all the significant peaks, as well as those samples which exhibit high signal variability. Finding zero-crossings works best when the signal variability is high but occurs less frequently. Otherwise too many zero crossings could increase number of segments and hence the computations to extract features. To illustrate this, Figures 3.20 and 3.21 depict the segmentation of a sample sleep actigraphy signal [16] using peaks and zero crossings respectively.

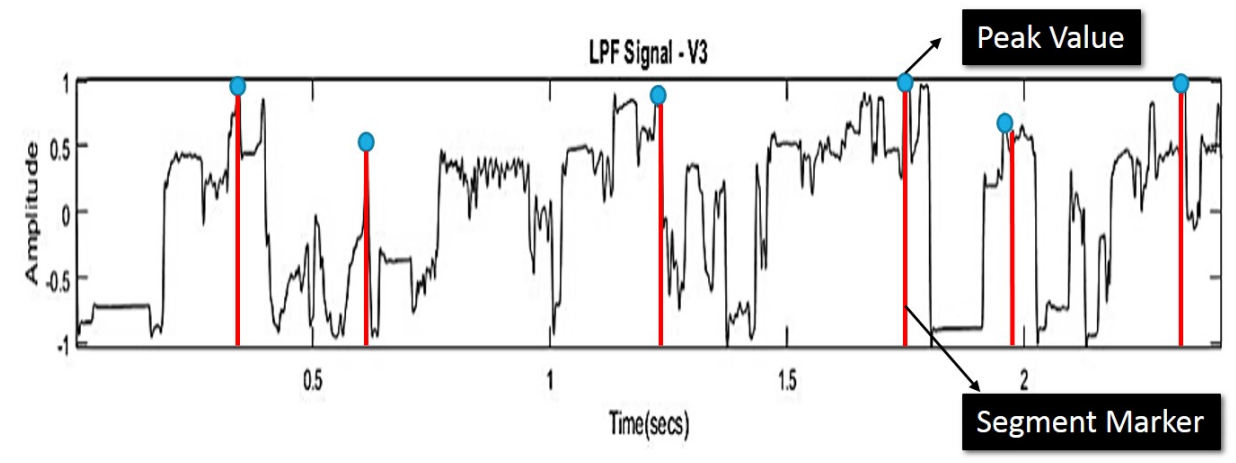

Figure 3.20: Peak detection based fixed segmentation

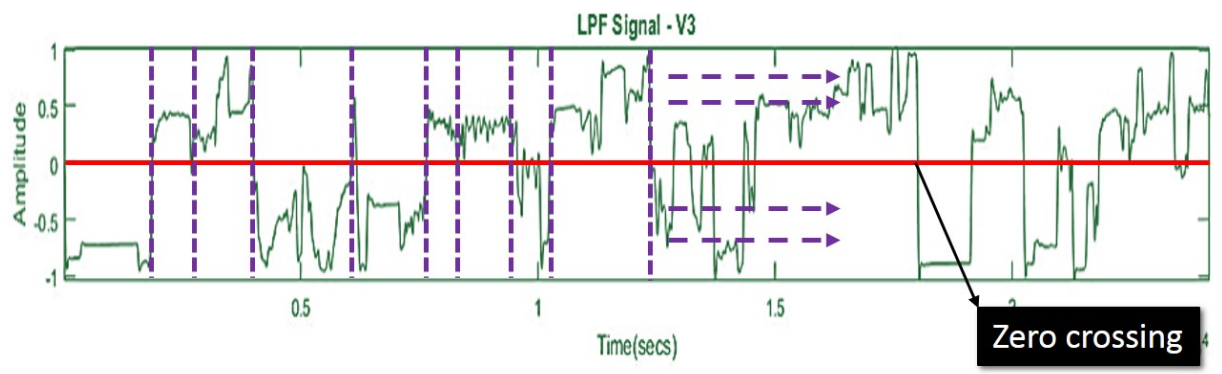

Figure 3.21: Zero-crossings based fixed segmentation

It should be noted that although segmenting using peaks or zero-crossings may not result in fixed-width windows, the segmentation is considered fixed in the sense that the decision rule to split the signal remains constant, and does not involve any windowing function.

Though fixed segmentation approaches present a promising avenue to signal analysis, they still lack in key areas when it comes to effective handling of non-stationarity and non-linearity of physiological signals. Some of these could be:

- The choice of fixed window function may not capture the true non-stationary nature of the signal. It is quite possible that the signal may be stationary longer than the window width. 
- The time-frequency distribution or localization within a signal segment would be limited and hence computing the overall PSD of the non-stationary signal is not suitable.

- Fixed window size may sometimes miss out instantaneous information or features which could be characteristic of the physiological signal behavior.

- Analyzing fixed-width segments could also result in redundant feature generation, and subsequently more number of computations to reduce the feature set to maximum relevance.

- Feature extraction could be computationally complex if it is needed to calculate the Fourier transform of each segment.

\subsubsection{Adaptive Segmentation}

A solution to these issues is the application of adaptive segmentation techniques which segment a signal based on detecting instantaneous changes, thereby tracking its non-stationarity effectively. In other words, a window function is developed which adapts its size to changes in signal patterns. There are numerous adaptive segmentation methods proposed in literature, which are mostly based on developing signal-specific or feature-based window functions which adaptively segment a given physiological signal. For a better understanding of adaptive segmentation to the reader, two commonly used methods have been described as follows $[27,91]$ :

\section{Detecting Spectral Changes:}

For a given signal $s(n)$, its PSD at $(n, \omega)$ is given as,

$$
P(n, \omega)=\frac{1}{2 \pi} \sum_{i=0}^{n} R_{i}(\tau) \exp (-j \omega \tau) d \tau
$$

where,

$$
R_{n}(\tau)=s^{*}\left(n-\frac{\tau}{2}\right) s\left(n-\frac{\tau}{2}\right)
$$

Here $R_{n}(\tau)$ is the instantaneous autocorrelation function of the signal $s(n)$. The logarithmic difference between the PSD in the current state and zero state defines the prediction error, and is computed using the instantaneous spectrum as,

$$
P_{e}(\omega)=\frac{P(n, \omega)}{P(0, \omega)}
$$

Now, if $\phi_{e}(m)$ represents the autocorrelation function of the prediction error, the spectral error measure 
$(S E M)$ at a given time instant $n$ can be defined as,

$$
\operatorname{SEM}(n)=\left[\frac{\phi_{e}(0,0)}{\phi_{e}(n, 0)}-1\right]^{2}+2 \sum_{k=1}^{m}\left[\frac{\phi_{e}(n, k)}{\phi_{e}(n, 0)}\right]^{2}
$$

Using this error measure and a pre-defined threshold based on the morphology or ground truth of the given physiological signal, the signal is segmented through the following steps:

- Start with a pre-defined window $h(n, m)$ such that $m \ll n$. Here the segmentation would be conducted using a varying size window function, $h(n, m)$.

- Scan along the windowed signal and for each sample compare if $S E M(n)>T_{1}$, where $T_{1}$ is the first threshold level.

- if the sample does not meet this condition, proceed to the next sample

- if the sample meets this condition, it means that a segment boundary has been found

- If the condition in previous step is met, the size of the window function is changed such that $h(n, m)=h\left(k=\operatorname{SEM}(n)>T_{1}, \omega\right)$

- The time is shifted by $(n+k)$ units and the first three steps are repeated till all the segment boundaries are found.

- Further to this, in order to avoid sharp transients, which could reduce the segmentation boundary to two adjacent time instants by defining another threshold $T_{2}$ which clips the prediction error as,

$$
e(n)=\left\{\begin{array}{cc}
e(n) & \text { if }|e(n)|<T_{2} \\
\operatorname{sgn}[e(n)] & \text { if }|e(n)|>T_{2}
\end{array}\right.
$$

where, $\operatorname{sgn}()$ is a signum or sign function of the prediction error $e(n)$.

\section{Autocorrelation Function (ACF):}

Using the ACF as statistical measure to detect changes in the signal is another way to define variable segment boundaries. The ACF helps detect rapid changes and transients in each moving window with respect to a fixed reference window [27]. Adaptively segmenting a non-stationary signal using its ACF can be done through the following steps:

- Assume that $\phi_{r}(k)$ is the ACF of the reference window, and $\phi_{T}(n, k)$ is the ACF of the moving test window at any time instant $n$. The ACF for zero lag is the power of the signal and is expressed as,

$$
P[n]=\frac{1}{(2 n+1)} \sum_{i=-n}^{n}|s(i)|^{2}
$$


- The normalized power distance between the two windows is then given as,

$$
d_{P}(n)=\frac{\left|\sqrt{\phi_{T}(n, 0)}-\sqrt{\phi_{r}(0)}\right|}{\min \left(\sqrt{\phi_{T}(n, 0)}, \sqrt{\phi_{r}(0)}\right)}
$$

- And the spectral distance only up to a certain lag $l$ is defined as,

$$
d_{F}(n)=\frac{\sum_{k=1}^{l}\left|\sqrt{\phi_{T}(n, k)}-\sqrt{\phi_{r}(k)}\right|}{0.5+\sum_{k=1}^{l} \min \left(\sqrt{\phi_{T}(n, k)}, \sqrt{\phi_{r}(k)}\right)}
$$

- Using the normalized and spectral distances the net ACF distance can be then computed as,

$$
d(n)=\frac{d_{P}(n)}{T_{p}}+\frac{d_{F}(n)}{T_{f}}
$$

where $T_{p}$ and $T_{f}$ are the scalar thresholds for power and spectral distances.

- A significant change in the ACF of the moving window with respect to reference window is observed when $d(n)>1$. The time instant at this change in the signal is then marked as a segment boundary. The entire process is repeated till all the boundaries are found.

As mentioned previously, it is evident that physiological signals exhibit different properties based on their source behavior. This being said, not all physiological signals are a result of evoked potentials generated due to neural or muscular activity, and some of them can also be captured through non-invasive contact sensors such as accelerometers [32]. These signals capture varied human movements and their intensity per activity. In this study of actigraphy signal analysis, an investigation into accelerometry signals was conducted and based on their properties as stated in Chapter 2 it was found that it would be best to devise adaptive segmentation algorithms which detect occurrence of peak movements. Based on extensive experiments conducted in this research and a survey of prior literature, two novel adaptive segmentation techniques for actigraphy signal analysis and compression at the acquisition source have been developed. The next couple of sections, will describe these methods in detail.

\subsubsection{Research Contribution: The Athavale-Krishnan (AK) segmentation algorithm}

Actigraphy data exhibits random, transient spikes which are recorded by the accelerometer sensor, every time a movement is sensed $[10,37,40]$. As per signal property tests, described in Chapter 2, this data is non-linear, non-stationary, non-Gaussian and sparse in short windows. 
Currently available actigraphs and other accelerometer-based wearables such as smart watches, quantify motion data more than required, and tend to sample data infrequently. Despite their capacity to provide long-term recording with good battery life, for activity monitoring applications, the inherent process of data acquisition, denoising and analysis uses conventional methods, which may not be suitable for edge computing applications within the IoMT (Internet of Medical Things) environment [10,20]. The AK algorithm helps to address these challenges by automatically denoising, compressing and adaptively segmenting of actigraphy data right at the source. This in turn would also address the issues with regards to computational overheads of processing long-duration data using cloud-based resources, which could also lower data security and information privacy.

It should be noted that, unlike other physiological signals such as ECG, actigraphy data upon visual inspection does not have any specific structure and only registers the amplitude of movements capture by the actigraph. These are randomly occurring values, exhibiting a non-normal nature. Figure 3.22 illustrates the flowchart of the AK segmentation algorithm.

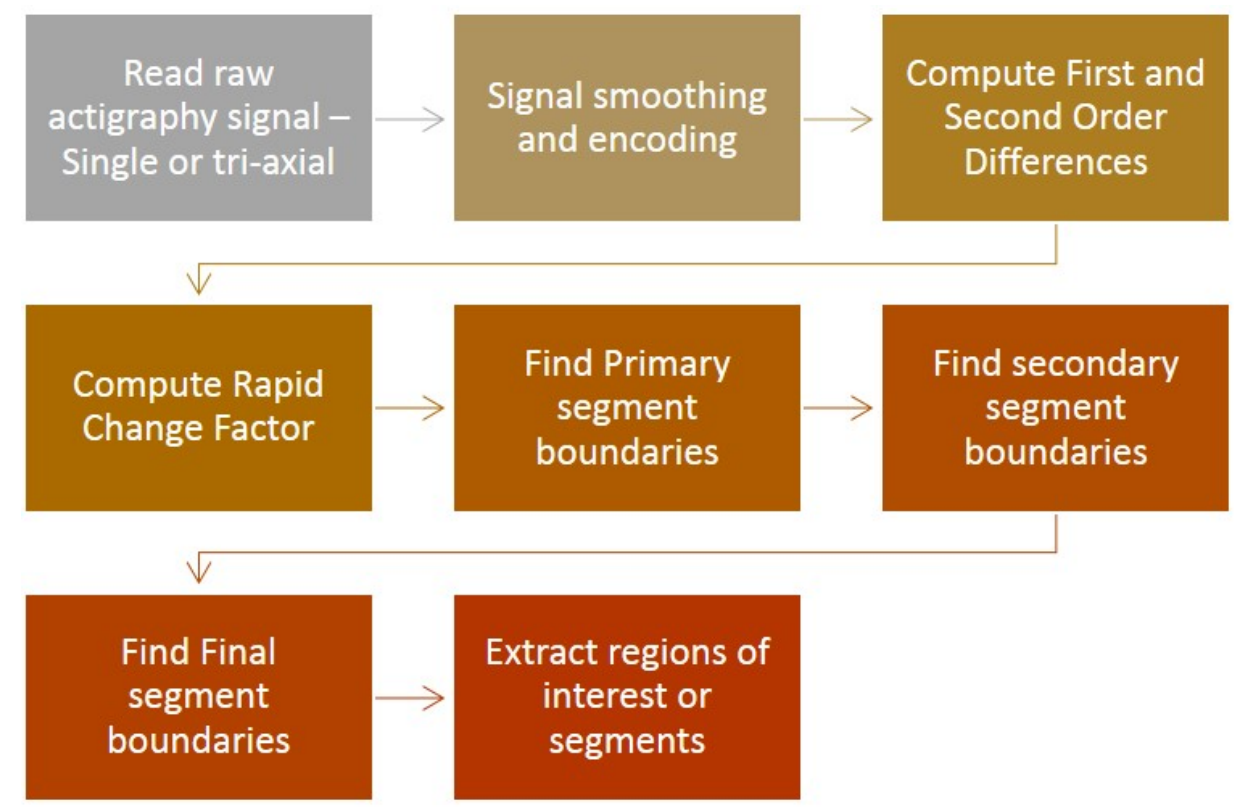

Figure 3.22: Flowchart of AK Algorithm

The AK algorithm basically performs additional encoding of already digitized data in the current state. The intention is to apply the AK algorithm directly at the acquisition source such that it would eliminate the need to digitize the actigraphy signal first, and then encode it. The objective is to encode the data directly into a low-level bit resolution, so as to avoid memory leakage, remove noise and increase computational efficiency. 
An actigraphy-specific parameter, known as the Rapid Change Factor is computed which detects events of interest from the spiky and transient actigraphy signals. This parameter is based on the second order statistics of the signal, and helps detect samples which depict drastic change in movement activity, with respect to past adjacent values. The AK algorithm uses the rapid change factor to detect segment boundaries, and then extract signal windows/frames only between those segment boundaries, which meet a certain criteria as provided by the user. It should be noted that the AK algorithm has been exclusively developed for actigraphy data. The AK algorithm is adaptive in nature, in the sense that the rapid change factor is characteristic of each signal, and hence the PSBs (primary segment boundaries) are computed accordingly. It should also be noted that although there is no direct feedback mechanism, the segmentation scheme adapts to the drastic changes in the signal by marking boundaries based on samples exceeding the rapid change factor. The detection of which segments to consider and which to discard is based on the application specific activity inputs provided by the user.

The AK signal segmentation technique has derived its origins from standard signal processing concepts in wireless communications as explained in Section 3.1.2, namely:

- Analog-to-Digital conversion and Pulse Code Modulation - signal quantization

- Delta Modulation - signal encoding

- Detection of slope overload in delta modulation - abrupt changes in quantization steps

For the reader's simplification, following block diagram in Figure 3.23 illustrates the AK algorithm.

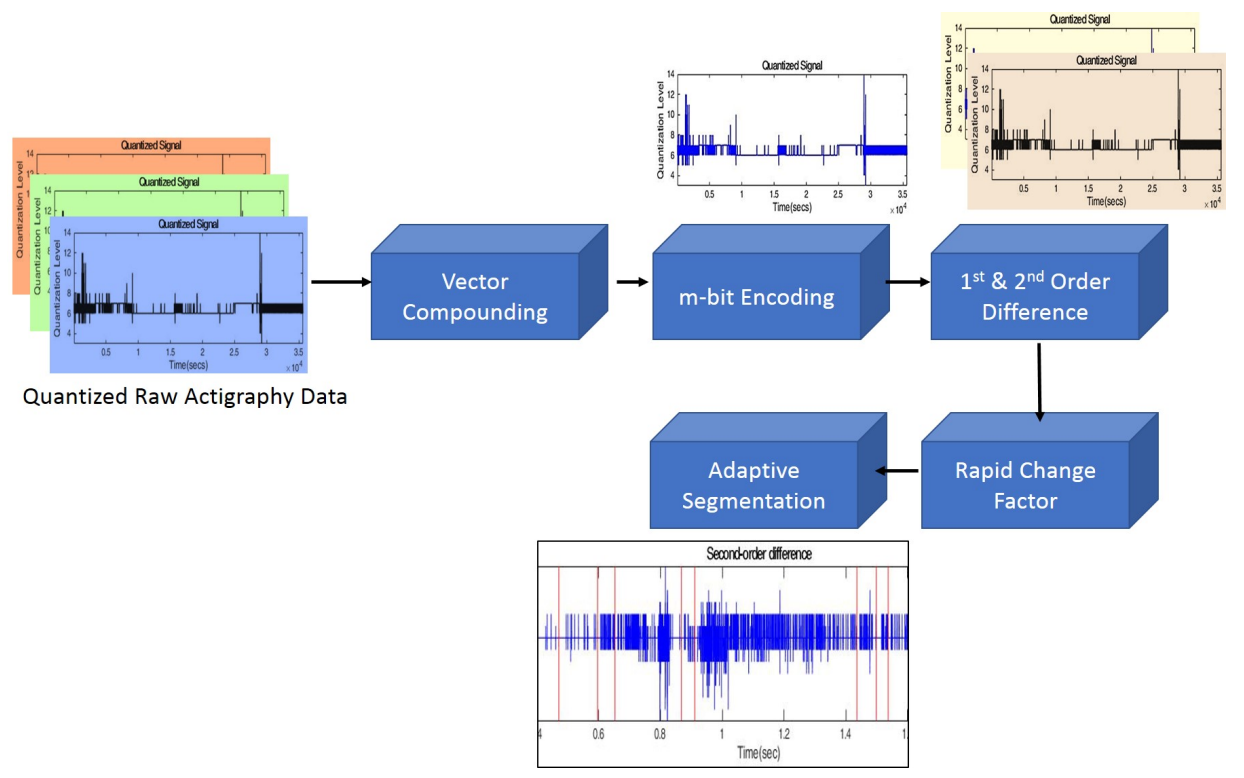

Figure 3.23: Actigraphy Segmentation using AK Algorithm 
Any actigraphy signal can be segmented using the AK algorithm through the following set of operations:

1. Signal Normalization: A given raw actigraphy signal $S_{r}$, is first normalized to remove the effect of gravitational force. This step removes the effect of earth's gravitational force on the accelerometry data, thereby enhancing sample amplitudes without losing any vital movement information. An actigraphy signal can be normalized using either of Eqns.3.1-3.3 in this study, depending on the device and dataset used. The resulting signal can be denoted as $S_{n}$.

2. Vector Compounding: This operation is applicable only to tri-axial actigraphy data. Given a normalized tri-axial signal $S_{n}=\left(x_{n}, y_{n}, z_{n}\right)$, it can be vector compounded using Eqn.3.9. The vector compounded signal can be denoted as $S_{v}$.

3. $m$-bit encoding: Following the vector compounding step, an $m$-bit encoding of the signal is performed such that $m \ll r$, where $r$ is the bit resolution of the raw actigraphy data. The signal can be encoded using Eqn.3.12 described in section 3.1.2. The resulting encoded signal can be denoted as $S_{e}$.

4. First \& Second Order Difference: After obtaining the encoded signal $S_{e}$, the first order difference version of the signal is calculated by subtracting every signal component from the value adjacent to it in the encoded signal.

$$
d S_{e}=\left[S_{e_{1}},\left(S_{e_{2}}-S_{e_{1}}\right), \ldots,\left(S_{e_{n}}-S_{e_{n-1}}\right)\right]
$$

where $n$ is the number of samples in the signal. The second order difference is then computed using the first order difference version as,

$$
2 d S_{e}=\left[d S_{e_{1}},\left(d S_{e_{2}}-d S_{e_{1}}\right), \ldots,\left(d S_{e_{n}}-d S_{e_{n-1}}\right)\right]
$$

5. Rapid Change Factor (RCF): The RCF detects rapid changes in the actigraphy signal and helps in developing segment boundaries. In other words, the RCF determines how fast an actigraphy signal changes its value from one discrete encoding step to another. Based on a prior survey of applications and concepts in PCM and DM [85,87-89], the development of RCF was inspired from the fundamentals of slope overload distortion, which mentions that if an input signal changes more than half of the step size within a sampling interval, slope overload distortion would occur. The condition to avoid this, as described in Section 3.1.2, is to ensure that $\left.\frac{d s(t)}{d t}\right|_{\max } \leq \frac{s}{t_{s}}$, where $\frac{d s(t)}{d t}$ is the first order difference of the input signal $s$ and $t_{s}$ is the sampling period [87]. This is holds true when the encoding is binary. In case the coding is more than two bits, the condition could be imposed as $\left.\frac{d s(t)}{d t}\right|_{\max } \leq \frac{s}{m \times t_{s}}$, where $m$ is the number of bits used for encoding. 
Using this reasoning, in case of actigraphy signals, one could impose a condition to detect drastic or rapid changes in encoded data when moving from one sample to another, by identifying all the samples in the second order difference signal, which are greater than $\frac{2 d s}{m \times t_{s}}$, where $2 d s$ is the second order difference of the encoded actigraphy signal $s$. Note that here instead of the first order difference, we have considered the second order difference version as it could provide insights into the second order statistics by showing the variance in signal amplitude changes. Additionally it would also provide highlights into significant peak movements which are of critical importance when recognizing a specific limb movement.

Thus, the RCF parameter is computed using three key steps:

- First, the spurious free dynamic range (SFDR) of the second order difference signal $2 d S_{e}$ is calculated. The SFDR is the difference in decibels between the amplitude of the fundamental or RMS (root mean square) value of the signal, and the amplitude of the largest spur or peak in the signal. It can also be expressed in terms of a logarithmic expression. The SFDR is computed using the expression $[85,87,89]$,

$$
R=\sqrt{\frac{1}{n}\left(2 d{S_{e_{1}}}^{2}+2 d{S_{e_{2}}}^{2}+\ldots+2 d{S_{e_{n}}}^{2}\right)}-\max _{n} 2 d S_{e}
$$

- The SFDR is then used for calculating the step-size of the signal encoder. It should be noted that step-size is different from the encoding factor computed in the first signal smoothing and encoding module. The step-size here denotes the interval with which the signal instantaneously change its value from one sample to another. Therefore, the step-size is computed as [85],

$$
S S=\frac{R}{\left(2^{m}-1\right)}
$$

- Finally using the SFDR and step-size the rapid change factor can be computed as,

$$
R C F=\frac{S S}{\left(m \times t_{s}\right)}
$$

where $t_{s}$ is the sampling period of the signal, and $t_{s}=\frac{1}{f_{s}}$, and $f_{s}$ is the sampling frequency of the signal.

6. Primary Segment Boundaries (PSBs):Next, the first set of segment boundaries in the encoded actigraphy signal can be found by identifying all the signal samples whose amplitude is greater than the rapid change factor. The reader may note the similarity here in the reasoning being similar to 
imposing a slope overload condition as described previously in Section 3.1.2 [87,88]. Corresponding to these samples, the time-stamps from the second order difference signal can also be identified. This can be expressed as,

$$
P S B_{i}=2 d S_{e_{n}} \forall\left\langle 2 d S_{e_{n}}>R C F\right\rangle
$$

where $i$ is the number of PSBs detected, and $n$ is the total number of signal samples. Therefore, as indicated in Eqn.3.35, the PSBs would be a subset of all samples in the second order difference $2 d S_{e_{n}}$, whose encoded amplitude is greater than the RCF. At this step, the user can either stop the segmentation process and split the signal using the corresponding time-stamp of each $P S B_{i}{ }^{t h}$ sample, or can proceed further to reduce the number of segments and extract only the regions of interest.

7. Secondary Segment Boundaries (SSBs): The adaptively segmented actigraphy signal can further be reduced using application or activity specific ground truth markers. The SSBs are activity-specific and their computation depends on the duration of the activity capture by the sensor. For example, if the activity is observed every 1 second, then only those primary segment boundaries need to be found whose time-stamps differ by at least 1 second. Only these boundaries will be considered as SSBs. In general, using a duration of activity threshold $t_{r e f}[27]$, the SSBs can be found as,

$$
S S B_{j}=P S B_{i} \forall\left\langle\left(t_{P S B_{i}}-t_{P S B_{i+1}}\right) \geq t_{r e f}\right\rangle
$$

where $j$ is the number of SSBs.

Therefore, as expressed in Eqn.3.39, the SSBs would be a subset of the PSBs such that the time period between the first sample marking the beginning of a PSB and the last sample indicating the end of that PSB, is greater than or equal to the reference duration of $t_{r e f}$.

8. Final Segment Boundaries (FSBs): This is an optional step in the AK algorithm, and can be implemented by user if the SSBs need to be reduced to a lower number of segment boundaries specific to a certain activity within an application. After reducing the PSBs to SSBs using a $t_{r e f}$, the FSBs can be found using another thresholding parameter based on the number of peaks within each SSB segment. That it, an SSB is marked as an FSB, if the number of peak values greater than the mean signal amplitude, between two consecutive SSBs is more than $C_{r e f}$. Here $C_{r e f}$ is a count parameter. Therefore,

$$
F S B_{l}=S S B_{j} \text { if }\left\langle\text { Count }\left(2 d S_{e_{j}}: 2 d S_{e_{j+1}}>\frac{\sum_{n} 2 d S e_{n}}{n}\right)>C_{r e f}\right\rangle
$$

where $l$ is the number of FSBs. 
The reader may again note here that the computations of SSBs and FSBs have been done in accordance with ground truth information which could be obtained from specific human movements recorded using an actigraph.

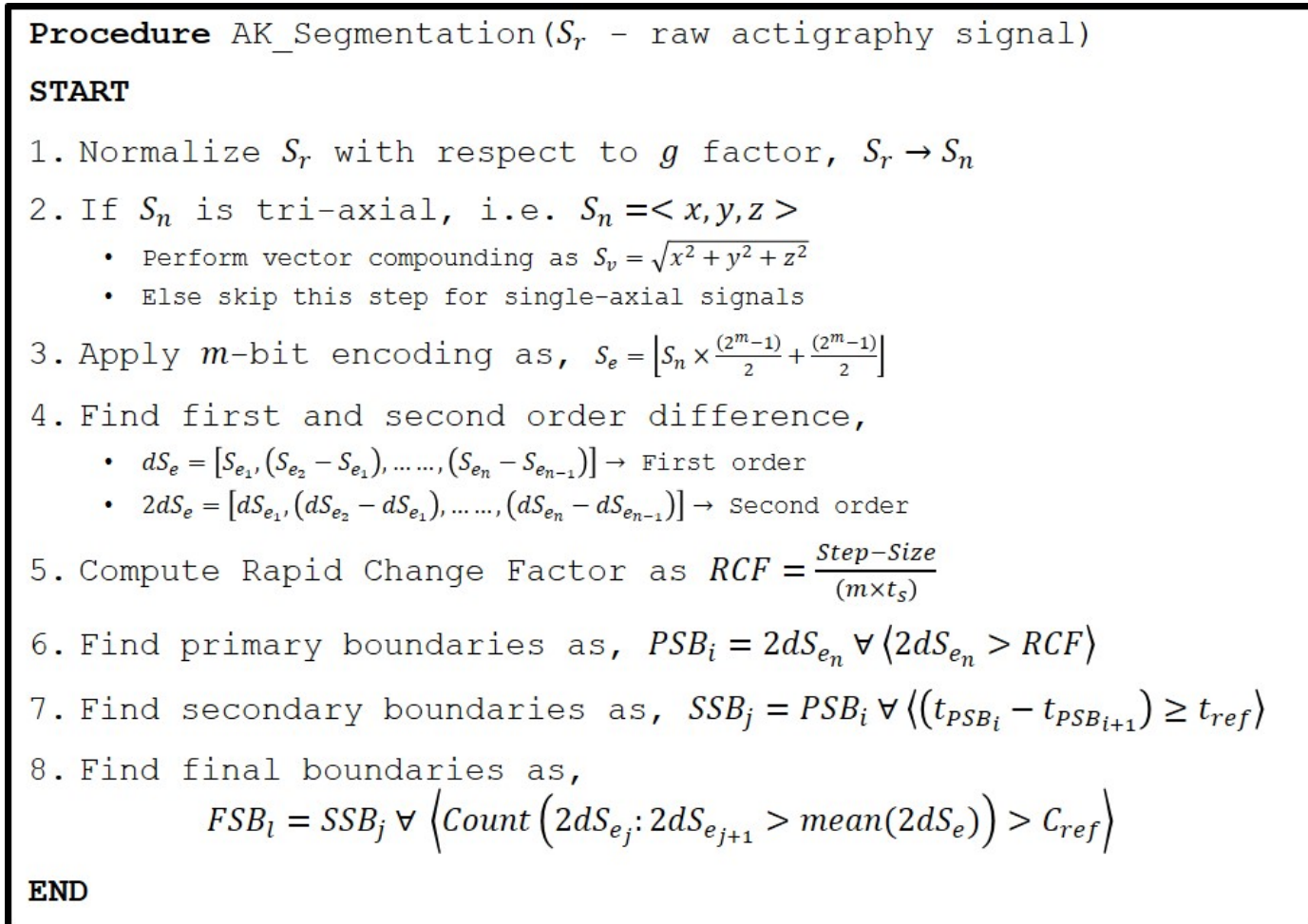

Figure 3.24: Pseudocode for AK Segmentation Algorithm

Using the afore mentioned steps, the AK algorithm not only denoises and encoded the actigraphy signal, but also adaptively segments and reduces it into a smaller number of segments, thereby compressing the signal. The purpose of this algorithm is to extract regions or activities of interest, and use only those segments for analysis and pattern classification. In the study, the AK algorithm was implemented on different actigraphy datasets and remarkable results were obtained in movement region extraction, as shown in subsequent chapters.

Additionally, this algorithm was also on a synthetic signal comprising of sine, tangent and chirp components. The intention of applying this algorithm on a synthetic signal was to show that the AK technique could not only be used on digitized actigraphy data, but could also be applied to encoding and segmenting analog signals emanating from any other physiological source. Figure 3.26 illustrates the segmentation of a synthetic signal using the AK algorithm. The synthetic signal was generated by concatenating simulated sine, tangent and chirp signals. Following flowchart in Figure 3.25 depicts the process of applying the AK algorithm to a synthetic signal. 


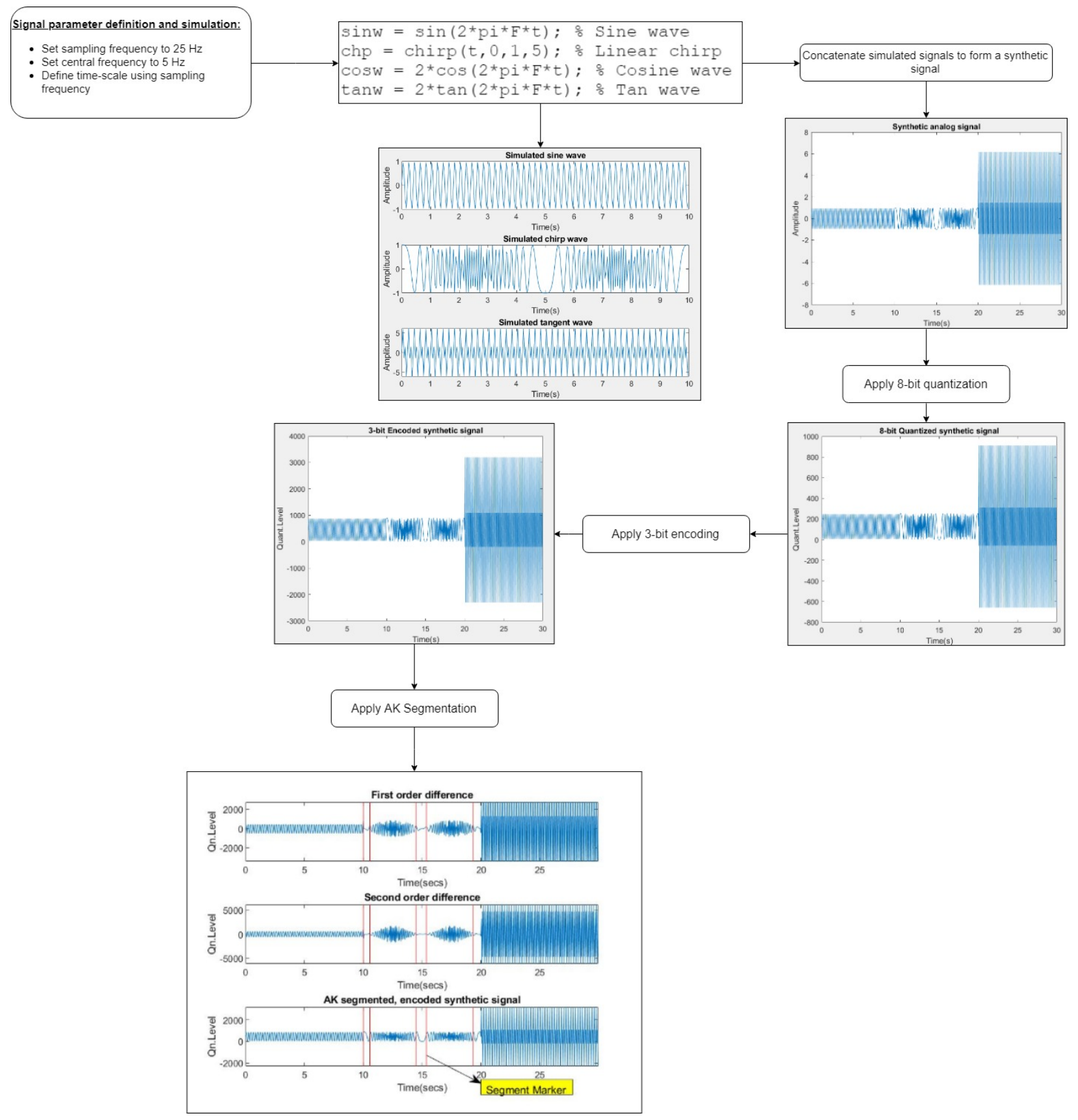

Figure 3.25: Segmenting a synthetic signal using AK Algorithm 


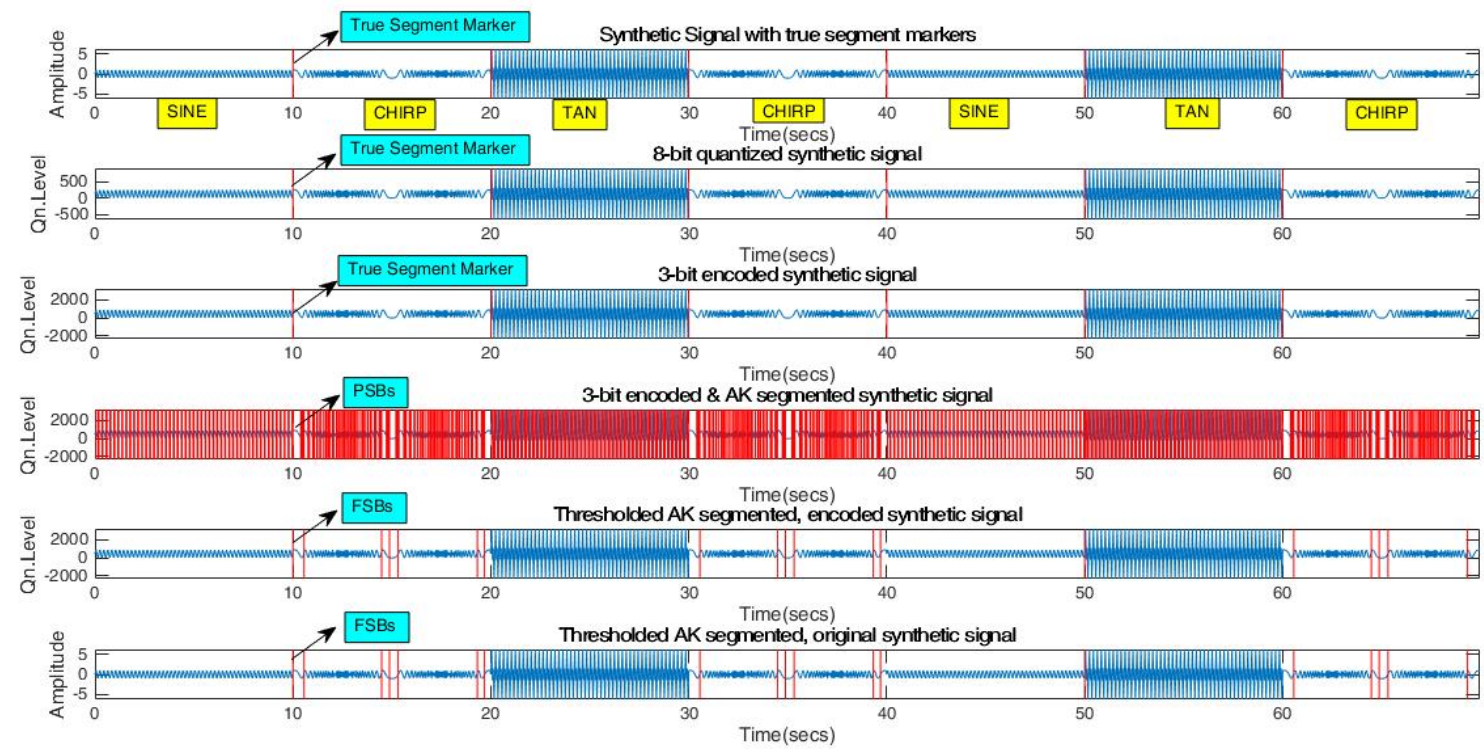

Figure 3.26: Testing AK Algorithm using a synthetic signal

It may be noted here that the application of the AK algorithm to synthetic signals was not conducted as a validation study, but to rather show the applicability of this algorithm directly to analog signals as well. Further to this, it may also be noted that the validation of the AK and the mobility-based algorithm was implicitly conducted since both were tested on over 900 actigraphy signals collected in sleep and wake states in this research work.

Following this, the next section proceeds to describing another novel adaptive segmentation technique which uses Hjorth's mobility parameter to identify regions of interest from an actigraphy signal.

\subsubsection{Research Contribution: Mobility-based adaptive segmentation algorithm}

From this study's experiments into actigraphy signals using the encoding process and the Athavale-Krishnan segmentation algorithm, it was realized that applying traditional physiological signal analysis methods could potentially provide good results on encoded data. A further investigation into known feature extraction methods, led to Hjorth's parameters [79,80], which have been used in characterizing multi-channel EEG (electroencephalogram) signals. Of these parameters, Hjorth's mobility could be very well suited for analyzing actigraphy data, since it measures the the square root ratio of variances of the signal and its first order difference [80]. The mobility depicts the mean frequency or the proportion of the standard deviation of the signal. Since mobility could be used to track drastic variations in signal, a unique, mobility-based segmentation algorithm for actigraphy analysis has been developed in this study. Before proceeding with the 
algorithmic steps, given an encoded actigraphy signal $S_{e}$ and its first order difference is $d S_{e}$, the mobility is defined as [80],

$$
M_{S_{e}}=\sqrt{\frac{\frac{1}{n} \sum_{i=1}^{n}\left(d S_{e_{i}}-\mu\right)^{2}}{\frac{1}{n} \sum_{i=1}^{n}\left(S_{e_{i}}-\mu\right)^{2}}}
$$

Following are the steps for performing mobility-based segmentation of an actigraphy signal:

1. Conditioning: Similar to the AK algorithm, the first four signal conditioning operations, namely normalization, vector compounding, $m$-bit encoding and computing the first and second order differences, are performed on the actigraphy signal.

2. Primary Segment Boundaries (PSBs): When the actigraphy signal is encoded, each sample is represented by one of the $\left(2^{m}-1\right)$ steps in the encoding process. This means that for a continuous time-series, each peak event would iterate between all the $\left(2^{m}-1\right)$ steps before getting assigned a specific quantization step. In order to find the first set of segment boundaries, the maxima (maximum peak value) of the second-order difference signal $2 d S_{e}$ is identified and divided it into $\left(2^{m}-1\right)$ steps. Next, all the samples in the second order difference signal are found such that,

$$
P S B_{i}=2 d S_{e_{n}} \forall\left\langle 2 d S_{e_{n}} \geq \frac{\max _{n} 2 d S_{e}}{\left(2^{m}-1\right)}\right\rangle
$$

where $P S B$ is a vector of actigraphy samples representing primary segment boundaries.

3. Secondary Segment Boundaries (SSBs): In order to find the next set of boundaries (SSBs), the intra-segment mobility within the primary segments is calculated, and an adjacent comparison is performed. It is evident that within an actigraphy signal random variations in signal amplitudes can be observed. Using variance ratios these changes can be captured and only those segments can be retained whose mobility values are unique from each other. Thus, the SSBs are computed as,

$$
S S B_{j}=P S B_{i} \forall\left\langle\left\lfloor M_{P S B_{i: i+1}}\right\rfloor \neq\left\lfloor M_{P S B_{i+1: i+2}}\right\rfloor\right\rangle
$$

where $P S B_{i}$ is the $i^{\text {th }}$ primary segment boundary and $j$ is the number of SSBs found.

4. Final Segment Boundaries (FSBs): In the last step of this algorithm, activity specific durations can be used to define time-scale thresholds, and to find the final segment boundaries. Similar to Eqn.3.36, the FSBs can be computed as,

$$
F S B_{l}=S S B_{j} \forall\left\langle\left(t_{S S B_{j}}-t_{S S B_{j+1}}\right) \geq t_{r e f}\right\rangle
$$




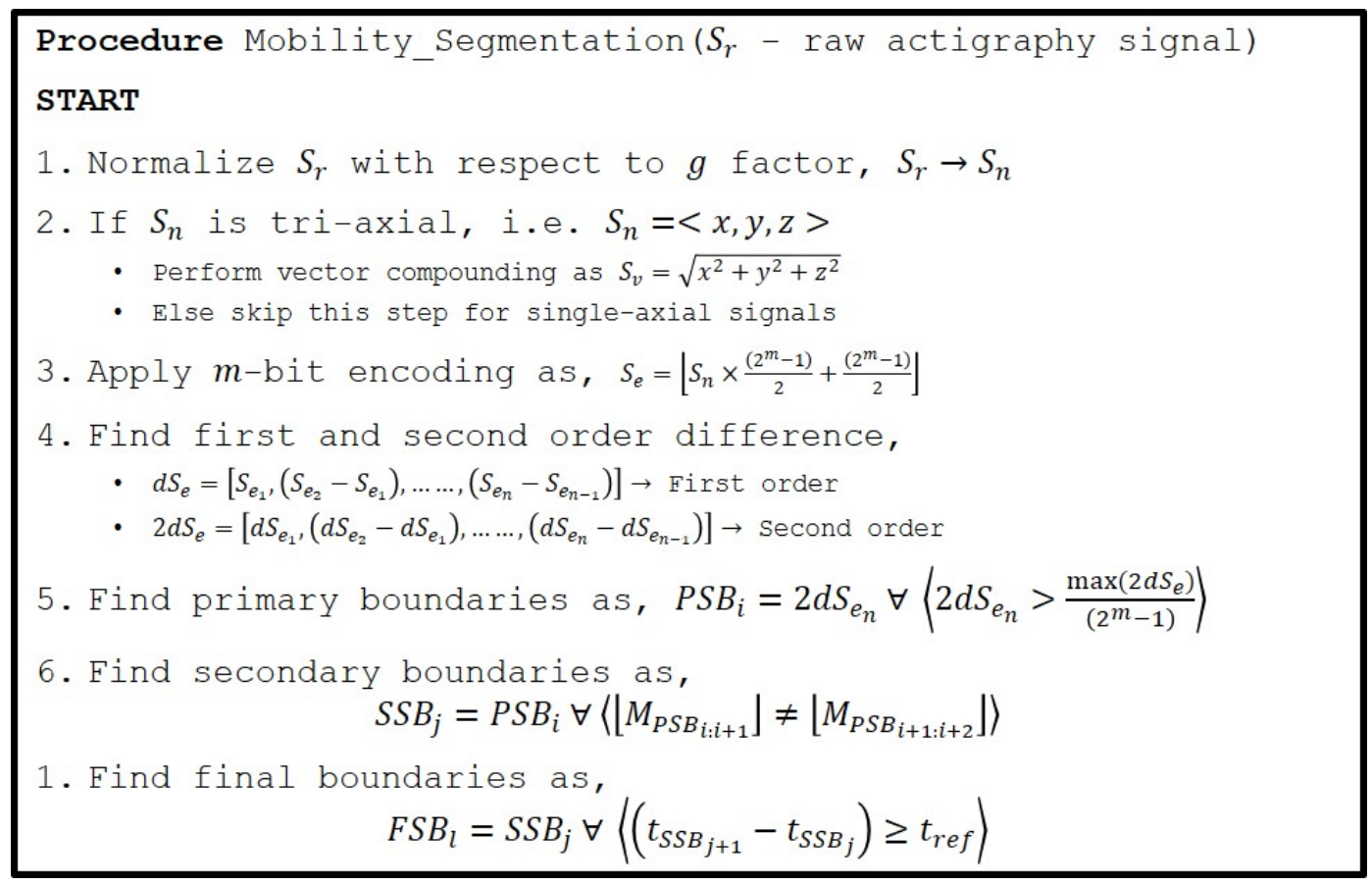

Figure 3.27: Pseudocode for Mobility Segmentation Algorithm

It should be noted that in case of the AK and mobility-based segmentation algorithms, all the PSBs, SSBs and FSBs are computed with respect to the time-scale of the signal. Similar to the AK algorithm, the mobility-based segmentation scheme was applied to different actigraphy signals and remarkable results were obtained. These results will be shown in subsequent chapters of this dissertation. This algorithm was also tested on a synthetic signal and it was found that similar to AK scheme, the mobility-based segmentation algorithm was able to separate out sine, tangent and chirp components from the synthetic signal. These results have been illustrated in Figure 3.28. 


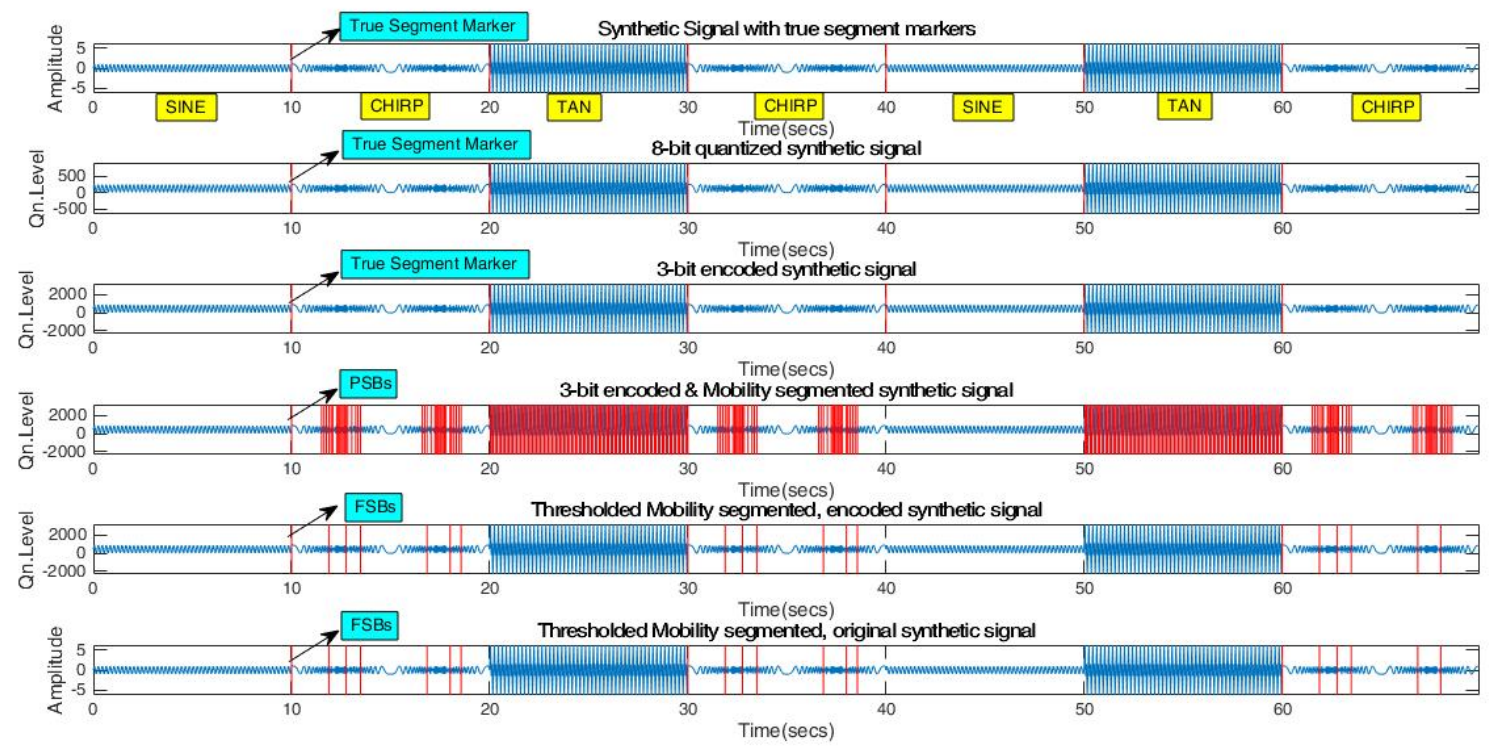

Figure 3.28: Testing Mobility-based segmentation on a synthetic signal

Following these methods, the next section now proceeds to discussing actigraphy feature extraction. In this section a review of various features known for pattern classification in actigraphy signals will be conducted along with the inclusion of two novel actigraphy-specific features and a new kernel-based method to reduce actigraphy data.

\subsection{Actigraphy Feature Extraction}

The process of identifying signal specific markers or properties exhibited in one phenomenon, and using them to distinguish from properties exhibited in another phenomenon, produced by the same source, is termed as Feature Extraction. In simpler terms, the process of reducing a non-stationary, real-world signal to a few key characteristic properties for pattern classification is called Feature Extraction. Feature extraction helps reduce the number of resources needed to analyze a signal. In the past few decades feature extraction methods have taken tremendous leaps - from being simple time and frequency domain techniques, to minimization based approaches using sparse representations and compressed sensing techniques [36].

Robust and relevant feature extraction from physiological signals is one of the hot topics in biomedical signal processing since it is affected many factors such as $[32,36]$ :

- Structure and morphology of biomedical signals and their source.

- Type of disorder, disease or movement pattern exhibited by the individual. 
- Inherent dynamic nature of physiological signals.

- Multi-modality nature of some biomedical signals such as EEG and EMG

- Methods by which a physiological signal is captured - electrode-based sensors or wearable sensors.

Raw physiological signals collected using wearable sensors, during movement activities are usually sampled over discrete time periods and contain a large number of data points or samples for analysis. A review of prior studies indicates that in most cases, feature analysis of physiological signals from sensors is mostly applied on the raw data [32]. Although these studies employ standard methods of noise and artifact filtering, when it comes to segmentation, most works have either used a fixed-width scheme, or treat the entire signal in one operation. This is true only in case of signals obtained using wearable devices, and is not applicable to clinically acquired physiological signals [32].

One of the biggest hurdles in analyzing actigraphy signals is to distinguish different types of human activities recorded by the sensor $[7,37,38,40,76,92]$. Ideally this process must be done automatically and with great speed and accuracy, considering the large amounts of data that need to be processed. In many prior studies, machine learning methods have been implemented to detect the type and level of human activity captured using actigraphs [5-7,92]. In most of these works, features were extracted from the raw signals at pre-defined times and then fed into a learning tool for classification. the most commonly used features for characterizing actigraphy signals were statistical in nature, or extracted from the time and frequency domains. Following sections briefly discuss these features.

\subsubsection{Time-domain Features}

Signal analysis in time-domain usually involves extracting features from a specific signal window of say $n$ samples. This segment can be randomly selected since the underlying source behavior would remain the same [36]. This would also account for the assumption that although the physiological signal may be nonlinear and non-stationary, within the segment it would still be considered as quasi-stationary, and therefore the features calculated from it would remain consistent for the entire signal [36]. The size of the segment or signal window not only depends on the specific activity, but also on the effect on classification accuracy. The window could be of fixed width and sliding in nature, or it could be designed to be activity specific. Activity-specific windows might require more pre-processing of the actigraphy signal and may be optimized using an adaptive segmentation technique such as the ones discussed in Sections 3.2.3 and 3.2.4. The purpose of time-domain features is to characterize the following measurements from the signal [36]:

- Signal structure 
- Visually evident patterns from corresponding human activity

- Signal strength

- Signal sample distribution and the associated probability density function

Of the many time-domain features, the most commonly used are the mean and standard deviation which describe the basic statistical behavior of an actigraphy signal. Although they may not be accurate to correctly classify an activity, nevertheless their inclusion does support the machine learning model in a better clustering of classes. Given an actigraphy signal $s$, Some of the commonly used time-domain features have been listed as follows [36, 93]:

1. Mean: The average amplitude of the actigraphy signal over $n$ discrete samples, given by

$$
\text { Mean }_{s}=\frac{1}{n} \sum_{i=1}^{n} s_{i}
$$

2. Standard deviation: The quantification of dispersion of a set of signal values over a given discrete time interval, expressed as,

$$
\Sigma_{s}=\sqrt{\frac{1}{n} \sum_{i=1}^{n}\left(s_{i}-\frac{1}{n} \sum_{i=1}^{n} s_{i}\right)}
$$

A low $\Sigma_{s}$ indicates that sample values are closely distributed around the mean, while a high $\Sigma_{s}$ shows a wider distribution of signal samples.

3. Variance: As a square root of the standard deviation, variance measures how far sample values are spread with respect to the mean. It essentially indicates the signal variability. Higher variance shows that sample values change drastically within a time window. It is calculated as,

$$
\operatorname{Var}_{s}=\sqrt{\Sigma_{s}}
$$

4. Hjorth's Parameters: Hjorth's statistical parameters were defined by Bo Hjorth in 1970 [80] and were based on the variance of a given signal and its first order derivative or first order difference. These parameters are defined as follows:

- Activity: This parameter represents the variance of a signal, and can be computed using Eqn.3.44. Hence,

$$
\text { Activity }_{s}=\text { Var }_{s}
$$


- Mobility: As mentioned in section 3.2.4, the mobility is the square root of the ratio of the variance of the first order difference $d s$ to the variance of the original signal $s$.

$$
\text { Mobility }_{s}=\frac{V a r_{d s}}{\operatorname{Var}_{s}}
$$

- Complexity: The complexity of a signal indicates the change in frequency and is the ratio of the mobility of the first order difference to the mobility of the original signal. Thus it can be expressed as,

$$
\text { Complexitys }_{s}=\frac{\text { Mobilityds }_{\text {obilitys }}}{\text { Mobits }}
$$

where, Mobility ds $=\frac{\operatorname{Var}_{2 d s}}{\operatorname{Var}_{d} s}$ and $2 d s$ is the second order difference of the actigraphy signal $s$.

5. Root Mean Square (RMS) value: The RMS of an actigraphy signal is the square root of the mean of the squares of the values, and is computed as,

$$
R M S_{s}=\sqrt{\frac{1}{n}\left(S_{1}^{2}+S_{2}^{2}+\ldots+S_{n}^{2}\right)}
$$

RMS could be used synonymously with standard deviation, when a signal has zero mean. This means that RMS would always be greater than the average value of the signal. RMS could be used to measure the deviation of a feature value from its usual or normal value. This also helps in feature discrimination $[94,95]$.

6. Maxima: This is the maximum peak value of the actigraphy signal

$$
\operatorname{Maxima}_{s}=\max _{s}\left(s_{n}\right)
$$

This is a highly useful statistical feature since it could be used for measuring peak activities in physiological signals. These peak activities usually depict an above normal signal behavior and their presence could very well characterize different phenomena from the biosignal data [5, 95].

7. Peak to Peak Difference: Difference between the maximum and minimum peak values given as,

$$
P 2 P=\max _{s}\left(s_{n}\right)-\min _{s}\left(s_{n}\right)
$$

Similar to maxima, the peak to peak difference also identifies signal peaks, and computes the difference between adjcent ones. This difference could be used as a surrogate parameter to measure how drastically 
a signal varies $[5,95]$.

8. Peak to RMS ratio: Ratio of the maximum value to the RMS of the signal, given as,

$$
\text { P2rms }=\frac{\text { Maxima }_{s}}{R M S_{s}}
$$

The peak to RMS ratio measures relative change in signal peak values with respect to its RMS value. This indicates the value with which a signal deviates from its normal value at zero mean.

9. Peak to Average ratio: Ratio of the maximum signal value to its mean.

$$
P 2 a v g=\frac{\text { Maxima }_{s}}{\text { Mean }_{s}}
$$

The peak to average ratio is similar to its peak to RMS counterpart, except that in this case, the absolute mean value of the signal is taken by assuming that it is stationary in nature [95].

10. Peak to Average power ratio: Ratio of the maximum signal value to its average power.

$$
P 2 p w r=\frac{\text { Maxima }_{s}}{\frac{1}{n} \sum_{i=1} n s_{i}^{2}}
$$

The peak to average power ratio is critical when a large number of peaks are present in the signal, and many of them may contribute to overlapping noise. In this case, the peak to average power ratio could differentiate between peak signal components and peak noise components [95].

11. Band Power: This is the average power of an actigraphy signal expressed as,

$$
B P_{s}=\lim _{n \rightarrow \infty} \frac{1}{2 n+1} \sum_{i=1} n s_{i}^{2}
$$

The band power essentially determines the contribution of a given frequency band to the overall power of the signal. In practical applications, the band power of a signal could be used for differentiating between sleep stages from EEG data [96].

12. Log-Energy: The logarithmic energy of the actigraphy signal calculated as,

$$
\log _{s}=\sum_{i=1}^{n} \log \left(s_{i}^{2}\right)
$$

The Log-energy of a signal is an improved quantitative measure of signal's energy, and helps in identifying regions of very small energy by preventing the values from becoming too small [97]. 
13. Accelerometer Counts: It is the sum of an actigraphy signal samples over a discrete period of time, expressed as,

$$
A C_{s}=\sum_{i=1}^{n} s_{i}
$$

The actigraphy counts signify how many important vibrations have been captured by the accelerometer, and which ones represent significant movements $[37,76]$.

14. Signal to Noise and Distortion (SINAD) ratio: This parameter measures the quality and clarity of an actigraphy signal, and indicates how it is affected by the presence of noise and distortion components [95]. It is usually expressed as,

$$
s n d r_{s}=\frac{P_{s}+P_{\text {noise }}+P_{\text {distort }}}{P_{\text {noise }}+P_{\text {distort }}}
$$

15. Zero-Crossings: The number of times an actigraphy signal crosses its median value.

Apart from these features, some accelerometry analysis studies have also used parameters such as sample occurrence percentiles, autocorrelation, cross-correlation, skewness and kurtosis, for activity recognition applications.

\subsubsection{Frequency-domain Features}

In most currently available signal processing methods, frequency domain transformation and feature extraction is a commonly occurring operation. While the time-domain features depict how a signal changes its values over time, the frequency domain transformation yields information on the rate of change in signal values and its spectral composition $[36,98]$. Frequency features are usually obtained through the Fast Fourier transform (FFT) of the physiological signal. The absolute values of an FFT of a signal, are the magnitudes of the corresponding frequency components. Therefore, the largest magnitude points towards the most dominant frequency component of the signal, which in turn indicates the activity intensity level. In actigraphy signals the spectral entropy has been a commonly extracted feature since it measures the intensity of each activity with a unique value. In an activity analysis study conducted by Bonomi et al. [99], the presence of low spectral entropy indicated the detection of simple human activities such as walking on a treadmill, while high entropy components directed towards complex movements such as freestyle walking or playing sports. This being said, the Fourier analysis of a physiological signal yields valuable feature information, and has been extensively used in biomedical signal classification and compression [36]. A review of prior studies yielded the following commonly used frequency-domain features of actigraphy signals [36, 98]: 
1. Dominant Frequency: The FFT of an actigraphy signal gives a distribution of various frequencies in the signal. The dominant frequency of the signal is defined as that frequency which carries the maximum energy when visualized in a spectrum.

2. Median Frequency: This is the frequency at which the power spectrum of the actigraphy signal is divided into two regions with equal amplitudes. It can be expressed as,

$$
M e d F=\frac{1}{2} \sum_{j=1} B P_{j}
$$

where, $P_{j}$ is the power spectrum at frequency bin $j$, and $B$ is the next power of 2 from the length of the actigraphy signal in time domain.

3. Mean Frequency: The mean frequency of an actigraphy signal is the ratio of the sum of product of the power spectrum and frequency, to the total sum of the signal's power spectrum, and is given as,

$$
\text { MeanF }=\frac{\sum_{j=1} B f_{j} \times P_{j}}{\sum_{j=1} B P_{j}}
$$

where $f_{j}$ is the frequency value at bin $j$. In many studies, the mean frequency has also been referred to as the central frequency of the physiological signal.

4. Entropy: The entropy of a signal is defined as the rate at which information or data is generated by the source. It is an indicator of the presence or lack of information, and is expressed as the negative logarithm (usually natural or base 2) of the probability mass function of each frequency component. The entropy of an actigraphy signal is therefore calculated as,

$$
H(s)=-\sum_{j=1}^{B} P\left(f_{j}\right) \log _{n} P\left(f_{j}\right)
$$

where $P\left(f_{j}\right)$ is the probability mass function of the $j^{\text {th }}$ frequency component of the signal.

Following these, the next section discusses in brief some demographic and application specific actigraphy features.

\subsubsection{Demographic and Domain-specific Features:}

Demographic and subjective features have shown to be very effective in conducting longitudinal analysis of human activity in many clinical and non-clinical actigraphy studies. Apart from anthropometric features, some studies also included clinical features which have also been listed as follows [5, 6,72$]$ : 
- Age, Gender, Height, Weight

- Body mass index (BMI), body composition

- Respiration index (RHI), Apnea-Hypopnea index (AHI)

- Heart rate (ECG), Oxygen Saturation (O2Sat)

- Periodic limb movement index (PLMSI), skin temperature

- Total Sleep time (TST), Sleep Efficiency (SE)

Additionally, following Table 3.1 highlights the various attributes of these features, which would be useful to the reader in getting a clearer picture of how these features could be used in a pattern classification experiment.

Table 3.1: Demographic features and their attributes

\begin{tabular}{|c|c|c|c|c|}
\hline Feature & Feature Type & Variable Type & Units & Sample Value \\
\hline Age & Categorical \& Continuous & Positive Integer & years & $20,55,67$ \\
\hline Gender & Categorical & String Character & $\mathrm{N} / \mathrm{A}$ & $\mathrm{M}, \mathrm{F}$ \\
\hline Height & Continuous & Float & $\mathrm{cms}$ & $175.2,150$ \\
\hline Weight & Continuous & Float & kgs, lbs & $100.4,88.6$ \\
\hline Body Mass Index (BMI) & Continuous & Float & $\mathrm{N} / \mathrm{A}$ & $24.5,19.5$ \\
\hline Respiration Index (RHI) & Continuous & Float & $\mathrm{N} / \mathrm{A}$ & $12.5,7.8$ \\
\hline Apnea-Hypopnea Index (AHI) & Categorical \& Continuous & Float & $\mathrm{N} / \mathrm{A}$ & $5.6,3.4$ \\
\hline Heart rate $(\mathrm{ECG})$ & Continuous & Positive Integer & beats per minute & $67,89,55$ \\
\hline Oxygen Saturation (O2Sat) & Categorical \& Continuous & Positive Integer & $\mathrm{mmHg}$ & $66,88,95$ \\
\hline Periodic Limb Movement Index (PLM Index) & Categorical \& Continuous & Positive Integer & movements/hour & $15,145,5$ \\
\hline Skin Temperature & Continuous & Float & $\mathrm{C}$ & $39.6,37.5,35.4$ \\
\hline Total Sleep Time & Continuous & Float & hours & $5.6,8.9,7.2$ \\
\hline Sleep Efficiency & Continuous & Positive Integer & $\%$ & $88,67,45$ \\
\hline
\end{tabular}

The impact of using these features is varied in different actigraphy studies, and although they provide a longitudinal view of an individual's motor activity health, their usage for pattern classification and disease severity estimation applications remains limited. This is also supported by the fact that very few clinical studies have employed the use of analyzing actigraphy data using signal processing techniques.

This being said, some clinical studies which focused on detecting periodic limb movements (PLM) in sleep using actigraphy and PSG (polysomnography), also proposed the inclusion of a PLM specific feature derived from the periodic limb movement index. This feature is termed as the Periodicity Index (PI) and 
it is defined as the ratio of the manually scored periodic limb movement index to the number of intervals between valid or true limb movements in sleep $[5,6,59]$. This is an application-specific parameter and is expressed as,

$$
P I=\frac{\text { Manually Scored PLM Index }}{\text { No. of Intervals between true movements }}
$$

The periodic limb movement index is defined as the number of true limb movements per hours in Non-REM (non rapid eye movement) sleep stage, and is manually scored by a sleep technologist by scanning through PSG readings in an overnight sleep study [100]. In recent clinical studies, the periodicity index has become a significant morphological parameter for assessing the severity of PLMs, and has been reported to provide a higher degree of stability and accuracy for their detection during sleep. It is also a desirable form of measurement as a smaller number of leg movements are required. A recent work published in $[5,6]$ extracted this feature from actigraphy data, and yielded remarkable results in visually classifying subjects experiencing mild and severe PLMs in sleep, as shown in Figure 3.29.

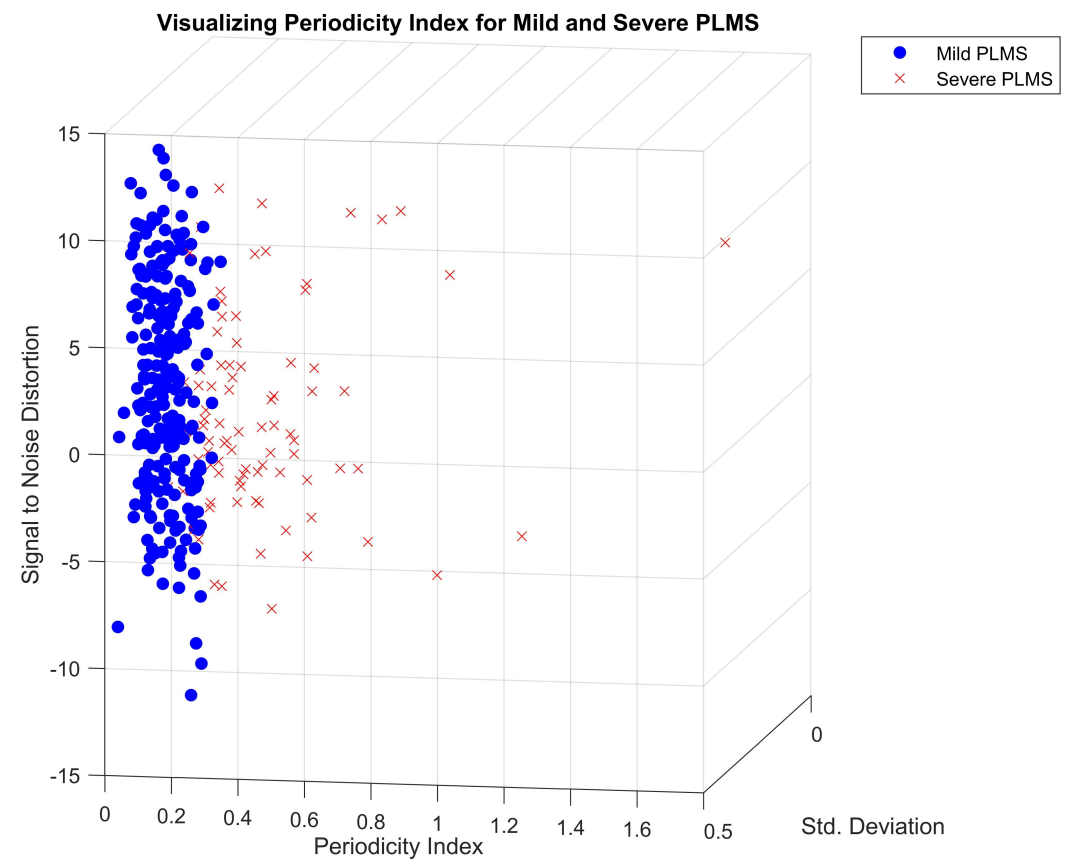

Figure 3.29: Periodicity Index as a classification feature [6]

The next section describes in detail the computation of two new actigraphy-specific features which will characterize movement data. Following this a new autocorrelation-based kernel function for feature extraction and dimensionality reduction of actigraphy will be introduced. 


\subsubsection{Research Contribution: Actigraphy-specific Features}

As discussed in Chapter 2, it is known that actigraphy signals are non-linear, non-stationary and register data only when a movement is sensed by the accelerometer. Conventionally, in order to identify movement specific markers from actigraphy data, the afore mentioned time and frequency domain features are extracted and fed to a machine learning tool for classification. Through this study's experiments of various actigraphy datasets it was observed that with increase in the number of classes or the number of actigraphy signals, the purpose of characterizing movements using generic time and frequency parameters becomes generalized, thereby reducing the classification accuracy. Additionally, not many prior studies have investigated into developing new features which are specific to actigraphy analysis. In this study, it was found that extracting rate of change information from actigraphy data and using it to deduce new parameters would be a useful approach in actigraphy signal classification. The inclusion of two new signal-specific parameters which describe the rapid changes and activity regions in an actigraphy signal is as follows:

1. Spiky Index (SI): The spiky index of an actigraphy signal is defined as the number of prominent peaks or movements detected within a total recording or activity time. A prominent peak is one whose amplitude is at least half of the maximum peak value of the actigraphy signal. The spiky index can be expressed as,

$$
\text { Spiky Index, SI }=\frac{\text { No. of prominent peaks }}{\text { Total activity time }}
$$

where, No. of prominent peaks $=$ Count $\left\langle s_{n} \geq \frac{\max _{n}\left(s_{n}\right)}{2}\right\rangle$. Similar to the PLM index mentioned in section 3.3.3, the spiky index is expressed in number of movements per hour, or any other units of time, depending on the duration of the recording.

2. Rapid Change Factor (RCF): As described earlier in Section 3.2.3, the rapid change factor detects drastic changes in an actigraphy signal using its quantization resolution and step-size. The purpose of this new parameter is to identify regions in the actigraphy signal which exhibit a significant change in human movement. It should be noted that actigraphy data is usually quantized or digitized during recording, and has a specific bits per sample resolution associated with it. The computation of RCF depends on this value, and is unique to each actigraphy signal. Quantization or digitization of an actigraphy signal results in the mapping of each analog sample value to a discrete real or integer value by the A-to-D converter. The range of these discrete values is determined by the bit resolution of the actigraph. For example, if the bit resolution of an actigraph is $m=12$ bits/sample, then the number of discrete steps would be $m=\left(2^{12}-1\right)=4095$ steps. From this it can be inferred that an actigraphy data sample would be assigned one of 4095 steps by the A-to-D converter. In simple words, actigraphy 
data can be visualized in the form of discrete steps. By definition, the Rapid Change Factor is the rate by which an actigraphy signal changes its discrete value from one step to another. It is the ratio of the step-size of the quantizer, to the product of the bit-resolution and the sampling period. The computation of this parameter has been explained in section 3.2.3. For a quick reference, the RCF is expressed as,

$$
R C F=\frac{S S}{\left(m \times t_{s}\right)}
$$

where $S S$ is the quantizer step-size given by $S S=\frac{R}{\left(2^{m}-1\right)}$, and $t_{s}$ is the sampling period. The spurious free dynamic range of the actigraphy signal $s$ is computed as shown in Eqn.3.32.

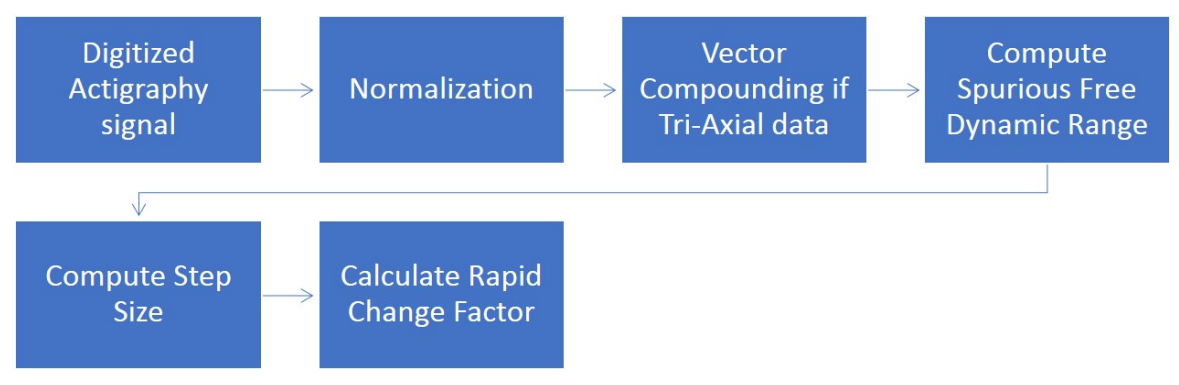

Figure 3.30: Computation of RCF

As per this study's experiments, the rapid change factor can be used in three distinct ways for actigraphy analysis:

- Signal Thresholding: Ignore signal amplitudes which are less than the RCF. Only consider those samples which are greater than $\mathrm{RCF}$ and represent peak activity.

- As a Feature: The RCF can be used as a unique feature for classifying actigraphy signals from each other. Note that the RCF will be unique for each type of activity recorded. If a sensor captures multiple types of activities, then the RCF will be computed for the entire time-series irrespective of the activity performed.

- Adaptive Signal Segmentation: As mentioned in section 3.2.3, When coupled with activity specific ground truth information, the RCF could be used to detect rapid changes in the actigraphy signal, and extract those regions which depict maximum amount of activity in a given application.

To give the reader a better idea, Figure 3.31 below illustrates how the RCF could be used for signal thresholding. For this a sample wrist actigraphy signal of a test subject walking on a flat surface for about 5 seconds has been chosen. 


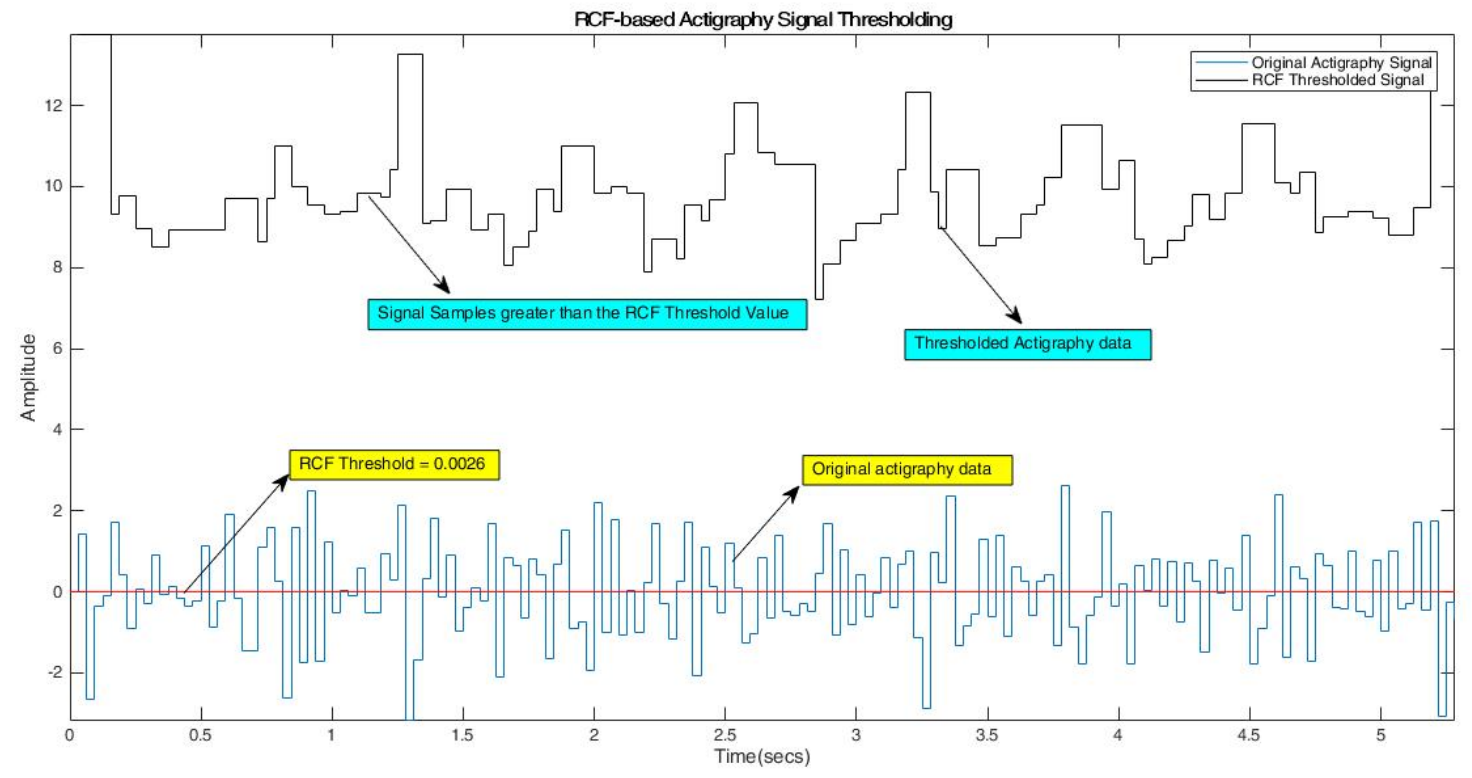

Figure 3.31: Thresholding Actigraphy Data using RCF

Following these two parameters, the next section proceeds to explaining a novel kernel-based actigraphy modeling method.

\subsubsection{Research Contribution: Auto-correlation Kernel for Actigraphy Modeling}

\section{Kernel-based modeling}

Deriving linear relationships within signal components is a desirable approach for developing machine learning tools, so that suitable features can be extracted from the input data for the learning algorithm. However, when the input signal exhibits properties of non-linearity and non-stationarity, simple signal processing and machine learning tools tend to produce non-linear separation of classes or patterns exhibited by a group of similar signals emanated from similar sources. For example, neuromuscular activities detected using accelerometry techniques are highly likely to exhibit random variations and differences when captured from different test subjects. This nullifies the possibility of applying simple signal processing tools for pattern classification [36].

One way to address this is by the application of kernel methods to the input signal, which would transform it into a higher dimensional space, with a compact representation, and where it would be easier to distinguish between signal classes $[36,101,102]$. This means, transforming the long-duration, one-dimensional input signal into a smaller set of features which could be visualized in higher dimensional space. Although the 
kernel mapping might be non-linear in nature, the inter-class separation itself would be linear. In general, kernel transformations involve two major steps [101,102]:

- Mapping the non-linearly separable input data from its current lower dimensional space (input space), to a linearly separable data in the (feature space), and,

- Application of a learning algorithm for finding patterns and classifying in the feature space.

A kernel can be represented as a function of the inner dot product of the input signal. Assume that the input signal or the training sample is of the form,

$$
s=\left[s_{1}, s_{2}, s_{3}, s_{4} \ldots, s_{n}\right]
$$

In order to explore its separability from other signals generated from similar sources, the signal $s$ can be transformed into higher dimensions using the following kernel trick,

$$
s=\left[s_{1}, s_{2}, s_{3}, \ldots, s_{n}\right] \rightarrow \Psi(s)=\left[\psi_{1}(s), \psi_{2}(s), \psi_{3}(s), \ldots, \psi_{n}(s)\right]
$$

Equation 3.66 indicates the mapping of the input signal from its original space $s$ into a new space $F$, which is also called Feature Space. In other words, the output of transformation $\Psi(s)$ for each signal, is actually a set of features or coefficients in higher dimensions, which in combination with features from the signal's original space, could be used for pattern classification [36,101,102]. Figure 3.32 would give the reader a better understanding of the kernel transformation process.

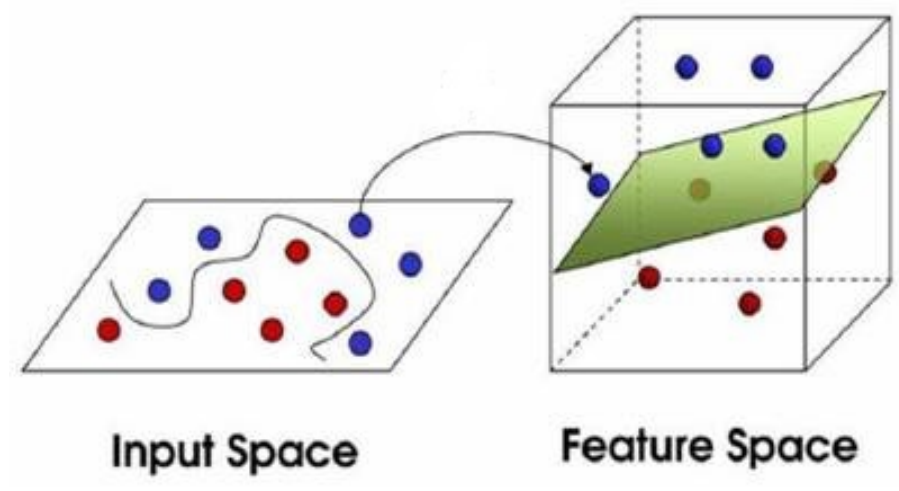

Figure 3.32: Dimension transformation using kernel methods [21]

The non-linear separation exhibited in the input space, as shown in Fig. 3.32, is largely attributed to the number of features extracted from each signal. For a given set of long-duration signals, increasing the 
number of features can lead to generalization of the classification algorithm, thus leading to poor performance [36]. This would also mean that a large number of features could hamper the learning capacity of the kernel function and subsequent learning algorithm. In terms of feature extraction, it may be noted that applying kernel transformation would not only transform the input signal to higher dimensions but would also considerably reduce the size of the feature set in kernel space $[36,102]$. In other words, kernel mapping would also enable the user to generate maximally relevant features, and help ignore insignificant or redundant features [101]. An inference from this, would be that kernel-methods can also help in appropriate feature selection by extracting only relevant information from each input signal.

Now, using equation 3.64, assume that the machine learning tool in the feature space or kernel space is governed by the following hypothesis for pattern classification,

$$
f(s)=\sum_{i=1}^{N} w_{i} \psi_{i}(s)+c
$$

where, $w_{i}$ is the weight associated with each signal component (or data point), $c$ is the constant bias of the system, and $\psi: s \rightarrow F$ is the kernel mapping from input space to the feature space. Corresponding to equation 3.66, the equivalent representation of pattern classification hypothesis in feature space can be written as,

$$
f(s)=\sum_{i=1}^{d} \alpha_{i} y_{i}<\psi(s) \cdot \psi(z)>+c
$$

where, $\alpha_{i}$ is the weight vector in feature space, $y_{i}$ in feature space represents each $s_{i}$ from input space, and $d$ represents the number of training points such that $d<<N$. The kernel function $K$ is then defined as,

$$
K(s, z)=<\psi(s) \cdot \psi(z)>\forall s, z \in s
$$

This implies that using kernels, a signal can be implicitly mapped into feature space by finding the inner dot products between two consecutive input samples or components, thus avoiding the computational complexity of finding the feature map. The kernel matrix or the feature matrix can be then expressed as,

$$
\mathbf{K}=\left(<s_{i}, s_{j}>\right)_{i, j=1}^{d}
$$

In order for a kernel to be fully admissible to pattern classification using a learning tool such as support vector machine, it must satisfy the following two conditions [101,102]:

- Mercer's Condition - It states that whether or not, a given kernel function is a dot product in some higher dimensional feature space. For a given kernel function $\mathbf{K}=\left(<s_{i}, s_{j}>\right)_{i, j=1}^{d}$, there 
exists a mapping $\psi$ in the feature space, if and only if, for any $g(s)$ such that $\int g(s)^{2} d s$ is finite, then $\int K(s, y) g(s) g(y) d s d y \geq 0$. It can be further shown that this condition is valid for $K(s, y)=(s . y)^{p}$ for any positive integer $p$. Therefore, any kernel function $K(s, y)=\sum_{p=0}^{\infty} b_{p}(s . y)^{p}$, where $b_{p}$ are positive real coefficients, and the series is uniformly convergent, satisfies Mercer's Theorem.

- Translation Invariance - For a kernel to be able to extract robust features, and subsequently train the learning algorithm, it must also be ensured that the kernel is translation invariant. This is possible only if its Fourier transform,

$$
F[K](\omega)=(2 \pi)^{-d / 2} \int_{R^{d}} \exp (-j(\omega . s)) K(s) d s
$$

is non-negative.

Note that in this study, it will be proven that the ACF kernel method satisfies the afore mentioned conditions of admissibility. The derivations briefly explained in this section indicate that kernel methods can prove to be highly suitable in analyzing characteristics or patterns in long-term biomedical signals, as they can significantly reduce computational complexity, improve hardware compatibility, and the generation of a compressed signal version for improved machine learning and classification. In kernel-based time series modeling methodologies, complex real-world signals undergo dimension transformation [101, 103-105]. The aim of a kernel function is to reduce the complex signal to simple set of features or representative values in (usually) higher dimensional space, so that identifying patterns (mostly through visualization) becomes easier.

In other words, kernel application is a dimension reduction process and greatly helps in pattern classification. Kernel based signal modeling methods have been extensively used in analyzing financial time series [101,103], biomedical time series such as gait signals [103], speech and speaker recognition [106-109]. In addition, kernel transformation methods can also double up as machine learning tools, and could be implemented in real-time applications.

The next sub-section proceeds towards discussing the contribution of an autocorrelation kernel function for actigraphy analysis.

\section{Autocorrelation Kernel Modeling}

Taking forward the afore-explained concepts of kernel-based feature extraction, an autocorrelation function based kernel [110] for extracting features from an actigraphy signal can be developed using the following steps. 
For a given signal $s=\left[s_{1}, s_{2}, \ldots, s_{n}\right]$, its Autocorrelation function $(\mathrm{ACF})$ is defined as,

$$
r_{s}(k, j)=E\left(s_{k} s_{k+j}\right)
$$

where, $n$ is the number of samples in the signal $s$, and $j$ is the time lag introduced between two samples. The ACF can then be expressed as,

$$
r_{s}(k, j)=\frac{1}{\sigma_{s}} \sum_{k=1}^{n-j}\left(s_{k}-\bar{s}\right)\left(s_{k+j}-\bar{s}\right)
$$

where, $\bar{s}$ is the mean value of the signal $s$, and $\sigma_{s}$ is the variance of the segment at zero lag. Note that the $\mathrm{ACF}$ is a symmetric function in nature, and hence $r_{s}(k+j)=r_{s}(-(k+j))$. As described in [110], the ACF can be decomposed as,

$$
r_{s}(k, j)=E\left(s_{k} s_{k+j}\right)=\lim _{n \rightarrow \infty} \frac{1}{\sigma_{s}} \sum_{k=1}^{n-j}\left(s_{k}-\bar{s}\right)\left(s_{k+j}-\bar{s}\right) \approx \frac{1}{\sigma_{s}} \sum_{k=1}^{n-j} \sum_{k=1}^{n-j}\left(s_{k}-\bar{s}\right)\left(s_{k+j}-\bar{s}\right)
$$

Therefore, $r_{s}(k, j)=\left(\psi\left(s_{k}\right) \cdot \psi\left(s_{k+j}\right)\right)$ can be considered as a kernel expression. Further to this, it can be shown that the ACF expressed in Eqn.3.73 follows Mercer's theorem and is always non-negative, as proved in [110]. Using the decomposed ACF derived in Eqn.3.73, a kernel-method which is a modification of works in [103] and [110] can be developed as follows.

Before proceeding with the derivation of score vectors or features, assume that for an actigraphy signal $s$ having $n$ number of samples, two types of signal components, namely: prominent peaks depicting movements and non-prominent peaks will be observed.

Based on this, the Expectation-Maximization (EM) algorithm [111] is applied to find the initial estimates given by the vector, $e_{l}=\left[w_{l}, \mu_{l}\right]$. The EM method allows the computation of maximum-likelihood estimates of truncated or segmented data, which in this case would be useful, when combining features obtained from all the segments, for suitable feature reduction. Note that in this case for $e_{l}, l$ will be $l=2$, since there are two types of observable components in an actigraphy signal. Here, $w_{l}$ is the weight vector and $\mu_{l}$ is the mean vector. The weight vector $w_{l}$ defines the tendency of an actigraphy signal sample to be a prominent peak or a non-prominent peak depending on its amplitude, and the $\mu_{l}$ is the mean value of prominent and non-prominent peaks.

Using $e_{l}$ and Eqn.3.73 an ACF model $M$ for the signal $s$ can be built as,

$$
M=R((k, j), l)=\frac{1}{\sigma_{s}} \sum_{k=1}^{n-j} \sum_{k=1}^{n-j}\left(s_{k}-\mu_{l}\right)\left(s_{k+j}-\mu_{l}\right)
$$


Next, the local covariance of each sample $s_{k}$ within the signal $s$ is computed using the probability density function for the $k^{t h}$ sample value and the weight estimate $w_{l}$, as

$$
P\left(s_{k} \mid M, e_{l}\right)=\sum_{l=1}^{2} w_{l} R((k, j), l)
$$

Using this vector of local covariances computed for each sample in a signal, the global log-likelihood of each signal is then calculated as,

$$
\log P\left(s \mid M, e_{l}\right)=\sum_{k=1}^{n-j} \log P\left(s_{k} \mid M, e_{l}\right)
$$

Using the global log-likelihood and the EM estimates $e_{l}$, the score vector or feature vector is then computed. The kernel scores are composed of partial derivatives with respect to each parameter in $e_{l}=$ $\left[w_{l}, \mu_{l}\right]$, and are expressed as,

$$
\begin{gathered}
\frac{\partial}{\partial w_{l}} \log P\left(s \mid M, e_{l}\right)=\sum_{k=1}^{n-j} \frac{R((k, j), l)}{P\left(s_{k} \mid M, e_{l}\right)} \\
\frac{\partial}{\partial \mu_{l}} \log P\left(s \mid M, e_{l}\right)=\sum_{k=1}^{n-j}\left(\frac{R((k, j), l)}{P\left(s_{k} \mid M, e_{l}\right)} \times \frac{w_{l}}{\sigma_{s}} \sum_{k=1}^{n-j} \sum_{k=1}^{n-j}\left(2 \mu_{l}-s_{k}-s_{k+j}\right)\right)
\end{gathered}
$$

From Eqns.3.77 and 3.78, it can be observed that each derivative has two components, since $l=2$ in this study. Thus, $\frac{\partial}{\partial w_{l}}=\left(\frac{\partial}{\partial w_{1}}, \frac{\partial}{\partial w_{2}}\right)$ and $\frac{\partial}{\partial \mu_{l}}=\left(\frac{\partial}{\partial \mu_{1}}, \frac{\partial}{\partial \mu_{2}}\right)$. Therefore, the feature vector can be written in a concise representation of Eqns.3.77 and 3.78 as,

$$
\Psi(s)=\left[\frac{\partial}{\partial w_{l}}, \frac{\partial}{\partial \mu_{l}}\right]^{T} \log P\left(C_{s} \mid M, e_{l}\right)
$$

Thus, for each actigraphy signal $s$, a 4 -score or 4 -feature vector was obtained. The algorithm's approximate order of complexity for an $n$-sampled signal $s$ is $\mathcal{O}(3 n+n \log n)$. Thus an actigraphy signal $s$ can be reduced as shown in following Eq.3.80,

$$
s \rightarrow \Psi(s)
$$

Note that in this study's experiments with different actigraphy signals, the use of ACF kernel has shown remarkable improvement in classifying human movements in wake and sleep states. The final section of this Chapter will now summarize all relevant actigraphy features and how they have been used in this research work. 


\subsubsection{Summary of modalities and features used in this research}

From the afore discussed known and newly developed features, a list of various features used in this study of actigraphy signal analysis can be defined. For the reader's reference, from Section 1.4 in Chapter 1, the list of actigraphy datasets used in this study for developing the actigraphy analysis system can be reiterated and they can be denoted alphabetically as follows:

- Database A: 191 short-duration, single-axial ankle actigraphy signals captured from 96 subjects in a clinical sleep study for detecting periodic limb movements (PLMs) in sleep.

- Database B: 332 long-duration, tri-axial ankle actigraphy signals acquired from 166 patients in a clinical sleep study for detecting PLMs in sleep.

- Database C: 92 long-duration, single-axial ankle actigraphy signals collected from 46 individuals undergoing stroke recovery in a clinical sleep study.

- Database D: 274 short-duration, tri-axial wrist actigraphy signals captured from 16 healthy volunteers performing 14 different activities of daily life (ADL).

- Database E: 89 short-duration, single-axial knee vibration signals acquired from 38 healthy and 51 unhealthy individuals for detecting severity of knee-joint cartilage degeneration.

For each database specific features were extracted, pertaining to each type of sleep and wake activity, for detecting movements using the approach depicted in Figure 2.7 of Chapter 2. Following Table 3.2 lists down the features used for analyzing each database in this research. The reader may note that the features highlighted in bold font are contributions from this research study on actigraphy signals. 
Table 3.2: Features used in this actigraphy study

\begin{tabular}{|c|c|c|c|c|c|c|c|}
\hline \multirow{2}{*}{ Domain } & \multirow{2}{*}{ No. } & \multirow{2}{*}{ Features Used } & \multicolumn{5}{|c|}{ Actigraphy Database } \\
\hline & & & $\mathbf{A}$ & B & $\mathbf{C}$ & $\mathbf{D}$ & $\mathbf{E}$ \\
\hline \multirow{10}{*}{ Time } & 1 & Mean & & & & & \\
\hline & 2 & Standard Deviation & & & $\checkmark$ & & \\
\hline & 3 & Variance & $\checkmark$ & & & & \\
\hline & 4 & RMS & & & & & \\
\hline & 5 & Maxima & & & & & \\
\hline & 6 & Peak-to-peak difference & & & & & \\
\hline & 7 & Peak-to-RMS ratio & & & & & \\
\hline & 8 & Peak-to-average ratio & & & & & \\
\hline & 9 & Peak-to-average power ratio & & & & & \\
\hline & 11 & Signal to noise \& distortion ratio & & & & & \\
\hline \multirow{2}{*}{ Frequency } & 12 & Median Frequency & & & & & \\
\hline & 13 & Mean Frequency & & & & & \\
\hline Kernel-based Features & $14-17$ & ACF Kernel Scores (4 Features) & & & & & \\
\hline \multirow{2}{*}{ Actigraphy-specific } & 18 & Rapid Change Factor & & & & & \\
\hline & 19 & Spiky Index & & $\checkmark$ & $\checkmark$ & & \\
\hline \multirow{4}{*}{ Morphological } & 20 & Hjorth's First order mobility & & & & & \\
\hline & 21 & Hjorth's Second order mobility & & & & & \\
\hline & 22 & Hjorth's complexity & & & $\checkmark$ & & \\
\hline & 23 & Periodicity Index & $\checkmark$ & $\checkmark$ & & & \\
\hline \multirow{4}{*}{ Clinical Labels } & 24 & Manual PLM Index & & $\checkmark$ & & & \\
\hline & 25 & Sleep Efficiency & & $\checkmark$ & & & \\
\hline & 26 & Oxygen Saturation & & & $\checkmark$ & & \\
\hline & 27 & Type of Knee Joint - Normal, Abnormal & & & & & $\checkmark$ \\
\hline Other Labels & 28 & Activity Type & & & & $\checkmark$ & \\
\hline
\end{tabular}

In addition to this, the following block diagram in Figure 3.33 shows an overall operation of the proposed actigraphy analysis system. Note that this flowchart also mentions which operations are conducted on-line and which are performed offline. "On-line" operations mean that these modalities could be implemented on the device with streaming data; whereas "offline" operations mean that the functionality needs to be implemented after acquiring sufficient modelling data from "on-line" operations. 


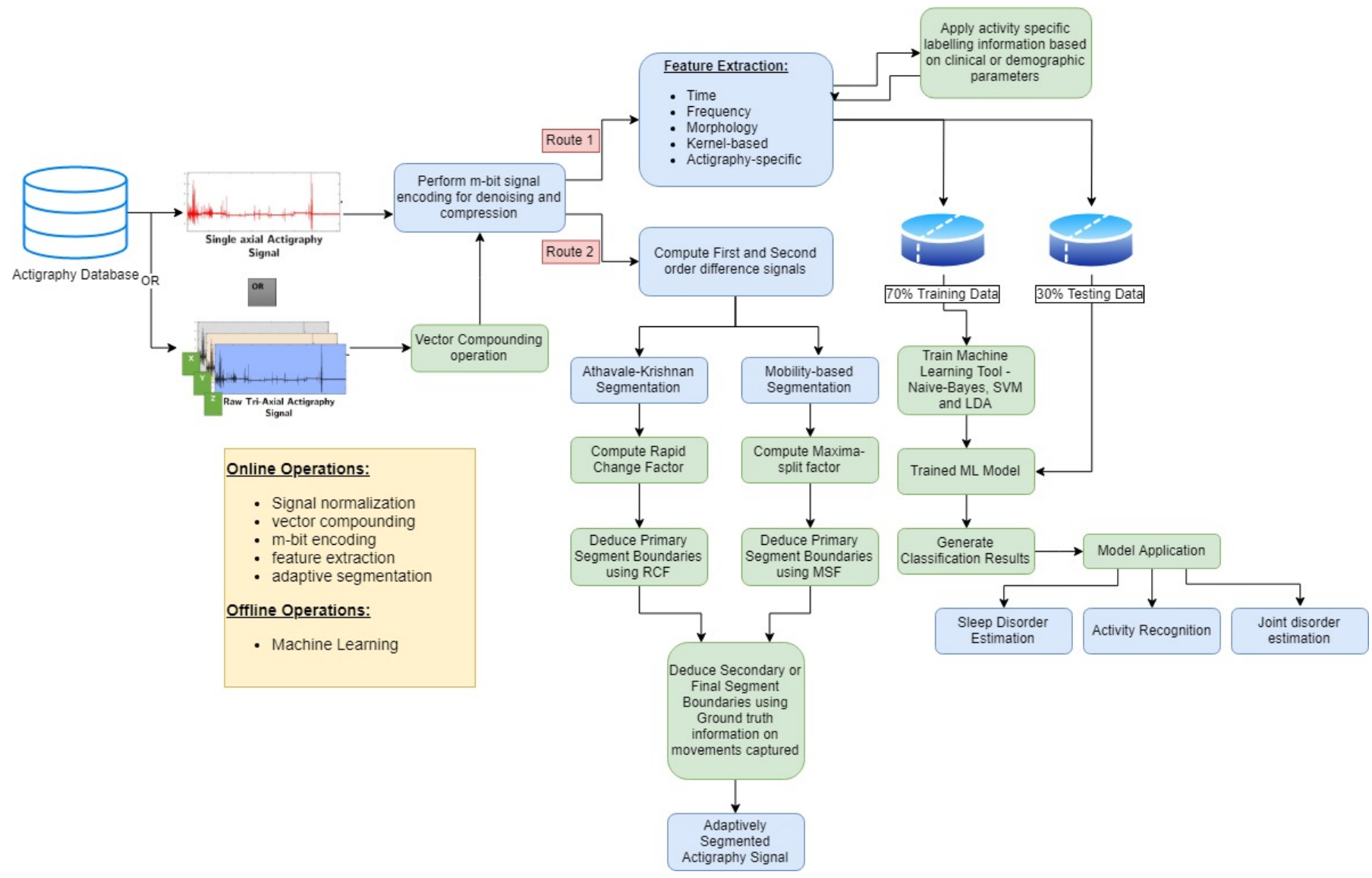

Figure 3.33: Overall Flowchart of Actigraphy Analysis Application

As illustrated in Figure 3.33, the reader may note that in this research two algorithmic routes have been taken to develop the actigraphy analysis application. Through Route 1, after encoding the actigraphy signal, it undergoes the process of feature extraction and pattern classification using machine learning tools for developing models for sleep disorder detection, activity recognition and joint-disorder severity estimation. It must be noted that in this approach, the signal does not undergo any segmentation to extract regions of significant movement activity. On the other hand in Route 2 methodology, after encoding an actigraphy signal undergoes adaptive segmentation using two key methods - the AK algorithm and the mobility-based algorithm. Using actigraphy specific parameters and activity-based ground truth information, segment boundaries are extracted for identifying regions of peak movement activities. Thus, to reiterate actigraphy signal classification and segmentation have been implemented as two separate modules within the same application, and could be combined in future research studies. Additionally, the reader may also note that in this research work the choice of machine learning algorithms, namely - the Naive-Bayes, LDA and SVM classifiers has been made with the intention of implementing light-weight or computationally inexpensive 
classifiers which could promote an edge computing approach to actigraphy analysis. The intention of machine learning in this research work is to therefore validate the accuracy and applicability of the proposed signal encoding, adaptive segmentation and actigraphy-specific feature extraction frameworks.

The next chapter will describe the application of various known and contributed actigraphy signal preprocessing, segmentation and feature extraction techniques, to sleep actigraphy studies for estimating the severity of neuromuscular disorders.

\subsection{Summary}

The third chapter of this dissertation has covered various aspects of actigraphy signal analysis. Taking the information on actigraphy signal behavior, this chapter begins by first understanding various signal pre-processing methods. In this section, a novel actigraphy signal encoding is described, which ensures signal denoising and compression at the acquisition source. Following this, two new adaptive segmentation techniques are discussed, namely - the Athavale-Krishnan algorithm and the Mobility-based algorithm for segmenting actigraphy signals for activity detection. Following this different feature extraction methods for actigraphy signals are described, which also includes two new actigraphy-specific features - the spiky index

and the rapid change factor, along with a novel ACF kernel method for feature extraction. This Chapter is then concluded by generating a table which summarizes which features have been used for different actigraphy datasets in this research. 


\section{Chapter 4}

\section{Sleep Actigraphy}

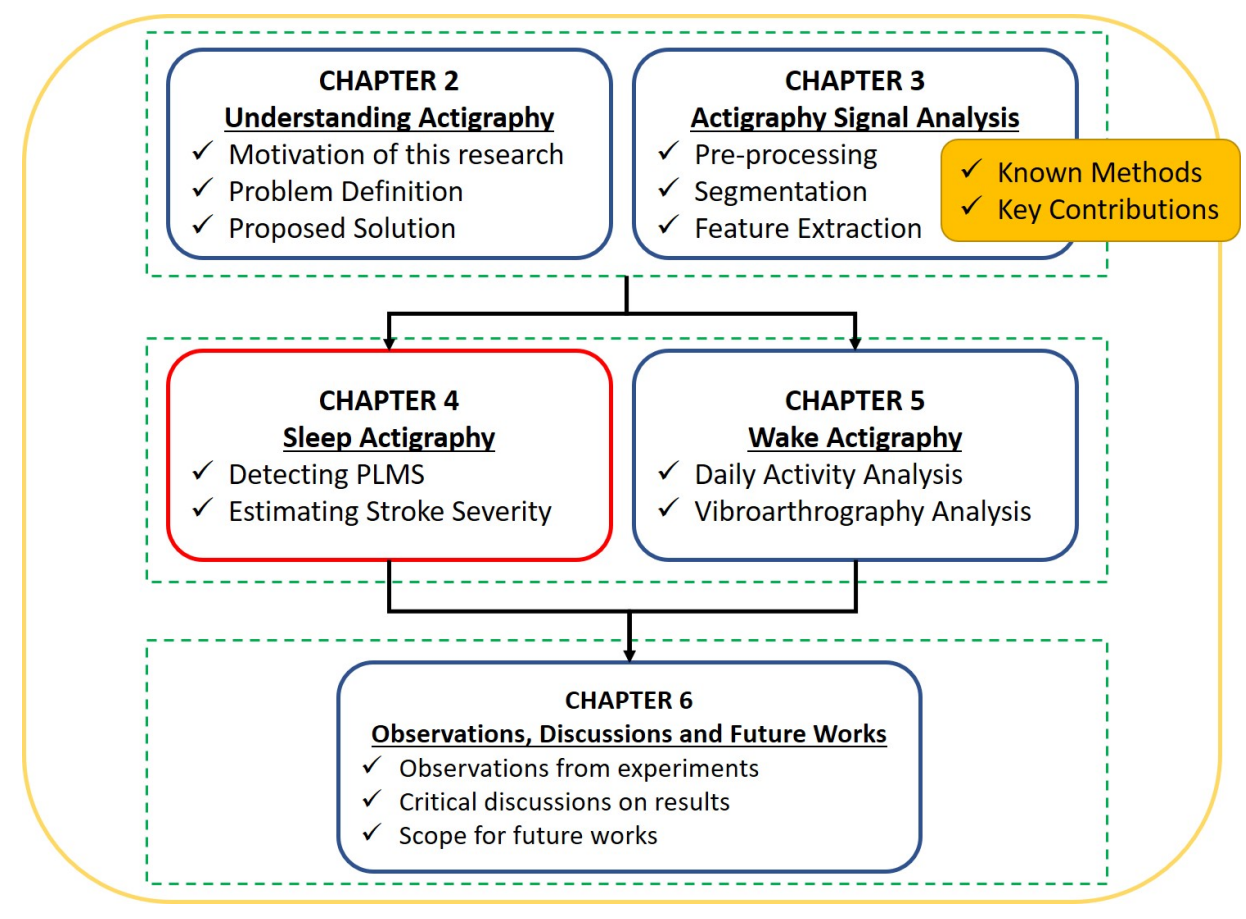

Figure 4.1: Organization of Dissertation

In the previous three chapters of this dissertation, extensive discussion was conducted on various aspects of this research in actigraphy analysis. Before applying the actigraphy analysis system to different datasets, the basics of actigraphy and its signal analysis were first revised. Various pre-processing, segmentation and feature extraction methods were discussed, which would be useful in developing the proposed system. Additionally, Chapter 3 also described the key contributions to this research in actigraphy analysis, and how they would potentially impact the outcomes of an actigraphy pattern classification problem. This chapter will 
now discuss the experiments in applying the afore said actigraphy analysis methods in various applications. Firstly, the developed methodology in this study is applied to sleep actigraphy analysis for estimating the severity of certain neuromuscular disorders. Before proceeding with describing the application of the proposed actigraphy analysis system to sleep actigraphy datasets, it is imperative to discuss sleep disorders in detail.

\subsection{Sleep Disorders}

Since the dawn of civilization, humans have constantly striven for improving their quality of life through various technological advances in tools pertaining to activities of daily life (ADL) $[5,7]$. These tools come from all facets of engineering design, be it infrastructure, energy systems, food supply, transportation, medicine, and communications. Advances in technology for civilization advancement has also bred its fair share of negative impact such as ever-increasing cost of living, stressful work routines and rising healthcare costs, to name a few. At an individual level, these trends have drastically impacted daily routines, especially sleep cycles.

It's quite common for an overall healthy individual to experience trouble sleeping once in a while, due to stress, sickness, travel or any other activity which might interrupt the person's daily routine. But if such episodes continue over a longer period, then that individual could be suffering from a sleep disorder. Sleep disorders can adversely affect an individual's physical and mental health such as fluctuating body weight, blood pressure, heart health, depression, anxiety, daytime sleepiness and fatigue $[2,3]$. Commonly prevalent disorders include insomnia, sleep apnea, periodic limb movements, restless leg syndrome and narcolepsy.

The last decade has seen a dramatic rise in the number of people being diagnosed with some sort of sleep related disorder, be it neurological or neuromuscular. Statistics indicate that sleep disorders are increasingly common in North America, in about $75 \%$ of individuals between the age group 20-59 in the United States and over $40 \%$ individuals in Canada [1,3]. As per experts from Sleep Disorders Program at University of British Columbia Hospital [1], about 15\% men and 5\% women in Canada suffer from sleep apnea, which is one of the most commonly diagnosed sleep disorders. Following Table highlights the prevalence of various sleep disorders in North America. 
Table 4.1: Neurological disorder statistics for North America [1-4]

\begin{tabular}{lc}
\hline \multicolumn{1}{c}{ Disorder } & \% Population \\
\hline Sleep Apfected in North America \\
Restless Leg Syndrome (RLS) & $3-7 \%$ \\
Periodic Limb Movements in Sleep (PLMs) & $4-11 \%$ \\
Parkinson's disease & $4-11 \%$ \\
Alzheimer's disease & $0.4 \%$ \\
Stroke & $2 \%$ \\
Depression & $2 \%$ \\
\hline
\end{tabular}

When it comes to diagnosing sleep related disorders, patients usually undergo a series of overnight sleep studies, wherein their sleep cycle is assessed by placing electrodes on specific regions of the body and recording vital physiological signals for analysis by the physician. This procedure is termed as polysomnography (PSG) and it involves the recording of EEG (electroencephalography), ECG (electrocardiogram), EMG (electromyography), respiration rate, and EOG (electrooculography) as shown in Figure 4.2.

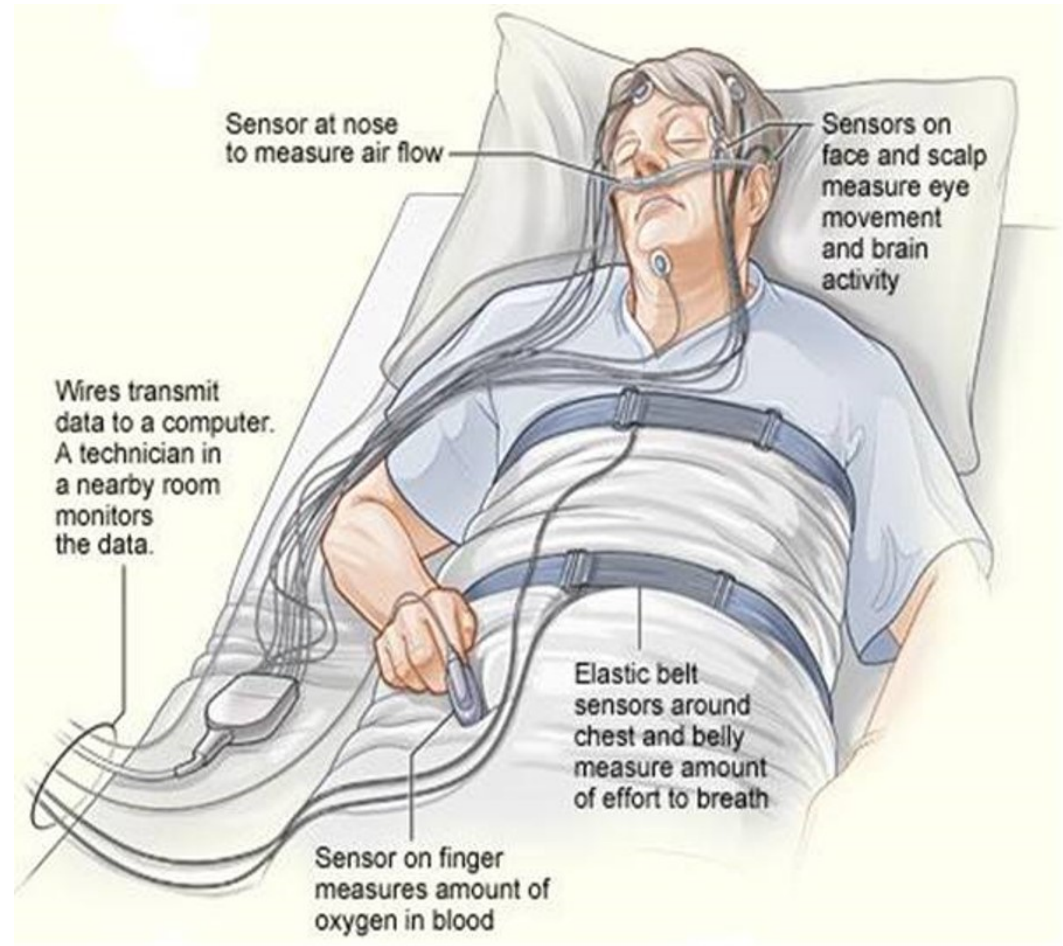

Figure 4.2: PSG Setup in a sleep clinic [22]

Advancements in sensor design and signal processing software has caused a boom in the development of wearable technologies which could potentially be used for vital sign monitoring via recently innovated 
wireless communication protocols and smart devices. These wearable technologies have also provided further motivation to investigate into their application for mobile or home-based sleep monitoring, as it would substantially reduce healthcare costs,and improve quality of life by encouraging a people-centered health monitoring model [32].

The objective in this chapter is to apply the proposed actigraphy analysis system to estimate the severity of sleep-related movement disorders using long-duration actigraphy signals. The initial algorithm development and subsequent actigraph signal analysis of around 96 patients (Database A) [5, 75], generated remarkable classification results which indicated the possibility of facilitating identification of PLMs across a wide spectrum of patient populations via the use of bilateral ankle actigraphy. This also provided an initial benchmark into the application of signal processing methods for analyzing actigraphy data to detect PLMs, since no prior studies were conducted from this perspective. A significant and potential outcome of this research would be the validation and implementation of wearable devices such as actigraphs for improved sleep monitoring, thus improving the quality of healthcare and life in general. The subsequent section will discuss a background survey in PLMs and the use of actigraphy in their estimation, which would be useful in applying the framework developed in this research.

\subsubsection{Periodic Limb Movements in Sleep (PLMS)}

Periodic Limb Movements (PLMs) is a common sleep-related neuromuscular disorder which occur in various patient populations and with increasing age. Growing evidence suggests a link between PLMs and vascular disease, and the study of PLMs remains an active area of investigation $[112,113]$. The ongoing research in sleep signal analysis for detecting and estimating periodic limb movements and associated cardiovascular disorders has given further inspiration to investigate into the use of accelerometer based sensors such as actigraphs which could potentially augment PSG analysis. Although a clinician's expertise in diagnosing diseases is unquestionable, analyzing large amount of physiological sleep signals for hidden information would require extensive informed aid from computer-based intelligent signal processing algorithms [5]. These algorithms not only extract hidden information from the signal but also help in classifying between normal and abnormal test subjects based on their respective signal analysis.

PLM in sleep are repetitive leg movements involving extension of the big toe coupled with stereotyped triple flexion of ankle, knee and hip. Each PLM occurs for a duration of at least 0.5 to 10 seconds, accumulating to a sequence of about four or more repetitive events at 5 to 90 second intervals $[100,114]$. The severity of these movements could vary from slight to strenuous and haphazard kicking, which can disrupt sleep and possibly lead to daytime sleepiness $[112,115,116]$. PLMs usually occur in non-Rapid Eye Movement 
(NREM) sleep.

During its initial discovery in the 1950s, PLMs were termed as nocturnal myoclonus, which meant rapid and rhythmic contraction of a muscle group (similar to that in seizures) during the night (usually when an individual is sleeping). Further investigations led to confirmation that PLMs did not correlate well with the term myoclonus, and hence this disorder was then disassociated with its original term [113]. Individuals with PLMs may also experience Restless Leg Syndrome (RLS) which causes discomfort of calves or thigh muscles, while at rest or times of inactivity [113]. RLS occurs in around 4\% of the North American population [11], and existing literature demonstrates that about $85 \%$ of individuals with RLS also experience PLMs [117]. In addition, PLMs commonly occur in individuals suffering from REM sleep behaviour disorder (RBD) $[112,115,116]$. It should be noted that RLS occurs only in wake state and exhibits sympotoms similar to PLMS. RLS usually causes a strong urge to move one's limbs without any neural control on the number or intensity of movements.

PLMs may be of primary or secondary nature. Some clinical experts have reported [118] that there's a strong evidence suggesting the pathophysiology of primary PLMs to be associated with neuronal hyperexcitability of gait generator, thus leading to decreased dopamine transmission. Consequently this theory supports the use of dopaminergic therapy to treat PLMs [118]. As the etiology doesn't seem to be clear, currently available treatments are only able to alleviate the symptoms but are unable to modify the disease. Secondary PLMs are usually attributed by causes related to some underlying medical problem such as diabetes, iron deficiency, spinal cord injury, narcolepsy and/or sleep apnea. An investigation of prior works indicates that many studies have reported the association of PLMs with attention deficit hyperactivity disorder (ADHD) and Tourette syndrome $[68,113,118,119]$. However, the latter has been widely debated due to different responses by patients to pharmacological treatments [118]. Other potential factors leading to secondary PLMs could be drug dependency, neuroleptic medication, anemia, uremia, Benzodiazepine withdrawal, Barbiturate withdrawal and Dopaminergic medication [118].

PLMs remain an active area of investigation for sleep specialists, with emerging evidence pointing to a potential link with cardiovascular disease $[64,73,74,120,121]$. Prior prospective studies have demonstrated that elevated PLM indices (of usually about 20 or more PLMs per hour) are associated with an increased risk of incident cardiovascular events $[74,120]$ and mortality $[73,120]$. Typically an individual experiencing PLMs may suffer from poor sleep or daytime sleepiness and fatigue $[112,113,115,118]$. As per a survey, it has been found that primary PLMs are usually chronic in nature, and although relapses/remissions occur, no treatment options currently available are able to modify the disease. Secondary PLMs on the other hand may subside with treatment of underlying cause $[116,118]$. Figure 4.3 shows PLMs of varying severity in different individuals during sleep. 

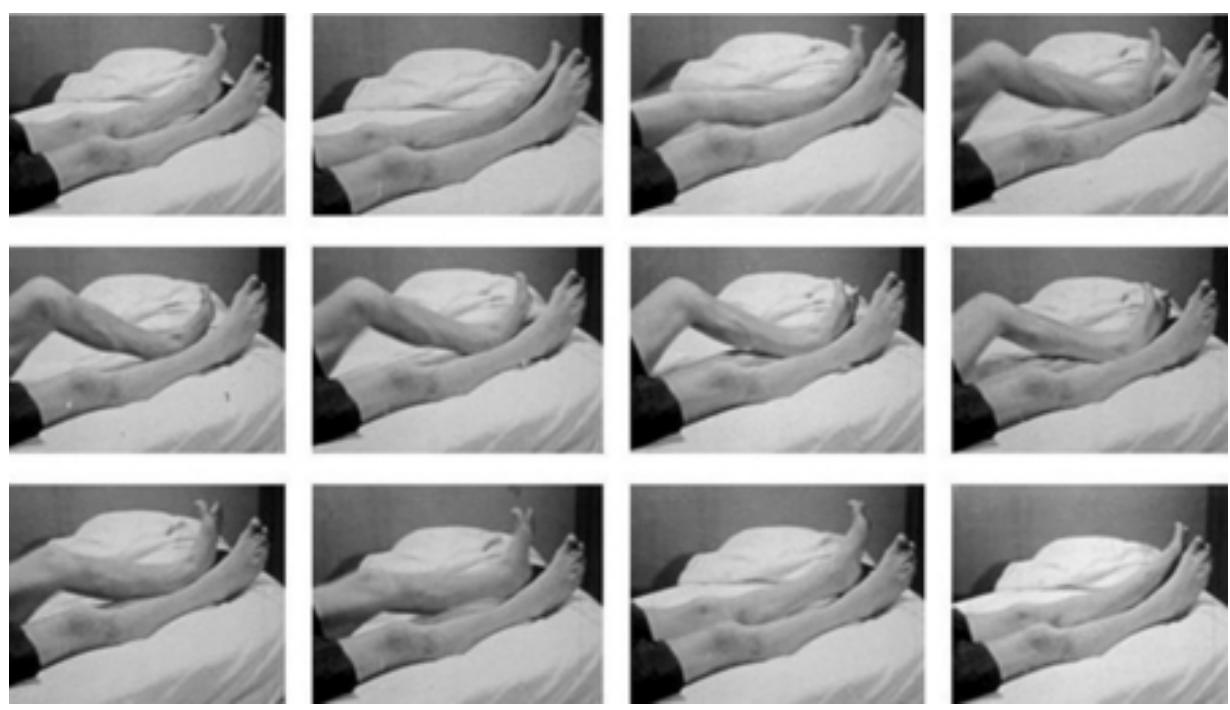

Figure 4.3: A Patient experiencing PLMs in lower left limb [23]

Although there are no lab tests or imaging studies which can help identify PLMs, there are certain clinical tests such as blood tests for hormones and haemoglobin whose deficiency could lead to secondary PLMs [118]. The only way to confirm the presence of PLMs is through Polysomnography (PSG), wherein an individual sleeps in a lab, and the leg movements are documented. As per clinical standards, a sleep specialist conducts a detailed, sleep study using Polysomnography (PSG) which captures electromyographic, electroencephalographic and respiratory breathing data for detecting PLMs [122]. The PSG study is usually carried out by a registered technologist in sleep clinic, who also performs data conditioning, filtering and labelling $[5,100]$. The extensive amount of biomedical signal data generated from PSG readings permits the detection of PLMs. Fig. 4.4 illustrates a sample EMG signal taken from a PSG reading of 90 second duration captured from a test subject in the age group 48-59 years and experiencing PLMs. 


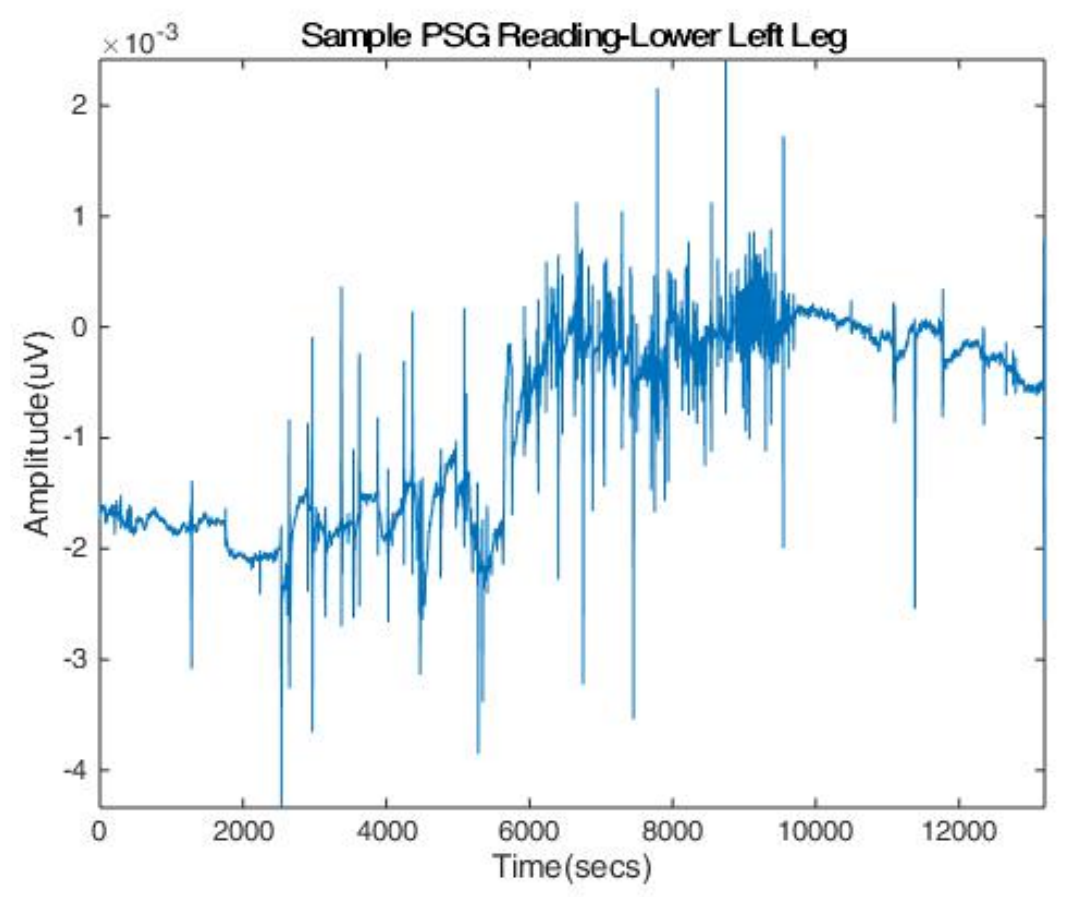

Figure 4.4: Sample PSG Signal taken from lower left leg

But considering the night-to-night variability in PLM data for any given patient, and the necessity to conduct a series of sleep studies, makes existing PSG techniques quite expensive, impractical and cumbersome. For example, in the province of Ontario, Canada, an overnight PSG study would cost about CAD $\$ 2000$ to the patient as out-of-pocket expenses if not covered by a third party insurance. Another factor which limits the use of PSG is the application of multiple electrodes placed at various locations on the patient's body, thus making the entire process of sleeping uncomfortable, and hence creating an unnatural environment for a sleep study. Accelerometer-based devices could prove to be useful tools in monitoring significant physiological signals such as leg movements in sleep, and their further analysis could potentially be benchmarked against existing gold standards for clinical validation. Of the many devices and sensors available in the market, actigraphy has been the most commonly used tool for assessing PLMs, and has been previously validated against PSG in multiple prior studies [59, 72, 123-126].

\section{Role of Actigraphy in Estimating PLMs}

As described in Chapter 2, actigraphs have been extensively used by researchers and clinicians to acquire and analyze motion data, be it during wake or sleep times. The primary intention of these works was to develop tools which could potentially be used by individuals for activity monitoring applications [42, 51 , $52,57,127,128]$. For example, actigraphy has been experimented for motion analysis in children diagnosed 
with Attention Deficit Hyperactivity Disorder (ADHD) [67], and in analyzing sleep patterns in children with autistic spectrum disorders [68]. Actigraphy has been extensively applied for studying individuals experiencing insomnia and depression [128-132], to detect motion abnormalities in activities of daily life (AOD). Similar studies have also been performed on elderly individuals suffering from Alzheimer's disease [55], Parkinson's disease [56,57], or in home-care or nursery-home settings [127,128]. A majority of these works have employed the use of wrist actigraphy, since the motion data was collected during wake times and periods of AOD $[38,40,42,51,55,132-134]$.

Apart from afore-mentioned short-comings, actigraphy has also been extensively used for monitoring abnormal motion activities in sleep. Specifically, exhaustive research has been done in evaluating the role of actigraph devices for monitoring disorders such as PLMs and Restless Leg Syndrome (RLS), for almost two decades $[5,59,64,74,126,135]$. The intention of these studies was to evaluate the performance of actigraphs with respect to existing PSG gold standards, and investigate the possibility of developing sleep/wake motion monitoring alternatives for patients suffering from specific sleep disorders. Additionally it has also been shown that, though there might be some differences in the data acquisition process between PSG and actigraphy, the latter allows for continuous recording of data for more than 24 hours without the need to replace sensors, batteries or its shelling [37]. PSG on the other hand requires extensive maintenance and consistency in operation, considering the number of electrodes required to be placed on the patient's body, and the amount of discomfort it causes.

One survey also indicated that in many sleep intervention trials when actigraphy was implemented on the same set of patients for multiple nights of testing, improved treatment effects were observed. Many studies have also been conducted to study the validity of actigraphy in otherwise healthy adults, on how they demonstrate the sedative effects of different medications while performing AOD [60]. Apart from traditional sleep studies, actigraphy has also been tested on military personnel to estimate severity of sleep deprivation and evaluate post-actigraphy treatment effects [69]. Additionally, actigraphy has also been extensively used evaluating post-treatment effects from critical diseases such as cardiac disorders and cancer [136-138], and has shown considerable improvement in patients' health through continuous activity monitoring. Finally, a survey of relevant literature also indicates that a lot of large-data studies have been conducted using actigraphy in community-based datasets, to identify salient differences in sleeping patterns, age, gender, ethnicity and sleep disorder prevalence [72,117].

Though, an ongoing review of prior works has reported numerous studies in estimating neuromuscular activities in sleep using actigraphs, very little consensus exists in determining which actigraphy data would yield best analysis for a researcher or clinician. Although several hospital-based studies have employed the use of actigraphs on lower extremities, such as bilateral ankle placement, for monitoring body movements in 
sleep, most of the actigraph devices are no longer available or they sample motion data too infrequently, thus leading to information loss. Also, it should be noted that previously reported actigraph studies did not apply extensive PSG and actigraphy signal analysis techniques, but rather depended on using simple statistical and morphological measures for generating informed decisions [6]. In conjunction to this, the investigation into applying actigraphs as home-based sleep monitoring wearables, is unable to reach a benchmarking standard due to debatable differences in device availability, demography, expert opinion and available research tools. Nevertheless, survey indicates that actigraphy has been highly promoted for evaluating sleep-movement disorders in conjunction to PSG over multiple trials and visits. Interestingly, in comparison to PSG, actigraphy has been found to generate moderately accurate results when assessing sleep disorders and as per some experts it could potentially be used for continuous sleep trails in a home-based setting, especially when the doctor expects the patient to undergo a normal sleep schedule.

This leads to inferring that there exists a big potential for developing generic, device-independent actigraphy screening tools and algorithms for monitoring motion activities in sleep [6]. To begin with, through this research, an actigraphy screening tool for estimating movement severity has been developed, which would aide clinicians in generating correct informed decisions. The system developed in this research is capable of analyzing long duration actigraph signals collected during sleep staging, by performing on-the-fly denoising, compression and activity-based adaptive segmentation. The algorithms developed in this study are capable of data dimension reduction, intelligent information extraction and pattern classification. Additionally, the results generated from this work also motivates clinical researchers to consider actigraphs as a complementary technology to the existing PSG standard, which is rather cumbersome and time-consuming to implement. It should be noted here that, in this study, a device-independent, actigraphy analysis system has been developed based on the flowchart depicted in Figure 2.7 of Chapter 2. The novelty of this research is that it addresses a potential gap created by the under-utilization of actigraphs in the clinical world.

To justify this research further, the contributed methods were compared with some significant clinical studies that validated actigraphy against in-laboratory PSG for estimating the severity of PLMs in sleep. Smith et al. [139] recently published a set of clinical guidelines for estimating PLMs in sleep in AASM (American Academy of Sleep Medicine), wherein it was suggested that actigraphy is premature method for evaluating PLMs in sleep since it exhibited very low to moderate correlation with PSG readings. This study was based exclusively on three prominent works by Gschliesser et al. [114], Kobayashi et al. [140] and Sforza et al. [141] mentioned in Table 4.1.1. Further investigation into these studies indicated that actigraphy-PSG comparison was primarily based on three aspects:

- Comparing subjective parameters between PSG and actigraphy 
- Technologist conducted computation of PLM indices from PSG and actigraphy data, and,

- Use of statistical tests such as Pearson's correlation coefficients, Bland-Altman plots and Wilcoxon signed rank test to compare PLM severity estimation between PSG and actigraphy

The PSG-actigraphy comparison from these studies yielded that actigraphy did not accurately identify PLMs and thus it was recommended not to be used in conjunction with PSG. But it should be noted that although these guidelines have given a strong opinion on the use of actigraphy, the included studies were exclusively based on clinical, statistical and subjective parameters. Thus, these guidelines did not consider the signal analysis aspect of actigraphy data. A detailed survey of other prior studies in actigraphy-based PLM assessment along with afore-mentioned works, have been briefly described in Table 4.1.1. As evident from Table 4.1.1, very few studies including the one developed in this study have employed the use of signal processing methods to analyze actigraphy data for PLM severity estimation. This research work intends to fill this gap and presents a new approach to actigraphy-based evaluation of PLMs using a computer aided application of analyzing actigraphy signals. The intention of this study is not to recommend actigraphy instead of PSG, but to rather explore the feasibility of using it as a screening tool in evaluating PLMs in sleep before a patient undergoes PSG-based sleep study [6]. It may also be noted that in comparison to prior actigraphy studies, this research work has performed extensive signal analysis on actigraphy data without ignoring subjective, morphological or statistical information from patient dataset.

In particular, experiments were conducted successfully on the following actigraphy datasets collected for a specific sleep disorder:

- Database A: Short-duration, bilateral ankle actigraphy dataset captured from 96 subjects in a clinical sleep study for detecting periodic limb movements (PLMs) in sleep $[5,17]$.

- Database B: Long-duration, tri-axial, bilateral ankle actigraphy signals acquired from 166 patients in a clinical sleep study for detecting PLMs in sleep $[6,16]$.

- Database C: Long-duration, single-axial ankle actigraphy signals collected from 46 individuals undergoing stroke recovery in a clinical sleep study [17].

Before proceeding with describing the developed system for estimating PLMs in sleep, a detailed discussion of an initial investigation into PLM severity estimation using Database A will be described. Additionally, the reader may also note the following about the research experiments in sleep actigraphy conducted in this study. Since this research involves developing an actigraphy based screening tool for detecting neuromuscular disorders, it should be noted that all of the actigraph data acquisition has been conducted simultaneously with PSG for each patient. The concurrent PSG-Actigraphy data acquisition was performed 
Table 4.2: Survey of prior studies in actigraphy for PLM estimation

\begin{tabular}{|c|c|c|c|c|}
\hline $\begin{array}{l}\text { Authors, refer- } \\
\text { ence number }\end{array}$ & $\begin{array}{l}\text { Actigraph/study } \\
\text { type }\end{array}$ & Settings & Placement & Study population \\
\hline $\begin{array}{l}\text { Athavale et al. [5] } \\
2017\end{array}$ & $\begin{array}{l}\text { Philips Respironics } \\
\text { ActicalTM (Philips } \\
\text { Respironics, 2015) }\end{array}$ & $\begin{array}{l}\text { Sampled } 32 \\
\mathrm{~Hz} ; 2 \text { s epochs }\end{array}$ & Bilateral ankles & $\begin{array}{l}96 \text { consecutive patients of } \\
\text { a sleep laboratory }(52.2 \pm \\
15.0 \text { y; } 57 \mathrm{M}, 39 \mathrm{~F})\end{array}$ \\
\hline $\begin{array}{l}\text { Athavale et al. [6] } \\
2019\end{array}$ & $\begin{array}{l}\text { GC DataConcepts } \\
\text { Model X16 }\end{array}$ & $\begin{array}{l}\text { Sampled } 25 \\
\mathrm{~Hz}\end{array}$ & Bilateral ankles & $\begin{array}{l}166 \text { consecutive patients } \\
(58.5 \pm 16.4 \mathrm{y} ; 98 \mathrm{M}, 68 \\
\mathrm{F})\end{array}$ \\
\hline
\end{tabular}

Analysis Method

Signal processing and machine

learning. Trained and Tested

a Naïve-Bayes classifier using actigraphy data features, to determine PLM severity and classify patients.

Signal encoding for data compression and denoising at source. Extracted time, frequency, morphological and actigraphy-specific features. Use of Naïve-Bayes, SVM and LDA tools for testing classification accuracies. Adaptive segmentation of signals to extract regions of true PLM activity from entire 6-8 hour of sleep actigraphy data.

\begin{tabular}{|c|c|c|c|c|}
\hline $\begin{array}{l}\text { Kye et al. [142] } \\
2017\end{array}$ & MetaWearC & $\begin{array}{l}\text { Sampled } 25 \\
\mathrm{~Hz}\end{array}$ & Foot & $\begin{array}{l}13 \text { participants ( } 11 \\
\text { male and } 2 \text { female) and } \\
\text { the total number of move- } \\
\text { ments } \\
\text { was } 520 \text { movements, } 260 \\
\text { PLMS and } 260 \text { non-PLMS. }\end{array}$ \\
\hline $\begin{array}{l}\text { Kobayashi et al. } \\
{[140] 2014}\end{array}$ & PAM-RL & $\begin{array}{l}\text { Sampled } \\
40 \mathrm{~Hz}\end{array}$ & Bilateral ankles & $\begin{array}{l}41 \text { Japanese patients with } \\
\text { suspected restless leg syn- } \\
\text { drome }(52.1 \pm 16.1 \mathrm{y}, 14 \\
\mathrm{M}, 27 \mathrm{~F})\end{array}$ \\
\hline
\end{tabular}

Analyzed foot actigraphy data using machine learning methods. The data was not collected in sleep but rather mimicked by participants in wake state

Use PAM-RL software to determine PLM thresholds and compute PLM Index. Compare with PSG using statistical tests - Bland-Altman plot, Wilcoxon signed rank test, Pearson product moment correlation and ROC Curve

\begin{tabular}{|c|c|c|c|c|c|}
\hline $\begin{array}{l}\text { Dias et al. [143] } \\
2013\end{array}$ & $\begin{array}{l}\text { Actigraph } \\
\text { (Actigraph } \\
\text { Pensacola } \\
\text { USA) }\end{array}$ & $\begin{array}{r}\text { GT3X } \\
\text { LLC, } \\
\text { Florida, }\end{array}$ & $\begin{array}{l}\text { Sampled } \\
30 \mathrm{~Hz}\end{array}$ & $\begin{array}{l}\text { Both legs at an- } \\
\text { kle level }\end{array}$ & $\begin{array}{l}2 \text { subjects referred for a } \\
\text { sleep study with suspect } \\
\text { sleep apnea }\end{array}$ \\
\hline
\end{tabular}

Perform visual similarity measurements between PSG and actigraphy data to detect regions of PLM activity in actigraphy plot. Used an open source software - Movyzer which includes a similarity function to compare actigraphy and PSG.

\begin{tabular}{|c|c|c|c|c|c|}
\hline $\begin{array}{l}\text { Gschliesser et al. } \\
\text { [114] } 2009\end{array}$ & $\begin{array}{l}\text { PAM-RL (IM Sys- } \\
\text { tems, USA); } \\
\text { Actiwatch } \\
: \text { (Cambridge } \\
\text { Neurotechnology, } \\
\text { Ltd.) } \\
\text { DTS }\end{array}$ & $\begin{array}{l}\text { Actiwatch } \\
: \\
\text { Sampled } \\
32 \mathrm{~Hz} \text {. } \\
\text { PAM-RL: } \\
\text { Sampled } \\
10 \mathrm{~Hz} \\
\text { Default set- } \\
\text { tings }\end{array}$ & $\begin{array}{l}\text { Actiwatch } \\
- \\
\text { bilateral } \\
\text { foot dorsa } \\
\text { PAM-RL- } \\
\text { bilateral ankle }\end{array}$ & $\begin{array}{l}24 \text { consecutive patients } \\
(57.5 \pm 12 \mathrm{y} ; 18 \mathrm{M}, 6 \mathrm{~F}) \\
\text { of these } 10(60.9 \pm 12.0 \mathrm{y} \\
\text { old; } 7 \mathrm{M}, 3 \mathrm{~F}) \\
\text { underwent additional } \\
\text { monitoring with PAM-RL }\end{array}$ & $\begin{array}{l}\text { Use of vendor software to detect } \\
\text { PLMs from actigraphy data. } \\
\text { Further comparison with PSG } \\
\text { was done using SPSS statis- } \\
\text { tics software. Tests included } \\
\text { Wilcoxon rank sum test, Spear- } \\
\text { man correlations and Bland } \\
\text { Altman plots }\end{array}$ \\
\hline $\begin{array}{l}\text { Kemlink } \\
\text { al. [144] } 2008\end{array}$ & $\begin{array}{l}\text { AW-64 } \\
\text { Actiwatch } \\
\text { (Cambridge } \\
\text { Neurotechnology } \\
\text { Ltd., } \\
\text { Cambridge UK); } \\
\text { DTS }\end{array}$ & $\begin{array}{l}32 \mathrm{~Hz} \text { resolu- } \\
\text { tion; } \\
2 \mathrm{~s} \text { epochs }\end{array}$ & $\begin{array}{l}\text { Bilateral } \\
\text { ankles } \\
\text { and dorsum } \\
\text { of foot }\end{array}$ & $\begin{array}{l}40 \text { consecutive nights in } \\
37 \text { adult patients with } \\
\text { RLS and/or SRBDs (age } \\
50.812 .1 \text { y; } 29 \mathrm{M}, 8 \mathrm{~F} \text { ) }\end{array}$ & $\begin{array}{l}\text { Manual analysis of actigraphy } \\
\text { data using vendor software, } \\
\text { and statistical comparison with } \\
\text { PSG data. Spearman's correla- } \\
\text { tion } \\
\text { test) and comparative tests } \\
\text { (pairwise sign test) } \\
\text { were employed to analyse the } \\
\text { data. The least square } \\
\text { non-parametric empirical esti- } \\
\text { mation was used to study } \\
\text { relationship between parame- } \\
\text { ters obtained by PSG and } \\
\text { actigraphy. }\end{array}$ \\
\hline $\begin{array}{l}\text { King et al. [145] } \\
2005\end{array}$ & $\begin{array}{l}\text { Actiwatch (Cam- } \\
\text { bridge } \\
\text { Neurotechnology } \\
\text { Ltd./Mini Mitter } \\
\text { Co., Inc.); } \\
\text { DTS }\end{array}$ & $\begin{array}{l}\text { Sampling } \\
\text { rate } 32 \mathrm{~Hz} \\
2 \mathrm{~s} \text { epochs }\end{array}$ & $\begin{array}{l}\text { Dorsum } \\
\text { of each } \\
\text { foot }\end{array}$ & $\begin{array}{l}50 \text { technically } \\
\text { acceptable } \\
\text { overnight hospital } \\
\text { PSG (demographics } \\
\text { unclear) }\end{array}$ & $\begin{array}{l}\text { Visual comparison and analy- } \\
\text { sis of actigraphy data with PSG } \\
\text { readings using vendor software } \\
\text { and Bland Altman analysis. }\end{array}$ \\
\hline $\begin{array}{l}\text { Sforza et al. [141] } \\
2005\end{array}$ & $\begin{array}{l}\text { PAM-RL (SOM- } \\
\text { NOmedics } \\
\text { GmHb, Germany); } \\
\text { DTS }\end{array}$ & $\begin{array}{l}\text { Sampling } \\
\text { rate } 40 / \mathrm{s}\end{array}$ & $\begin{array}{l}\text { Bilateral ankles } \\
108\end{array}$ & $\begin{array}{l}43 \text { consecutive adult } \\
\text { patients }(57.6 \pm 3.7 \mathrm{y} \text { old; } \\
33 \mathrm{M} \text { and } 10 \mathrm{~F}) \text { referred } \\
\text { for PSG for insomnia } \\
\text { and/or EDS; }\end{array}$ & $\begin{array}{l}\text { Actigraphy data analyzed using } \\
\text { vendor algorithm, and then } \\
\text { compared statistically with } \\
\text { PSG to determine closeness in } \\
\text { PLM indices. Use of Pearson's } \\
\text { correlation test and Bland } \\
\text { Altman analysis technique. }\end{array}$ \\
\hline
\end{tabular}


on consenting patients undergoing routine overnight sleep studies at the Sunnybrook Sleep Laboratory, Toronto, Canada. It should also be noted that all the sleep studies conducted in this research have been approved by Sunnybrook Health Sciences Centre Research Ethics Board, and written consent was given by all participants $[5,6]$.

The PSG readings were recorded and scored by a registered sleep technologist, who monitored the overnight sleep studies. During each study, apart from acquisition of actigraphy signals, following PSG data was also recorded from each patient [22]:

- EEG - electroencephalogram

- Reference leads at the mastoids (bone behind the ear)

- EOG - electrooculogram

- Surface electromyography from mentalis/submentalis, and anterior tibialis

- Respiratory measures - abdominal and thoracic efforts using respiratory inductive plethysmography belts

- Nasal/oral pressure, and nasal/oral flow

- Oxygen saturation, and a 2-lead ECG - electrocardiogram

Each sleep study was manually staged as per the criteria set out by the American Academy of Sleep Medicine, and all the studies were interpreted by trained sleep physicians and scored by a registered PSG technologist [100]. The PLM index which determines severity of limb movements in sleep is defined as the ratio of number of PLMs detected during sleep divided by the total sleep time. The reader should note at this juncture that for the preliminary experiments and contributed actigraphy analysis tool, the manual PLM indices provided by the registered technologist would be used for labeling the signal and feature data, based on PLM severities (as set out by standards in American Academy of Sleep Medicine [100]). This aspect would only be used for training the application on estimating PLMs.

\subsection{Preliminary Experiments using Database A}

In an initial set of studies, experiments were conducted with short-duration segments clipped from long actigraphy data recorded simultaneously with PSG during overnight sleep studies conducted for 96 consenting test subjects [5]. The objective of this preliminary study was to test the effectiveness of using a signal processing approach to analyzing actigraphy data for estimating the severity of periodic limb movements in 
sleep and establish a baseline for analyzing long-duration actigraphy data. Furthermore, this was also done to explore the possibility of using actigraphs as a screening tool before the patient undergoes PSG. Being a validation study, the objective was to test the efficiency of using bilateral ankle actigraphy in detecting limb movements and compare their detection with technologist-marked PSG segments. Following flowchart in Figure 4.5 illustrates the approach in the preliminary experiments for PLM estimation using Database A.

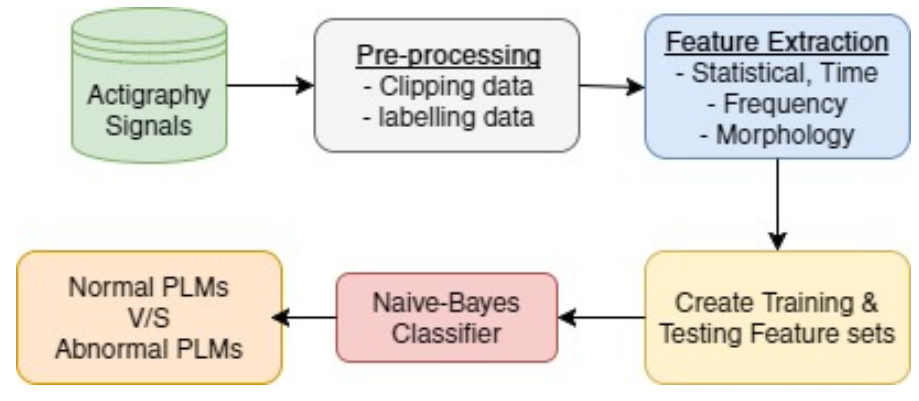

Figure 4.5: A Patient experiencing PLMs in lower left limb [23]

The next set of sections will describe the preliminary experiments starting with data acquisition and following it with the signal processing methods adopted in these experiments.

\subsubsection{Materials and Methods}

\section{Data Acquisition and Pre-processing}

During the preliminary studies, simultaneous PSG and single-axial actigraphy data acquisition was conducted on 96 consecutive consenting patients, undergoing routine overnight sleep studies, at the Sunnybrook Sleep Laboratory. The PSG readings were acquired using a standard setup whereas the actigraphy signals were acquired using Philips Respironics ${ }^{\circledR}$ Actical $^{\text {TM }}[17]$ wearable devices placed on both ankles as shown in Figure 4.6. For additional information on demographics of the study population, the reader may refer to the publication [5] and Table 4.3. 


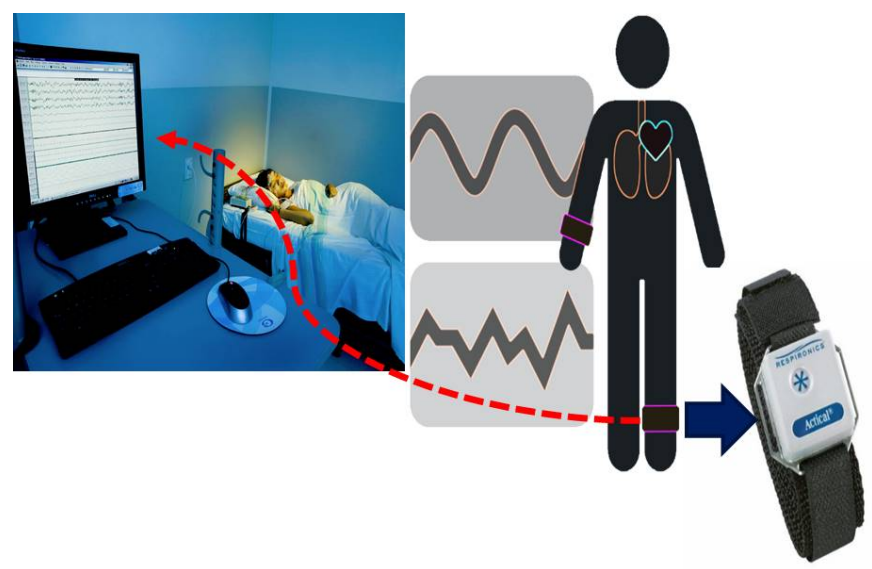

Figure 4.6: Actigraph Data Acquisition Setup

Table 4.3: Demographics and PSG parameters - Actical Data, Database A [5]

\begin{tabular}{lccccc}
\hline \multicolumn{1}{c}{ Parameters } & All & PLM $<\mathbf{5 . 0}$ & PLM $\geq \mathbf{5 . 0}$ & PLM $<\mathbf{1 5 . 0}$ & PLM $\geq \mathbf{1 5 . 0}$ \\
& $(\mathbf{N}=\mathbf{9 6})$ & $\mathbf{( N = 5 9 )}$ & $\mathbf{( N = \mathbf { 3 7 } )}$ & $\mathbf{( N = 6 5 )}$ & $\mathbf{( N = 3 1 )}$ \\
\hline Age (years) & $52.2 \pm 15.0$ & $47.7 \pm 14.4$ & $59.3 \pm 13.1$ & $48.6 \pm 14.4$ & $59.5 \pm 13.6$ \\
Male gender & $57(59.4 \%)$ & $30(50.8 \%)$ & $27(73.0 \%)$ & $34(52.3 \%)$ & $23(74.2 \%)$ \\
Body mass index $\left(\mathrm{kgm}^{2}\right)$ & $29.4 \pm 5.8$ & $29.1 \pm 6.6)$ & $29.8 \pm 4.3$ & $29.4 \pm 6.6$ & $29.2 \pm 4.0$ \\
Sleep efficiency & $71.1 \pm 20.2$ & $72.3 \pm 19.8$ & $69.1 \pm 21.0$ & $73.2 \pm 19.2$ & $66.6 \pm 21.8$ \\
AHI & $11.8 \pm 19.5$ & $13.8 \pm 23.2$ & $8.5 \pm 10.9$ & $12.9 \pm 22.3$ & $9.5 \pm 11.6$ \\
RDI & $18.1 \pm 20.7$ & $20.1 \pm 24.1$ & $14.9 \pm 13.4$ & $18.9 \pm 23.3$ & $16.3 \pm 14.0$ \\
Lowest oxygen saturation & $85.8 \pm 8.2$ & $86.2 \pm 9.1$ & $85.1 \pm 6.7$ & $86.2 \pm 8.9$ & $84.9 \pm 6.5$ \\
\hline
\end{tabular}

AHI, apnea-hypopnea index; PLM, periodic limb movement; RDI, respiratory disturbance index; SD, standard deviation.

The Actical ${ }^{\mathrm{TM}}$ is a readily available actigraph, which non-invasively captures single-axial motion signals using an embedded accelerometer. It is a light-weight and waterproof wearable device which offers real-time data acquisition and monitoring for research and clinical purposes. With a $32 \mathrm{MB}$ (megabytes) of storage, the Actical $^{\mathrm{TM}}$ is capable of acquiring limb movements at a sampling rate of $32 \mathrm{~Hz}$, with an option of reporting data samples in epochs of 1, 2 and 5 seconds. At about 16 grams, the device is low-weight and can be comfortably worn using a velcro strap on ankles. In its raw mode, it is capable of recording and exporting up to 12 hours of motion data [17]. For the experiments in this research, the device was calibrated to collect actigraphy signals at $32 \mathrm{~Hz}$ with an epoch of 2 seconds, which yielded an effective sampling rate of $16 \mathrm{~Hz}$ during the simultaneous PSG recording.

As shown in Fig. 4.7, the actigraphy signals were found to be highly correlated with respect to the regions of activity exhibited in PSG readings. As per standard signal property tests using MATLAB ${ }^{\mathrm{TM}}$, it was found that the actigraphy signals were non-stationary, non-linear and non-Gaussian in nature and were 
sparsely distributed between the "lights off" and "lights on" time interval (which is about 6-8 hour long). These tests inferred that the actigraphy signals could be analyzed using stationary time-based windows, in order to avoid losing information.

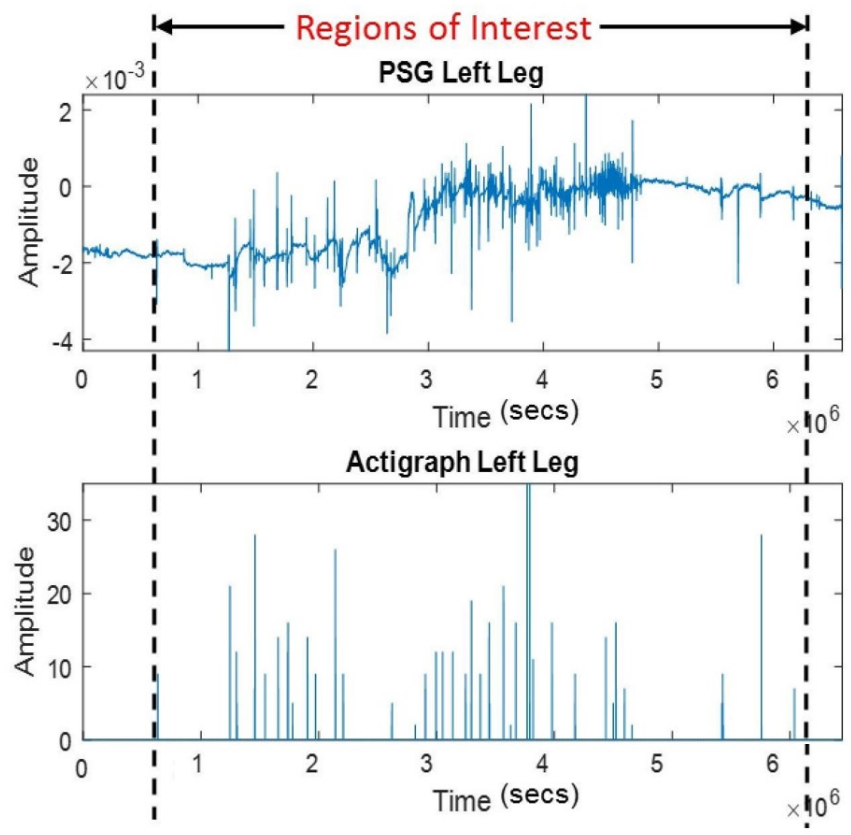

Figure 4.7: Visual correlation between PSG and Actigraphy

The actigraphy signals provided by the registered technologist were annotated as per regions of peak movement activity in PSG readings. In order to capture significant PLM regions, and to address the nonstationarity, each signal was further truncated to an 85-second window. In this operation, those PLMs were removed which were below the average amplitude of the entire actigraphy signal, thus ensuring the retention of those events which could be construed as "true limb movements" by the technologist. Figure 4.8 explains a step-by-step methodology in developing the feature extraction and pattern classification system for classifying the actigraphy data in Database A using manual PLM index. 


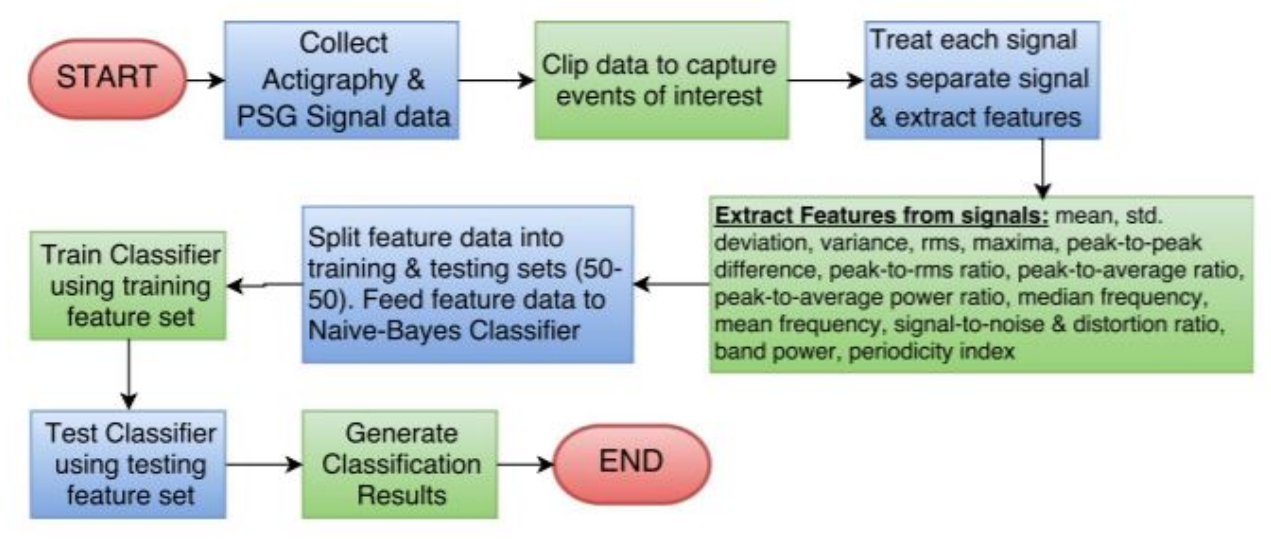

Figure 4.8: Algorithm for preliminary studies

\section{Feature Extraction and Classification}

The preliminary set of experiments comprised of implementing the Actical for collecting and analyzing actigraphy signals, as well as validating its usage and results with regards to PSG gold standards. As described in previous section, the 85-second long, annotated and truncated signals were used for extracting characteristic features for pattern classification. The first approach was to classify actigraphy signals into normal (patients not experiencing PLMs or healthy) and abnormal (patients experiencing PLMs or unhealthy) using manually scored PLM indices as labels for the signal data.

In this analysis the actigraphy signal data was categorized as either 'normal' or 'abnormal', irrespective of which leg (i.e. right or left) each signal belonged to. For the 96 patients, 191 signals (i.e. left and right leg data for each patient) were obtained; one right leg actigraphy file was corrupted during signal acquisition and was discarded. Based on current and previous scoring standards for PLM indices, the data was grouped as follows:

- Group 1: Initially the signal data was grouped based on a PLM cut-off of 5 [146] (i.e. actigraphy signals associated with a PLM Index $\geq 5$ were classified as 'abnormal' and those with a PLM Index $<5$ were classified as 'normal'). Using this classification, 131 'normal' actigraphy signals and 60 'abnormal' actigraphy signals were obtained.

- Group 2: In next phase the data was grouped on a PLM cut-off of 15 [100] (i.e. actigraphy signals associated with a PLM Index $\geq 15$ were classified as 'abnormal' and those with a PLM Index $<15$ were classified as 'normal'). Using this classification, 150 'normal' actigraphy signals and 41 'abnormal' actigraphy signals were obtained.

It should be noted here that the PLM cut-off of 5 movements per hour was an old standard used for 
categorize PLMs, used in a prior study [5,146]. As per current scoring standards cited in [6, 100], the new PLM cut-off has been shifted to 15 movements per hour.

For both of these groups, robust signal features were extracted, and a supervised pattern classification algorithm was trained to classify 'normal' and 'abnormal' actigraphy signals. About 14 time-based [93], frequency-based [98] and signal morphology-based features [123,124] were extracted from each actigraph signal for identifying characteristic PLM activity information. Table 4.4 describes all these features.

Table 4.4: Features extracted from Actigraphy Signals in Database A

\begin{tabular}{llll}
\hline \multicolumn{3}{c}{ Features } & \\
\hline Mean & Standard Deviation & Variance & RMS \\
Maxima & Peak to Peak Difference & Peak to RMS Ratio & Peak to Average Ratio \\
Peak to Avg. Power Ratio & Median Frequency & Mean Frequency & Band Power \\
Signal to Noise \& Distortion ratio & Band Power & Periodicity Index & \\
\hline
\end{tabular}

Except for the periodicity index (PI), all of the remaining 13 features were extracted using standard functions available in MATLAB ${ }^{\mathrm{TM}}$. The PI has recently become an important application-specific feature for assessing PLMs, and is reported to provide a higher degree of stability and accuracy for the detection of PLMs during sleep $[123,124,147,148]$. It is also a desirable form of measurement as a smaller number of leg movements are required. The PI is calculated using the number of intervals in the truncated, pre-processed actigraphy signal (Figure 4.7). An interval is defined as a period of inactivity that was disrupted by a leg movement [124]. In this study, the number of intervals (or periods of inactivity) in the segments between true PLMs (i.e. the PLM activity or signal components whose amplitude was greater than or equal to the average amplitude of the truncated signal) were found. The PI is then computed using the following equation 4.1

$$
P I=\frac{\text { Recorded PLM Index }}{\text { No. of Intervals between PLM events }}
$$



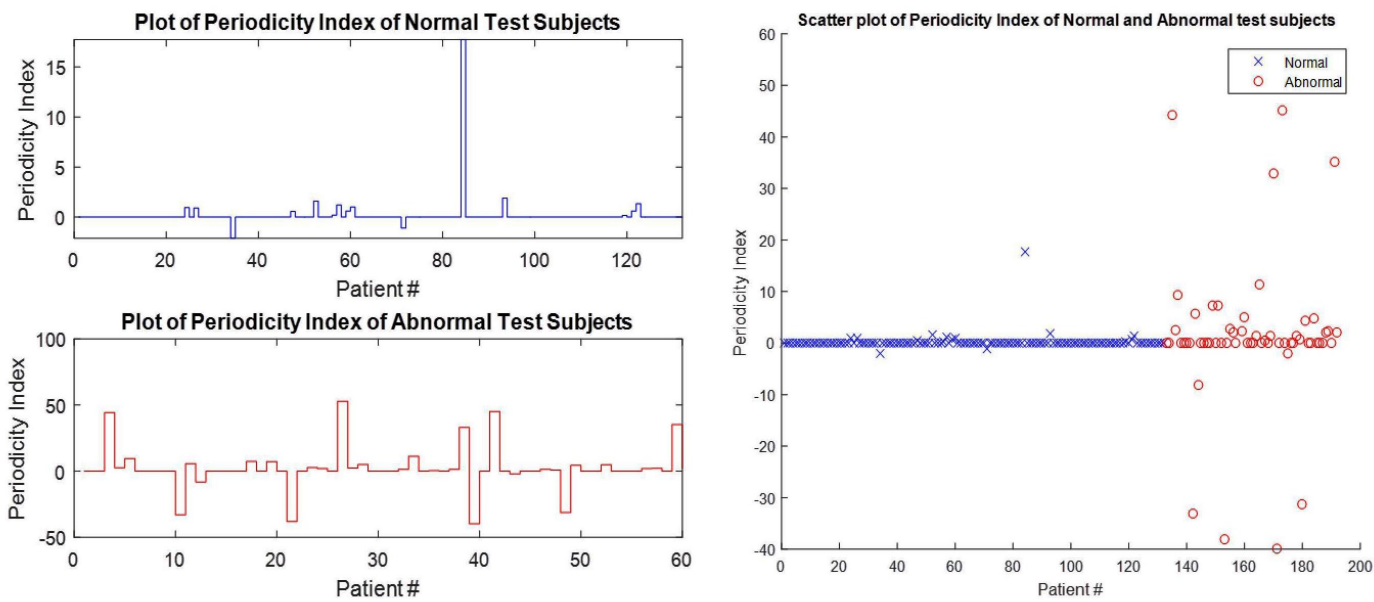

Figure 4.9: Plots of PIs for normal and abnormal patients

Once the features were extracted from the signals, the feature dataset was split evenly into training and testing sets, and fed into a Naïve-Bayes classifier [149]. The Naïve-Bayes classifier is based on the Bayesian theorem and is usually applied for linear pattern classification when the dimensionality (number of features) for a signal is high. The Bayesian theorem is based on prior probabilities and their application in predicting current outcomes. In this study, the theorem was applied to prior probabilities of feature values and was used to predict the outcomes of classification in the testing data set [149]. A Naïve-Bayes classifier can handle a random number of continuous or categorical (discrete) variables, which makes it able to reduce a high-dimensional dataset into a more simple decision output $[149,150]$. The training and testing sets did not have any overlap in the samples used. The algorithm was used for both Groups 1 and 2, and the classification results are reported in Table 4.5.

In order to test the robustness of the Naïve-Bayes classifier and validate it, its performance was compared with other pattern classifiers such as a linear discriminant analysis (LDA) and support vector machine (SVM) with a quadratic kernel function [151]. In addition, the Naive-Bayes classifier was also cross-validated using the leave-one-out method [151]. The algorithm was executed for both Groups 1 and 2 using a Windows ${ }^{\mathrm{TM}}$ 7 computer.

\subsubsection{Results from Preliminary Experiments}

Using the afore described feature extraction and classification scheme, the following results were obtained as shown in Table 4.5. The Naive-Bayes classifier outperformed the SVM and LDA in classifying both Group 1 and 2. For Group 1, the algorithm took 6.57 seconds to generate classification results, and for Group 2 it took 9.78 seconds. 
Table 4.5: PLM Classification results using Database A

\begin{tabular}{clccc}
\hline Group & Classifier & Accuracy(\%) & Sensitivity(\%) & Specificity(\%) \\
\hline \multirow{2}{*}{ Group 1 } & Naive-Bayes & 78.9 & 80.3 & 73.7 \\
PLM cut-off at 5 & LDA & 70.5 & 71.1 & 60.0 \\
& SVM & 73.7 & 73.0 & 83.3 \\
\hline \multirow{2}{*}{ Group 2 } & Naive-Bayes & 80.2 & 84.1 & 57.1 \\
PLM cut-off at 15 & LDA & 74.0 & 78.4 & 25.0 \\
& SVM & 78.1 & 82.1 & 50.0 \\
\hline
\end{tabular}

In addition, upon cross validation, it was found that the classification losses for Groups 1 and 2 were low with values of about $18.8 \%$ and $15.8 \%$, respectively. In other words, the Naïve-Bayes classifier yielded a cross-validated classification accuracy of about $81.2 \%$ for Group 1, and about $84.2 \%$ for Group 2. Overall, these experiments indicated that the Naïve-Bayes classifier worked best for the proposed application, and provided the highest classification accuracies, sensitivities and specificities. This is also evident from the receiver operating characteristic (ROC) curves for LDA and Naïve-Bayes classifiers, illustrated in Figures 4.10 and 4.11 .

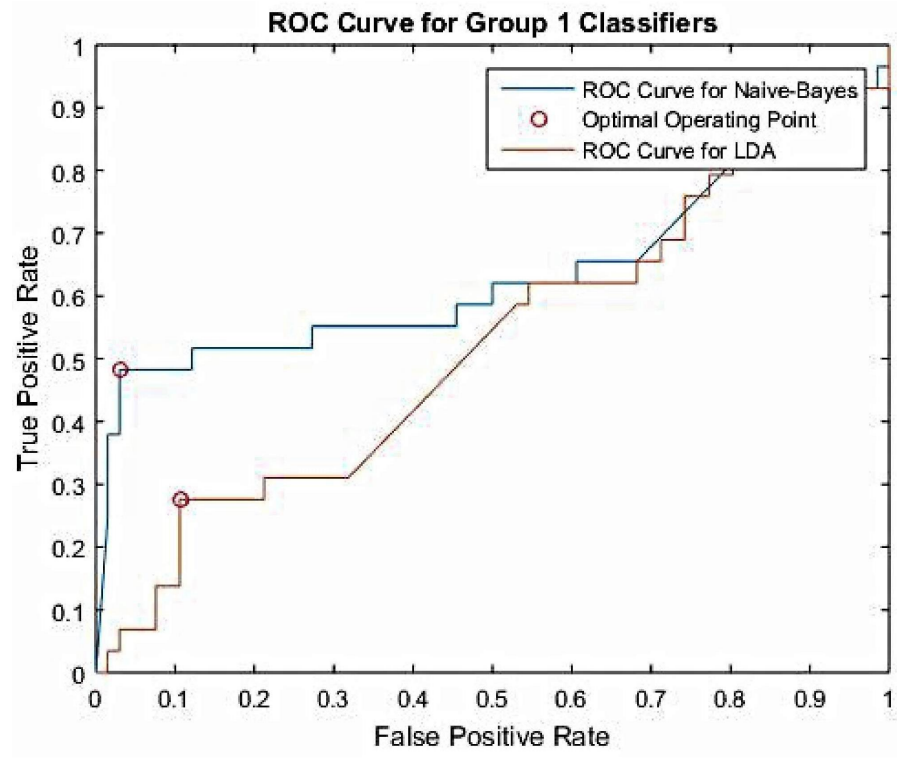

Figure 4.10: ROC Curve for Group 1 Classification 


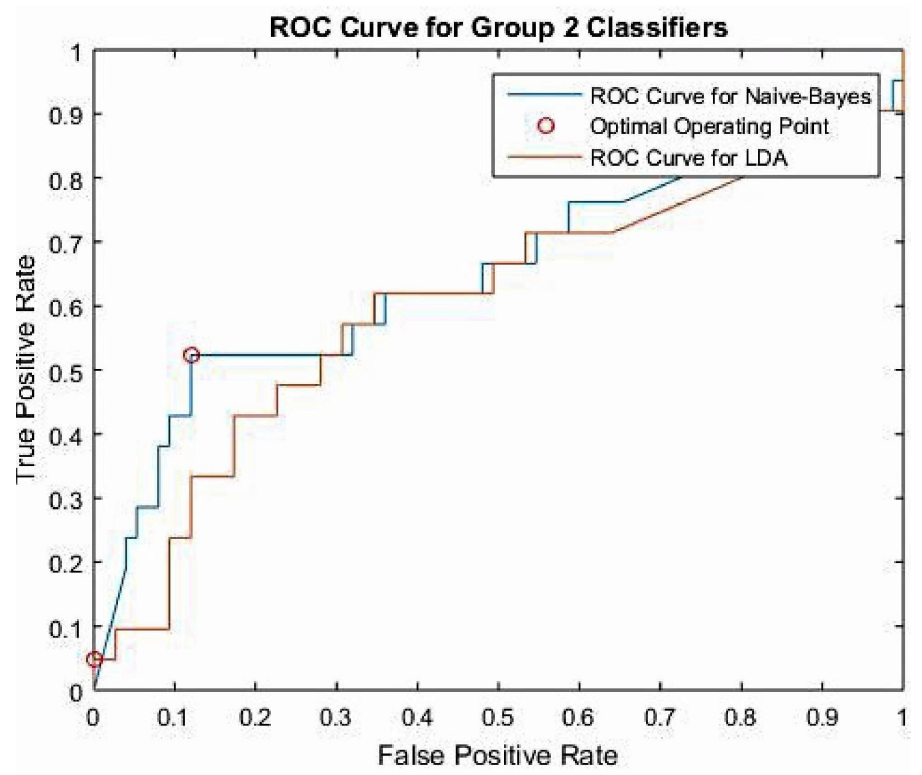

Figure 4.11: ROC Curve for Group 2 Classification

Although actigraphy signals are non-linear and non-stationary, it was observed that they can be relatively accurately classified by extracting simple time and frequency domain features, from short signal windows, as shown in Table 4.5. In the next section a new set of experiments will be described on analyzing tri-axial, long-duration sleep actigraphy data recorded simultaneously with PSG from 166 test subjects. This section will highlight on how the signal encoding and segmentation methods, coupled with actigraphy-specific and kernel-based features are distinctly able to identify and quantify periodic limb movements in sleep.

\subsection{Sleep Actigraphy Analysis using Database B}

Following the study on short-duration actigraphy signals from 96 test subjects in Database A, another study was conducted with actigraphy data acquired from 166 patients. In this study bilateral, long-duration (about 6-8 hours) sleep actigraphy signals were obtained using a generic tri-axial device. The subsequent sections will discuss the data acquisition and pre-processing methods, followed by adaptive segmentation, feature extraction and pattern classification for PLM severity estimation from long-duration actigraphy data. The methodology used in this study has been illustrated in the following Figure 4.12, reiterated from Section 2.4 in chapter 2 . 


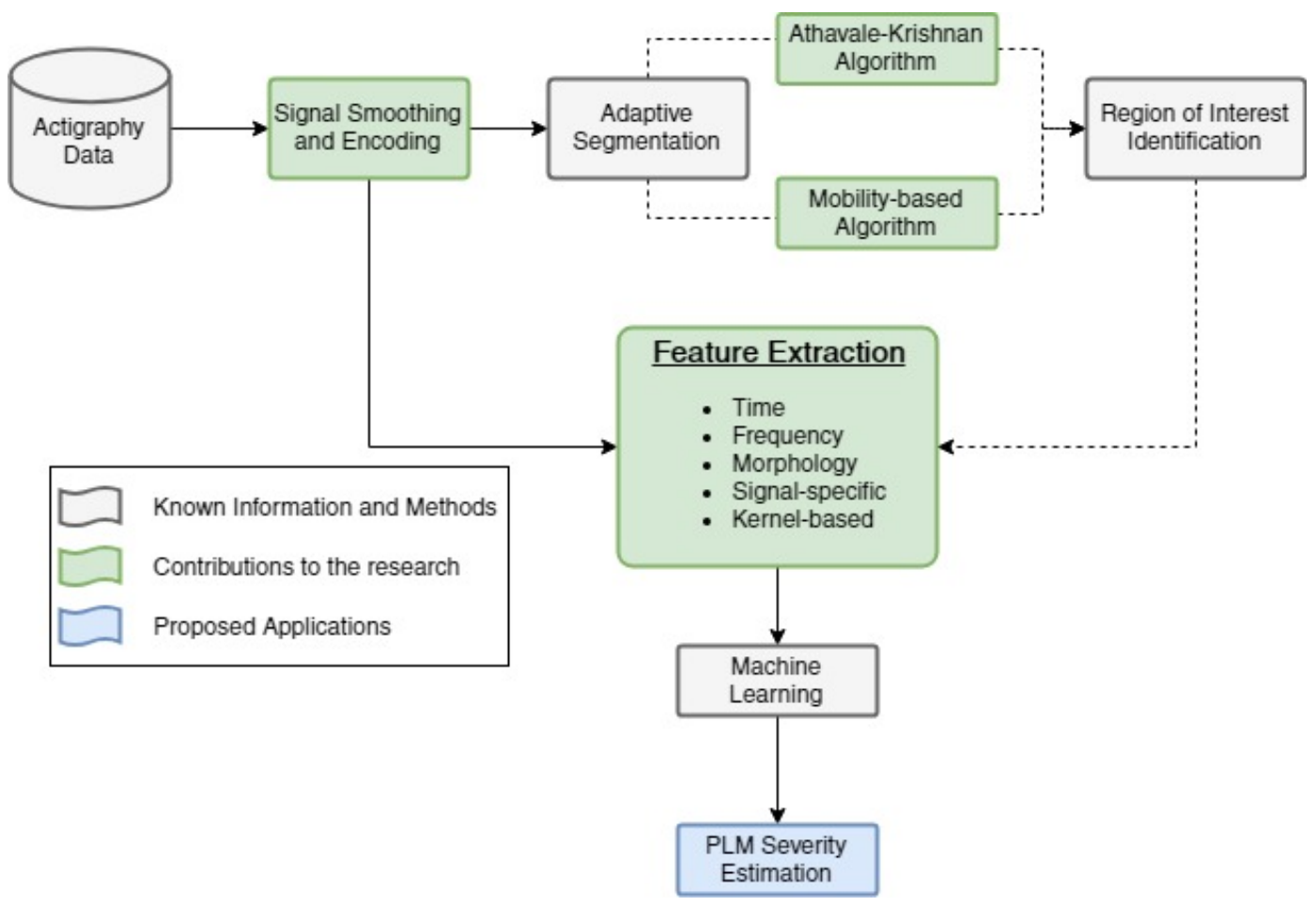

Figure 4.12: Sleep Actigraphy Analysis System

\subsubsection{Materials and Methods}

\section{Data Acquisition}

In the next set of studies, the GC Data Concepts ${ }^{\circledR}$ 's USB Accelerometer Model X16-mini ${ }^{\mathrm{TM}}[16]$ was used for collecting long-duration (6-8 hours) of tri-axial, actigraphy data from 166 patients. In this case each signal was sampled at $25 \mathrm{~Hz}$ with a 16-bit resolution. Thus, a total of 332 actigraphy signals were collected from both limbs of all patients.

In comparison to previously used Actical ${ }^{\mathrm{TM}}$ [17], the Model X16-mini ${ }^{\mathrm{TM}}$ [16] has better specifications in terms of data acquisition \& storage, universal compatibility, and user preferred sampling rate. The Model X16-mini ${ }^{\mathrm{TM}}$ [16] is a small, 17 gram, tri-axial accelerometer-based device which can record human motion/activity signals in three-dimensions by operating on an internal rechargeable battery, and can record $\&$ store data up to $8 \mathrm{~GB}$ using a removable microSD ${ }^{\mathrm{TM}}$ card. With a 16 -bit resolution, the device gives the user an option to select the sampling rate from 12, 25, 50,100,200, 400 and $800 \mathrm{~Hz}$. The data can be exported via a USB connection, and is stored in ".csv" (comma separated values) format in a readable text file, as shown in Figure 4.13. 


\begin{tabular}{|c|c|c|c|c|c|c|c|}
\hline \multicolumn{2}{|c|}{ A1 } & \multirow[t]{2}{*}{$\vdots$} & \multirow{2}{*}{$\begin{array}{c}x \\
\mathrm{c} \\
\mathrm{c}\end{array}$} & \multirow{2}{*}{$f_{\boldsymbol{x}}$} & $\operatorname{Ax}(\mathrm{g})$ & \multirow[b]{2}{*}{$\mathrm{F}$} & \multirow[b]{2}{*}{ G } \\
\hline 4 & A & & & & $E$ & & \\
\hline 1 & $\mathrm{Ax}(\mathrm{g})$ & Ay $(\mathrm{g})$ & $\mathrm{Az}(\mathrm{g})$ & & & & \\
\hline 2 & 0.879395 & -0.16748 & $8-0.42383$ & & & & \\
\hline 3 & 0.879395 & -0.16846 & $\begin{array}{ll}6 & -0.43018\end{array}$ & & & & \\
\hline 4 & 0.888184 & -0.16504 & -0.43896 & & & & \\
\hline 5 & 0.891602 & -0.17041 & -0.44385 & & & & \\
\hline 6 & 0.884766 & -0.17285 & $5 \quad-0.44043$ & & & & \\
\hline 7 & 0.881348 & -0.16846 & $6 \quad-0.42383$ & & & & \\
\hline 8 & 0.881836 & -0.17285 & -0.42383 & & & & \\
\hline 9 & 0.881836 & -0.17725 & -0.4292 & & & & \\
\hline 10 & 0.880371 & -0.18311 & -0.41895 & & & & \\
\hline 11 & 0.875977 & -0.18506 & $\begin{array}{ll}5 & -0.43018\end{array}$ & & & & \\
\hline 12 & 0.879395 & -0.17188 & $8 \quad-0.43945$ & & & & \\
\hline 13 & 0.884766 & -0.16699 & $\begin{array}{ll}9 & -0.43018\end{array}$ & & & & \\
\hline 14 & 0.880371 & -0.16748 & $\begin{array}{ll}8 & -0.43457 \\
\end{array}$ & & & & \\
\hline 15 & 0.882813 & -0.16602 & $\begin{array}{ll}2 & -0.43799\end{array}$ & & & & \\
\hline 16 & 0.884766 & -0.16943 & B -0.43262 & & & & \\
\hline 17 & 0.875 & -0.17188 & $\begin{array}{ll}8 & -0.43457\end{array}$ & & & & \\
\hline 18 & 0.872559 & -0.1709 & $\begin{array}{ll}9 & -0.44043\end{array}$ & & & & \\
\hline 19 & 0.872559 & -0.1709 & 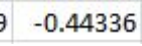 & & & & \\
\hline
\end{tabular}

Figure 4.13: CSV format data from X16-mini ${ }^{\mathrm{TM}}$ Actigraph

Using the on-board rechargeable battery, the device is capable of recording continuous data for up to 24 hours at a sampling rate of $25 \mathrm{~Hz}$. Figs. 4.14 and 4.15 illustrate the Model X16-mini ${ }^{\mathrm{TM}}$ [16] device and it corresponding actigraphy signal.

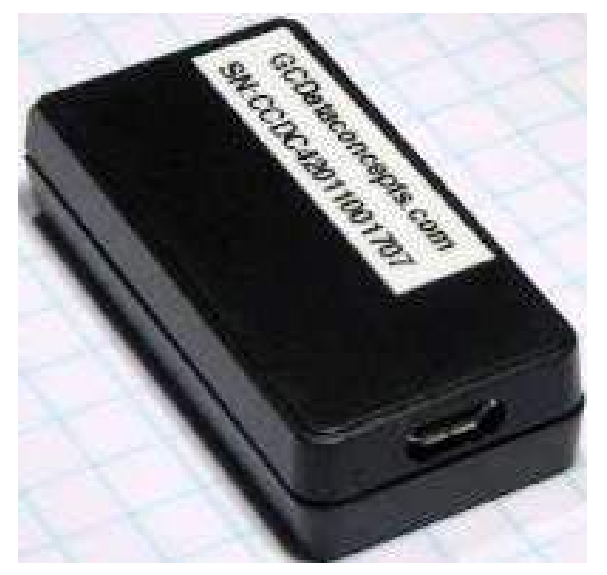

Figure 4.14: Model X16-mini ${ }^{\mathrm{TM}}$ Actigraph 

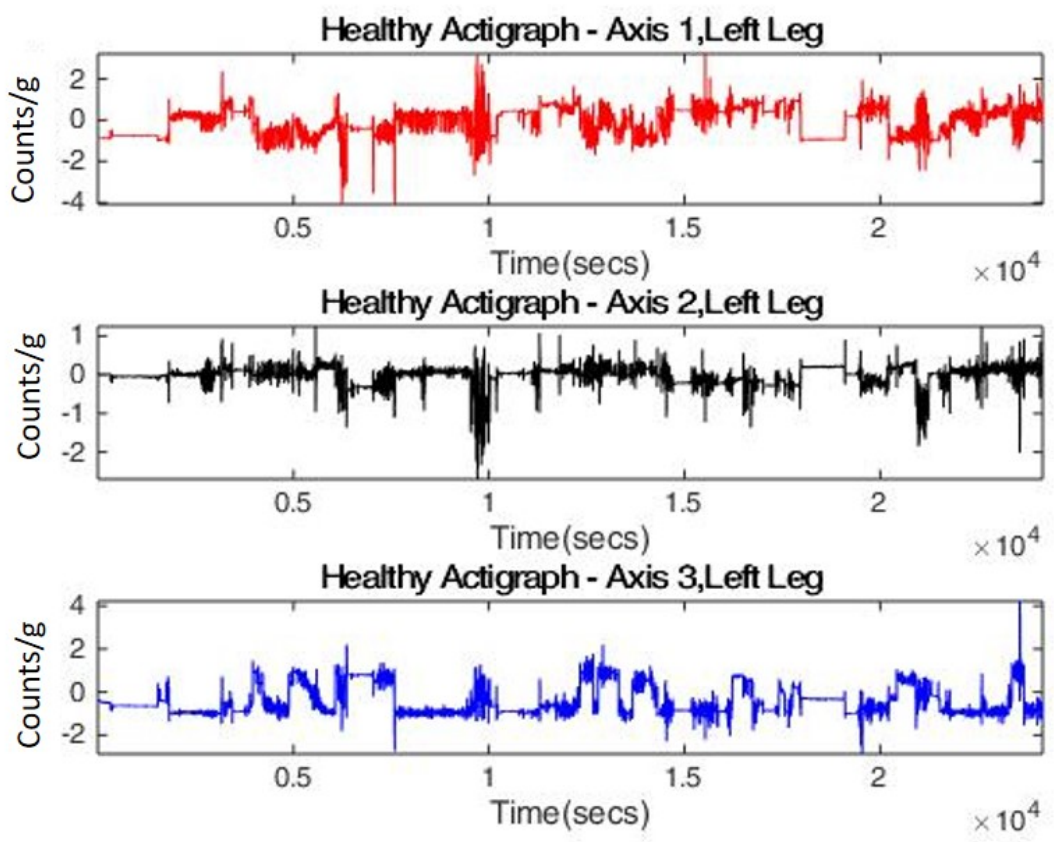

Figure 4.15: Sample Tri-axial actigraphy signal from a healthy subject's left leg

In comparison, to previously acquired Actical [17] data, in these experiments no signal truncation was done, as the intention was to develop a long-term, signal monitoring algorithm, capable of handling largedata without compromising feature robustness. The raw PSG and actigraphy signals were recorded between "lights off" and "lights on" intervals, typically between 6-8 hours of sleep duration for each patient. The PLM indices were manually scored by a registered technologist, in this study as well. Similar to the Actical ${ }^{\text {th }}$ [17], the Model X16 [16] was placed on both ankles using comfortable velcro straps for data recording.

It should be noted here that Actical has been validated for in-laboratory usage and screening after the preliminary set of experiments, and through the newly developed algorithm, the intention is to do the same validation for the Model X16 [16], including pre-labeling of signal and feature data based on manual PLM indices. Similar to the labeling scheme used in Section 4.2.1, the 332 tri-axial actigraphy signals were further segregated into two groups by pre-labeling each signal as either Normal with PLM Index $\leq$ 15 movements/hour or Abnormal with PLM Index > 15 movements/hour [100], irrespective of which leg (left/right) each signal belonged to. Following Table 4.6 highlights the demographics of the study population in this study, also denoted as Database B. 
Table 4.6: Demographics and PSG parameters - Tri-Axial X16 ${ }^{\mathrm{TM}}$ Data, Database B [6]

\begin{tabular}{lc}
\hline \multicolumn{1}{c}{ Parameters } & All Subjects, $\mathbf{N}=\mathbf{1 6 6}$ \\
\hline Age, mean \pm sd & $58.5 \pm 16.4$ \\
Male to Female ratio & $98: 68$ \\
Body Mass Index, BMI,mean \pm sd & $29.9 \pm 6.25$ \\
Sleep Efficiency, mean \pm sd & $68.4 \pm 19.8$ \\
AHI, mean \pm sd & $13.5 \pm 24.0$ \\
RDI, mean \pm sd & $22.7 \pm 27.7$ \\
Lowest oxygen saturation, mean \pm sd & $86.6 \pm 8.4$ \\
\hline PLM $<5.0=82 ;$ PLM $\geq 5.0=67 ; P L M<15.0=95 ; ~ P L M \geq 15.0=54$ \\
\hline AHI, apnea-hypopnea index; PLM, periodic limb movement; RDI, respiratory disturbance index \\
SD, standard deviation
\end{tabular}

\section{Actigraphy Pre-processing}

In order to effectively analyze actigraphy signals for PLM severity, they are first conditioned to remove noise, artifacts and redundant components. In order to show the efficiency of the signal encoding scheme described in Section 3.1.2, it is necessary to compare its performance with conventional pre-processing methods described in Section 3.1.1. For this, two groups of data, namely Group $\operatorname{Tr} i_{F}$ and Group $\operatorname{Tr} i_{E}$ were created, each consisting of the same 332 tri-axial actigraphy signals from Database B. It should be noted that in this study a one-on-one comparison of signal analysis results from Group $\operatorname{Tri}_{F}$ and Group $\operatorname{Tri}_{E}$ datasets was performed, in order to show the efficacy of the contributed methods in Chapter 3. Specific to each group, the following set of pre-processing operations are applied:

\section{- Group $\operatorname{Tr}_{F}$ :}

Each actigraphy signal in this group is conditioned using the following steps:

1. The signal is first normalized using the device specifications to remove the effect of $g$ factor. In this study, since the Model X-16 ${ }^{\mathrm{TM}}$ was used, a given tri-axial signal $S_{r}=\left(x_{n}, y_{n}, z_{n}\right)$ was normalized using the relation

$$
S_{\text {norm }}=\frac{S_{r}}{2048 \text { counts } / g}
$$

2. Following this, it was then detrended by subtracting the mean from each sample using the relation,

$$
S_{d c}=S_{\text {norm }_{i}}-\left(\frac{\sum_{i=1}^{n} S_{\text {norm }_{i}}}{n}\right)
$$

where $n$ is the number of samples in the signal. 
3. Next, a low-pass Butterworth filter with a passband of $0.4 \mathrm{~Hz}$ and a stopband of $1.6 \mathrm{~Hz}$ was applied using the relation,

$$
S_{f}(t)=S_{d c}(t) \circledast h_{l p f}(t)
$$

where $h_{l p f}(t)$ is the transfer function of the low pass filter.

4. The filtered signal $S_{f}=\left(x_{f}, y_{f}, z_{f}\right)$ is finally vector compounded to quantify the effect of the three axis as,

$$
S_{v}=\sqrt{x_{f}^{2}+y_{f}^{2}+z_{f}^{2}}
$$

\section{- Group $\operatorname{Tri}_{E}$ :}

Each actigraphy signal in this second group is conditioned using the following steps:

1. The signal $S_{r}=\left(x_{n}, y_{n}, z_{n}>\right.$ was first normalized using Eqn.4.1.

2. Next, a vector compounding of the signal was performed using Eqn.4.4, so that $S_{r} \rightarrow S_{v}$

3. Assuming that $r$ bits/sample is the bit resolution of the raw actigraphy signal, it was further encoded using $m$ bits $/$ sample such that $m \ll r$, via the floor operation,

$$
S_{E}=\left\lfloor\left(S_{v} \times \frac{\left(2^{m}-1\right)}{2}+\frac{\left(2^{m}-1\right)}{2}\right)\right\rfloor
$$

The encoding operation assigns each sample of $S_{v}$ to a discrete integer value from one of the $\left(2^{m}-1\right)$ steps.

Following through the afore-mentioned pre-processing steps for each group, one would get Group Tri ${ }_{F}$ with vector compounded and filtered actigraphy signals, and Group Tri $i_{E}$ with vector compounded and encoded signals. The reader must note that the creation of Group $\operatorname{Tri}_{F}$ and Group $\operatorname{Tri}_{E}$ has been done in order to show the efficiency of signal encoding with respect to signal denoising, SNR enhancement, higher clarity and better classification accuracy.

The next section will show how the Athavale-Krishnan and Mobility-based adaptive segmentation algorithms were applied to identify regions of activity from the encoded group of signals, $\operatorname{Tr} i_{E}$.

\section{Encoding-based Segmentation}

In this study of long-duration actigraphy signals, the Athavale-Krishnan and Mobility-based segmentation algorithms were tested out on encoded signal data in Group $\operatorname{Tri}_{E}$, and their efficiency in identifying regions of true periodic limb movements in sleep was determined. 
The Athavale-Krishnan algorithm to segment an encoded signal $S_{E}$ was applied using the following steps. As shown from Eqn.4.6, after obtaining the encoded and vector compounded actigraphy signal $S_{E}$, the first order and the subsequent second order difference signals were computed as,

$$
d S_{e}=\left[S_{e_{1}},\left(S_{e_{2}}-S_{e_{1}}\right), \ldots,\left(S_{e_{n}}-S_{e_{n-1}}\right)\right]
$$

and,

$$
2 d S_{e}=\left[d S_{e_{1}},\left(d S_{e_{2}}-d S_{e_{1}}\right), \ldots,\left(d S_{e_{n}}-d S_{e_{n-1}}\right)\right]
$$

Next, the rapid change factor (RCF) of the signal was calculated using its second order difference version as,

$$
R C F=\frac{\text { Step }- \text { Size }}{\left(m \times t_{s}\right)}
$$

where $t_{s}$ is the sampling period. The PLM segments were then found using the following ground truth information explained in Section 4.1.1:

1. Valid periodic leg movements occur within a 5-90 second window

2. Each movement lasts for about 0.5 to 10 seconds

3. A group of 4 or more consecutive limb movements within 90 seconds is a true PLM sequence.

First the primary segment boundaries (PSBs) were identified by finding all the signal samples in $2 d S_{E}$ which are greater than the $\mathrm{RCF}$,

$$
P S B_{i}=2 d S_{E_{n}} \forall\left\langle 2 d S_{E_{n}}>R C F\right\rangle
$$

where $i$ is the number of PSBs. Next, using the afore stated ground truth on PLMs, it can be inferred that two PLM sequences (of 4 or more movements) can be separated by at least 90 seconds. Using this fact, the secondary segment boundaries (SSBs) were found such that,

$$
S S B_{j}=P S B_{i} \forall\left\langle\left|t_{P S B_{i}}-t_{P S B_{i+1}}\right|>90\right\rangle
$$

where $j$ is the number of SSBs. Finally, the final segment boundaries were found by detecting only those samples, which contain 4 or more consecutive leg movements, and whose amplitude is at least half of the maximum peak found between two SSBs,

$$
F S B_{l}=S S B_{j} \forall\left\langle\operatorname{Count}\left(2 d S_{E_{j}}: 2 d S_{E_{j+1}}>\frac{\max _{n} 2 d S_{E_{n}}}{2}\right)>4\right\rangle
$$


where $l$ is the number of FSBs. Using the FSBs, regions of PLM activity from the entire actigraphy signal can be extracted in order to use them further for estimating PLM severity in the test subject.This algorithm was applied on various test subject signals, and remarkable segmentation results were obtained as illustrated in Section 4.3.2.

Following this, the mobility-based segmentation scheme was then applied on sleep actigraphy data using following steps. Using Eqns.4.7 and 4.8 the second order difference signal of the encoded data $S_{E}$ was computed. The primary segment boundaries were then found by detecting all the samples in $2 d S_{E}$ which were greater than a factor of $\frac{\max _{n} 2 d S_{E}}{\left(2^{m}-1\right)}$, such that,

$$
P S B_{i}=2 d S_{E_{n}} \forall\left\langle 2 d S_{E_{n}}>\frac{\max _{n} 2 d S_{E}}{\left(2^{m}-1\right)}\right\rangle
$$

Using these PSBs, the SSBs were found such that the Hjorth's mobility values of two segments were unique from each other.

$$
S S B_{j}=P S B_{i} \forall\left\langle\left\lfloor M_{P S B_{i: i+1}}\right\rfloor \neq\left\lfloor M_{P S B_{i+1: i+2}}\right\rfloor\right\rangle
$$

Using the ground truth information on PLMs, the final segment boundaries (FSBs) were computed such that two SSBs were at least 90 seconds apart.

$$
F S B_{l}=S S B_{j} \forall\left\langle\left|t_{S S B_{j}}-t_{S S B_{j+1}}\right|>90\right\rangle
$$

Using the FSBs the regions of high activity in sleep could then be detected. Though the mobility-based segmentation scheme is focused on detecting the variance in the actigraphy signal with only one inclusion of ground truth information on PLMs, it was still implemented to compare with the Athavale-Krishnan method. The results of these experiments with the mobility-based segmentation on Database B have been recorded in Section 4.3.2. In addition, these results also depict the efficacy of the contributed segmentation approaches with respect to a fixed segmentation of sleep actigraphy signals.

In the next Section, the feature extraction and classification methodologies adopted for Groups $\operatorname{Tr}_{F}$ and $\operatorname{Tr}_{E}$ will be described, followed by highlighting the results from pre-processing, segmentation and classification experiments.

\section{Feature Extraction and Classification}

Following the pre-processing and segmentation operations conducted on Group $\operatorname{Tr} i_{F}$ and Group $\operatorname{Tr} i_{E}$ datasets, the next step was to proceed extracting features from the signals in both groups. Using these features and ground truth labeling information machine learning tools were used to classify the signals based 
on PLM severity.

The feature extraction and classification methodology was conducted for each signal in Groups $T r i_{F}$ and $\operatorname{Tri}_{E}$ using the following steps:

- Using the manually scored PLM indices, the actigraphy signals in each group were first labelled either as Normal if PLM Index $<15$ movements/hour, or as Abnormal if PLM Index $\geq 15$ movements/hour [100].

- Next, about 23 time, frequency, kernel-based, actigraphy-specific and morphological features were extracted from each signal, as described in Table 4.7.

Table 4.7: Features captured from Groups $T r i_{F}$ and $T r i_{E}$ signals

\begin{tabular}{|c|c|c|}
\hline Domain & No. & Features Used \\
\hline \multirow{11}{*}{ Time } & 1 & Mean \\
\hline & 2 & Standard Deviation \\
\hline & 3 & Variance \\
\hline & 4 & RMS \\
\hline & 5 & Maxima \\
\hline & 6 & Peak-to-peak difference \\
\hline & 7 & Peak-to-RMS ratio \\
\hline & 8 & Peak-to-average ratio \\
\hline & 9 & Peak-to-average power ratio \\
\hline & 10 & Band power \\
\hline & 11 & Signal to noise \& distortion ratio \\
\hline \multirow{2}{*}{ Frequency } & 12 & Median Frequency \\
\hline & 13 & Mean Frequency \\
\hline Kernel-based Features & $14-17$ & ACF Kernel Scores (4 Features) \\
\hline \multirow{2}{*}{ Actigraphy-specific } & 18 & Rapid Change Factor \\
\hline & 19 & Spiky Index \\
\hline \multirow{4}{*}{ Morphological } & 20 & Hjorth's First order mobility \\
\hline & 21 & Hjorth's Second order mobility \\
\hline & 22 & Hjorth's complexity \\
\hline & 23 & Periodicity Index \\
\hline \multirow{3}{*}{ Clinical Labels \& Features } & 24 & Manual PLM Index \\
\hline & 25 & Gender (Male/Female) \\
\hline & 26 & Age \\
\hline
\end{tabular}

- Using a combination of various features, a 70-30 partition of training and testing sets was created for 
feature data in Groups $\operatorname{Tri}_{F}$ and $T r i_{E}$, and used for modelling three machine learning tools, namely - a Naive-Bayes classifier, a LDA tool and a linear SVM $[150,151]$. The reader may note that the purpose of using three different machine learning tools was not only to cross-validate the classification results, but also to show that at a device level, employing simple machine learning would be easier than a deep neural network on the cloud. This also aligns with the principles of edge computing and light-weight machine learning.

- The reader may note that in this study the choice of creating different combinations of feature sets was based on three key aspects:

1. The intention was to see how the machine learning models would perform when the classification is done only using generic time, frequency and statistical features.

2. Another approach was to combine generic features with actigraphy specific features to determine if any classification improvement took place.

3. This was further verified by using only the proposed actigraphy-specific, morphology and kernelbased features to classify mild and severe PLMs.

It may also be noted that similar aspects were also considered for actigraphy experiments with Databases C, D and E as well. Additionally, an mRMR (minimum redundancy maximum relevancy) feature selection technique was applied on all the 23 features, and it was found that the periodicity index, the rapid change factor and the mean frequency of an actigraphy signal could provide the best classification or discrimination between mild and severe PLMs.

- It should be noted that in these experiments, a set of features which does not include the Periodicity Index (which is a significant parameter) has also been considered. Instead of the periodicity index, the spiky index was applied for both Groups $\operatorname{Tri}_{F}$ and $T r i_{E}$ feature data, and the effect of using a non-clinical parameter in classifying test subjects based on PLM index severity was observed. This was done in order to show that, in the absence of clinical information, it is still possible to determine the severity of PLMs in sleep. Additionally, the effect of using only the proposed kernel-based, actigraphyspecific and morphological parameters to classify actigraphy signals, without considering any time or frequency parameters was also illustrated. All the experimental results have been highlighted in Section 4.3.2. 


\subsubsection{Results from Experiments on Database B}

This Section will illustrate the findings of various pre-processing, segmentation, feature extraction and classification experiments with actigraphy signals in Database B.

\section{Signal Filtering and Encoding Results}

As described in Section 4.3.1, two groups of actigraphy signals - $\operatorname{Tr}_{F}$ and $\operatorname{Tr}_{E}$ were obtained using two different pre-processing approaches respectively. As mentioned in Section 4.3.1, the raw actigraphy signals in Group $\operatorname{Tri}_{F}$ were low-pass filtered using a passband of $0.4 \mathrm{~Hz}$ and a stopband of $1.6 \mathrm{~Hz}$. In case of Group $\operatorname{Tri}_{E}$ actigraphy data, for the 16-bit resolution raw actigraphy, a $m=3$-bit encoding scheme was applied using Eq.4.6. To illustrate the effects of both filtering and encoding approaches, a sample tri-axial raw actigraphy signal obtained from a test subject experiencing severe limb movements in sleep was used.

Figure 4.16 illustrates the effect of low-pass filtering on a sample, tri-axial sleep actigraphy signal.
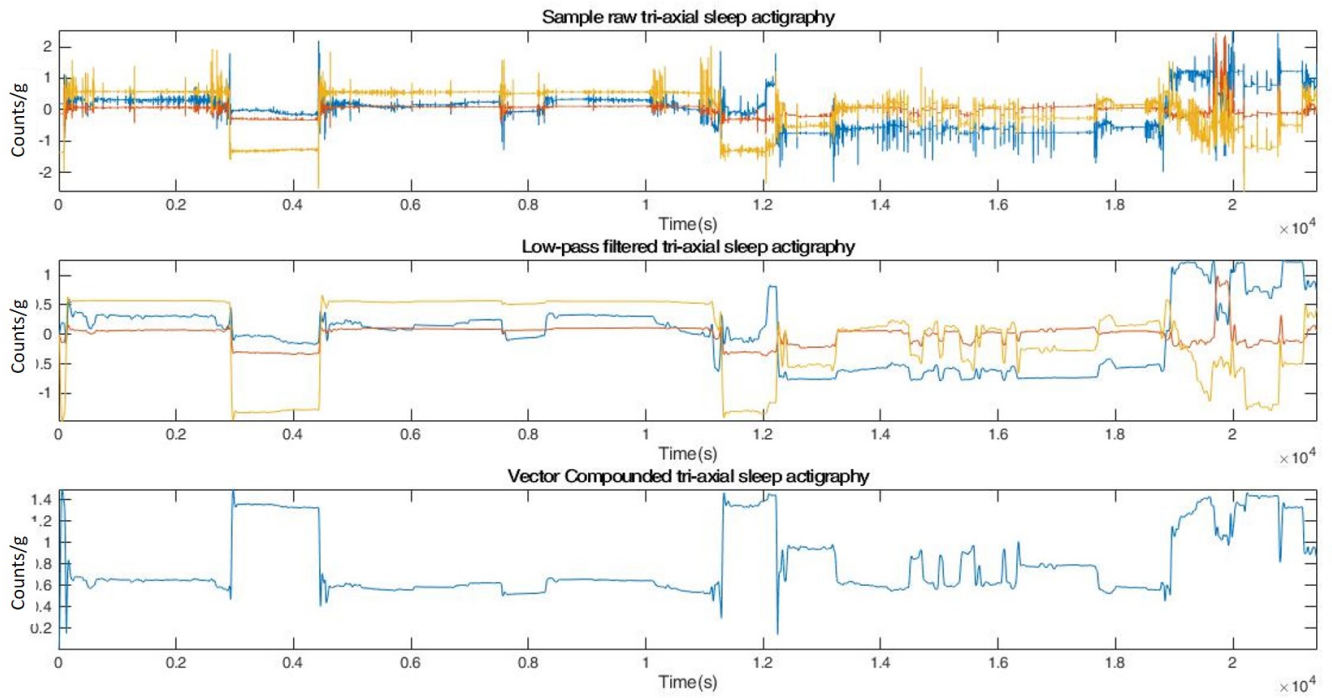

Figure 4.16: Low-pass filtering of tri-axial sleep actigraphy

Figure 4.17 illustrates the effect of 3-bit encoding on a sample, tri-axial sleep actigraphy signal. 

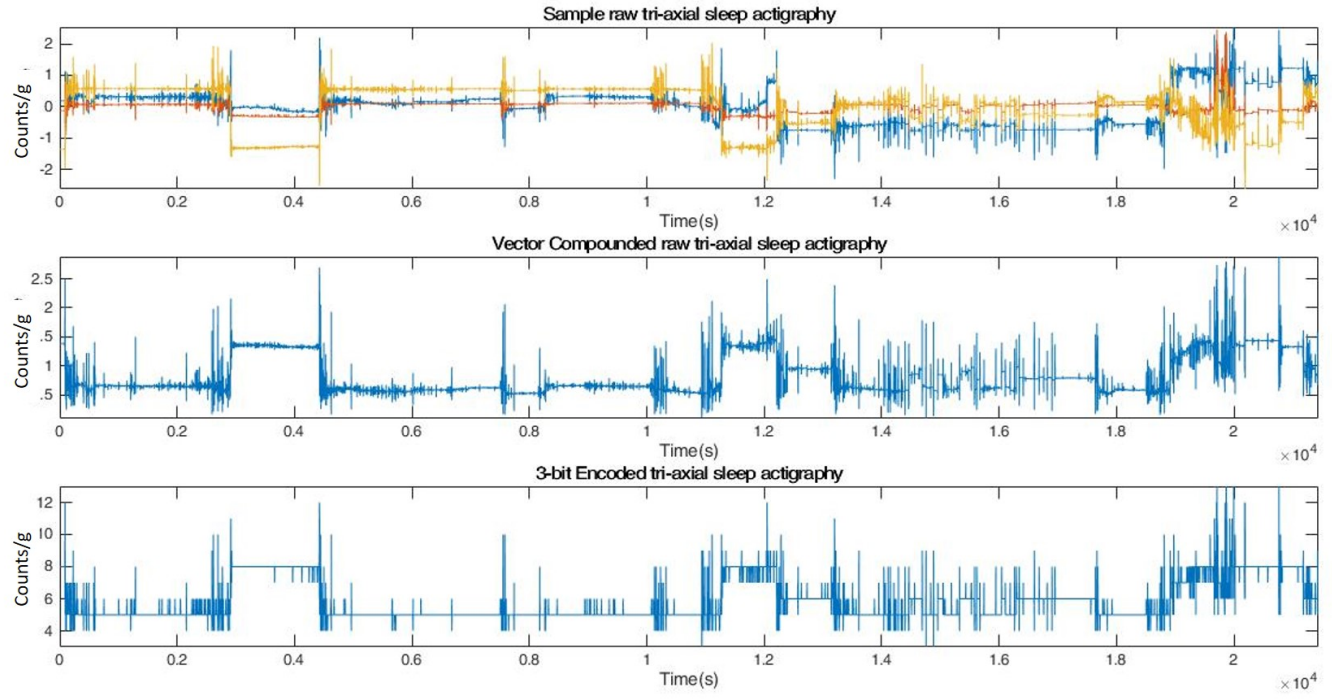

Figure 4.17: 3-bit Encoding of tri-axial sleep actigraphy

Additionally, a parametric comparison between raw, filtered and encoded tri-axial sleep actigraphy signals was also performed as highlighted in Table 4.8. Note that these results have been averaged for different parameters, depending on the raw actigraphy file size and its data content.

Table 4.8: Parametric comparison between raw, filtered and encoded signals

\begin{tabular}{lccc}
\multicolumn{1}{c}{ Parameter } & Raw Signal & Filtered Signal & Encoded Signal \\
\hline Avg. File Size (Mb) & 19 & 4 & 1.4 \\
Bit Rate (bits/sec) & 400 & 400 & 75 \\
Avg. File Read Time (secs) & 1.3 & 0.9 & 0.5 \\
Avg. SNR (dB) & -18 & 10.5 & 38.82 \\
\% Space Savings & NA & $79 \%$ & $92 \%$ \\
\hline
\end{tabular}

As evident from Table 4.8 and following Figure 4.18, signal encoding with a lower bit resolution, provides the highest signal clarity in terms of SNR (signal to noise ratio), coupled with a high space savings and lowered bit rate. 


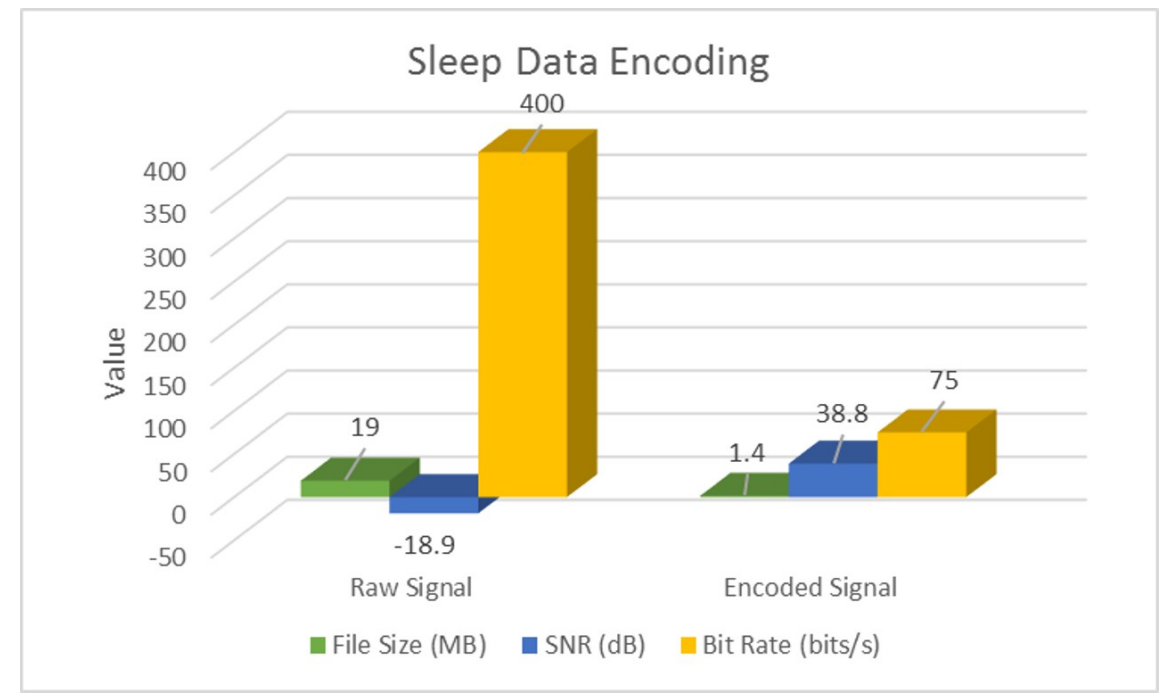

Figure 4.18: 3-bit Encoding of tri-axial sleep actigraphy

In addition to these results, in order to show that vector compounding of tri-axial information was an effective way of capturing vital movement in sleep, the same encoding procedure was also to individual axial data, and found that although there was an increase in SNR by $23 \mathrm{~dB}$ as compared to $20 \mathrm{~dB}$ increase in encoding of vector compounded data, the average space savings was comparatively lower at $82 \%$.

Following these pre-processing experiments, the next Section illustrates the experiments on segmenting encoded data using the Athavale-Krishnan and Mobility-based adaptive segmentation algorithms.

\section{$\underline{\text { Encoding-based Segmentation Results }}$}

After encoding the sleep actigraphy signals, to a lower resolution using the encoding approach in Section 4.3.1, the next step was to adaptively segment the signal into smaller frames for analysis or activity recognition. As discussed previously, both AK and Mobility-based segmentation techniques were applied to actigraphy signals. Figures 4.19-4.22 illustrate the application of these segmentation approaches to two actigraphy signals (left and right leg) captured from a patient experiencing mild PLMs in sleep. Note that for each case, a fixed segmentation scheme has been shown, which splits the actigraphy signal into smaller frames, each of 250 second duration.

To give the reader a clearer picture of the segmentation process, let us consider the AK algorithm's segmentation results depicted in Figure 4.19. The first subplot in this figure illustrates the vector compounded version of the normalized and detrended tri-axial actigraphy signal. The second subplot shows a 3-bit encoded version of the vector compounded signal. This version then undergoes the adaptive segmentation process described in Section 4.3.1, and the final segment boundaries are identified and marked onto the encoded signal 
as shown in Figure 4.19's third subplot. Finally, the fourth subplot in this figure depicts the application of a fixed segmentation scheme to encoded data, wherein the signal is segmented every 250 seconds as shown by the segment markers.
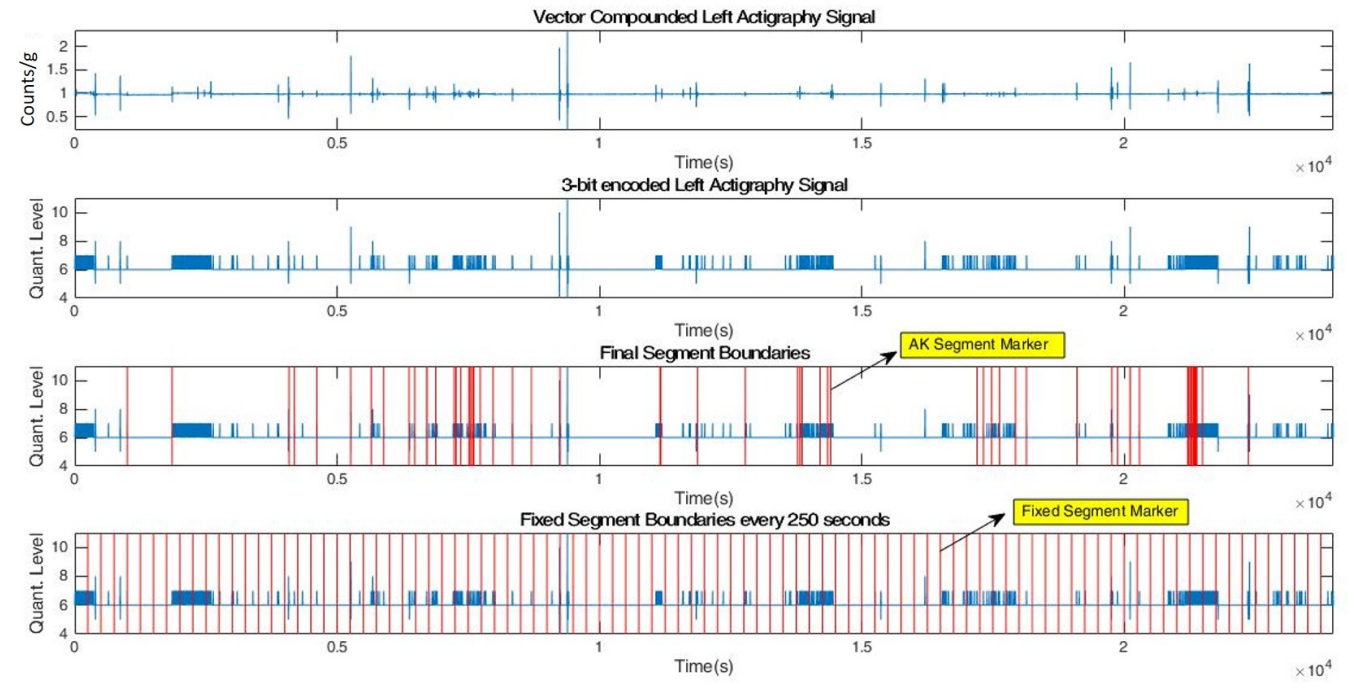

Figure 4.19: AK Segmentation of left actigraphy signal in sleep
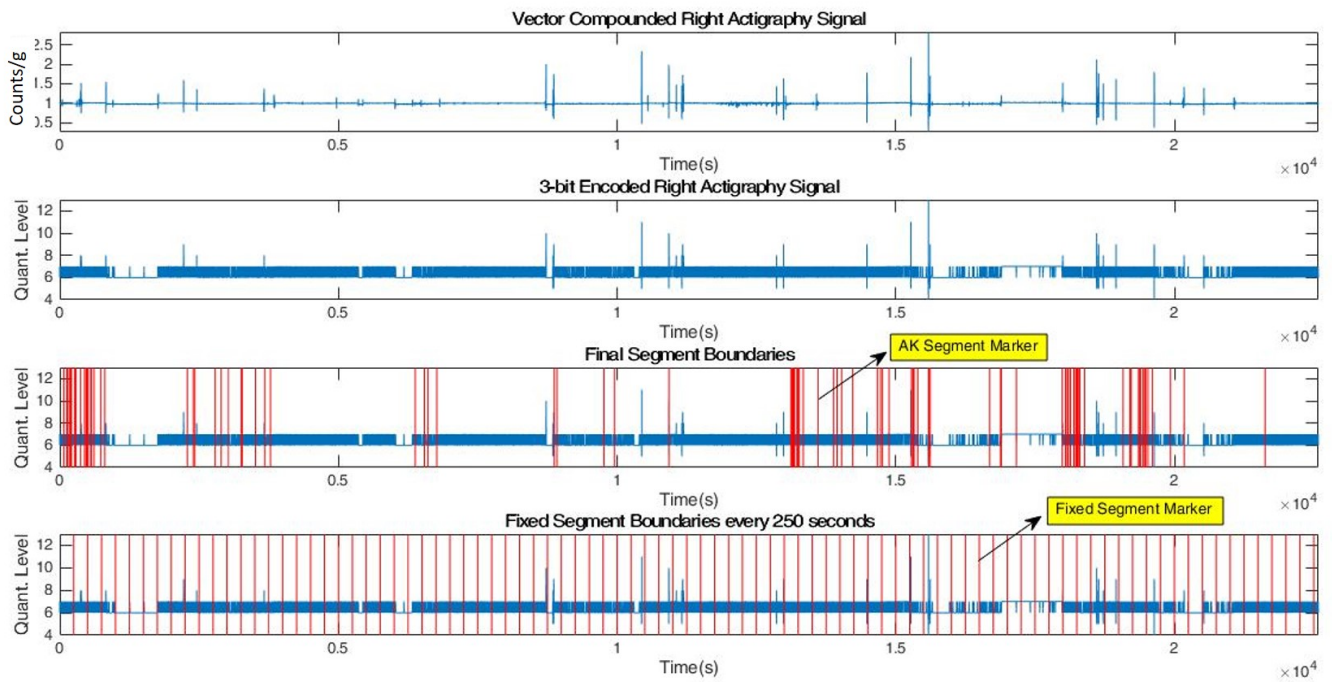

Figure 4.20: AK Segmentation of right actigraphy signal in sleep 

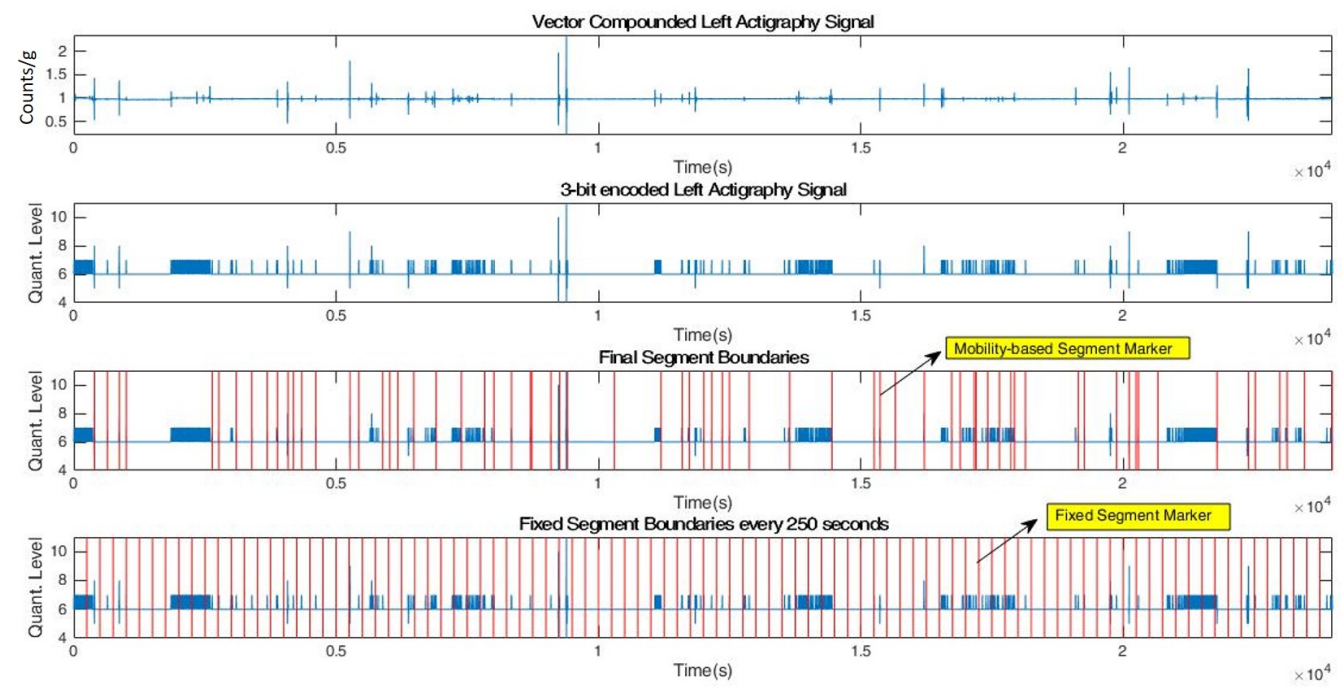

Figure 4.21: Mobility-based Segmentation of left actigraphy signal in sleep
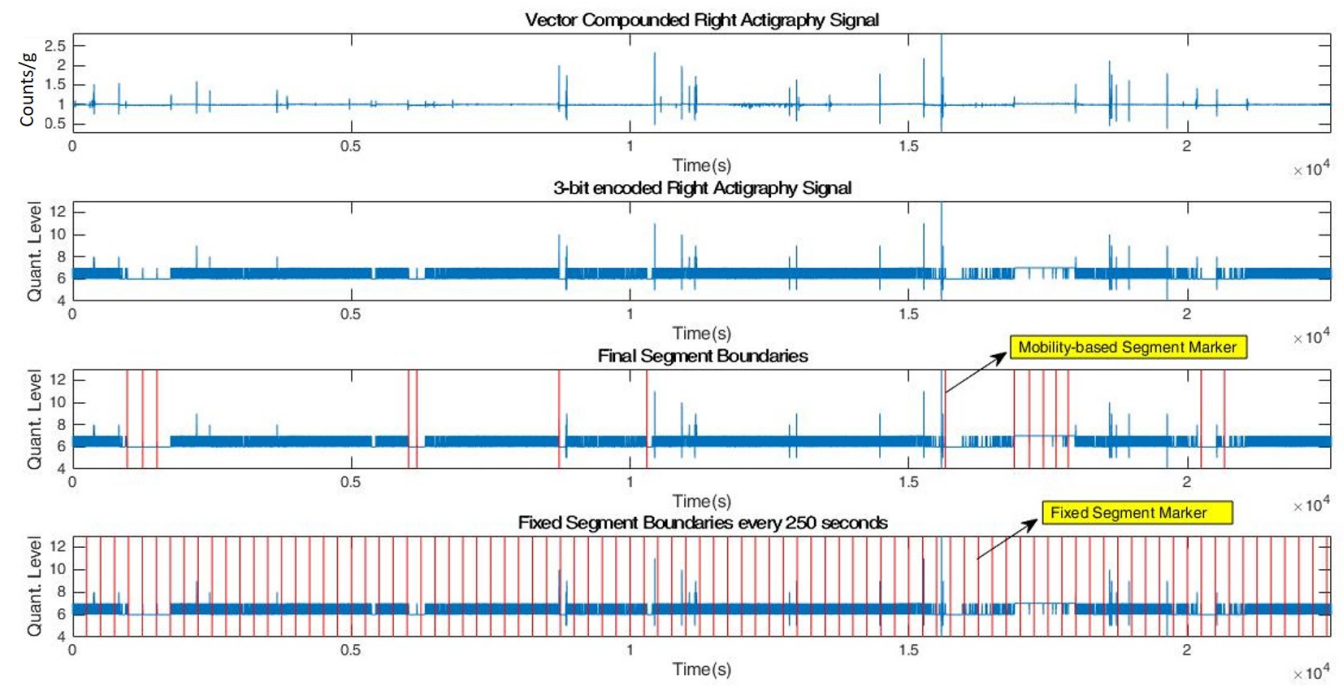

Figure 4.22: Mobility-based Segmentation of right actigraphy signal in sleep

Additionally, a quantitative comparison of the number of segments created in each case was also done, as shown in Table 4.9, for the same sample actigraphy signal. It is quite evident from these results, that the AK segmentation scheme addresses PLM morphology most accurately, by segmenting the actigraphy signal as per regions of high limb activity. 
Table 4.9: Fixed, AK and Mobility-based segments for sample actigraphy

\begin{tabular}{cccc}
\hline Signal & No. Fixed Segments & No. AK Segments & No. Mobility-based Segments \\
\hline Left ankle actigraphy & 95 & 58 & 67 \\
Right ankle actigraphy & 90 & 38 & 15 \\
\hline
\end{tabular}

Further to these results, the relationship between the manually scored PLM index for each patient, and the number of segments created using the AK algorithm was also observed as shown in Figures 4.23-4.25. It is quite evident from these results that the PLM index and number of signal segments present an approximately linear relationship with each other. That is, the higher the PLM index severity, more the number of segments would be created using the AK-algorithm on the corresponding actigraphy signal.

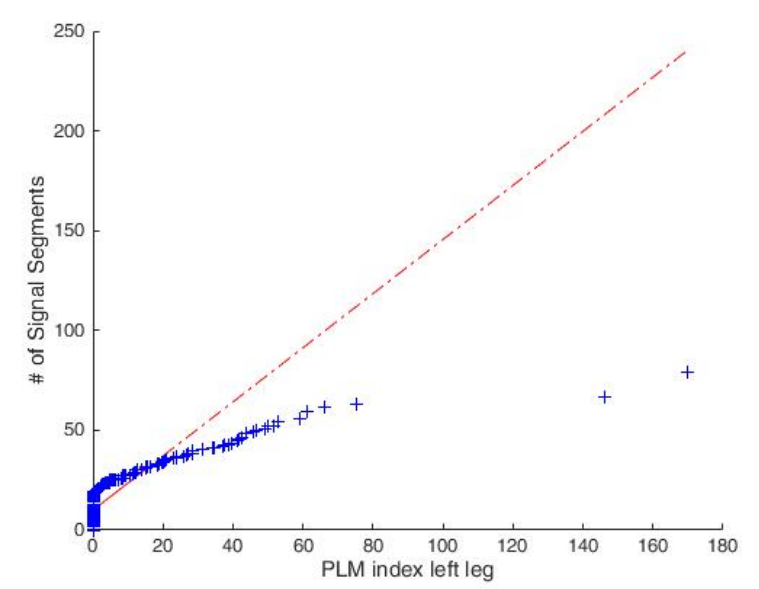

Figure 4.23: Left Leg PLM Index v/s No. of Segments

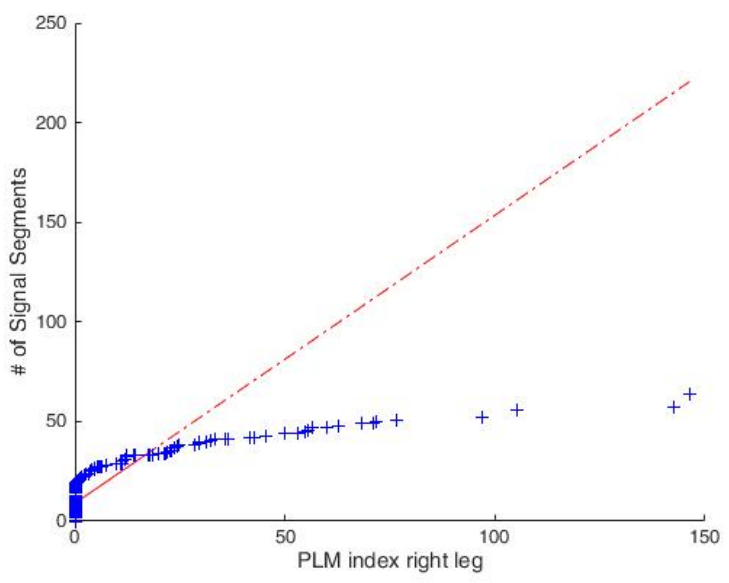

Figure 4.24: Right Leg PLM Index v/s No. of Segments 

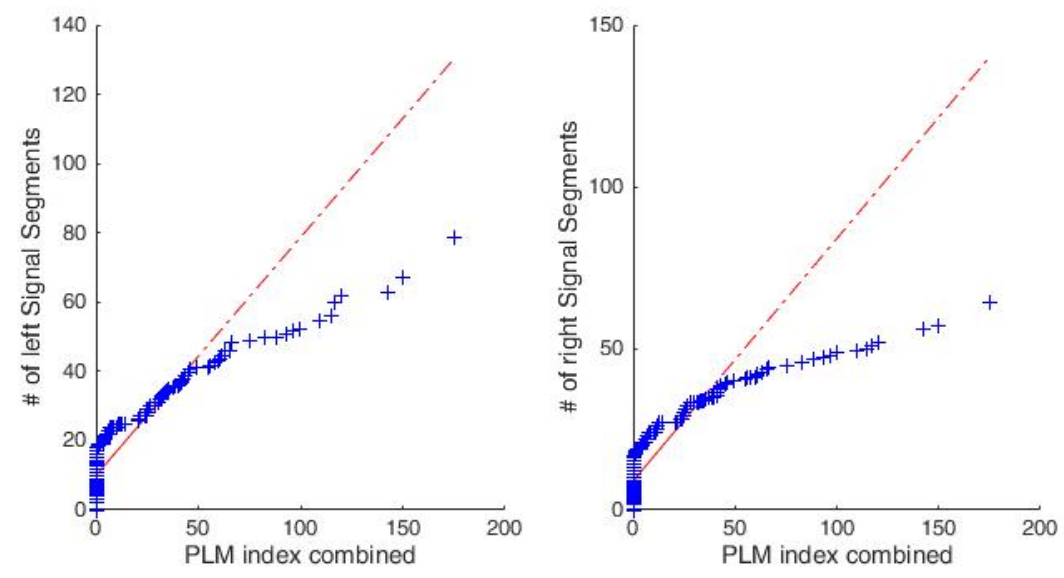

Figure 4.25: Combined PLM Index v/s No. of Segments

In addition to this, a longitudinal comparison of patient population based on the sleep efficiency was also conducted. The sleep efficiency is defined as the ratio of the actual time spent sleeping to the total time in bed. Using a combination of sleep efficiency, number of AK segments generated from a sleep actigraphy signal, and the respective limb's PLM index, it was possible visualize patient populations into three distinct clusters as illustrated in Figures 4.26 and 4.27 .

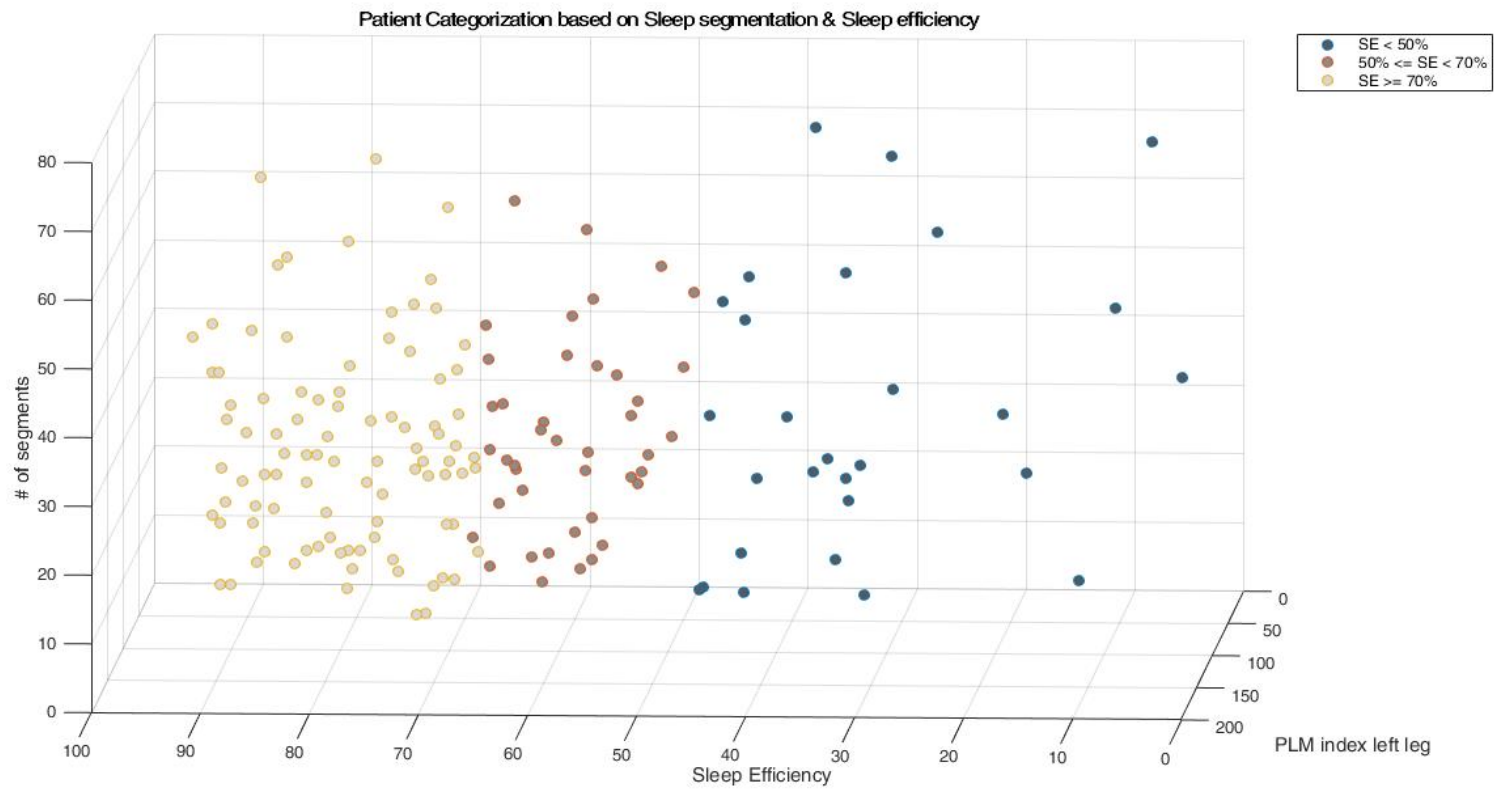

Figure 4.26: Left Leg Actigraphy segregation of patients using sleep efficiency 


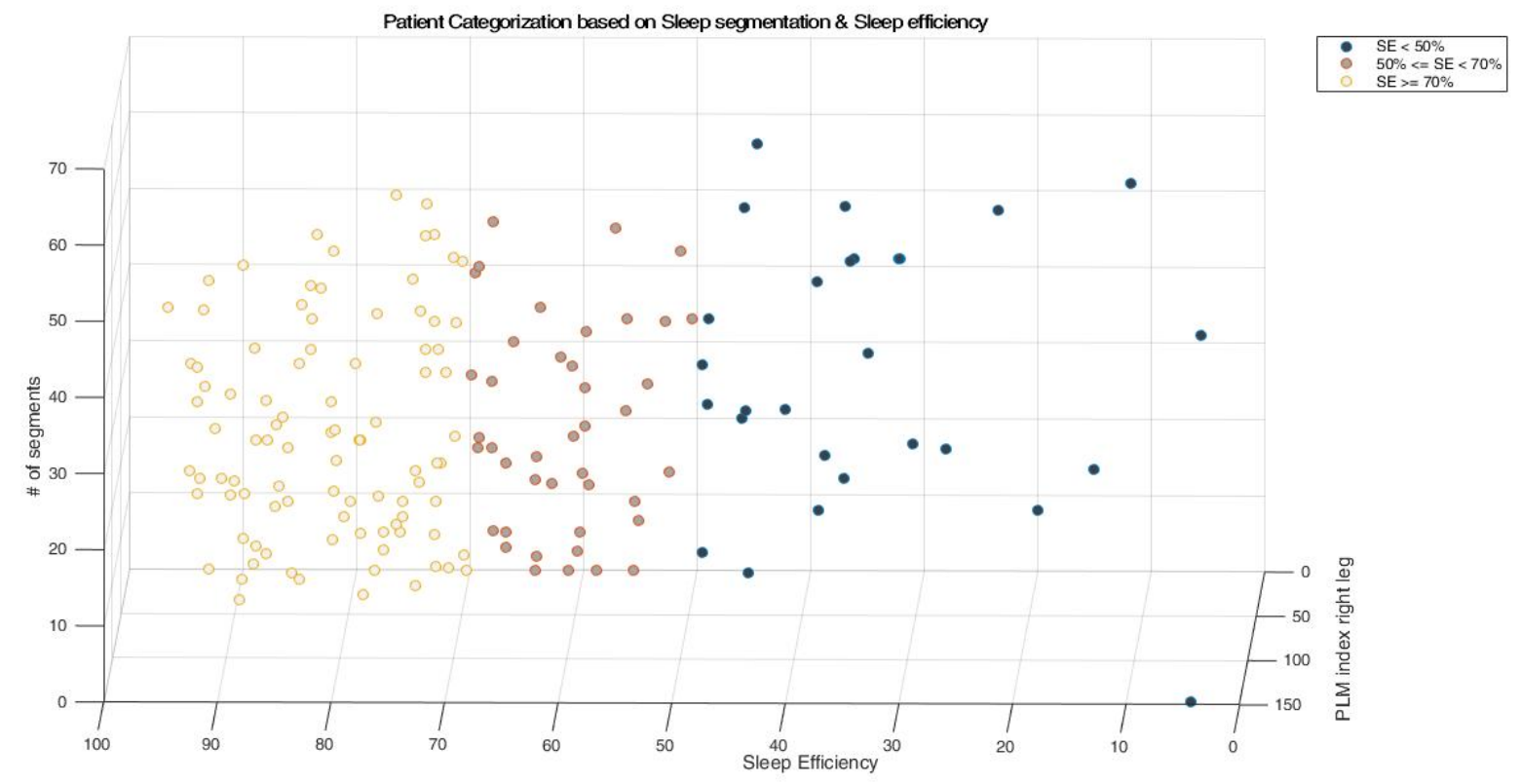

Figure 4.27: Right Leg Actigraphy segregation of patients using sleep efficiency

Thus, it can be inferred from the afore-illustrated segmentation results that the contributed adaptive schemes work remarkably well with long-duration actigraphy data, and can help detect regions of interest for a sleep technologist and a clinician. Following the encoding-based segmentation, the next section highlights the results from the feature extraction and classification experiments on long-duration actigraphy data.

\section{Feature Extraction and Classification Results}

As stated in Section 4.3.1, after extracting 24 time, frequency, kernel-based, actigraphy-specific, morphological and clinical features, different sets were created using a combination of these features. These sets were further split into a 70-30 training-testing partition, for evaluating a machine learning tool, and generating classification results based on PLM index severity. Following Tables 4.10-4.15 highlight the classification results between Normal and Abnormal actigraphy signals, based on a specific combination of features. 
Table 4.10: Classification of PLMs using 14 Features

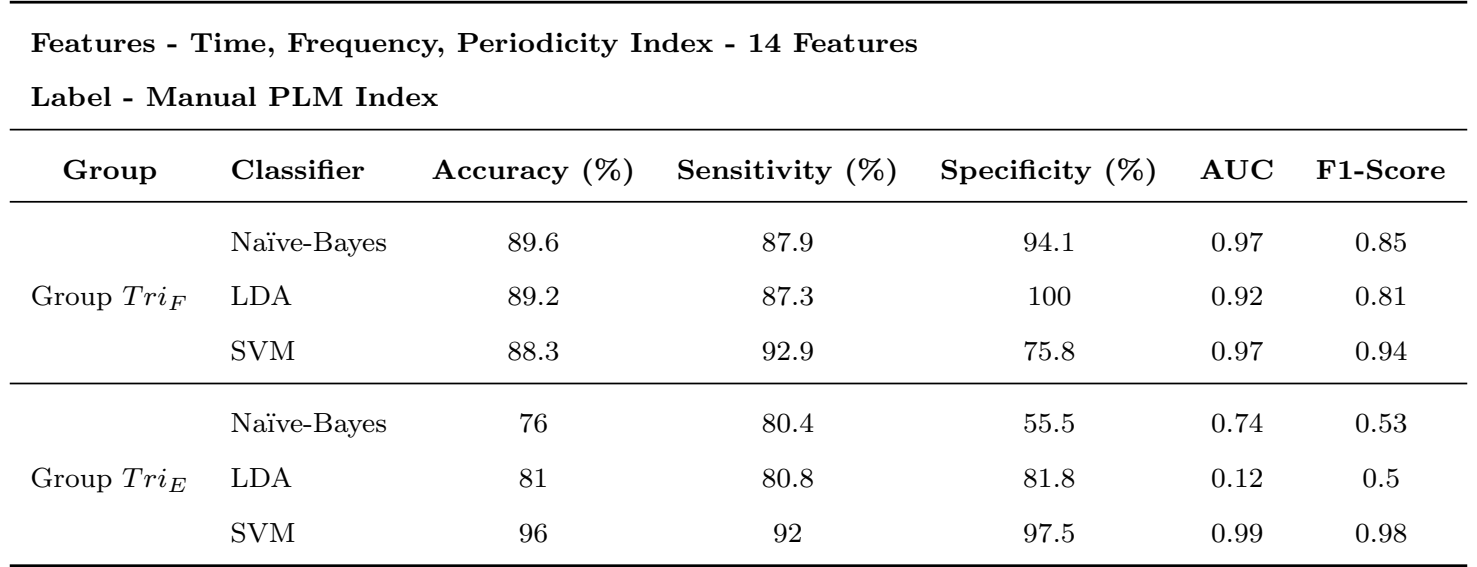

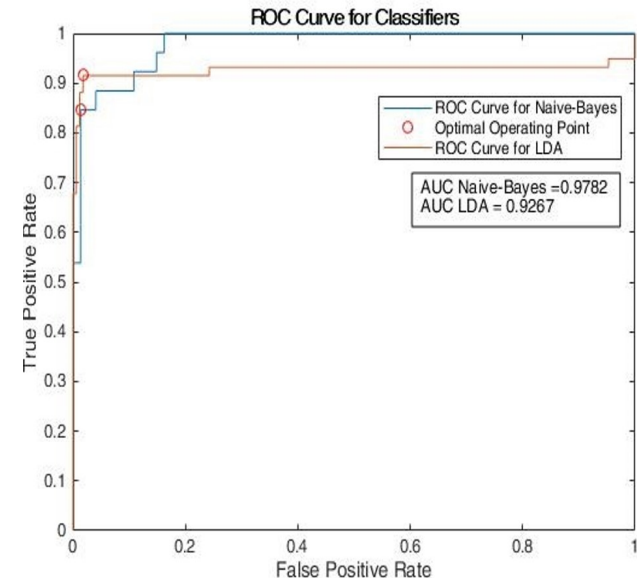

A

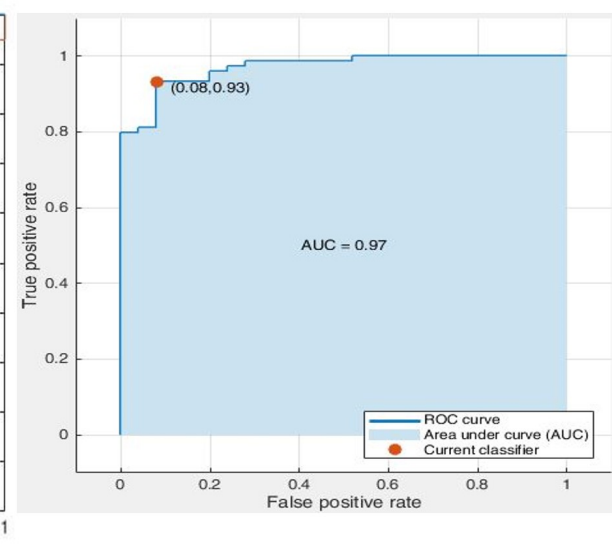

B

Figure 4.28: ROC Curves for Group $\operatorname{Tr}_{F}$ in Table 4.10: (A) Naive-Bayes and LDA, (B) SVM 


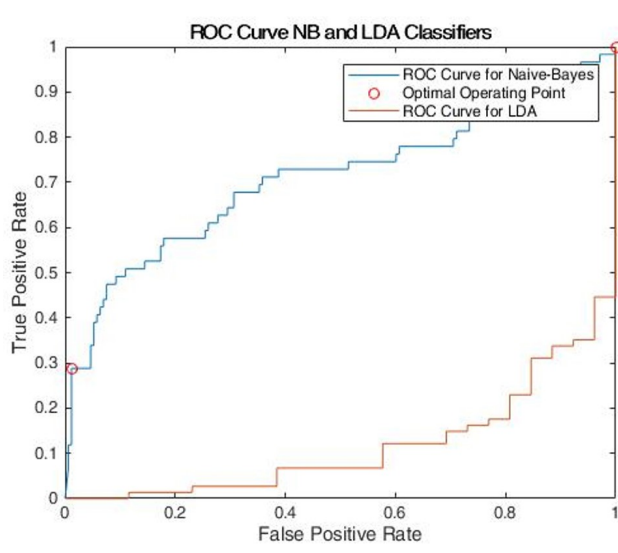

A

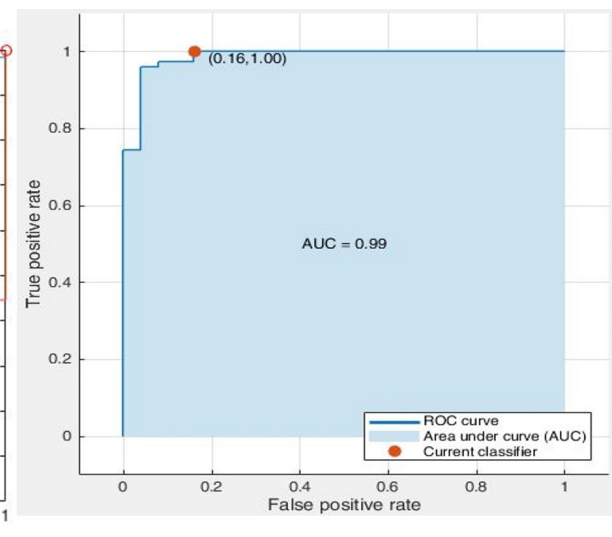

B

Figure 4.29: ROC Curves for Group Tri $i_{E}$ in Table 4.10: (A) Naive-Bayes and LDA, (B) SVM

Table 4.11: Classification of PLMs using 23 features

Features - Time, Frequency, Kernel-based, Actigraphy-specific, Morphological -

23 Features

Label - Manual PLM Index

\begin{tabular}{clccccc}
\hline Group & Classifier & Accuracy (\%) & Sensitivity (\%) & Specificity (\%) & AUC & F1-Score \\
\hline \multirow{2}{*}{ Group Tri $F$} & Naïve-Bayes & 91 & 90.1 & 94.7 & 0.97 & 0.8 \\
& LDA & 87 & 85 & 100 & 0.05 & 0.66 \\
& SVM & 77.6 & 83.8 & 56.6 & 0.92 & 0.74 \\
\hline \multirow{2}{*}{ Group Tri } & Naïve-Bayes & 73 & 80.5 & 47.8 & 0.7 & 0.52 \\
& LDA & 85 & 84.7 & 86.6 & 0.17 & 0.53 \\
& SVM & 89.9 & 85.5 & 87.5 & 0.91 & 0.93 \\
\hline
\end{tabular}




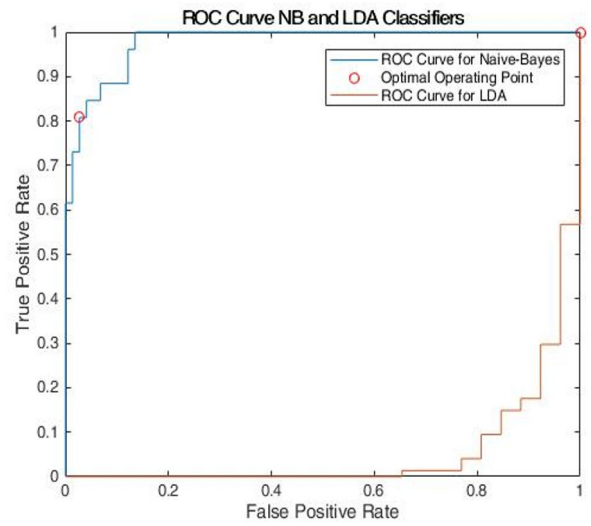

A

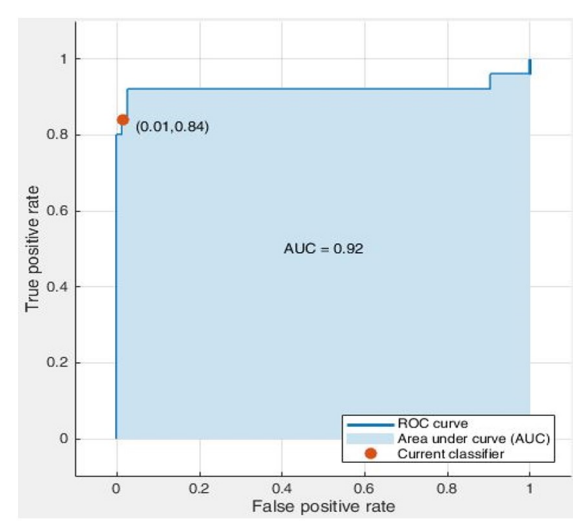

B

Figure 4.30: ROC Curves for Group $\operatorname{Tr}_{F}$ in Table 4.11: (A) Naive-Bayes and LDA, (B) SVM

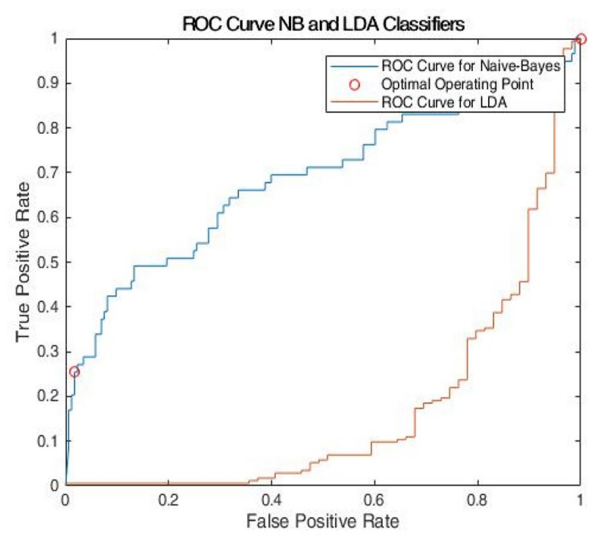

A

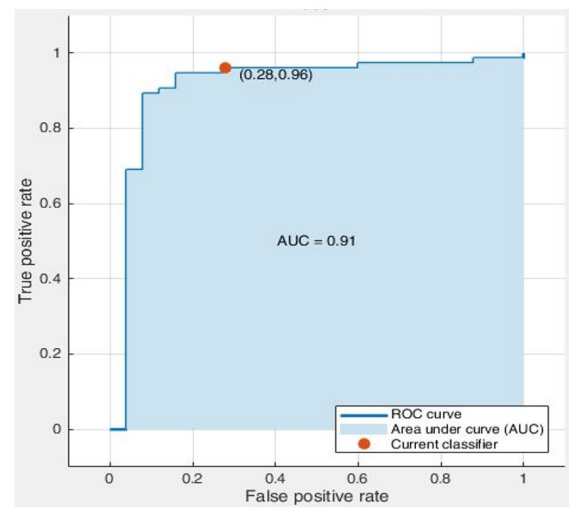

B

Figure 4.31: ROC Curves for Group Tri $i_{E}$ in Table 4.11: (A) Naive-Bayes and LDA, (B) SVM

Similar experiments with conducted by encoding the individual axial data for each tri-axial actigraphy signal, and it was observed that although a higher increase in SNR was obtained through signal encoding, the pattern classification accuracy using the afore-mentioned 23 features was lower, as compared to the results shown in Table 4.11.

In addition to these experiments, a mRMR (minimum Redundancy and Maximum Relevancy) feature selection [152] algorithm was executed to generate the top five discriminatory features which were - the Periodicity Index, the Rapid Change Factor the Mean Frequency, spiky index and Hjorth's mobility. Using these, a scatter plot was generated as shown in Figure 4.32, which indicated that these features gave the maximum discrimination between mild and severe PLMs. This also inferred that the Rapid Change Factor was a key parameter in discriminating between PLM events, aside from Periodicity Index. Additionally, it 
was also found that the classification accuracy using only these five features was higher by about $0.1-2 \%$ as compared to results cited in Table 4.11. Table 4.12 highlights the classification results using mRMR technique.

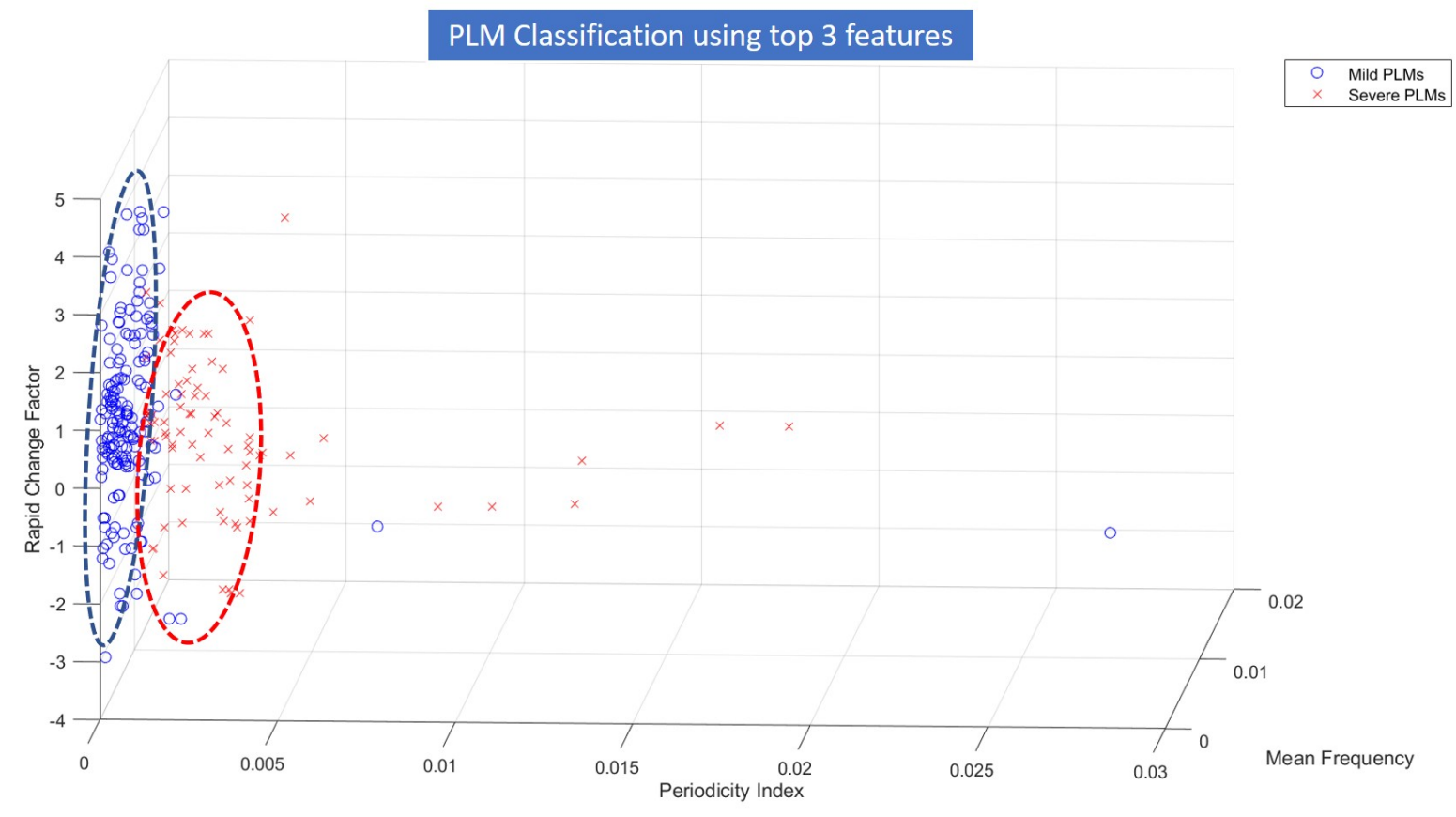

Figure 4.32: Top 3 Features Classifying PLMs

Table 4.12: Classification of PLMs using top 5 mRMR features for encoded signals

Features - Time, Frequency, Kernel-based, Actigraphy-specific, Morphological -

5 Features

Label - Manual PLM Index

\begin{tabular}{clccccc}
\hline \multirow{2}{*}{ Group } & Classifier & Accuracy (\%) & Sensitivity (\%) & Specificity (\%) & AUC & F1-Score \\
\hline \multirow{2}{*}{ Group Tri $_{E}$} & Naïve-Bayes & 80 & 80.4 & 69.2 & 0.72 & 0.58 \\
& LDA & 83.2 & 82.2 & 91.1 & 0.21 & 0.67 \\
& SVM & 89.8 & 84.1 & 86.9 & 0.87 & 0.88 \\
\hline
\end{tabular}


Table 4.13: Classification of PLMs using 22 features, excluding periodicity index

\begin{tabular}{|c|c|c|c|c|c|c|}
\hline \multicolumn{7}{|c|}{ Label - Manual PLM Index } \\
\hline Group & Classifier & Accuracy (\%) & Sensitivity (\%) & Specificity (\%) & AUC & F1-Score \\
\hline \multirow{3}{*}{ Group $\operatorname{Tri}_{F}$} & Naïve-Bayes & 73 & 73 & 0.15 & 0.56 & 0.34 \\
\hline & LDA & 72 & 73.9 & 25 & 0.41 & 0.2 \\
\hline & SVM & 59.9 & 74.6 & 25.7 & 0.5 & 0.24 \\
\hline \multirow{3}{*}{ Group $\operatorname{Tri}_{E}$} & Naïve-Bayes & 70 & 79.7 & 42.3 & 0.63 & 0.42 \\
\hline & LDA & 75 & 76.9 & 55.5 & 0.36 & 0.28 \\
\hline & SVM & 74 & 74.5 & 50 & 0.62 & 0.84 \\
\hline
\end{tabular}
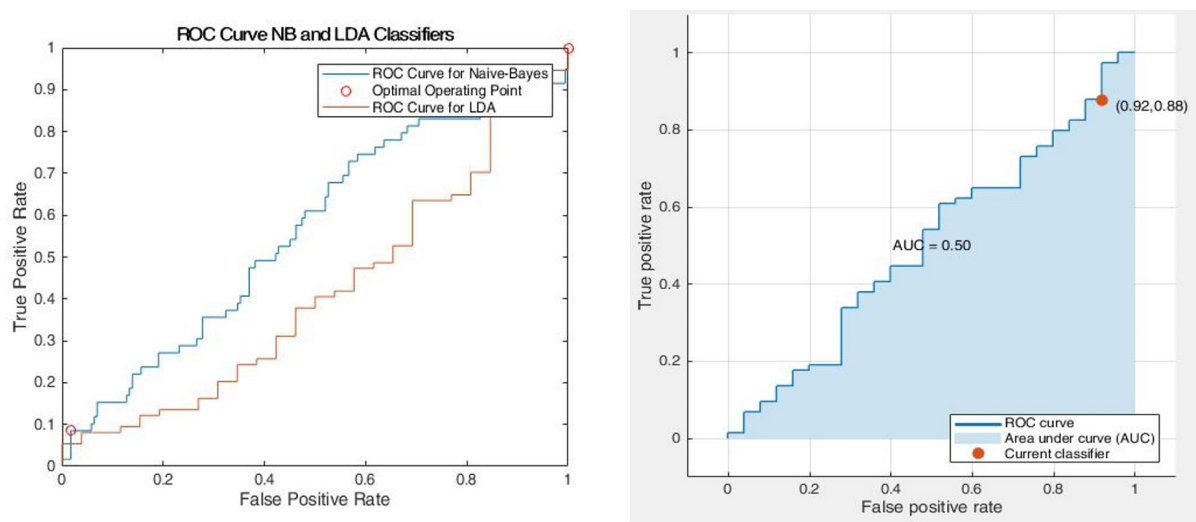

A

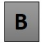

Figure 4.33: ROC Curves for Group $\operatorname{Tri}_{F}$ in Table 4.13: (A) Naive-Bayes and LDA, (B) SVM
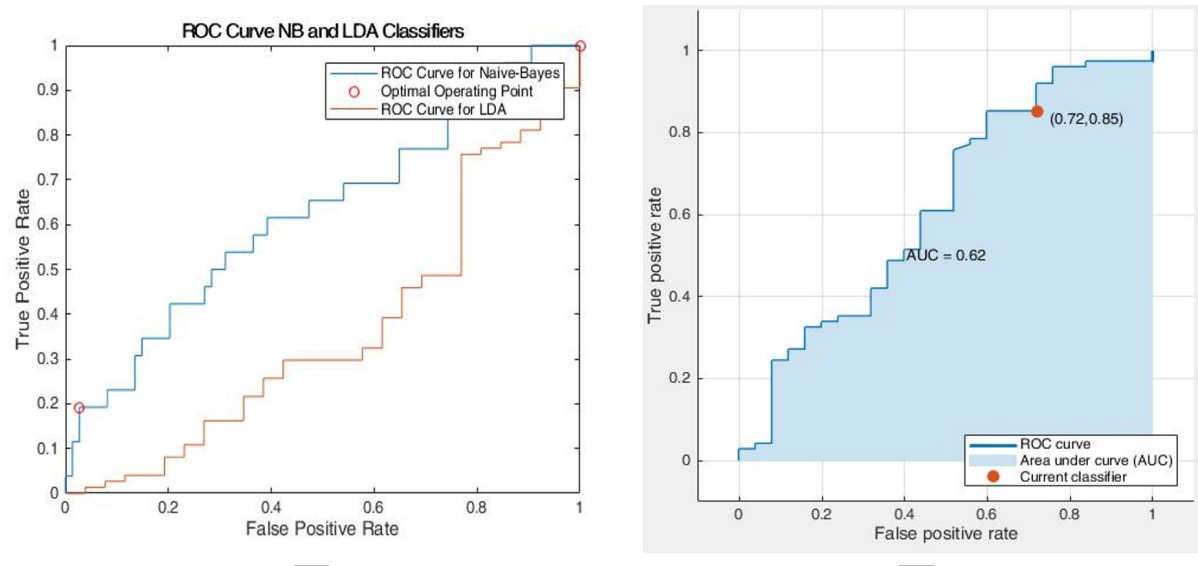

A

B

Figure 4.34: ROC Curves for Group Tri $i_{E}$ in Table 4.13: (A) Naive-Bayes and LDA, (B) SVM 
Table 4.14: Classification of PLMs using 10 proposed features

\begin{tabular}{|c|c|c|c|c|c|c|}
\hline Group & Classifier & Accuracy (\%) & Sensitivity (\%) & Specificity (\%) & AUC & F1-Score \\
\hline \multirow{3}{*}{ Group $T r i_{F}$} & Naïve-Bayes & 96 & 100 & 86.6 & 0.99 & 0.92 \\
\hline & LDA & 86 & 84 & 100 & 0.01 & 0.63 \\
\hline & SVM & 91 & 95.7 & 79.3 & 0.98 & 0.83 \\
\hline \multirow{3}{*}{ Group $\operatorname{Tr} i_{E}$} & Naïve-Bayes & 81 & 86 & 64.1 & 0.78 & 0.61 \\
\hline & LDA & 84 & 82.6 & 95.8 & 0.17 & 0.55 \\
\hline & SVM & 92.9 & 88.5 & 92.5 & 0.95 & 0.94 \\
\hline
\end{tabular}
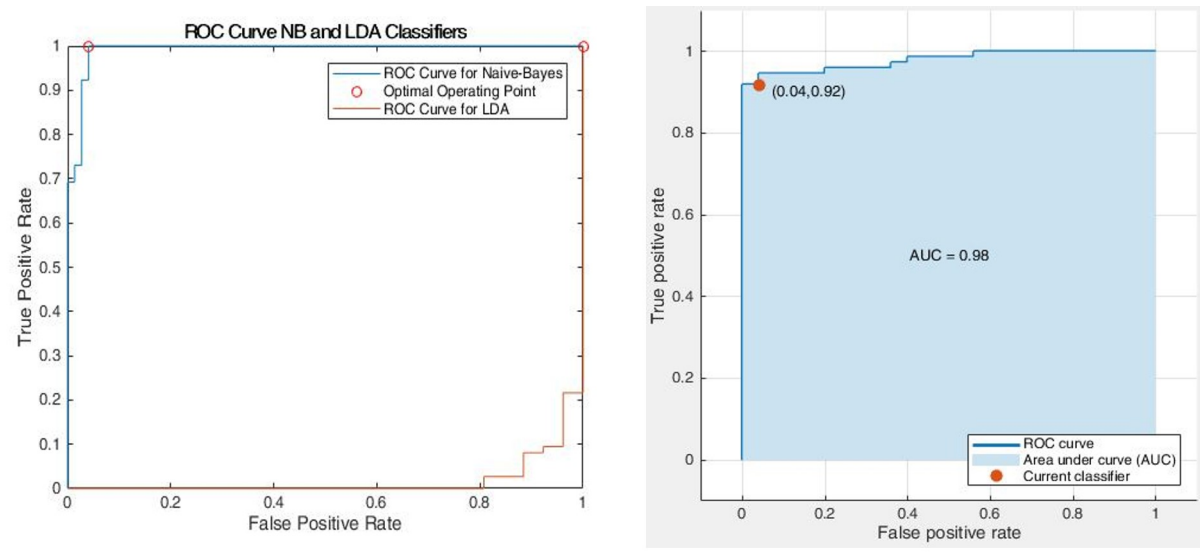

A

Figure 4.35: ROC Curves for Group $\operatorname{Tri}_{F}$ in Table 4.14: (A) Naive-Bayes and LDA, (B) SVM
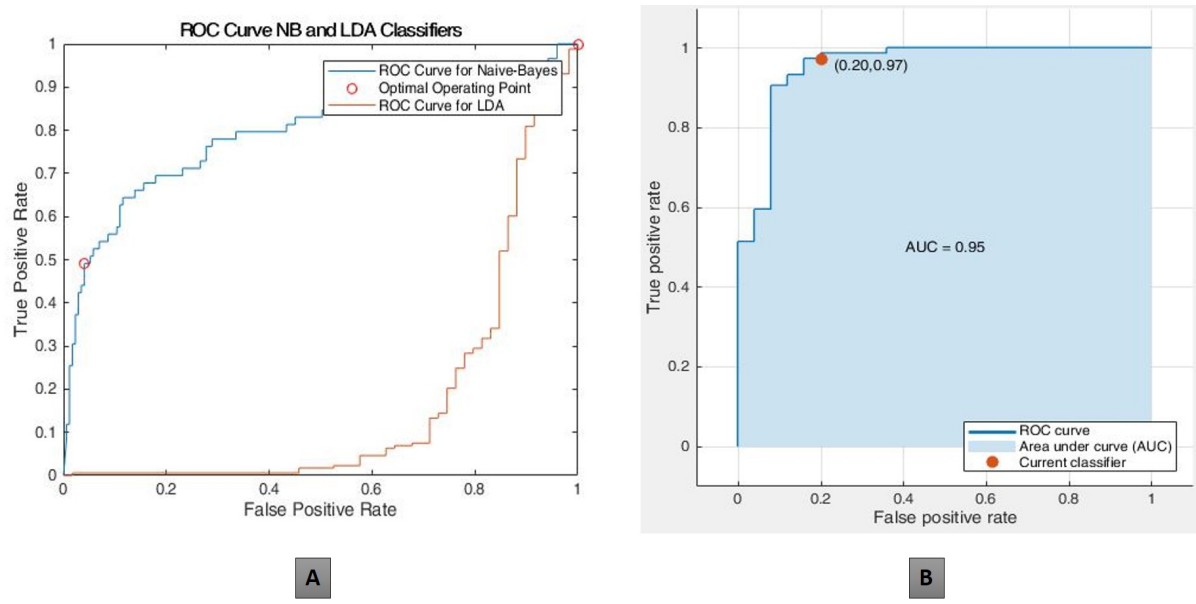

Figure 4.36: ROC Curves for Group $T r i_{E}$ in Table 4.14: (A) Naive-Bayes and LDA, (B) SVM 
Table 4.15: Classification of PLMs using 9 proposed features

\begin{tabular}{|c|c|c|c|c|c|c|}
\hline \multicolumn{7}{|c|}{$\begin{array}{l}\text { Features - Kernel-based, Actigraphy-specific, Morphological } \\
\text { excluding Periodicity Index } \\
\text { Label - Manual PLM Index }\end{array}$} \\
\hline Group & Classifier & Accuracy (\%) & Sensitivity (\%) & Specificity (\%) & AUC & F1-Score \\
\hline \multirow{3}{*}{ Group $T r i_{F}$} & Naïve-Bayes & 63 & 77.6 & 33.3 & 0.58 & 0.37 \\
\hline & LDA & 72.8 & 75 & 33.3 & 0.45 & 0.11 \\
\hline & SVM & 71.7 & 74.3 & 25 & 0.45 & 0.31 \\
\hline \multirow{3}{*}{ Group $\operatorname{Tr} i_{E}$} & Naïve-Bayes & 63 & 72.8 & 21 & 0.57 & 0.2 \\
\hline & LDA & 75 & 76.7 & 53 & 0.4 & 0.23 \\
\hline & SVM & 76.8 & 78.2 & 72 & 0.68 & 0.85 \\
\hline
\end{tabular}

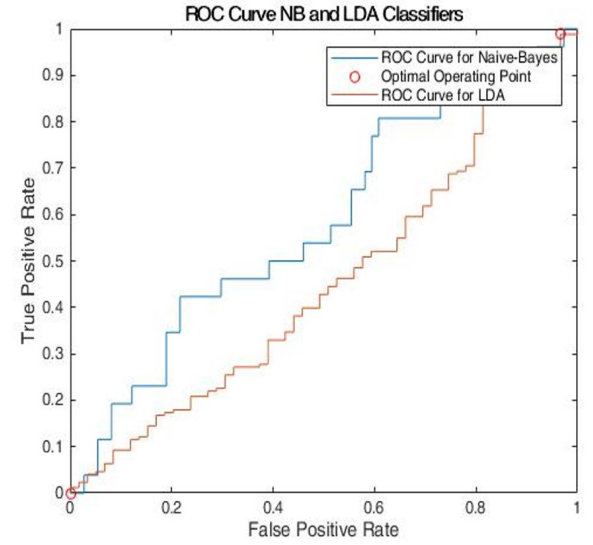

A

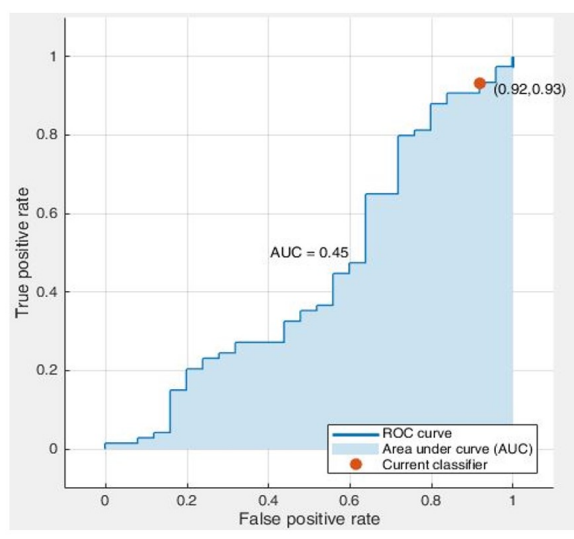

B

Figure 4.37: ROC Curves for Group $\operatorname{Tri}_{F}$ in Table 4.15: (A) Naive-Bayes and LDA, (B) SVM 


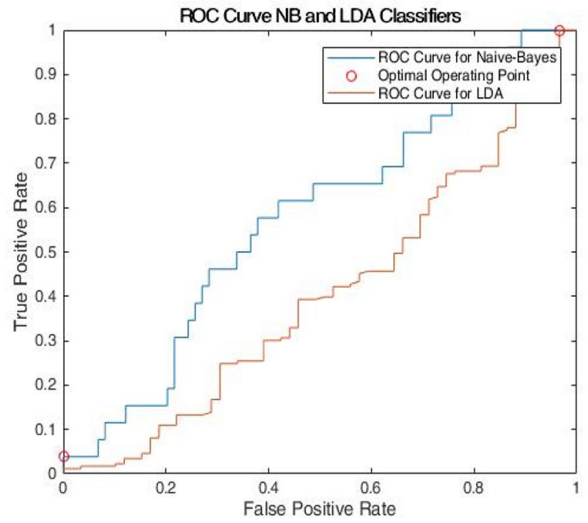

A

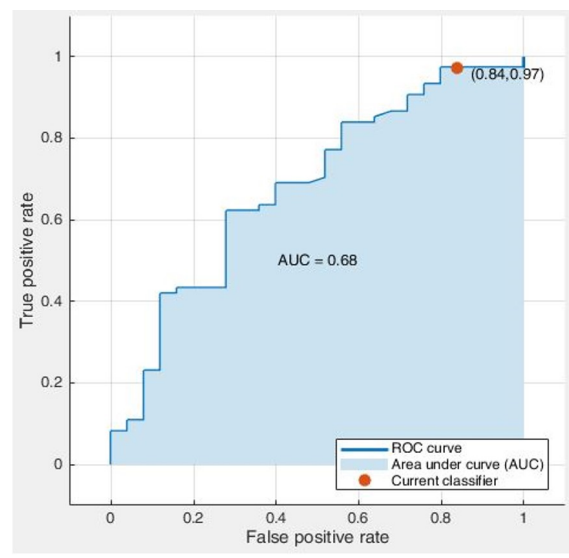

B

Figure 4.38: ROC Curves for Group Tri ${ }_{E}$ in Table 4.15: (A) Naive-Bayes and LDA, (B) SVM

Of all the features extracted from the sleep actigraphy signals (see Table 4.7, the Periodicity Index (PI) has proven to be one of the most discriminant parameters in classifying PLMs. Being directly derived from the manually scored PLM index, the PI aims to encapsulate true limb movements in deep sleep. In order to show the classification capability, a simple visualization of PI with respect to SNDR (signal to noise and distortion ratio) and standard deviation was performed, as shown in Figure 4.39, and it was found that PI was effectively able to separate normal and abnormal subjects in two distinct clusters.

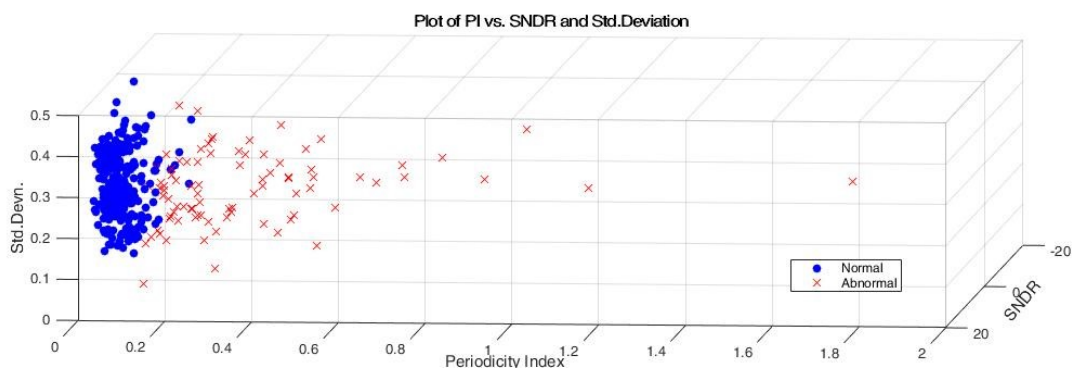

Figure 4.39: Visualizing PI for Normal and Abnormal Subjects

As described previously, employing a 3-bit encoding of actigraphy signals in Group $T r i_{E}$ led to an increase in classification accuracy of the proposed actigraphy analysis algorithm. In order verify the effectiveness of the 3-bit encoding, similar feature extraction and classification experiments were conducted with $6,8,10$ and 12-bit encoded sleep actigraphy data, and their classification rates were compared as shown in Figure 4.40 . 


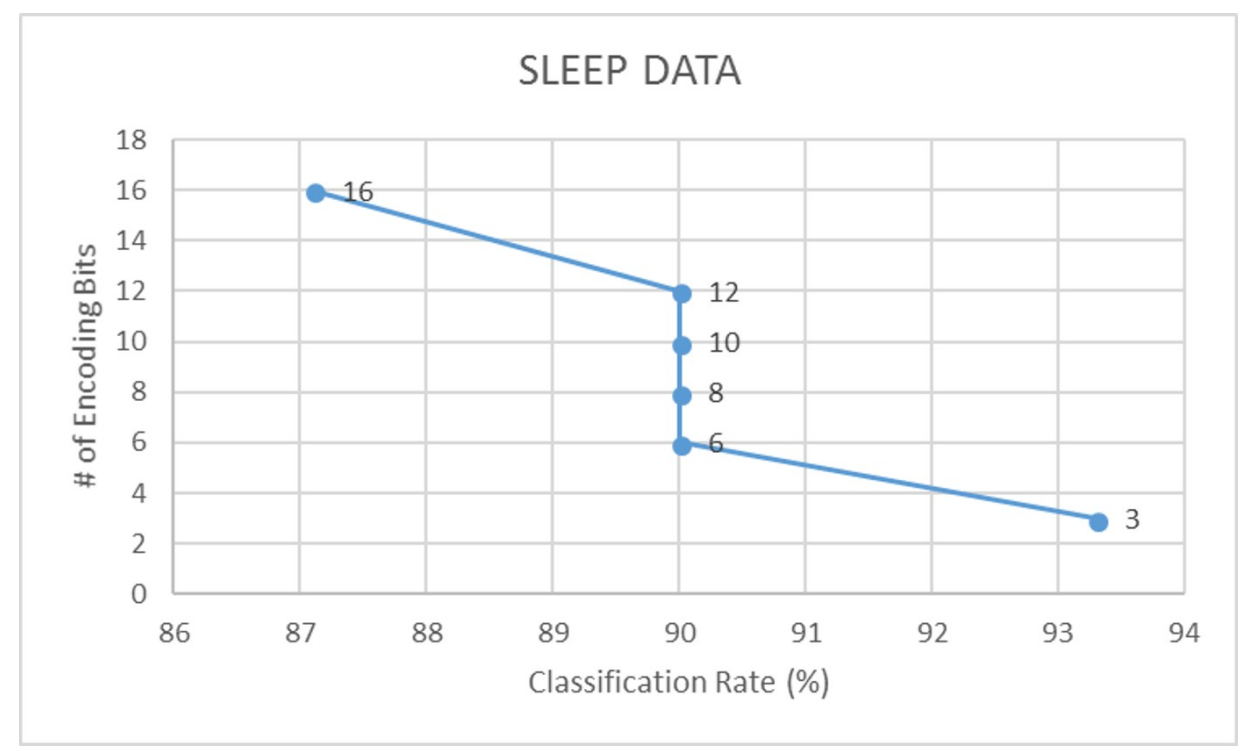

Figure 4.40: Classification Accuracy vs Encoding Levels

From Fig. 4.40, it would be also evident to the reader that 6, 8, 10 and 12-bit encoding resulted in nearly the same classification rate for PLMs in Database B. Upon further investigation, it was found that the reason for this similarity was the presence of small or insignificant spurious vibrations captured by the actigraph, which were retained in the encoding process, and would have been otherwise approximated to zero amplitude in case of 3-bit encoding. For example, a sample with an amplitude of 0.0005 in a 16-bit quantized actigraphy signal, would still retain a value if encoded with $6,8,10$ or 12-bits, but would reduce to zero when encoded with 3-bits per sample.

As evident from Figure 4.40, the 3-bit encoding of sleep actigraphy signals provided the highest classification accuracy of identifying normal PLMs from abnormal ones. In addition to classifying the longduration sleep actigraphy signals using the afore-mentioned features in Table 4.7, a similar pattern classification methodology was also experimented using two clinical parameters - Age as a feature, and Gender (male/female) as a class label. Since the experiments in this study involved conducting bilateral ankle actigraphy for each patient, the feature extraction and classification of PLMs was done separately for both legs per patient. Following Tables 4.16-4.19 highlight these results. 
Table 4.16: Classification of PLMs based on Gender using 24 features - Left Leg

\begin{tabular}{|c|c|c|c|c|c|c|}
\hline \multicolumn{7}{|c|}{ Label - Gender (Male vs Female) } \\
\hline Group & Classifier & Accuracy (\%) & Sensitivity (\%) & Specificity (\%) & AUC & F1-Score \\
\hline \multirow{2}{*}{ Group $\operatorname{Tri}_{F}$} & LDA & 63.3 & 60 & 63 & 0.56 & 0.61 \\
\hline & SVM & 65.3 & 66.6 & 64.8 & 0.6 & 0.66 \\
\hline \multirow{2}{*}{ Group $\operatorname{Tri}_{E}$} & LDA & 46.9 & 35.2 & 53.1 & 0.54 & 0.37 \\
\hline & SVM & 61.2 & 100 & 59.5 & 0.58 & 0.16 \\
\hline
\end{tabular}

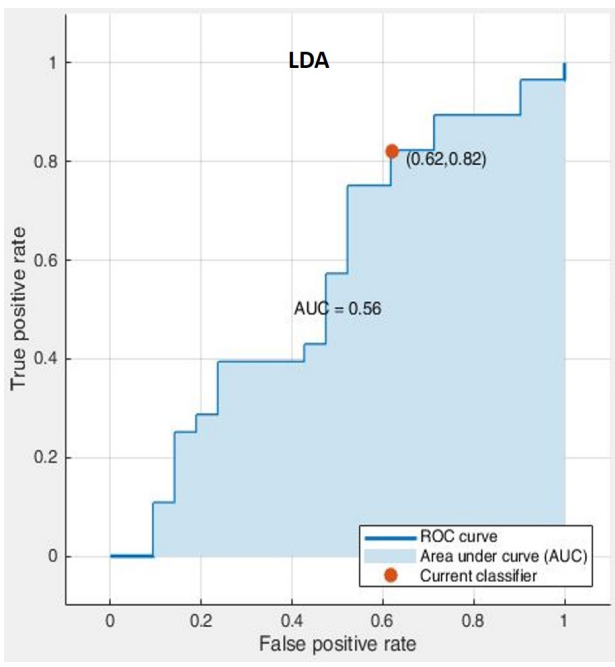

A

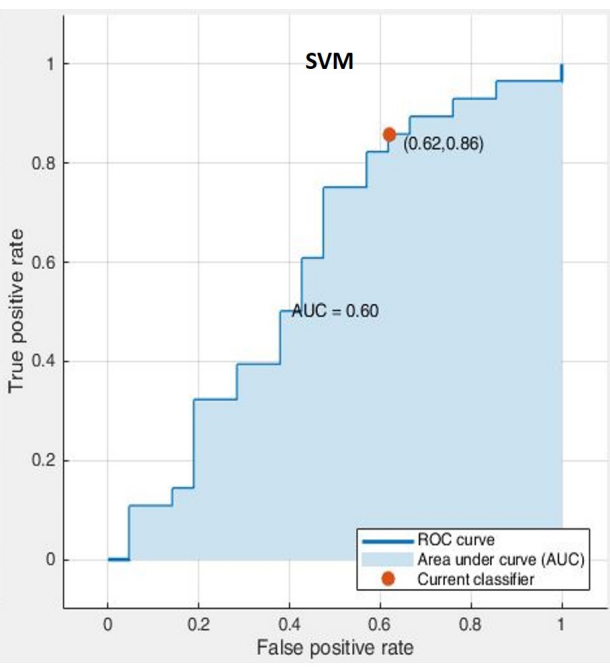

B

Figure 4.41: ROC Curves for Group Tri $i_{F}$ in Table 4.16: (A) LDA, (B) SVM 


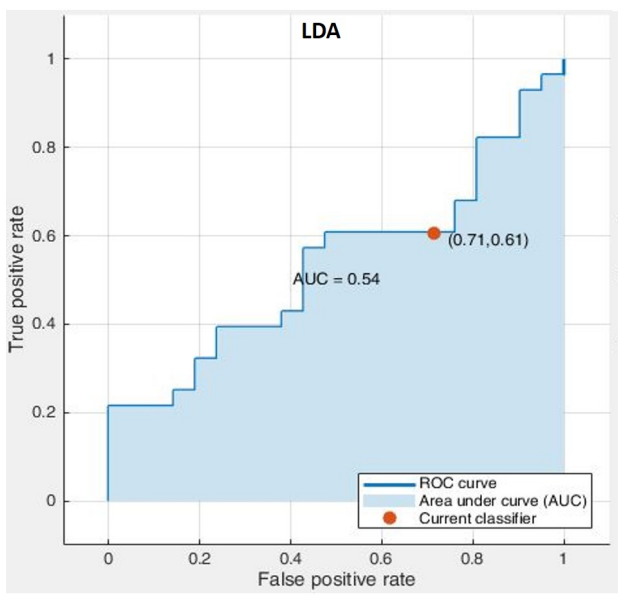

A

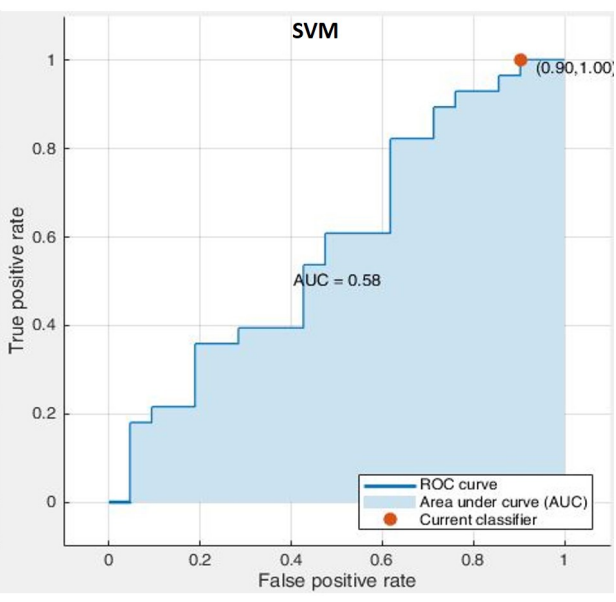

B

Figure 4.42: ROC Curves for Group Tri $i_{E}$ in Table 4.16: (A) LDA, (B) SVM

Table 4.17: Classification of PLMs based on Gender using 24 features - Right Leg

Features - Time, Frequency, Kernel-based, Actigraphy-specific, Morphological and Clinical (age) - 24 Features

Label - Gender (Male vs Female)

\begin{tabular}{|c|c|c|c|c|c|c|}
\hline Group & Classifier & Accuracy (\%) & Sensitivity (\%) & Specificity (\%) & AUC & F1-Score \\
\hline \multirow{2}{*}{ Group $\operatorname{Tri}_{F}$} & LDA & 57.1 & 50 & 62.9 & 0.63 & 0.51 \\
\hline & SVM & 59.2 & 53.3 & 61.7 & 0.58 & 0.44 \\
\hline \multirow{2}{*}{ Group $T r i_{E}$} & LDA & 61.2 & 55 & 65.5 & 0.64 & 0.53 \\
\hline & SVM & 61.2 & 55 & 65.3 & 0.57 & 0.52 \\
\hline
\end{tabular}




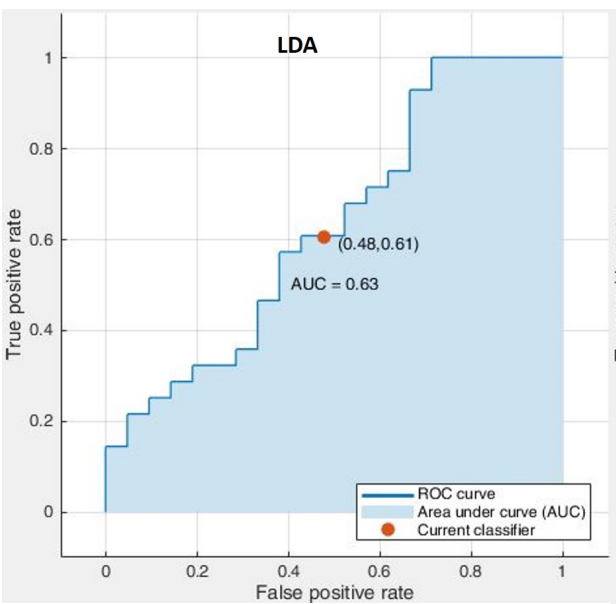

A

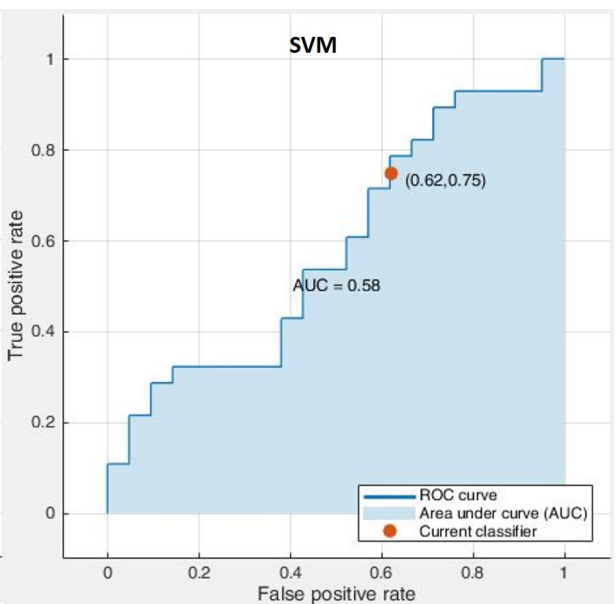

B

Figure 4.43: ROC Curves for Group Tri $i_{F}$ in Table 4.17: (A) LDA, (B) SVM

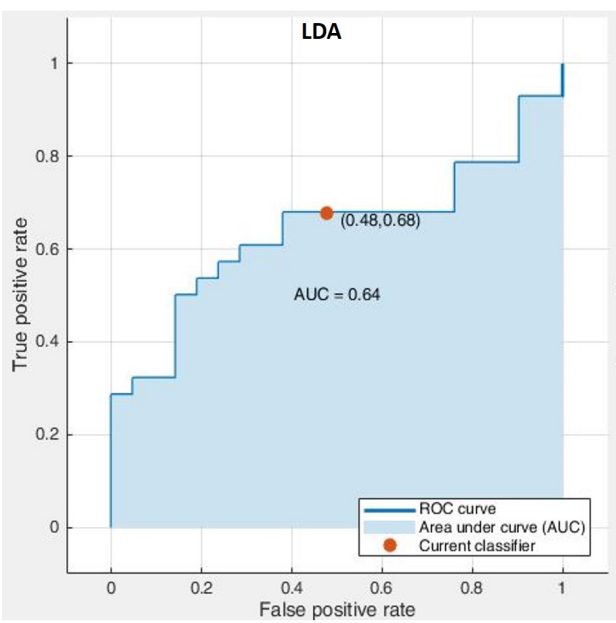

A

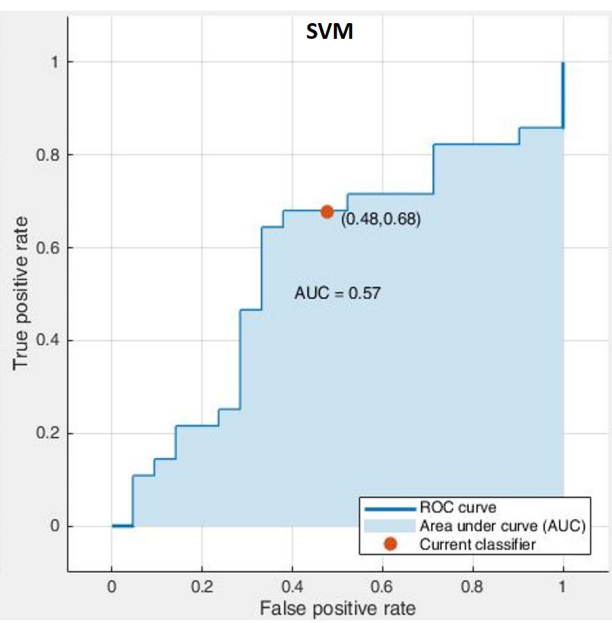

B

Figure 4.44: ROC Curves for Group $T r i_{E}$ in Table 4.17: (A) LDA, (B) SVM 
Table 4.18: Classification of PLMs using 24 features including Age - Left Leg

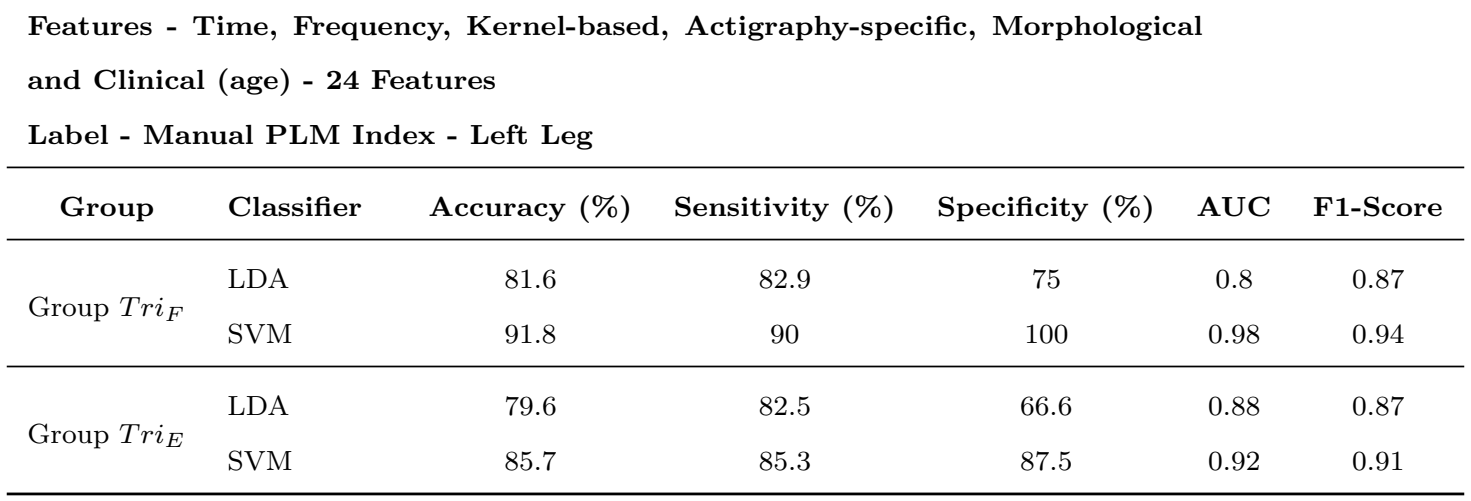

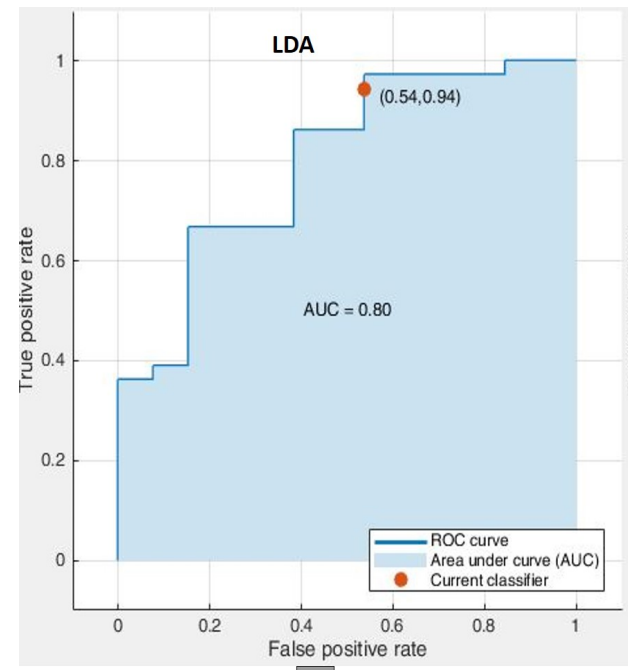

A

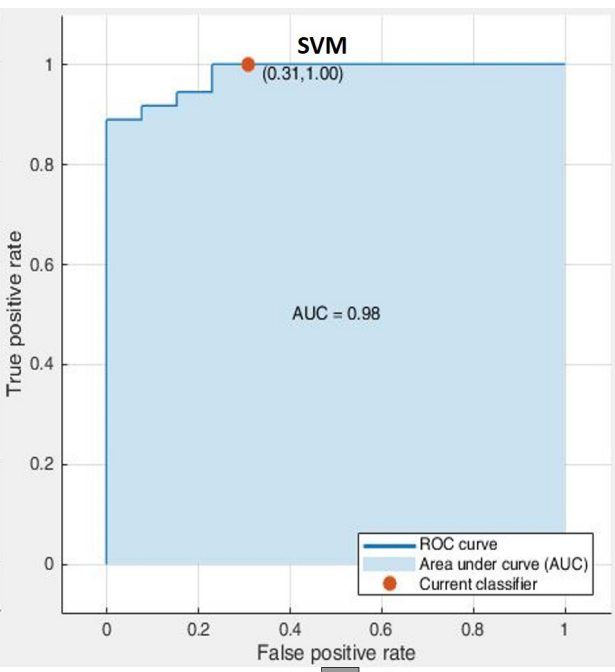

B

Figure 4.45: ROC Curves for Group Tri $i_{F}$ in Table 4.18: (A) LDA, (B) SVM 


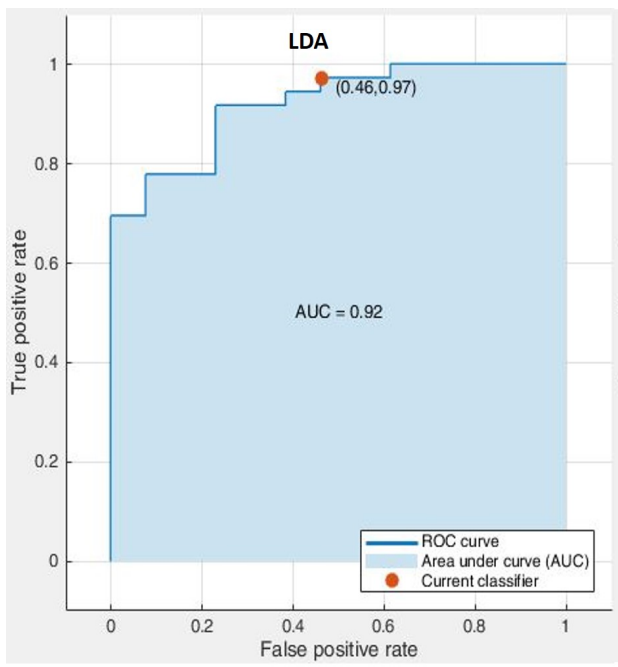

A

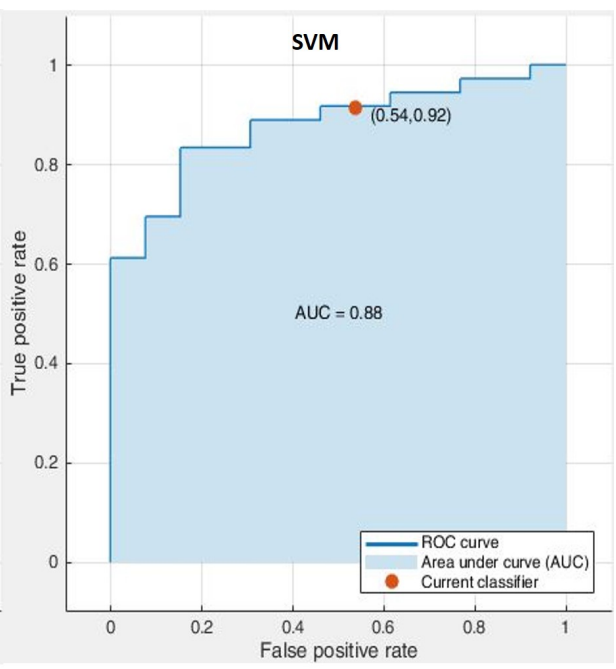

B

Figure 4.46: ROC Curves for Group Tri $i_{E}$ in Table 4.18: (A) LDA, (B) SVM

Table 4.19: Classification of PLMs using 24 features including Age - Right Leg

\begin{tabular}{|c|c|c|c|c|c|c|}
\hline \multicolumn{7}{|c|}{ Label - Manual PLM Index - Right Leg } \\
\hline Group & Classifier & Accuracy (\%) & Sensitivity (\%) & Specificity (\%) & AUC & F1-Score \\
\hline \multirow{2}{*}{ Group $\operatorname{Tri}_{F}$} & LDA & 93.9 & 92.5 & 100 & 0.99 & 0.94 \\
\hline & SVM & 91.8 & 94.5 & 83.3 & 0.89 & 0.93 \\
\hline \multirow{2}{*}{ Group $\operatorname{Tr} i_{E}$} & LDA & 87.8 & 91.8 & 75 & 0.88 & 0.91 \\
\hline & SVM & 91.8 & 92.3 & 90 & 0.95 & 0.94 \\
\hline
\end{tabular}




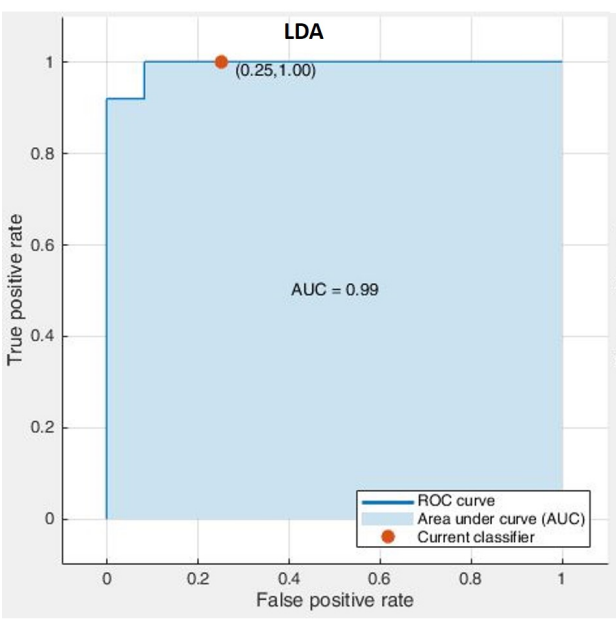

A

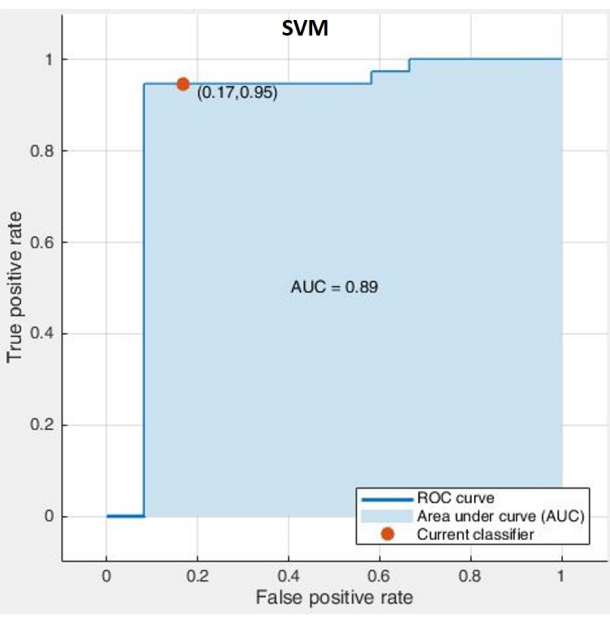

B

Figure 4.47: ROC Curves for Group Tri $i_{F}$ in Table 4.19: (A) LDA, (B) SVM

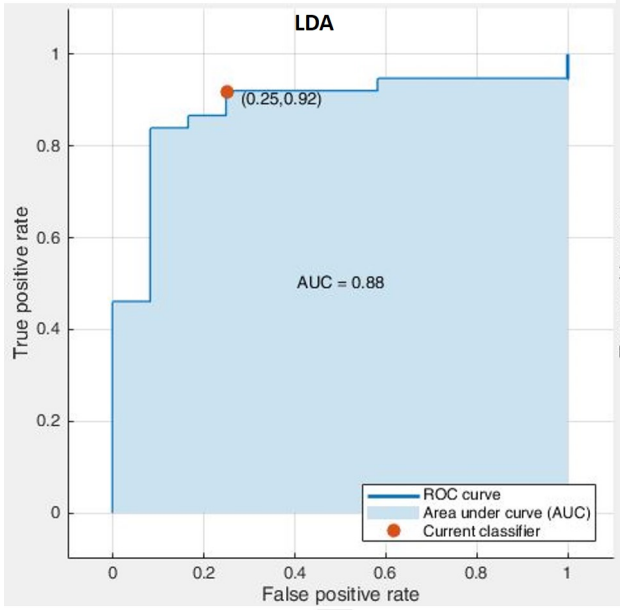

A

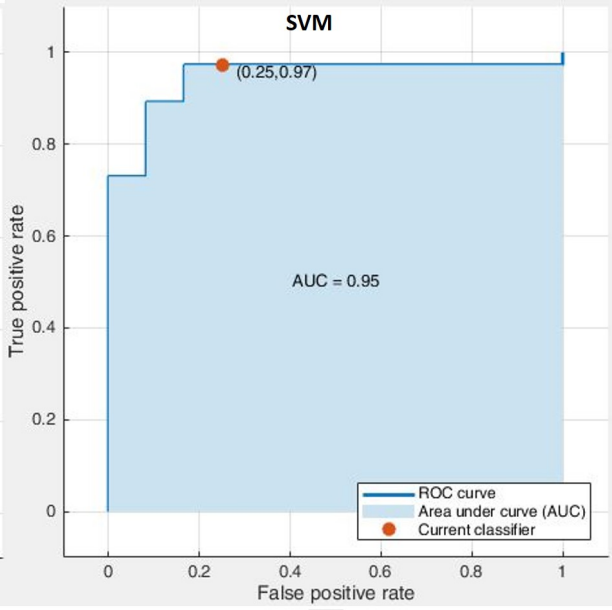

B

Figure 4.48: ROC Curves for Group Tri $i_{E}$ in Table 4.19: (A) LDA, (B) SVM

Following these remarkable results, the next step was to proceed in conducting similar actigraphy analysis experiments with long-duration sleep actigraphy data collected from stroke recovery patients in Database $\mathbf{C}$, as described in the next Section. 


\subsection{Sleep Actigraphy Analysis for Stroke Subjects using Database C}

In a most recent sleep actigraphy studies conducted with Sunnybrook Health Sciences Center, single-axial, long-duration sleep actigraphy data was obtained from 45 test subjects undergoing stroke recovery. As described in Section 4.1, a prior review of studies have indicated a potential link between PLMs and cardiovascular events in sleep [63-65,73,74,120,121]. By definition, a stroke event happens when blood stops flowing to any part of the human brain, thereby leading to an imbalance in heart rhythm and blood flow to the brain $[24,63,153]$. Depending on which region the stroke impacts the brain, strokes can be categorized into 3 types:

- Ischemic Stroke: This is caused by a blockage or blood clot in a specific region of the brain. In most cases, the blockage is caused due to formation of plaque in the artery wall. Figure 4.49 illustrates an ischemic stroke.

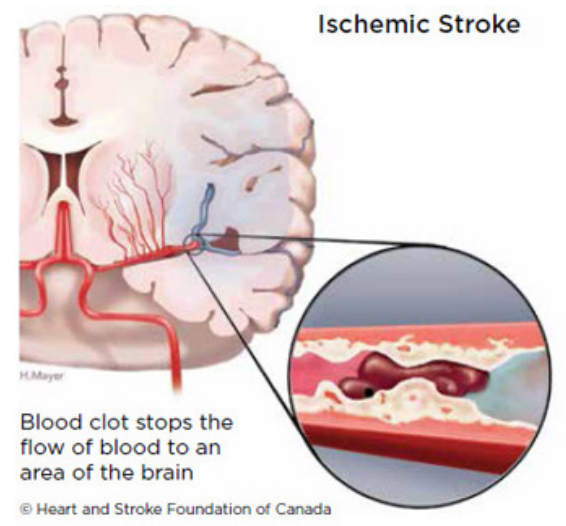

Figure 4.49: Ischemic Stroke [24]

- Hemorrhagic Stroke: A hemorrhagic stroke is detected when an artery bursts open in the brain leading to major interruption in the blood flow. The presence of a persistent high blood pressure could result in the weakening of the arteries thereby leading to their breaking and causing this type of stroke (see Figure 4.50) 


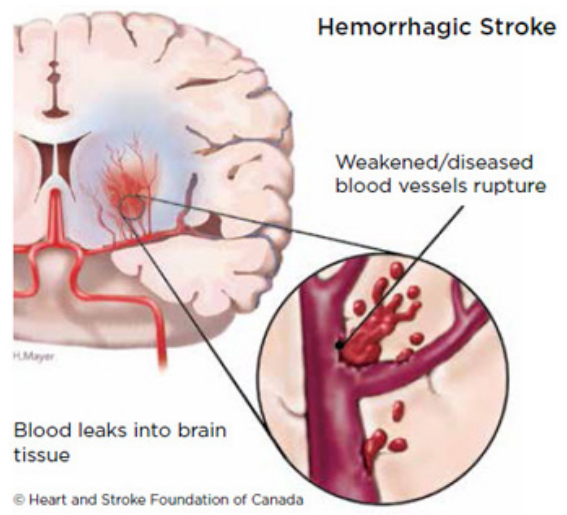

Figure 4.50: Hemorrhagic Stroke [24]

- Transient Ischemic Attack (TIA): A TIA is usually interpreted as a small stroke or a warning sign of an impeding major stroke, caused due to small blockages in the artery. Symptoms of such an event usually last for few minutes and may subside within an hour. Figure 4.51 depicts a TIA.

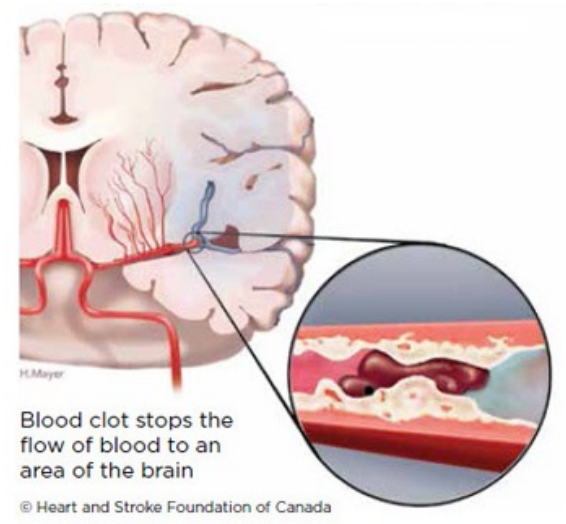

Figure 4.51: Transient Ischemic Attack [24]

A recent statistical survey indicates that stroke affects about 62,000 individuals annually in Canada, with about $80 \%$ survival rate [24]. Of these only $16 \%$ patients transfer to rehabilitation right away after getting acute hospital care, and only $19 \%$ within first month of leaving the hospital. Age is a major risk factor for stroke, and about $80 \%$ of individuals experiencing it are over 60 years [24]. Recent clinical studies have shown that many individuals experiencing neuromuscular disorders in sleep such as PLMs, also suffer from stroke or stroke-like symptoms [63,64]. As stated in Section 4.1.1, there has been a debate in connecting the lack of dopamine levels related to PLMs and the occurrence of stroke in sleep. In simple words, many clinical experts perceive that individuals experiencing stroke in sleep may also show signs of severe PLMs (PLM Index $\geq 15$ movements/hour). 
In this study, long-duration sleep actigraphy data was collected from individuals undergoing stroke recovery. The purpose of this study was to analyze actigraphy data to determine limb movement and stroke severity based on the Spiky Index, the Rapid Change Factor and the oxygen saturation (O2Sat) level of each patient. It should be noted that the O2Sat parameter is an indicator of blood oxygen levels. A blood oxygen level above 88 millimeters of mercury $(\mathrm{mm} \mathrm{Hg})$ is considered to be normal. A value under $60 \mathrm{~mm} \mathrm{Hg}$ indicates the need for supplemental oxygen $[24,64,153]$. Many clinical studies have pointed out that ensuring a high blood oxygen level in patients suffering from acute stroke, ensures faster recovery [63,64]. The following sections will describe the experiments conducted on sleep actigraphy signals from stroke recovery patients in this study.

\subsubsection{Materials and Methods}

\section{Data Acquisition}

In this study single-axial, bilateral, long-duration (about 6-8 hours) of sleep actigraphy signals simultaneously recorded with PSG, were obtained from 45 consenting patients using the Actical ${ }^{\text {TM }}$ device [17]. This yielded a total of 90 actigraphy signals in this study (2 per subject). Note that in this study, the same Actical ${ }^{\text {TM }}$ device, which was used in the preliminary experiments described in Section 4.2, was applied again to stroke recovery subjects for movement recordings. The actigraphy signals were recorded at an effective sampling rate of $16 \mathrm{~Hz}$ with an 8-bit resolution. Following Table 4.20 highlights the demographics of the study population.

Table 4.20: Demographics - Single Axial Actical ${ }^{\mathrm{TM}}$ Data, Database C

\begin{tabular}{lc}
\hline \multicolumn{1}{c}{ Parameter } & All Subjects, $\mathbf{N}=\mathbf{4 5}$ \\
\hline Age, mean $\pm s d$ & $65.5 \pm 12.8$ \\
Gender, M:F & $28: 17$ \\
Sleep Efficiency, mean $\pm s d$ & $82.2 \pm 11$ \\
Total Sleep Time, mean $\pm s d$ & $550.4 \pm 212.1$ \\
BMI, mean $\pm s d$ & $29.6 \pm 8.4$ \\
AHI, mean $\pm s d$ & $10.7 \pm 12.3$ \\
O2Sat, mean $\pm s d$ & $81.8 \pm 7.8$ \\
\hline AHI, apneahyopnea index; BMI, body mass index; O2Sat, oxygen satu- \\
ration
\end{tabular}




\section{$\underline{\text { Actigraphy Pre-processing }}$}

Similar to the data grouping in Section 4.3.1, the single axial data in this study was divided in two groups, namely Group $S t r_{R}$ and Group $S t r_{E}$.

- Group $\operatorname{Str}_{R}$ comprised of 90 raw actigraphy signals which were used as is, without any additional conditioning. It should be noted that the 8-bit quantized, single-axial actigraphy signals generated from Actical ${ }^{\mathrm{TM}}$, were already filtered using its proprietary software [17].

- Group $\operatorname{Str}_{E}$ contained $90 \mathrm{~m}$-bit encoded actigraphy signals. Assuming that $r$ bits/sample is the bit resolution of the raw actigraphy signal $S_{r}$, it was further encoded using $m$ bits/sample such that $m \ll r$, using the floor operation,

$$
S_{E}=\left\lfloor\left(S_{r} \times \frac{\left(2^{m}-1\right)}{2}+\frac{\left(2^{m}-1\right)}{2}\right)\right\rfloor
$$

The encoding operation assigns each sample of $S_{r}$ to a discrete integer value from one of the $\left(2^{m}-1\right)$ steps.

\section{$\underline{\text { Encoding-based segmentation }}$}

Using the encoded, single axial actigraphy data in Group $S t r_{E}$, the Athavale-Krishnan and the Mobilitybased segmentation schemes were applied to each signal, in order to determine regions of high limb movements in sleep. Since this study involved deducing a correlation between stroke severity and PLMs in sleep, the segmentation schemes described in Section 4.3.1 were applied again as follows:

First the Athavale-Krishnan algorithm was applied to segment an encoded signal $S_{E}$ (obtained from Eq.4.16 using the following steps. As shown in Eq.4.16, after computing the encoded actigraphy signal $S_{E}$, the first order and the subsequent second order difference signals were found as,

$$
d S_{e}=\left[S_{e_{1}},\left(S_{e_{2}}-S_{e_{1}}\right), \ldots,\left(S_{e_{n}}-S_{e_{n-1}}\right)\right]
$$

and,

$$
2 d S_{e}=\left[d S_{e_{1}},\left(d S_{e_{2}}-d S_{e_{1}}\right), \ldots,\left(d S_{e_{n}}-d S_{e_{n-1}}\right)\right]
$$

The rapid change factor $(\mathrm{RCF})$ of the signal using its second order difference version was calculated as,

$$
R C F=\frac{\text { Step }- \text { Size }}{\left(m \times t_{s}\right)}
$$


where $t_{s}$ is the sampling period. Next, the signal segments were determined using the following ground truth information explained in Section 4.1.1:

1. Valid limb movements occur within a 5-90 second window

2. Each movement lasts for about 0.5 to 10 seconds

3. A group of 4 or more consecutive limb movements within 90 seconds is a true sequence.

The primary segment boundaries (PSBs) were defined by finding all the signal samples in $2 d S_{E}$ which were greater than the RCF,

$$
P S B_{i}=2 d S_{E_{n}} \forall\left\langle 2 d S_{E_{n}}>R C F\right\rangle
$$

where $i$ is the number of PSBs. Next, using the afore stated ground truth on limb movements in sleep, it could be inferred that two sequences (of 4 or more movements) can be separated by at least 90 seconds. Using this information, the secondary segment boundaries (SSBs) were found such that,

$$
S S B_{j}=P S B_{i} \forall\left\langle\left|t_{P S B_{i}}-t_{P S B_{i+1}}\right|>90\right\rangle
$$

where $j$ is the number of SSBs. Finally, the final segment boundaries (FSBs) were identified by detecting only those samples, which contained 4 or more consecutive leg movements, and whose amplitude was at least half of the maximum peak found between two SSBs,

$$
F S B_{l}=S S B_{j} \forall\left\langle\operatorname{Count}\left(2 d S_{E_{j}}: 2 d S_{E_{j+1}}>\frac{\max _{n} 2 d S_{E_{n}}}{2}\right)>4\right\rangle
$$

where $l$ is the number of FSBs. Using the FSBs, the regions of high limb movements from the entire actigraphy signal could be then extracted for estimating PLM severity in the stroke recovery participant.

The next experiment was to apply the mobility-based segmentation scheme on stroke actigraphy data through the following steps. Using Eqs.4.17 and 4.18 the second order difference signal of the encoded data $S_{E}$ was computed. The PSBs were found by detecting all the samples in $2 d S_{E}$ which were greater than a factor of $\frac{\max _{n} 2 d S_{E}}{\left(2^{m}-1\right)}$, such that,

$$
P S B_{i}=2 d S_{E_{n}} \forall\left\langle 2 d S_{E_{n}}>\frac{\max _{n} 2 d S_{E}}{\left(2^{m}-1\right)}\right\rangle
$$

Using these PSBs, the SSBs were identified such that the Hjorth's mobility values of two segments were unique from each other.

$$
S S B_{j}=P S B_{i} \forall\left\langle\left\lfloor M_{P S B_{i: i+1}}\right\rfloor \neq\left\lfloor M_{P S B_{i+1: i+2}}\right\rfloor\right\rangle
$$


Using the ground truth information on limb movements in sleep, the FSBs were computed such that two SSBs were at least 90 seconds apart.

$$
F S B_{l}=S S B_{j} \forall\left\langle\left|t_{S S B_{j}}-t_{S S B_{j+1}}\right|>90\right\rangle
$$

The results of these segmentation schemes on single-axial stroke actigraphy data have been illustrated in Section 4.4.2.

\section{Feature Extraction and Classification}

Similar to the feature extraction methodology described in Section 4.3.1, the following steps have been performed on actigraphy signals in Groups $\operatorname{Str}_{R}$ and $\operatorname{Str}_{E}$ :

- Using the O2Sat levels of each patient, the respective signal data in Groups $\operatorname{Str}_{R}$ and $\operatorname{Str}_{E}$ was segregated into two groups as - Normal if O2Sat $\geq 88 \mathrm{~mm} \mathrm{Hg}$, and Abnormal if O2Sat $<88 \mathrm{~mm} \mathrm{Hg}$.

- Following this, 22 time, frequency, kernel-based, actigraphy-specific and morphological features were extracted from each actigraphy signal in Groups $S t r_{R}$ and $S t r_{E}$, as shown in Table 4.21.

- The feature-set generated from signals in Groups $S t r_{R}$ and $S \operatorname{tr}_{E}$ was then split into 70-30 trainingtesting ratio, and fed to three different machine learning tools - a Naive-Bayes classifier, a LDA and a SVM tool $[150,151]$, to classify signals based on stroke severity and number of limb movements in sleep. It should be noted that in these experiments, two different combinations of feature sets were considered for determining the classification accuracy of the kernel-based, actigraphy-specific and morphological features. 
Table 4.21: Features extracted from Groups $S t r_{R}$ and $S t r_{E}$ actigraphy signals

\begin{tabular}{|c|c|c|}
\hline Domain & No. & Features Used \\
\hline \multirow{11}{*}{ Time } & 1 & Mean \\
\hline & 2 & Standard Deviation \\
\hline & 3 & Variance \\
\hline & 4 & RMS \\
\hline & 5 & Maxima \\
\hline & 6 & Peak-to-peak difference \\
\hline & 7 & Peak-to-RMS ratio \\
\hline & 8 & Peak-to-average ratio \\
\hline & 9 & Peak-to-average power ratio \\
\hline & 10 & Band power \\
\hline & 11 & Signal to noise \& distortion ratio \\
\hline \multirow{2}{*}{ Frequency } & 12 & Median Frequency \\
\hline & 13 & Mean Frequency \\
\hline Kernel-based Features & $14-17$ & ACF Kernel Scores (4 Features) \\
\hline \multirow{2}{*}{ Actigraphy-specific } & 18 & Rapid Change Factor \\
\hline & 19 & Spiky Index \\
\hline \multirow{3}{*}{ Morphological } & 20 & Hjorth's First order mobility \\
\hline & 21 & Hjorth's Second order mobility \\
\hline & 22 & Hjorth's complexity \\
\hline Clinical Labels & 23 & Oxygen Saturation (O2Sat) \\
\hline
\end{tabular}

Following these steps, the next section highlights the results from the experiments on stroke actigraphy.

\subsubsection{Results from Experiments on Database C}

\section{Signal Encoding Results}

As described in Section 4.4.1, two distinct signal groups were created from the single-axial actigraphy signals in Database C, namely - Group $S t r_{R}$ and Group $S t r_{E}$. While the Group $S t r_{R}$ comprised of 90 raw actigraphy signals which were already filtered using Actical ${ }^{\mathrm{TM}}$ 's proprietary software, the Group $\operatorname{Str}_{E}$ 's 90 signals were further encoded using a 3-bit resolution. Following Figure 4.52 highlights a sample, raw, single-axial sleep actigraphy signal captured from the left leg of a stroke recovery subject, along with its encoded version. 

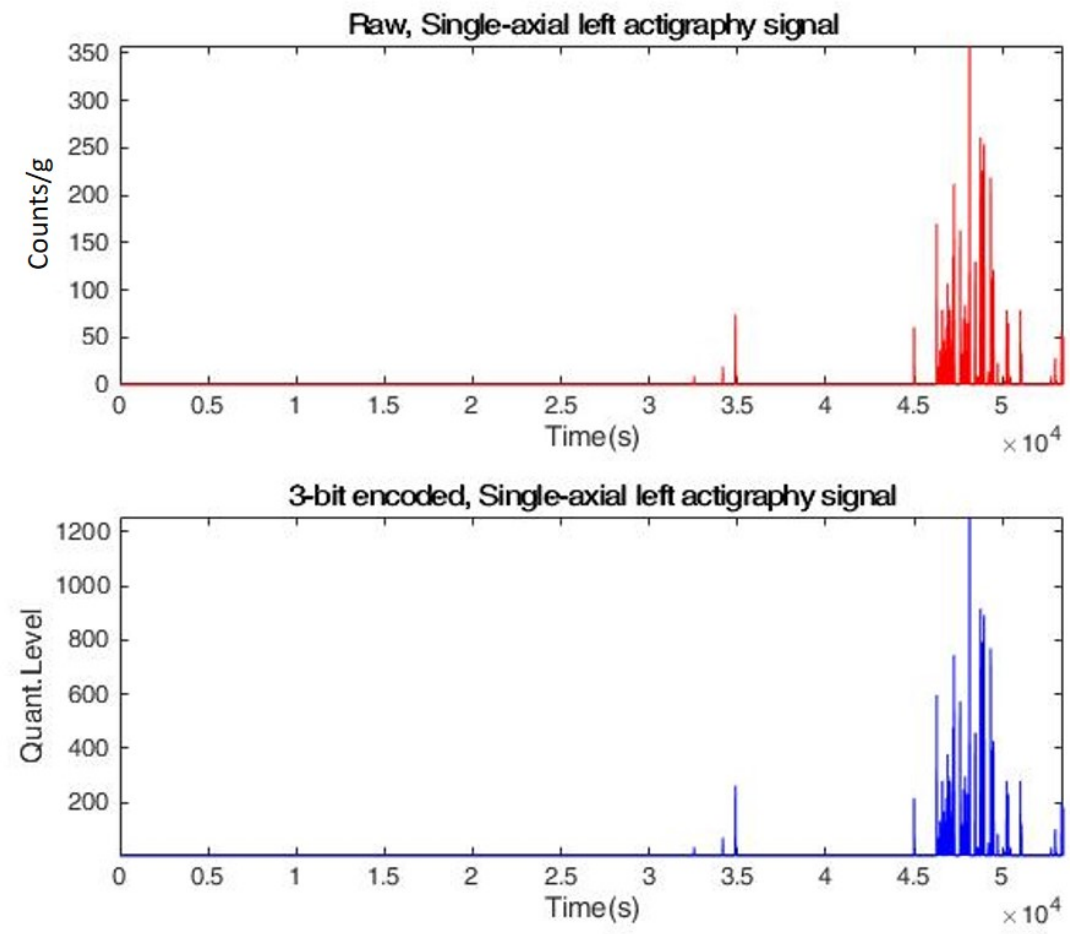

Figure 4.52: Raw and Encoded sleep actigraphy signal from stroke recovery subject

To compare the efficacy of the proposed encoding scheme with respect to raw data, a parametric comparison has been shown in Table 4.22 .

Table 4.22: Parametric comparison between raw and encoded stroke actigraphy signals

\begin{tabular}{lcc}
\hline \multicolumn{1}{c}{ Parameter } & Raw Signal & Encoded Signal \\
\hline Avg. File Size (Mb) & 3.07 & 2.05 \\
Bit Rate (bits/sec) & 128 & 48 \\
Avg. File Read Time (secs) & 0.8 & 0.5 \\
Avg. SNR (dB) & -20 & 38.82 \\
\% Space Savings & NA & $34 \%$ \\
\hline
\end{tabular}




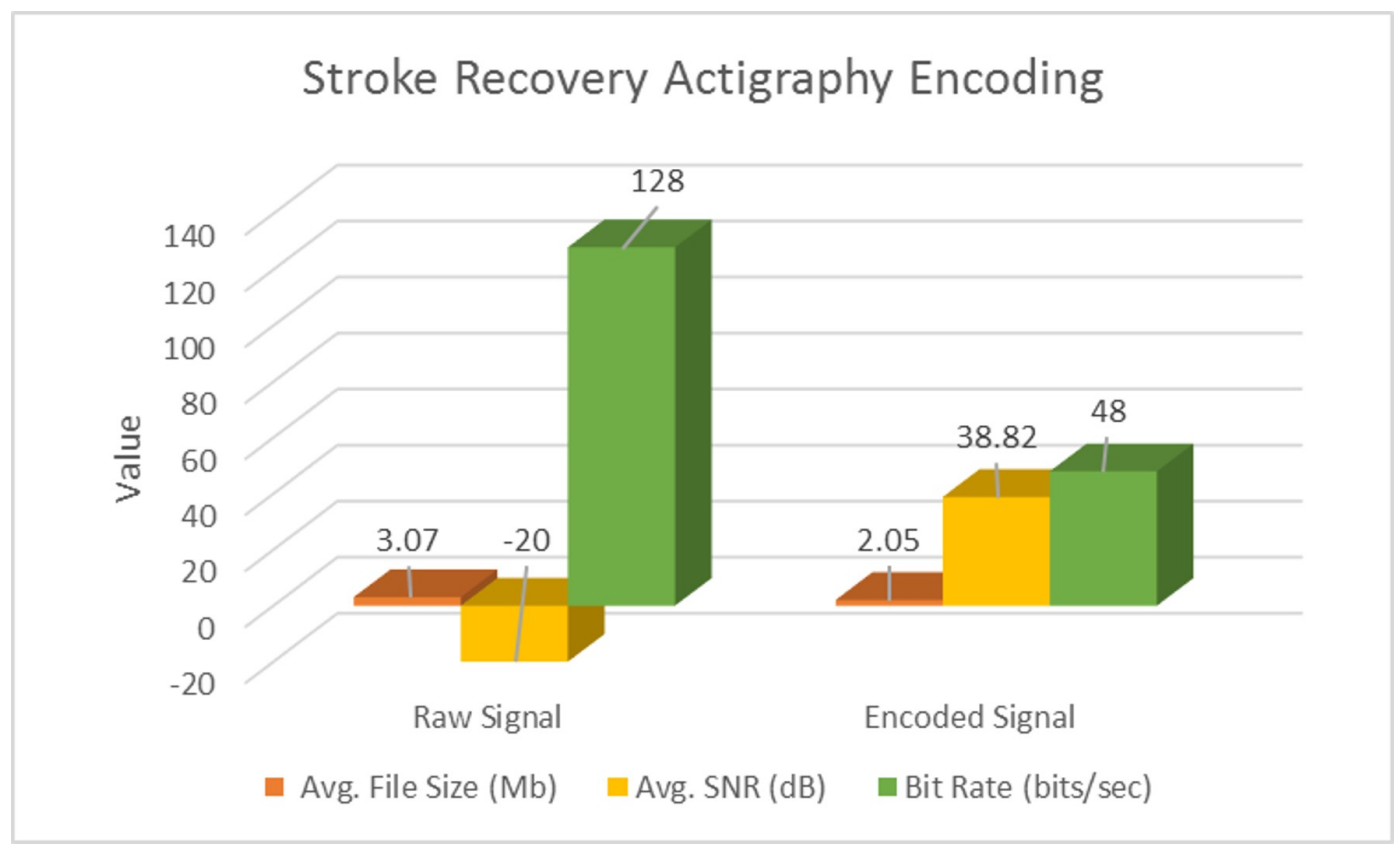

Figure 4.53: 3-bit encoding of stroke recovery actigraphy

As evident from Table 4.22 and Figure 4.53, the 3-bit encoding of actigraphy signals from stroke recovery subjects, ensured a considerable increase in SNR coupled with about $30 \%$ space savings, and a lowered bit rate. These results are similar to the ones exhibited in Table 4.8. Following the encoding-based preprocessing of the single-axial actigraphy signals, the next Section will highlight the results from applying the AK and mobility-based segmentation methods to the encoded actigraphy signals, to identify regions of peak limb activity in sleep, from a stroke patient.

\section{Encoding-based Segmentation Results}

In order to detect regions of high limb movements in sleep from actigraphy data in stroke recovery subjects, the AK and Mobility-based segmentation schemes were applied as described in Section 4.4.1. Upon application, it was found that in comparison to a fixed segmentation method which splits the actigraphy signal into frames of 1000 second duration, the AK and mobility-based methods were able to segment the actigraphy signals more effectively using smaller number of frames. Following Figures 4.54-4.57 highlight these results for a sample left and right actigraphy signal taken from a stroke recovery patient. 

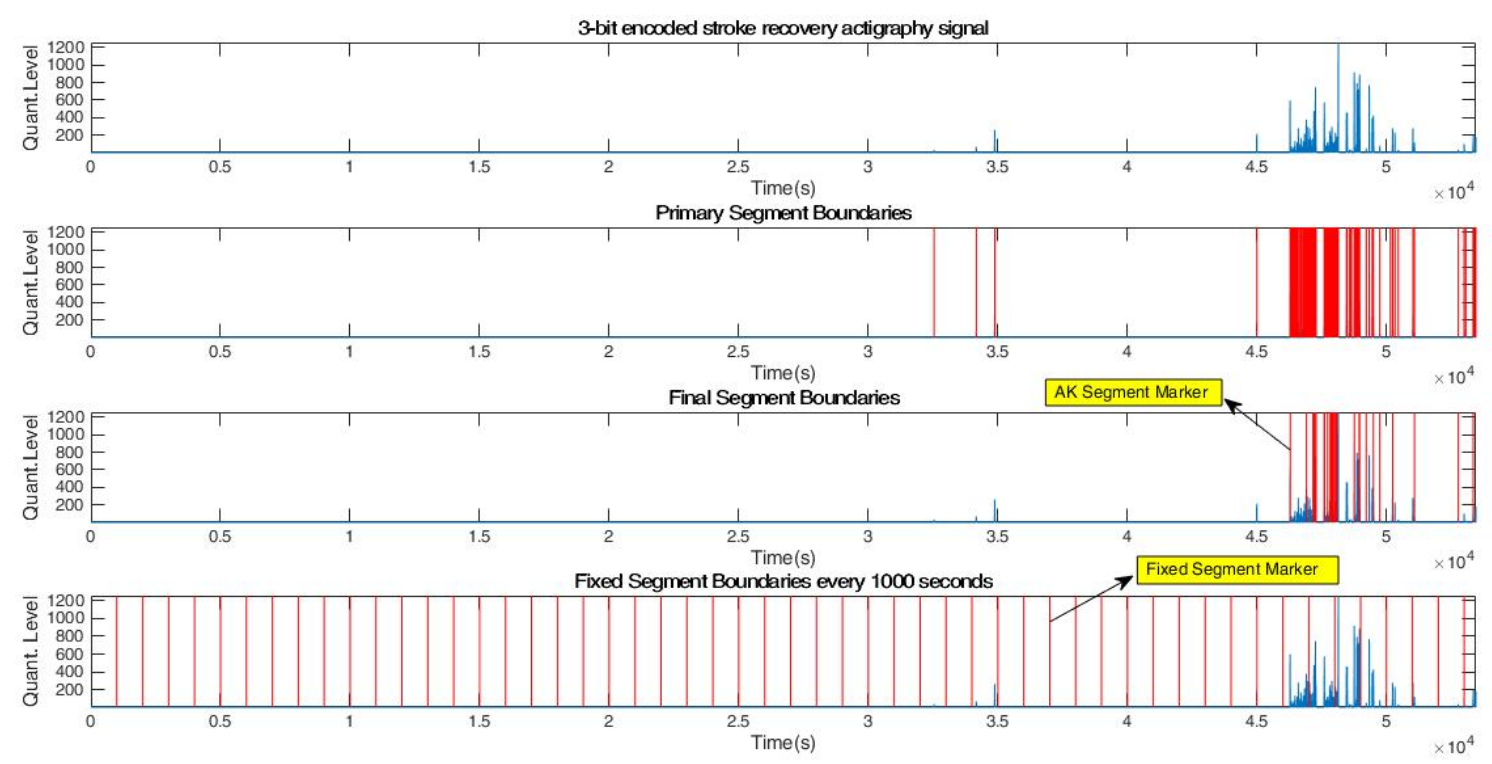

Figure 4.54: AK Segmentation of Left Leg Stroke Actigraphy
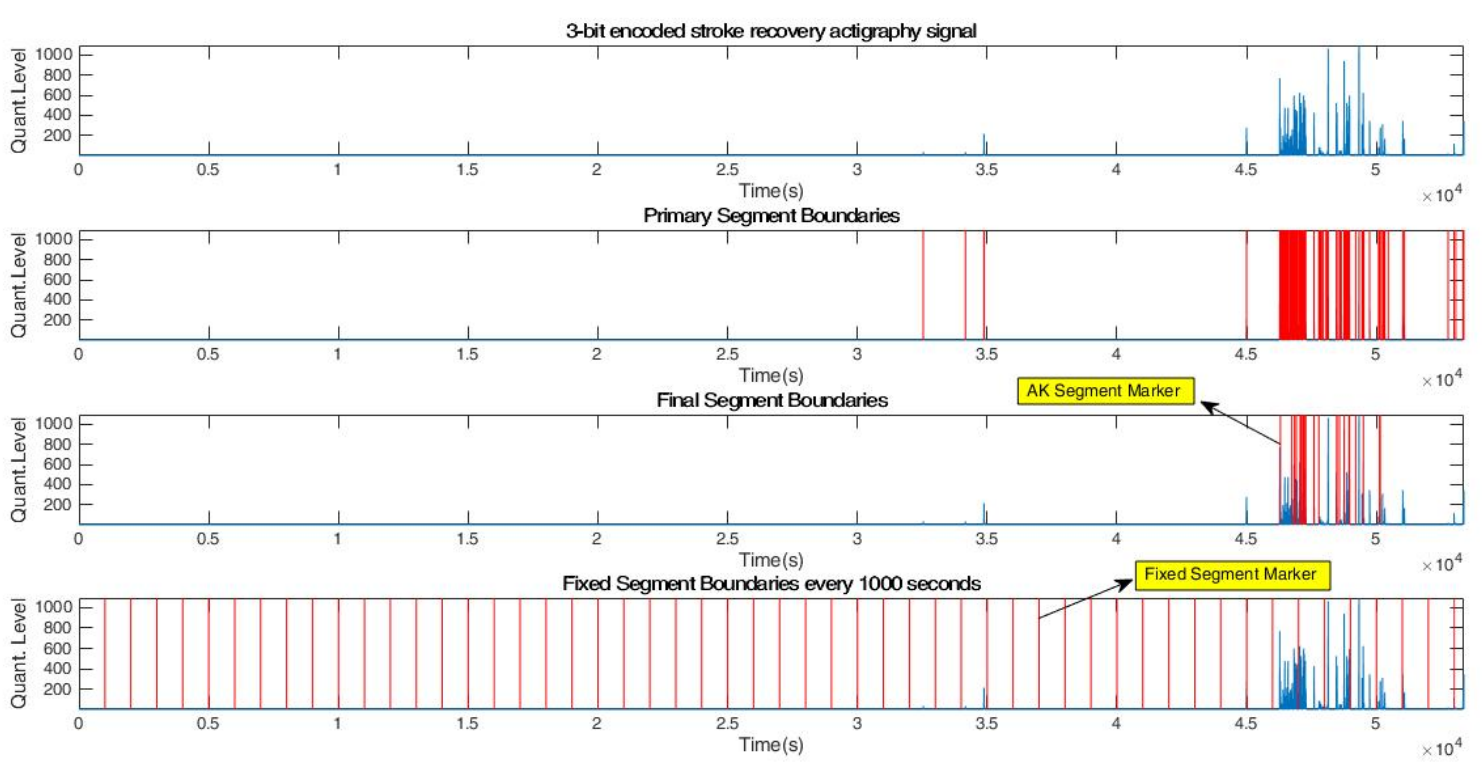

Figure 4.55: AK Segmentation of Right Leg Stroke Actigraphy 

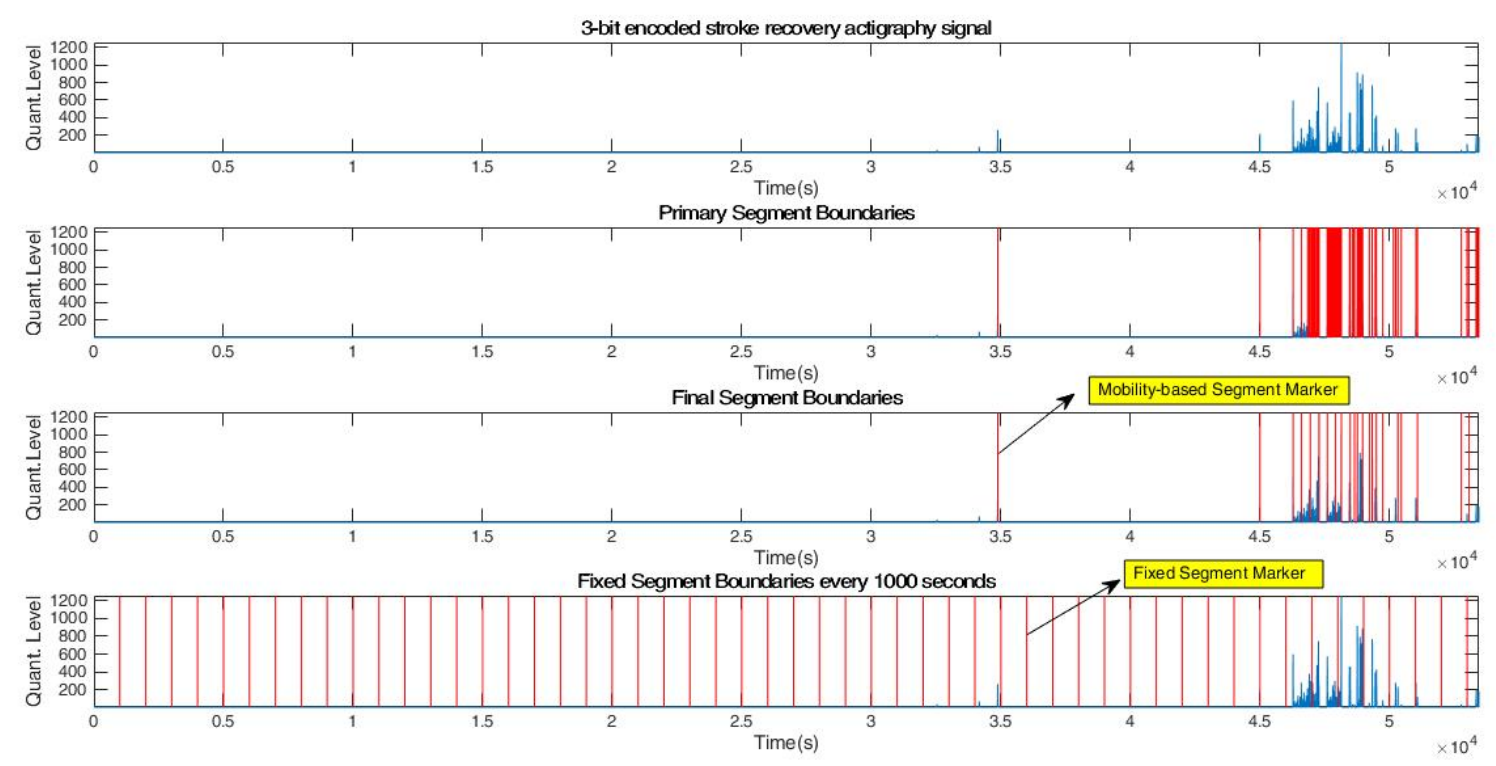

Figure 4.56: Mobility-based Segmentation of Left Leg Stroke Actigraphy
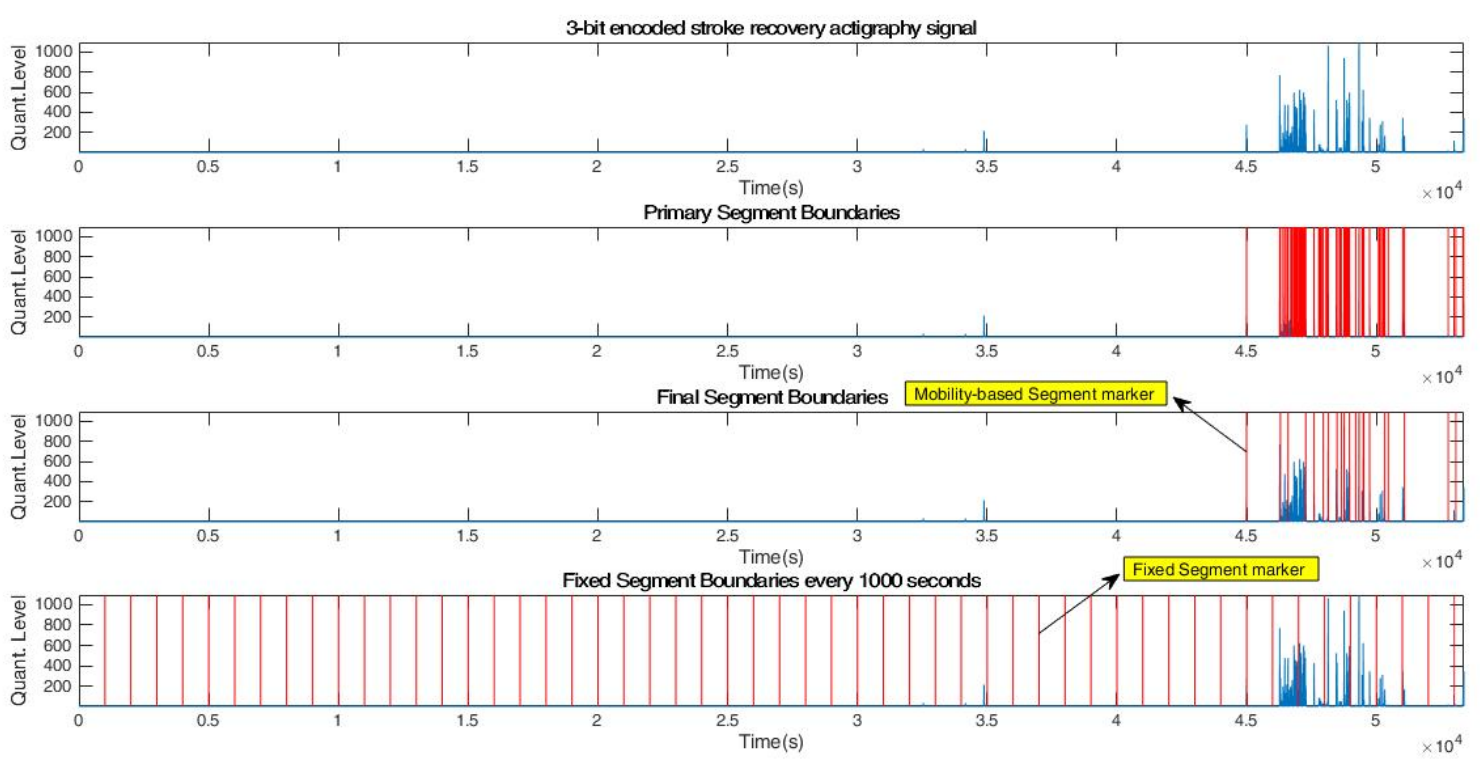

Figure 4.57: Mobility-based Segmentation of Right Leg Stroke Actigraphy 
Table 4.23: Fixed, AK and Mobility-based segments for sample actigraphy

\begin{tabular}{cccc}
\hline Signal & No. Fixed Segments & No. AK Segments & No. Mobility-based Segments \\
\hline Left ankle actigraphy & 54 & 28 & 29 \\
Right ankle actigraphy & 54 & 23 & 29 \\
\hline
\end{tabular}

As evident from Table 4.23, the AK segmentation technique outperforms the mobility-based as well as the fixed segmentation scheme, by ensuring that peak limb movements are captured using a smaller number of signal frames. This further indicates that while analyzing long-duration actigraphy signals, the AK segmentation method allows for a smaller portion of the signal to be analyzed, thereby reducing computational overheads. The next Section 4.4.2 will discuss the feature extraction and classification results of Groups $\operatorname{Str}_{R}$ and $\operatorname{Str}_{E}$ signals into normal and abnormal classes, as described in Section 4.4.1.

\section{$\underline{\text { Feature Extraction and Classification Results }}$}

As stated in Section 4.4.1, after extracting 22 time, frequency, kernel-based, actigraphy-specific and morphological features, three different sets were created using a combination of these features. These were further split into a 70-30 training-testing partition, for evaluating a machine learning tool, and generating classification results based on oxygen saturation (O2Sat) levels into normal and abnormal categories. Following Tables 4.24-4.27 exhibit the classification results based on a specific combination of features.

Table 4.24: Classification of Stroke subjects using 22 features

\begin{tabular}{|c|c|c|c|c|c|c|}
\hline \multicolumn{7}{|c|}{$\begin{array}{l}\text { Features - Time, Frequency, Kernel-b } \\
23 \text { Features } \\
\text { Label - Oxygen Saturation (O2Sat) }\end{array}$} \\
\hline Group & Classifier & Accuracy (\%) & Sensitivity (\%) & Specificity (\%) & AUC & F1-Score \\
\hline \multirow{3}{*}{ Group $S t r_{R}$} & Naïve-Bayes & 57.1 & 45.4 & 100 & 0.86 & 0.62 \\
\hline & LDA & 64.3 & 50 & 66.6 & 0.36 & 0.28 \\
\hline & SVM & 74.1 & 73.9 & 75 & 0.61 & 0.92 \\
\hline \multirow{3}{*}{ Group $S t r_{E}$} & Naïve-Bayes & 46.4 & 39.1 & 80 & 0.71 & 0.54 \\
\hline & LDA & 77.8 & 76.1 & 83.3 & 0.64 & 0.84 \\
\hline & SVM & 66.7 & 70 & 57.1 & 0.69 & 0.75 \\
\hline
\end{tabular}




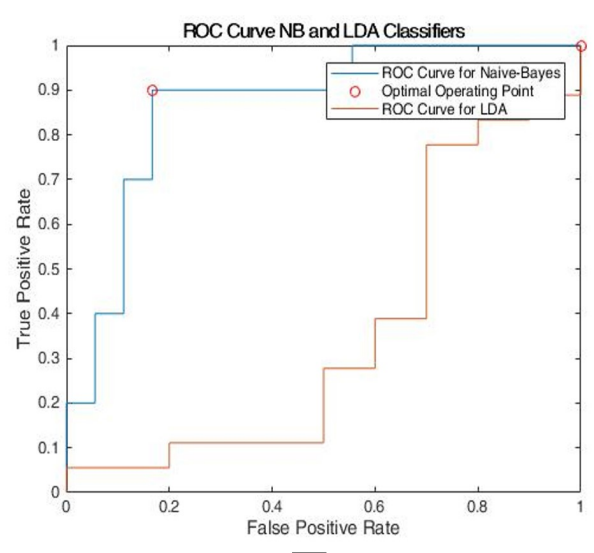

A

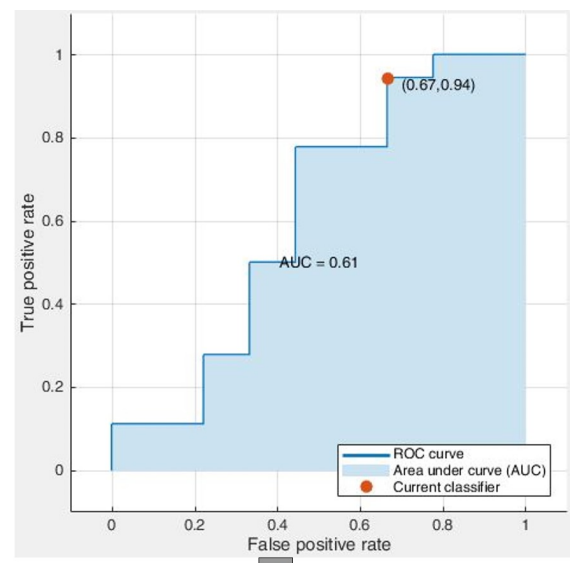

B

Figure 4.58: ROC Curves for Group $\operatorname{Str}_{R}$ in Table 4.24: (A) Naive-Bayes \& LDA, (B) SVM

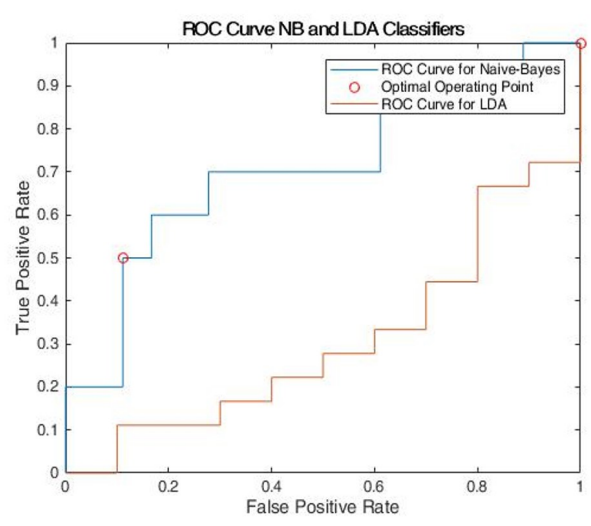

A

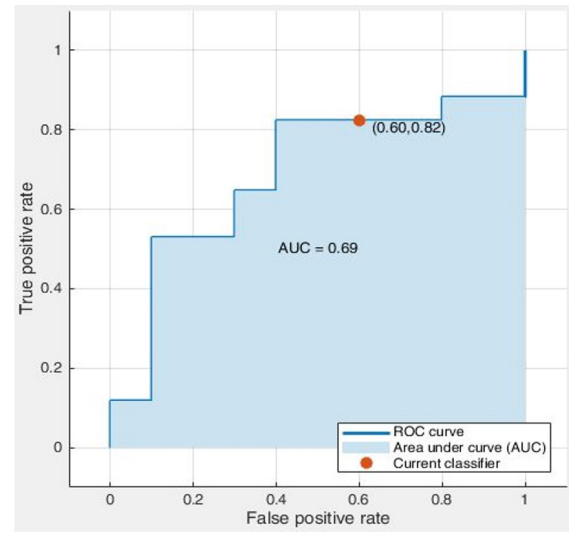

B

Figure 4.59: ROC Curves for Group $S t r_{E}$ in Table 4.24: (A) Naive-Bayes \& LDA, (B) SVM

In addition to these experiments, a mRMR feature selection [152] algorithm was executed to generate the top five discriminatory features which were - the Spiky Index, the Hjorth's complexity, the Mean Frequency, rapid change factor and Hjorth's mobility. Using these, a scatter plot was generated as shown in Figure 4.60, which indicated that these features gave the maximum discrimination between normal and abnormal stroke subjects. This also inferred that the Spiky Index was a key parameter in discriminating between PLM events, aside from Periodicity Index. Additionally, it was also found that the classification accuracy using only these five features was higher by about $4-22 \%$ as compared to results cited in Table 4.24. Table 4.25 highlights the results on mRMR classification in stroke actigraphy. 


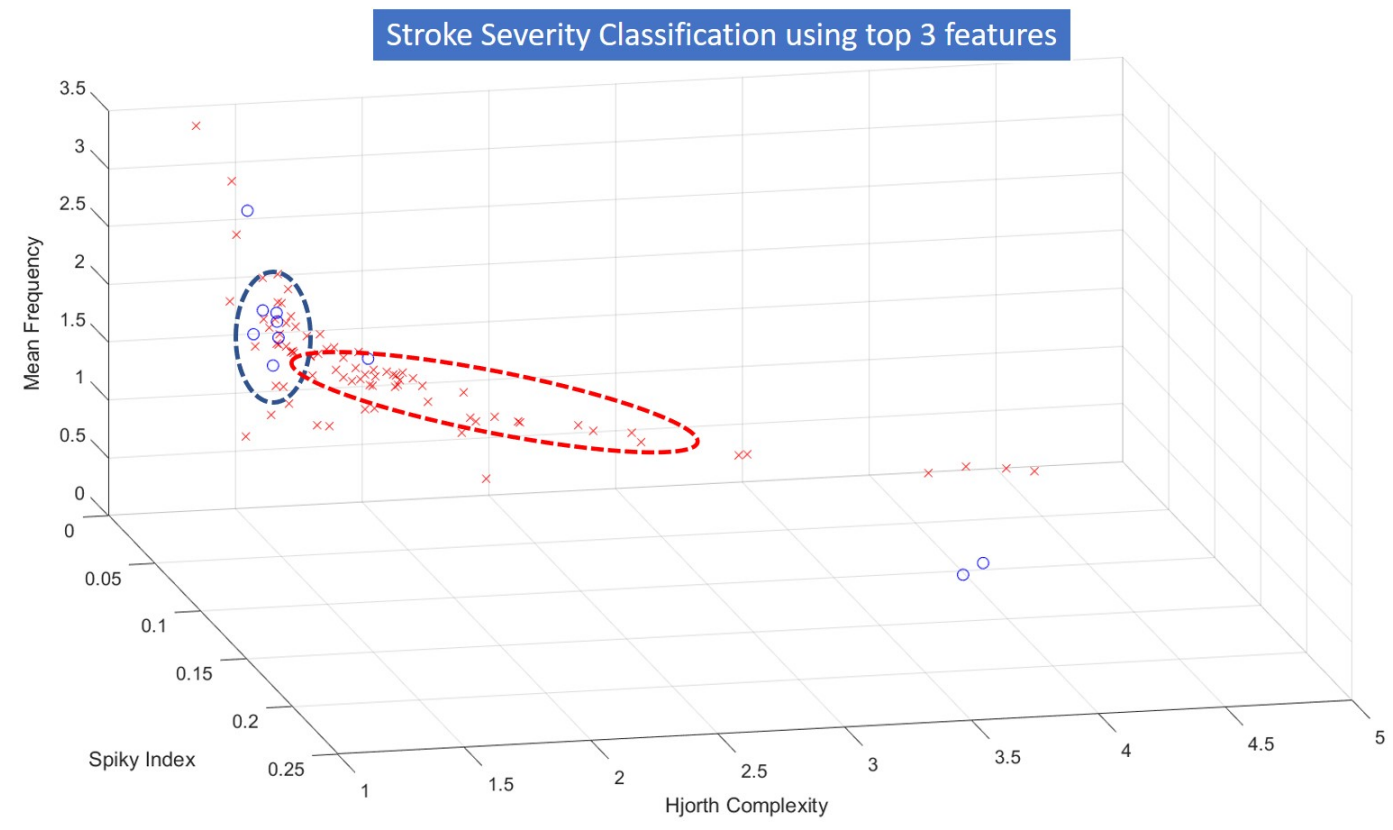

Figure 4.60: Top 3 Features Classifying Stroke Subjects

Table 4.25: Classification of Stroke Subjects using top 5 mRMR features for encoded signals

Features - Time, Frequency, Kernel-based, Actigraphy-specific, Morphological -

5 Features

Label - O2Sat

\begin{tabular}{clccccc}
\hline Group & Classifier & Accuracy (\%) & Sensitivity (\%) & Specificity (\%) & AUC & F1-Score \\
\hline \multirow{2}{*}{ Group Tri $_{E}$} & Naïve-Bayes & 88.8 & 50 & 92 & 0.12 & 0.56 \\
& LDA & 82.5 & 16.6 & 89.4 & 0.17 & 0.57 \\
& SVM & 88.9 & 51.7 & 89 & 0.52 & 0.68 \\
\hline
\end{tabular}


Table 4.26: Classification of Stroke subjects using 15 features

\begin{tabular}{|c|c|c|c|c|c|c|}
\hline Group & Classifier & Accuracy (\%) & Sensitivity (\%) & Specificity (\%) & AUC & F1-Score \\
\hline \multirow{3}{*}{ Group $S t r_{R}$} & Naïve-Bayes & 64.2 & 50 & 68.1 & 0.74 & 0.37 \\
\hline & LDA & 67.8 & 57.1 & 71.4 & 0.38 & 0.47 \\
\hline & SVM & 74.1 & 81.2 & 63.6 & 0.79 & 0.78 \\
\hline \multirow{3}{*}{ Group $S t r_{E}$} & Naïve-Bayes & 42.8 & 37.5 & 85.7 & 0.65 & 0.53 \\
\hline & LDA & 75 & 80 & 73.9 & 0.25 & 0.76 \\
\hline & SVM & 70.4 & 68 & 100 & 0.74 & 0.81 \\
\hline
\end{tabular}
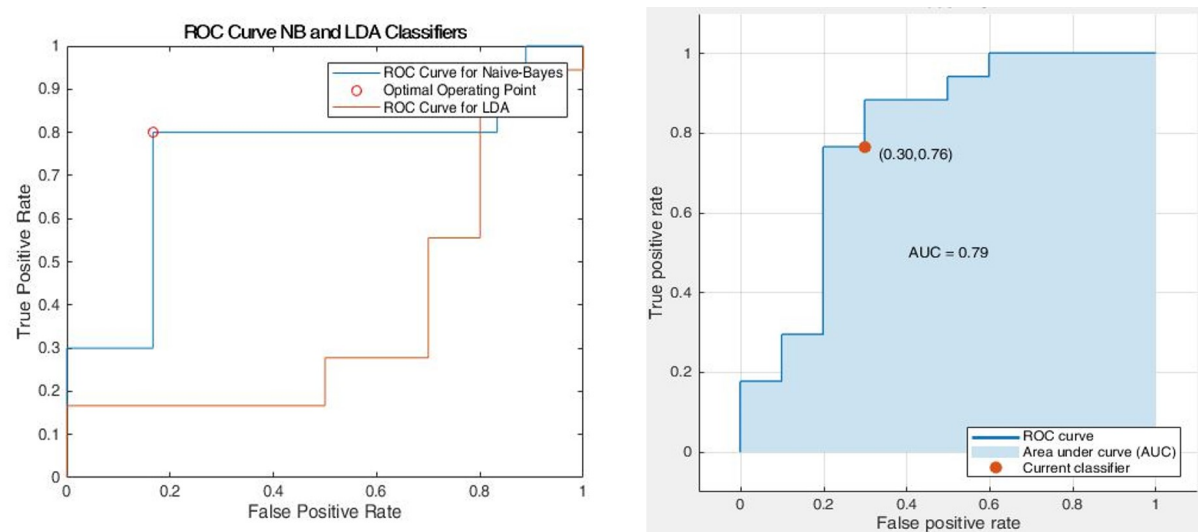

A

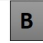

Figure 4.61: ROC Curves for Group $S t r_{R}$ in Table 4.26: (A) Naive-Bayes \& LDA, (B) SVM
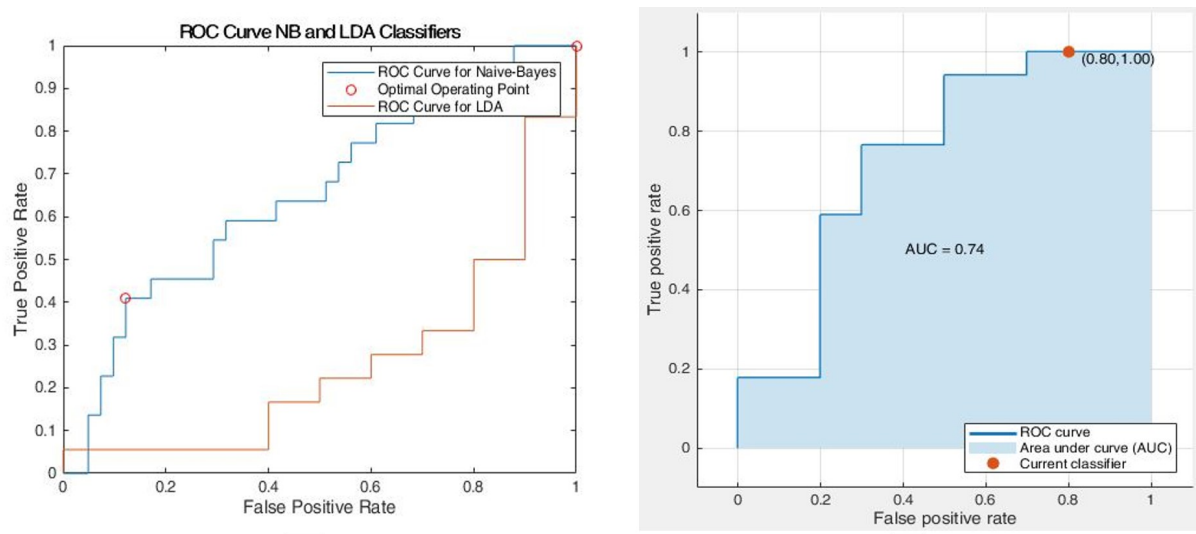

A

Figure 4.62: ROC Curves for Group $\operatorname{Str}_{E}$ in Table 4.26: (A) Naive-Bayes \& LDA, (B) SVM 
Table 4.27: Classification of Stroke subjects using 9 features

\begin{tabular}{|c|c|c|c|c|c|c|}
\hline Group & Classifier & Accuracy (\%) & Sensitivity (\%) & Specificity (\%) & AUC & F1-Score \\
\hline \multirow{3}{*}{ Group $S t r_{R}$} & Naïve-Bayes & 63.5 & 46.1 & 68 & 0.58 & 0.34 \\
\hline & LDA & 60.7 & 40 & 65.2 & 0.53 & 0.26 \\
\hline & SVM & 63 & 64 & 50 & 0.54 & 0.76 \\
\hline \multirow{3}{*}{ Group $S t r_{E}$} & Naïve-Bayes & 67.8 & 57.1 & 71.4 & 0.81 & 0.47 \\
\hline & LDA & 66.6 & 52.6 & 72.7 & 0.31 & 0.48 \\
\hline & SVM & 70.4 & 70.8 & 66.6 & 0.67 & 0.8 \\
\hline
\end{tabular}

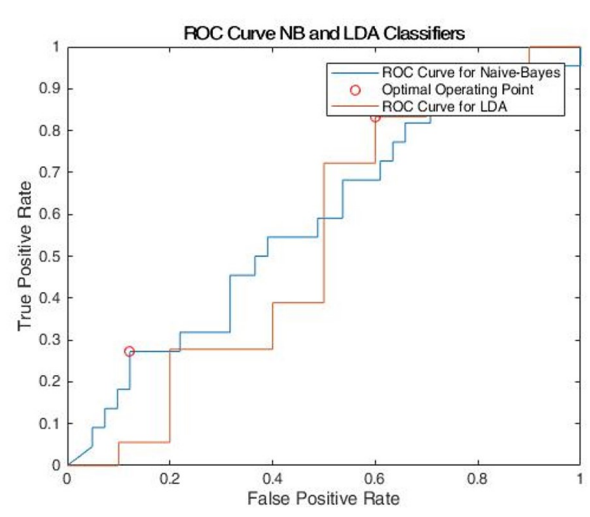

A

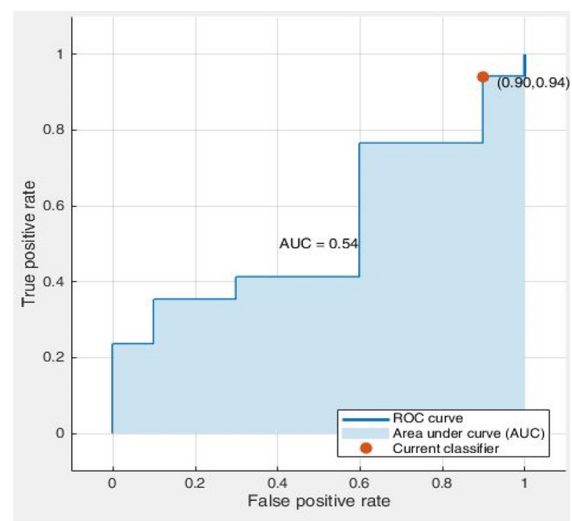

B

Figure 4.63: ROC Curves for Group $S t r_{R}$ in Table 4.27: (A) Naive-Bayes \& LDA, (B) SVM

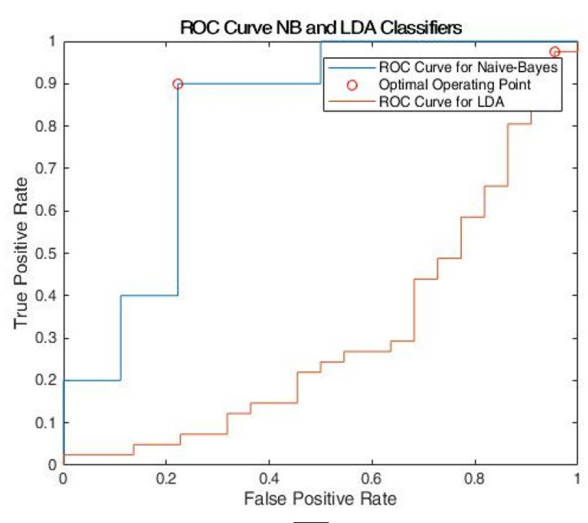

A

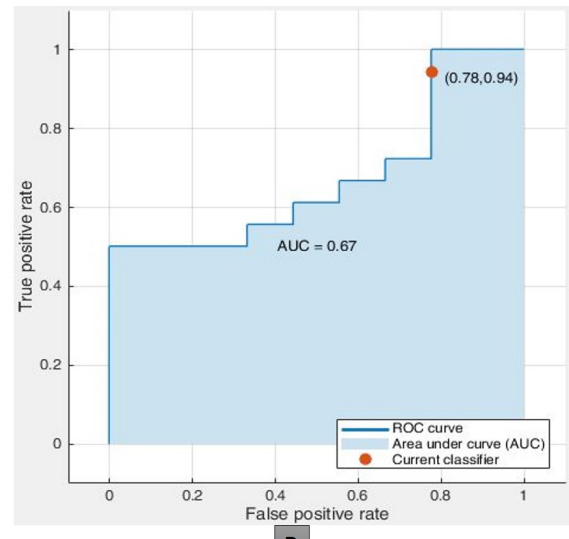

B

Figure 4.64: ROC Curves for Group $S t r_{E}$ in Table 4.27: (A) Naive-Bayes \& LDA, (B) SVM

In addition to these experiments, feature extraction and classification of 5 and 7 bit encoded data was 
also performed, and it was found again that the 3-bit encoding gave the highest classification accuracy even for long-duration, single-axial, stroke actigraphy signals. Figure 4.65 illustrates these results.

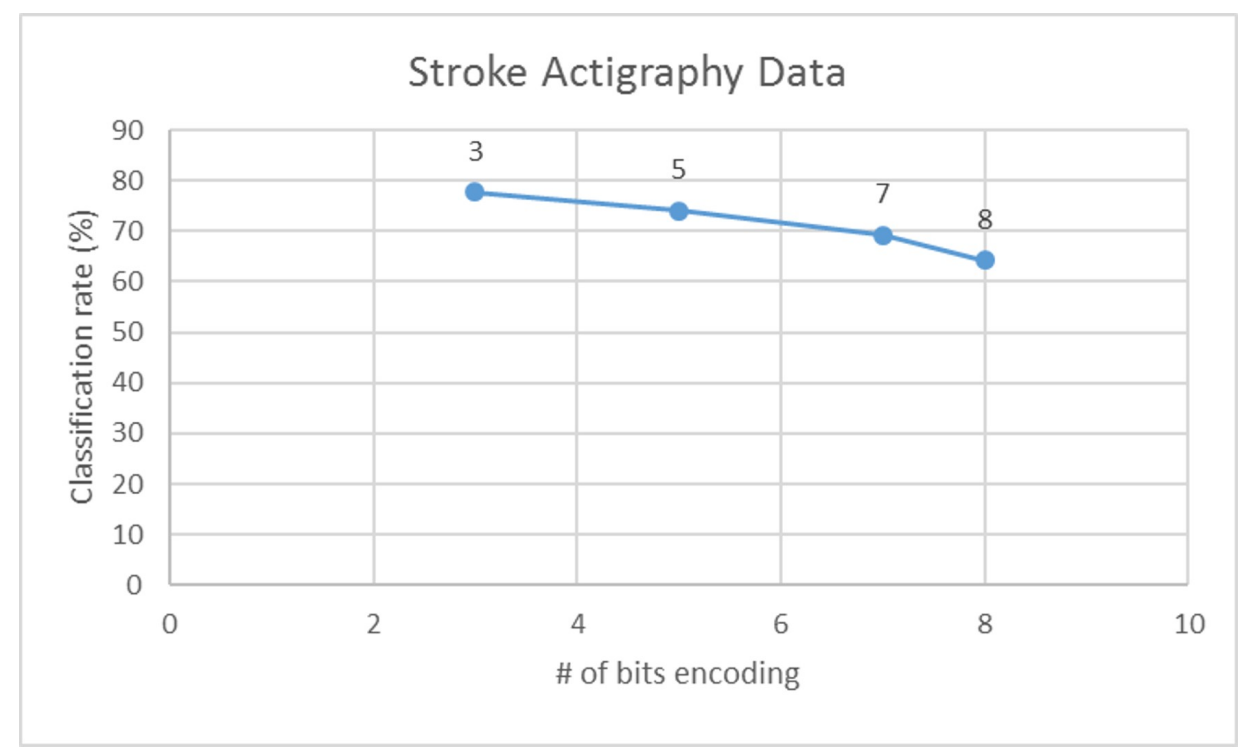

Figure 4.65: Classification Accuracy vs Encoding Levels

Following these remarkable results from the experiments on Datasets A, B and C, the next Section will discuss the observations on how the encoding, segmentation and feature extraction methods performed on analyzing sleep actigraphy signals.

\subsection{Observations from Experiments on Datasets A, B and C}

\subsubsection{Observations from Database A experiments}

In the preliminary set of experiments with single-axial, short-duration actigraphy signals in Database A, the following results were observed:

- The algorithm for feature extraction and classification of PLMs from these actigraphy signals was computationally efficient and fast.

- Though from the initial investigation into actigraphy signal properties it was observed that actigraphy data is non-linear and non-stationary, analyzing these signals in short-windows could be done efficiently by extracting simple time and frequency features.

- The first encounter with a PLM specific feature, the Periodicity Index (PI), was using signals in Database A. From Figure 4.9, it can be inferred that PI is the most robust and discriminant parameters 
in classifying patients with mild and severe PLMs. Being a function of the manually scored PLM index and the amount of time spent in deep sleep, PI captures significant limb movements in sleep which aggregate to form true PLM sequences for each test subject.

- From these experiments it was also observed that Naive-Bayes classifier performed the best in classifying actigraphy signals. It should be noted that Naive-Bayes classifier assumes that all the input features are of equal importance, and performs the learning accordingly. Although this factor may create dubious results from non-stationary actigraphy signals, one must also note that the analysis of signals in Database A, was performed on short-duration windows (about 85 seconds). Additionally, these results were also cross-validated using LDA and SVM tools, which further justified the high accuracy of the Naive-Bayes classifier.

\subsubsection{Observations from Database B experiments}

Following the analysis of short-duration, single-axial signals in Database A, similar experiments were conducted with long-duration, tri-axial actigraphy data in Database B. The objective of this study was to extrapolate the work on short-duration actigraphy data, by applying similar techniques to long-duration signals. From the experiments with Database B signals, the following were observed:

- Compared to previous study of Database A, this work also included the extraction of actigraphyspecific, kernel-based and morphological features, along with the conventional time and frequency features, in order to better characterize PLMs from actigraphy data.

- Further to this, the application of a low-bit encoding of actigraphy signals led to a high increase in SNR, automatic denoising and about $92 \%$ in space-savings of the signals, as compared to a $79 \%$ improvement in space savings using low-pass filtering methods. This pre-processing step also eliminated the need to apply conventional low-pass or band-pass filters, which otherwise sometimes fail to capture all the significant limb movements. Additionally, the signal encoding scheme also improved signal clarity through higher classification accuracy of PLMs. It should also be noted that the vector compounding of tri-axial signals also accounts for information retention, SNR enhancement, and simplified computations.

- Experiments using the AK and Mobility-based adaptive segmentation schemes revealed that it was indeed possible to extract only regions of interest from the actigraphy data, and discard the rest, which also significantly improved computation efficiency. As shown in Figures 4.19-4.22, and Table 4.9, it was quite evident that the AK technique segmented long-duration actigraphy data into a smaller number of 
segments as compared to the Mobility-based method. Additionally, Figures 4.23-4.25 indicated that the number of segments created using the AK method were nearly linearly correlated to the corresponding PLM index of each subject. That is, the higher the PLM index, the more limb movements will be captured by the actigraphy, and correspondingly the more number of segments will be created by the AK algorithm.

- An inclusion of Sleep Efficiency (SE) as longitudinal clinical parameter indicated that it was possible to segregate patients based on their sleep efficiency, when coupled with the number of AK segments, and their manually scored PLM index, as illustrated in Figures 4.26 and 4.27.

- As shown in Table 4.7, 24 time, frequency, actigraphy-specific, kernel-based, morphological and clinical features were extracted from the long-duration, tri-axial, sleep actigraphy data. Various combinations of these features were then fed into a Naive-Bayes, LDA and SVM tools for classification. Tables 4.10-4.15 highlight the classification of PLMs using various combinations of afore-mentioned features coupled with the manual PLM index as the class label. From these tables and the corresponding ROC curves, it was observed that the Naive-Bayes classifier did not perform well with encoded signals in many feature combinations. In fact, consistently good results were obtained with a linear SVM as indicated by the classification accuracies and corresponding F1-scores. Note that, the classification results were also compared with those obtained from low-pass filtered signals.

- The periodicity index extracted for these signals also showed high discriminant power in distinguishing mild and severe PLMs, as shown in Figure 4.39. Further to this, an investigation into using different bit-levels to encode the actigraphy data indicated that the 3-bit encoding applied in this study, provided the highest classification accuracy, as shown in Figure 4.40.

- In the next subset of experiments, a longitudinal classification of PLM test subjects was performed based on their gender (male or female) using a combination of various features cited in Table 4.7. Note that these experiments were conducted on actigraphy data obtained from individual legs per patient. The results from these have been recorded in Tables 4.16 and 4.17, coupled with their corresponding ROC curves. Although, compared to previous set of experiments, sufficiently high results weren't obtained in some cases, it should be noted that linear SVM still gave the best classification accuracy and F1-score for encoded signals.

- Additionally, a classification of normal and abnormal PLM subjects using manual PLM index of their respective legs as a class label was also performed along with age as an additional clinical feature, as 
shown in Tables 4.18 and 4.19. The linear SVM performed significant well in this case as well, giving an average classification accuracy of about $90 \%$ in classifying normal and abnormal test subjects.

- The classification results recorded from the analysis of signals in Database B, strongly indicate the efficacy of the novel encoding, adaptive segmentation and feature extraction methods to actigraphy data for both local and longitudinal applications.

\subsubsection{Observations from Database C experiments}

In the final set of experiments, the actigraphy conditioning and analysis techniques were applied to longduration, single-axial sleep actigraphy signals recorded from test subjects undergoing stroke recovery. Although the sample population used in this study was limited to 45 participants, the results obtained from signal encoding, segmentation and classification, strongly support the algorithms developed in this study. Following are some key observations recorded from the experiments:

- Compared to the previous encoding experiments in Database B, which showed a $92 \%$ space savings after compressing the actigraphy data, in this study the compression ratio of single-axial data was much lower, and resulted in an average space savings of only $34 \%$. Further investigation in these signals revealed that most of the data space was occupied by zero or close to zero amplitudes, and major peak activity was concentrated in specific regions only. This was also confirmed through a Giniindex computation, which indicated that the actigraphy signals in Database $\mathrm{C}$ were exhibiting sparsity in short windows.

- Further to this, upon applying the AK and mobility-based segmentation schemes to the single-axial signals, it was observed that these algorithms were able to identify peak regions of activity in most cases, as illustrated in Figures 4.54-4.57.

- These peak regions of activity were further analyzed through feature extraction and classification, as evident from results in Tables 4.24-4.27. Although the linear SVM performed fairly well in classifying encoded signals using various combinations of features, it was observed that the LDA performed significantly better. Note that in this study as well, the classification results were compared with raw signal data in Group $\operatorname{Str}_{R}$.

- Figure 4.65 also illustrates how the signal encoding scheme provided highest classification accuracy at a 3-bit resolution.

- Though this study of monitoring stroke recovery subjects using actigraphy is currently in the nascent 
stage of extensive data collection, the preliminary results from a set of 45 patients indicate a strong potential of the applicability of the contributed signal analysis algorithm to a larger dataset.

In the next chapter, an investigation into applying the contributed algorithms to actigraphy data recorded in wake states for activity recognition and applications in monitoring joint disorders will be conducted.

\subsection{Summary}

In this chapter of the dissertation, an investigation into the application of the actigraphy encoding, adaptive segmentation and classification methodologies to short- and long-duration sleep actigraphy data for assessing the severity of neuromuscular disorders has been done. This chapter begins by applying an initial set of signal analysis algorithms to short-duration, single-axial actigraphy data to test the efficacy of assessing actigraphy signals for estimating the severity of PLMs in sleep. Following this, in the next section the novel signal encoding and analysis algorithm is applied to long-duration, tri-axial sleep actigraphy data, which generates remarkable classification results in estimating PLM severity. Finally, this study is extrapolated further into classifying patients based on their stroke severity using their oxygen saturation levels, and single-axial sleep

actigraphy data. This chapter is then concluded by presenting the results for each set of experiments, and recording some key observations from them. 


\section{Chapter 5}

\section{Wake Actigraphy}

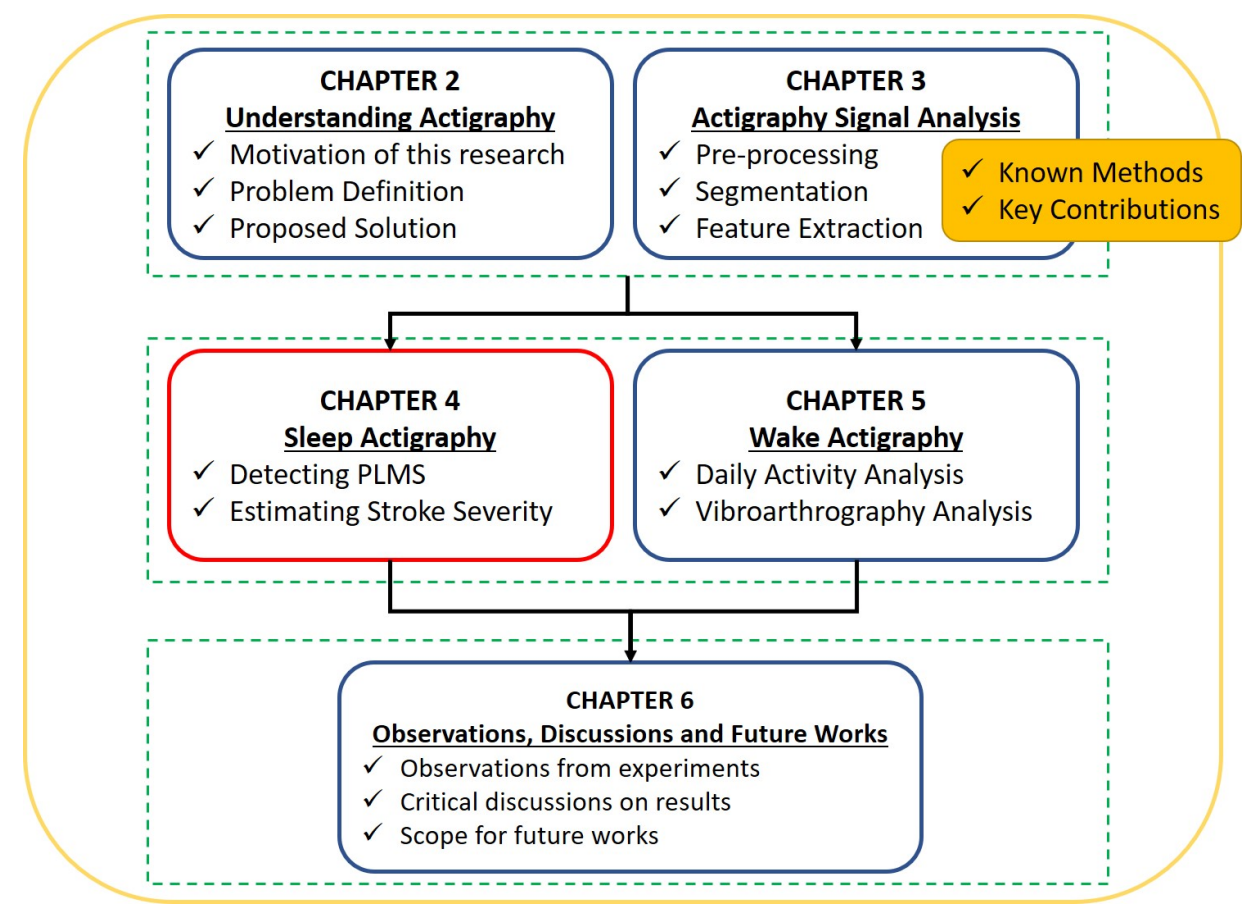

Figure 5.1: Organization of Dissertation

Following an extensive investigation into analyzing different actigraphy datasets captured in sleep, for detecting the severity of neuromuscular disorders, this chapter now proceeds to applying the proposed algorithm to actigraphy data collected in wake states. Although this research is primarily is focused on sleep actigraphy analysis applications, the purpose of applying the contributed methods in this study to wake actigraphy, is to show their applicability to a myriad of actigraphy signals captured from different locations on the human body. Compared to previous studies using Databases A, B and C, wherein bi-lateral ankle actigraphy was 
analyzed, in this chapter human activities captured using wrist and knee actigraphs will be explored.

\section{$5.1 \quad$ Introduction}

As discussed earlier in Chapters 1 and 2, actigraphs or accelerometer-based wearables have been one of the most effective means of activity monitoring in daily life. Recent advancements within the IoT paradigm have led to the inclusion of smart health monitoring solutions in a home-based environment, which can help an individual maintain optimum health and fitness levels. Motion or movement activity modeling has become an integral part of ambient intelligence applications in both smart living environments and surveillance $[7,38,92]$. Monitoring daily human activities such as sitting, sleeping, climbing stairs, drinking, eating, walking, bathing and lifting objects, using actigraphs could potentially help in measuring the autonomy with which an individual can function [7,92]. Additionally, this could also lead to potential applications in monitoring individuals suffering from a neuromuscular, or joint-related or injury-related movement disability, and improve their quality of life through proper feedback and alarm generation [32].

Wake actigraphy solutions could form an integral part of a home automation system, generating streaming activity data which could be used to convert qualitative human behaviour into quantitative models. To reiterate a few examples, currently available devices such as the Apple ${ }^{\mathrm{TM}}$ Watch, FitBit $^{\mathrm{TM}}$ and Samsung ${ }^{\mathrm{TM}}$ Gear Watch employ the use of accelerometers and gyroscopes to track human activity pertaining to daily routine and fitness $[33,34]$. The algorithms installed on these devices, analyze the input signals for generating user-friendly feedback such as step count, calories burnt, miles ran and pulse rate variability, which is useful in maintaining individual fitness levels [35]. Though these devices do not provide vital health information from sensor data, a systematic investigation indicates that motion data could be a primal contributing factor in assessing movement-related disorders or disabilities, and could also be used for developing surrogate markers for detecting or predicting the onset of various diseases such as Parkinson's disease [57].

Although there are other sensor-based modalities which could be used for detecting human activity, such as camera-based tracking and touch sensors on everyday appliances, employing a wearable sensor such as actigraph, ensures constant activity monitoring and feedback. A survey of prior works suggests that numerous studies have been conducted in the context of IoT/IoMT models, for determining the conduciveness of using wearables for activity monitoring $[11,13,14]$. These studies not only dealt with quantitative sensor data, but also included other aspects such as subjective parameters (age, gender etc.) and other sensorial data captured from an individual in smart environment. Reviewing these works, it was inferred that there are few key factors which must be considered when collecting and analyzing motion data from accelerometer-based wearables [32]. These are: 
- Sensor Placement: Detecting movements or activities correctly highly depends on the wearable device's placement on the human body. For example, a wrist actigraph is used for detecting hand and upper body gestures, whereas a hip or leg actigraph could be more suitable for full body activities.

- Sensor Count: Determining the number of actigraphs needed for detecting complete human activity in a smart environment has been a debatable topic in many prior studies. While some researchers proposed that analyzing data from multiple body locations could give a more holistic approach to activity recognition, a general consensus indicates that applying the right mathematical model could potentially limit the number of wearables to a single device. Employing single-device or single-sensor solutions can help detect most daily activities while ensuring wearable comfort and design.

- Modelling Approach: Analyzing actigraphy or accelerometry data could be done either using a ground-truth logic-based approach wherein a set of established rules govern activity recognition, or by employing a probability-based approach wherein the data is mathematically modelled using signal processing techniques to determine the type of activity conducted. While the former could save on data computation costs, the latter ensures an incremental machine learning pathway which takes into consideration human factors such as intensity of activity, fitness levels and rigorous comparison with training data.

A review of recent works in activity monitoring suggests the use of light-weight signal analysis and machine learning methods embedded on the device, for real-time, streaming data analysis without much need to compromise on memory and power consumption $[10,14,20,32]$. However, in reality not many such wearables are available in the market which have encompassed the IoT's layered architecture by ensuring an edge computing approach [77]. Nevertheless, the potential to develop novel signal analysis algorithms for wearables is huge [32]. Through this research, the intention is to leverage this opportunity by applying the novel encoding, segmentation and feature extraction schemes to wake actigraphy data for efficient data compression and activity recognition. In the following Sections, it will be shown how the contributed methods in this study could be applied to two wake actigraphy datasets, namely:

- Database D: Short-duration, tri-axial, wrist actigraphy signals captured form 16 healthy subjects, each performing 14 different activities of daily life (ADL). This data has been acquired from UCI's Machine Learning Repository [7,92].

- Database E: Short-duration, single-axial, vibroarthrography signals captured from 89 health and unhealthy test subjects undergoing knee-joint screening using actigraphs. This data has been acquired from Krishnan et al.'s research at University of Calgary [27]. 
Before proceeding further, the reader must note that although in wake actigraphy, analyzing tri-axial data may be conducted more efficiently by processing individual axial data $[40,55]$. But in this particular study all the tri-axial data was vector compounded in order to study only the intensity of the activity performed, as well as compare the efficiency of the proposed encoding, segmentation and feature extraction modules with regards to applications in sleep actigraphy. Concurrently, tri-axial factors such as Euler's angle and phase shift due to change in movement's direction have not been accounted for individually in the ADL dataset, but rather a vector compounding operation has been done to measure only the intensity of the activity performed.

\subsection{Experiments with Database D}

In this set of experiments, the key contributed actigraphy analysis algorithms were applied to classifying daily activity captured using wrist actigraphy from 16 healthy subjects. Before delving further, the reader may take a quick glance at the methodology illustrated in following Figure 5.2 reiterated from Chapters 2 and 4 .

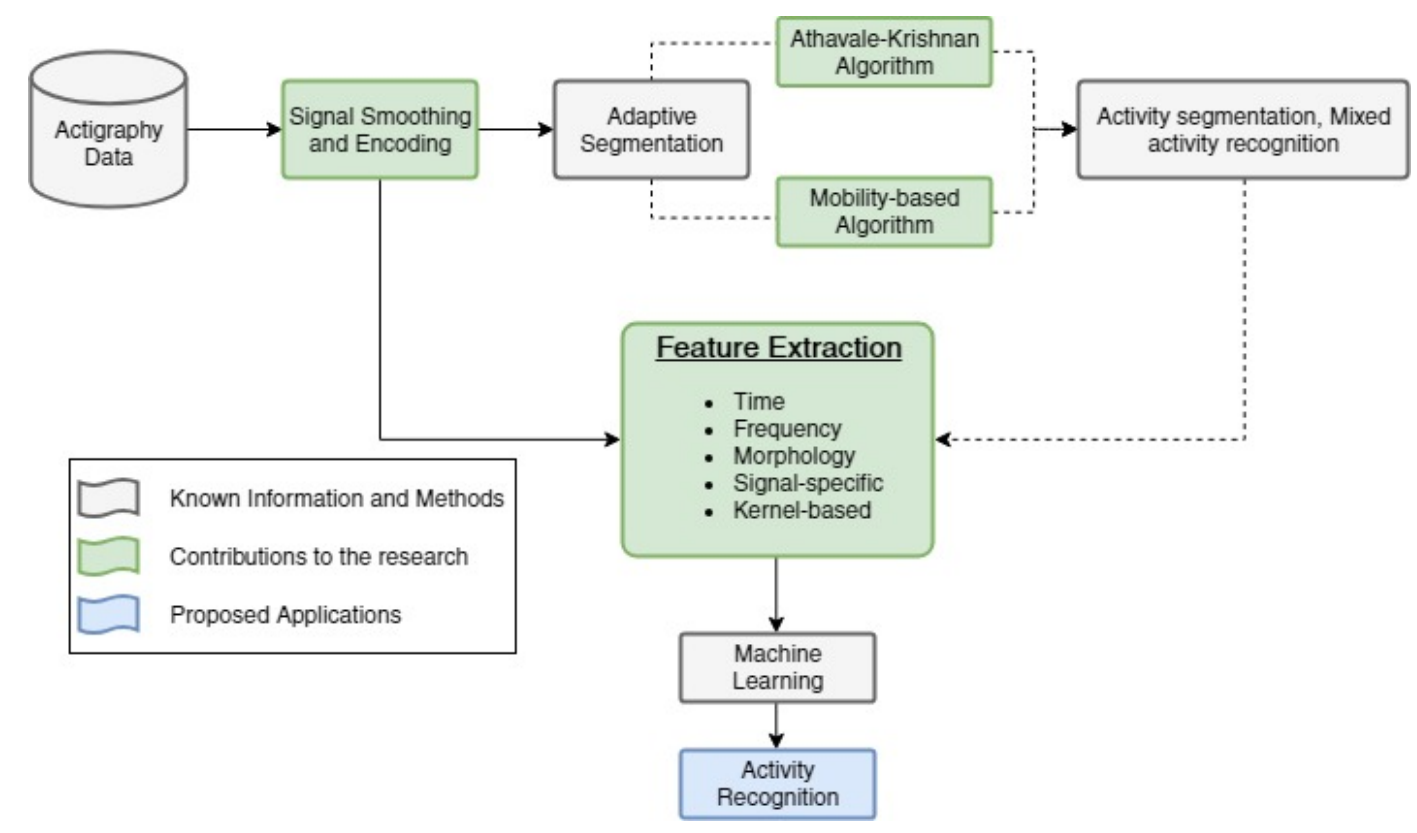

Figure 5.2: Proposed Daily Activity Analysis System 


\subsubsection{Materials and Methods}

\section{Data Acquisition}

The Database D comprised of wrist actigraphy signals for human motion primitives recognition, from 16 healthy subjects performing 14 different activities of daily life (see Table 5.1).

Table 5.1: Demographics - ADL Data, Database D

\begin{tabular}{cccccccc}
\hline \multicolumn{3}{c}{ Gender } & \multicolumn{3}{c}{ Age } & \multicolumn{3}{c}{ Weight } \\
\hline M & F & Min & Max & Avg & Min & Max & Avg \\
\hline 11 & 5 & 19 & 81 & 57.4 & 56 & 85 & 72.7 \\
\hline
\end{tabular}

The dataset contained 274 signals in total, recorded using a generic wrist actigraph with a sampling rate of $32 \mathrm{~Hz}$ and 6-bit resolution [8]. Following Table 5.2 highlights the number of signals per activity, along with a class label assigned for each activity for machine learning purpose.

Table 5.2: ADL Dataset with Class labels for classification $[7,8]$

\begin{tabular}{lcc}
\hline \multicolumn{1}{c}{ Activity } & No. of Signals & Class Label \\
\hline Brush teeth & 12 & 0 \\
Climb Stairs & 20 & 1 \\
Comb Hair & 31 & 2 \\
Descend Stairs & 42 & 3 \\
Drink from Glass & 20 & 4 \\
Eat meat & 5 & 5 \\
Eat soup & 3 & 6 \\
Get up from bed & 20 & 7 \\
Lie down on bed & 28 & 8 \\
Pour water & 20 & 9 \\
Sit down & 20 & 10 \\
Stand up & 20 & 11 \\
Telephone Talk & 13 & 12 \\
Walking & 20 & 13 \\
\hline TOTAL & $\mathbf{2 7 4}$ & $\mathbf{1 4}$ \\
\hline
\end{tabular}

To give the reader a better idea, following Figure 5.3 illustrates sample actigraphy signals captured from four different activities. 

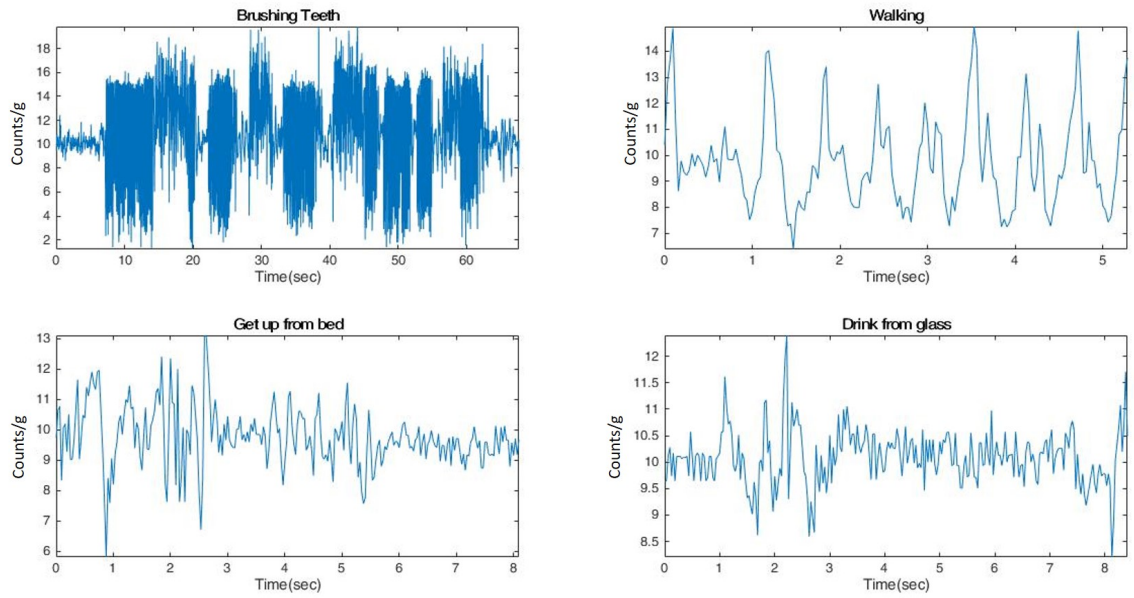

Figure 5.3: Sample daily activity signals

Similar to the approach in previous chapter, wherein sleep actigraphy signal properties were tested, it was found that the wrist actigraphy signals comprised in the ADL dataset also exhibited non-linearity and non-stationarity [7]. In the next Section, the actigraphy encoding method for on-the-fly denoising and compression of the ADL signals will be described.

\section{Actigraphy Encoding}

In order to show the efficacy of the encoding scheme, two groups of actigraphy data from the ADL dataset as Group $A D L_{R}$ and Group $A D L_{E}$, each consisting of the same 274 wrist actigraphy signals were created. Note that, the subsequent sections will compare the analysis results between Groups $A D L_{R}$ and $A D L_{E}$, to show the efficacy of this study's approach in activity segmentation and recognition. For each signal in Groups $A D L_{R}$ and $A D L_{E}$, the following pre-processing steps were performed:

\section{- $\underline{\text { Group } A D L_{R} \text { : }}$}

Each actigraphy signal in this group was pre-processed as follows:

1. For each signal $S_{R}$, any missing or NaN (Not a Number) values were removed.

2. As indicated in Equation 3.2 from Chapter 3, the actigraphy signal was then normalized using the specifications cited in [8] as,

$$
S_{N}=-1.5 g+\frac{S_{R}}{63} \times 3 g
$$

where $g=9.8 \mathrm{~m} / \mathrm{s}^{2}$ 
3. Following this, the tri-axial signal $S_{n}=\left(x_{n}, y_{n}, z_{n}\right)$ was vector compounded as,

$$
S_{V}=\sqrt{x_{n}^{2}+y_{n}^{2}+z_{n}^{2}}
$$

\section{- Group $A D L_{E}$ :}

In this group, each signal was conditioned as follows:

1. After normalizing and vector compounding each signal using Equations 5.1 and 5.2, a $m$-bit encoding of the actigraphy signal $S_{V}$ was performed such that $m \ll r$, where $r$ is the bit resolution of the raw actigraphy signal. This encoding was performed using the floor operation as,

$$
S_{E}=\left\lfloor\left(S_{V} \times \frac{\left(2^{m}-1\right)}{2}+\frac{\left(2^{m}-1\right)}{2}\right)\right\rfloor
$$

Using the afore-mentioned set of pre-processing steps resulted in generating normalized and vector compounded raw actigraphy signals in Group $A D L_{R}$, and encoded actigraphy data in Group $A D L_{E}$. Note that the purpose of the encoding process in Equation 5.3 is to ensure signal compression, denoising and SNR enhancement, for improving further signal analysis. The results of this have been tabulated and illustrated in Section 5.2.2. The following section will describe the application of the contributed adaptive segmentation schemes in this research, to activity recognition from the encoded group of signals $A D L_{E}$.

\section{$\underline{\text { Encoding-based Segmentation }}$}

In this study of short-duration, wrist actigraphy signals, the AK and mobility-based adaptive segmentation techniques were tested on encoded signals for two applications:

- Single activity segmentation

- Segmentation of a synthetic signal comprising of 14 different activities

First, the AK segmentation algorithm was applied to an encoded signal $S_{E}$ through the following steps. As shown from Equation 5.3, after encoding the vector compounded signal, the first order and the subsequent second order difference signals were computed as,

$$
d S_{e}=\left[S_{e_{1}},\left(S_{e_{2}}-S_{e_{1}}\right), \ldots,\left(S_{e_{n}}-S_{e_{n-1}}\right)\right]
$$

and,

$$
2 d S_{e}=\left[d S_{e_{1}},\left(d S_{e_{2}}-d S_{e_{1}}\right), \ldots,\left(d S_{e_{n}}-d S_{e_{n-1}}\right)\right]
$$


The rapid change factor (RCF) of the signal was calculated using its second order difference version, using the expression,

$$
R C F=\frac{\text { Step }- \text { Size }}{\left(m \times t_{s}\right)}
$$

where $t_{s}$ is the sampling period. The primary segment boundaries (PSBs) were found by finding all the signal samples in $2 d S_{E}$ which were greater than the RCF,

$$
P S B_{i}=2 d S_{E_{n}} \forall\left\langle 2 d S_{E_{n}}>R C F\right\rangle
$$

where $i$ is the number of PSBs. From an extensive investigation of different activity signals in the ADL dataset, the following observations were found to be useful in deducing the secondary and final segment boundaries $[7,92]$ :

1. Most human activity exhibited significant bouts which lasted for a time-period of 1.5 to 2 seconds.

2. The duration of each peak on an average was about 0.5 seconds.

Using these observations, the secondary segment boundaries (SSBs) were found by identifying PSBs which were separated by at least 0.5 seconds, as,

$$
S S B_{j}=P S B_{i} \forall\left\langle\left|t_{P S B_{i}}-t_{P S B_{i+1}}\right|>0.5\right\rangle
$$

where $j$ is the number of SSBs. Following this, the final segment boundaries (FSBs) were found by extracting only those SSBs which were at least 2 seconds apart,

$$
F S B_{l}=S S B_{j} \forall\left\langle\left|t_{S S B_{j}}-t_{S S B_{j+1}}\right|>2\right\rangle
$$

Following this, the next experiment was the application of the mobility-based adaptive segmentation scheme to encoded actigraphy data, using the steps as follows. Using Eqns.5.4 and 5.5 we compute he second order difference signal of the encoded data $S_{E}$ was computed. The PSBs were found by detecting all the samples in $2 d S_{E}$ which were greater than a factor of $\frac{\max _{n} 2 d S_{E}}{\left(2^{m}-1\right)}$, such that,

$$
P S B_{i}=2 d S_{E_{n}} \forall\left\langle 2 d S_{E_{n}}>\frac{\max _{n} 2 d S_{E}}{\left(2^{m}-1\right)}\right\rangle
$$

Using these PSBs, the SSBs were identified such that the Hjorth's mobility values of two segments were unique from each other.

$$
S S B_{j}=P S B_{i} \forall\left\langle\left\lfloor M_{P S B_{i: i+1}}\right\rfloor \neq\left\lfloor M_{P S B_{i+1: i+2}}\right\rfloor\right\rangle
$$


Using the ground truth information on the ADL data, the FSBs were found such that two SSBs were at least 2 seconds apart.

$$
F S B_{l}=S S B_{j} \forall\left\langle\left|t_{S S B_{j}}-t_{S S B_{j+1}}\right|>2\right\rangle
$$

The results of activity segmentation using the AK and mobility-based algorithms have been illustrated in Section 5.2.2. The following section will describe the feature extraction and classification methodologies adopted for Groups $A D L_{R}$ and $A D L_{E}$ respectively, and then follow that to highlight the results from the pre-processing, segmentation and classification approaches.

\section{Feature Extraction and Classification}

Following the pre-processing and segmentation operations conducted on Groups $A D L_{R}$ and $A D L_{E}$ datasets, the next step was to proceed to extract features from the signals in both groups. Using these features and the labeling information from Table 5.2 machine learning tools were used to classify the signals based on their respective activity. The feature extraction and classification methodology was conducted for each signal in Groups $A D L_{R}$ and $A D L_{E}$. From each pre-labelled signal,22 time, frequency, kernel-based, actigraphyspecific and morphological features were extracted as described in Table 5.3. 
Table 5.3: Features extracted from Groups $A D L_{R}$ and $A D L_{E}$ activity signals

\begin{tabular}{|c|c|c|}
\hline Domain & No. & Features Used \\
\hline \multirow{11}{*}{ Time } & 1 & Mean \\
\hline & 2 & Standard Deviation \\
\hline & 3 & Variance \\
\hline & 4 & RMS \\
\hline & 5 & Maxima \\
\hline & 6 & Peak-to-peak difference \\
\hline & 7 & Peak-to-RMS ratio \\
\hline & 8 & Peak-to-average ratio \\
\hline & 9 & Peak-to-average power ratio \\
\hline & 10 & Band power \\
\hline & 11 & Signal to noise \& distortion ratio \\
\hline \multirow{2}{*}{ Frequency } & 12 & Median Frequency \\
\hline & 13 & Mean Frequency \\
\hline Kernel-based Features & $14-17$ & ACF Kernel Scores (4 Features) \\
\hline \multirow{2}{*}{ Actigraphy-specific } & 18 & Rapid Change Factor \\
\hline & 19 & Spiky Index \\
\hline \multirow{3}{*}{ Morphological } & 20 & Hjorth's First order mobility \\
\hline & 21 & Hjorth's Second order mobility \\
\hline & 22 & Hjorth's complexity \\
\hline Other Labels & 23 & Activity Type \\
\hline
\end{tabular}

Using a combination of various features, a 70-30 partition of training and testing sets for features were created in Groups $A D L_{R}$ and $A D L_{E}$, and further used to model two machine learning tools, namely - a LDA tool and a SVM $[150,151]$. The results of these classification experiments have been documented further in Section 5.2.2.

\subsubsection{Results from Experiments on Database D}

\section{Actigraphy Encoding Results}

As described in Section 5.2.1, two groups of signals, namely $A D L_{R}$ and $A D L_{E}$ were created. While Group $A D L_{R}$ contained normalized and vector compounded signals, Group $A D L_{E}$ contained 3-bit encoded signals. As explained earlier, the purpose of the 3-bit encoding is to achieve data compression and denoising at the source, without compromising information loss. Figure 5.4 illustrates a sample actigraphy signal captured 
from a healthy individual while climbing stairs, and its corresponding 3-bit encoded version.
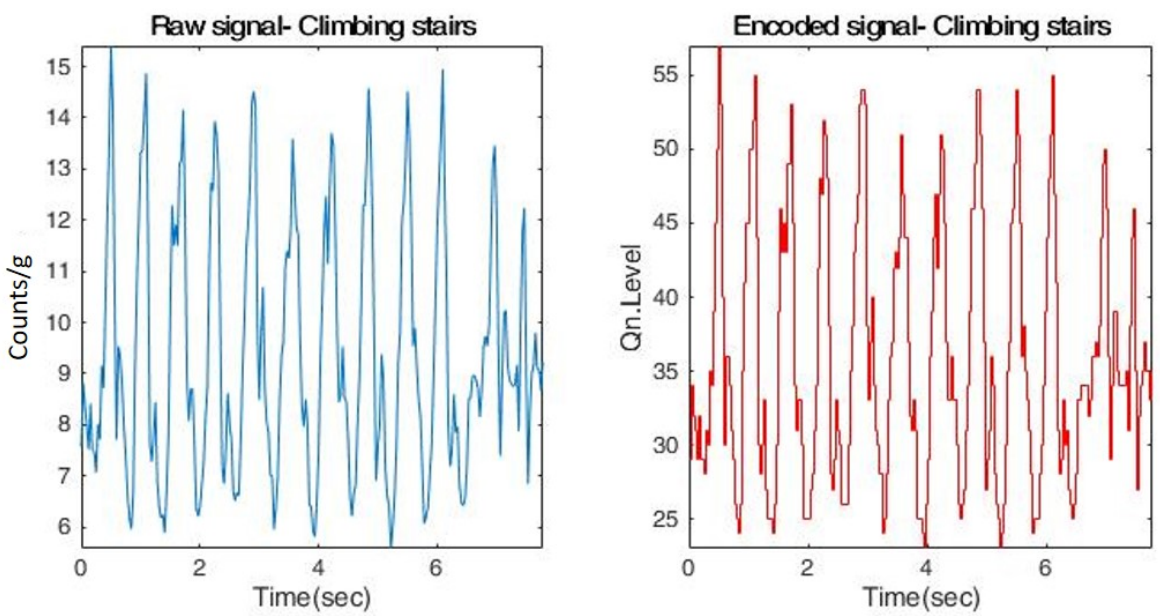

Figure 5.4: Example of 3-bit encoding of ADL signals

The encoding of all the available 274 activity signals was performed, and their average parametric results were compared with those of the raw signals, as shown in Table 5.4 and Figure 5.5.

Table 5.4: Parametric comparison between raw and encoded ADL signals

\begin{tabular}{lcc}
\multicolumn{1}{c}{ Parameter } & Raw Signal & Encoded Signal \\
\hline Avg. File Size (kB) & 32 & 10 \\
Bit Rate (bits/sec) & 192 & 96 \\
Avg. File Read Time (secs) & 0.9 & 0.4 \\
Avg. SNR (dB) & -8.4 & 28.2 \\
\% Space Savings & NA & $68 \%$ \\
\hline
\end{tabular}




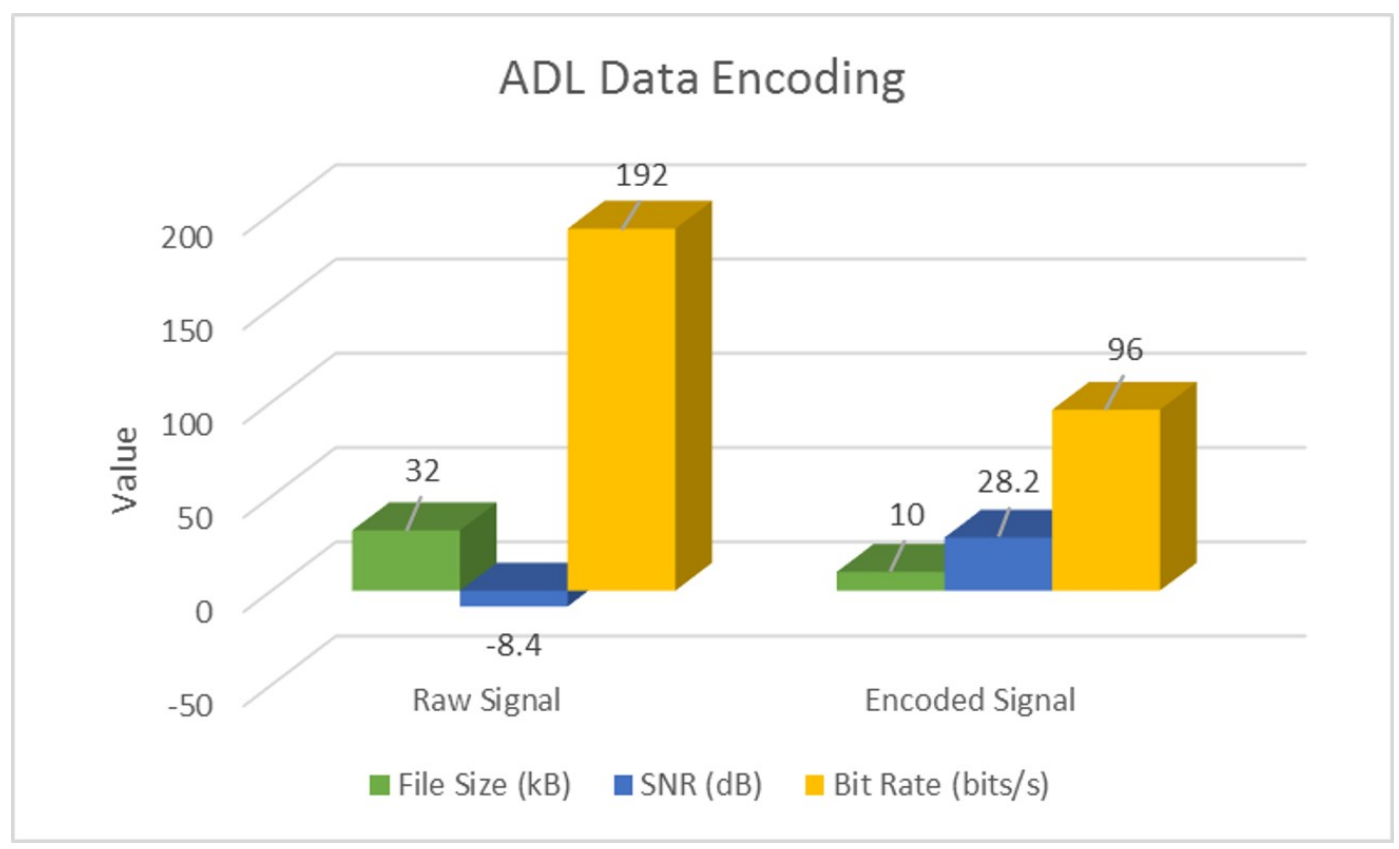

Figure 5.5: 3-bit encoding of ADL actigraphy

As evident from these results, the 3-bit encoding not only ensures a high SNR, but also helps in a $68 \%$ space savings of file memory usage, without compromising the movement information in each activity signal. In the next Section, a closer look will be taken on the effect of applying the AK and mobility-based methods to segmenting ADL data.

\section{Encoding-based Segmentation Results}

Following the encoding of ADL data in Group $A D L_{E}$, the adaptive segmentation schemes were applied for following applications, as cited in Section 5.2.1:

- Single activity segmentation

- Segmentation of synthetic signals comprising of 11 and 14 different activities

Following set of Figures 5.6-5.19 illustrate the application of the AK and mobility-based schemes to segmenting individual activity data in the ADL database. Note that each of the following figures illustrate a sample activity taken from its corresponding set in the ADL database. 

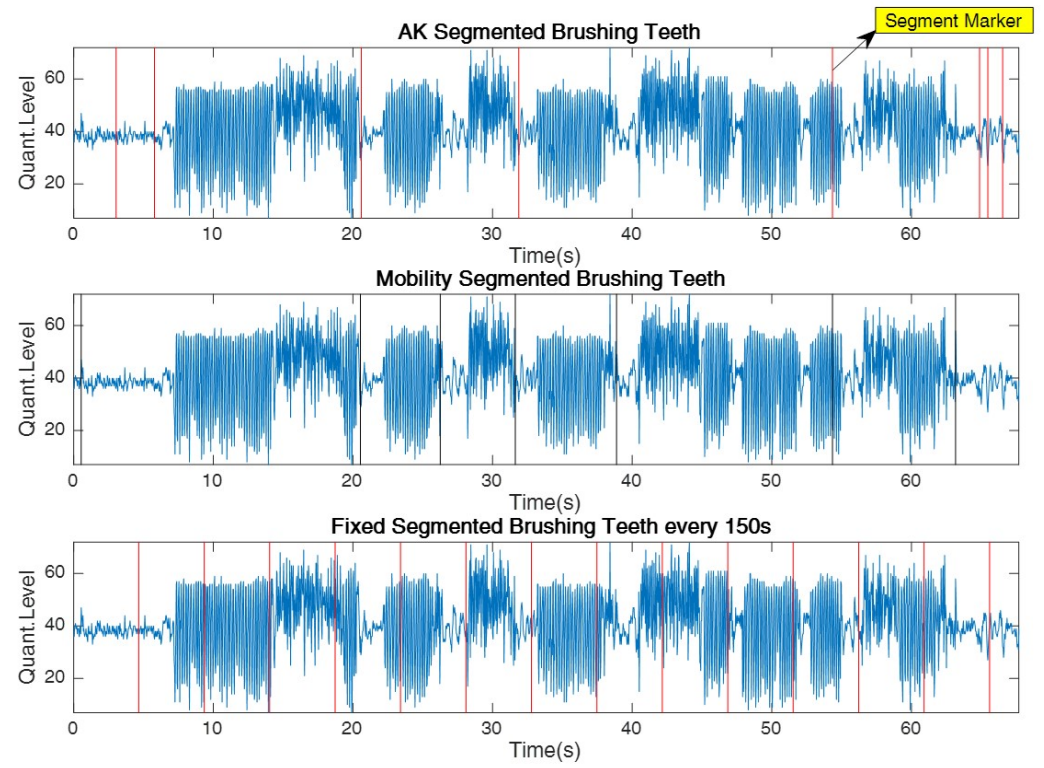

Figure 5.6: Adaptive Segmentation: Brushing Teeth
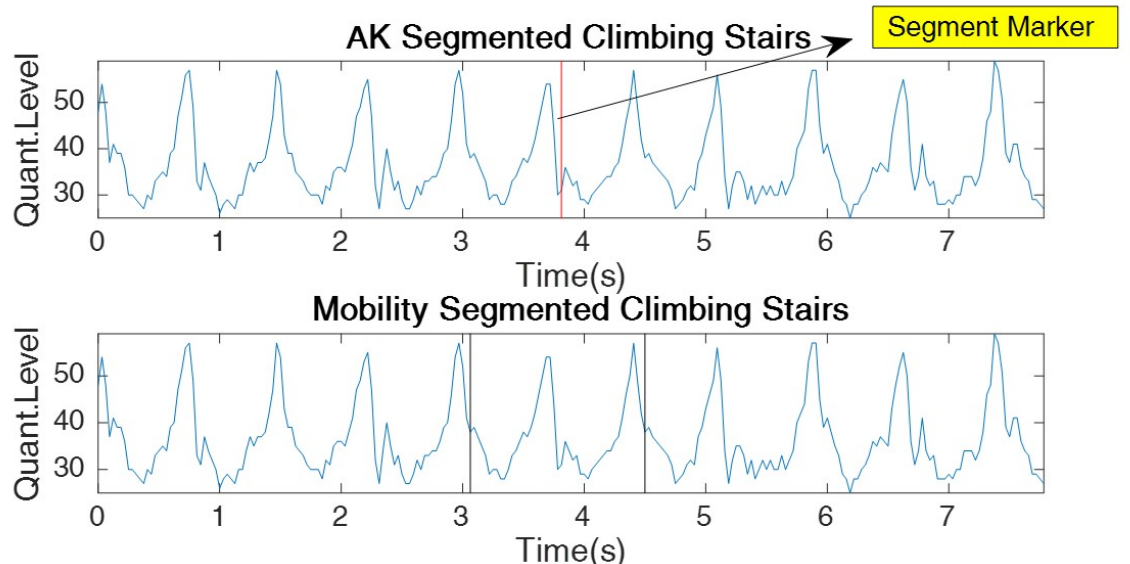

Fixed Segmented Climbing Stairs

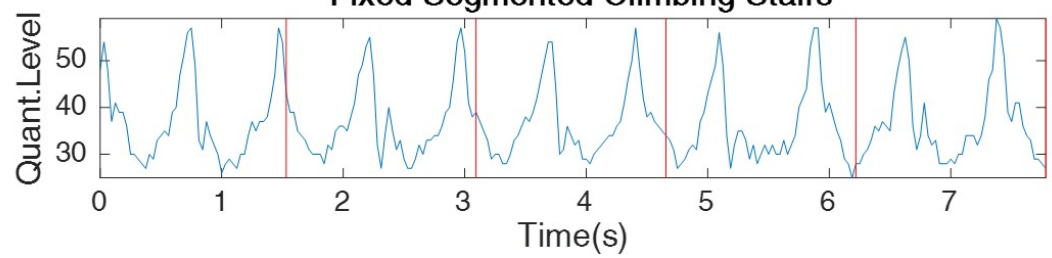

Figure 5.7: Adaptive Segmentation: Climbing Stairs 


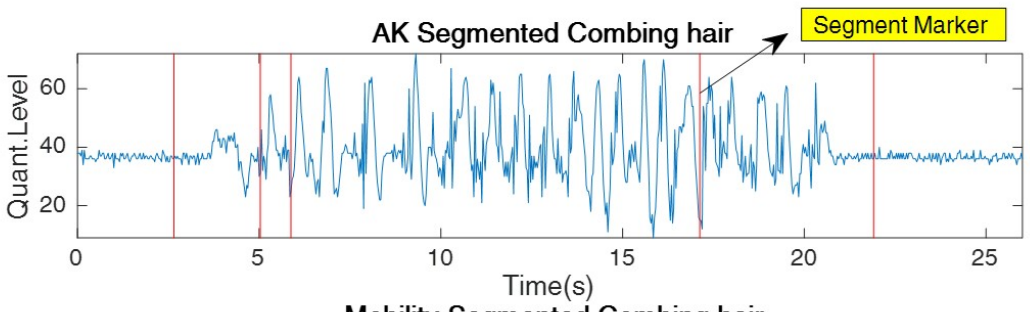

Mobility Segmented Combing hair

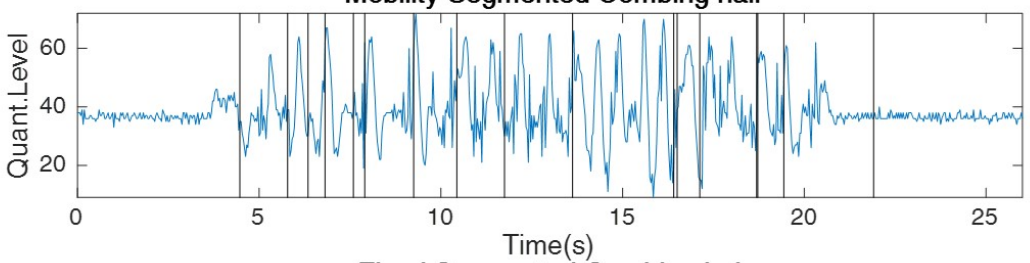

Fixed Segmented Combing hair

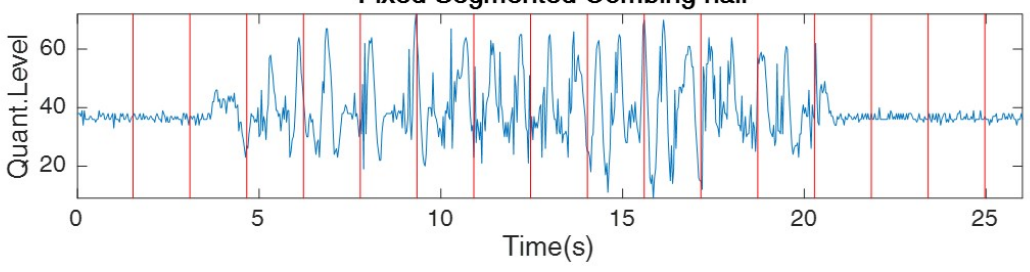

Figure 5.8: Adaptive Segmentation: Combing Hair
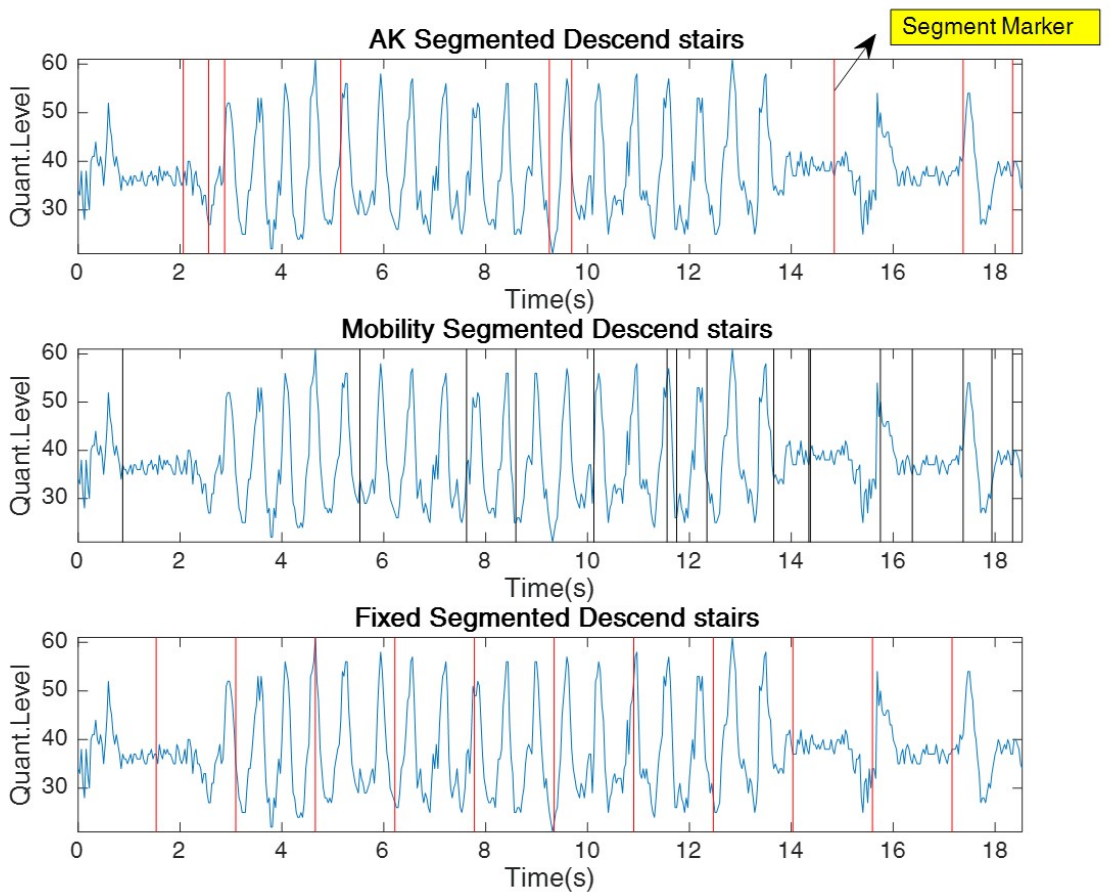

Figure 5.9: Adaptive Segmentation: Descending Stairs 

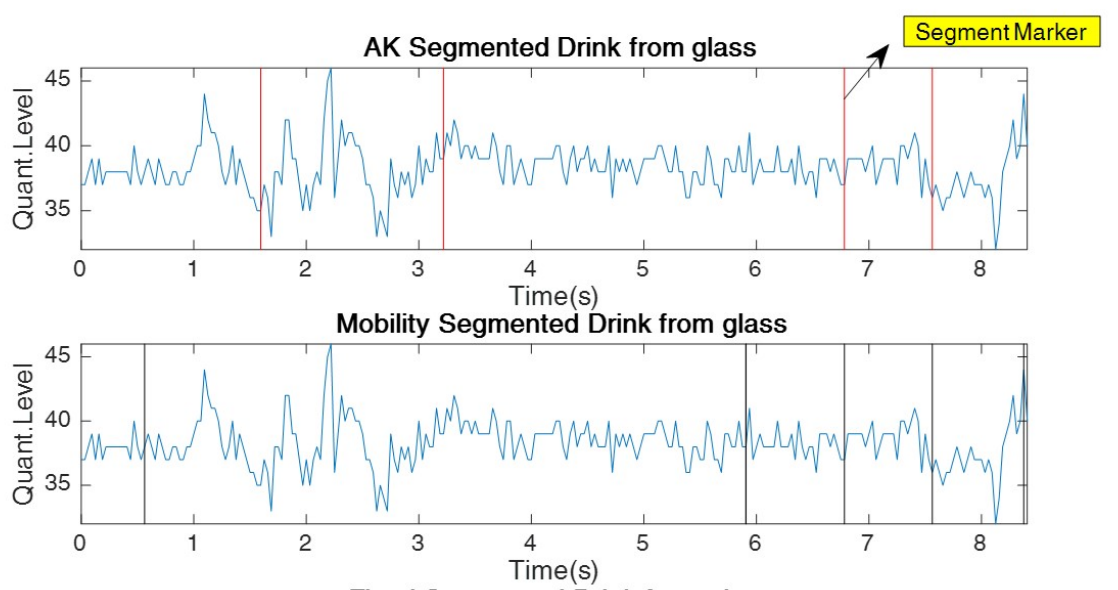

Fixed Segmented Drink from glass

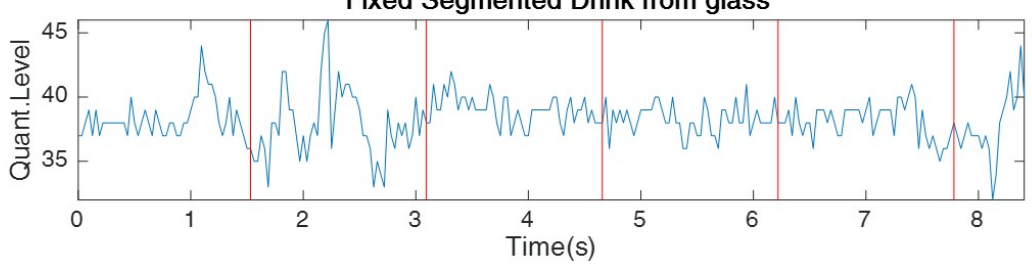

Figure 5.10: Adaptive Segmentation: Drink from Glass
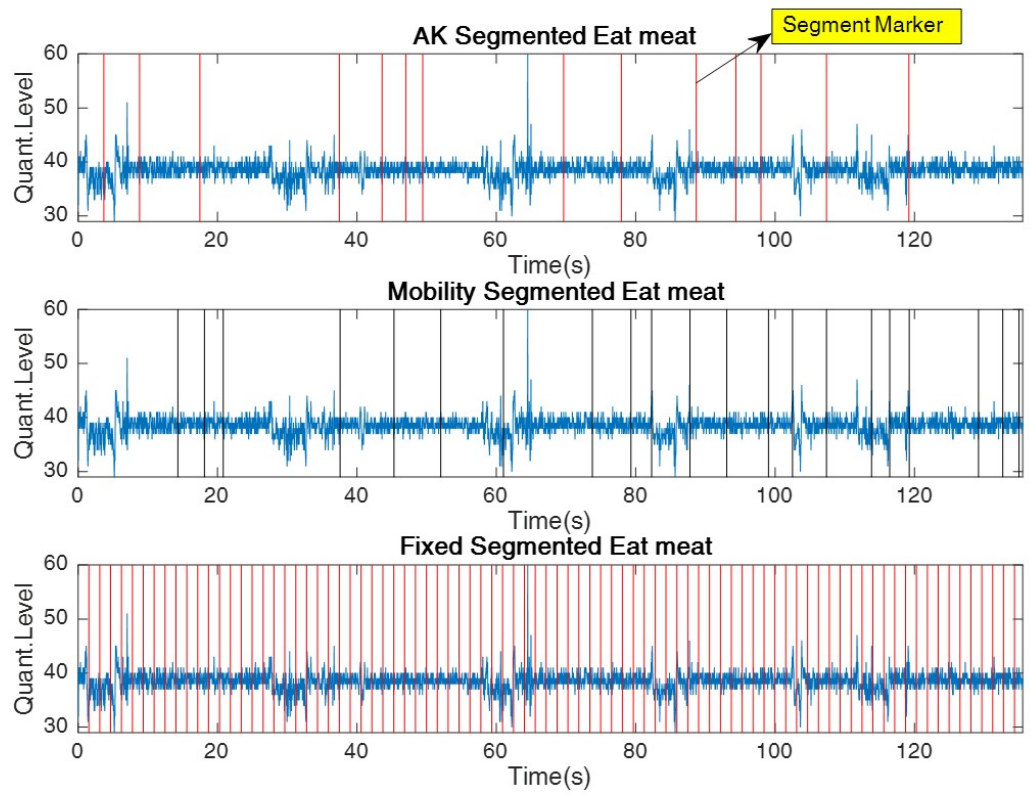

Figure 5.11: Adaptive Segmentation: Eating Meat 

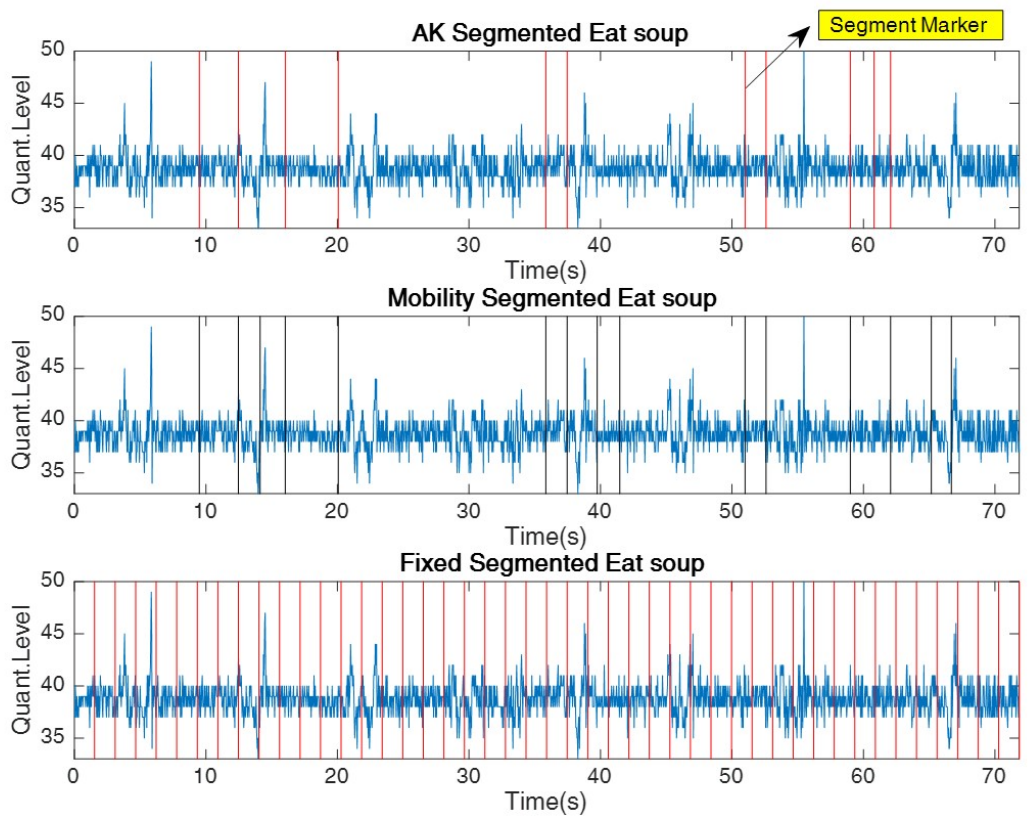

Figure 5.12: Adaptive Segmentation: Eating Soup
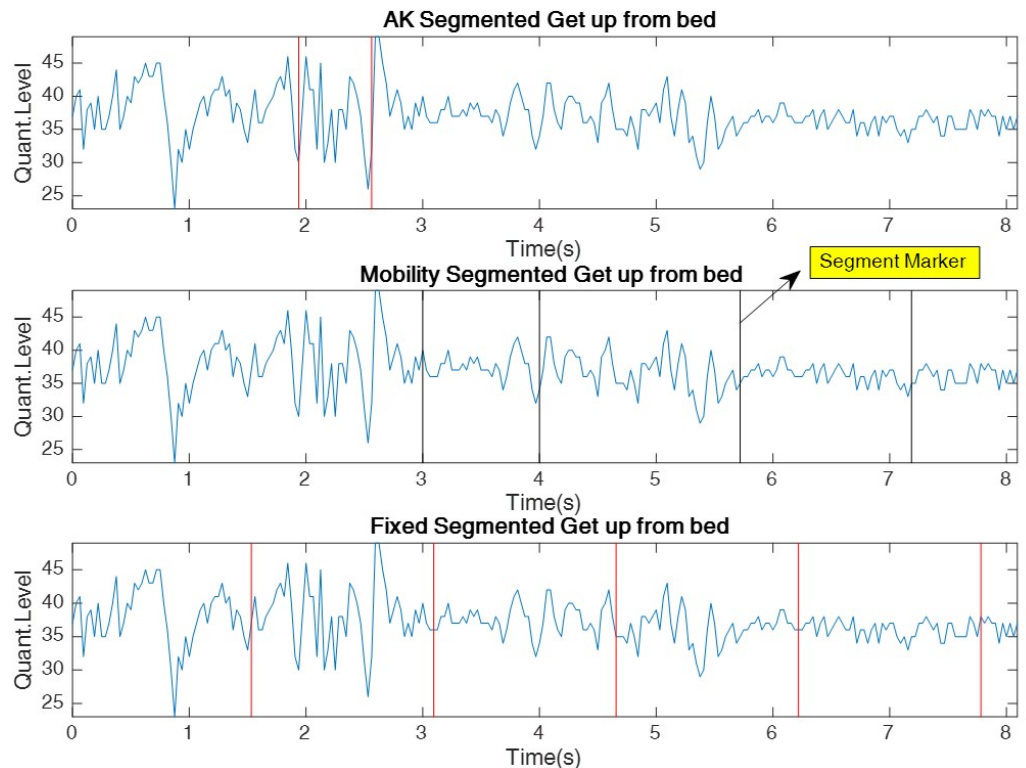

Figure 5.13: Adaptive Segmentation: Get up from bed 

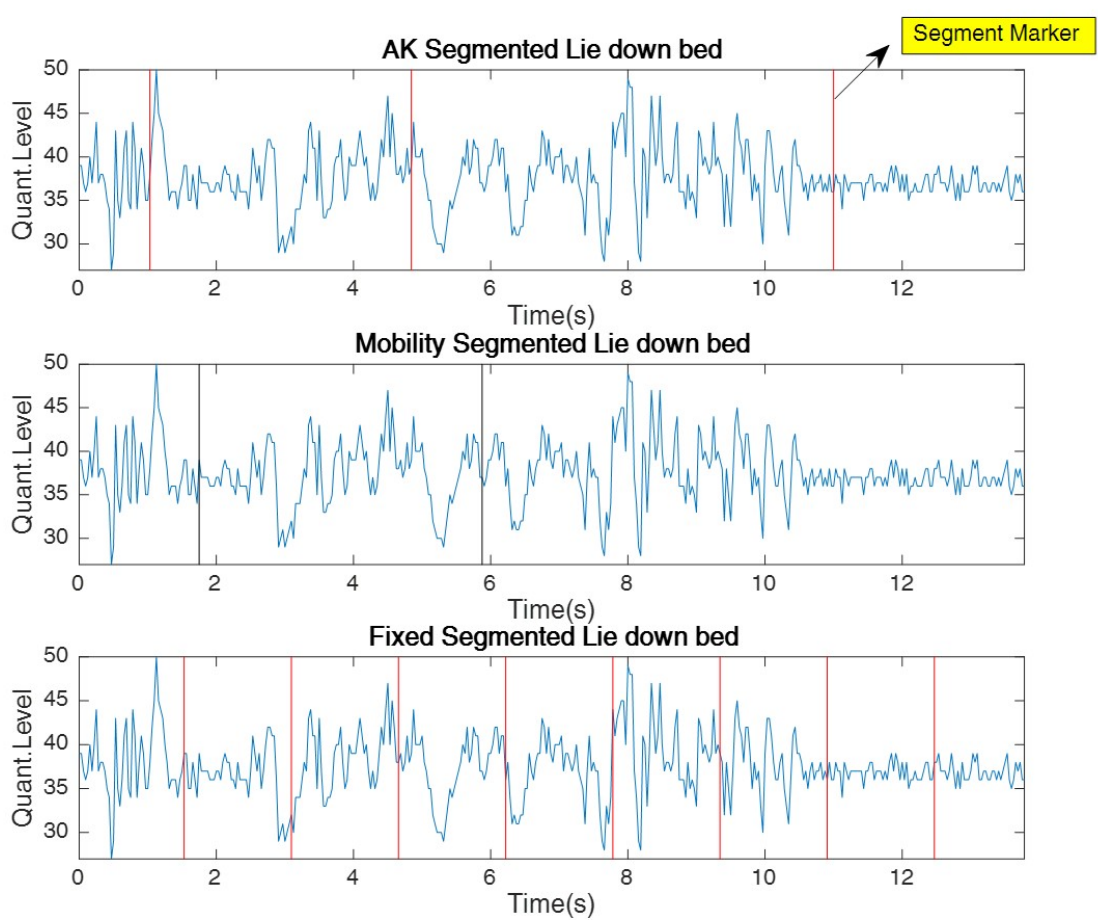

Figure 5.14: Adaptive Segmentation: Lie Down on bed
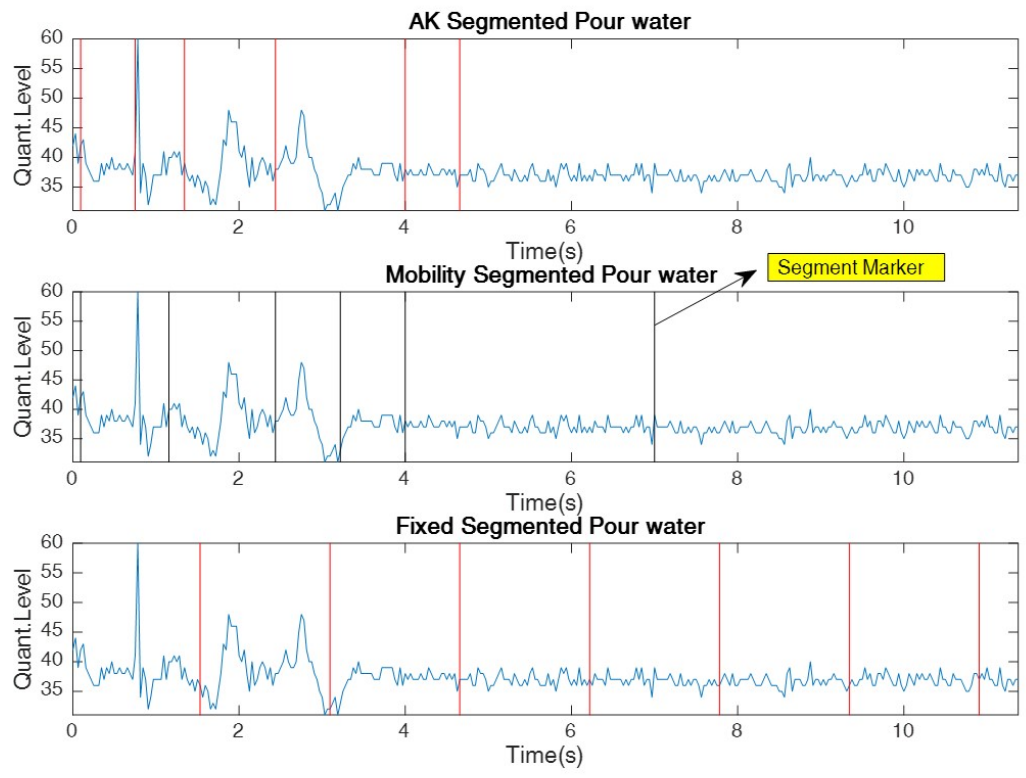

Figure 5.15: Adaptive Segmentation: Pouring water 

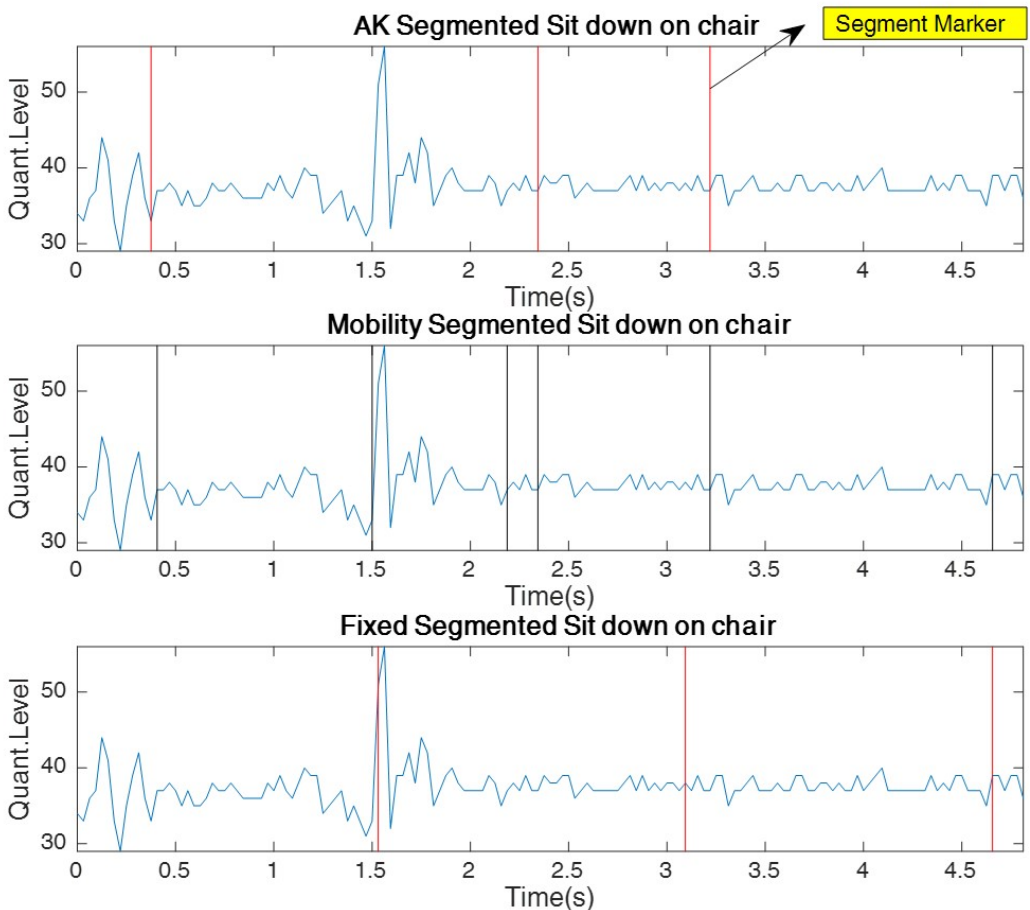

Figure 5.16: Adaptive Segmentation: Sit Down
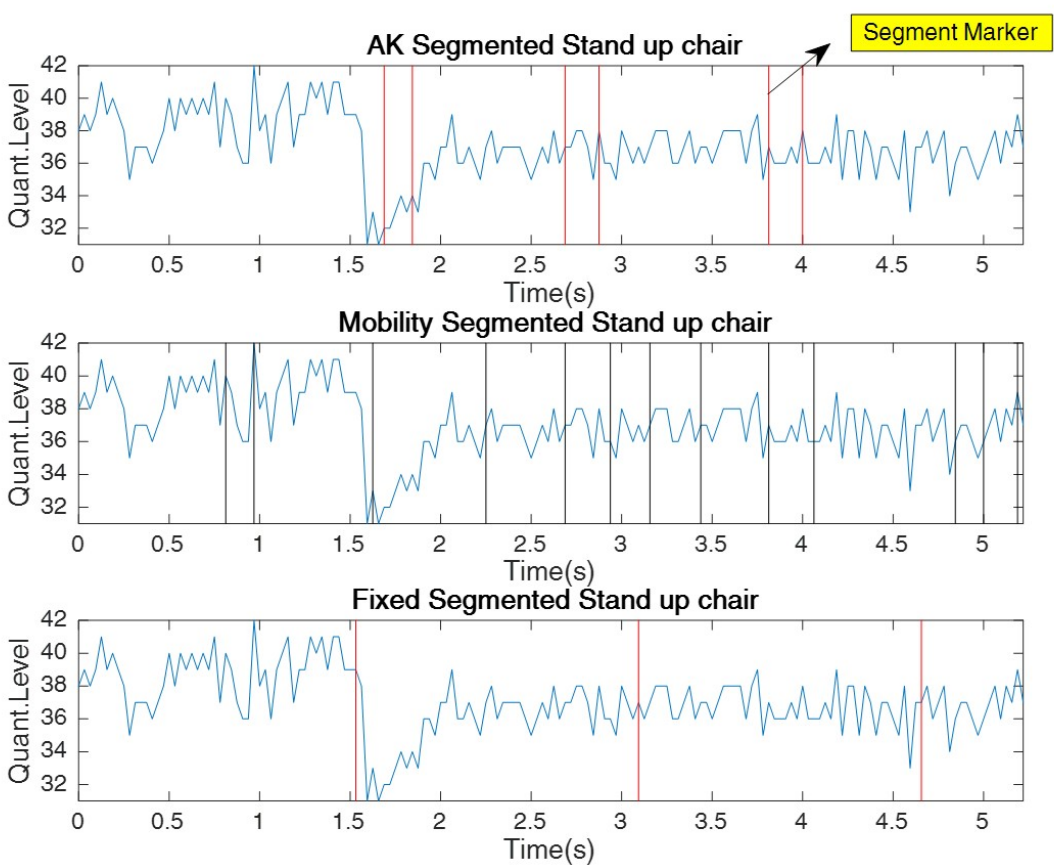

Figure 5.17: Adaptive Segmentation: Stand up 

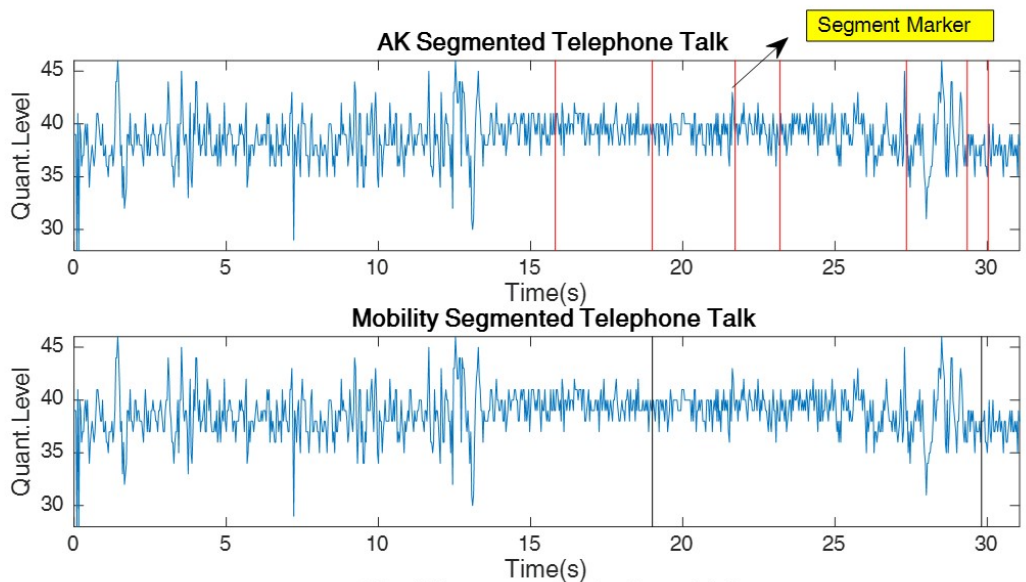

Fixed Segmented Telephone Talk

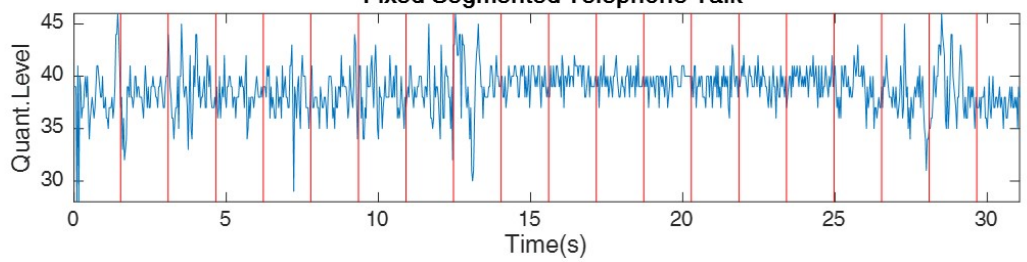

Figure 5.18: Adaptive Segmentation: Telephone Talk
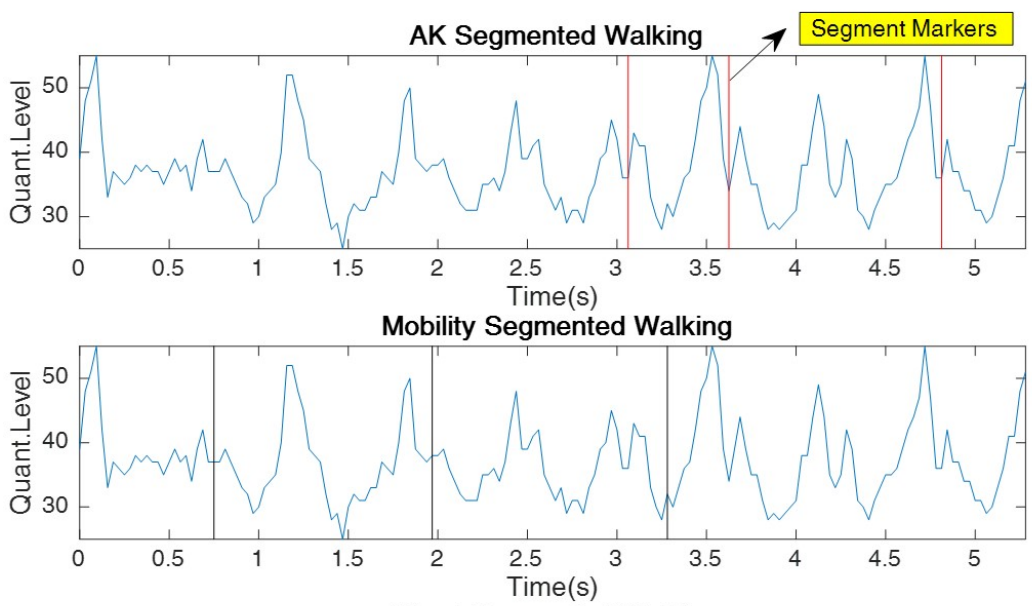

Fixed Segmented Walking

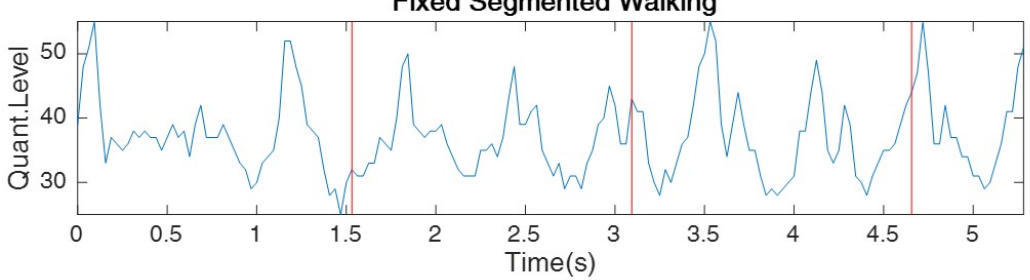

Figure 5.19: Adaptive Segmentation: Walking 
Further to this, the number of segments generated for each sample signal (see Figs. 5.6 - 5.19) segmented by the AK and Mobility algorithms respectively was recorded as shown in Table 5.5.

Table 5.5: No. of Segments between AK and Mobility algorithms on ADL data

\begin{tabular}{lcc}
\multicolumn{1}{c}{ Activity } & No. of AK Segments & No.of Mobility-based Segments \\
\hline Brush teeth & 9 & 8 \\
Climb Stairs & 2 & 3 \\
Comb Hair & 6 & 18 \\
Descend Stairs & 10 & 17 \\
Drink from Glass & 6 & 7 \\
Eat meat & 15 & 22 \\
Eat soup & 12 & 16 \\
Get up from bed & 3 & 5 \\
Lie down on bed & 4 & 3 \\
Pour water & 7 & 7 \\
Sit down & 4 & 7 \\
Stand up & 7 & 14 \\
Telephone Talk & 8 & 3 \\
Walking & 4 & 4 \\
\hline
\end{tabular}

As evident from Table 5.5, the AK algorithm performed fairly well as compared to the Mobility-based scheme by segmenting a given daily activity signal into lower number of segments, in most cases. This being said, the mobility-based algorithm also performed well, by capturing relevant movement variance from the actigraphy data.

Following this, two synthetic activity signals were generated by concatenating different activity data from the ADL database, and then the AK and mobility-based algorithms were re-applied for activity recognition. The results of this have been illustrated in following Figures 5.20 and 5.21. 

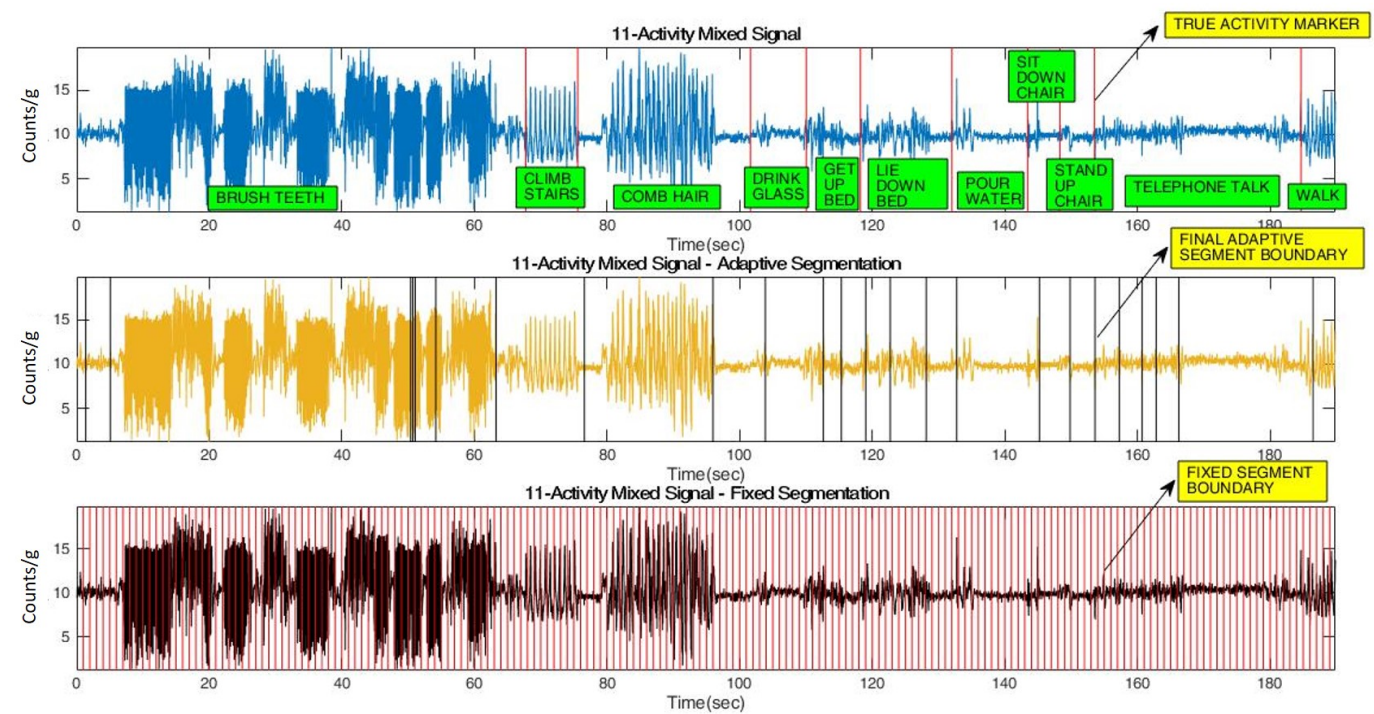

Figure 5.20: AK Algorithm applied to a synthetic signal of 11 concatenated activities

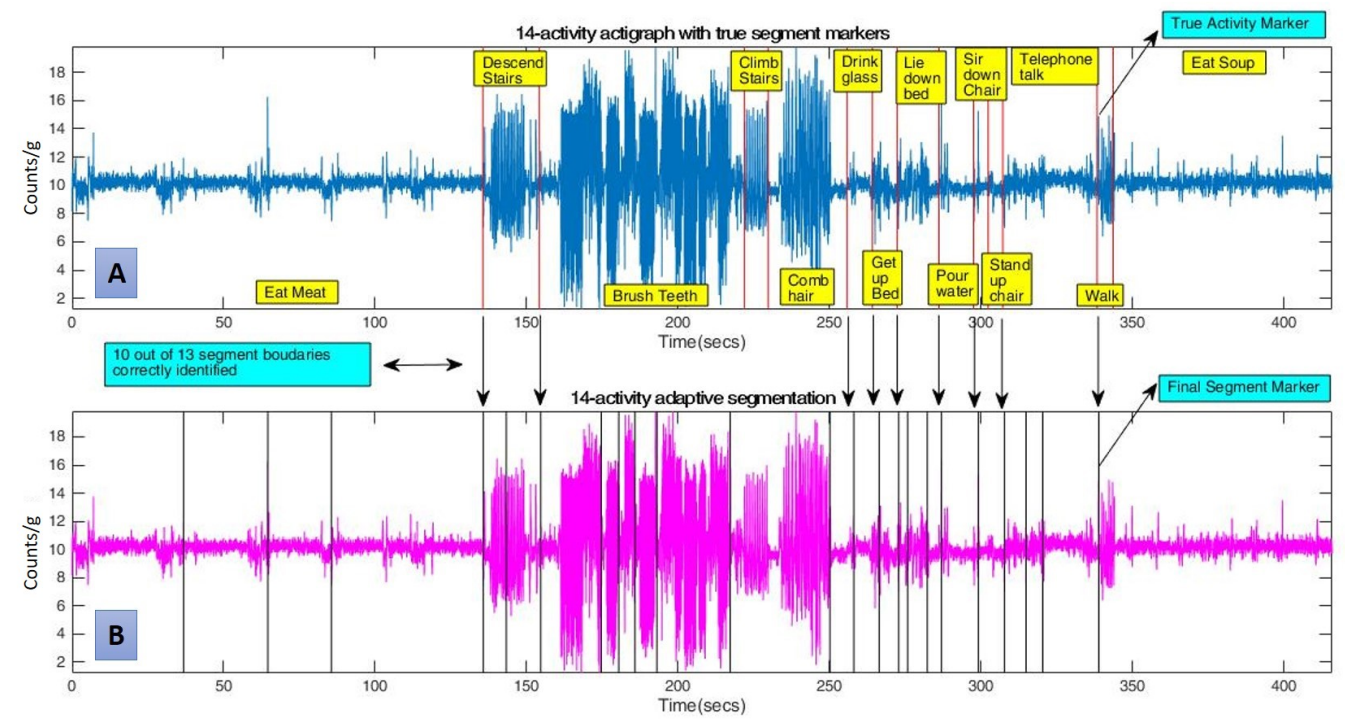

Figure 5.21: Mobility-based Algorithm applied to a synthetic signal of 14 concatenated activities - (A) Ground truth signal, (B) Segmented signal

Figures 5.20 and 5.21 clearly indicate the efficacy of the AK and mobility-based techniques to real and synthetic activity data, by correctly identifying regions of different movements with an average accuracy of $70 \%$. Following this, the next Section will highlight various feature extraction and classification results obtained from the experiments on the ADL data. 


\section{$\underline{\text { Feature Extraction and Classification Results }}$}

As described in Section 5.2.1, 22 time, frequency, kernel-based, actigraphy-specific and morphological features were extracted from each signal in Groups $A D L_{R}$ and $A D L_{E}$. Three different combinations of these features were used for modelling a LDA and a SVM tool for activity recognition. Following Tables 5.6-5.9 highlight the activity classification results using two classifiers. Correspondingly, Figures 5.22-5.27 illustrate the ROC curves and the confusion matrices for these activity recognition results.

Table 5.6: Activity Recognition using 22 features

\begin{tabular}{|c|c|c|c|c|c|c|}
\hline \multicolumn{7}{|c|}{$\begin{array}{l}\text { Features - Time, Frequer } \\
22 \text { Features } \\
\text { Label - Activity Type }\end{array}$} \\
\hline Group & Classifier & Accuracy (\%) & Sensitivity (\%) & Specificity (\%) & AUC & F1-Score \\
\hline \multirow{2}{*}{ Group $A D L_{R}$} & LDA & 89.2 & 91.3 & 91 & 0.98 & 0.94 \\
\hline & SVM & 89.2 & 83 & 91.2 & 0.84 & 0.83 \\
\hline \multirow{2}{*}{ Group $A D L_{E}$} & LDA & 91.4 & 85.4 & 91.7 & 0.97 & 0.84 \\
\hline & SVM & 93.5 & 90.7 & 94.3 & 0.99 & 0.92 \\
\hline
\end{tabular}



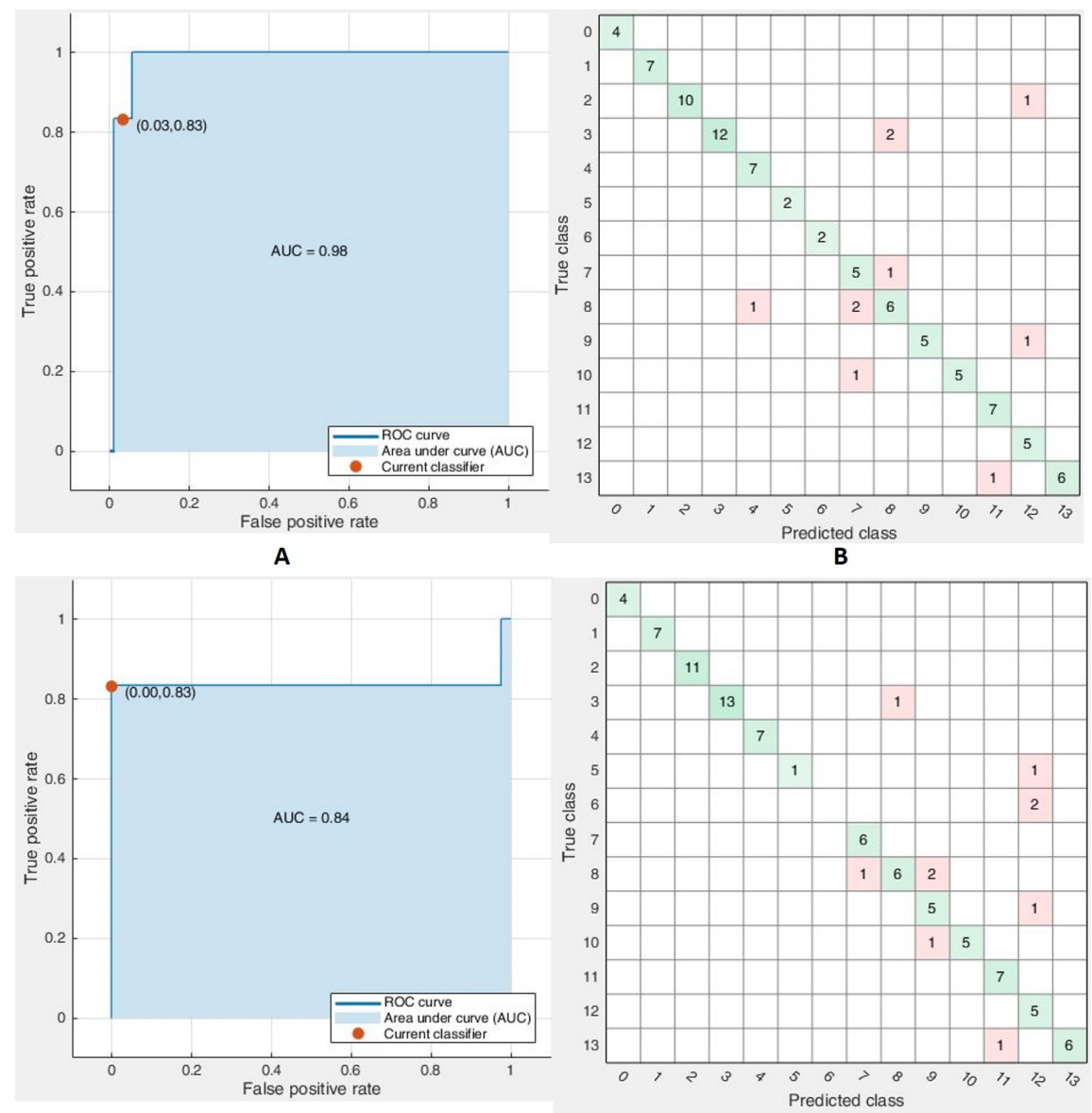

C

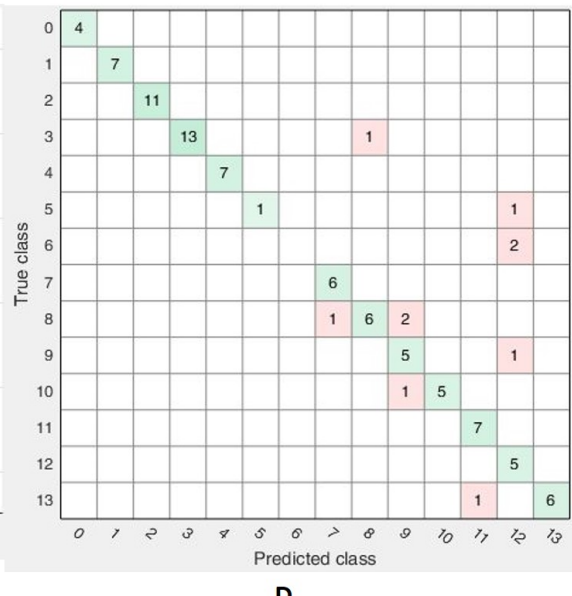

Figure 5.22: ROC Curves for Group $A D L_{R}$ in Table 5.6: (A) ROC for LDA, (B) Confusion Matrix for LDA, (C) ROC for SVM and (D) Confusion Matrix for SVM 

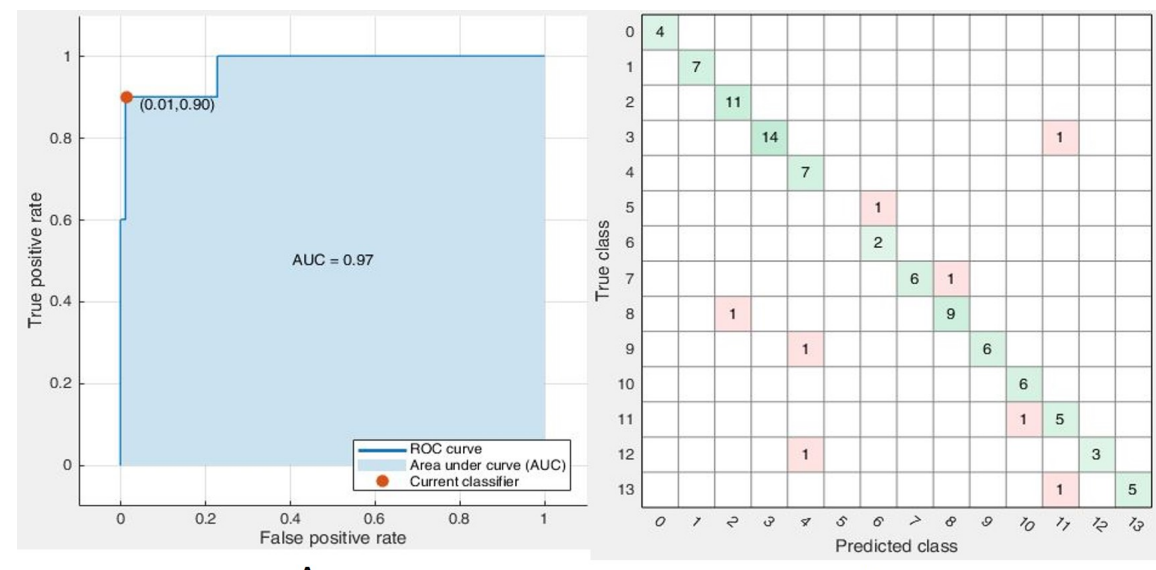

A

B

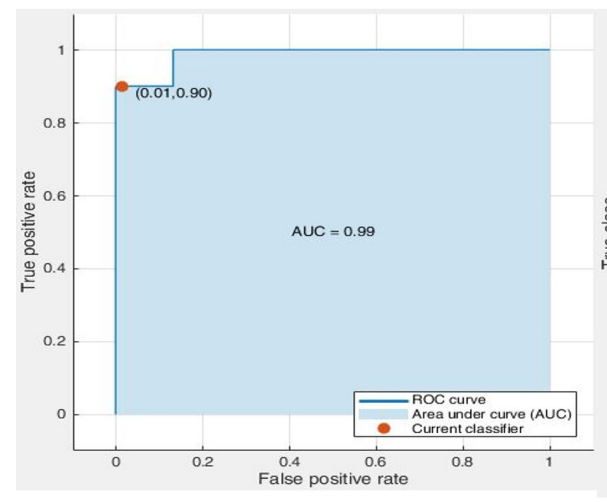

C

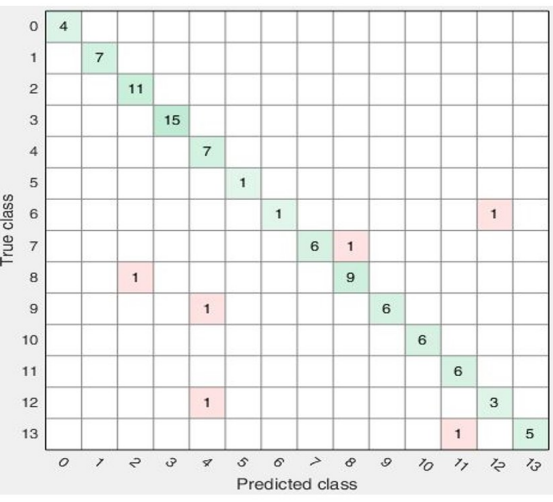

D

Figure 5.23: ROC Curves for Group $A D L_{E}$ in Table 5.6: (A) ROC for LDA, (B) Confusion Matrix for LDA, (C) ROC for SVM and (D) Confusion Matrix for SVM

In addition to these experiments, a mRMR feature selection [152] algorithm was executed to generate the top three discriminatory features which were - the Spiky Index, the standard deviation and the Peak-to-Peak difference. Table 5.7 highlights the classification results using these 5 features.

Table 5.7: Activity Recognition using top 5 mRMR features for encoded signals Features - Time, Frequency, Kernel-based, Actigraphy-specific, Morphological -

5 Features

Label - Activity Type

\begin{tabular}{clccccc}
\hline Group & Classifier & Accuracy (\%) & Sensitivity (\%) & Specificity (\%) & AUC & F1-Score \\
\hline \multirow{2}{*}{ Group $A D L_{E}$} & LDA & 57.3 & 46.2 & 54.6 & 0.75 & 0.53 \\
& SVM & 81.3 & 76.3 & 77 & 0.88 & 0.77 \\
\hline
\end{tabular}


Table 5.8: Activity Recognition using 9 features

Features - Kernel-based, Actigraphy-specific and Morphological- 22 Features

Label - Activity Type

\begin{tabular}{clccccc}
\hline Group & Classifier & Accuracy (\%) & Sensitivity (\%) & Specificity (\%) & AUC & F1-Score \\
\hline \multirow{2}{*}{ Group $A D L_{R}$} & LDA & 54.8 & 55 & 54 & 0.92 & 0.54 \\
& SVM & 82.8 & 77.5 & 80.4 & 0.97 & 0.78 \\
\hline \multirow{2}{*}{ Group $A D L_{E}$} & LDA & 52.7 & 50.5 & 53.7 & 0.9 & 0.48 \\
& SVM & 80.6 & 75.4 & 75.7 & 0.97 & 0.75 \\
\hline
\end{tabular}
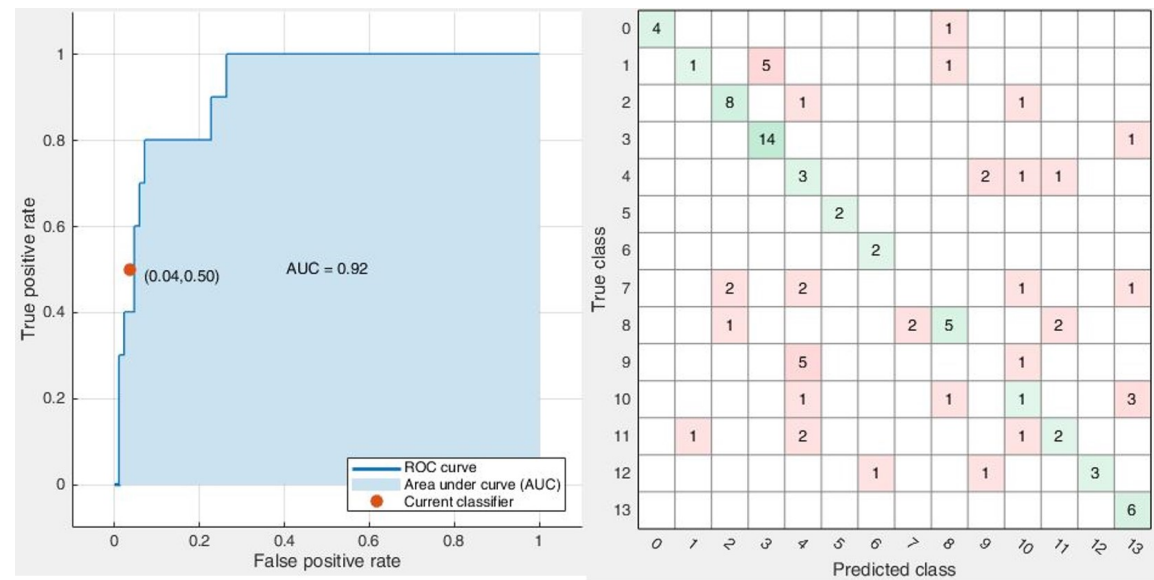

A
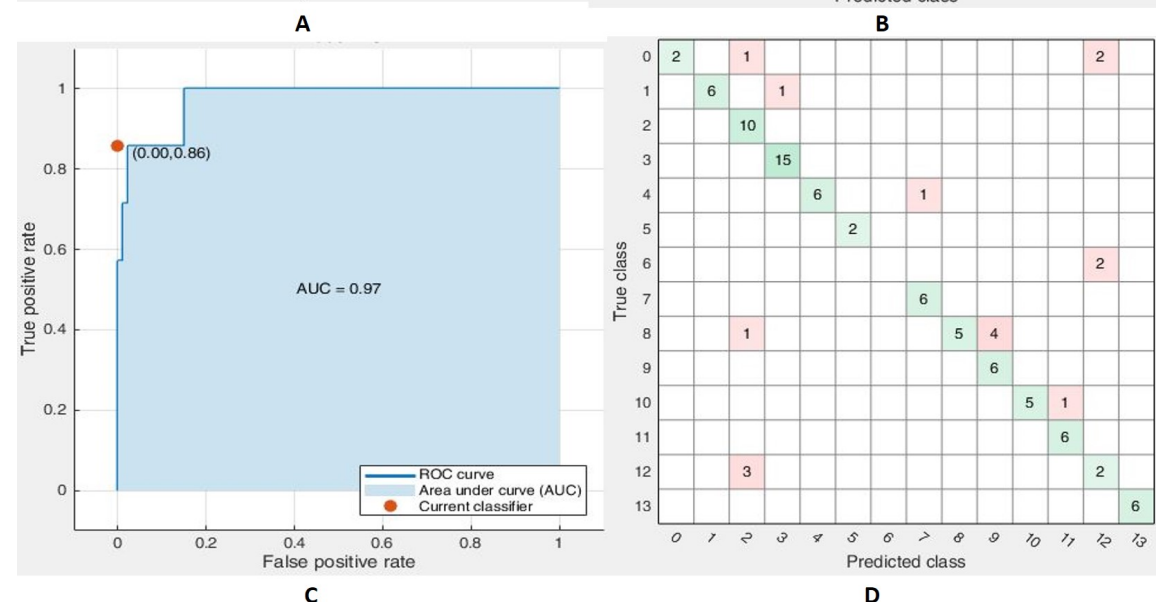

C

Figure 5.24: ROC Curves for Group $A D L_{R}$ in Table 5.8: (A) ROC for LDA, (B) Confusion Matrix for LDA, (C) ROC for SVM and (D) Confusion Matrix for SVM 

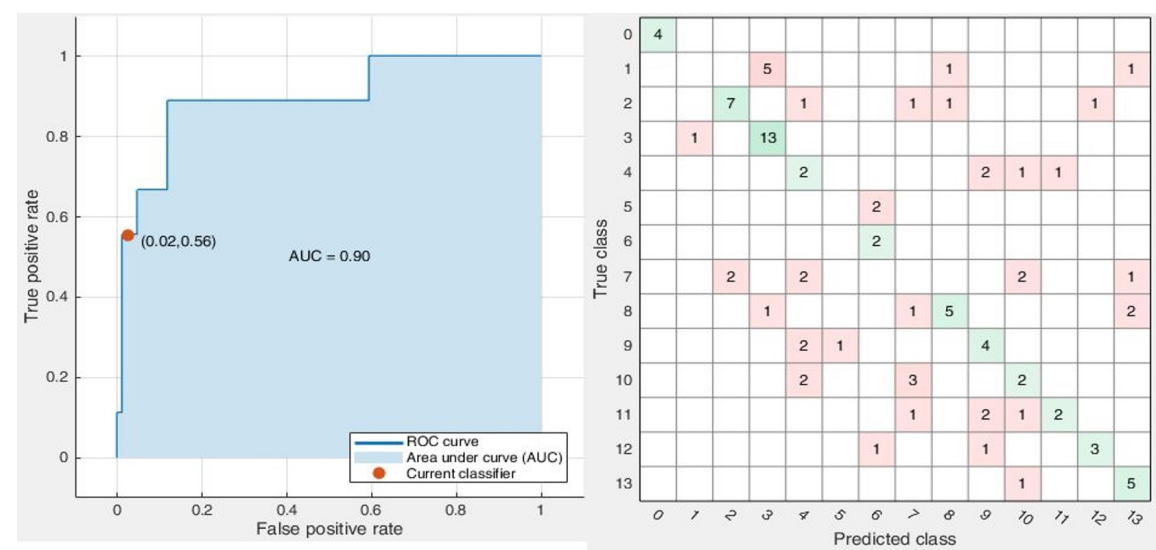

A

B

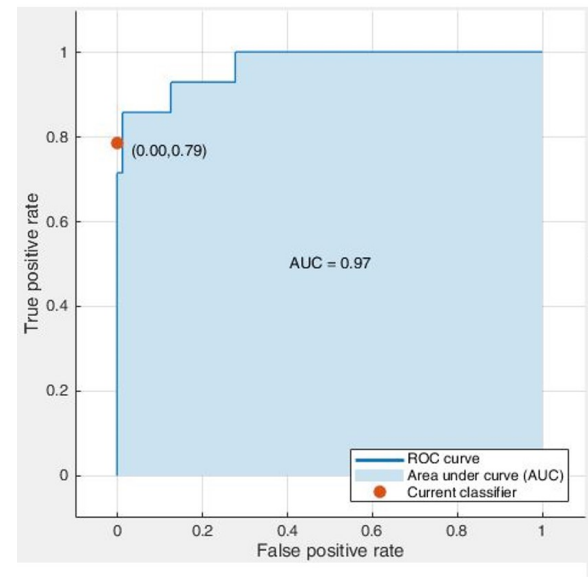

C

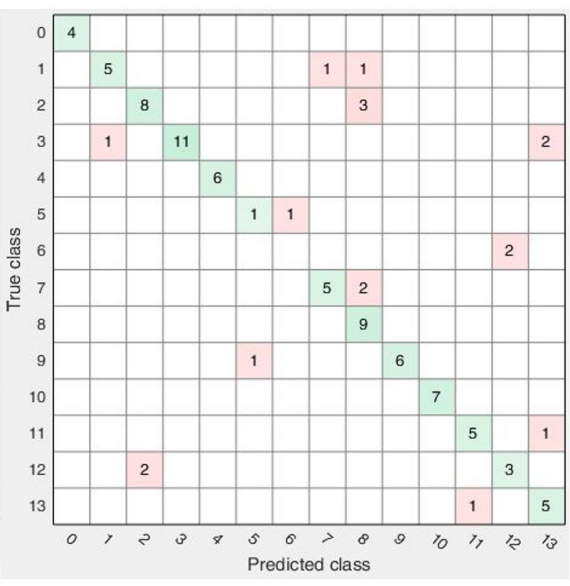

D

Figure 5.25: ROC Curves for Group $A D L_{E}$ in Table 5.8: (A) ROC for LDA, (B) Confusion Matrix for LDA, (C) ROC for SVM and (D) Confusion Matrix for SVM

Table 5.9: Activity Recognition using 15 features

Features - Time, Frequency and Actigraphy-specific - 15 Features

Label - Activity Type

\begin{tabular}{clccccc}
\hline Group & Classifier & Accuracy (\%) & Sensitivity (\%) & Specificity (\%) & AUC & F1-Score \\
\hline \multirow{2}{*}{ Group $A D L_{R}$} & LDA & 87.1 & 89.5 & 88 & 0.95 & 0.88 \\
& SVM & 89.2 & 88.6 & 93.1 & 0.99 & 0.91 \\
\hline \multirow{2}{*}{ Group $A D L_{E}$} & LDA & 87.1 & 89 & 88.9 & 0.99 & 0.89 \\
& SVM & 83.9 & 73.4 & 85.7 & 0.98 & 0.72 \\
\hline
\end{tabular}




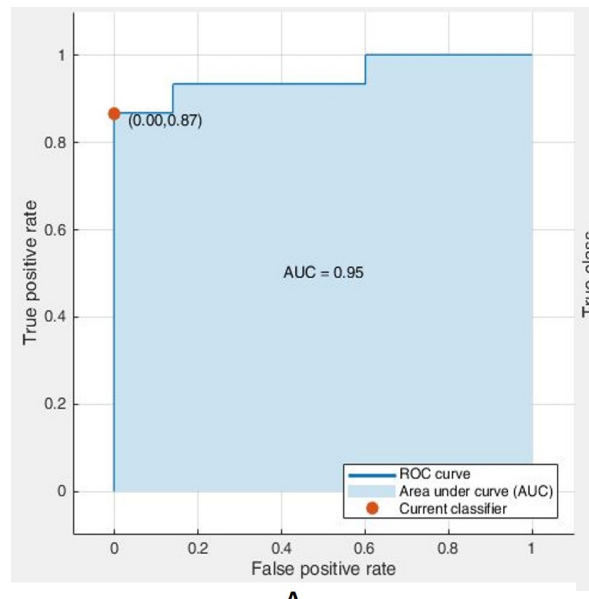

A

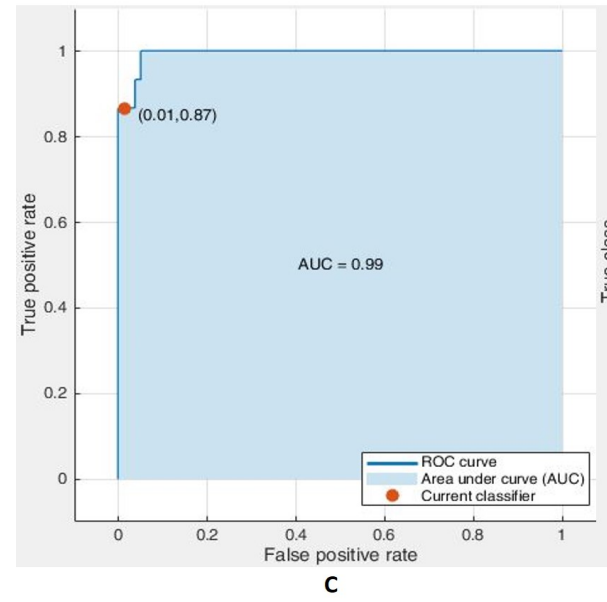

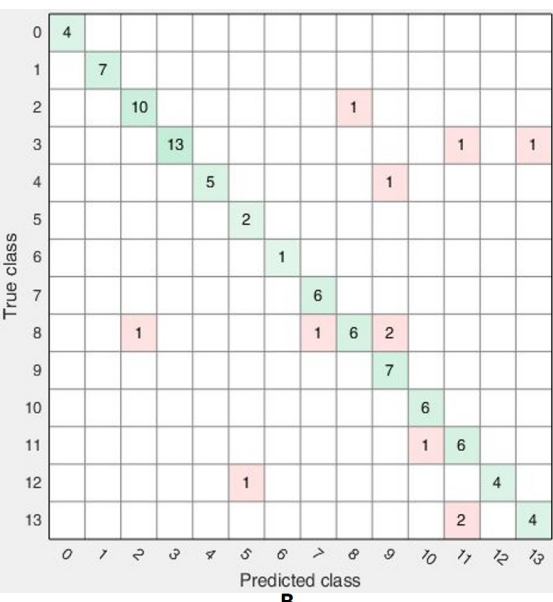

B

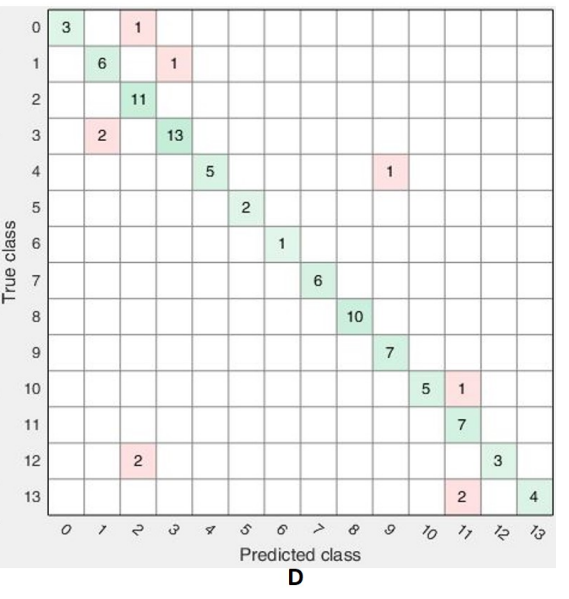

Figure 5.26: ROC Curves for Group $A D L_{R}$ in Table 5.9: (A) ROC for LDA, (B) Confusion Matrix for LDA, (C) ROC for SVM and (D) Confusion Matrix for SVM 

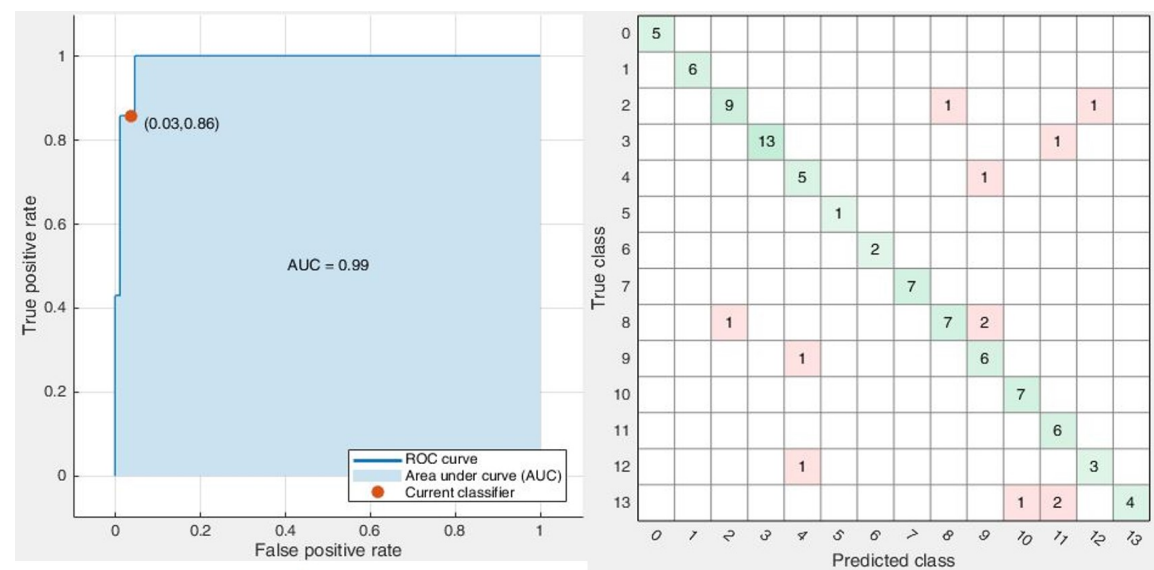

A

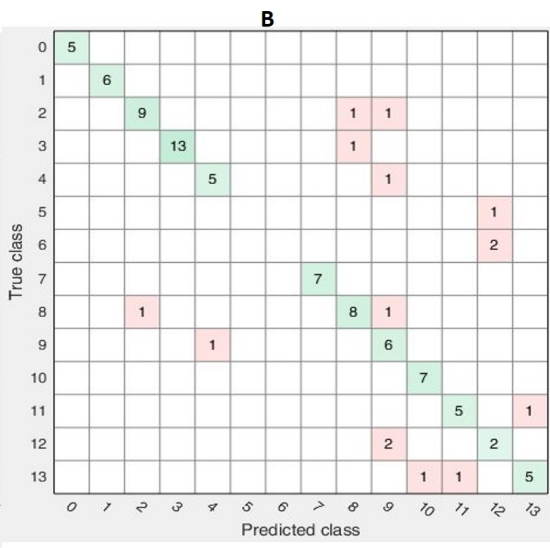

D

Figure 5.27: ROC Curves for Group $A D L_{E}$ in Table 5.9: (A) ROC for LDA, (B) Confusion Matrix for LDA, (C) ROC for SVM and (D) Confusion Matrix for SVM

To show that the signal encoding scheme improved the classification accuracy, classification experiments were done with different encoding levels from 3-6 bits, and it was found that the 3-bit encoding again ensured highest classification accuracy using a combination of 22 features. Figure 5.28 highlights this trend. 


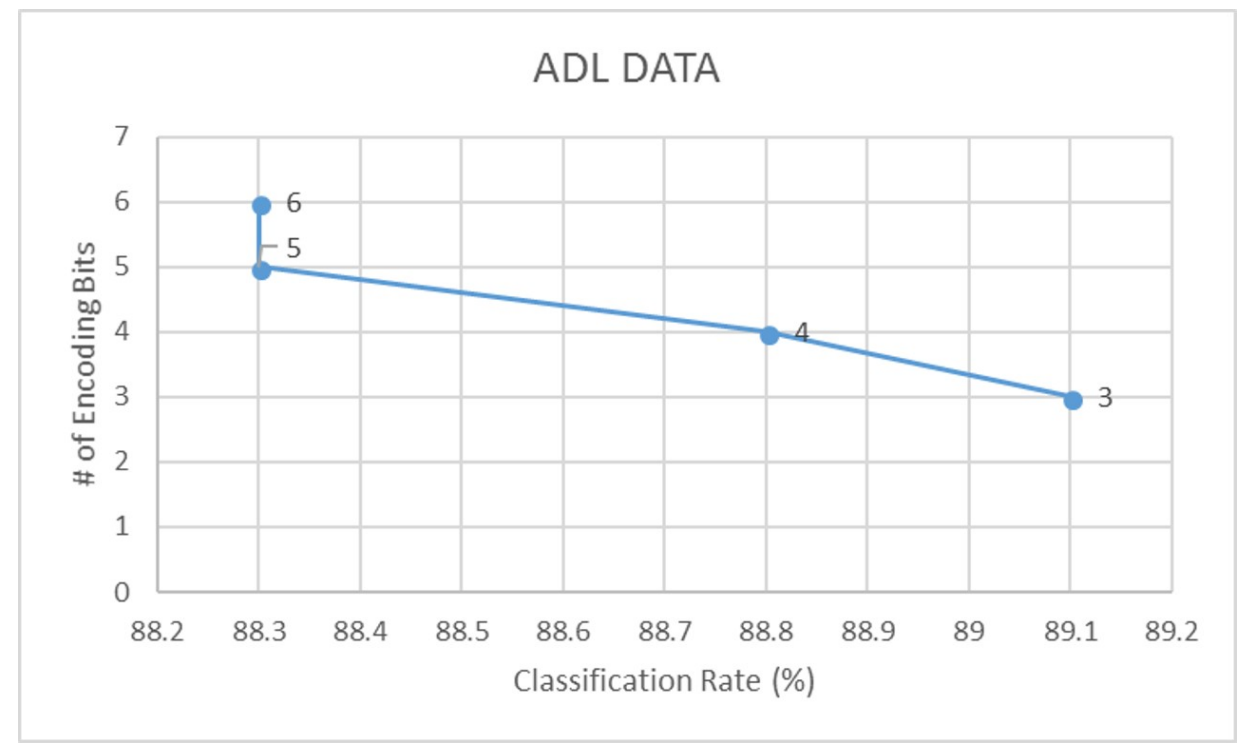

Figure 5.28: Classification Accuracy vs Encoding Levels for ADL Dataset

As evident from these results, though there has been a slight shift in the classification accuracy between raw and encoded signals, it can be inferred that given the right combination of features, signal encoding does improve activity recognition accuracy using linear SVMs. Following these remarkable results in daily activity recognition, the next Section 5.3 will now proceed to describing the application of the actigraphy analysis algorithms to vibroarthrography data for estimating the severity of knee-joint degeneration.

\subsection{Experiments with Database E}

In the final set of experiments demonstrated in this dissertation, the actigraphy analysis system was applied to classifying knee-joints based on the severity of their degeneration. Before proceeding with the experiments, the reader must first understand osteoarthritis and joint degeneration in brief.

\subsubsection{Joint Degeneration Disorders}

Joint-related disorders cause pain, movement disabilities and reduce the quality of life. One of the most common forms is Osteoarthritis (OA), and it affects about $10 \%$ men and $13 \%$ women aged more than 60 years in North America [154]. OA mostly affects common body parts such as knees, hips and hand joints. Acute pain during daily movements is a prominent symptom in OA, and is also a major factor in initiating its diagnosis. Our review of the literature indicates that most individuals experiencing difficulty in climbing stairs or walking, have shown signs of degeneration in their working joints [26,155]. Severe cases also account 
for a large number of individuals undergoing knee or hip replacement surgeries. Recent statistics in North America indicated that the lifetime risk of developing joint degeneration was about $40 \%$ in men and $47 \%$ in women. This percentage rose to about $60 \%$ in individuals having body mass index (BMI) greater than 30 [154]. Demographic trends from Canada indicated that the number of individuals (especially elderly citizens) undergoing joint replacement surgeries rose by about $20 \%$ between 2014-2015. This has risen in the recent years and accounts for the rehabilitation and physiotherapy care for almost 120,000 Canadians annually [156]. Similarly, a recent study conducted by Kremers et al. in 2010 yielded that over 4.7 million Americans have undergone knee replacement surgery and 2.5 million have undergone hip replacements. This includes general population, war veterans, athletes and elderly citizens [157].

Being one of the most significant support for human structure, the knee joints are a critical lower-body part, and support basic human movements such as walking, running and sitting. Knee joints are connected to each other via the cartilage, which is about $70 \%$ liquid state protein and $30 \%$ connective tissue $[101,155]$. Cartilage degeneration, and hence onset of OA occurs when knee joints are extensively used during one's lifespan, thus reducing the liquid protein in the cartilage, thereby leading to friction between tissue and bone. A complete loss of cartilage due to aggravated bone-tissue friction, could lead to severe OA and may even lead to a knee replacement surgery. This disorder has a multi-factor etiology, and could be considered the outcome of a combination of systemic and environmental factors. Factors causing joint degeneration include: age, diet, overweight and obesity, knee injury, occupation involving repetitive use of joints, bone density, muscle weakness and joint laxity [154]. Figure 5.29 illustrates the degeneration of cartilage in knee joints leading to OA.

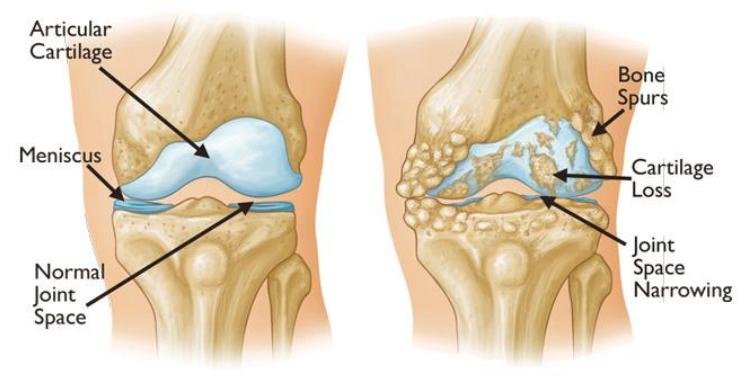

Figure 5.29: Effects of Osteoarthritis [25]

\section{Detection and Treatment of Joint disorders}

Detecting joint disorders using simple laboratory tests is not possible and requires the use of invasive methods. Two standard practices are usually employed in detecting joint disorders, namely: Arthrocentesis and 
Arthroscopy $[27,101]$. Both techniques are invasive, and require the examination of joint fluid content, to estimate the severity of degeneration. In case of arthrocentesis, the joint fluid is extracted using a surgical needle and analyzed for infections and damage. This is followed by injection of corticosteroids into the joints, which relieve pain, swelling and inflammation. It should be noted that use of steroids is beneficial in treating cartilage damage, if detected in early stages. These steroids help in rebuilding the fluid protein and maintaining overall cartilage health. On the other hand, in case of arthroscopy, the physician inserts a viewing tube between the joints to determine degeneration severity, and is able to repair any issues without the need of extensive surgery $[101,155]$.

Apart from invasive methods, joint disorders could also be detected using computer-aided, non-invasive techniques such as X-ray, CT scan, ultrasound or a radiograph. For example, X-ray can help detect the loss of cartilage fluid by showing the knee cap and joint bones more visible. Additionally, it also helps in excluding other causes of joint pain, thereby helping the physician in deciding if surgery is needed [27,101]. Figure 5.30 illustrates an X-ray image of an OA affected joint.

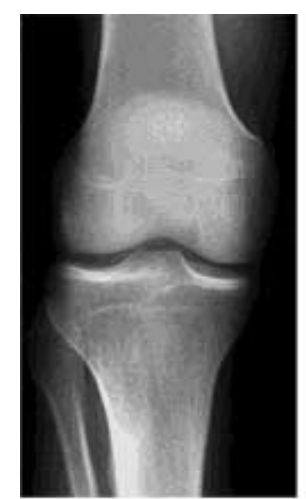

Normal knee

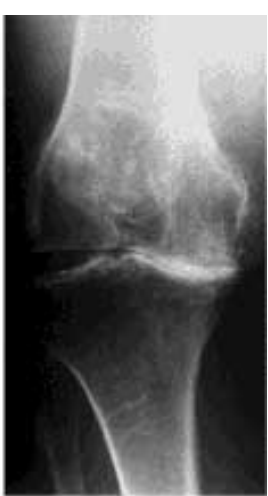

Osteoarthritic

Figure 5.30: X-ray of OA joints [26]

Also in prior studies, some researchers have also employed the use of vibroarthrography (VAG) as a non-invasive monitoring tool to determine the severity of $\mathrm{OA}[27,101]$. In this procedure, the subject is made to perform specific movements whose amplitude is recorded using accelerometer-based sensor affixed to the joint under observation. This movement data is usually recorded for a short-duration (usually less than a minute) for each activity, and is then subjected to audification; i.e. the movement amplitudes are converted to audible frequencies. The physician then listens to these sounds, and is able to identify the severity of OA $[27,101]$. Usually, the presence of a cracking sound in joint movements indicates cartilage damage and possible arthritis. In addition, these VAG signals also yield information such as joint density, 
roughness and cartilage lubrication. Also in comparison to standard methods, vibroarthrography stands out in the following ways [27]:

- Imaging techniques such as X-ray are unable to capture minute cartilage changes, which otherwise significantly change the properties in a VAG signal.

- Standard clinical methods to detect OA symptoms are quite expensive for repeated use. Whereas, VAG signal acquisition is easy to setup and cost effective, as it requires only the use of a strap-on actigraph to capture joint-movement data.

- The portability of VAG actigraph and its ease of data acquisition, makes it conducive for a physician to operate it with minimal training.

- In conjunction to this, it is highly feasible to use actigraphy as an initial OA screening tool in a home-based environment as it would help in monitoring post-surgery recovery for individuals.

Despite these benefits, vibroarthrography studies need more data and clinical trials before being accepted as a viable tool for detecting joint disorders or disabilities. For the reader's simplification, vibroarthrography is the audified version of actigraphy. Figure 5.31 shows how a VAG signal is acquired.

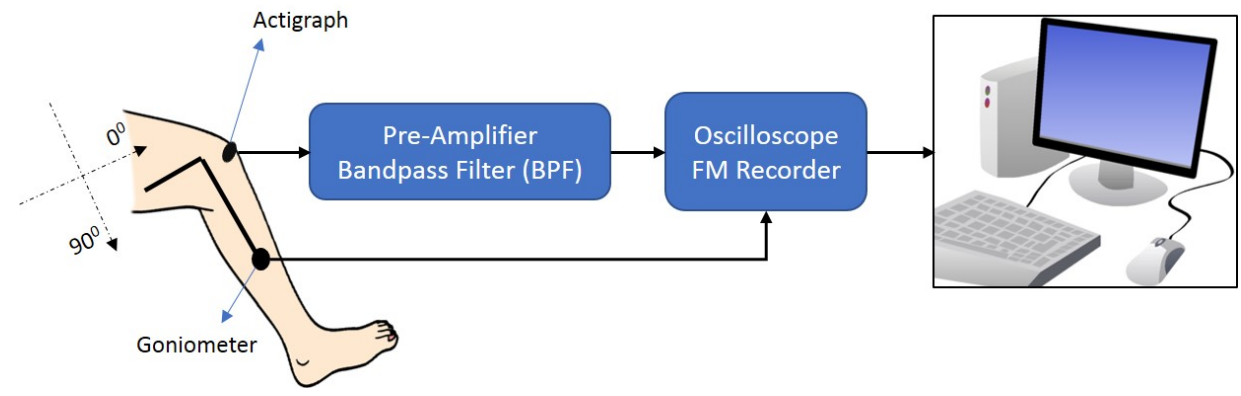

Figure 5.31: VAG signal acquisition [27]

\section{$\underline{\text { Prior studies employing VAG signal analysis }}$}

Our literature review indicates that numerous works have been conducted in VAG signal analysis using intelligent signal processing techniques, which have yielded promising results. These works mostly involved extracting characteristic features from VAG signals, and feeding them to a machine learning algorithm to identify joint disorder severity. For example, Krishnan et al. implemented a time-frequency distribution algorithm, which transformed one-dimensional VAG data into 2D time-frequency plane which led to identifying VAG severity $[27,158,159]$. Many research groups such as those of Boniatis et al. [160], Gregory et al. [161], Wu et al. [162] and Duryea et al. [163] implemented neural networks for classifying arthritic patients 
as well as identifying gait patterns. On the other hand, some researchers such as Levinger et al. [164] and Lai et al. [165] employed the use of support vector machines for estimating OA pain severity, and also in detecting recovery from knee replacement surgery using quantitative gait measures. Other prior studies, such as those conducted by Rangayyan et al. $[166,167]$ and Deluzio et al. [168], extracted biomechanical and statistical features from VAG signals for OA severity analysis. In a previous study [101], Fisher kernels were applied to map VAG signals into higher dimensions for pattern classification and visualization. From these experiments, Fisher kernels were able to correctly classify between normal and abnormal VAG signals with an accuracy of $84 \%$. In recent years, knee sound acoustics were also analyzed by Inan et al. [70,71,169-171] for assessing knee joint health in war veterans and professional athletes during rehabilitation, as well as in real-time activity classification applications using wearables.

\subsubsection{Contributed solution}

Although these works produced fruitful outcomes in vibroarthrography analysis, many of them exhibited a few shortcomings:

- Methods implemented directly using neural networks or other transform-based techniques, do not have an explanatory power. These techniques primarily perform a mathematical transformation of input VAG data into a reduced feature space. Inclusion of morphological parameters and hence the ground truth is minimal, and so it becomes trivial to explain the science behind correct or incorrect classification. For example, although the previous research in applying Fisher kernels to VAG actigraphy data yielded promising classification results, it did not consider disease morphology and simply transformed a 1D time-series into higher dimensions for pattern visualization [101].

- The algorithms implemented in prior studies were mostly developed for offline data analysis and hence may not be readily suitable for IoMT based applications.

- The feasibility of implementing these methods along with suitable accelerometer sensors on mobile devices is low, and needs much improvement in terms of algorithm efficiency as well as hardware design. Hence it becomes trivial to apply them in a smart monitoring environment.

In this study, the intention was to address these obstacles by applying a combination of actigraphy-specific encoding, segmentation and feature extraction techniques for analyzing VAG signals to estimate the severity of cartilage degeneration. It should be noted that although in this research work the analysis techniques have been applied to short duration VAG signals, previous experiments (see Chapter 4) in actigraphy signal analysis suggest that this work could also be extrapolated to analyse long-duration VAG data. The algorithm 
in this research work could potentially be used as a monitoring tool in a home-based environment, as well as for augmenting and validating a physician's diagnosis. Following flowchart in Figure 5.32 illustrates the methodology in this study.

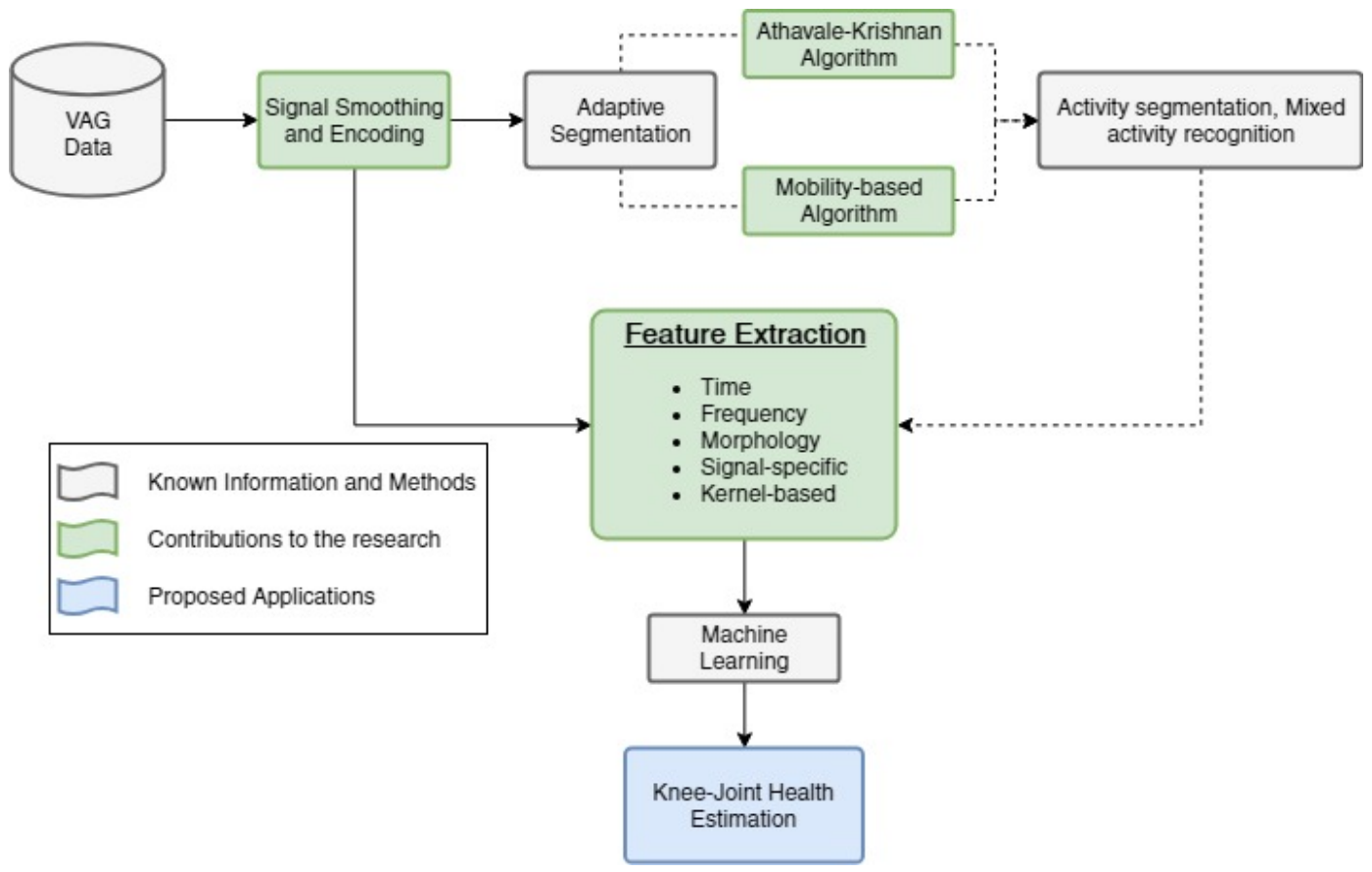

Figure 5.32: Proposed Knee Vibroarthrography Analysis System

\subsubsection{Materials and Methods}

\section{Data Acquisition}

In this study, the actigraphy-based vibroarthrography (VAG) dataset was collected by Krishnan et al. [27] from 89 individuals. 38 subjects were labelled as normal (or healthy) and 51 were labelled as abnormal (or unhealthy) based on the severity of their cartilage degeneration (see Table 5.10).

Table 5.10: Demographics - VAG Data, Database E

\begin{tabular}{lccc}
\hline Joint Condition & No. of Participants & Age $(\mathbf{m e a n} \pm \mathbf{s d})$ & Male:Female Ratio \\
\hline Normal & 51 & $28 \pm 9.54$ & $22: 29$ \\
Abnormal & 39 & $35 \pm 13.89$ & $21: 18$ \\
\hline
\end{tabular}

For each subject, the VAG data was acquired using the following procedure [27]:

1. Each subject was strapped on the knee-joint surface with a single-axial actigraph and made to sit on a table with legs suspended. 
2. The subject was then asked to perform a swing motion of the knee from $135^{\circ}$ to $0^{\circ}$ and back to $135^{\circ}$ within a period of 4 seconds. This motion was recorded by the surface actigraph.

3. The knee movement angles were recorded using an electronic goniometer, attached to the lateral side of the knee. The labelling of an individual was done on the basis of the goniometer angle and the corresponding knee sound recorded in VAG.

4. The VAG signals were digitized with a sampling frequency of $2 \mathrm{kHz}$ and a quantization resolution of 12 bits/sample.

Figure 5.33 illustrates sample VAG signals acquired from a normal (healthy) and an abnormal (unhealthy) knee joint movements. Note that the average duration for each VAG signal is about 4 seconds, and its signal to noise ratio $(\mathrm{SNR})$ is in the range of -0.1 to $-16.2 \mathrm{~dB}$.
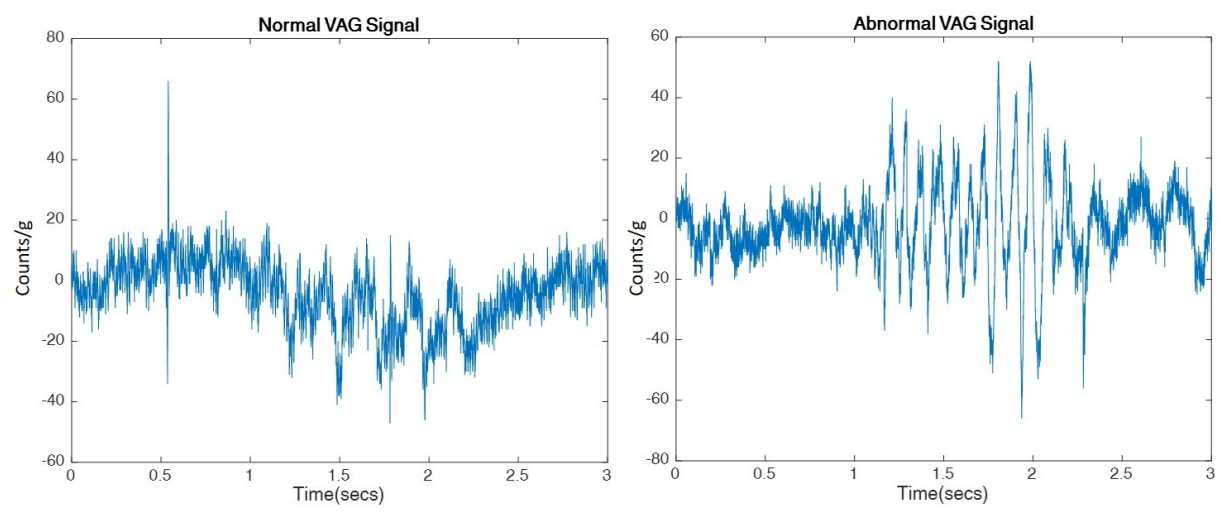

Figure 5.33: Sample VAG signals [27]

\section{VAG data encoding}

Using the 89 actigraphy signals, two distinct groups namely- $V A G_{R}$ and $V A G_{E}$ were created, with each comprising of 89 signals conditioned in the following ways:

\section{- Group $V A G_{R}$ :}

Each VAG signal in this group was pre-processed using the following steps:

1. For each signal $S_{R}$ the missing or $\mathrm{NaN}$ (Not a Number) values were removed.

2. Next, the signal was normalized using the expression,

$$
S_{N}=\frac{\left(\max \left(S_{R}\right)-S_{R}\right)}{\left(\max \left(S_{R}\right)-\min \left(S_{R}\right)\right)}
$$


3. The normalized signal $S_{N}$ was then de-trended by subtracting the mean from each sample as,

$$
S_{D}=S_{N}-\frac{\sum_{n} S_{N}}{n}
$$

\section{- Group $V A G_{E}$ :}

For the second group, each VAG signal was conditioned using the following steps:

1. After removing the missing values, normalizing the signal and de-trending it using Equations 5.13 and 5.14, a $m$-bit encoding of each actigraphy signal $S_{D}$ was conducted, such that $m \ll r$, where $r$ is the quantization resolution of the raw signal $S_{R}$, and $r=12$ bits/sample. The encoding was performed using the expression,

$$
S_{E}=\left\lfloor\left(S_{D} \times \frac{\left(2^{m}-1\right)}{2}+\frac{\left(2^{m}-1\right)}{2}\right)\right\rfloor
$$

Using these steps two groups of actigraphy signals were obtained from the VAG dataset, as - $V A G_{R}$ containing 89 normalized and de-trended actigraphy signals, and $V A G_{E}$ containing 89 encoded signals. For the reader's reference, in this study as well, a 3-bit encoding was applied to the VAG data in Group $V A G_{E}$. A parametric comparison between raw and encoded VAG signals has been illustrated in Section 5.3.4. Following this, the next section proceeds towards applying the AK and mobility-based segmentation algorithms to encoded VAG signals in Group $V A G_{E}$.

\section{Encoding-based segmentation}

In this study of short-duration, VAG signals, the AK and Mobility-based adaptive segmentation techniques were tested on the encoded signals for identifying those regions of knee-joint movements which generate crackling-like sounds indicating the severity of OA in a test subject. First, the AK segmentation algorithm was applied to an encoded signal $S_{E}$ through the following steps. The encoded and vector compounded actigraphy signal $S_{E}$ was obtained as shown from Equation 5.15. Using this the first order and the subsequent second order difference signals were computed as,

$$
d S_{e}=\left[S_{e_{1}},\left(S_{e_{2}}-S_{e_{1}}\right), \ldots,\left(S_{e_{n}}-S_{e_{n-1}}\right)\right]
$$

and,

$$
2 d S_{e}=\left[d S_{e_{1}},\left(d S_{e_{2}}-d S_{e_{1}}\right), \ldots,\left(d S_{e_{n}}-d S_{e_{n-1}}\right)\right]
$$


The RCF of the signal was then calculated using its second order difference version, using the expression,

$$
R C F=\frac{\text { Step }- \text { Size }}{\left(m \times t_{s}\right)}
$$

where $t_{s}$ is the sampling period. Next, the PSBs were identified by finding all the signal samples in $2 d S_{E}$ which were greater than the RCF,

$$
P S B_{i}=2 d S_{E_{n}} \forall\left\langle 2 d S_{E_{n}}>R C F\right\rangle
$$

where $i$ is the number of PSBs. From an extensive analysis of VAG signals, the following set of observations were obtained for deducing the secondary and final segment boundaries:

1. Most prominent knee-joint movements last for about 0.05 seconds. These capture the knee-joint activity when the subject moves the leg up from resting position, and when the subject brings down the leg back to resting position.

2. The time-period between peak knee movements is about 0.25 seconds.

Using these observations, the SSBs were detected by identifying PSBs which were separated by at least 0.05 seconds, as,

$$
S S B_{j}=P S B_{i} \forall\left\langle\left|t_{P S B_{i}}-t_{P S B_{i+1}}\right|>0.05\right\rangle
$$

where $j$ is the number of SSBs. Following this, the FSBs were computed by extracting only those SSBs which were at least 0.25 seconds apart,

$$
F S B_{l}=S S B_{j} \forall\left\langle\left|t_{S S B_{j}}-t_{S S B_{j+1}}\right|>0.25\right\rangle
$$

Using the FSBs, one could then segment the encoded VAG signal into smaller regions of interest.

Following this, the next of experiments were done with the application of the mobility-based adaptive segmentation scheme to encoded VAG data, using the steps as described. Using Eqns.5.18 and 5.19 the second order difference signal of the encoded data $S_{E}$ was computed. The PSBs were identified by detecting all the samples in $2 d S_{E}$ which were greater than a factor of $\frac{\max _{n} 2 d S_{E}}{\left(2^{m}-1\right)}$, such that,

$$
P S B_{i}=2 d S_{E_{n}} \forall\left\langle 2 d S_{E_{n}}>\frac{\max _{n} 2 d S_{E}}{\left(2^{m}-1\right)}\right\rangle
$$

Using these PSBs, the SSBs were computed such that the Hjorth's mobility values of two segments were 
unique from each other.

$$
S S B_{j}=P S B_{i} \forall\left\langle\left\lfloor M_{P S B_{i: i+1}}\right\rfloor \neq\left\lfloor M_{P S B_{i+1: i+2}}\right\rfloor\right\rangle
$$

Using the afore-mentioned observations on VAG data, the FSBs were found such that two SSBs were at least 0.05 seconds apart.

$$
F S B_{l}=S S B_{j} \forall\left\langle\left|t_{S S B_{j}}-t_{S S B_{j+1}}\right|>0.05\right\rangle
$$

The results of VAG segmentation using the AK and Mobility-based algorithms have been illustrated in Section 5.3.4. In the next few sections, the feature extraction and classification methodologies adopted for Groups $V A G_{R}$ and $V A G_{E}$ respectively will be described, followed by highlighting the results from the encoding, segmentation and classification approaches applied to VAG data.

\section{Feature Extraction and Classification}

The next step in VAG signal analysis after pre-processing was to conduct feature extraction and perform machine learning modelling. From both the sets of 89 VAG signals in Groups $V A G_{R}$ and $V A G_{E}, 21$ time, frequency, kernel-based, actigraphy-specific and morphological features were extracted, as highlighted in Table 5.11. 
Table 5.11: Features extracted from Groups $V A G_{R}$ and $V A G_{E}$ VAG signals

\begin{tabular}{|c|c|c|}
\hline Domain & No. & Features Used \\
\hline \multirow{10}{*}{ Time } & 1 & Mean \\
\hline & 2 & Standard Deviation \\
\hline & 3 & Variance \\
\hline & 4 & RMS \\
\hline & 5 & Maxima \\
\hline & 6 & Peak-to-RMS ratio \\
\hline & 7 & Peak-to-average ratio \\
\hline & 8 & Peak-to-average power ratio \\
\hline & 9 & Band power \\
\hline & 10 & Signal to noise \& distortion ratio \\
\hline \multirow{2}{*}{ Frequency } & 11 & Median Frequency \\
\hline & 12 & Mean Frequency \\
\hline Kernel-based Features & $13-16$ & ACF Kernel Scores (4 Features) \\
\hline \multirow{2}{*}{ Actigraphy-specific } & 17 & Rapid Change Factor \\
\hline & 18 & Spiky Index \\
\hline \multirow{3}{*}{ Morphological } & 19 & Hjorth's First order mobility \\
\hline & 20 & Hjorth's Second order mobility \\
\hline & 21 & Hjorth's complexity \\
\hline Other Labels & 22 & Type of Knee Joint - Normal, Abnormal \\
\hline
\end{tabular}

Using the pre-defined normal and abnormal labels, and a 70-30 partition of training and test sets, a Naive-Bayes, a LDA and a SVM machine learning tool were modelled for pattern classification. It should be noted that in these set of experiments, two different combinations of features were used for modeling the machine learning tools and comparing their performances. The results from these experiments have been shown in the next Section 5.3.4.

\subsubsection{Results from Experiments on Database E}

\section{VAG Encoding Results}

As described in Section 5.3.3, the purpose of the $m$-bit encoding was to ensure on-the-fly data compression and denoising of VAG data, without losing significant information. Figure 5.34 shows a sample VAG signal and its 3-bit encoded version. 

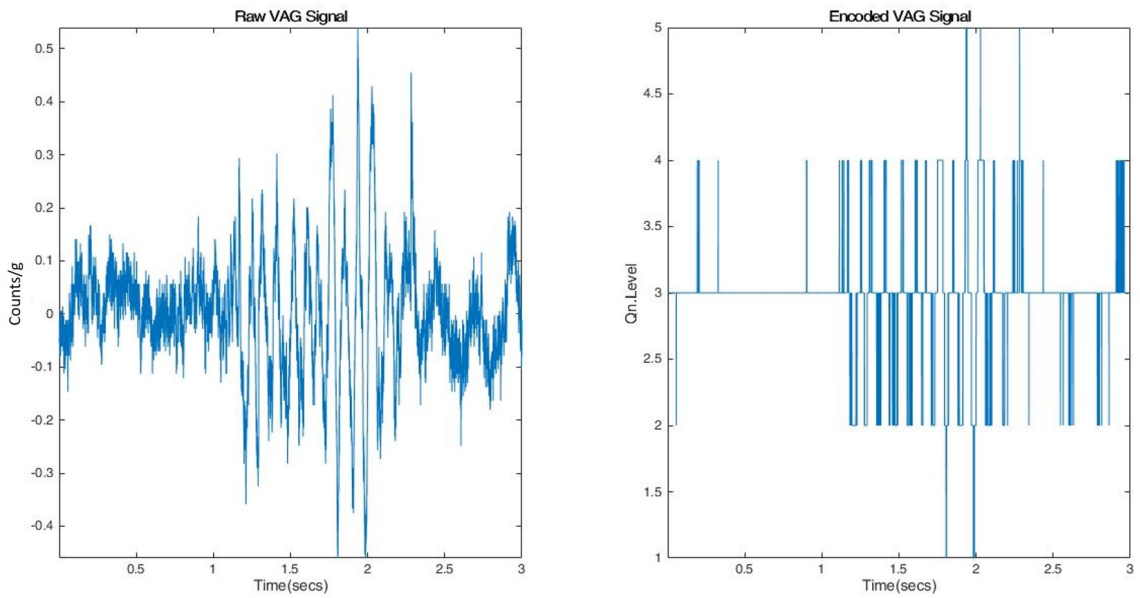

Figure 5.34: Sample VAG signal and its 3-bit encoded version

The 3-bit encoding was applied to all the 89 VAG signals, and an averaged parametric comparison was performed with their raw versions, as highlighted in Table 5.12 and Figure 5.35.

Table 5.12: Parametric comparison between raw and encoded VAG signals

\begin{tabular}{lcc}
\hline \multicolumn{1}{c}{ Parameter } & Raw Signal & Encoded Signal \\
\hline Avg. File Size (kB) & 133 & 16 \\
Bit Rate (kilobits/sec) & 30 & 7.5 \\
Avg. File Read Time (secs) & 0.5 & 0.2 \\
Avg. SNR (dB) & -0.1 & 19.9 \\
\% Space Savings & NA & $88 \%$ \\
\hline
\end{tabular}




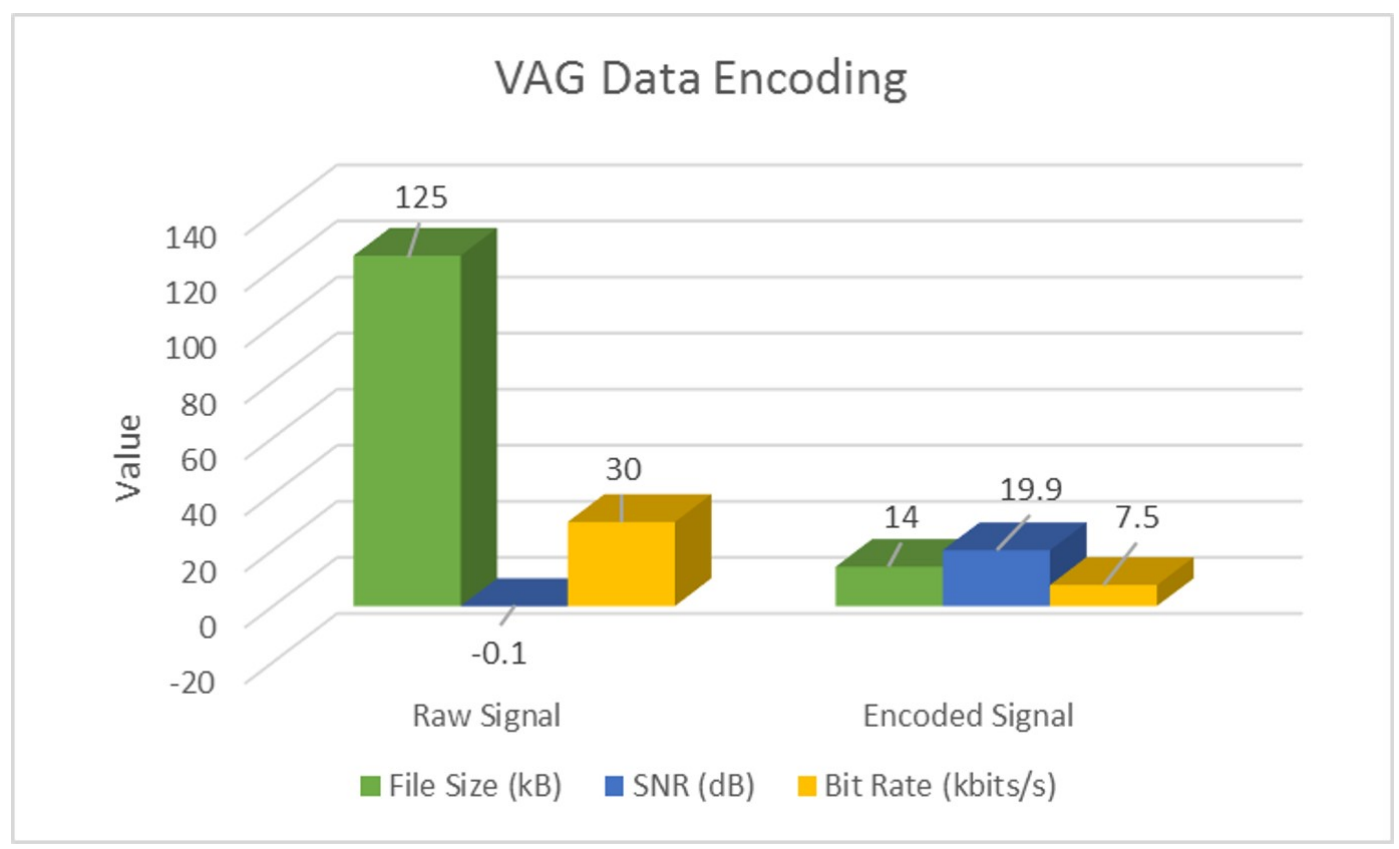

Figure 5.35: 3-bit encoding of VAG Data

As evident from Figure 5.35, the 3-bit encoding proposed in this research, worked exceptionally well in the case of VAG signals. A lower-bit encoding ensured a high space savings coupled with improved SNR and lowered bit rate. In the Section, the segmentation results obtained after applying the AK and mobility-based algorithms on VAG signals will be illustrated.

\section{Encoding-based Segmentation Results}

As described in Section 5.3.3, the AK and mobility-based adaptive segmentation schemes were applied to VAG signals, for identifying regions of peak movements. Specifically, the knee-bending process producing crackling-like sounds and their average time duration was recorded for further analysis. These observations were plugged into the AK and mobility-based algorithms to segment each VAG signal. Following Figures 5.36 and 5.37 illustrate the results from adaptive segmentation experiment on a sample VAG signal. 

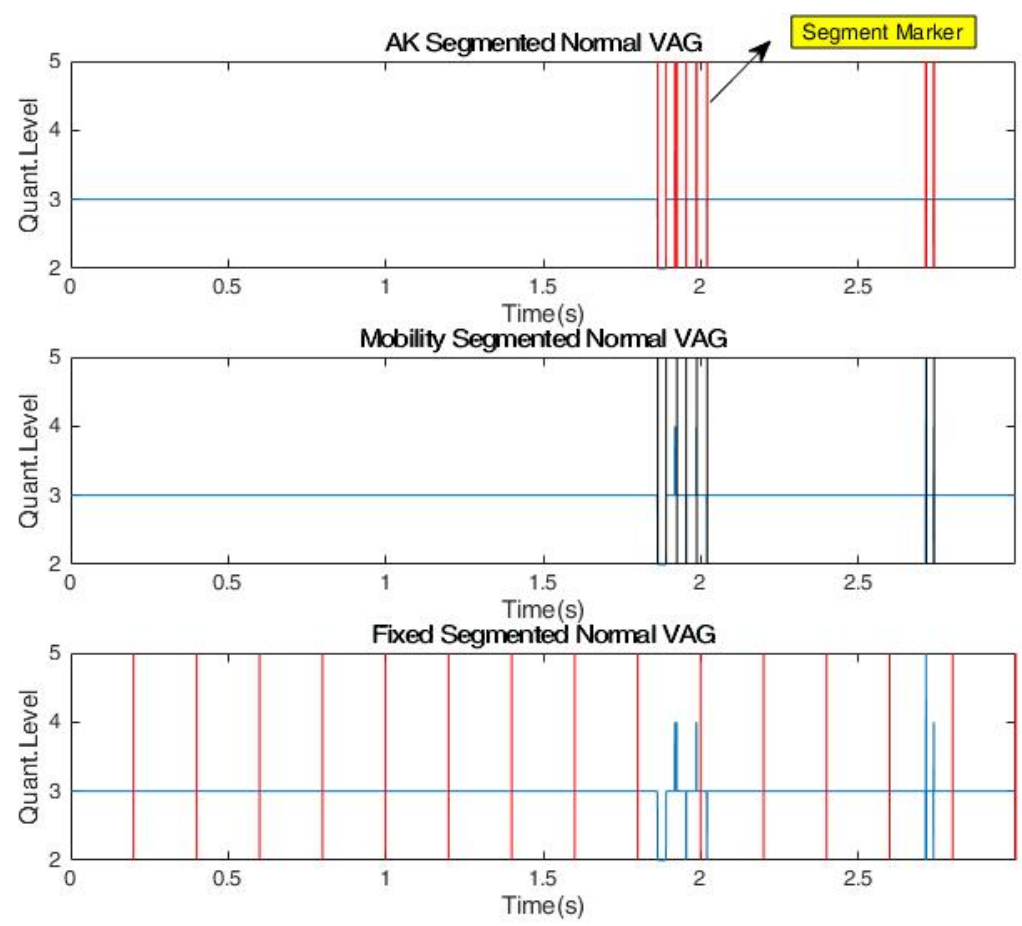

Figure 5.36: Adaptive Segmentation of a sample normal VAG signal
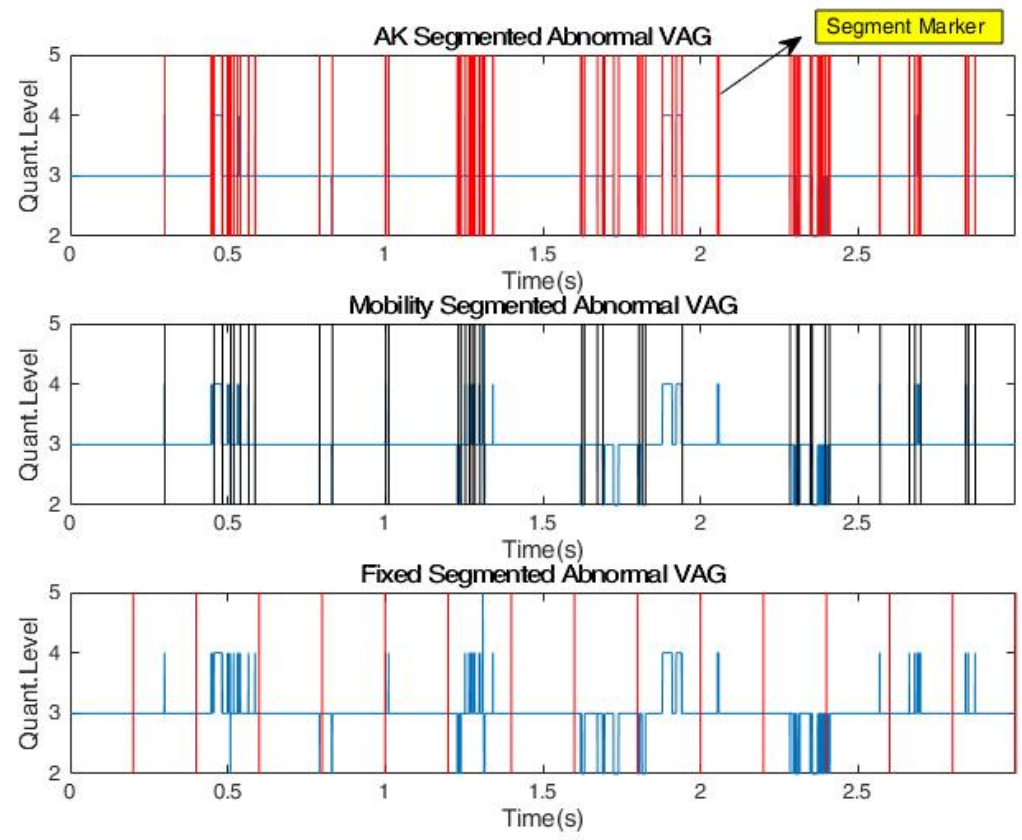

Figure 5.37: Adaptive Segmentation of a sample abnormal VAG signal 
Additionally, the number of segments generated by each method were also recorded and compared with a fixed segmentation scheme for the sample signal in Table 5.13 .

Table 5.13: Fixed, AK and Mobility-based segments for sample VAG signals

\begin{tabular}{cccc}
\hline Signal & No. Fixed Segments & No. AK Segments & No. Mobility-based Segments \\
\hline Normal VAG & 16 & 9 & 10 \\
Abnormal VAG & 16 & 33 & 34 \\
\hline
\end{tabular}

As evident from Figures and Table, the AK method performed better than the mobility-based algorithm for most VAG signals by segmenting them into smaller number of segments. This also indicated that adaptive segmentation of actigraphy signals would also help in data compression at the source, by retaining only regions of interest for further analysis.

\section{Feature Extraction and Classification Results}

Using three different combinations of the 21 features from VAG signals, a LDA and a SVM machine learning tool were modelled to classify normal and abnormal subjects. The modeling was done for feature sets created for both Groups $V A G_{R}$ and $V A G_{E}$. Following Tables 5.14-5.17 highlight the classification results of VAG data using three machine learning models. Corresponding to this, the ROC curves have been illustrated in Figures 5.38-5.43.

Table 5.14: Classification of VAG data using 21 features

\begin{tabular}{|c|c|c|c|c|c|c|}
\hline Group & Classifier & Accuracy (\%) & Sensitivity (\%) & Specificity (\%) & AUC & F1-Score \\
\hline \multirow{3}{*}{ Group $V A G_{R}$} & Naïve-Bayes & 80.7 & 75 & 85.7 & 0.8 & 0.78 \\
\hline & LDA & 73.1 & 72.2 & 75 & 0.66 & 0.77 \\
\hline & SVM & 76.9 & 71.4 & 100 & 0.78 & 0.83 \\
\hline \multirow{3}{*}{ Group $V A G_{E}$} & Naïve-Bayes & 69.2 & 61.5 & 76.9 & 0.66 & 0.71 \\
\hline & LDA & 74.2 & 74.1 & 74.1 & 0.84 & 0.78 \\
\hline & SVM & 98.9 & 98 & 100 & 1 & 0.98 \\
\hline
\end{tabular}



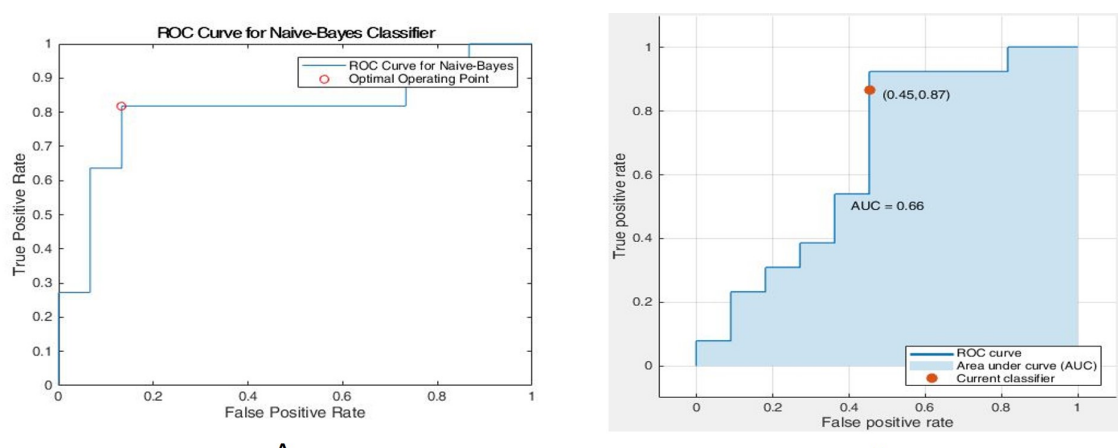

A

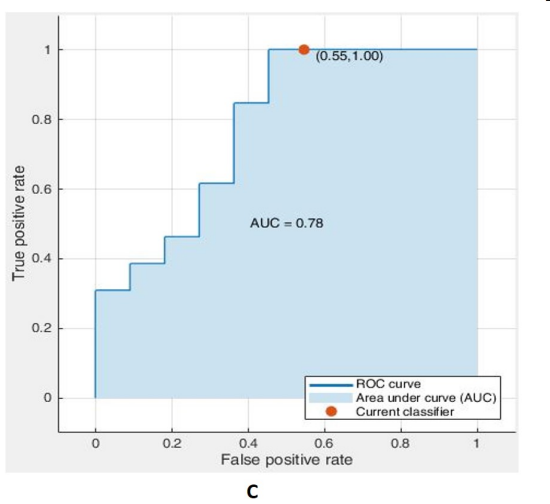

Figure 5.38: $V A G_{R}$ ROC Curves-21 Features: (A) Naive-Bayes, (B) LDA, (C) SVM 


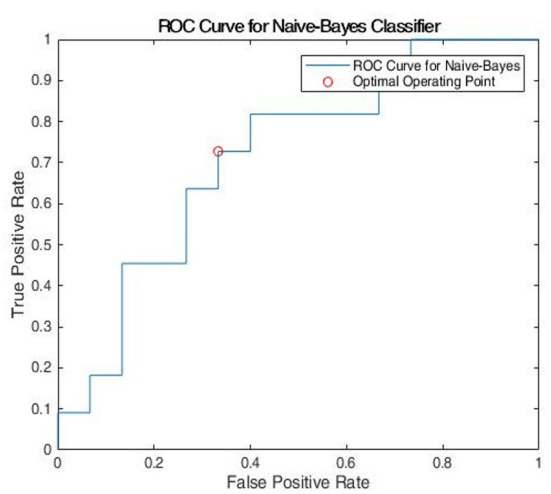

A

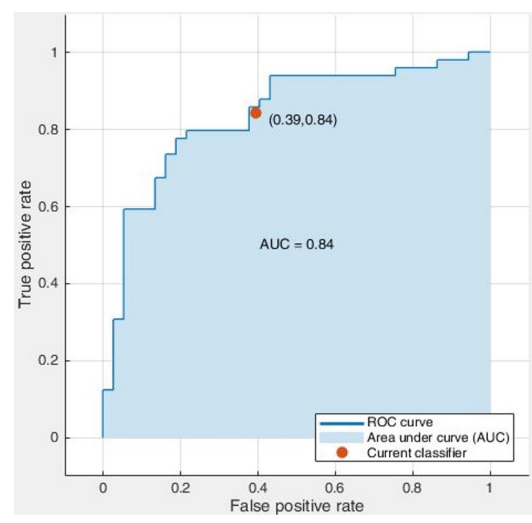

B

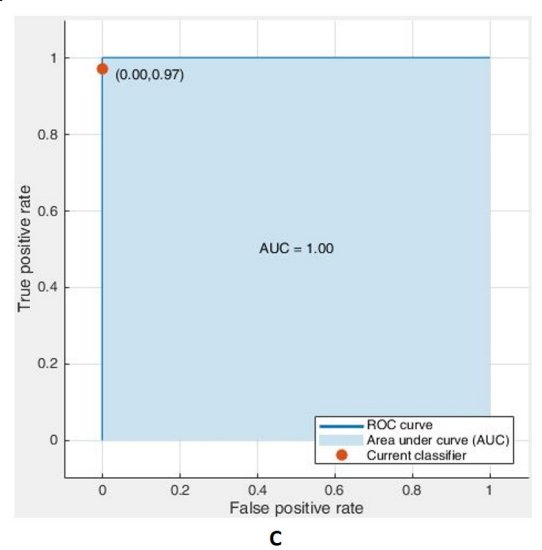

Figure 5.39: $V A G_{E}$ ROC Curves-21 Features: (A) Naive-Bayes, (B) LDA, (C) SVM

In addition to these experiments, a mRMR feature selection [152] algorithm was executed to generate the top three discriminatory features which were - the ACF scores with respect to weight vectors and the ACF score with respect to mean vector. Table 5.15 highlights these results.

Table 5.15: Classification of VAG Data using top 5 mRMR features for encoded signals

Features - Time, Frequency, Kernel-based, Actigraphy-specific, Morphological -

5 Features

Label - Knee Joint Type

\begin{tabular}{clccccc}
\hline Group & Classifier & Accuracy (\%) & Sensitivity (\%) & Specificity (\%) & AUC & F1-Score \\
\hline \multirow{2}{*}{ Group Tri $_{E}$} & Naïve-Bayes & 69.6 & 83.3 & 50 & 0.46 & 0.66 \\
& LDA & 61.7 & 66.6 & 51.2 & 0.30 & 0.70 \\
& SVM & 97 & 95.6 & 100 & 0.98 & 0.96 \\
\hline
\end{tabular}


Table 5.16: Classification of VAG data using 9 features

$\begin{aligned} & \text { Features - Kernel-based, Actigraphy-specific and Morphological - 9 Features } \\
& \text { Label - Type of Knee Joint }\end{aligned}$
\begin{tabular}{clcccccc} 
Group & Classifier & Accuracy (\%) & Sensitivity (\%) & Specificity (\%) & AUC & F1-Score \\
\hline \multirow{2}{*}{ Group $V A G_{R}$} & Naïve-Bayes & 61.5 & 66.6 & 60.8 & 0.74 & 0.28 \\
& LDA & 61.5 & 66.6 & 54.5 & 0.56 & 0.66 \\
& SVM & 61.5 & 63.1 & 57.1 & 0.59 & 0.70 \\
\hline \multirow{2}{*}{ Group $V A G_{E}$} & Naïve-Bayes & 61.9 & 71.4 & 54.2 & 0.68 & 0.61 \\
& LDA & 62.9 & 65.5 & 58 & 0.73 & 0.69 \\
& SVM & 97.8 & 96 & 100 & 1 & 0.97
\end{tabular}

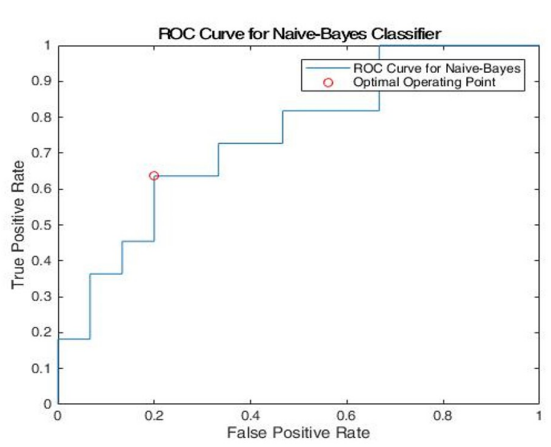

A

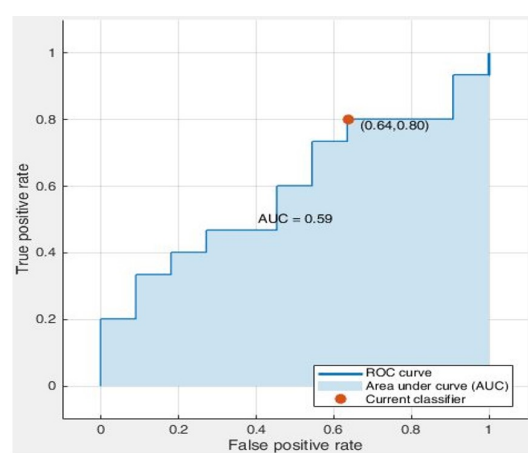

B

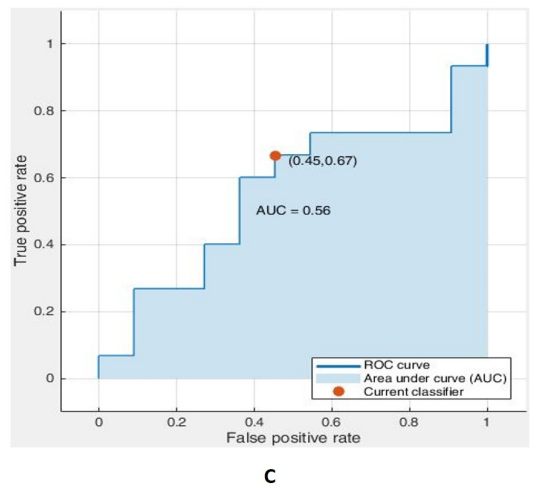

Figure 5.40: $V A G_{R}$ ROC Curves-9 Features: (A) Naive-Bayes, (B) LDA, (C) SVM 

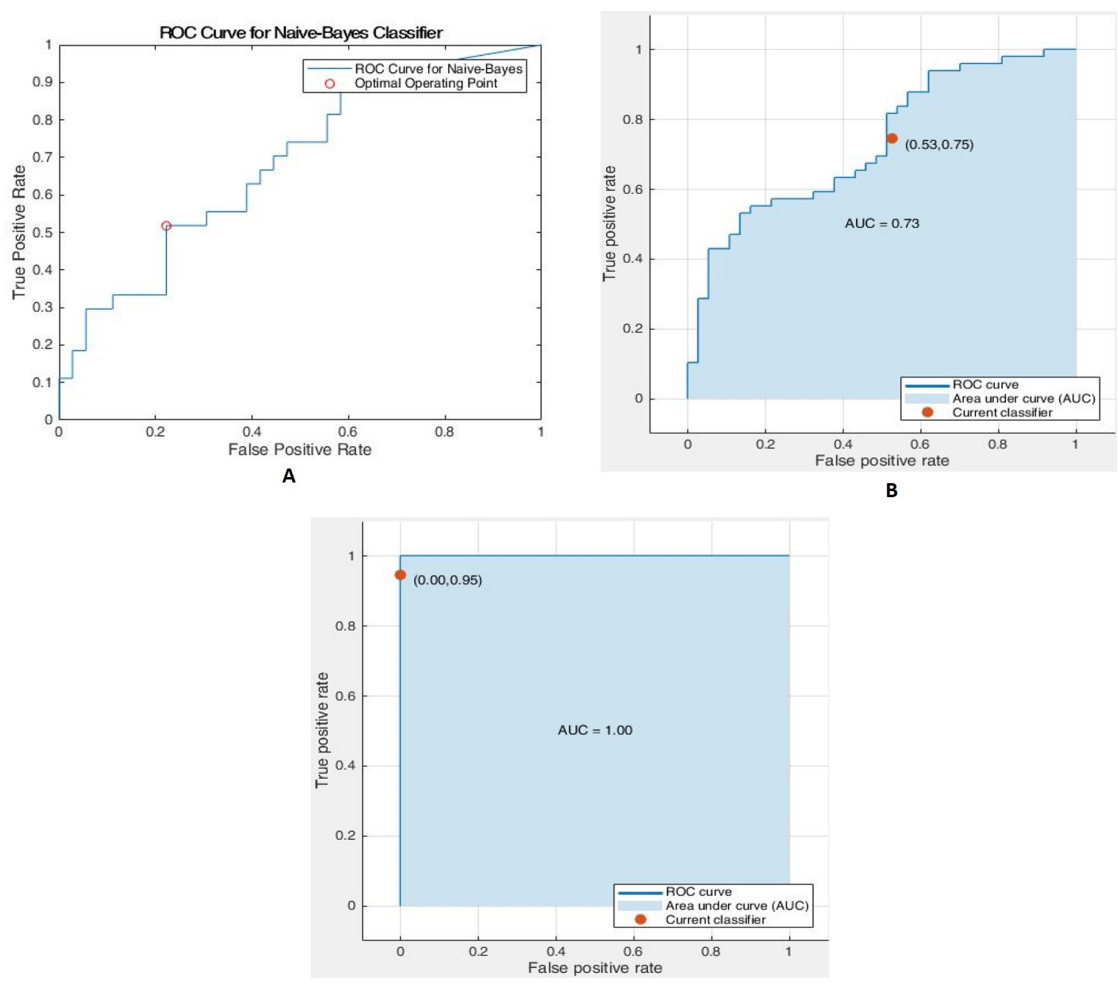

C

Figure 5.41: $V A G_{E}$ ROC Curves-9 Features: (A) Naive-Bayes, (B) LDA, (C) SVM

Table 5.17: Classification of VAG data using 14 features

Features - Time, Frequency and Actigraphy-specific - 14 Features

Label - Type of Knee Joint - Normal, Abnormal

\begin{tabular}{clccccc}
\hline Group & Classifier & Accuracy (\%) & Sensitivity (\%) & Specificity (\%) & AUC & F1-Score \\
\hline \multirow{2}{*}{ Group $V A G_{R}$} & Naïve-Bayes & 69.2 & 60 & 81.8 & 0.81 & 0.69 \\
& LDA & 84.6 & 78.9 & 100 & 0.79 & 0.87 \\
& SVM & 69.2 & 66.6 & 80 & 0.76 & 0.77 \\
\hline \multirow{2}{*}{ Group $V A G_{E}$} & Nä̈ve-Bayes & 61.5 & 72.7 & 53.3 & 0.71 & 0.61 \\
& LDA & 69.7 & 70.6 & 67.7 & 0.76 & 0.74 \\
& SVM & 98.9 & 98 & 100 & 1 & 0.98 \\
\hline
\end{tabular}




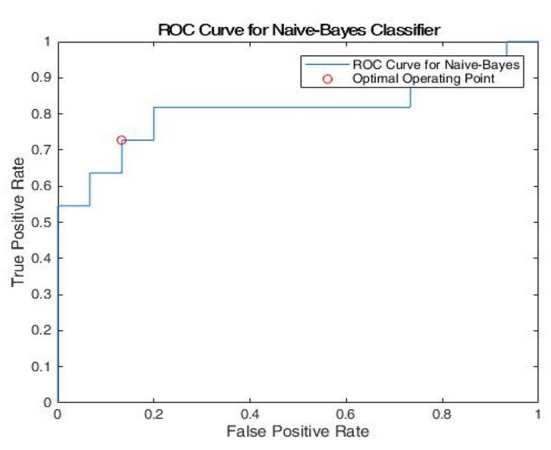

A
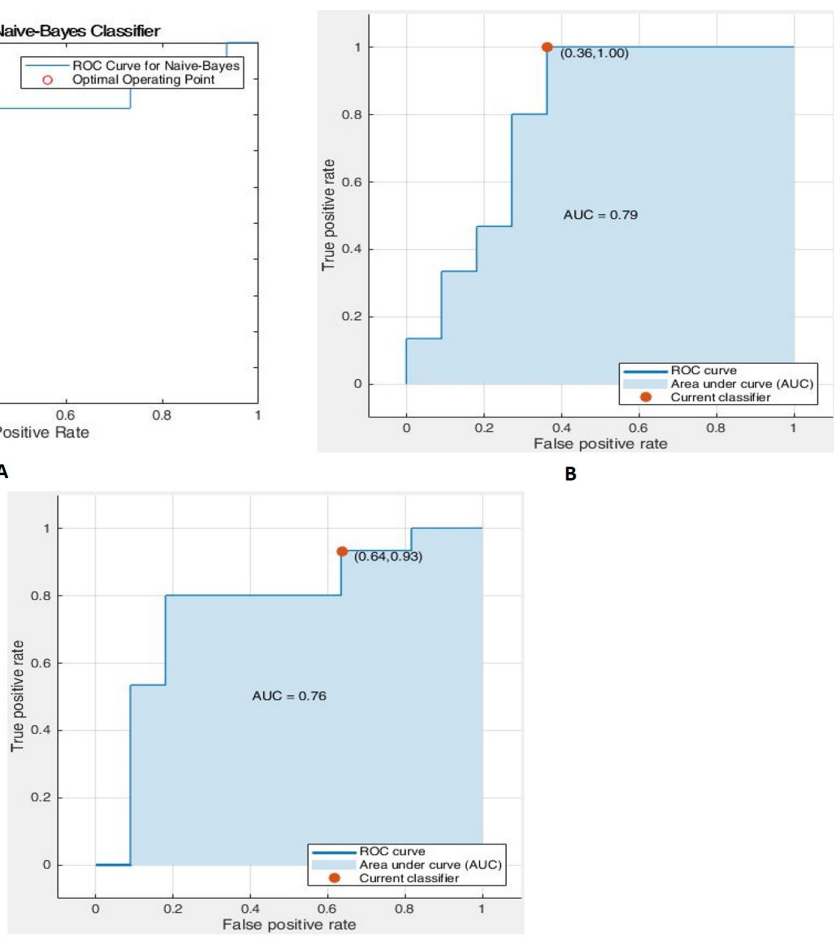

c

Figure 5.42: $V A G_{R}$ ROC Curves-14 Features: (A) Naive-Bayes, (B) LDA, (C) SVM 


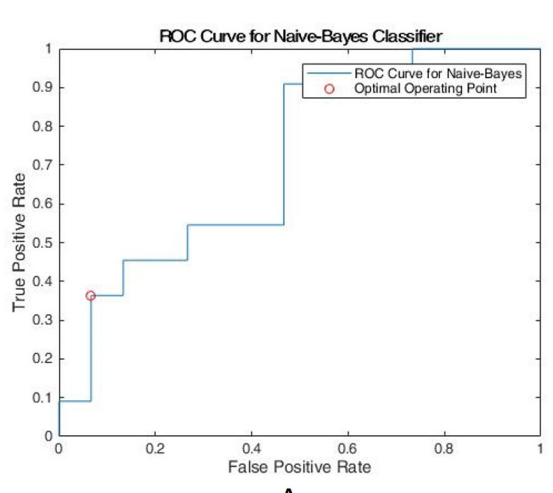

A

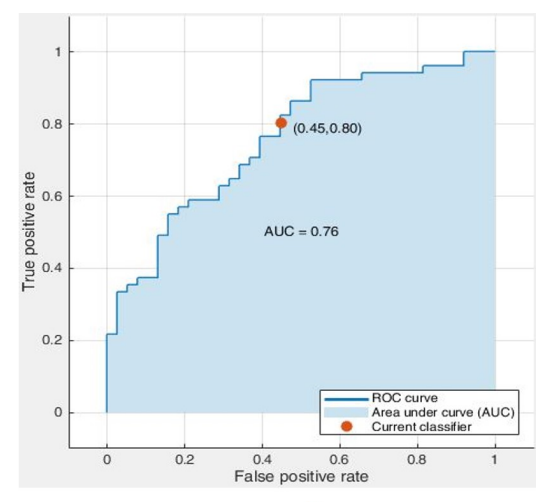

B

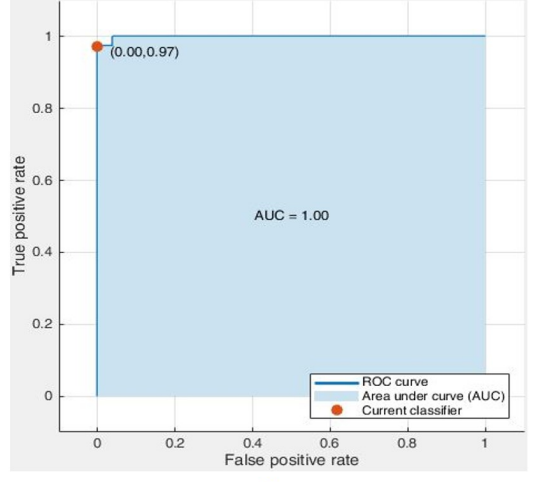

C

Figure 5.43: $V A G_{E}$ ROC Curves-14 Features: (A) Naive-Bayes, (B) LDA, (C) SVM

Further to this, experimentation was also done with different levels of signal encoding, and it was found that using 21 features, the 3-bit encoding of VAG signals ensured highest classification accuracy along with a high F1-score. This is evident from Figure 5.44 as shown below. 


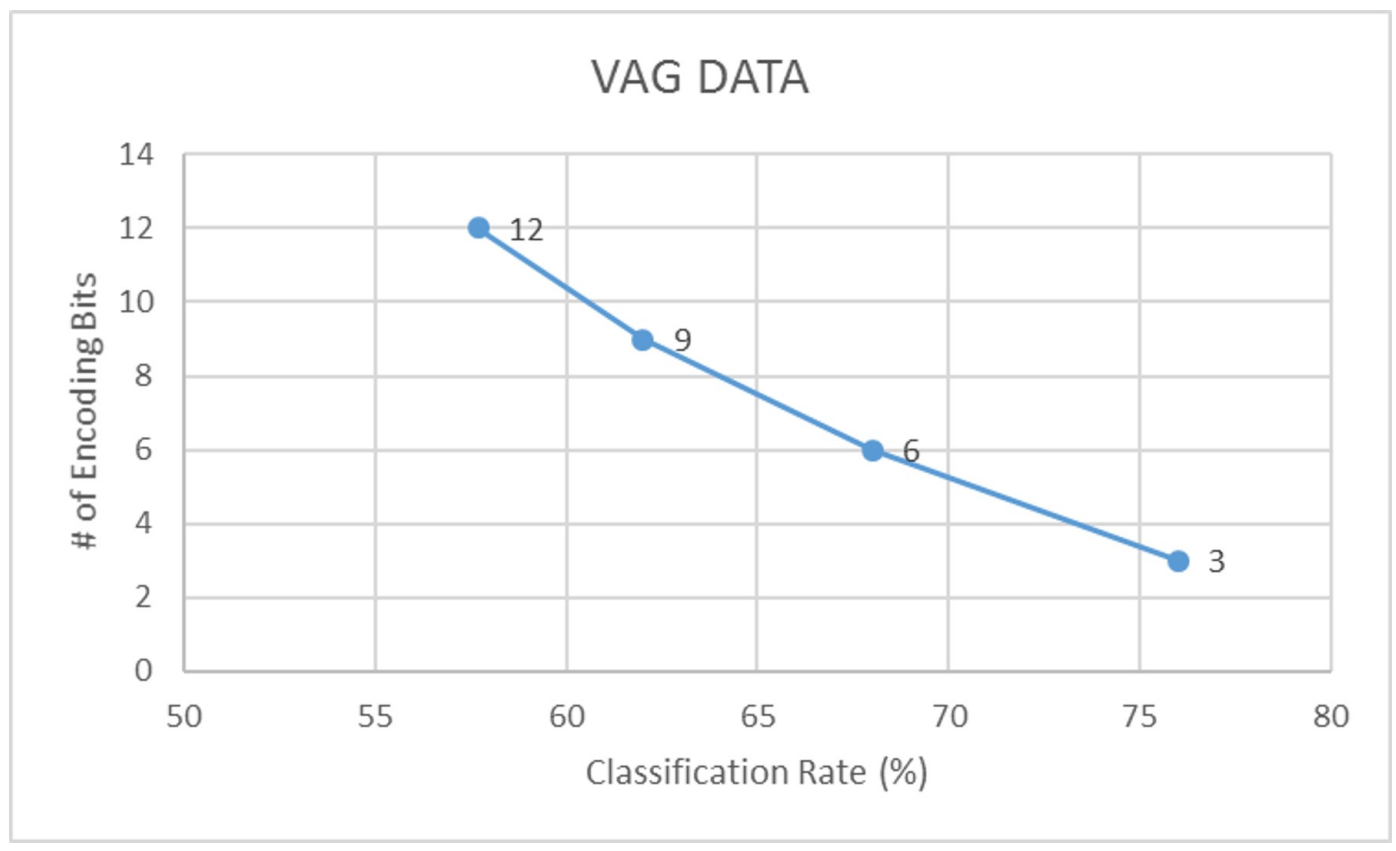

Figure 5.44: Classification Accuracy vs Encoding Levels for VAG Dataset

From extensive experiments with VAG and daily activity data, it was found that the actigraphy analysis system developed in this research work is not only applicable to sleep disorder analysis, but could also be implemented into wake actigraphy modalities for developing home-based activity monitoring solutions. This chapter concludes by presenting key observations and inferences from the experiments on Datasets D and E.

\subsection{Observations from Experiments on Datasets D and E}

\subsubsection{Observations from Database D experiments}

From the first set of experiments in wake actigraphy using the ADL data in Database D, the following was observed:

- Although the proposed encoding resulted only in about $68 \%$ space savings in 3-bit encoded activity signals, a very high SNR coupled with lowered data read time was obtained, as evident from Table 5.4 and Figure 5.5.

- Upon applying the AK and mobility-based algorithms to different activity signals in the ADL dataset, it was observed that the AK algorithm performed better by generating lower number of segments, as well as identifying regions of peak movements in each type of activity. Though the results have been obtained only from short-duration activity data, the previous experiments with long-duration sleep 
actigraphy data indicate that the AK method could also work well with long-duration wrist actigraphy signals. Results illustrated in Figures 5.6-5.19 and Table 5.5 show that the AK method performs comparatively better than the mobility-based algorithm.

- Additionally, both segmentation schemes were also tested on two different synthetic activity signals generated by concatenating various activity signals from the ADL dataset. While the AK algorithm was tested on a synthetic signal comprising of 11 activities, the mobility-based method was applied on a 14-activity synthetic signal. This was done in order to check the robustness of both the algorithms in classifying different activities in a real-world scenario.

- Following this, three pattern classification experiments were conducted, by using three different combinations of features from the ADL signal groups $A D L_{R}$ and $A D L_{E}$. A LDA and a SVM were modelled for each classification experiment, and it was found that the SVM gave a better recognition accuracy when analyzing encoded ADL signals. This is quite evident from the results recorded in Tables 5.6-5.9, and the corresponding ROC curves and confusion matrices in Figures 5.22-5.27.

- Finally, it was also observed that the 3-bit encoding ensured highest classification accuracy of the ADL data, as depicted in Figure 5.28.

\subsubsection{Observations from Database E experiments}

In the final set of experiments, the actigraphy analysis application was applied to vibroarthrography (VAG) signals captured from knee-joint movements. This study was conducted to estimate the severity of OA-based cartilage degeneration in 89 consenting test subjects. The following observations were obtained from these experiments:

- Firstly, upon applying the proposed 3-bit encoding scheme to VAG signals, it was found that the SNR increased by about $19.9 \mathrm{~dB}$, and lowered the bit rate from $30 \mathrm{kbits} / \mathrm{sample}$ to $7.5 \mathrm{kbits} / \mathrm{sample}$. Additionally, a space savings of about $88 \%$ was obtained by encoding the VAG signal, as evident from Table 5.12 and Figure 5.35. As shown in Figure 5.34, the encoding process not only normalizes signal amplitudes to discrete levels, it also retains peak movement information with minimal loss.

- Following the 3-bit encoding, the AK and mobility-based segmentation schemes were applied to a sample normal and abnormal VAG signal, and it was found that both algorithms performed remarkably well in segmenting the VAG data as per region of activity. Although the fixed segmentation approach, illustrated in third plot of each Figure 5.36 and 5.37, depicts a lower number of segments, it does not include the observations on knee-joint movements as described in Section 5.3.3. 
- Next, 21 features were extracted from the VAG signals in Groups $V A G_{R}$ and $V A G_{E}$, and using three different combinations of these features, a Naive-Bayes, a LDA and a SVM were modelled to classify between normal and abnormal knee joints. Upon a closer observation from the results in Tables 5.145.17 , it can be inferred that the linear SVM gave us the best classification accuracy for encoded VAG signals in Group $V A G_{E}$. This has also been consistent with experiments on Datasets $\mathrm{B}, \mathrm{C}$ and $\mathrm{D}$.

- Finally, it was also found that in case of VAG signals as well, the 3-bit encoding ensured highest classification accuracy, when the SVM was modelled using all 21 features, as evident from Figure 5.44.

- The results from this study indicated that the proposed actigraphy analysis system could potentially be applied to long-term monitoring of joint-related disorders in a home-setting.

The concluding chapter of this dissertation will revisit the observations collected from all the experiments in this and previous chapters, and use them to conduct some critical discussions on the advantages and limitations of the actigraphy analysis application developed in this research. Further, this chapter will also explore the potential of applying the application onto novel wearable platforms which could be enabled in an IoT environment with a focus on edge computing approaches.

\subsection{Summary}

In this penultimate chapter of the dissertation, the proposed actigraphy analysis application has been applied to publicly available datasets in short-duration, wake actigraphy for activity recognition and joint-disorder severity estimation. This chapter begins by applying the proposed algorithms to wrist-actigraphy data captured from 16 healthy subjects performing routine activities such as walking, drinking and sleeping. Following this, a study is conducted on estimating and classifying the severity of osteoarthritis in knee joints from vibroarthrography (sonified actigraphy) data taken from 89 test subjects, using the proposed actigraphy analysis methods. Finally, this chapter is concluded by presenting the experimental results and observations from each study. 


\section{Chapter 6}

\section{Discussions, Conclusions and Future}

\section{Works}

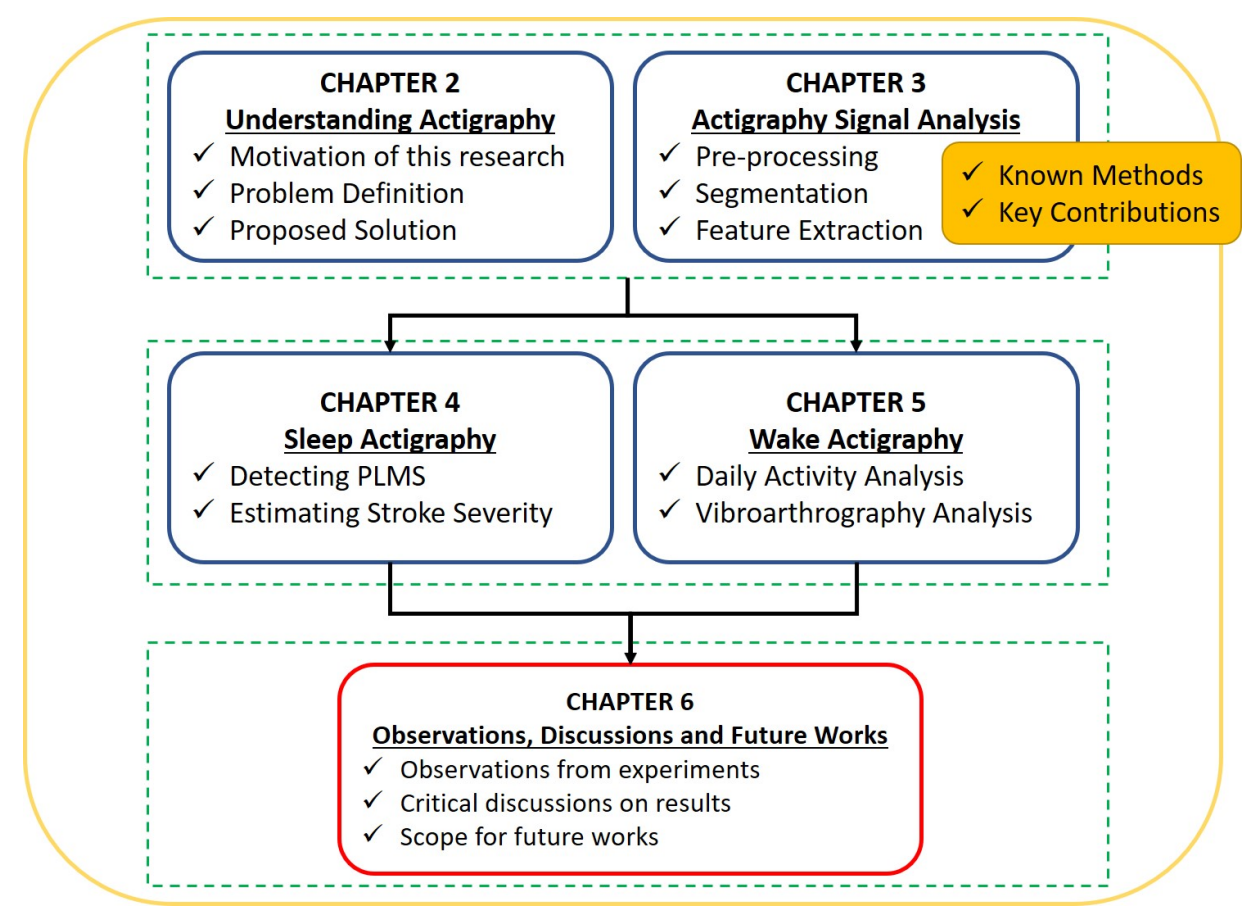

Figure 6.1: Organization of Dissertation

The core objective of this research work was to perform a systematic investigation into various modalities of actigraphy signal analysis, and develop novel algorithms which promoted the idea of edge computing in an IoMT paradigm. Through this study, the following algorithms were developed, whose combination formed 
the proposed actigraphy analysis application for sleep and wake activity monitoring.

\section{Contributions to Actigraphy Analysis:}

\section{- Actigraphy Encoding Algorithm:}

In order to ensure on-the-fly signal compression, denoising, bit-rate reduction and SNR enhancement, a $m$-bit encoding algorithm was developed for actigraphy data. This algorithm proposed a unique approach to signal pre-processing at the data acquisition source. As per the results recorded in Tables $4.8,4.22,5.4$ and 5.12 , the technique outperforms conventional techniques of signal filtering for denoising and SNR enhancement. Extensive experiments indicate that only 3-bits per sample are needed to capture vital movement information from actigraphs without compromising further segmentation and pattern classification modalities. The choice of 3-bit encoding was reached after conducting extensive classification experiments with values of $m$ in the range $[3 \leq m \leq r]$, where $r$-bits per sample is the quantization resolution of the raw actigraphy signal. The proposed actigraphy encoding scheme was able to achieve a $45-51 \%$ increase in SNR (signal-to-noise ratio), $60-80 \%$ bit rate reduction, 35-92\% data compression, and an increase in classification accuracy by $12 \%$ in case of sleep actigraphy. For wake actigraphy, the SNR increased by $29 \%$ in activity recognition and by $90 \%$ in vibroarthrography. This was also coupled by a $25-50 \%$ bit rate reduction, $68-88 \%$ data compression and an increase in classification accuracy by $5 \%$ in activity classification and $22 \%$ in vibroarthrography analysis. It should be noted that unlike conventional biomedical signals such as ECG (electrocardiogram), which have a specific structure or pattern, actigraphy signals are mainly transient in nature with spiky movement information recorded at random time periods. Therefore, alternating or lowering the quantization of this data would not affect the morphology and hence its analysis for pattern detection.

\section{- Adaptive Segmentation Algorithms:}

As discussed in Chapter 2, actigraphy signals are known to have a random, non-linear and nonstationary behavior, with sparsity shown in small windows. The accelerometer sensor embedded inside an actigraphy device is highly sensitive to motion and registers the smallest movements captured by the device. This creates a trivial situation for data analysis, when one is looking for specific movements within a sporadic array of movement amplitudes. Though fixed segmentation techniques can help narrow down regions of interest with moderate accuracy, they are not able to capture the non-stationary nature of actigraphy data and may lose information in the process. An alternative is to adaptively segment the actigraphy data into variable-sized small segments based on observations from significant movements. This study's investigation into various types of actigraphy signals recorded during sleep and wake states indicated that, peak movement activity leads to a drastic rise in the signal 
amplitude before settling down to zero or close to zero vibrations. This peak movement is characteristic of the human activity performed. Identifying these drastic change points can thus lead to detecting regions of interest from an actigraphy signal, thereby segmenting it into small windows, and discarding portions which are of insignificant value. In this study, the following two novel adaptive segmentation algorithms were developed, based on the afore-mentioned signal encoding scheme, and incorporated the drastic change principle:

\section{Athavale-Krishnan (AK) Segmentation Algorithm:}

The AK algorithm first computes the rapid change factor (RCF) of the given $m$-bit encoded actigraphy signal, and then uses it to detect primary segment boundaries which exhibit peak movements in the data. Further to this, the algorithm also then reduces the primary boundaries to a final set of segments by incorporating ground truth information on specific human movements. From various experiments it was found that the AK algorithm worked exceptionally well in detecting over $70 \%$ of correct regions of interest in different types of actigraphy data. These results were also justified by testing the same on synthetic signals as shown in Figure 3.26.

\section{Mobility-based Segmentation Algorithm:}

The Mobility-based algorithm is primarily based on using Hjorth's mobility parameter for detecting regions of interest in an actigraphy signal. Instead of using the RCF, the algorithm directly

finds signal values which are greater than ${\frac{1}{\left(2^{m}-1\right)}}^{\text {th }}$ of the maximum amplitude in the signal. These constitute the primary segment boundaries. Next, the algorithm computes the intra-segment mobilities, and deduces the secondary boundaries by identifying those segments whose mobilities are different from each other. The final segments are then computed using ground truth information on specific activity. The experiments using the mobility-based segmentation gave a good segmentation with over $60 \%$ correct segment identification. The mobility-based algorithm was also tested on synthetic data as depicted in Figure 3.28.

A comparison between the two schemes indicated that the AK algorithm outperformed the mobilitybased method in the following key ways:

1. Lower number of segments

2. Scope for including more ground truth information on human movements

3. RCF computed in AK algorithm also acts as a good discriminatory feature while classifying actigraphy signals

These findings are also justified by the segmentation results shown in Tables 4.9, 4.23, 5.5 and 5.13. 


\section{- Actigraphy-specific Features:}

A detailed survey of actigraphy analysis techniques in prior studies have indicated that most pattern classification problems involved the use of conventional time and frequency features from accelerometry signals. An increase in data dimensionality could lead to feature generalization and poor classification when the actigraphy data collected from large population. An effective way to overcome this is to include actigraphy-specific features, which characterize human movements and can be extracted easily from the non-stationary accelerometry data. Through this research, two actigraphy-specific features were derived, namely - the spiky index (SI) and the rapid change factor (RCF), which focus on characterizing human movements, by detecting rapid or drastic changes in actigraphy data, as well as quantifying rate of change in limb movements. Subsequently, the experiments with sleep and wake actigraphy data, revealed that these features when extracted from encoded data, led to a remarkable pattern recognition accuracy using linear classifiers. The RCF is also a critical parameter in adaptively segmenting actigraphy data using the afore-mentioned AK or Mobility-based algorithms. It is a unique value for each given actigraphy signal, and requires few steps for computation. The SI indicates rate of change in the signal and usually measured in movements per second or movements per hour. A systematic investigation also revealed that the SI could potentially be used as a surrogate parameter for quantifying sleep-related movement disorders, along with being a discriminatory feature.

\section{- Kernel-based Features:}

In this actigraphy-based research, methods which could reduce signal dimension by mapping nonstationary data to higher feature space were also investigated. While doing so, a novel autocorrelation function-based $(\mathrm{ACF})$ kernel was developed, which reduces a given actigraphy signal to four distinct features. As described in Chapter 3, the ACF kernel computes four features based on partial derivatives of the signal with respect to mean and weight estimates calculated using Expectation-Maximization algorithm. These features allow for better feature space visualization and pattern classification, as evident from the experiments in Chapters 4 and 5 .

- Upon performing extensive mRMR feature selection [152] using different combinations of time, frequency, morphological, actigraphy-specific and kernel-based features, it was found that the at least one of the contributed actigraphy-specific features and the kernel-based features were ranked in the top 5 features for classification of each type of actigraphy data. This is evident from results tabulated in Tables 4.12, 4.25, 5.7 and 5.15. Additionally from results illustrated in Figures 4.32 and 4.60, it was also inferred that the Rapid change factor and the spiky index proved to be good discriminatory features for classifying sleep actigraphy signals, which also indicated their efficiency to quantify limb movements 
during sleep. This being said, it should be noted that the Periodicity Index was also selected again as a top discriminatory feature along with the RCF and mean frequency, which showed that PLMs can be easily classified using a combination of morphological and actigraphy-specific parameters.

- It may also be noted that, in this study of sleep actigraphy,the combination of generic time, frequency features and the actigraphy-specific, morphological and kernel-based features was done for the first time, in comparison to an extensive survey of prior studies in actigraphy-PSG analysis. Thus, experiments with Databases A [5] and B [6] could be considered as benchmarking studies with regards to application of novel signal processing methods to sleep actigraphy data for estimating the severity of PLMs. This has also been extensively covered in Section 4.1.1.

A quick glance of the afore described quantitative results from each contribution to this research indicates, that from an IoMT perspective, the developed actigraphy analysis application fits well within the context of wearable implementation and could potentially promote edge computing applications, with focus on data compression, denoising and activity recognition at the source. Considering the various applications described in this research work, the reader may ponder over the following salient points for each vertical:

\section{Sleep Actigraphy:}

Though most prior research on the use of actigraphy to analyse neuromuscular disorders such as PLMs and stroke, provided low to moderate agreement with conventional PSG standards, it should be noted that many of these works did not employ extensive signal processing techniques to develop insights from actigraphy data. They rather depended on comparing actigraphy outputs with PSG readings using statistical measures such as Pearson's correlation coefficients, p-values, t-tests and Bland-Altman plots. Also, the registered technologists used the same methods to analyze actigraphy and PSG data for disease severity estimation. Many of these prior studies analyzed short-duration actigraphy signals. This research on the other hand was directed towards analyzing both short and long-duration actigraphy data using novel signal analysis techniques. Additionally, it also ensured that no patients were excluded from the study population, based on their medication, or stroke severity or other neuromuscular disorders. The remarkable encoding, segmentation and classification results obtained in the experiments highlighted in Chapter 4, indicate that the proposed actigraphy analysis application could be transformed into a generic screening tool for detecting neuromuscular disorders in sleep. It could potentially be implemented on low-cost (typically around USD 100) actigraphy devices for home-based monitoring of sleep disorders.

\section{Wake Actigraphy:}

Furthermore to this study in sleep actigraphy, similar experiments were conducted on wrist and leg 
actigraphy data obtained in wake state for activity recognition and joint degeneration severity estimations. The results from these shown in Chapter 5, infer that the proposed system could also be used for long-term, home-based wake activity monitoring and rehabilitation solutions in an ambient assisted-living environments.

Most healthcare systems around the world still rely on frequent patient visits to the healthcare providers, who rely on using advanced yet cumbersome equipment to diagnose various diseases and disorders. Of these PSG setup is quite expensive to operate, and poses higher out-of-pocket expenses for the patient. Recent trends in rising healthcare costs have pushed the general populous in adopting remote health monitoring technologies such as smart devices and wearables in daily life, thereby promoting the people-centered healthcare paradigm. This has also in-turn motivated healthcare providers to look for treatment alternatives through telemedicine frameworks.

Though the proposed actigraphy analysis system in this study presents a novel solution to activity monitoring in a home-based environment, this research work still has certain shortcomings which could be met as part of future works:

- Combining the adaptive segmentation and feature extraction modules, in order to perform pattern classification only on segments of interest from a given actigraphy signal.

- Access to more patient-consented actigraphy data from wake and sleep states.

- Migrating the proposed system onto a commercially viable, open-source platform.

- Developing a front-end user interface for wearable and smart-computing devices.

Additionally, a lesson for future works suggests that smart and non-invasive signal analysis algorithms embedded in smart devices and wearables such as actigraphs, could enable the practical implementation of telemonitoring of patients and people in general. The research work described in this dissertation aims to motivate further studies in wearable signal analysis for developing novel solutions for smart environment, thereby improving our quality of life.

\subsection{Summary}

In this final chapter of the dissertation, a brief discussion has been conducted on various implications of this research work. Quantitative results from each modality of the proposed actigraphy analysis application have been included, in order to show how this research could be transformed into a novel actigraphy analysis solution for home-based IoT environments. This dissertation then concludes with citing the references used 
in this study which would give the reader additional insights on different aspects of this research work on actigraphy signal analysis. 


\title{
List of Abbreviations
}

\author{
Symbol \\ Definition \\ LTE \\ Long Term Evolution \\ NFC \\ Near field communication \\ WSN \\ Wireless Sensor Networks \\ VR \\ Virtual Reality \\ AR \\ Augmented Reality \\ IoT \\ Internet of Things \\ RFID \\ Radio Frequency Identification \\ CSV \\ Comma Separated Values \\ DBMS \\ Database Management Systems \\ CPU \\ Central Processing Unit \\ IoMT \\ Internet of Medical Things \\ ECG \\ Electrocardiogram \\ $\mathrm{ADC}$ \\ Analog-to-Digital Converter \\ IMU \\ Intertial Motion Unit \\ PLMs \\ Periodic Limb Movements \\ WSS \\ Wide Sense Stationary \\ KPSS \\ Kwiatkowski-Phillips-Schmidt-Shin \\ KS \\ Kolmogorov-Smirnov test \\ PSG \\ Polysomnography \\ ADHD \\ Attention Deficit Hyperactivity Disorder \\ PLMSI \\ Periodic Limb Movement in Sleep Index \\ RLS \\ Restless Leg Syndrome \\ $\mathrm{TF}$ \\ Time-Frequency \\ EMG \\ Electromyography \\ DC \\ Direct current \\ SDK \\ Software Development Kit \\ ADL \\ Activities of Daily Life \\ SNR \\ Signal-to-Noise Ratio \\ DWT \\ Discrete Wavelet Transforms \\ PCM \\ Pulse Code Modulation
}


DM

RMS

$\mathrm{ACF}$

PSD

STFT

SEM

$\mathrm{RCF}$

SFDR

PSB

SSB

FSB

EEG

SINAD

FFT

BMI

RHI

AHI

O2Sat

TST

SE

PI

SI

SS

EMS

EOG

NREM

CAD

AOD

AASM

RDI

LDA

SVM
Delta Modulation

Root Mean Square

Autocorrelation Function

Power spectral density

Short-time Fourier transform

Spectral error measure

Rapid Change Factor

Spurious Free Dynamic Range

Primary Segment boundary

Secondary Segment boundary

Final Segment boundary

Electroencephalogram

Signal to Noise and Distortion Ratio

Fast Fourier Transform

Body Mass Index

Respiration index

Apnea-Hypopnea index

Oxygen Saturation

Total Sleep Time

Sleep Efficiency

Periodicity Index

Spiky Index

Step Size

Expectation Maximization

Electrooculography

Non-Rapid Eye Movement

Canadian Dollar

Activities of Daily Life

American Academy of Sleep Medicine

Respiratory diturbance index

Linear Discriminant Analysis

Support vector machine 
mRMR

ROC

USB

CSV

TIA

$\mathrm{NaN}$

$\mathrm{OA}$

$\mathrm{CT}$

VAG minimum Redundancy Maximum Relevancy

Receiver operating characteristics

Universal Serial Bus

Comma Separated Values

Transient Ischemic Attack

Not a Number

Osteoarthritis

Computerized Tomography

Vibroarthrography 


\section{Bibliography}

[1] "Sleep disorders will affect $40 \%$ of canadians, pose serious health risks - british columbia - cbc news," http://www.cbc.ca/news/canada/british-columbia/sleep-disorder-ubc-1.3294306, (Accessed on $02 / 21 / 2017)$.

[2] "Sleep disorders and problems: Symptoms, treatment, and self-help for common sleeping problems," https://www.helpguide.org/articles/sleep/sleep-disorders-and-sleeping-problems.htm, (Accessed on $02 / 21 / 2017)$.

[3] "Sleep disorders: Causes, diagnosis \& treatments," http://www.healthline.com/health/sleep/ disorders, (Accessed on 02/21/2017).

[4] "Causes of sleep problems," http://www.webmd.com/sleep-disorders/sleep-disorders-causes, (Accessed on $02 / 21 / 2017)$.

[5] Y. Athavale, S. Krishnan, D. D. Dopsa, A. G. Berneshawi, H. Nouraei, A. Raissi, B. J. Murray, and M. I. Boulos, "Advanced signal analysis for the detection of periodic limb movements from bilateral ankle actigraphy," Journal of sleep research, vol. 26, no. 1, pp. 14-20, 2017.

[6] Y. Athavale, S. Krishnan, A. Raissi, N. Kirolos, T. Jairam, B. J. Murray, and M. I. Boulos, "Actigraphic detection of periodic limb movements: development and validation of a potential device-independent algorithm. a proof of concept study," Sleep, 2019.

[7] B. Bruno, F. Mastrogiovanni, A. Sgorbissa, T. Vernazza, and R. Zaccaria, "Analysis of human behavior recognition algorithms based on acceleration data," in 2013 IEEE International Conference on Robotics and Automation. IEEE, 2013, pp. 1602-1607.

[8] "Uci machine learning repository: Dataset for adl recognition with wrist-worn accelerometer data set," https://archive.ics.uci.edu/ml/datasets/Dataset+for+ADL+Recognition+with+Wrist-worn+ Accelerometer\#, (Accessed on 07/21/2019). 
[9] P. Sethi and S. R. Sarangi, "Internet of things: architectures, protocols, and applications," Journal of Electrical and Computer Engineering, vol. 2017, 2017.

[10] Y. Athavale and S. Krishnan, "A device-independent efficient actigraphy signal-encoding system for applications in monitoring daily human activities and health," Sensors, vol. 18, no. 9, 2018. [Online]. Available: http://www.mdpi.com/1424-8220/18/9/2966

[11] D. Miorandi, S. Sicari, F. De Pellegrini, and I. Chlamtac, "Internet of things: Vision, applications and research challenges," Ad hoc networks, vol. 10, no. 7, pp. 1497-1516, 2012.

[12] B. L. R. Stojkoska and K. V. Trivodaliev, "A review of internet of things for smart home: Challenges and solutions," Journal of Cleaner Production, vol. 140, pp. 1454-1464, 2017.

[13] J. J. Rodrigues, D. B. Segundo, H. A. Junqueira, M. H. Sabino, R. M. Prince, J. Al-Muhtadi, and V. H. C. de Albuquerque, "Enabling technologies for the internet of health things," IEEE Access, 2018.

[14] E. McMahon, R. Williams, M. El, S. Samtani, M. Patton, and H. Chen, "Assessing medical device vulnerabilities on the internet of things," in Intelligence and Security Informatics (ISI), 2017 IEEE International Conference on. IEEE, 2017, pp. 176-178.

[15] (2019) Illustrations — john bradley graphics, last accessed: 2019-06-18. [Online]. Available: http://www.johnbradleygraphics.com/illustrations

[16] "Gcdc usb accelerometer x16-mini," http://www.gcdataconcepts.com/x16-mini.html, (Accessed on $03 / 22 / 2017)$.

[17] "Philips respironics - actical," http://www.actigraphy.com/solutions/actical, (Accessed on 01/24/2017).

[18] M. Schimmack, S. Nguyen, and P. Mercorelli, "Anatomy of haar wavelet filter and its implementation for signal processing," IFAC-PapersOnLine, vol. 49, no. 6, pp. 99-104, 2016.

[19] d. Created and nurtured by Eric Weisstein at Wolfram Research. (2019) Haar function. [Online]. Available: http://mathworld.wolfram.com/HaarFunction.html

[20] Y. Athavale, S. Krishnan, A. Raissi, N. Kirolos, B. J. Murray, and M. I. Boulos, "Integrated signal encoding and analysis system for actigraphy-based long-term monitoring of periodic limb movements in sleep," in 2018 40th Annual International Conference of the IEEE Engineering in Medicine and Biology Society (EMBC). IEEE, 2018, pp. 4436-4439. 
[21] "The kernel trick - towards data science," https://towardsdatascience.com/ the-kernel-trick-c98cdbcaeb3f, (Accessed on 06/22/2019).

[22] "Sleep medicine part 2 - doctor in progress," https://doctorinprogress.com/2018/09/02/ sleep-medicine-part-2/, (Accessed on 06/22/2019).

[23] "Periodic limb movements in sleep - clinical gate," http://clinicalgate.com/ periodic-limb-movements-in-sleep/, (Accessed on 03/21/2017).

[24] (2019) Types of stroke,heart and stroke foundation of canada. [Online]. Available: https: //www.heartandstroke.ca/stroke/what-is-stroke/types-of-stroke

[25] "How to cope with osteoarthritis and the knee - victoria wellness professionals," http://www. victoriawellness.com/how-to-cope-with-osteoarthritis-and-the-knee/, (Accessed on 06/07/2018).

[26] "Arthritis of the knee - orthoinfo - aaos," https://orthoinfo.aaos.org/en/diseases--conditions/ arthritis-of-the-knee/, (Accessed on 06/07/2018).

[27] S. Krishnan, Adaptive signal processing techniques for analysis of knee joint vibroarthrographic signals. Calgary, 1999.

[28] M. Wollschlaeger, T. Sauter, and J. Jasperneite, "The future of industrial communication: Automation networks in the era of the internet of things and industry 4.0," IEEE Industrial Electronics Magazine, vol. 11, no. 1, pp. 17-27, 2017.

[29] S. Saha, "Ubiquitous sensors, iot-impacts and the data analysis, pulse, linkedin," https://www.linkedin. com/pulse/ubiquitous-sensors-iot-impacts-data-analysis-soham-saha/, (Accessed on 03/27/2018).

[30] "What is edge computing? (+how it's revolutionizing businesses)," https://learn.g2.com/trends/ edge-computing, (Accessed on 05/28/2019).

[31] G. J. Joyia, R. M. Liaqat, A. Farooq, and S. Rehman, "Internet of medical things (iomt): Applications, benefits and future challenges in healthcare domain," Journal of Communications, vol. 12, no. 4, 2017.

[32] Y. Athavale and S. Krishnan, "Biosignal monitoring using wearables: Observations and opportunities," Biomedical Signal Processing and Control, vol. 38, pp. 22 - 33, 2017.

[33] "Wearable technology database," http://vandrico.com/wearables/, (Accessed on 06/16/2016).

[34] "Wearable tech could soon reach 385m people - business insider," http://www.businessinsider.com/ wearable-tech-could-soon-reach-385m-people-2015-7, (Accessed on 06/16/2016). 
[35] "The Numbers Don’t Lie: Wearables are the Future of Healthcare."

[36] S. Krishnan and Y. Athavale, "Trends in biomedical signal feature extraction," Biomedical Signal Processing and Control, vol. 43, pp. 41-63, 2018.

[37] C. Acebo, "Actigraphy," Sleep: A comprehensive handbook, pp. 1035-1038, 2005.

[38] S. G. Trost, Y. Zheng, and W.-K. Wong, "Machine learning for activity recognition: hip versus wrist data," Physiological measurement, vol. 35, no. 11, p. 2183, 2014.

[39] I. C. Gyllensten, Physical Activity Recognition in Daily Life Using a Traxial Accelerometer. Skolan för datavetenskap och kommunikation, Kungliga Tekniska högskolan, 2010.

[40] J. L. Martin and A. D. Hakim, "Wrist actigraphy," Chest Journal, vol. 139, no. 6, pp. 1514-1527, 2011.

[41] M. de Almeida Mendes, I. C. da Silva, V. V. Ramires, F. F. Reichert, R. C. Martins, and E. Tomasi, "Calibration of raw accelerometer data to measure physical activity: A systematic review," Gait \& Posture, vol. 61, pp. 98 - 110, 2018. [Online]. Available: http://www.sciencedirect.com/science/article/pii/S0966636217310573

[42] S. Zhang, A. V. Rowlands, P. Murray, T. L. Hurst et al., "Physical activity classification using the genea wrist-worn accelerometer," Ph.D. dissertation, Lippincott Williams and Wilkins, 2012.

[43] D. Kwiatkowski, P. C. Phillips, P. Schmidt, and Y. Shin, "Testing the null hypothesis of stationarity against the alternative of a unit root: How sure are we that economic time series have a unit root?" Journal of econometrics, vol. 54, no. 1-3, pp. 159-178, 1992.

[44] W. A. Fuller, "Introduction to statistical time series, new york: Johnwiley," FullerIntroduction to Statistical Time Series1976, 1976.

[45] A. Ghasemi and S. Zahediasl, "Normality tests for statistical analysis: a guide for non-statisticians," International journal of endocrinology and metabolism, vol. 10, no. 2, p. 486, 2012.

[46] D. Zonoobi, A. A. Kassim, and Y. V. Venkatesh, "Gini index as sparsity measure for signal reconstruction from compressive samples," IEEE Journal of Selected Topics in Signal Processing, vol. 5, no. 5, pp. 927-932, 2011.

[47] M. de Zambotti, A. Goldstone, S. Claudatos, I. M. Colrain, and F. C. Baker, "A validation study of fitbit charge $2^{\mathrm{TM}}$ compared with polysomnography in adults," Chronobiology international, pp. 1-12, 2017. 
[48] I. W. Muns, Y. Lad, I. G. Guardiola, and M. Thimgan, "Classification of rest and active periods in actigraphy data using pca," Procedia Computer Science, vol. 114, pp. 275-280, 2017.

[49] Y. El-Manzalawy, O. Buxton, and V. Honavar, "Sleep/wake state prediction and sleep parameter estimation using unsupervised classification via clustering," in Bioinformatics and Biomedicine (BIBM), 2017 IEEE International Conference on. IEEE, 2017, pp. 718-723.

[50] A. Domingues, T. Paiva, and J. M. Sanches, "Sleep and wakefulness state detection in nocturnal actigraphy based on movement information," IEEE Transactions on Biomedical Engineering, vol. 61, no. 2 , pp. 426-434, 2014.

[51] J. Hedner, G. Pillar, S. D. Pittman, D. Zou, L. Grote, and D. P. White, "A novel adaptive wrist actigraphy algorithm for sleep-wake assessment in sleep apnea patients," Sleep, vol. 27, no. 8, pp. 1560-1566, 2004.

[52] A. C. Brown, M. H. Smolensky, G. E. D'Alonzo, and D. P. Redman, "Actigraphy: a means of assessing circadian patterns in human activity," Chronobiology international, vol. 7, no. 2, pp. 125-133, 1990.

[53] V. Natale, G. Plazzi, and M. Martoni, "Actigraphy in the assessment of insomnia: a quantitative approach," Sleep, vol. 32 , no. 6, pp. 767-771, 2009.

[54] T. Blackwell, S. Redline, S. Ancoli-Israel, J. L. Schneider, S. Surovec, N. L. Johnson, J. A. Cauley, and K. L. Stone, "Comparison of sleep parameters from actigraphy and polysomnography in older women: the sof study," SLEEP-NEW YORK THEN WESTCHESTER-, vol. 31, no. 2, p. 283, 2008.

[55] E. F. Camargos, F. M. Louzada, and O. T. Nóbrega, "Wrist actigraphy for measuring sleep in intervention studies with alzheimer's disease patients: application, usefulness, and challenges," Sleep medicine reviews, vol. 17 , no. 6 , pp. $475-488,2013$.

[56] J. E. Maglione, L. Liu, A. B. Neikrug, T. Poon, L. Natarajan, J. Calderon, J. A. Avanzino, J. CoreyBloom, B. W. Palmer, J. S. Loredo et al., "Actigraphy for the assessment of sleep measures in parkinson's disease," Sleep, vol. 36, no. 8, p. 1209, 2013.

[57] J. Barth, J. Klucken, P. Kugler, T. Kammerer, R. Steidl, J. Winkler, J. Hornegger, and B. Eskofier, "Biometric and mobile gait analysis for early diagnosis and therapy monitoring in parkinson's disease," in 2011 Annual International Conference of the IEEE Engineering in Medicine and Biology Society. IEEE, 2011, pp. 868-871. 
[58] S. Kye, J. Moon, T. Lee, S. Lee, K. Lee, S. c. Shin, and Y. S. Lee, "Detecting periodic limb movements in sleep using motion sensor embedded wearable band," in 2017 IEEE International Conference on Systems, Man, and Cybernetics (SMC), Oct 2017, pp. 1087-1092.

[59] D. T. Plante, "Leg actigraphy to quantify periodic limb movements of sleep: a systematic review and meta-analysis," Sleep medicine reviews, vol. 18, no. 5, pp. 425-434, 2014.

[60] M. J. Grap, C. T. Borchers, C. L. Munro, R. Elswick, and C. N. Sessler, "Actigraphy in the critically ill: correlation with activity, agitation, and sedation," American Journal of Critical Care, vol. 14, no. 1, pp. 52-60, 2005.

[61] T. Penzel, K. Kesper, I. Pinnow, H. F. Becker, and C. Vogelmeier, "Peripheral arterial tonometry, oximetry and actigraphy for ambulatory recording of sleep apnea," Physiological measurement, vol. 25, no. 4, p. $1025,2004$.

[62] C. Burton, B. McKinstry, A. S. Tătar, A. Serrano-Blanco, C. Pagliari, and M. Wolters, "Activity monitoring in patients with depression: a systematic review," Journal of affective disorders, vol. 145, no. 1, pp. 21-28, 2013.

[63] V. Reiterer, C. Sauter, G. Klösch, W. Lalouschek, and J. Zeitlhofer, "Actigraphy-a useful tool for motor activity monitoring in stroke patients," European neurology, vol. 60, no. 6, pp. 285-291, 2008.

[64] L. Ferini-Strambi, A. S. Walters, and D. Sica, "The relationship among restless legs syndrome (willisekbom disease), hypertension, cardiovascular disease, and cerebrovascular disease," Journal of neurology, vol. 261, no. 6, pp. 1051-1068, 2014.

[65] T. Kendzerska, M. Kamra, B. J. Murray, and M. I. Boulos, "Incident cardiovascular events and death in individuals with restless legs syndrome or periodic limb movements in sleep: A systematic review," Sleep, vol. 40, no. 3, 2017.

[66] R. L. Benz, M. R. Pressman, E. T. Hovick, and D. D. Peterson, "Potential novel predictors of mortality in end-stage renal disease patients with sleep disorders," American Journal of Kidney Diseases, vol. 35, no. 6, pp. 1052-1060, 2000.

[67] F. De Crescenzo, S. Licchelli, M. Ciabattini, D. Menghini, M. Armando, P. Alfieri, L. Mazzone, G. Pontrelli, S. Livadiotti, F. Foti et al., "The use of actigraphy in the monitoring of sleep and activity in adhd: A meta-analysis," Sleep medicine reviews, vol. 26, pp. 9-20, 2016. 
[68] L. Wiggs and G. Stores, "Sleep patterns and sleep disorders in children with autistic spectrum disorders: insights using parent report and actigraphy," Developmental Medicine 83 Child Neurology, vol. 46, no. 06, pp. 372-380, 2004.

[69] N. L. Miller, L. G. Shattuck, and P. Matsangas, "Longitudinal study of sleep patterns of united states military academy cadets," DTIC Document, Tech. Rep., 2010.

[70] H. Töreyin, S. Hersek, C. N. Teague, and O. T. Inan, "A proof-of-concept system to analyze joint sounds in real time for knee health assessment in uncontrolled settings," IEEE Sensors Journal, vol. 16, no. 9, pp. 2892-2893, May 2016.

[71] H. Töreyin, H. K. Jeong, S. Hersek, C. N. Teague, and O. T. Inan, "Quantifying the consistency of wearable knee acoustical emission measurements during complex motions," IEEE Journal of Biomedical and Health Informatics, vol. 20, no. 5, pp. 1265-1272, Sept 2016.

[72] E. Morrish, M. A. King, S. N. Pilsworth, J. M. Shneerson, and I. E. Smith, "Periodic limb movement in a community population detected by a new actigraphy technique," Sleep medicine, vol. 3, no. 6, pp. 489-495, 2002.

[73] M. Mirza, W.-K. Shen, A. Sofi, A. Jahangir, N. Mori, A. J. Tajik, and A. Jahangir, "Frequent periodic leg movement during sleep is associated with left ventricular hypertrophy and adverse cardiovascular outcomes," Journal of the American Society of Echocardiography, vol. 26, no. 7, pp. 783-790, 2013.

[74] B. B. Koo, T. Blackwell, S. Ancoli-Israel, K. L. Stone, M. L. Stefanick, S. Redline, O. F. in Men (MrOS) Study Group et al., "Association of incident cardiovascular disease with periodic limb movements during sleep in older menclinical perspective," Circulation, vol. 124, no. 11, pp. 1223-1231, 2011.

[75] Y. Athavale, M. Boulos, B. J. Murray, and S. Krishnan, "Classification of periodic leg movements through actigraphy signal analysis," CMBES Proceedings, vol. 39, no. 1, 2016.

[76] M. A. Khabou and M. V. Parlato, "Classification and feature analysis of actigraphy signals," in Southeastcon, 2013 Proceedings of IEEE. IEEE, 2013, pp. 1-5.

[77] "Edgeml: Edge machine learning," https://www.microsoft.com/en-us/research/project/ resource-efficient-ml-for-the-edge-and-endpoint-iot-devices/, (Accessed on 05/28/2019).

[78] "What is edge computing? advantages of edge computing - alibaba cloud knowledge base," https: //www.alibabacloud.com/knowledge/what-is-edge-computing, (Accessed on 05/28/2019). 
[79] B. Hjorth, "The physical significance of time domain descriptors in eeg analysis," Electroencephalography and clinical neurophysiology, vol. 34, no. 3, pp. 321-325, 1973.

[80] —_ "Eeg analysis based on time domain properties," Electroencephalography and clinical neurophysiology, vol. 29, no. 3, pp. 306-310, 1970.

[81] V. T. van Hees, M. Pias, S. Taherian, U. Ekelund, and S. Brage, "A method to compare new and traditional accelerometry data in physical activity monitoring," in 2010 IEEE International Symposium on" A World of Wireless, Mobile and Multimedia Networks"(WoWMoM). IEEE, 2010, pp. 1-6.

[82] S. Sprager and D. Zazula, "Impact of different walking surfaces on gait identification based on higherorder statistics of accelerometer data," in 2011 IEEE International Conference on Signal and Image Processing Applications (ICSIPA). IEEE, 2011, pp. 360-365.

[83] S. Haykin, Communication systems. John Wiley \& Sons, 2008.

[84] S. S. Haykin, M. Moher, and T. Song, An introduction to analog and digital communications. Wiley New York, 1989, vol. 1.

[85] "Vi. data acquisition," http://www.ccs.fau.edu/ bressler/EDU/STSA/Modules/II.pdf, (Accessed on $07 / 22 / 2019)$

[86] "https://nptel.ac.in/courses/webcourse-contents/iit kharagpur/digi comm/pdf-m-3/m3l14.pdf," https://nptel.ac.in/courses/Webcourse-contents/IIT $\ \% 20 K h a r a g p u r / D i g i ~ \% 20 C o m m / p d f-m-3 /$ m3114.pdf, (Accessed on 06/22/2019).

[87] "m3l12 - module 3 quantization and coding version 2 ece iit kharagpur lesson 12 logarithmic pulse code modulation(log pcm and companding version 2 ece," https://www.coursehero.com/file/7116006/ m3112/, (Accessed on 07/22/2019).

[88] J. G. Proakis and M. Salehi, Fundamentals of communication systems. Pearson Education India, 2007.

[89] "Digital signal processing using matlab - file exchange - matlab central," https://www.mathworks.com/ matlabcentral/fileexchange/2189-digital-signal-processing-using-matlab?s_tid=gn_loc_drop, (Accessed on $07 / 22 / 2019)$.

[90] "Mt-229," https://www.analog.com/media/en/training-seminars/tutorials/MT-229.pdf, (Accessed on $07 / 22 / 2019)$ 
[91] Y. Athavale and S. Krishnan, "Time-frequency distributions in biomedical signal processing," 2019.

[92] B. Bruno, F. Mastrogiovanni, A. Sgorbissa, T. Vernazza, and R. Zaccaria, "Human motion modelling and recognition: A computational approach," in 2012 IEEE International Conference on Automation Science and Engineering (CASE). IEEE, 2012, pp. 156-161.

[93] D. Tkach, H. Huang, and T. A. Kuiken, "Study of stability of time-domain features for electromyographic pattern recognition," Journal of neuroengineering and rehabilitation, vol. 7, no. 1, p. 21, 2010.

[94] d. Created and nurtured by Eric Weisstein at Wolfram Research. (2019) Root-mean-square. [Online]. Available: http://mathworld.wolfram.com/Root-Mean-Square.html

[95] S. K. Mitra and Y. Kuo, Digital signal processing: a computer-based approach. McGraw-Hill New York, 2006, vol. 2.

[96] D. V. Moretti, C. Babiloni, G. Binetti, E. Cassetta, G. Dal Forno, F. Ferreric, R. Ferri, B. Lanuzza, C. Miniussi, F. Nobili et al., "Individual analysis of eeg frequency and band power in mild alzheimer's disease," Clinical Neurophysiology, vol. 115, no. 2, pp. 299-308, 2004.

[97] S. Aydın, H. M. Saraoğlu, and S. Kara, "Log energy entropy-based eeg classification with multilayer neural networks in seizure," Annals of biomedical engineering, vol. 37, no. 12, p. 2626, 2009.

[98] A. Phinyomark, C. Limsakul, H. Hu, P. Phukpattaranont, and S. Thongpanja, The usefulness of mean and median frequencies in electromyography analysis. INTECH Open Access Publisher, 2012.

[99] G. Plasqui, A. Bonomi, and K. Westerterp, "Daily physical activity assessment with accelerometers: new insights and validation studies," obesity reviews, vol. 14, no. 6, pp. 451-462, 2013.

[100] A. S. Walters, G. Lavigne, W. Hening, D. L. Picchietti, R. P. Allen, S. Chokroverty, C. A. Kushida, D. L. Bliwise, M. W. Mahowald, C. H. Schenck et al., "The scoring of movements in sleep," Journal of Clinical Sleep Medicine, vol. 3, no. 02, pp. 155-167, 2007.

[101] Y. R. Athavale, "Pattern classification of time-series signals using fisher kernels and support vector machines."

[102] J. Shawe-Taylor and N. Cristianini, Kernel Methods for Pattern Analysis. Cambridge University Press, June 2004.

[103] Y. Athavale, S. Krishnan, and A. Guergachi, "Pattern classification of signals using fisher kernels," Mathematical Problems in Engineering, vol. 2012, 2012. 
[104] T. Farooq, A. Guergachi, and S. Krishnan, "Chaotic time series prediction using knowledge based green's kernel and least-squares support vector machines," in 2007 IEEE International Conference on Systems, Man and Cybernetics, Oct 2007, pp. 373-378.

[105] Y. Athavale, S. Krishnan, P. Hosseinizadeh, and A. Guergachi, "Identifying the potential for failure of businesses in the technology, pharmaceutical and banking sectors using kernel-based machine learning methods," in Systems, Man and Cybernetics, 2009. SMC 2009. IEEE International Conference on, Oct 2009, pp. 1073-1077.

[106] M. Sewell, "The fisher kernel: A brief review," http://www.cs.ucl.ac.uk/fileadmin/UCL-CS/research/ Research_Notes/RN_11_06.pdf, (Accessed on 10/04/2016).

[107] Y. Tian, L. He, Z. y. Li, W. l. Wu, W. Q. Zhang, and J. Liu, "Speaker verification using fisher vector," in Chinese Spoken Language Processing (ISCSLP), 2014 9th International Symposium on, Sept 2014, pp. 419-422.

[108] V. Wan and S. Renals, "Evaluation of kernel methods for speaker verification and identification," in Acoustics, Speech, and Signal Processing (ICASSP), 2002 IEEE International Conference on, vol. 1, May 2002, pp. I-669-I-672.

[109] — - "Speaker verification using sequence discriminant support vector machines," IEEE Transactions on Speech and Audio Processing, vol. 13, no. 2, pp. 203-210, March 2005.

[110] R. Kong and B. Zhang, "Autocorrelation kernel functions for support vector machines," in Third International Conference on Natural Computation (ICNC 2007), vol. 1. IEEE, 2007, pp. 512-516.

[111] A. P. Dempster, N. M. Laird, and D. B. Rubin, "Maximum likelihood from incomplete data via the em algorithm," Journal of the royal statistical society. Series B (methodological), pp. 1-38, 1977.

[112] "Periodic limb movement disorder causes, symptoms, treatments," http://www.webmd.com/ sleep-disorders/periodic-limb-movement-disorder, (Accessed on 01/24/2017).

[113] "Plmd (periodic limb movement disorder) - national sleep foundation," https://sleepfoundation. org/sleep-disorders-problems/sleep-related-movement-disorders/periodic-limb-movement-disorder, (Accessed on 01/24/2017).

[114] V. Gschliesser, B. Frauscher, E. Brandauer, R. Kohnen, H. Ulmer, W. Poewe, and B. Högl, "Plm detection by actigraphy compared to polysomnography: a validation and comparison of two actigraphs," Sleep medicine, vol. 10, no. 3, pp. 306-311, 2009. 
[115] "Periodic leg movements during sleep (plms) \& (plmd)," https://www. sleepassociation.org/patients-general-public/periodic-leg-movements-during-sleep-plms/ about-periodic-leg-movements-during-sleep/, (Accessed on 01/24/2017).

[116] "Periodic limb movements - overview and facts," http://www.sleepeducation.org/ sleep-disorders-by-category/sleep-movement-disorders/periodic-limb-movements/overview-facts, (Accessed on 01/24/2017).

[117] H. Scofield, T. Roth, and C. Drake, "Periodic limb movements during sleep: population prevalence, clinical correlates, and racial differences," Sleep, vol. 31, no. 9, pp. 1221-1227, 2008.

[118] "Periodic limb movement disorder: Background, etiology, epidemiology," http://emedicine.medscape. com/article/1188558-overview, (Accessed on 03/21/2017).

[119] M. Hagenbuchner, D. P. Cliff, S. G. Trost, N. Van Tuc, and G. E. Peoples, "Prediction of activity type in preschool children using machine learning techniques," journal of Science and Medicine in Sport, vol. 18, no. 4, pp. 426-431, 2015.

[120] T. Kendzerska, A. S. Gershon, G. Hawker, R. S. Leung, and G. Tomlinson, "Obstructive sleep apnea and risk of cardiovascular events and all-cause mortality: a decade-long historical cohort study," PLoS Med, vol. 11, no. 2, p. e1001599, 2014.

[121] T. Willemen, D. Van Deun, V. Verhaert, M. Vandekerckhove, V. Exadaktylos, J. Verbraecken, S. Van Huffel, B. Haex, and J. Vander Sloten, "An evaluation of cardiorespiratory and movement features with respect to sleep-stage classification," IEEE journal of biomedical and health informatics, vol. 18, no. 2, pp. 661-669, 2014.

[122] "9 types of eeg tests - everything about brainwave monitoring - medicwiz," https://www.medicwiz. $\mathrm{com} /$ medtech/diagnostics/9-types-of-eeg-tests-everything-about-brainwave-monitoring, (Accessed on 03/21/2017).

[123] R. Ferri, M. Zucconi, M. Manconi, O. Bruni, S. Miano, G. Plazzi, and L. Ferini-Strambi, "Computerassisted detection of nocturnal leg motor activity in patients with restless legs syndrome and periodic leg movements during sleep." Sleep, vol. 28, no. 8, pp. 998-1004, 2005.

[124] R. Ferri, "The time structure of leg movement activity during sleep: the theory behind the practice," Sleep medicine, vol. 13, no. 4, pp. 433-441, 2012. 
[125] A. Sadeh, P. J. Hauri, D. F. Kripke, and P. Lavie, "The role of actigraphy in the evaluation of sleep disorders." Sleep, vol. 18, no. 4, pp. 288-302, 1995.

[126] A. Sadeh, "The role and validity of actigraphy in sleep medicine: an update," Sleep medicine reviews, vol. 15, no. 4, pp. 259-267, 2011.

[127] Z. Zhou, W. Dai, J. Eggert, J. T. Giger, J. Keller, M. Rantz, and Z. He, "A real-time system for inhome activity monitoring of elders," in 2009 Annual International Conference of the IEEE Engineering in Medicine and Biology Society. IEEE, 2009, pp. 6115-6118.

[128] M. M. Sánchez-Ortuño, J. D. Edinger, M. K. Means, and D. Almirall, "Home is where sleep is: an ecological approach to test the validity of actigraphy for the assessment of insomnia," Journal of clinical sleep medicine: JCSM: official publication of the American Academy of Sleep Medicine, vol. 6, no. 1, p. $21,2010$.

[129] K. L. Lichstein, K. C. Stone, J. Donaldson, S. D. Nau, J. P. Soeffing, D. Murray, K. W. Lester, and R. N. Aguillard, "Actigraphy validation with insomnia," SLEEP-NEW YORK THEN WESTCHESTER-, vol. 29, no. 2, p. 232, 2006.

[130] A. Vallieres and C. M. Morin, "Actigraphy in the assessment of insomnia," SLEEP-NEW YORK THEN WESTCHESTER-, vol. 26, no. 7, pp. 902-906, 2003.

[131] B. Sivertsen, S. Omvik, O. E. Havik, S. Pallesen, B. Bjorvatn, G. H. Nielsen, S. Straume, and I. H. Nordhus, "A comparison of actigraphy and polysomnography in older adults treated for chronic primary insomnia," SLEEP-NEW YORK THEN WESTCHESTER-, vol. 29, no. 10, p. 1353, 2006.

[132] I. Verbeek, J. Arends, G. Declerck, and L. Beecher, "Wrist actigraphy in comparison with polysomnography and subjective evaluation in insomnia," Sleep-Wake Research in the Netherlands, vol. 5, pp. 163-169, 1994.

[133] J. A. Slater, T. Botsis, J. Walsh, S. King, L. M. Straker, and P. R. Eastwood, "Assessing sleep using hip and wrist actigraphy," Sleep and Biological Rhythms, vol. 13, no. 2, pp. 172-180, 2015.

[134] A. Kosmadopoulos, C. Sargent, D. Darwent, X. Zhou, and G. D. Roach, "Alternatives to polysomnography (psg): A validation of wrist actigraphy and a partial-psg system," Behavior research methods, vol. 46, no. 4, pp. 1032-1041, 2014.

[135] M. Hornyak, B. Feige, D. Riemann, and U. Voderholzer, "Periodic leg movements in sleep and periodic limb movement disorder: prevalence, clinical significance and treatment," Sleep medicine reviews, vol. 10, no. 3, pp. 169-177, 2006. 
[136] D. R. Epstein and S. R. Dirksen, "Randomized trial of a cognitive-behavioral intervention for insomnia in breast cancer survivors." in Oncology nursing forum, vol. 34, no. 5, 2007.

[137] N. S. Redeker, J. Ruggiero, and C. Hedges, "Patterns and predictors of sleep pattern disturbance after cardiac surgery," Research in nursing 83 health, vol. 27, no. 4, pp. 217-224, 2004.

[138] A. C. Volkers, J. H. Tulen, W. W. van den Broek, J. A. Bruijn, J. Passchier, and L. Pepplinkhuizen, "Motor activity and autonomic cardiac functioning in major depressive disorder," Journal of Affective Disorders, vol. 76 , no. 1, pp. 23-30, 2003.

[139] M. T. Smith, C. S. McCrae, J. Cheung, J. L. Martin, C. G. Harrod, J. L. Heald, and K. A. Carden, "Use of actigraphy for the evaluation of sleep disorders and circadian rhythm sleep-wake disorders: an american academy of sleep medicine clinical practice guideline," Journal of Clinical Sleep Medicine, vol. 14, no. 07, pp. 1231-1237, 2018.

[140] M. Kobayashi, K. Namba, E. Ito, S. Nishida, M. Nakamura, Y. Ueki, N. Furudate, T. Kagimura, A. Usui, and Y. Inoue, "The validity of the pam-rl device for evaluating periodic limb movements in sleep and an investigation on night-to-night variability of periodic limb movements during sleep in patients with restless legs syndrome or periodic limb movement disorder using this system," Sleep medicine, vol. 15, no. 1, pp. 138-143, 2014.

[141] E. Sforza, M. Johannes, and B. Claudio, "The pam-rl ambulatory device for detection of periodic leg movements: a validation study," Sleep medicine, vol. 6, no. 5, pp. 407-413, 2005.

[142] S. Kye, J. Moon, T. Lee, S. Lee, K. Lee, S.-c. Shin, and Y. S. Lee, "Detecting periodic limb movements in sleep using motion sensor embedded wearable band," in 2017 IEEE International Conference on Systems, Man, and Cybernetics (SMC). IEEE, 2017, pp. 1087-1092.

[143] A. Dias, L. Gorzelniak, J. Rudnik, D. Stojanovic, and A. Horsch, "Detecting periodic limb movements with off-the-shelf accelerometers: a feasibility study." in MedInfo, 2013, pp. 152-156.

[144] D. Kemlink, M. Pretl, K. Sonka, and S. Nevsimalova, "A comparison of polysomnographic and actigraphic evaluation of periodic limb movements in sleep," Neurological research, vol. 30, no. 3, pp. $234-238,2008$.

[145] M. A. King, M.-O. Jaffre, E. Morrish, J. M. Shneerson, and I. E. Smith, "The validation of a new actigraphy system for the measurement of periodic leg movements in sleep," Sleep medicine, vol. 6, no. 6 , pp. 507-513, 2005. 
[146] C. Iber and C. Iber, The AASM manual for the scoring of sleep and associated events: rules, terminology and technical specifications. American Academy of Sleep Medicine Westchester, IL, 2007, vol. 1.

[147] S. Fulda, N. Szesny, M. Ising, A. Heck, A. Grübl, R. Lieb, and S. Reppermund, "Further evidence for executive dysfunction in subjects with rls from a non-clinical sample," Sleep medicine, vol. 12, no. 10, pp. 1003-1007, 2011.

[148] M. Manconi, I. Zavalko, C. L. Bassetti, E. Colamartino, M. Pons, and R. Ferri, "Respiratory-related leg movements and their relationship with periodic leg movements during sleep." Sleep, vol. 37, no. 3, pp. 497-504, 2014.

[149] I. Rish, "An empirical study of the naive bayes classifier," in IJCAI 2001 workshop on empirical methods in artificial intelligence, vol. 3, no. 22. IBM New York, 2001, pp. 41-46.

[150] S. J. Russell and P. Norvig, "Artificial intelligence: a modern approach (international edition)," 2002.

[151] G. McLachlan, Discriminant analysis and statistical pattern recognition. John Wiley \& Sons, 2004, vol. 544.

[152] "minimum-redundancy maximum-relevance feature selection - file exchange - matlab central," https://www.mathworks.com/matlabcentral/fileexchange/ 14916-minimum-redundancy-maximum-relevance-feature-selection, (Accessed on 07/23/2019).

[153] "What is stroke? - national stroke association," https://www.stroke.org/understand-stroke/ what-is-stroke/, (Accessed on 06/23/2019).

[154] Y. Zhang and J. M. Jordan, "Epidemiology of osteoarthritis," Clinics in geriatric medicine, vol. 26, no. 3, pp. 355-369, 2010.

[155] E. Nic, "Osteoarthritis: national clinical guideline for the care and management of osteoarthritis in adults (draft for consultation)," 2007.

[156] "Joint replacements — cihi," https://www.cihi.ca/en/joint-replacements, (Accessed on 06/18/2018).

[157] "First nationwide prevalence study of hip and knee arthroplasty shows 7.2 million americans living with implants - for medical professionals - mayo clinic," https://www.mayoclinic.org/medical-professionals/clinical-updates/orthopedic-surgery/

study-hip-knee-arthroplasty-shows-7-2-million-americans-living-with-implants, (Accessed on 06/18/2018). 
[158] S. Krishnan, R. M. Rangayyan, G. D. Bell, and C. B. Frank, "Adaptive time-frequency analysis of knee joint vibroarthrographic signals for noninvasive screening of articular cartilage pathology," IEEE Transactions on Biomedical Engineering, vol. 47, no. 6, pp. 773-783, 2000.

[159] Y. Wu, S. Krishnan, and R. M. Rangayyan, "Computer-aided diagnosis of knee-joint disorders via vibroarthrographic signal analysis: a review," Critical Reviews ${ }^{T M}$ in Biomedical Engineering, vol. 38, no. $2,2010$.

[160] I. Boniatis, L. Costaridou, D. Cavouras, I. Kalatzis, E. Panagiotopoulos, and G. Panayiotakis, "Assessing hip osteoarthritis severity utilizing a probabilistic neural network based classification scheme," Medical Engineering and Physics, vol. 29, no. 2, pp. 227-237, 2007.

[161] J. S. Gregory, R. Junold, P. E. Undrill, and R. M. Aspen, "Analysis of trabecular bone structure using fourier transforms and neural networks," IEEE transactions on information technology in biomedicine, vol. 3, no. 4, pp. 289-294, 1999.

[162] W.-L. Wu and F.-C. Su, "Potential of the back propagation neural network in the assessment of gait patterns in ankle arthrodesis," Clinical Biomechanics, vol. 15, no. 2, pp. 143-145, 2000.

[163] J. Duryea, S. Zaim, and F. Wolfe, "Neural network based automated algorithm to identify joint locations on hand/wrist radiographs for arthritis assessment," Medical physics, vol. 29, no. 3, pp. 403-411, 2002.

[164] P. Levinger, D. T. Lai, K. Webster, R. K. Begg, and J. Feller, "Support vector machines for detecting recovery from knee replacement surgery using quantitative gait measures," in Engineering in Medicine and Biology Society, 2007. EMBS 2007. 29th Annual International Conference of the IEEE. IEEE, 2007, pp. 4875-4878.

[165] D. T. Lai, P. Levinger, R. K. Begg, W. L. Gilleard, and M. Palaniswami, "Automatic recognition of gait patterns exhibiting patellofemoral pain syndrome using a support vector machine approach," IEEE Transactions on Information Technology in Biomedicine, vol. 13, no. 5, pp. 810-817, 2009.

[166] R. M. Rangayyan and Y. Wu, "Screening of knee-joint vibroarthrographic signals using statistical parameters and radial basis functions," Medical $\&$ biological engineering $\&$ computing, vol. 46, no. 3, pp. 223-232, 2008.

[167] R. M. Rangayyan, S. Krishnan, G. D. Bell, C. B. Frank, and K. O. Ladly, "Parametric representation and screening of knee joint vibroarthrographic signals," IEEE Transactions on Biomedical Engineering, vol. 44, no. 11, pp. 1068-1074, 1997. 
[168] K. Deluzio and J. Astephen, "Biomechanical features of gait waveform data associated with knee osteoarthritis: an application of principal component analysis," Gait $\&$ posture, vol. 25, no. 1, pp. 86-93, 2007.

[169] H. K. Jeong, M. B. Pouyan, D. C. Whittingslow, V. Ganti, and O. T. Inan, "Quantifying the effects of increasing mechanical stress on knee acoustical emissions using unsupervised graph mining," IEEE Transactions on Neural Systems and Rehabilitation Engineering, vol. 26, no. 3, pp. 594-601, March 2018.

[170] C. N. Teague, S. Hersek, H. Töreyin, M. L. Millard-Stafford, M. L. Jones, G. F. Kogler, M. N. Sawka, and O. T. Inan, "Novel methods for sensing acoustical emissions from the knee for wearable joint health assessment," IEEE Transactions on Biomedical Engineering, vol. 63, no. 8, pp. 1581-1590, Aug 2016.

[171] H. Töreyin, H. K. Jeong, S. Hersek, C. N. Teague, and O. T. Inan, "Real-time activity classification in a wearable system prototype for knee health assessment via joint sounds," in 2016 38th Annual International Conference of the IEEE Engineering in Medicine and Biology Society (EMBC), Aug 2016, pp. 3113-3116. 